\title{
Index of
}

\section{Metallic and Nonmetallic}

\section{Mineral Deposits of Alaska}

Compiled From Published Reports of Federal and State Agencies

\section{Through 1959}

By EDWARD H. COBB and REUBEN KACHADOORIAN

GE OLOGICAL SURVEY B ULLE T I N 1139

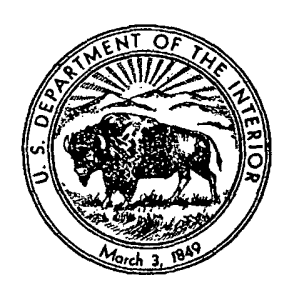




\title{
UNITED STATES DEPARTMENT OF THE INTERIOR
}

\author{
STEWART L. UDALL; Secretary
}

GEOLOGICAL SURVEY

Thomas B. Nolan, Director

The U.S. Geological Survey Library has cataloged this publication as follows:

Cobb, Edward Huntington, 1916-

Index of metallic and nonmetallic mineral deposits of Alaska, compiled from published reports of Federal and State agencies through 1959, by Edward $\mathrm{H}$. Cobb and Reuben Kachadoorian. Washington, U.S. Govt. Print. Off., 1961.

$\nabla, 363$ p. map (in pocket) diagr. $24 \mathrm{~cm}$. (U.S. Geological Survey. Bulletin 1139)

"List of reports cited": p. 345-363.

1. Mines and mineral resources-Indexes. 2. Mines' and mineral resources-Alaska. I. Kachadoorian, Reuben, 1921-, joint author. II. Title. (Series)

i: 


\section{CONTENTS}

Introduction

Reports cited . .

Methods of compilation and acknowledgments $\ldots \ldots$

Arrangement of index...

Coordinate system_:_-

Commodity listing-1.0. 4

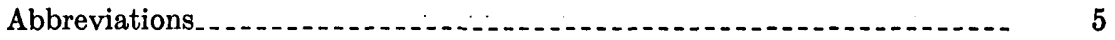

Quadrangle index........... 5

Afognak quadrangle

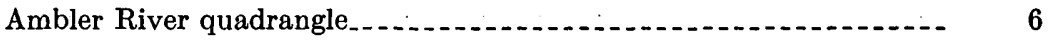

Anchorage quadrangle.......... 8

Atka quadrangle . . . .

Baird Mountains quadrangle...

Beaver quadrangle................. 21

Bendeleben quadrangle

Bering Glacier quadrangle. .

Bethel quadrangle . .

Bettles quadrangle........ 33

Big Delta quadrangle _._.

Blying Sound quadrangle_._._.

Bradfield Canal quadrangle

Candle quadrangle............ 42

Chandalar quadrangle........ 46

Chandler Lake quadrangle_._.

Charley River quadrangle.

Chignik quadrangle_._._.

Circle quadrangle........ 53

Coleen quadrangle..... 59

Cordova quadrangle

Craig quadrangle.... 64

De Long Mountains quadrangle._._.

Dillingham quadrangle

Dixon Entrance quadrangle._. 83

Eagle quadrangle:_._. 86

Fairbanks quadrangle...

Fort Yukon quadrangle.

Goodnews quadrangle... 111

Gulkana quadrangle . . .

Hagemeister Island quadrangle

Healy quadrangle........... 115

Holy Cross quadrangle

Howard Pass quadrangle...... 121

Hughes quadrangle

Iditarod quadrangle 
Quadrangle index-Continued Page

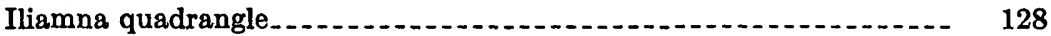

Juneau quadrangle.................. 130

Kantishna River quadrangle.......

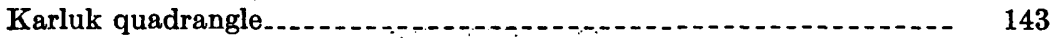

Kenai quadrangle............ 144

Ketchikan quadrangle...

Kodiak quadrangle

Kotzebue quadrangle _.

Lake Clark quadrangle..

Livengood quadrangle

McCarthy quadrangle.....

McGrath quadrangle....... 188

Medfra quadrangle

Melozitna quadrangle

Middleton Island quadrangle

Misheguk Mountain quadrangle._._.

Mount Fairweather quadrangle.....

Mount Hayes quadrangle....

Mount Katmai quadrangle... _.

Mount McKinley quadrangle.

Mount Michelson quadrangle...

Nabesna quadrangle . . .

Naknek quad́rangle._.

Nome quadrangle....... 209

Norton Bay quadrangle _._.

Nulato quadrangle............... 223

Ophir quadrangle....

Petersburg quadrangle .

Port Alexander quadrangle._._._. 232

Port Moller quadrangle _....._.

Prince Rupert quadrangle

Rat Islands quadrangle

Ruby quadrangle

Russian Mission quadrangle

St. Lawrence quadrangle _ _

Seldovia quadrangle... 243

Seward quadrangle

Shungnak quadrangle

Sitka quadrangle _.

Skagway quadrangle._.

Sleetmute quadrangle

Solomon quadrangle.

Stepovak Bay quadrangle...... 294

Sumdum quadrangle......

Survey Pass quadrangle.

Taku River quadrangle.... 297

Talkeetna quadrangle

Talkeetna Mountains quadrangle._. 304

Tanacross quadrangle

Tanana quadrangle... 306

Taylor Mountains quadrangle

Teller quadrangle... 317 
Quadrangle index-Continued Page

Trinity Island quadrangle..... 328

Tyonek quadrangle........... 328

Unalakleet quadrangle...

Unalaska quadrangle

Unimak quadrangle

Valdez quadrangle........... 329

Wiseman quadrangle...

Yakutat quadrangle. . . .

List of reports cited.

U.S. Geological Survey_.._.

Annual reports. . _ .

Professional papers.

Bulletins... .

Water-supply paper.1. 359

Circulars _.......... 359

Geologic quadrangle maps . .

Miscellaneous geologic investigations maps_._._.

U.S. Bureau of Mines....

Bulletins. . -

Information circulars._-_.

Reports of investigations _...

Territory of Alaska Department of Mines pamphlets............ 363

\section{ILLUSTRATIONS}

Plate 1. Map showing quadrangles of Alaska Reconnaissance Topographic Series $(1: 250,000) \ldots \ldots$ In pocket

Figure 1. Examples of coordinate system 



\title{
INDEX OF METALLIC AND NONMETALLIC MINERAL DEPOSITS OF ALASKA COMPILED FROM PUBLISHED REPORTS OF FEDERAL AND STATE AGENGIES THROUGH 1959
}

\author{
By Edward H. Cobb and Reuben Kachadoorian
}

\section{INTRODUCTION}

Since World War II the U.S. Geological Survey has been receiving an increasing number of requests from private and public sources concerning the mineral resources of Alaska. To help fill these re, quests in part, a card index of data on the occurrences of valuable or potentially:valuable minerals was compiled and placed in 11 depositories for consultation by the public. To increase the availability of these data, this index has been prepared for publication in the bulletin series of the Geological Survey.

This index contains page references to data on mines, prospects, and reported occurrences of valuable or potentially valuable minerals in Alaska, exclusive of mineral fuels, fissionable materials, and bulk construction materials, such as sand and gravel.

\section{REPORTS CITED}

This index comprises references to reports published before January 1, 1960, by the U.S. Geological Survey, the U.S. Bureau of Mines, and the Territory of Alaska Department of Mines (now the Division of Mines and Minerals in the Alaska Department of Natural Resources). Abbreviated references and page numbers are listed in the "Quadrangle index;" complete references used in the index are given under the "List of reports cited." Recurring statistical reports, such as the annual Minerals Yearbook of the U.S. Bureau of Mines and the biennial Report of the Commissioner of Mines to the Governor of Alaska, have not been included, as these reports, in general, contain relatively small amounts of scientific or technical information on individual properties and mineral occurrences. Also omitted are preliminary reports that have been placed only in open files of the Geological Survey, for such reports are normally superseded by formal reports within a few years. References to the five Special Publica- 
tions of the Geological Survey that deal with Alaska subjects have been omitted. ${ }^{1}$ These reports were published between 1898 and 1901, and they contain very little information not included in other reports.

\section{METHODS OF COMPILATION AND ACKNOWLEDGMENTS}

The card index was compiled by systematically searching the source publications for references to mines, prospects, and other mineral occurrences and entering page references to each occurrence on worksheets, from which the data were transferred to index cards. In 1954, 11 copies of the card index were prepared and placed in selected Geological Survey offices and the Juneau office of the Territory of Alaska Department of Mines, where they were available for consultation by interested persons. As users of the card files found errors and reported them to the compilers, the master file, from which this bulletin was prepared, was corrected. Donald M. Ford, D. Shannon Ryan, and Frank J. Markewicz assisted in the compilation. Checking within the Geological Survey was done mainly by Cobb, Donald W. Radbruch, Laing J. DeWitt, and Ruth E. Twitchen. In addition, Jean L. Crosby, mineral analyst, Alaska Division of Mines and Minerals, checked a set of cards against original references. Reports published since April 30, 1953, were indexed and the data added to the master file by Cobb. The compilers wish to acknowledge also the aid of Helmuth Wedow, Jr., in devising a system of binomial coordinates for locating geographic features.

\section{ARRANGEMENT OF INDEX}

The primary breakdown of this index is by area; the areal units are the quadrangles into which the Geological Survey has subdivided Alaska for topographic mapping at a scale of 1:250,000 (pl. 1). Names of mines and other mineral occurrences are listed alphabetically under each quadrangle with no further breakdown. Proper names applied to mines and claims and the names of property owners and operators are given for those occurrences for which such names appear in the reports examined. If an occurrence is unnamed, but is on or near a named geographic feature, the name of that feature is shown in parentheses in lieu of a proper name. In general, all references to placer operations on one stream are entered together under the name of the stream. For an occurrence which has no proper name or recorded owner or operator, and is not near a named geographic feature, no name has been applied; a list of the commodities present follows a series of dashes in parentheses. References to a single occurrence are listed only once, even though it may have

\footnotetext{
${ }^{1}$ Listed in "Publications of the Geological Survey," May 1958, p. 244.
} 
been known by several names and may have had several owners or operators. All applicable names appear in the alphabetical list with a reference to the name under which the page references are listed. Conversely, the name under which references are listed is followed by a note giving other names under which the occurrence appears in the literature. Minor obvious variations in spelling have been ignored, as, for example, Saltchuck and Salt Chuck. If a mine and an operator have the same name except for a few additional words in the operator's name, the additional words appear in parentheses after the mine name, as, for example, Nabesna (Min. Corp.).

\section{COORDINATE SYSTEM}

A simplified binomial coordinate system was devised to locate occurrences on maps of the Alaska Reconnaissance Topographic Series. These coordinates are given in parentheses after the name of each occurrence for which page references are listed. The first number is the perpendicular distance in inches from the west boundary of the map to the location of the occurrence. The second number is the distance from the southwest corner of the map along the west boundary to its intersection with a perpendicular from the occurrence. Thus, an occurrence with coordinates $(7.5,9.6)$ would be 7.5 inches from the west boundary along a line, normal to the boundary, 9.6 inches above the southwest corner of the map (fig. 1A). If an occurrence covers an area (for example, a stream on which there has been extensive placer mining), coordinates include the extreme limits of the area. Thus, a stream with coordinates (6.1-12.2, 4.4-9.5) would be in the area between lines normal to the west boundary 4.4 and 9.5 inches above the southwest corner of the map and between 6.1 and 12.2 inches east of the west boundary (fig. $1 B$ ).

Some occurrences cannot be located closely enough to assign accurate coordinates. In such instances the coordinates of the area in which the occurrence lies are given to the nearest inch, or there is merely a note that the occurrence is in some part of the quadrangle.

Coordinates given in this index are for use with maps of the Alaska Reconnaissance Topographic Series. As these maps are superseded by maps of the new Alaska Topographic Series (scale 1:250,000), locations should still be close; though refinements in mapping may. result in some significant changes in planimetry, especially in areas far from population centers and old mining camps. If the west boundary of any quadrangle is changed when a new edition is issued, the amount of change will have to be added to, or subtracted from, the first figure of the coordinates to determine the approximate location of an occurrence. 


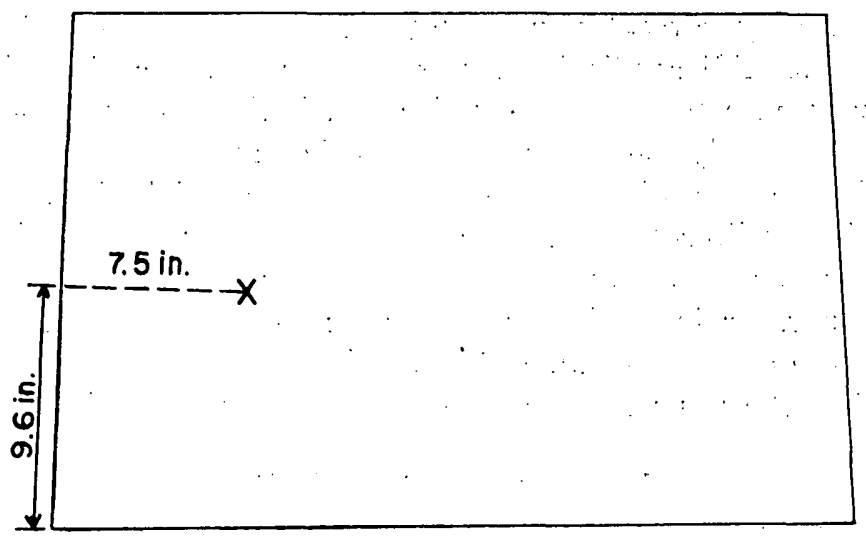

$A$

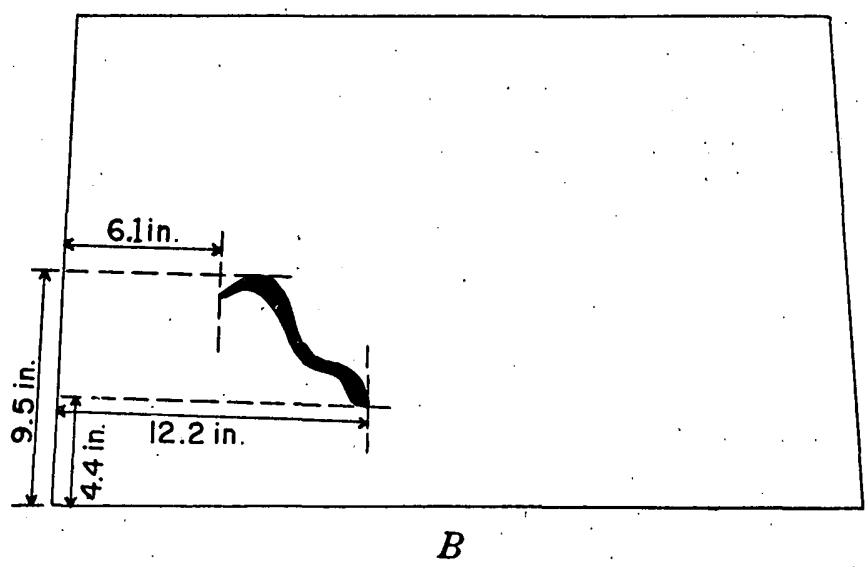

Figorn 1.-Examples of coordinate system. . A. For a point (7.5, 9.6). $B$. For an area $(6.1-12.2,4.4-9.5)$.

\section{COMMODITY LISTING}

Mineral commodities in each mine, prospect, or occurrence are listed, in capital letters, after the coordinates. The commodity name used is generally that of the valuable element present in each mineral if treatment of ore results in the production of an element; otherwise the name of the mineral or rock is given. Exceptions are chromite rather than chromium, and barium rather than barite or witherite.

In this index sulphosalts of antimony have not generally been considered as ore minerals of antimony. Sulfides such as pyrite and chalcopyrite, with a few exceptions, have not been listed for their sulfur content, because the data are generally too inconclusive to give any basis for a decision as to the possible value of the sulfide content 
of a mineral deposit except in terms of the metallic component. All platinum-group metals are listed as platinum, though very few, if any, of the Alaska deposits have yielded a product of essentially pure platinum. Likewise, no attempt has been made to list rareearth metals by name; all are lumped under the name "rare earths."

If sufficient data are given in descriptions of an occurrence to determine the economically most important mineral commodity in a deposit, that commodity is listed first and the others follow in descending order of importance. If data are lacking or are contradictory, commodities are listed alphabetically.

\section{ABBREVIATIONS}

The following abbreviations, in addition to those in common usage, have been used in this index :

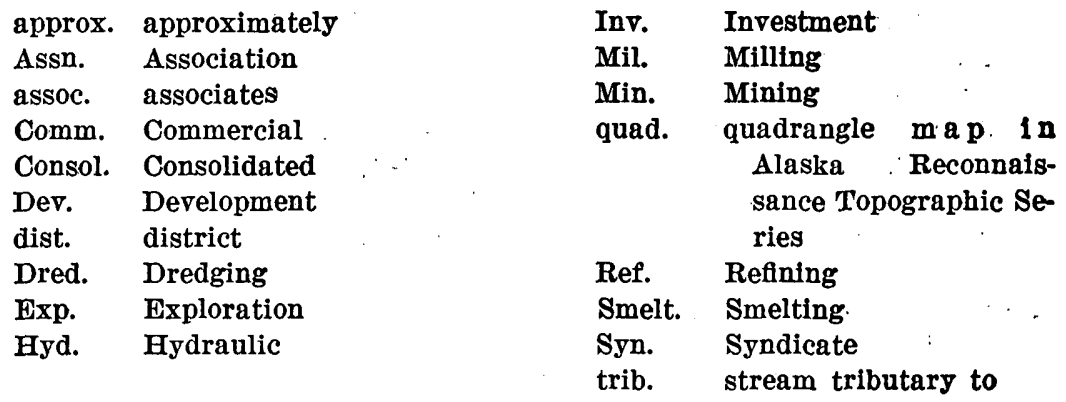

Abbreviations used for publication series are:

$\begin{array}{ll}\text { AR } & \text { U.S. Geological Survey Annual Report } \\ \text { B } & \text { U.S. Geological Survey Bulletin } \\ \text { BMB } & \text { U.S. Bureau of Mines Bulletin } \\ \text { C } & \text { U.S. Geological Survey Circular } \\ \text { GQ } & \text { U.S. Geological Survey Geologic Quadrangle Map } \\ \text { I } & \text { U.S. Geological Survey Miscellaneous Geological Investigations Map } \\ \text { IC } & \text { U.S. Bureau of Mines Information Circular } \\ \text { P } & \text { U.S. Geological Survey Professional Paper } \\ \text { RI } & \text { U.S. Bureau of Mines Report of Investigation } \\ \text { TDM } & \text { Territory of Alaska Department of Mines Pamphlet } \\ \text { W } & \text { U.S. Geological Survey Water-Supply Paper }\end{array}$

\section{QUADRANGLE INDEX}

\section{AFOGNAK QUADRANGLE}

(Malina Bay) - $(8: 75,4.15)$; GOLD, SILVER.

B 520, p. 28

B 880-C, p. 176

B 542, p. 133-134

(Raspberry Beach) - (6.15-6.2, 1.8-1.9) ; GOLD.

B 622, p. 47

B $880-C$, p. 172 


\section{AMBLER RIVER QUADRANGLE}

(Änes Cr. ) - (10.7-11.6, 3.1-6.8) ; GOLD.

B 773, p. 51

B 910-A, p. 73

B 836, p. 51 .

B 917-A, p. 71

B 844-A, p. 52

(Ambler R.) - (E 1/2 quad.) ; GOLD.

B 714, p. 95

Arctic Circle Exp. Co., Inc. See (Asbestos Mtn.), (Cosmos Cr.), (Dahl Cr.). (Asbestos Mtn.) - $(14.85,0.95)$; ASBESTOS, JADE, QUARTZ, TALC.

RI 4414, p. 2, 7; 11-13, 19-20, TDM 3-R, p. 14-18, 25 22-24

TDM 5-R, p. 14-15

(Aurora Mtn.) - $(12.9,1.3)$; COPPER, SILVER, GOLD, LEAD. Includes references to lodes head of Cosmos $\mathrm{Cr}$.
B 379, p. 31
B 714, p. 36
B 480 , p. $300-303$
B 815 , p. $339-342$
B 536, p. 149-150, 153
TDM 5-R, p. 24

(Bismark Mtn.) - $(11.95,1.35)$; ASBESTOS, JADE. See also (Shungnak R.). RI 4414, p. 10, 16-19 TDM 5-R, p. 15

TDM 3-R, p. 5-7, 9, 13, 19-20, 24-26

(Boulder Cr.) - (SE 1/16 quad.) ; GOLD. NoTE.-Trib. Kögoluktuk R.
B 864-A, p. 53
B 910-A, p. 73
B 868-A, p. 55-56
B 917-A, p. 71
B 880-A, p. 58
B 926-A, p. 67
B 897-A, p. 68

(Cosmos Cr.) - (12.85, 0.45) ; ASBESTOS, JADE. See also (Aurora Mtn.).

RI 4414, p. 11, 21-22

TDM 5-R, p. 15

TDM 3-R, p. 5, 7, 9, 13, 20-21, 26

(Dahl Cr.) - (14.45-14.5, 0.2-0.5) ; ASBESTOS, CADMIUM, CHROMITE, COP-

PER, GOLD, JADE, NICKEL, QUARTZ, SILVER. Includes references to (Dahl Cr.) in the Shungnak quad.; see also (Asbestos Mtn.).

B 379 , p. 59

B 442 , p. 46

B 480, p. 292-294, 304

B 520, p. 42

B 536, p. 126-129, 154

B 592 , p. 72

B 642, p. 71

B 692, p. 42

B 714, p. 95

B 722, p. 67

B 755, p. 49

B 773, p. 51

B 792, p. 23

B 797, p. 28

B 810, p. 37

B 813, p. 44
B 815, p. 322-324, 344-345

B 824, p. $49^{\circ}$

B 836 , p. $51^{\circ}$

B 844-A, p. 51-52

B 857-A, p. 48

B 864-A, p. 53

B 868-A, p. 55

B 880-A, p. 58

B 897-A, p. 68

B 910-A, p. 73

B 917-A, p. 71

B 926-A, p. 67

B 933-A, p. 64

IC 7379, p. 75-76

TDM 3-R, p. 5, 7, 13, 17-26

TDM 5-R, p. 18, 23, 36, 47-48

Garland. See (Cosmos Cr.), (Dahl Cr.).

(Hunt R.) - (SW 1/4 quad.) ; GOLD, ASBESTOS.

B 536, p. 146

TDM 5-R, p. 16

TDM 3-R, p. 11

Ing-Ihk. See (Asbestos Mtn.). Nort.-Other spellings are Ing-hik and Ingik. 
(Iron Mtn.) - (\$ 1/2 13, 1) ; IRON.

B 536, p. 153-154

TDM 5-R, p. 26

B 815, p. 343

(Jade Cr.) - (6.0-6.7, 3.25-4.2) ; ASBESTOS, JADE.

TDM 3-R, p. 5-6, 24-26

TDM 5-R, p. 16

(Jade Hills) - $(6.35,3.85)$ approx; ASBESTOS, JADE, NICKEL. See also (Jade Mtn.).

B 536, p. 155

B 815, p. $344-346$

B 714, p. 55

TDM 3-R, p. 5, 24

(Jade Mtn.) - $(6.35,3.85)$ approx; ASBESTOS, JADE. See also (Jade Hills). IC 7379 , p. 82

TDM 3-R, p. 5, 7, 13, 22-25.

RI 4414, p. 13-14

TDM 5-R, p. 16

Kobuk Alaska Mines. See (Shungnak R.).

Malfiatti - $(0.0,9.8)(?)$; COPPER.

B 592, p. 72

(Midas Cr.) - (16.5-19.8, 15.0-17.0) ; GOLD.

B 520 , p. $336-337$

B 536, p. 141-142

B 773, p. 51

B 815, p. 332

Northwest Alaska Mines. See (Shungnak R.).

(Pearl Cr.) - (14-15, 1-2) ( ?) ; GOLD.

B 864-A, p. 53

(Riley Cr.) - (14.7-14.8, 1.05-1.15) ; GOLD.

B 442, p. 46

B 480, p. 294-296, 299

B 880-A, p. 58

B 536, p. $129-131$

B 692, p. 42

B 897-A, p. 68

B 910-A, p. 73

B 815 , p. 324-325

B $917-A$, p. 71

B $864-\mathrm{A}$, p. 53

B 926-A, p. 67

B $868-$ A, p. $55-56$

B 933-A, p. 64

(Ruby Cr.) - $(13.95,1.5)$; COPPER, GOLD, LEAD.
B 480 , p. $300-303$
B 815, p. 339-342
B 536, p. 147-149, 153
TDM 5-R, p. 24

B 714, p. 36

(Shungnak R.) - (12.0-12.3, 0.75-1.2) ; GOLD, SILVER, COPPER, ASBESTOS. See also (Bismark Mtn.).

B 379, p. 59

B 442, p. 46

B $857-$ A, p. 48

B 480, p. 296-298

B 864-A, p. 53

B 536, p. 131-132

B 868-A, p. 55-56

B 592, p. 72

B $880-A$, p. 58

B 642, p. 71

B $897-A$, p. 68

B 692, p. 42

B 910-A, p. 73

B 792, p. 23

B $917-A$, p. 71

B 815, p. 321-322

B $926-A$, p. 67

B 824, p. 49

B 933-A, p. 64

B 844-A, p. 51-52

TDM 5-R, p. 15-16, 23

(Stockley Cr.) - $(14.6,0.75)$; ASBESTOS, NICKEĹ.

TDM 3-R, p. 18

(Wesley Cr.) - $(13,0)$; LEAD, JADE, ASBESTOS.

B 536, p. 153

TDM 3-R, p. 11, 13, 21-22, 28

B 815, p. 343

(-----) (gold) - (14.5, 0.95) ; GOLD.

B 480, p. 299

B 815, p. 336 
(-----) (metals) -

B 536, p. 150

B 815, p. 342
$(17-18,8)$ ( ?) ; LEAD, COPPER; GOLD, SILVER.

TDM 5-R, p. 23

\section{ANCHORAGE QUADRANGLE}

Agostino - $(7.55,0.85)$; GOLD, SILVER, ZINC, LEAD, COPPER, MOLYB-

DENUM. Includes references to Agra, Alaska Gold Exp. \& Dev. Co., Anna, Barnes, Edlund (gold), Monarch Min. Co., Ruth, Stella, Styles, Tony.

B 520, p. 153-155

B 587, p. 173-176

B 642, p. 188-191

B 722 , p. $40-41$
B 797, p. 12

B 810, p. 17

B 849-G, p. 407, 409, 413-417

B 926-C, p. 187-188

Agra. See Agostino.

Alaska Crow Cr. Min. Co. See (Crow Cr.).

Alaska Free Gold. See Martin.

Alaska Free Gold Co. See Alaska Homestake.

Alaska Free Gold Min. Co. See Gold Cord, Martin, Rae-Wallace.

Alaska Gold Exp. \& Dev. Co. See Agostino.

Alaska Gold Quartz (Min. Co.) See Independence.

Alaska Gypsum Products Corp. See (Gypsum Gulch), (Yellow Jacket Gulch). Alaska Gypsum Queen Corp. See (Gypsum Gulch), (Yellow Jacket Gulch). Alaska Homestake (Min. Co.) - (13-15, 0-1.) (?) ; GOLD. May be in Seward quad.

B 692, p. 150

B 712, p. 33

Alaska Hoosier Co. See (Willow Cr.).

Alaska-Pacific Consol. Min. Co. See Independence.

Alaska Placer Gold Min. Co. See (Alfred Cr.).

Alaska Quartz - $(6,14)$; GOLD. (?).

B 692, p. 185

Alaska-Willow Cr. - (SE $1 / 4,6,14)(?)$; GOLD (?).

B 773, p. 42

Alaskan Wonder. See Simonton \& Mills.

(Alfred Cr.) - (20.2-21.3, 16.9-17.2) ; GOLD, PLATINUM. Includes reference to (Caribou Cr.).

B 542, p. 44

B 755, p. 28

B 592, p. 278-279, 281

B 773, p. 30

B 668, p. 62-63

American Roofing Co. - $(1.7,1.2)$; CLAY.

RI 4932, p. 30

Anchorage Gold Mines Co. See Lane.

Anchorage Gypsum Products Co. See (Gypsum Gulch), (Yellow Jacket Gulch).

Anchorage Sand \& Gravel Co. See (Kings R.) (haydite).

Anna. See Agostino.

Arch - $(6.4,14.14)$; GOLD.

B 592, p. 263-264

B 642, p. 200

B 607 , p. $70-71$

B 692 , p. 185

Archangel - $(6.8,14.2)$ approx; GOLD.

B 642, p. 200

Austin. See Snow King.

Babcock-McCoy. See McCoy.

Bahrenberg - $(7.65,1.0)$; GOLD. Includes references to Treasure Box.

B 587 , p. $176-177$

B $849-G$, .p. $417-418$

B 642 , p. 191 
Barnes. See Agostino.

(Barry Arm) (antimony) - $(15.85,1.65)$; ANTIMONY.
B 443 , p. 78
B 649, p. 61

(Barry Arm) (gold) - $(16.05,1.55)(?)$; GOLD (?).

B 662, p. 189

Bartholf, B. \& C. See Gold Cord.

Bartholf, C. See Rae-Wallace.

Bartholf, W. See Gold Bullion.

Bartholf, W. \& E. See (Willow Cr.)

Bartholf, Horning \& Black. See Gold Cord.

Bartholf-Isaacs - $(6.2,14.5)$ ( ?) ; GOLD.

B 592, p. 264

B 607, p. 76

Basic Building Products, Inc. See (Kings R.) (haydite).

(Big L.) - (0.3-1.25, 9.3-9.5) ; MARL.

B 1039-A, p. 17-18

Big Mogul. See (Pioneer Cr.).

(Bird Cr.) - (4.9-6.4, 0.0-1.1) ; GOLD.

AR 20, p. 320-321

B 917-A, p. 41

B 642 , p. 187

Black. See Paymaster.

Black \& Hogan - $(14.7,1.6)$; GOLD, LEAD, ZINC.

B 592 , p. 228

B 622, p. 139

Blue Quartz Min. Co. - $(4-5,15)$ (?) ; GOLD.

B 714, p. 203

Bluebird - $(5,13-14)(?)$; GOLD.

B 692, p. 185

Bralaska Min. Co. See Independence, Martin.

Brassel Bros. See Gold King, Leona.

Brenner - $(7.5,0.8)$; GOLD.

B $849-G$, p. $406,408-410,419$

Brooklyn (Dev. Co.). See Kelly-Willow.

Brooklyn-Willow Cr. Gold Min. Co. See Kelly-Willow.

Brown. See (Wet Gulch).

Bruno Agostino (Min. Co.). See Agostino.

Bullion. See Gold Bullion.

Burr. See Mary Ann.

Cameron - $(16.3,0.35)$; GOLD. See also Last Chance No. 2. B 592 , p. $226-227$

Cann \& Miner - $(17.8,1.9)$; GOLD. B 592, p. 218

Capitol Hill - $(15,1-2)$; GOLD, SILVER, COPPER. NOTE.-North shore of Barry Arm.

B 712, p. 33

(Caribou Cr.). See (Alfred Cr.).

Carle. See Independence.

(Castle Mtn.) - $(12.0,15.1)$; LIMESTONE.

RI 4932, p. 121-122

(Chickaloon Cr.) or (R.) - (12.65-13.15, 13.85-14.65) ; GOLD.

May be in Talkeetna Mtns. quad.

AR 20, p. 322

B 520, p. 37

B 622, p. 47,49 
Clay Products, Inc. See (Pt. Woronzof).

Conroy. See Webfoot.:

Conroy \& Marion. See (Jim Cr.).

Consol. (Min. Co.) See Talkeetna.

Consol. Gold Mines. See Alaska-Willow Cr., Newman \& Miller, Talkeetna.

Conwest Min. Co. See Willow Cr.

(Crow Cr.) - (7.55-7.9, 0.05-0.5) ; GOLD, SILVER, COPPER.

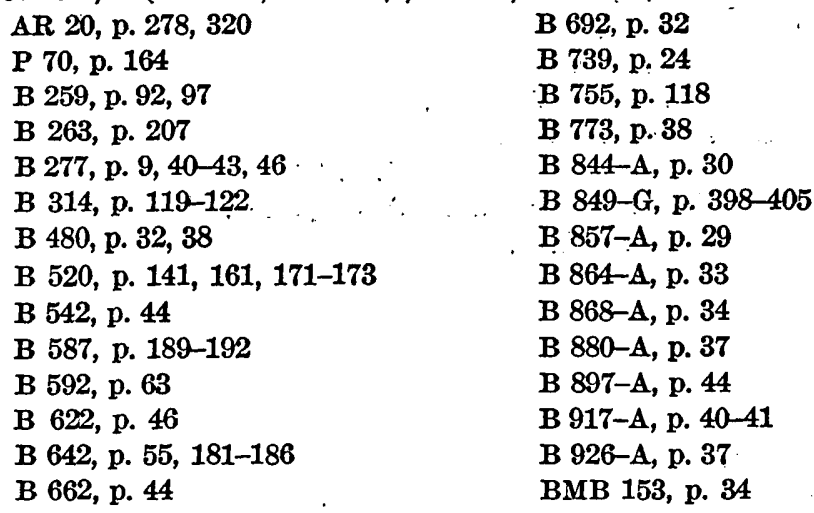

Crow Cr. Consol. Min. Co. See (Crow Cr.).

Crow Cr. Gold Corp. See Monarch.

Crow Cr. Min. Co., Inc. See Agostino.

Dixie - $(5.8,14.4)$; COPPER.

B 714, p. 202-203

Dodson. See Fern.

Doheny \& Thomson. See Gold Bullion.

Dolores. See Grimes.

Eagle (Girdwood dist.) See Agostino.

Eagle (Willow Cr. dist.). See Jap.

Eagle R. - (7.7, 2.4); GOLD, SILVER, LEAD, ZINC, COPPER. Includes references to Mayflower.

B 587, p. 178

B 642, p. 193-194

B 849-G, p. 409, 419-420

C 196, p. 10

Edlund (gold). See Agostino.

Edlund (marl) $\because(5.1,10.2)$; MARL.

B 1039-A, p. 9-10, 12-13, 15, 20

(Eklutna) - $(5.4,8.2)$; BUILDING STONE.

C 18, p. 3

(Eklutna R.) : (NE 1/4 SW 1/4 quad.) ; TIN(?)

B 662, p. 47

Elder \& Thorpe. See Thorpe (Grubstake Gulch).

Eldorado. See Martin.

(Eska Cr.) - (9.1, 12.85) ; CLAY.

RI 4932, p. 54

Eureka. See Griset \& Benson.

Evening Star. See Rae-Wallace.

Fairangel. See Talkeetna.

Fennimore. See (Gypsum Gulch), (Yellow Jacket Gulch). 
Fern - $(6.2,14.5)$; GOLD, TUNGSTEN, LEAD. Includes references to Fern \& Goodell, Fern :Gold Leasing Co., Fern Gold Min. Co., Rutland; ree also Talkeetna.

B 692, p. 186
B 712, p. 176
B 714, p. 204
B 755, p. 31
B 773, p. 15,42
B 783, p. 8
B 792, p. 11
B 797, p. 12
B 810, p. 14
B 813, p. 16
B 836, p. 18
B $844-$ A, p. 18
B $849-$ C, p. $169,187-188,195,202$,
$222-226$

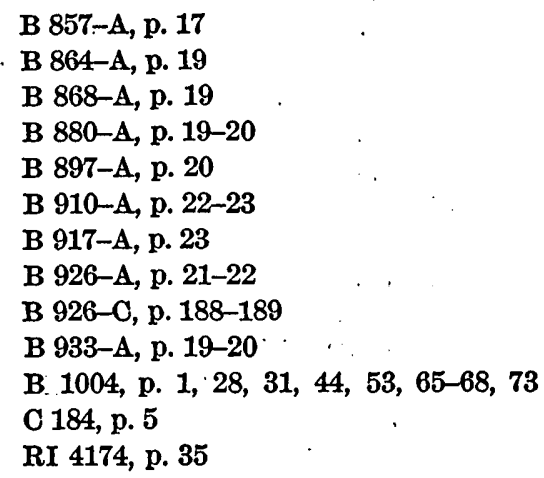

Fern \& Goodell. See Fern.

Fern Gold Leasing Co. See Fern.

Fern Gold Min. Co. See Fern, Talkeetna. Note.-Acquired Talkeetna mine in $\mathbf{1 9 2 5}$.

Fern, Taulman \& Goodell. See Arch.

(Finger L.) - (6.0-6.2, 10.5-10.75) ; MARL.

B 1039-A, p. 16, 19

(Fishhook Cr.) - $(6.35,14.3)$; GOLD.

B 592, p. 253

B 607 , p. 55

Fiske \& Reed. See Reed \& Fiske.

Free Gold (Min. Co.). See Martin.

Gaikema \& Conroy. See Fern, Webfoot.

Galena - Gold - $(5.8,14.4)$ ( ?) ; COPPER, GOLD, LEAD. See also Dixie. B 692 , p. 186

Gem. See Little Gem.

Giant Gold Min. Co. See Marmot.

Gilbert. See (Grubstake Gulch)..

Girdwood. See (Crow Cr.).

Glacier. See Lane.

Globe - $(23,0)(?)$; COPPER.

B 692, p. 146

Gold Bullion (Min. Co.) - (5.55-5.6, 13.8-13.85) ; GOLD, MERCURY, COPPER.

Includes reference to New Bullion.
B 442, p. 35
B 480 , p. 146-147
B 722, p. 41
B 520, p. 29
B 739, p. 25
B 542, p. 39
B 755 , p. 30
B 592, p. 65, 260-262
B 773, p. 15, 40
B 607 , p. 50, 66-69
B 792, p. 11
B 622, p. 48
B 813 , p. 16
B 642, p. 197
B 662, p. 48
B 824, p. 18
B $849-$ C, p. $169,186,188,192,204$,
B 692, p. 32, 178-179 213-214
B 712, p. 34, 173
B 714, p. 77, 202
B 897-A, p. 20
B 917-A, p. 23
B 926-A, p. 21

593018-61-2 
Gold Bullion (Min. Co.)-Continued

$$
\begin{aligned}
& \text { B 933-A, p. 19, } 21 \\
& \text { B 1004, p. 1, } 83
\end{aligned}
$$

BMB 142, p. 44, 52

BMB 153, p. 40-42

Gold Center. See Kelly-Willow.

Gold Cord (Min., Mil. \& Power Co.) - $(5.9,14.0)$; GOLD TUNGSTEN, LEAD, COPPER, ZINC. Includes reference to Golden Bear Min. Co.
B 692, p. 32, 180-181, 185
B 864-A, p. 19
B 712, p. 34, 174-175
B 868-A, p. 19
B 714, p. 204
B 897-A, p. 20
B 773, p. $39-40$
B 917-A, p. 23
B 813, p. 16
B 926-A, p. 21
B 824, p. 19
B 933-A, p. 19, 21
B 844-A, p. 17-18
B $849-$ C, p. 186, 188, 192, 215, 217- 220
B 1004, p. 1, 31-32, 54-58
C 184, p. 5
RI 4174, p. 35

B 857-A, p. 17

Gold Dust. See Gold Bullion.

Gold King - $(5.55,14.3)$; GOLD.

B 714, p. 202

Gold Mint. See Lonesome.

Gold Quartz (Min. Co.) (Independence). See Independence.

Gold Quartz (not Independence) - (SE 1/4 SW 1/4 6, 14) ; GOID.

B 642, p. 200

Gold Top Min. Co. - $(5,13)$; GOLD.

B 442, p. 19

B 520, p. 31

Gold Top Syn. See Kempf.

Golden Bear Min. Co. See Gold Cord.

Golden Light - $(5.3,13.8)$; GOLD.

B 714, p. 202

Golden Top. See Kempf.

Golden Wonder. See Gold Bullion.

Good Hope - (S 1/2 SE 1/4 6, 14); GOLD.

B 692 , p. 186

(Government Hill) - $(1.0,4.1)$ approx ; CLAY.

$$
\text { C } 18, \text { p. } 9
$$

Grimes - $(6,13-14)$; GOLD.

B 592, p. 265

Griset \& Benson - (16.5, 0.55) ; GOLD.

B 607 , p. 77

B 592;,p. 227

(Grubstake Gulch) or (Cr.) - (4.75-4.85, 13.1-13.3) ; GOLD.
P 70, p. 165
B 314, p. 116-118
B 327 , p. $65-66$
B 442, p. 42
B 480 , p. 139, 150-151
B 592, p. 250-253
B 607 , p. 52-54
B 622 , p. 48

B 642, p. 200

B 824, p. 31

B 836, p. 31

B $849-$ C, p. 188,228

B $910-A$, p. 44

B 917-A, p. 41

B 1004, p. 83

C 184, p. 5

Gunnysack - $(7.5,1.1)$; GOLD.

B 813, p. 18

(Gypsum Gulch) - $(20.5,14.6) \cdot$; GYPSUM.

B $989-$ C, p. $39,41,54,57-60$

RI 4932, p. 126-129 
Hanson \& Richter. See Homebuilder.

Hatcher. See Little Gem, Lonesome.

Herning. See (Grubstake Gulch), (Willow Cr.).

High Grade - $(5.85,14.05)$; GOLD. Includes references to Kloss (\& Snyder),

B 836, p. 18

B $849-$ C, p. 220

B 868-A, p. 19

B $857-\mathrm{A}, \mathrm{p} .17$

B $880-A$, p. 20

B 1004, p. 1, 76

Highway - $(6.6,8.3)$; CHROMITE.

RI 4356, p. 1-5

Hill. See (Grubstake Gulch).

Hill \& Cope. See Lonesome.

Hillis. See Fern.

Holland. See Little Willie.

Holmgren. See Monarch.

Homebuilder - $(6.6,14.4)$; GOLD.
B 755, p. 31
B 773, p. 42

Homestake. See Martin. .

Homesteader-Martha - (4-6, 13-14) ( ?) ; GOLD.

B 755, p. 31

Horning \& Bartholf. See Gold Cord.

Horning, Bartholf, Miller \& Rock. : See War Baby.

Hottentot. See Bahrenberg.

Idamar - $(6.65,14.45)$; GOLD:

B 714, p. 205

Independence - $(5.85,13.9)$; GOLD, TUNGSTEN, LEAD, ZINC. Includes references to Alaska Gold Quartz (Min. Co.), Alaska-Pacific Consol. Min. Co., Gold Quartz, Independent.
B 442 , p. 35
B 480 , p. 147-148
B 520, p. 29
B 542, p. 39
B 592, p. 65, 257-260
B 607 , p. 50, 62, 64-66
B 622, p. 48
B 642, p. 197-198
B 662, p. 48
B 692, p. 180
B 722, p. 41
B 755, p. 30
B 773, p. 40
B 824, p. 19

B 849-C, p: $169,188,215-216$

B 868-A, p. 19

B 880-A, p. 20

B 897-A, p. 20

B 917-A, p. 23

B 926-A, p. 21

B 933-A, p. 19-20.

B 933-C, p. 204-216

B 1004, p. 1, 32, 51-53, 58-65

C 184, p. 5

BMB 142, p. 44, 52

BMB 153, p. 40

RI 4174, p. 34

Independence Gold Miries Co. See Independence.

Independence (Gold) Min. Co. See Independence.

Independent. See Independence.

(Indian Cr.) - (4.3-4.6, 0.0-0.95) ; GOLD.

B 917-A, p. 41

Jap - $(5.7,13.8)$ approx ; GOLD.

B 642, p. 199

B 692, p. 184-185

B 773, p. 41

B 1004, p. 81

Jenning. See Rae.

Jessie B. - $(5.9,15.1)$ ( ?) ; GOLD.

B 692 , p. 186 
Jewel. See Monarch.

(Jim Cr.) - $(9.95,10.05)$; COPPER, ZINC, SILVER.

B 792, p. 58, 69-70

Jọhnson. See Lonesome.

Kelly (Mines Co.). See Independence, Kelly-Willow, Martin.

Kelly Gold Mint - (NE 1/4 5, 13) ( ?) ; GOLD.

B 739, p. 25

Kelly-Willow (Cr. Min. Co.) - (5.7-5.8, 13:75); GOLD: Includes references to Brooklyn Dev. Co., Brooklyn-Willow Cr. Gold Min. Co., Gold Center.

B 442, p. $35-36$

B 480 , p. 147

B 520, p. 29

B 592, p. 264-265

B 607, p. 71

B 692, p. 182
B 714, p. 204

B 722, p. 41

B 755, p. 30

B 1004, p..1, 78, 80-82

BMB 153, p. $40-42$

Kempf - $(5.1,13.95)$; GOLD. Includes references to Golden Top.
B 857-A, p. 16-17
B 868-A, p. 18

B 864-A, p. 18

(King R.) (copper) - (11, 13-16) (?) ; COPPER (?).

B 662, p. 47

(Kings R.) (haydite) - (10.45-10.5, 12.9-12.95) ; HAYDITE, CLAY.

B 1039-C, p. 35, 37, 40-42, 47-56 RI 4932, p. 33-34, 54-60, 62, 80

Klondike Boston (Min.) Co. See (Grubstake Gulch), (Willow Cr.).

Kloss (\& Snyder). See High Grade.

Lane - $(6.35,14.85)$; GOLD. Includes reference to Anchorage Gold Mines Co. B 712 , p. 176

B 1004, p. 82

Larsen. See Idamar.

Last Chance No. 2 - $(16.3,0.75)$; GOLD.

B 592, p. 227

Leona - $(5.55,14.3)$; GOLD.

B 714, p. 202

Le Roi Min. Co. - $(6.9,14.05)$; GOLD.

B 712, p. 176

B 714, p. 205

Lime Products, Inc. See Edlund (marl).

Lindblad. See (Crow Cr.).

Little Gem - $(6.3,14.6)$; GOLD. Includes references to Gem, Hatcher on Archangel Cr.

B 592, p. 266

B 607, p. 76

B 692, p. $185-186$

B 642 , p. 200

B 712, p. 176

Little Gem Gold Min. Co. See Little Gem, Webfoot.

Little Willie - $(5.9,14.4)$; GOLD, MOLYBDENUM, COPPER. Includes references to Holland.

B 714, p. 203

B $864-B$, p. $109-110$

B 1004, p. 82-83

(Little Willow Cr.) - (3.75-4.75, 13.3-13.4) ; GOLD. These references are probably to the part of Willow Cr. near the mouth of Grubstake Gulch; See also (Willow Cr.)

B 910-A, p. 44

B $917-$ A, p. 41 
Lonesome - (7.25, 13.55) ; GOLD, SILVER. Includes references to Gold Mint. Marion Twin (Gold Min. Co.) (Little Susitna R.), Mint.

B 714, p. 205-206

B 755, p. 31

B 773, p. 41

B 797, p. 12

B 810 , p. 14

B 824 , p. 19

B 836, p. $17-18$

Long \& Holland. See Little Willie.

(Long Bay) - $(23,0)$; COPPER.

B $963-B$, p. 77

Loveland-Alaska Min. Co. See Mabel.

(Lucille L.) - (4.2-4.65, 10.0-10.15) ; MARL.

B 1039-A, p. 17, 19

Lucky Shot - $(4.9,13.65)$; GOLD. Includes references to Willow Cr. Mines (, Inc.) ; see also War Baby.

B 712 , p. 174

B 714, p. 202

B 739, p. 25

B 755, p. 30

B 773 , p. $15,40-41$

B 792, p. 11

B 797, p. 12

B 810, p. 14

B 813 , p. 16

B 824, p. 18

B 836 , p. $16-17$

B 844-A, p. 17
B 844-A, p. 18

B $849-$ C, p. $169,188,227-228$

B 857-A, 17

B 864-A, p. 19

B 868-A, p. 19

B $897-A$, p. 21

B 1004, p. 1, 70-73
B $849-C$, p. $169,181-183,185-186$, $188,190,192,194-198,200,202$, 204213, 219, 225

B 857-A, p. 16

B 864-A, p. 18

B 868-A. p. 18

B 880-A, p. 19

B 897-A, p. $19-20$

B $910-A$, p. 22

B $917-$ A, p. 23

B $933-A$, p. $19-20$

B 1004, p. 1, 31, 42, 83

Lydell - $(5.75,13.5)$; GOLD (?).

B 480 , p. 149

Mabel - $(6.5,13.9)$; GOLD, LEAD, ZINC, COPPER, MOLYBDENUM(?). Includes references to Mabel Min. (, Mil. \& Power) Co.

B 592, p. 263

B 607 , p. $69-70$

B 642 , p. 198

B 662 , p. 48

B 692 , p. $32,181-182$

B 712 , p. $34,175-176$

B 714, p. 77,204

B 739, p. 25

B 755 , p. 31

B 773, p. 15,41

B 783, p. 8

B 792, p. 11

Mabel Min. (, Mil. \& Power) Co. See Mabel.

McCallie. See Moose Cr.

McCoy - $(6,14)$; GOLD. Includes reference to Babcock-McCoy.

B 592, p. 266

B 607 , p. 76

McDougall. See Fern.
B 797, p. 12

B 813, p. 16

B 824, p. 19

B 836, p. 18

B 844-A, p. 18

B $849-$ C, p. $169,188,194,220-222$

B 897-A, p. 21

B 917-A, p. 23

B 926-A, p. 21

B 933-A, p. 19

B 1004, p. $1,14,21,31-32,68-70$

BMB 153, p. 40 
Mammoth - $(5,65,13.55)$; GOLD.

B 592, p. 266-267

B 642, p. 199

B 607, p. 71-72

Marion Twin (Gold Min. Co.) (Craigie Cr.) - (5.8, 14.25) ; GOLD, LEAD.

B 813 , p. 16

B 824, p. $18-19$

B 864-B, p. 109

B 836, p. 17

B 868-A, p. 18-19

B $857-$ A, p. 17

B $880-$ A, p. 20

B $864-A$, p. 18

B 897-A, p. 21

B 1004, p. 76

Marion Twin (Gold Min. Co.) (Little Susitna R.). See Lonesome.

Marmot - $(6.2,14.6)$; GOLD.

B 714, p. 204

Martin - $(5.85,13.85)$; GOLD. Includes all references to Alaska Free Gold, Free Gold, Thomas, and pertinent references to Alaska Free Gold Min. Co.

B 480 , p. 148

B 722, p. 41

B 520, p. 29

B 755, p. 30

B 542, p. 39

B 773, p. 40

B 592, p. $65,255-257$

B 797, p. 12

B 607, p. 50, 60-64, 66

B 824 , p. 19

B 622, p. 48

B 849-C, p. 169, 188, 215-216

B 642 , p. $196-197$

B 897-A, p. 20

B 662, p. 48

B 692 , p. 32, 179-180, 199

B 1004, p. 1, 31, 83

B 712, p. 34,174

BMB 142, p. 43-44, 52

B 714, p. 77, 204

BMB 153, p. 37

Martin, W. P. See Gold Cord, Martin.

Mary. See Jap.

Mary Ann - $(3.9,14.4)$; GOLD.

B 714, p. 205

Matanuska (Gold Min. Co.) See Talkeetna.

Maverick - $(7.45,13.95)$; GOLD.

B 714, p. 206

Mayflower. See Eagle R.

(Metal Cr.) - (12.5-13.5, 7.8-10.3) ; GOLD, PLATINUM.

P 70, p. 165

B 314, p. 118

B 792, p. 33, 71

B 327, p. 67

B 797, p. 40

B 442, p. 42

B 810 , p. 53

B 755, p. 32

B 813, p. 60

B 783, p. 25

B $897-A$, p. 84

Miller - $(6.45,12.95)$; GOLD

B 480, p. 149

B 607, p. 74

B 592, p. 268-269

Miller-Newman. See Newman \& Miller.

(Miners Bay) - $(21.3,1.6)$; COPPER.

B 963-B, p. 77

(Miner's R.) - $(21.3,1.6)$ approx ; NICKEL(?).

B 284, p. 86

B 443, p. 77

Mint. See Lonesome.

Mitchell \& Myers - $(15.5,0.62)$; GOLD, LEAD.

B 592, p. 227-228 
Mogul - $(6.85,14.7)$; GOLD.

B. 592, p. 269 B 607, p. 74

Mohawk (Min. Co.) - $(6.55,14.1)$; GOLD.

B 692, p. 183

Monarch (-Jewel) - $(7.55,0.75)$; GOLD, SILVER. Includes references to Jewel.
B 739, p. 24
B 868-A, p. 24
B 755, p. 30
B $880-A$, p. 27
B 813, p. 18
B $897-A$, p. 33
B 844-A, p. 21
B 910-A, p. 29-30
B $849-$ G, p. $409,418-419$
B $917-A$, p. 28
B 864-A, p. 22
B 926-A, p. 25-26

Monarch Min. Co. See Agostino.

Moon. See Rae-Wallace.

(Moose Cr.) See Moose Cr.

Moose Cr. - (7.8-7.9, 13.9-13.95); COPPER, GOLD, SILVER, ZINC. See also Northwestern.

B 662, p. 47

Morning Star. See Rae-Wallace.

Morris \& Herndon. See (Willow Cr.).

Myers - $(5.2,7.45)$; LEAD, ZINC, COPPER.

B 792, p. 71

New Bullion. See Gold Bullion.

Newman \& Miller - $(5.8,14.1)$; GOLD. Includes reference to Miller-Newman.

B 714, p. 202
North Homestake. See Martin.

B 714, p. 206

Northwestern - (7.8-7.9, 13.9-13.95) ; GOLD, SILVER, COPPER, ZINC, NICKEL.

See also Moose $\mathrm{Cr}$.

B 692, p. 183-184

Nugget - $(5.7,13.8)(?)$; GOLD.

BMB 153, p. 40-42

Nutter-Dawson (Min.) Co. See (Crow Cr.).

Opal - $(6.55,14.35)$; GOLD.

B 714, p. 205 B 773, p. 42

B 755, p. 31

Oregon. See Stiles.

Osceola - (17-18, 1-2) ( ?) ; GOLD. NotE.-On College Fiord. B 662, p. 189

B 692, p. 150

(Otter L.) - (2.2-2.3, 5.15-5.25) ; MARL.

B 1039-A, p. 2, 18

Panhandle - $(5.05,13.85)$; GOLD.

B 712, p. 174

B 714, p. 202

Patchell. See Bahrenberg.

Paymaster - $(16.05,1.55)$; GOLD.

B 592, p. 225

Pearl. See Lane.

(Peters Cr.) - (7.7-7.9, 4.0-4.1) ; GOLD, LEAD, COPPER, OHROMITE. B 642, p. 192-193

B 712, p. 23, 34

Ploneer. See (Pioneer Cr.).

(Pioneer Cr.) - $(6.85,8.2)$; CHROMITE.

RI 4356, p. 2-5 
(Pt. Woronzof) - (0.0-0.4, 3.5-3.55) ; CLAY.
O18, p. 10
RI 4932, p. 22-30, 34-35, 38, 47, 53-54

(Poorman Cr.) - $(22.5,17.85)$; GOLD. B 622, p. 128-129

(Potter) - $(1.7,1.2)$ approx ; LIMESTONE.

C 18, p. 5, 8

Q \& Q - (17-18, 1-2) (?) ; GOLD. Nore.-On College Fiord.

B 712, p. 33

Rae - $(6.15,13.65)$; GOLD, LEAD, COPPER. B 592, p. 269

B 607, p. 77

Rae-Wallace (Min. Co.) - $(6.3,13.85)$; GOLD. Includes references to RayWallace (Min. Co.), Rosenthal.
B 592, p. 270
B 692, p. 183
B 607, p. 74-75
B 755 , p. 31
B 622, p. 48
B 824, p. 19

B 642, p. 198-199

(Rainbow Cr.) - (3.1-3.55, 0.0-0.35) ; GOLD.
B 314, p. 120
B 917-A, p. 41
B 910-A, p. 43

Rapp \& Till. See Gold Bullion.

(Raven Cr.) - (7, 1-2) ; GOLD.

B $849-G$, p. 406

Ray-Wallace (Min. Co.). See Rae-Wallace.

Red Hills. See Fern.

Reed \& Fiske - $(6.15,14.3)$ approx ; GOLD.

B 480, p. 148

(Reid Cr.) - (E 1/2 6, 14) ; MOLYBDENUM.

B 692, p. 23

B 926-C, p. 189.

Reiter \& OIson - $(15.55,1.3)$; GOLD, ANTIMONY, LEAD, COPPER. B 592, p. 228

Renshaw. See Gold Cord.

Rhodes. See (Potter).

Richter. See Homebuilder.

Rosenthal. See Rae-Wallace.

Ruth. See Agostino.

Rutland. See Fern.

Ryan and assoc. See Highway.

San Juan - (NW 1/4 6, 13) ; GOLD.

B 592, p. 270

B 607, p. 77-78

(Schoonoven Cr.) - (13.15-16.5, 14.6-16.9) ; GOLD. New name is Boulder Cr. AR 20, p. 322

Schroff-O'Neil - $(5.85,14.25)$; GOLD, LEAD, COPPER, ZINC.

B 1004, p. 44, 73, 75-76

(Sheep Mtn.) (copper) - $(21.0,15.0)$; COPPER.
B 542, p. 39
B 622, p. 47
B 592, p. 281-282
B 791, p. 73

(Sheep Mtn.) (nonmetals). See (Gypsum Gulch), (Yellow Jacket Gul(h).

Sherry - $(6.85,14.65)$; GOLD.

B 773, p. 43

Shough. See Stiles.

Simonton \& Mills - $(16.15,1.0)$; GOLD, LEAD, COPPER.

B 592, p. 226 
Skarstad (\& Laubner). See Opal.

Skinner, Johnson \& Ohlson. See (Pioneer Cr.).

Smith, G. B. See Gold Cord.

Smith, H. - $(5.55,14.5)$; GOLD.

B 864-B, $\ddot{\text { p. }} 110$

Smith \& Sutherland - $(6.3,13.9)$; GOLD.

B 692, p. 185

Smith \& Swan. See Gold Cord.

Snow King - $(6.6,14.45)$; GOLD.

B 714, p. 205

Snowbird - $(6.6,14.65)$; GOLD.

B 1004, p. 1, 32, 73-75

(South Cr.) - (22.0-22.3, 17.55-17.7) ; GOLD.

B 592, p. 278

South Homestake. See Martin.

Spruce. See Griset \& Benson.

Stella. See Agostino.

Stiles - $(6.4,13.7)$; GOLD, COPPER, LEAD. Includes references to Shough. B 542, p. 39

B 592 , p. 270

B 642, p. 199

B 607 , p. 75-76

B $849-$ C, p. $183,200,226-227$

Styles. See Agostino.

Sun. See Rae-Wallace.

(Sutton) - (8.65-8.8, 12.05-12.2) ; HAYDITE, CLAY.

B 1039-C, p. 35, 37-38, 43-45, 47, : RI 4932, p. 33-34, 47, 60-61, 80 $50-56$

Talkeetna (Gold Min. Co.) - (6.05, 14.45) ; GOLD, MOLYBDENUM. Includes references to Consol. Min. Co., Matanuska Gold Min. Co.

B 480 , p. 148

B 714, p. 77, 204

B 520, p. 29

B 755, p. 31

B 592 , p. $267-268$

B 773, p. 42

B 607 , p. 50, 72-73

B 792, p. 11

B 642, p. 200

B $849-$ C, p. $169,183,188,222-223$

B 662 , p. 48

B 692, p. 32,182

B 926-C, p. 188-189

B 712, p. 34, 176

B 1004, p. 65, 83

Tar Flat. See Black \& Hogan.

Teddy. See Lane.

Thomas. See Martin.

Thorpe (Craigie Cr.) - $(5.35,14.1)$; GOLD.

B 824, p. 19

Thorpe (Grubstake Gulch) - $(4.85,13.0)$; GOLD. Includes references to Elder \& Thorpe.

B 783, p. 8

B 792 , p. 11

B $849-\mathrm{C}$, p. 228

Tony. See Agostino.

Treasure Box. See Barhrenberg.

Trickster. See Rae-Wallace.

Van Dolan. See American Rooflng Co.

Wadman. See (Crow Cr.).

Waldron \& Harrison. See (Kings R.) (haydite).
B 933-A, p. 21

B 1004, p. $1,11,78$ 
Walters, Brasslin \& Atkinson - $(16.35,1.1)$; GOLD, LEAD, ZINC. B 592, p. 225-226

War Baby - $(5.0,13.7)$; GOLD. Includes references to Willow Cr. Mines (, Inc.) ; see also Lucky Shot.

B 712, p. 34-35, 173-174

B 714, p. 77, 202

B 755, p. 30

B 773, p. 15, 40-41

B 783, p. 8

B 792, p. 11

B 797, p. 12

B 810, p. 14

B 813, p. 16

B 824, p. 18
B $849-$ C, p. 169, 177-179, 181-183, 186, $188 ; 190,192,204-205,212-213 ; 219$

B 864-A, p. 18

B 868-A, p: 18

B 880-A, p. 19

B 897-A, p. 19-20

B 910-A, p. 22 .

B 917-A, p. 23

B 933-A, p. 19-20

B 1004, p. 1, 83

(Wasilla L.) - (5.0-5.05, 10.15-10.25) ; MARL.

B 1039-A, p. 15

Webfoot - $(6.25,14.15)$; GOLD. Includes references to Conroy.

B 480 , p. 148-149

B 714, p. 204

B 692, p. 185

B 712 , p. 176

B 755, p. 31

B 1004, p. 1, 78

(Wells Bay) - (21-22, 0) ; COPPER (?).

B 963-B, p. 77

(Wet Gulch) - (4.2-4.5, 12.65-13.35) ; GOLD.

B 1004, p. 83

Willow. Cr. - (4.9-5.0, 13.65-13.7) ; GOLD. See also Lucky Shot, War Baby. B 926-A, p. 21

(Willow Cr.) - (3.75-4.75, 13.3-13.4) ; GOLD. See also (Little Willow Cr.). P 70, p. 165

B 284, p. 7

B 592, p. 250-253

B 314, p. 116-117

B 607 , p. 52-53

B 327, p. 65-66

B 622, p. 27-28

B 480, p. $139,150-151$

B 642 , p. 200

B 520, p. 37

B 662, p. $47-48$

B 542, p. 44

B $849-$ C, p. 188, 228

Willow Cr. Mines Co. See Gold Bullion, Kelly-Willow, Nugget.

Willow Cr. Mines (, Inc.). See Gold Bullion, Lucky Shot, Panhandle, War

Baby. Note.-B 810, p. 46, reports copper from this company, but does not specify from which mine.

Willow Cr. Mines, Ltd. See Gold Bullion, Lucky Shot, War Baby.

Willow Cr. Min. Co. See Gold Bullion, Lucky Shot, War Baby.

Wilson. See Maverick.

Yago. See Jap.

(Yellow Jacket Gulch) - (20.55-20.7, 14.65-14.75) ; GYPSUM, CLAY. Includes references to gypsum deposit between Gypsum and Yellow Jacket gulches. B 989-C, p. 39, 41, 49-60 $\quad$ RI 4932, p. 34-39, 47, 126-129

(-.--) (lead, silver, gold) - (SE 1/4 7, 2) ; LEAD, SILVER, GOLD. B 897-A, p. 33

\section{ATKA QUADRANGLE}

(Salt I.) - (14.5-14.8, 11.8-12.0) ; COPPER.

B 857-D, p. 149 


\section{BAIRD MOUNTAINS QUADRANGLE}

(Bear Cr.) - (11.4-11.5, 2.8-2.9) ; GOLD.

B 868-A, p. 55

(Center Cr.). See (Central Cr.).

(Central Cr.) - (11.05-11.1, 2.15-2.25) ; GOLD. Includes. references to (Center Cr.).
B 864-A, p. 53
B $880-$ A, p. 58
B 868-A, p. 55
B $897-$ A, p. 67

(Kiana). See (Klery Cr.). NoTE.-General name applied to placer district.

(Klery Cr.) - (10.5-10.9, 2.55-4.75) ; GOLD, COPPER(?), LEAD (?), TIN(?). Nork.-Includes references to (Kiana), (Squirrel R.); the district includes the following creeks: Bear; Boldrin, Canyon, Central, Cross, Klery, Mink, Peluk, Spruce, Timber.
B 480, p. $312-318$
B 520 , p. 42
B 536, p. 134-138
B 592, p. 72
B 642, p. 71
B 692, p. 42
B 714, p. 95
B 722, p. 67
B 739, p. 44
B 755, p. 49
B 773, p. 51
B 783, p. 17
B 792, p. 23
B 797, p. 27-28
B 810, p. 37

B 813, p. 44

B 815, p. $327-330$

B 824, p. 49

B. 836, p. 50

B 844-A, p. 51

B 857-A, p. 48

B 864-A, p. 53

B 868-A, p. 55

B $880-$ A, p. 58

B $897-A$, p. 67

B 910-A, p. 71-72

B 917-A, p. 70-71

B 926-A, p. 67 -

B 933-A, p. 63-64

TDM 5-R, p. 23, 32, 41

Klery Placers, Inc. See (Klery Cr.).

(Squirrel R.). See (Klery Cr.) - Note-General name applied to placer district.

\section{BEAVER QUADRANGLE}

(Trout Cr.) - (NE 1/4 NW 1/4 quad.) ; ZINC, MOLYBDENUM.

B 926-C, p. 197

\section{BENDELEBEN QUADRANGLE}

(Admiral Cr.). See (Camp Cr.) (trib. Tubutulik R.). Note.-Not creek shown as Admiral Cr. on Bendeleben quad., but next downstream tributary from east. Alaska Dred. Assn. See (Candle Cr.). May be in Candle quad.

Alaska Kougarok (Dred.) Co. See (Kougarok R.), (Taylor Cr.).

Alaska Taylor Min. Co. See (Kougarok R.).

(Albion Gulch) or (Cr.) - (9.5-9.6, 0.5-0.55) ; GOLD.
B 328, p. 254
B 792, p. 24
B 345, p. 217
B 810, p. 40
B 442 , p. 362
B $844-A$, p. 49
B 449, p. 121
B 868-A, p. 51-52
B 622, p. 371
B 880-A, p. 53
B 712, p. 189
B 897-A, p. 65
B 783, p. 18
(American Cr.) - (13.0-13.35, 14.45-15.1) ; GOLD, TIN.
B 247, p. 57
TDM 5-R, p. 41 
Anderson. See (Macklin Cr:).

(Anderson Gulch) - $(2.0,8.0)$ approx ; GOLD.

B 314, p. 178

B 328 ; p. 321

(Angeles Cr.) - (3.65-3.75, 7.9-8.15) ; GOLD.

P 2, p. 65

Arctic Circle Exp. Co. See (Candle Cr.)." NoTE-Bulk of holdings in Candle quad.

(Arizona Cr.) - (0.8-1.55, 10.5-10.85) ; GOLD.
B 314, p. 179
B 379, p. 296
B 328, p. 324
B 712, p. 189

(Balm of Gilead Gulch) - $(9.7,0.4)$; GOLD: Includes reference to (Balmof Gulch).

\section{B 328, p. 254}

B 449, p. 121

(Balmof Gulch). See (Balm of Gilead Gulch).

Be(h) ring Dred. Corp. See (Kougarok R.), (Taylor Cr.).

(Bella Cr.) - (0.9-1.1, 14.3-14.8) ; GOLD.

P 2, p. 55

(Big Bar Cr.) - (19.5-20.4, 8.4-9.7) ; GOLD (?).

B 449, p. 114

(Birch Cr.) - $(7-8,4)$; MICA. NoTE.-Near head of Niukluk $R$.

B 449, p. 69

TDM 5-R, p. 46

(Black Cr.) or (Gulch) - (5.95-6.0, 9.2-9.5) ; GOLD.
B 314, p. 175
B 917-A, p. 65
B 328, p. 314
B 926-A, p. 61
B 910-A, p. 66
B 933-A, p. 58

Blue Goose (Min. Co.). See (Ophir Cr.). May be in Solomon quad.

Bodis. See (Dick Cr.).

(Boulder Cr.) - (4.9-5.25, 9.1-10.2) ; GOLD.
P 2, p. 65-67
B 314, p. 175
B 379, p. 297
B 328, p. 314
B 442 , p. $365-366$
B 345, p. 228
B 712, p. 189
B 933-A, p. 58

(Bryan Cr.) - (0.15-0.25, 14.8-15.1) ; GOLD (?).

P 2, p. 55

(Buzzard Gulch) or (Cr.) - (6.0-6.3, 9.45-9.7) ; GOLD:
B 328, p. 314
B 917-A, p. 65
B $897-A$; p. 63
B 926-A, p. 61
B 910-A, p. 66
B 933-A, p. 58

(California Cr.) - (0.95-1.45, 11.0-11.2) ; GOLD.

B 314, p. 179

B 328, p. 324

(Camp Cr.) (trib. Coffee Cr.) - (1.5-3.0, 5.1-5.7) approx ; GOLD. B 824, p. 47

(Camp Cr.) (trib. Tubutulik R.) - $(21.35,0.95)$; GOLD. NoTE.-Now called Admiral Cr.

B 442, p. 341

B 449, p. 115-116

(Candle Cr.) - (20.9-21.6, 13.75-15.1) ; GOLD. Note.-Mining has been dóne in both Bendeleben and Candle quads.

P 10, p. 51

B 225, p. 77-78

B 247, p. 60-63

B 284, p. 140

B 314, p. 35
B 379, p. 355, 364, 368

B 442, p. 369-370

B 622, p. 371

B 662 , p. $451-452,454-455$

B 692, p. $380,382-383,385,387$ 
(Candle Cr.) - Continued

B 712 , p. $187 \div 188$

B 714, p. 232-233

B. 844-A, p. 46-47

B 722, p. 62

B 773, p. 27

B 783, p. 17

B 792, p. 22,24

B 797, p. 30

B 810, p. 34,40

B 813, p. 40,47

B 824, p. 45, 53

B. 857-A, p. 43,51

B 864-A, p. 49, 57

B 868-A, p. 50, 59

B. $880 \div$ A, p. $53-54,61$

B 897-A, p. 61-62

B 910-A, p. 66-67, 76-77 .

B 917-A, p. 66

B 926-A, p. 62

B 933-A; p. 58-59

B 836, p. 46

BMB 142, p. 28

Candle Cr. Dred. Co. See (Candle Cr.). May be in Candle quad.

Candle Cr. Min. Co. See (Candle Cr.). May be in Candle quad.

Candle Ditch Co. See (Candle Cr.). May be in Candle quad.

Carroll. See (Kougarok R.).

(Chicago Cr.) - (18.55-19.7, 16.1-17.25) ; GOLD.

P 10, p. 51

B 314, p. 35

B 247, p. 67

B. 442, p. 369

Coal Cr. Dred. Co. See (Kougarok R.)

(Coarse Gold Cr.) - (0.6-1.75, 9.4-10.5) ; GOLD.

B 314, p. 179

B 379, p. 296

B 328, p. 323

(Coffee Cr.) - (1.55-3.0, 5.15-5.5) ; GOLD, MERCURY.
P 2, p. 61-62
B 810, p. 35
B 314, p. 171, 174
B 328, p. 308, 311, 313
B 813, p. 42
B 345, p. 228
B 824, p. 47
B 379, p. 295
B $844-A$, p. 48
B 442 , p. 364
B $857-$ A, p. 45
B 592, p. 394
B 897-A, p. 63
B 622 , p. 372
B $910-A$, p. 66
B 662, p. 455
B 933-A, p. 58
B 712, p. 189
BMB 142, p. 28
TDM 5-R, p. 34

B 773, p. 19

Coffee Cr. Min. Co. See (Camp Cr.) (trib. Coffee Cr.), (Coffee Cr.).

(Collins Cr.) - $(13.0,15: 85)$; GOLD, LEAD.

B 247, p. 54

TDM 5-R, p. 31

Continental Gold Min. Co. See (Henry Cr.), (Merritt Gulch).

Cordovado (Min. Co.). See (Pinnell R.).

(Crooked Cr.) - (9.55-9.9, 0.35-0.6) ; GOLD; MERCURY.
B 259, p. 23
B 722, p. 63
B 263, p. 14, 53, 209
B 739, p. 8
B 284, p. 138-139
B 755, p. 14
B 314, p. 150-151, 155
B 813, p. $40-41$
B 328, p. 244, 251-253, 262
B 857-A, p. 45
B 345, p. 217
B 864-A, p. 50
B 449 , p. 120-121, 128
B 868-A, p. 52
B 592, p. 392
B 880-A, p. 53
B 622, p. 371
B $897-A ;$ p. 65
B 662, p. 452
BMB 142, p. 28

B 714, p. 232-233 
Crooked Cr. Dred. Co. See (Albion Gulch), (Crooked Cr.).

(Cunningham Cr.) or (Gulch) $\therefore(11.7-12.15,16.2-16.45)$; GOLD, LEAD, SILVER.

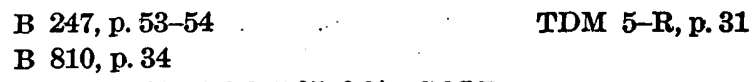

(Dahl Cr.) - (1.65-2.3, 5.95-6.3) ; GoLD.

P 2, p. 61

B 773, p. 22

B 225, p. 54

B 810 , p. 35

B 259, p. 24

B 844-A, p. 48

B 284, p. 140

B 857-A, p. 45

B 314, p. 169, 171, 173-175

B 868-A, p. 53

B 328, p. 307-308, 311-312

B 880-A, p. 55

B 345, p. 228

B $897-$ A, p. 63

B 379, p. 295

B $926-A$, p. 61

B 622, p. 372

B. 933-A, p. 58

B 662 , p. 455

BMB 142, p. 28

B 712, p. 189

Dahl Cr. Min. Co. See (Dahl Cr.).

Dashley. See (Inmachuk R.) (placer).

Dearborn Inv. Co. See (Kugruk R.) (gold).

Deering Dred. \& Min. Co. See (Inmachuk-R.) (placer).

(Dick Cr.) - $(0.2-0.35,13.65-14.8)$; GOLD, TIN, TUNGSTEN.
B 284, p. 139
B 836, p. 48
B 328, p. 282
B $857-\mathrm{A}$, p. 46
B 592, p. 388,393
B 864-A, p. 51
B 622, p. 372
B 868-A, p. 53
B 662, p. 455
B 880-A, p. 55
B 692 , p. 353
B 897-A, p. 63
B 712, p. 189
B 910-A, p. 66
B 783, p. 17
B 917-A, p. 65
B 792, p. 22
B 926-A, p. 61
B 810, p. 35
B $933 \div$ A, p. 58
B 813, p. 42
C 265, p. 4, 6
B 824, p. 47
TDM 5-R, p. 41, 43-44

Dick Cr. Min. Co. See (Dick Cr.).

(Discovery Gulch) or (Cr.) - (11-14, 15-17) ; GOLD. Near Inmachuk R.
B 897-A, p. 62
B 917-A, p. 66
B 910-A, p. 68
B 926-A, p. 63

(Dixie Cr.) - (19.0-19.2, 9.1-9.6) ; GOLD.

B 247 , p. 64-65

(Dome Cr.) - $(20.75-21.75,11.8-12.4)(?)$; GOLD(?). May be in Candle quad. P 10, p. 51

(Dreamy Gulch) - (1.4-1.45, 11.65-11.7) ; GOLD.

B 328, p. 324

B 592, p. 394

Dry Cr. Dred. Co. or Min. Co. See (Inmachuk R.) (placer), (Pinnell R.).

(Eagle Gulch) - (1.5-3.0, 5.1-5.7) ; GOLD.

B 844-A, p. 48

B $857-A$, p. 45

(Esperanza Cr.) - (8.15-9.0, 14.39-14.42) ; GOLD.

B 379, p. 369

B 442, p. 366

(Eureka Cr.) - (2.2-2.6, 10.5-10.6) ; GOLD.

B 314, p. 178

B 328, p. 321

Fairhaven Ditch (\& Hyd.) or (\& Water) Co. See (Inmachuk R.) (placer). 
Fairhaven Gold Dred. Co. See (Candle Cr.). Probably also in Candle quad. Fernegal \& Hanson. See (Crooked Cr.).

Flodin \& Hutton. See (Dick Cr.).

Flume Dred. Co. See (Crooked Cr.), (Ophir Cr.).

Forsgren (\& Vollmer) Dred. Co. See (Inmachuk R.) (placer).

Fox Bar Dred. Co. See (Kougarok R.).

French. See (Jump Cr.). May be in Candle quad.

Fries Dred. Co. See (Inmachuk R.) (placer).

(Frost Cr.) - (6.2-6.5, 10.15-10.35) ; GOLD.

B 328, p. 314

(Garfield Cr.) - (3.3-4.6, 7.3-8.65) ; GOLD.

P 2, p. 65

B 314, p. $169,171,175$

B 345, p. 228

B 328, p. 306, 309, 313-314

B 622, p. 372

B 712, p. 189

(Glacier Cr.) - (20.95-21.2, 10.75-11.0) ; GOLD.

B 379, p. 368-369

B 442 , p. 369-371

B 810, p. 34

Godfrey. See (Kougarok R.).

(Gold Run) - (21.1-21.8, 9.7-10.35) ; GOLD, TUNGSTEN, KYANITE. NoTE.Much of mining may have been in Candle quad.

B 263, p. 209

B 442, p. 369, 371

B 864-A, p. 49

B 810, p. 34

B 813, p. 40

B 868-A, p. 50

B 824, p. 45

B $880-A$, p. 54

B 836, p. 46

B $897-A$, p. 62

B 844-A, p. 47

B 910-A, p. 67

B 857-A, p. 44

(Goldbottom Cr.) - (8.75-8.9, 0.5-0.6) ; GOLD.

B 259, p. 23

B 926-A, p. 62

B 314, p. 147, 151

B 933-A, p. 59

TDM 5-R, p. 45, 47

B 328, p. 236, 254-255

B 442 , p. 343

B 449 , p. 117, 122

Golden Center Mines, Inc. See (Candle Cr.). May be in Candle quad.

Golofnin Bay Min. Co. See Omilak.

(Goose Cr.) or (Gulch) - (6.2-6.35, 9.8-9.9) ; GOLD.

P 2, p. 65, 67

B 314, p. 175

B 328, p. 314

B $926-$ A, p. 61

Grant Min. Co. See (Coffee Cr.).

(Grouse Cr.) (trib. Noxapaga R.) - (5.5-5.7, 9.0-9.4) ; GOLD. B 314, p. 175

B 328, p. 314

(Grouse Cr.) (trib. Tubutulik R.) - (20.6-20.7, 1.1-1.5) ; GOLD. C 300, p. 3

(Hannum Cr.) - (11.9-13.2, 15.55-16.3) ; GOLD, LEAD, TIN.
P 10, p. 51
B 225, p. 77
B 284, p. 140
B 247, p. $49,51-54$
B 263, p. 209
B 379, p. $\mathbf{3 5 5}, 357$
B 442, p. 367
TDM 5-R, p. 31, 41

(Harris Cr.) - (2.95-3.8, 10.7-12.0) ; GOLD, LEAD, SILVER.
P 2, p. 63
B 225, p. 54
B 328, p. 306, 309, 321-323
B 259, p. 24
B 379 , p. 296
B 662, p. 455
B 314, p. $169,171,178$
B 712, p. 189 
(Harris Cr.)-Continued
B 810, p. 35
TDM 5-R, p. 31
C 265, p. 6

(Harry Cr.) - (11.95, 16. 25) ; LEAD.

B 247, p. 54

(Henry Cr.) - (0.3-1.4, 10.95-11.55) ; GOLD.
B 314, p. 179
B 328, p. 324
B 810, p. 35
B 345, p. 228
B $897-$ A, p. 63
B $910-A$, p. 66

Henry Cr. Gold Dred. Co. See (Kougarok R.).

Homestake. See (Inmachuk R.) (placer).

(Homestake Cr.) - (0.65-1.3, 12.0-12.15) ; GOLD, TUNGSTEN.
B 225, p. 54
B 263, p. 209
B 712, p. 189
B 314, p. 179
B 810, p. 35
B 328, p. 325-326
C 265, p. 4
B 379, p. 279
TDM 5-R, p. 43

Hoogendorn. See (Discovery Gulch), (Inmachuk R.) (placer).

(Hot Springs). See (Serpentine Hot Springs).

(Hot Springs Cr.) - (1-2, 14-15) ; MERCURY.

\section{265, p. 4}

(Humboldt Cr.) - (4.9-5.5, 15.25-16.95) ; GOLD, TIN.
B 345, p. 266
B 358, p. 63
B 857-A, p. 44
B 442 , p. 367
B 864-A, p. 49
B 712, p. 22, 52, 189, 195-196
B 868-A, p. 51
B 714, p. 71,236
B 880-A, p. 54
B 810, p. 36
B $897-A$, p. 62
B 813, p. 42
B 824, p. 45
B 910-A, p. 68
C 265, p. 4, 6, 11
TDM 5-R, p. 41

B 844-A, p. 47

(Imuruk L.) - (11-13, 8-9) (?) ; GOLD (?).

B 910-A, p. 68

Independence. See Perkeypile \& Ford.

(Independence Cr.). See Perkeypile \& Ford.

Inmachuk Gold Dred. Co. See (Inmachuk R.) (placer).

(Inmachuk R.) (lode) - $(12,15)$; SILVER, LEAD, IRON. B 662, p. 446

TDM 5-R, p. 30

(Inmachuk R.) (placer) - (12.95-14.75, 15.45-17.4) ; GOLD, MERCURY.

Includes references to Fairhaven Ditch Co., (Ipnichuk R.) ; references probably include production data for some tributaries, particularly Pinnell $\mathbf{R}$.
P 10, p. 50
B 662 , p. $452,454-455$
B 225 , p. $76-77$
B 712, p. $187-189$
B 247, p. 58-60
B 783, p. 17
B 259, p. 24
B 792, p. 22
B 263 , p. 209
B 797 , p. 26
B 379, p. $355,357-361$
B 810, p. 34
B 442 , p. $367-368$
B. 813 , p. 40
B 480 , p. 42
B 824, p. 45
B 592, p. $388,394-395$
B 844-A, p. 47,54
B 622 , p. 372
B $857-A$, p. $\cdot 43-44,51$ 
(Inmachuk R.) (placer)-Continued

B $864-A$, p. 49,57

B 917-A, p. 66

B $868-A$, p. 51,59

B 926-A, p. 62-63, 71

B $880-A$, p. 53-54, 61

B $933-A$, p. 59,68

B $897-$ A, p. $61-62,71$

BMB 142, p. 28.

B 910-A, p: 67-68, 76

TDM 5-R, p: $34^{\prime}$

(Ipnichuk R.). See (Inmachuk R.) (placer).

(Joe Cr.) - (1.1, 6.4) ; GOLD.

B 328, p. 311-312

Johnson. See (Candle Cr.) ; (Kugruk R.) (gold). NotE.-Some operations may have been in Candle quad.

Johnston. See (Cunningham Cr.).

(Joseph Cr.) - (NE 1/4 quad.) (?) ; GOLD.

B 662 , p. 454

(Jump Cr.) - (21.25-21.5, 15.6-15.85) ; GOLD. Many references may apply to part of creek in Candle quad.
B 247 , p. 50
B 379 , p. 364
B 844-A, p. 46
B 622 , p. 371
B 857-A, p. 43
B 692, p. 392
B $864-A$, p. 49
B 712 , p. 189
B 897-A, p. 62
B 810 , p. 34
B 910-A, p. 67
B 813 , p. 40
B 917-A, p. 66
B 824, p. 45
B 926-A, p. 62
B 933-A, p. 59

B 836 , p. 46

Kanari. See (Kougarok R.).

Keenan \& Castleton. See (Kougarok R.).

Kelliher Dred. Co. See (Kougarok R.).

Kimball \& Saupe. See (Ophir Cr.).

Kougarok Consol. Placers, Inc. See (Kougarok R.).

Kougarok Min. Co. See (Kougarok R.).

(Kougarok R.) - (0.0-3.55, 4.45-13.0) ; GOLD, MERCÚRY, TIN, COPPER:

Includes reference to (Kugruk R.) in $\mathbf{P} 2$.

P 2, p. 62, 64

B 314, p. $169,171-172,176-178$

B 328 , p. $306-309,315-320$

B 792, p. 22, 24

B 345 , p. $228,244,266$

B 797, p. 27,30

B 813, p. 42

B 379, p. 296

B 442, p. 364-365

B 836, p. $48-49$

B 480, p. 42

B 592, p. 388, 394

B 622, p. 372

B 662, p. 452, 455

B 712, p. 189

B 714, p. 233

B 722, p. 63,181

B 739, p. 9

B 755, p. 14

B 773, p. 27

B 783, p. 18

B 844-A, p. 48,54

B 857-A, p. 45, 51

B 864-A, p. 51

B 868-A, p. 53, 59

B $880-A$, p. 55,62

B 897-A, p. 63; 71

B 910-A, p. 65-66, 76

B 917-A, p. 64

B 926-A, p. 61, 71

B 933-A, p. 58, 68

C 265, p. 4, 6

BMB 142, p. 27-28

TDM 5-R, p. 22 
(Kougarok R., North Fork) - (2.05-3.75, 10.35-10.8) ; GOLD.
P 2, p. 62-63
B 328, p. 321-323
B 225, p. 54
B 379, p. 296
B 314, p. 178
B 810, p. 35

Kugruk (Mines, Inc.). See (Kugruk R.) (gold).

(Kugruk R.) (gold). - (18.2-18.75; 15.0-16.3) ; GOLD. Includes reference to Kugruk.
P 10, p. 50
B 810, p. 34
B 379, p. 361
B $880-$ A, p. 54
B 442, p. 369
B 897-A, p. 62
B 592, p. 388, 394-395
B $910-A$, p. 67
B 622 , p. 372
B. $917-A$, p. 66
B 662, p. 451
B 926-A, p. 62
B 792, p. 22
B $933-A$, p. 59

(Kugruk R.) (silver, lead). See Perkeypile \& Ford.

Lammers-Fitzpatrick Min. \& Exp. Co. See (Buzzard Gulch).

Laurin Bros. See (Macklin Cr.).

(Macklin Cr.) - (1.25-2.05, 12.95-13.3) ; GOLD.

$\begin{array}{ll}\text { P } 2, \text { p. } 62,64 & \text { B } 712, \text { p. } 189 \\ \text { B } 314, \text { p. } 179 & \text { B } 810, \text { p. } 35 \\ \text { B } 328, \text { p. } 326 & \text { B } 844-\text { A, p. } 48 \\ \text { B } 345, \text { p. } 228 & \text { B } 857-A, \text { p. } 45 \\ \text { B } 379, \text { p. } 297 & \text { B } 864-\text { A, p. } 51 \\ \text { B } 442, \text { p. } 365 & \text { B } 910-A, \text { p. } 66 \\ \text { B 592, p. } 394 & \text { B } 933-\text { A, p. 58 } \\ \text { B } 622, \text { p. } 372 & \text { BMB 142, p. } 28 \\ \text { B } 662, \text { p. } 455 & \end{array}$

(Mascot Cr.) or (Gulch) - $(0.4-0.45,12.65-12.85)$; GOLD, TIN. B 314, p. 179

B 328, p. 320

Mascot Min. Co. See (Kougarok R.).

Mebes \& Hansen. See (Albion Cr.).

(Merritt Gulch) $-\cdot(\mathrm{SW} 1 / 4 \mathrm{4}$ 0, 11) ( ?) ; GOLD. B 792, p. $22 \quad$ B 857-A, p. 46 B 810 , p. 35

Midnight Sun Min. Co. See (Boulder Cr.).

(Milroy Cr.) - (12.1-12.3, 16.35-16.85) ; LEAD. TDM 5-R, p. 31

(Mina Cr.) - (18.7-19.2, 13.0-13.45) ; GOLD. B 442 , p. 369

(Minnehaha Cr.) - (20.7-21.5, 15.9-16.4) ; GOLD. P 10, p. 51

Nashenweng. See (Dahl Cr.), (Quartz Cr.).

(Nelson Gulch) - $(12.85,14.95)$ approx ; GOLD. B 247, p. 56-57

B 442, p. 368

(Neva Cr.) - (2.3-2.4, 8.35-8.45) ; GOLD. P 2, p. 62-63 : B 328, p. 321

North American Mines, Inc. See (Inmachuk R.) (placer).

North Fork Dred. Co. See (Harris Cr.).

Northern Light Min. Co. See (Ophir Cr.). May be in Solomon quad. 
(Old Glory Cr.) - (12.7-13.35, 14.8-15.1) ; GOLD, TIN.

P 10, p. 51

B 225, p. 77-78

B 229, p. 29

B 247 , p. 54-56

B 260 , p. 181
B 284, p. 140

B 379, p. 355,357

B 692 , p. 353

TDM 5-R, p. 41

Omalik. See Omilak.

Omilak - $(17.15,1.35)$; SILVER, LEAD, ANTIMONY, GOLD. Includes references to Omalik, Oonilak.
B 314, p. 156
B 328, p. 236
B 533, p. 127
B 345, p. 245-246
B 622, p. 20
B 379 , p. 28
B 642, p. 69
B 394, p. 201
B 649, p. 50,57
B 442 , p. $345-349$
B 662 , p. 440,446
B 449 , p. 130-133
B 722, p. 176, 183, 185, 224
B 480, p. 88,92
B $938-A$, p. 79
TDM 5-R, p. 12, 27-28

Omilak Gold \& Silver Min. Co. See Omilak.

Oonilak. See Omilak.

(Ophir Cr.) - (9.4-10.0, 0.1-1.25) ; GOLD. See also (Ophir Cr.) under Solomon quad.

$\begin{array}{ll}\text { B } 213, \text { p. } 46 & \text { B } 755, \text { p. 14 } \\ \text { B 225, p. 49, 54 } & \text { B 773, p. 19, 27 } \\ \text { B 259, p. 23 } & \text { B 783, p. 18 } \\ \text { B 263, p. 37, 42, 194, 209 } & \text { B 797, p. 30 } \\ \text { B 314, p. 147, 150-151 } & \text { B } 810, \text { p. 40 } \\ \text { B 328, p. 235-236, 242-250, 261-263 } & \text { B } 813, \text { p. 40, 47 } \\ \text { B 345, p. 216-217 } & \text { B 824, p. 46, 53 } \\ \text { B 379, p. 293-294 } & \text { B 836, p. 47, 54 } \\ \text { B 442, p. 343, 360-363 } & \text { B 844-A, p. 49, 54 } \\ \text { B 449, p. 117-121, 128 } & \text { B 857-A, p. 45, 71 } \\ \text { B 520, p. 342 } & \text { B 864-A, p. 50, 57 } \\ \text { B 662, p. 452-453, 455 } & \text { B } 868-A, p .51-52,59 \\ \text { B 712, p. 187, 189 } & \text { B 897-A, p. 65, 71 } \\ \text { B 714, p. 233 } & \text { BMB 142, p. 28 } \\ \text { B 722,p. } 63,206-207 & \text { BMB 153, p. 56 } \\ \text { B 739, p. } 9 & \end{array}$

Ophir Gold Dred. Co. See (Ophir Cr.). May be in Solomon quad.

Ost. See (Pargon Mtn.).

(Oxide Cr.). See (Ophir Cr.).

(Pargon Mtn.) - (N 1/2 10, 2) (?) ; MICA.

TDM 5-R, p. 46

(Patterson Cr.) - (21.15-21.6, 15.25-15.3) ; GOLD, LEAD, SILVER. NoTE.-

Lead occurrence may be in Candle quad.
B 379, p. 366
B 692, p. 382, 385, 391-392
B 844-A, p. 46
B 712, p. 189
B 857-A, p. 43
B 783, p. 17
B 864-A, p. 49
B 810, p. 34
B 910-A, p. 67
B 917-A, p. 66
B 813, p. 40
B 926-A, p. 62
B 824, p. 45
B 933-A, p. 59
B 836 , p. 46
TDM 5-R, p. 31 
Perkeypile \& Ford - $(18.5,11.95)$; SILVER, LEAD, GOLD, ZINC. Includes references to Independence, (Independence Cr.), (Kugruk R.) (silver, lead).

B 692, p. 399

B 739, p. 43

B 712, p. 194-195

B 755, p. 47

B 714, p. 236-237

TDM 5-R, p. 31-32

B 722, p. 183

(Perry Cr.) - (13.3-13.85, 14.45-14.6) ; GOLD.

P 10, p. 51

B 442 , p. 368

B 247 , p. 58

(Pinnell R.) - (13.8-13.85, 15.4-15.65) ; GOLD. Includes references to (Purnell R.).
B 247 , p. 57-58
B 857-A, p. 44
B 284, p. 140
B 864-A, p. 49
B 442 , p. $367-368$
B 868-A, p. 51
B 824, p. 45
B 880-A, p. 54
B 836, p. 46
B 897-A, p. 62
B 844-A, p. 47
B 917-A, p. 66-67

(Pish R.) - $(2-3,15)$; TIN.

TDM 5-R, p. 41

Polar Bear. See (Inmachuk R.) (placer).

(Purnell R.) Bee (Pinnell R.).

(Quartz Cr.) - (2.2-3.0, 5.25-6.4) ; GOLD, TUNGSTEN.
P 2, p. 60-62
B 592, p. 394
B 284, p. 140
B 712, p. 189
B 314, p. 169, 173, 175
B $933-A$, p. 58
B 328, p. 306, 311-312
BMB 142, p. 28
B 379, p. 295
TDM 5-R, p. 43

Russian-American Min. Exp. Co. - See Omilak.

(Salmon Cr.) - See (Solomon Cr.).

(Schlitz Or.) - (1.05-1.35, 14.3-14.95) ; GOLD( ?).

P 2, p. 55

(Serpentine Hot Springs) - $(2.35,15.0)$ approx; TIN(?). Includes reference to (Hot Springs).

B 229, p. 17, 28

Smith \& Shane. See (Jump Cr.). May be in Candle quad.

(Solomon Cr.) - (2.9-3.0, 12.45-12.55) ; GOLD. NoTE.-Now called Salmon Cr. B 314, p. 171, 179

B 328, p. 308, 325

Stick \& Co. See (Albion Cr.).

(Taylor Cr. - (1.5-3.9, 12.0-13.1) ; GOLD. See also (Kougarok R.).
B 314, p. 179
B 328, p. 324-325
B 345, p. 228
B 379, p. 296
B 520, p. 342

B 712, p. 187

B 722, p. 63

B 773, p. 27

BMB 142, p. 28

Taylor Cr. Ditch Co. See (Kougarok R.).

(Timber Cr.) - $(19.35,5.1)$ approx; COPPER, SILVER, GOLD. NOTE.-Reported as copper lode on upper branches of Koyuk R. in B 345.
B 345, p. 244
B 449, p. 134
B 722, p. 181
TDM 5-R, p. 20

(Trinity Cr.) - $(0.8,12.9)$; GOLD.

B 314, p. 179 
(Trio Cr.) - $(21,10)(?)$; GOLD. May be in Candle quad. B 442, p. 371

(Tubutulik R.) - (20.5-21.7, 0.4-1.1) ; GOLD. B 449 , p. 115-116 B 712, p. 189

(Turner Cr.) or (Gulch) - (4.9-5.36, 8.55-10.21) ; GOLD. P 2, p. 65-67 B 917-A, p. 65

Utica. See (Inmachuk R.) (placer).

Waldhelm. See (Dahl Cr.).

Well Bros. See (Henry Cr.), (Merritt Gulch). (Windy Cr.) - (1.35-2.3, 8.0-8.15) ; GOLD.
P 2, p. 62-63
P 10, p. 51
B 345 , p. 228
B 314 , p. 171
B 379 , p. $295-296$
B 328 , p. $308,320-321$
B 442 , p. 365

(Wonder Gulch) - (1.95-2.0, 5.45-5.6) ; GOLD, LEAD, MERCURY:
B 712 , p. 189
B 857-A, p. 45
B $844-A$, p. 48
TDM 5-R, p. 28, 34

Xavier. See (Gold Run.)

(-.--) (copper, gold) - (8.0, 3.0) approx; COPPER, GOLD. Nore.-On divide between Kingsland and Nugget creeks.

B 449, p. 135

B 722 , p. 181

TDM 5-R, p. 20

BERING GLACIER QUADRANGLE

(Army Cr.) - (2.6-2.8, 17.3-17.55) (?) ; GOLD. May be in McCarthy quad. P 41, p. 120

(Cape Yaktag). See (Yakataga).

(Kiagna R.) - $(14,16)$ (?) ; GOLD.

B 642, p. 135

(White R.) - (15.7-16.4, 1.45-1.55) ; GOLD.
B 520, p. 37
B 622, p. 43
B 542, p. 43
B 642, p. 54

B 592 , p. 138-141

White R. Min. Co. See (White R.).

(Yakataga) - (13.7-17.2, 0.65-1.3) ; GOLD. Includes references to (Cape Yak-

tag), (Yakatag Beach).
B 259 , p. $88-89$
B 783 , p. 12
B 314, p. 34
B 792, p. 14-15
B 379, p. 52
B 797, p. 16
B 442 , p. 41
B 810 , p. 21
B 480 , p. 37
B 813, p. 24
B 520, p. 37
B 824 , p. $27-28$
B 542 , p. 43
B 864-A, p. 29
B 592, p. 60, 134-138
B 868-A, p. 30
B 622, p. 43
B $880-A$, p. 34
B 642, p. 45
B 897-A, p. 40
B 662, p. 41
B $910-A$, p. 38
B 722, p. 34
B 917-A, p. 36-37
B 755, p. 26
B 773, p. 23, 37
B $926-A$, p. 33
B 933-A, p. 32-33

(Yakatag Beach). See (Yakataga). 


\section{BETHEL QUADRANGLE}

Anderson Bros. See (Canyon Cr.).

(Arsenic Cr.) - (16.6, 0.5) ; MERCURY.

RI 4065, p. 50-54

RI 4361, p. 2, 4-6

Camp Robber. See (Marvel Cr.).

(Canyon Cr.) - (17.5-17.75, 3.15-3.55) ; GOLD.

B 622, p. 67, 303-304, 356-357

B 844-A, p. 44

B 642, p. 22,68

B 864-A, p. 46.

B 662, p. 60

B 868-A, p. 47

B 722, p. 60

B 880-A, p. 49-50

B 783, p. 15

B 897-A, p. 59

B 797, p. 24

B 910-A, p. 62

B 810 , p. 32

B 917-A, p. 60

B 813, p. 37

B 926-A, p. 57

B 824, p. 42

B 933-A, p. 54

B 836, p. 43-44

BMB 153, p. 55

Corrigal. See (Arsenic Cr.).

(Cripple Cr.) - (20.65-20.9, 13.2-13.8) ; GOLD.

B 520, p. 41

B 592, p. 70

B 792, p. 20

B 622, p. 347-351

B 933-A, p. 53-54

Dahl \& Wilson. See (Marvel Cr.).

Davidson. See (Cripple Cr.).

(Dominion Cr.) - (22.0-22.15, 17.2-17.25) ; GOLD.

B 622, p. 336-338

Eek R. Min. Co. See (Rainy Cr.).

(Eureka Cr.) - (20.2-21.1, 17.2-17.65) ; GOLD.

B 622, p. 339

(Fisher Cr.) - (20.2-20.85, 14.4-14.9) ; GOLD.

B 622, p. 346-347

(Fork Cr.) - (16.4-17.6, 3.0-5.4) ; GOLD.

B 642 , p. 68

Garrison Co. See (Granite Cr.).

(Granite Cr.) - $(16.2,17.3)$; GOLD.

B 622, p. 331

B 926-A, p. 57

B 917-A, p. 60

B 933-A, p. 53

Hess. See (Marvel Cr.).

Hornet. See (Marvel Cr.).

Jones. See (Rainy Cr.).

(Kapon Cr.) - (15.2-16.9, 1.1-3.0) ; GOLD, MERCURY.

B 622, p. 67,357

B 642, p. 68

Koamme \& Co. See (Canyon Cr.).

Kramme \& Anderson. See (Canyon Cr.).

Kvamme \& Co. See (Canyon Cr.).

McCann. See (Arsenic Cr.).

(Marvel Cr.) - (20.2-20.3, 16.0-16.6) ; GOLD.

B 542, p. 46-47

B 722, p. 60

B 592 , p. 70

B 792, p. 20

B 622 , p. $67,303,339-346$

B 810, p. 32

B 642 , p. 68

B 813, p. 37

B 662 , p. 60

B 824, p. 42

B 714, p. 93

B 836, p. $43-44$ 
(Marvel Cr.)-Continued

$\begin{array}{ll}\text { B } 844-A, \text { p. } 44 & \text { B } 897-A, \text { p. } 59 \\ \text { B } 857-A, \text { p. } 41 & \text { B } 910-A, \text { p. } 62 \\ \text { B } 864-A, \text { p. } 46 & \text { B } 917-A, \text { p. } 60-61 \\ \text { B } 868-A, \text { p. } 47 & \text { B } 926-A, \text { p. } 57 \\ \text { B } 880-A, \text { p. } 49-50 & \text { B 933-A, p. } 53\end{array}$

Marvel Cr. (Min. \& Dev.) Co. See (Marvel Cr.).

(Marvel Dome) - (19-22,16-17) ; GOLD.' See also (Marvel Cr.).

B 810, p. 32

B 864-A, p. 46

B 813, p. 37

B 868-A, p. 47

B.836, p. 44

B 880-A, p. 50

B 844-A, p. 44

B 892-A, p. 59

B 857-A, p. 41

(Mary Cr.) - (15.75-16.0, 17.6-17.8) (?) ; GOLD. NoTE.-Location uncertain, but probably is the same as Mary Lou Gulch on maps.

B 722, p. 60

Peck \& Rice. See (Cripple Cr.).

Pioneer. See.(Marvel Cr.).

(Rainy Cr.) - (16.3-16.6, 0.35-0.6) (?) ; GOLD, MERCURY. May be in Goodnews quad.

B 622, p. 67, $357 \quad$ B 933-A, p. 54-55

B 642, p. $68 \quad$ RI 4361, p. 1-3, 7

Ready Money. See (Marvel Cr.).

(Riglagalik R.) - $(\mathbf{1 5 . 6 5 , 9 . 0 ) ~ ( ? ) ~ ; ~ T I N ~ ( ? ) . ~ N o t e . - O l d ~ n a m e ~ f o r ~ K i s a r a l i k ~ R . ~}$ B 692, p. 7,20

(Robin Cr.) - (20.3-20.6, 17.55-17.7) ; GOLD.

B 622 , p. 337-339

Royal Quartz - (15.65, 9.05) ; GOLD(?), SILVER( ?), ANTIMONY(?).

B 520, p. 35

(Tuluksak R.) - $(14.9,16.9)$; GOLD.

B 622, p. 335.

Wild Horse. See (Marvel Cr.).

Wilson. See (Marvel Cr.).

(Windy Cr.) - (S. central 1/4 quad.) ; GOLD. NotE.-Probably near Fork Cr. B 642, p. 68

BMB 153, p. 55

Yellow Jacket. See (Marvel Cr.).

\section{BETTLES QUADRANGLE}

(Davis Cr.) - $(17.65,17.8)$; GOLD.

B 442, p. 292, 312

B 532, p. 70, 107

(Dome Cr.) - (S 1/2 quad.) (?) ; GOLD.

B 520, p. 38

(Prospect Cr.) - (N 1/4 19, 13) (?) ; GOLD.

B 442, p. 311

B 532, p. 105

\section{BIG DELTA QUADRANGLE}

American Smelt. \& Ref. Co. - $(21.3,6.6)$; GOLD. See also Blue Lead. B $897-$ A, p. 22-23

(Banner Cr.) - (49-5.05, 5.05-5.35) ; GOLD. See also (Democrat Cr.).

B 345 , p. 44

B 379 , p. 55

B 442, p. 245

B 480, p. 167
B 525 , p. 113, 141-142

B 592, p. 361

B 662 , p. 60

BMB 142, p. 25 
(Beaver Cr.) - (4.0-6.5, 13.3-13.5) ; GOLD, TIN.

B 542, p. 208

TDM 1, p. 34

Blue Lead - $(21.3,6.6)$; GOLD. See also American Smelt. \& Ref. Co. B 917-A, p. 30

(Boulder Cr.) - $(21.7,6.85)$; MOLYBDENUM. TDM 1, p. 29

(Buckeye Cr.) $(5.05-5.6,5.35-5.85)$; GOLD, TUNGSTEN, LEAD, TIN (?). Note.-Much of production was from tributaries; see also (Hinkley Gulch), (Moore Cr.).

$\begin{array}{lll}\text { B 525, p. 113, } 142 & & \text { B 824, p. } 34 \\ \text { B } 662, \text { p. } 60 & \cdots & \text { C 331, p. 13 } \\ \text { B 813, p. } 29 & \cdots & \text { BMB 142, p. 25 }\end{array}$

(Butte Cr.) - (9.1-9.6, 12.55-13:0) ; GOLD.
B 284, p. 123-125
B 442, p. 245
B 295, p. 26
B 480, p. 167
B 345, p. 43
B 538, p. 80

(Caribou Cr.) - (9.5-10.0, 11.6-12.45) ; GOLD, TIN, TUNGSTEN.
B 284, p. 123-125
B 739 ; . p. 33
B 295 , p. 26
B 783, p. 15
B 345 , p. 43
B $910-A$, p. 48
B 442 , p. 245
B $917-A$, p. $44,46-47$
B 480 , p. 167
B 926-A, p. 40,43
B 538 , p. 80
TDM 1, p. 34, 39
B 642 , p. 67

Caribou Gold Dred. Co. See (Caribou Cr.).

Caribou Min. Co. See (Caribou Cr.)

(Central Cr.) - $(15-19,6)$; GOLD. NotE.-In valley of Goodpaster R. B 810 , p. 26

Democrat - $(4.9,5.9)$ approx; GOLD. Nore.-Not mentioned by name in B 692 . B 692, p. 39

B 739 , p. 33

(Democrat Cr.), (Gulch), or (Pup) - (4.9-5.0, 5.85-5.95) ; GOLD.
B 379 , p. 55
B 783, p. 15
B 442 , p. 245
B 797, p. 20
B 480 , p. 167
B 810 , p. 26
B 525, p. 113,142
B 813 , p. 29
B 542, p. 222
B 824, p. 34
B 592 , p. 361
B 836, p. 34
B 622 , p. 235
BMB 142, p. 25
B 739 , p. 33

Frazier \& Reed. See (Harding L.).

(Goodpaster R.) - (SE 1/4 quad.) ; GOLD(?). See also (Central Cr.), (Michigan Cr.).

B 797, p. 20

(Granite Cr.) - (20.55-20.9, 7.2-7.5) ; GOLD.

B 662 , p. 60

Grizzly Bear - $(21.05,6.75)$; GOLD.

B 917-A, p. 30

(Harding L.) - $(0.65-0.75,7.45)$; CLAY.

RI 4932 , p. $43-45$

(Hinkley Gulch) - (N 1/2 5, 5) ; GOLD. See also (Buckeye Cr.).

B 836 , p. 34 
Kaolin Assn. See (Harding L.).

(Kenyon Cr.) - (SE 1/8 quad.) (?) ; GOLD. May be in Mt. Hayes quad. B 642, p. 67

(Michigan Cr.) - (SE $1 / 8$ quad.) ; GOLD. B 662 , p. $60 \quad$ B $810-A$, p. 26

(Mineral Cr.) - (4-6, 4-6) (?) ; GOLD.

B 755, p. 38

(Moore Cr.) - (N 1/2 5, 5) ; GOLD. See also (Buckeye Cr.). B 810 , p. 26

(Nickel Cr.) - (14-15, 12-13) ; NICKEL

TDM 1, p. 18

(No Grub Cr.) - (9.9-10.5, 11.7-12.5) ; GOLD.

B 480 , p. 167

B 642 , p. 67

B 538, p. 80

B 933-A, p. 39

Puntilla. See (Tenderfoot Cr.).

(Pyne Cr.) - (NW 1/4 quad.) ; TIN

TDM 1, p. 34

(Redmond Cr.) - (3.0,6.5) (?) ; GOLD (?).

B 442, p. 245

Salcha Min. Co. See (Caribou Cr.).

(Salcha R.) (chromite) - $(15.3,13.3)$ approx ; CHROMITE. TDM 1, p. 16

(Salcha R.) (gold) - (NW 1/2 quad.) ; GOLD. Most references include data on

Butte, Caribou, and other tributary creeks.
B 284, p. 7
B 864-A, p. 36
B 480, p. 167
B 868-A, p. 37
B 844-A, p. 34
B 880-A, p. 41

B 857-A, p. 31

Shuff. See Democrat.

Stevens \& Johnson. See (Caribou Cr.).

(Tenderfoot Cr.) - (5.6-6.0, 4.65-5.1) ; GOLD.

\begin{tabular}{|c|c|c|}
\hline B 284 , p. $123-124$ & & B 642, p. 67 \\
\hline B 314, p. 37 & & B 662, p. 60 \\
\hline B 337, p. 29 & & B 739, p. 33 \\
\hline B 345, p. 44 & & B 797, p. 20 \\
\hline B 379, p. 55 & & B 810, p. 26 \\
\hline B 442, p. 245 & & B 813, p. 29 \\
\hline B 480, p. 167 & & B 824, p. 34 \\
\hline B 525, p. 113,141 & & B 836, p. 34 \\
\hline B 542, p. 222 & & B 917-A, p. 47 \\
\hline В 592, p. 361 & & BMB 142, p. 25 \\
\hline
\end{tabular}

B 622,235

Terra Cotta Assn. See (Harding L.).

(Tibbs Cr.) - $(20.55,6.3)$; GOLD, ANTIMONY, LEAD, MOLYBDENUM. B 926-A, p. 28

TDM 1, p. 14, 29

(Twentymile Cr.) - (West central 1/4 quad.) (?), GOLD (?)

B 480 , p. 167

( Volkmar R.) - (SE 1/8 quad.) ; GOLD(?)

B 797, p. 20 


\section{BLYING SOUND QUADRANGLE}

(Elrington I.) - (16.5, 16.9) ; COPPER.

$$
\text { I } 273
$$

Ellsworth. See Iron Mask.

Fairview - $(6.4,15.8)$; CÖPPER.
B 379, p. 105-106
B 587, p. 235-236
B 442 , p. 170

Feather Bed - (6.25-6.6, 17.2-17.25) ; COPPER.
B 379, p. 104
B 587, p. 234-235
B 442, p. 170

Iron Mask - $(6.3,15.85)$; COPPER, ZINC.
B 379, p. 104-105
B 587, p. 235

Latouche Consol. Copper Co. - $(17.3,16.8)$; COPPER.
B 379, p. 89
I 273

Lietzke - (6.3-6.4, 15.35-15.5) ; COPPER.
B 379, p. 106
B 587, p. 236

Peterson - $(6.3,16.95)$; COPPER.

B 379, p. 104

B 587, p. 235

Pitman \& Gould. See Fairview, Feather Bed.

Reynolds-Alaska Dev. Co. - $(6.7,17.5)$; COPPER. See also Iron Mask.
B 379, p. 104
B 587, p. 234

Seattle-Alaska Copper Co. - $(17.7,17.1)$; COPPER, GOLD, ZINC. Includes reference to Seattle-Alaska Min. Co.
B 379, p. 89
B 480, p. 31
B 662, p. 201, 209-210
B 520, p. 28
B 622, p. 132
B 692, p. 145
B $963-$ B, p. 67
I 273

Seattle-Alaska Min. Co. See Seattle-Alaska Copper Co.

\section{BRADFIELD CANAL QUADRANGLE}

Alaska. See Alaska-Premier, Hyder Lead, Monarch. Note.-Three claims of same name.

Alaska-Comstock. See Hyder Lead.

Alaska Marble. See (Ham I.).

Alaska-Premier - $(19.25,0.7)$; GOLD, ZINC, LEAD, SILVER, COPPER, TUNGSTEN.
B 773, p. 74, 78-79
B 1024-F, p. 140

B 807, p. 85-86

American. See Stoner.

Andrews \& Stoner. See Glacier.

(Banded Mtn.) - (15.5-16.4, 0.5-1.1) ; GOLD.
B 824, p. 17
B 836, p. 16
B 857-A, p. 16
B 864-A, p. 16

B 844-A, p. 17

Bankovich - $(17.0,0.9)$ approx ; Commodity, if any, not known.

B 1058-A, p. 27

Bartholf - $(18.9,1.75)$; COPPER, LEAD, BARIUM.

B 807, p. 92-93

Berg - $(0.7,7.0)$; GOLD (?).
B 642, p. 78
B 662 , p. 75

Bertha - (19.1-19.25, 0.65-0.85) ; LEAD, ZINC, COPPER. See also Daly-Alaska. B 642, p. 97 
Bervaqua. See Engineer, North Star (Texas Cr.).

Bevaque - $(17.4,1.05)$; GOLD, SILVER, LEAD. See also North Star (Texas Cr.).

B 783, p. 53

(Blake I.). See (Ham I.).

Blasher -. $(17.0,1.15)$; LEAD, ZINC, COPPER, MOLYBDENUM. NOTE.Blasher also held Morning prospect; which see also:

B. 807 , p: $100^{-}$

B.1024-F, p. 140

B 926-C, p. 171

Bluebird - $(19.1,0.55)$; COPPER, LEAD, MOLYBDENUM, TUNGSTEN. B 1024-F, p. 139-140

Border - $(19.25,1.0)$; LEAD, ZINC, COPPER. B 807 , p. 90

(Bradfield Canal) - $(1.05-1.15,3.45-4.0) ;$ LIMESTONE.
B 542, p. 72
B 682, p. 95

Brigadier - $(18.95,0.5)$; GOLD, LEAD, SILVER, TUNGSTEN. Note.-No connection with Brigadier Min. Co.; includes references to Butte (group), Hyder Butte.

B 807, p. 81

Brigadier Min. Co. See Cripple Cr.

Butte (claim). See Fish Cr. (mine) under Ketchikan quad.

Butte (group). See Brigadier.

Cantu (Min. Co.) - $(18.95,1.55)$; LEAD, SILVER, ZINC, GOLD, COPPER, BARIUM.
B 792, p. 30
B $864-A$, p. 16
B 800 , p. 324
B $879-$ A, p. 19
B 807, p. $43,91-92$
B 824, p. 17
B $1024-B$, p. 30
B 1024-F, p. 140

Carlson \& Hewitt. See Homestake, Ibex.

Charles, Nelson \& Pitcher - $(18.8,1.2)$; ZINC, LEAD, GOLD, SILVER, COPPER. B 722, p. 129, 139-140 B 773, p. 87

Chickamin - $(16.9,1.4)$; LEAD, ZINC, COPPER. B 807 , p. 100

Climax. See Fish Cr. (mine) under Ketchikan quad.

Comstock. See Hyder Lead.

Connors. See Silver Bell.

Copper. Sce Dugas.

Crest - $(19.0,0.5)$; GOLD, LEAD, COPPER. B 807 , p. 81-82

Cripple Cr. - $(18.95,0.65)$; LEAD, ZINC, COPPER.

B 773, p. $74,82-83$

B 1024-F, p. 140

B 807, p. 83-84

Daly-Alaska - (19.1-19.25, 0.65-0.85) ; SILVER, GOLD, LEAD, ZINC, COPPER. Includes references to Elevenmile, New Alaska (Min. Co.); see also Bertha, Iron, Western.
B 642, p. 97, 99
B 792 , p. 30
B 714, p. 142
B 800 , p. 318,327
B 722, p. 128, 131-133
B 807, p. $43,86-88$
B 739, p. 21
B 1024-B, p. 31, 33
B 773, p. 74, 83-84
B 1024-F, p. 140

B 783, p. 41,53

Day Bros. See Ibex. 
Doggat. See Border.

Double Anchor - $(17.05,1.3)$; ZINC, LEAD, COPPER.

B 773, p. 91

Dugas - $(16.9,1.25)$; ZINC, LEAD, COPPER.

B 807 , p. 98-99

B 807, p. 99

Edelweiss - $(16.15,0.7)$; GOLD, SILVER, LEAD. B 807, p. 101

B 1024-F, p. 140

Elevenmile. See Daly:Alaska.

Engineer - $(17.45,1.0)$; SILVER, GOLD, LEAD, TUNGSTEN, COPPER. B 807, p. 109-110

B 1024-F, p. 127, 140.

Evening Star - $(17.9,1.4)$; LEAD, SILVER(?). Includes references to Morning Star.

B 773, p. 90

B 807, p. 94

Extenuate. See Monarch.

Fish Cr. (mine). See Fish Cr. (mine) under Ketchikan quad.

Fitzgerald - (W 1/2 19, 0) (?). SILVER(?), LEAD(?). May be in Ketchikan quad.

B 739, p. 21

Fortuna. See Hyder Lead.

Frey, Goldborgh \& Davidson. See Double Anchor.

Glacier - $(15.7,1.0)$ approx ; SILVER, GOLD, COPPER, LEAD. B 807 , p. $120-121$

Gold Cliff Premier - $(19.15,1.1)$; GOLD, SILVER, LEAD, ZINC, COPPER. B 807 , p. 90

Greenpoint - (W $1 / 2$ 19, 0 ) ( $?$ ) ; LEAD. May be in Ketchikan quad. IC 7379, p. 42

(Ham I.) - $(0.65-0.85,3.75-4.0)$; MARBLE. NotE.-Called Blake I. on recent maps.
B 284 , p. 57
B 379 , p. $84-85$
B 314 , p. 77
B 542, p. 72
B 345 , p. 122
B 682, p. $91,93-95$

B 347, p. 198

Heckla - (16.4, 0.35) ; SILVER, GOLD, LEAD, ZINC, COPPER. Includes reference to Hummel, Blasher \& Moss.
B 792, p. 30
B 807 , p. $101-102$

Hewitt \& Carlson. See Homestake, Ibex.

Hobo - $(19.15,0.6)$; GOLD, ZINC, LEAD, COPPER, SILVER.
B 807 , p. $84-85$
B 1024-B, p. 31, 33

Homestake - $(17.85,1.5)$; LEAD, SILVER, GOLD, ZINC, COPPER.
B 773, p. $75,88-89$
B 807 , p. $43,94-95$
B 792 , p. 30
B 1024-B, p. 30

B 800, p. $317,320,358$

Hoosier. See Daly-Alaska.

Hummel - $(17.2,1.15)$; ZINC, LEAD, COPPER, SILVER, GOLD.

B 807, p. 48,98

Hummel, Blasher \& M(r) oss. See Edelweiss, Heckla, Jumbo (Banded Mtn.)

Hummel, Mross \& Blasher. See Heckla.

Hyder Butte. See Brigadier.

Hyder Jumbo - (19.4, 0.65) approx; Barren.

B 807, p. 72 
Hyder Lead (Mines, Inc.), (Min. Co.) - (16.95-17.2, 0.75-1.1) ; SILVER, LEAD, ZINC, COPPER, GOLD, MOLYBDENUM, BARIUM. Includes references to Comstock, Fortuna, Texas Cr. Comstock.
B 773 , p. $75,77,91-93$
B $844-A$, p. 17
B 800, p. 317
B $1024-B$, p. 30
B 807 , p. $43,102-108$
B $1024-F$, p. 140
B 824 , p. 17
IC 7379 , p. 42

Hyder Skookum - $(19.25,0.7)$; COPPER.

B 807, p. $72 \quad$ B $1024-F$, p. 140

Ibex - $(17.8,1.5)$; ZINC, LEAD, SILVER, COPPER.

B 773, p. 75, 87-89 B 800, p. 317, 324

B 783, p. 53-54 B 807, p. 96-97

Ickis \& Moa. See Portland.

Iron - (19.1-19.25, 0.65-0.85); SILVER, LEAD, COPPER, ZINC. See also Daly-Alaska.

B 642, p. 97, 99

B 714, p. 142

Iron Cap - $(17.5,1.4)$; ZINC, SILVER, GOLD, COPPER.

B 773 , p. 95

B 1024-B, p. 31

B 807, p. 44,98

Jackson. See Liberty (Jackson).

Jackson-Hummel. See Hyder Lead.

Joe-Joe: See Hyder Lead.

Johnson, Bryne \& Hummel. See Hyder Lead.

Jumbo (Banded Mtn.) - $(16.2,0.5)$; Commodity, if any, not known.

B 807, p. 101

Jumbo (Texas Cr.) - (17.55, 1.1). LEAD, COPPER, ZINC.

B 807, p. 111

B 1024-F, p. 140

Jumbo Mining. See Jumbo (Texas Cr.).

Juneau - $(17.5,0.7)$; COPPER, LEAD.

B 807, p. $108-109$

Kennedy, See Silver King.

Kennedy \& Provinse. See Jumbo ('Texas Cr.).

Keno - $(17.35,0.7)$; LEAD, SILVER, GOLD, COPPER, ZINC, BARIUM. B 773, p. 74, 87, 94

B 807, p. 108

Lake - $(16.9,1.15)$; LEAD.

B 773, p. 74, 87, 91

B 1024-F, p. 140

B 807, p. 101

Last Chance - $(19.15,0.25)$; GOLD, LEAD, COPPER, ZINC, TUNGSTEN. See also Fish Cr. (mine) (Ketchikan quad.).

B 880-A, p. 18-19 B 1024-F, p. 128, 139

Liberty (Jackson) - $(17.9,1.15)$; LEAD, TUNGSTEN.

B 807, p. $93-94$
B $1024-B$, p. 33

B 1024-F, p. 140

Liberty (Stoner-Clegg-O'Rourke), See Stoner-Clegg-O'Rourke.

Lindeborg. See Riverside.

Lodge. See Stoner.

McDonald \& Cronholm. See Cantu.

McGraw - $(18.6,1.15)$; commodity, if any, not known.

B 773, p. 94

McVey, Daniel. Bee Silver Bar. 
McVey, David. See Iron Cap.

McVey \& Connors. See Evening Star, Silver Star.

Marietta - $(16.95,1.4)$; GOLD. May be same as Silver King, which see.also. B 844-A, p. 17

Meagher \& Snyder. See Silver Coin.

Metcalf \& Findley. See (Banded Mtn.).

Miller. See (Ham I.).

Moa. See Bluebird.

Moa, Ickis \& Hill. See Crest.

Moa, Ickis \& Horland. See Monarch.

Monarch - (19.1, 0.25) ; GOLD, SILVER, LEAD, TUNGSTEN, ZINC, COPPER,

BARIUM. Note.-Part of area covered by claims is probably in Ketchikan quad.
B 807 , p. 74-75
B $1024-$ F, p. 139
IC 7379, p. 68
RI 4174 , p. 4
Morning $(16.95,1.1)$; LEAD.
B 807, p. 101
B 1024-F, p. 140

Morning Star. See Evening Star.

Mountain View. See Fish Cr. (mine) under Ketchikan quad.

Murphy \& Stevens. See Sunset.

New Alaska (Min. Co.). See Daly-Alaska.

Ninety-six - $(18.95,1.0)$; LEAD, ZINC, COPPER. Includes reference to Snyder.

B 773, p. 87

B 1024-F, p. 140

B 807, p. 93

North Star (Monarch). See Monarch.

North Star (Texas Cr.) - (17.4, 1.05) ; LEAD. See also Bevaque.

B 807, p. 110

Nothiger - $(17.85,1.1)$; LEAD.

B 807 , p. 94

Olympia Extension. See Last Chance.

Olympia Fraction. See Fish Cr. (mine) under Ketchikan quad., Last Chance.

Pederson \& Johnson. See Hobo.

Peterson \& Olson. 'See Brigadier.

Portland - $(19.1,0.6)$; LEAD, ZINC, COPPER.

B 807 , p. 84

Ready Money. See Alaska-Premier.

Red Rose. See Hobo.

Riverside (Min. \& Mil. Co.) - $(18.95,0.35)$; LEAD, SILVER, GOLD, TUNG: STEN, ZINC, COPPER. NotE.-Part of area covered by claims is in Ketchikan quad.; includes references to Lindeborg, Riverview.
B 642 , p. 97
B 714, p. 141
B 722 , p. 139
B 739, p. 21
B 773, p. 74-77, 79-82
B 783, p. $23,41,53$
B 792, p. 30
B 797, p. 37
B 800 , p. $317,324,330,347$
B 807, p. $43,77-81$
B 810 , p. $12,46,49,51$

B 813, p. 15

B 824 , p. 17

B 857-A, p. 16

B $864-A ;$, p. 16

B 933-A, p. 94

B 1024-B, p. $30,33,35,42,44$.

B 1024-F, p. 123, 125-126, 128-136

IC 7379, p. $68 \div 69$

IC 7844, p. 13

RI 4174, p: 4-5, 36-44 
Riverview. See Riverside.

Scott, J. H., Co. See Riverside.

Silver Bar - $(18.15,1.25)$; COPPER, LEAD, BARIUM. Includes reference to McVey, Daniel.

B 773, p. 88

B 807, p. 93

Silver Bell - $(17.6,1.5)$; LEAD, ZINC, COPPER.

B 807, p. 44,97

B 1024-B, p. 31

Silver Coin - $(17.85,1.6)$; LEAD, COPPER.

B 773, p. 90

B 807, p. 95

Silver King - $(16.95,1.4)$; ZINC, LEAD, GOLD, SILVER, COPPER, BARIUM. See also Marietta.

B 807, p. 99-100

Silver Star - $(17.75,1.35)$; SILVER, LEAD, ZINC.

B 773, p. $89-90$

B 807, p. 97

Single Rose. See Fish Cr. (mine) under Ketchikan quad.

Snyder. See Ninety-six.

Snyder \& Wilson. See Ninety-six.

Stoner-Clegg-O'Rourke - $(19.25,0.75)$; ZINC, LEAD, COPPER. B 807 , p: 88

Stoner (Gold \& Silver Min. Co.) - $(19.3,0.9)$; GOLD, SILVER, ZINC, LEAD. B 722, p. 131-132

B 807, p. $43,89-90$

B 773 , p. 74,83

B 1024-B, p. 31-33

Strong (, Barber) \& Black. See Riverside.

Summit. See Fish Cr. (mine) under Ketchikan quad.

Sunset - $(17.5,0.65)$; LEAD, BARIUM.

B 807 , p. 109

Swede - $(19.1,0.6)(?)$; LEAD, ZINC, COPPER. NoTE.-May be old name for Hobo, Portland, or both; see also Hobo, Portland.

B 773, p. 74, 78

Texas (Cr.) Comstock. See Hyder Lead.

Texas Discovery - $(17.6,1.3)$; LEAD, GOLD, SILVER, COPPER.

B 773, p. 74, 90-91 $\quad$ B 1024-F, p. 140

B 807, p. 98

Titan (\& Salmon R. Syn.) - (19.35, 0.4); ZINC, GOLD, SILVER, LEAD, COPPER.

B 773, p. 74, 77 $\quad$ B 1024-B, p. 33; 44

B 807, p. 72-74 $\quad$ B 1024-F, p. 140

B 813, p. 15

(Unuk R.) - $(11.9,5.9)$ approx ; GOLD(?).

B 347 , p. 186

Vermont Marble Co. See (Bradfield Canal), (Ham I.)

Virginia - $(19.2 ; 0.95)$; GOLD, ZINC, LEAD, COPPER.

B 773, p. 74, 84

B 1024-B, p. 31

B 807, p. $43,88-89$

Wano. See Monarch.

Western - (19.1-19.25, 0.66-0.83); LEAD, ZINC, COPPER. See also Daly-Alaska. B 642, p. 97

B 714, p. 142

Woodbridge \& Lowery. See (Ham I.). 


\section{CANDLE QUADRANGLE}

(Alameda Cr.) - (7.25-7.4, 0.95-1.1) ; GOLD.
B 442, p. 336-339
B 449 , p. $110-113$

Alaska Dred. Assn. See (Candle Cr.). May be in Bendeleben quad.

Arctic Circle Exp. Co. See (Candle Cr.). Note.-Some of holdings probably in Bendeleben quad.

(Bear Cr.) - (6.8-7.1, 9.5-9.75) ; GOLD, PLATINUM.
B 225, p. 78-79
B 810, p. 34
B 247, p. 63-64
B 813 , p. 40
. B 345, p: 226
B 379, p. 369
B 824, p. 45
B 442, p. 344
B 836, p. 47
B 449 , p. $125-126$
B 857-A, p. 43-44
B 622 , p. 371-372
B 864-A, p. 49
B 662, p. $21,24,455,458$
B 868-A, p. 51
B 666, p. 96
B 880-A, p. 53-55
B 692 , p. 41, 381, 386, 392-394
B 897-A, p. 61-63
B 712, p. 23, 52, 189
B 917-A, p. 67
B 714, p. 38, 71, 236
I 287
B 739, p. 13, 158
C 250, p. 3, 22, 24-25
B 773, p. 30
BMB 142, p. 28
B 783, p. 17
TDM 5-R, p. 39
(Bear Gulch) - (4.9-5.0, 6.0-6.1) ; GOLD PLATINUM.
B 692 , p. 395
O 250, p. 3-4, 8

$\mathrm{Be}(1) \mathrm{tz}-(6.4,9.5)$; COPPER. Note.-Not referred to by name in B 722 .
B 692, p. 399
B 722, p. 181
C 250, p. 22
TDM 5-R, p. 22-23

(Boulder Cr.) - (6.4-6.9, 6.95-7.25) ; GOLD.

O 250, p. 22, 24

(Candle Cr.) - (0.0-0.6, 14.65-16.1) ; GOLD, LEAD, COPPER. NoTE.-Mining has been in both Bendeleben and Candle quads.
B 225, p. 77-78
B 810, p. 34, 40
B 247, p. 60-63
B 813, p. 40, 47
B 263, p. 209
B 314, p. 35
B 824, p. 45,53
B 379 , p. $355,364-368$
B 836, p. 46, 54
B 442, p. 369-370
B 844-A, p. 46-47,54
B 857-A, p. 43, 51
B 864-A, p. 49, 57
B 449, p. 126-127
B 868-A, p. 50, 59
B 592, p. 388, 394-395
B $880-A$, p. 53-54, 61
B 622, p. 371
B 662, p. 451-452, 454-455
B 897-A, p. 61-62
B 692 , p. $382-383,385-386$, 391- B 910-A, p. 66-67; 76-77 392
B 712, p. 187-189
B 714, p. 232-233, 235
B 722, p. 62
B 773, p. 27
B 783, p. 17
B 792, p. 22, 24
B 797, p. 30
B 917-A, p. 66
B 926-A, p. 62, 71
B 933-A, p. 58-59, 68
I 287
C 250, p. 11-14
BMB 142, p. 28
TDM 5-R, p. 31, 39 
Candle Cr. Dred Co. See (Candle Cr.). May be in Bendeleben quad.

Candle Cr. Min. Co. See (Candle Cr.). May be in Bendeleben quad.

Candle Ditch Co. See (Candle Cr.).

(Canoe Cr.) - (0.0-1.4, 8.75-10.0) ; SILVER, LEAD.

TDM 5-R, p. 31

Circle. See (Sweepstakes Cr.).

(Cub Cr.) - (7.1-7.3, 8.9-9.1) ; GOLD.

B 225, p. 78-79

B 247 , p. 64

B 379 , p. 369

B 442 , p. 344
B 449, p. 125

B 692 , p. 392-394

I 287

C 250, p. 22

(Diamond Cr.) See (Rube Cr..). Diamond is old name.

(Dime Cr.) - (6.25-6.4, 2.9-3.9) ; GOLD, PLATIUM, CHROMITE(?). Includes references to (Haycock), Haycock Min. Co.

B 642, p. 22,70

B 662, p. 21, 24, 454,458

B 692 , p. 21, 40-41, 380-381, 386387, 396-398

B 712, p. $23,52,188-189,196$

B 714, p. 38, 71, 94, 236

B 722, p. $23,183-184$

B 739, p. 13, 158

B 773, p. 27, 30, 50

B 783, p. 17-18, 25

B 792, p. 24, 33

B 797, p. 27, 30, 39

B 810, p. 36, 40

B 813, p. $41-42,47,59$

B 824, p. $46,53,65-66$
B 836, p. $48,54,68$

B 844-A, p. $49,55,67$

B 857-A, p. 46,63

B $864-A$, p. 52,68

B 868-A, p. 53, 59, 70

B $880-A$, p. $57,62,74$

B 897-A, p. 66, 71, 84

B $910-A$, p. $70,76,89$

B 917-A, p. 68-69, 83

B 926-A, p. $64,71,77-78$

B 933-A, p. $61,68,74$

I 287

BMB 142, p. 27

BMB 153, p. 56

TDM 5-R, p. 18, 39

Dime Cr. Dred Co. See (Dime Cr.).

Dime Cr. Min. Co. See (Dime Cr.).

(Dome Cr.) - $(0.0-1.05,11.85-12.05)(?)$; GOLD(?). May be in Bendeleben quad.

P 10, p. 51

Eagle. See (Sweepstakes Cr.).

(Eldorado Cr.) - $(6.1-6.35,3.7-4.35)$; GOLD. Includes reference to (Little Eldorado Cr.).

B 692, p. $380,396,398 \quad \therefore \quad$ I 287

Fairbanks. See (Sweepstakes Cr.).

Fairhaven Gold Dred Co. See (Candle Cr.). May be in Bendeleben quad.

(Farmer Cr.) - (6.3-6.7, 6.6-6.75) ; GOLD.

B 692, p. 394-395

French. See (Jump Cr.).

(Gold Run) - (0.0-1.1, 9.95-11.5) ( ?) ; GOLD. Mining in Bendeleben quad. also.

B 263, p. 209

B 442, p. 369,371

B 810, p. 34

B 813, p. 40

B 824, p. 45

B 836, p. 46

B 844-A, p. 47

B 864-A, p. 49

B 868-A, p. 50-51

B 880-A, p. 54

B $897-$ A, p. 62

B 910-A, p. 67

B $926-A$, p. 62

B 933-A, p. 59

$593018-61-4$ 
Golden Center Mines, Inc. See (Candle Cr.). May be in Bendeleben quad. (Greenstone Cr.) - (6.3-6.6, 3.0-3.5) ; PLATINUM.

B 662, p. 458

(Haycock). See (Dime Cr.).

Haycock Min. Co. See (Dime Cr.).

Hoxie, Tendness \& Barr. See (Bear Cr.).

(Hunter Cr.) - (2.5-3.8, 12.65-13.65) ; GOLD.
I 287
C 250, p. 22

(Hunter Cr., Right Fork) - $(3.8-5.5,12.6-13.4)$; GOLD (?).

C 250, p. 22

Jack. See (Quartz Cr.).

(John Bull Hill) - $(0.5,15.9)$ approx; GOLD.
B 379, p. $366-367$
B 692, p. 392
B 442, p. 370
B 712, p. 189

Johnson, A. See (Sweepstakes Cr.).

Johnson, I. See (Candle Cr.). May be in Bendeleben quad.

(Jump Cr.) - (0.0-0.25, 15.4-15.6) ; GOLD. · Mining in Bendeleben quad. also.
B 379, p. 364
B $857-A$, p. 43
B 622, p. 371
B 864-A,p. 49
B 692 , p. 392
B $897-$ A, p. 62
B 712, p. 189
B 910-A, p. 67
B 810, p. 34
B $917-A$, p. 66
B 813, p. 40
B 926-A, p. 62
B 824, p. 45
B 933-A, p. 59
B 836, p. 46
C 250, p. 11

B 844-A, p. 46

Keewalik Min. Co. See (Candle Cr.), (John Bull Hill).

(Kiwalik R.) - $(0,16)$; GOLD.

$$
\text { I } 287
$$

(Koopuk R.) - (north central 1/8 quad.) ; GOLD.
B 773, p. 50
B 792, p. 22
B $783, \mathrm{p} .17$
B 810, p. 34

(Little Eldorado Cr.). See (Eldorado Cr.)

(Meinzer Cr.) - (5.0-5.9, 13.5-15.3) ; GOLD.

C 250, p. 22

Moon. See (Sweepstakes Cr.).

Moon \& Ryan. See (Dime Cr.).

(Mud Cr.) - (0.1-0.7, 16.1-17.2) ; LEAD, MERCURY.

TDM 5-R, p. 31, 34

New York-Alaska Gold Dred. Co. See (Candle Cr.).

(Peace R.) - (6.35-7.0, 5.8-7.85) ; GOLD, LEAD, TUNGSTEN, COPPER, ZINC. NotE.-Most of production from Peace R. drainage has been from (Bear Gulch) and (Sweepstakes Cr.), which see also.
B 442 , p. 340
B 449 , p. 114
C 196, p. 2,5
B 692 , p. 380-382
C 250, p. $9,26,28-31$
TDM 5-R, p. 23

I 287

(Placer Cr.) - (SW1/4 quad.) ; GOLD.

B 542, p. 49

Porter. See (Bear Cr.), (Sweepstakes Cr.). 
(Quartz Cr.) - (4.8-5.55, 8.55-8.8) ; GOLD; PLATINUM:

B 449, p. 115

B 810, p. 34

B' 813 , p. 40

B 824, p. 45

B 836 , p. 46

B 844-A, p. 47

B 857-A, p. 44, 63

B 864-A, p. 49,68

B 868-A, p. 50, 70
B 880-A, p. 54, 74

B 897-A, p. 62, 84

B $910-A$, p. 67,90

B 917-A, p. 66, 83

B 926-A, p. 62, 78

B 933-A, p. 59, 74

I 287

C 250, p. 3, 15

Rampart. See (Sweepstakes Cr.).

(Rock Cr.) - (6.3-6.95, 6.95-7.5) ; GOLD(?). C 250, p. 22

(Rube Cr.) - (6.25-6.8, 6.5-6.6) ; GOLD, PLATINUM (?), CHROMITE( ?).

B 692 , p. $380-381,386,394-395$

I 287

C 250, p. 3, 22

TDM 5-R, p. 39

(Scotch Cr.) - (5.65-6.0, 12.05-12.2) ; GOLD( ?).

C 250, p: 22

(Sherdon Cr.). See (Sheridan Cr.).

(Sheridan Cr.) - $(6.4-6.65,10.3-10.35)$; GOLD. Includes references to (Sherdon Cr.).
B 225, p. 78-79
B 247, p. 64
B 379, p. 369
B 442 , p. 344

B 449 , p. 125

B 622 , p. 371-372

B 692, p. 392-394

I 287

Smith. See (Dime Cr.).

Smith \& Shane. See (Jump Cr:).

(Spring Cr.) - (5.4-5.9, 6.3-7.4) ; GOLD. C 250, p. 3-4

(Spruce Cr.) - (3.9-4.3, 11.7-12.1) ; GOLD(?). C 250, p. 22

(Sugar Loaf Cr.) - (5.7-6.4, 12.9-13.0) (?) ; GOLD(?). C 250, p. 22

(Sweepstakes Cr.) - (4.95-5.2, 5.9-7.05) ; GOLD, PLATINUM. See also (Bear Gulch).

B 542, p. 49

B 622, p. 371

B 692 , p. $21,41,380-381,383-384$; 386,395

B 712, p. 23, 189

B 714, p. $38,71,236$

B 739, p. 13, 158

B 783, p. 17

B 797, p. 27

B 810, p. 36

B 813, p. 42

B 824 , p. 46

B 836, p. 48

B 844-A, p. 49
B 857-A, p. 46

B 864-A, p. 52

B 868-A, p. 53

B $880-A$, p. 57

B 897-A, p. 66

B $910-A$, p. 70

B 917-A, p. 69

B 926-A, p. 64

B 933-A, p. 61

I 287

C 250, p. 1, 3-5, 22

BMB 153, p. 56

TDM 5-R, p. 39

(Trio Cr. $)-(0,10)(?)$; GOLD. May be in Bendeleben quad.

B 442 , p. 371

Weinard. See (Mud Cr.). 
(Willow Cr.) - (3.5-4.5, 0.0-2.0) approx; $\operatorname{GOLD}(?)$.

B 449, p. 114

Winder. See (Sweepstakes Cr.).

(_-_-.) (gold) - (6.4;9.9) approx; GOLD. Note-Between Polar and Split Creeks.

C 250, p. 22

\section{ChaNdataR QUADRANGIE}

Alaska-Chandalar Min. Co. See Little Squaw.

Anderson. See (Tobin Cr.).

(Bettles R.) - (4-7, 9-12) approx; GOLD. NoTE.-Most of production from the following tributary creeks : Eightmile, Emory, Garnet, Mule, Phoebe, Robert, Sheep, Spruce.

B 532, p. 104-105

B 926-A, p. 52

B 813, p. 33

B 933-A, p. 47

B 897-A, p. 54

(Big Cr.) - (SW 1/4 12, 9) ; GOLD, TUNGSTEN, SILVER, LEAD, ZINC, AN-

TIMONY. Includes general references to lodes near head of creek.

B 314, p, 38

B 345, p. 46

B 824, p. 40

B 379, p. 30-31, 57

B 836, p. 40

B 442, p. 315

B 844-A, p. 40

B 520, p. 38

B 857-A, p. 37

B 532, p. 112, 114-116

B 864-A, p. 41

B 622, p. 65

B 642 , p. 67

B 868-A, p. 43

B 722, p. 58

B 880-A, p. 46

B 739, p. 41

B 897-A, p. 55

B 755, p. 45

B 910-A, p. 58

B 773, p. 260-261, 263

B 917-A, p. 57

B 926-A, p. 54

B 783, p. 14

B 933-A, p. 50

B 797, p. 22

C 195, p. 8

B 813, p. 34

C 348 , p. 16

(Big Squaw Cr.) - $(12.25,10.0)$ approx ; GOLD, ANTIMONY, LEAD, MOLYBDENUM.
B 773, p. 259-260, 263
B 813, p. 34

B 783, p. 14

C $348 ;$ p. 18

(Boer Gulch) or (Cr.) - (2.5-3.4, 8.0-8.4) (?) ; GOLD.

B 442, p. 312

B 532, p. 108

Buckley. See (Little Squaw Cr.).

(California Cr.) - (3.35-3.5, 7.8-8.7) ( ?) ; GOLD.

P 20, p. 102

B 662, p. 59

B 442, p. 292, 312

B 824, p. 38

B 532, p. 70, 108

Carlson (, Amero) \& Buckley. See (Little Squaw Cr.).

Carlson \& Freshman. See (Tobin Cr.).

Carter - (SW. 1/4 12, 9) (?) ; GOLD. Note.- Lode near divide between Big and Little Squaw Creeks.

B 592, p. 69

Chandalar Min. Co. See Little Squaw. 
Christenson. See (Jim Pup).

Creecy. See (Gold Cr.).

Crystal - (NW 1/4 12, 9) (?) ; GOLD( ?).

B 773, p. 262

(Dictator Cr.) - (W 1/2 12, 6) approx ; GOLD.

B 813, p. 34

B 864-A, p. 41

B 836, p. 40

Eclipse - (NW 1/4 12, 9) (?) ; GOLD(?).

B 773, p. 262

(Eightmile Cr.) - (4.25-5.0, 9.5-10.1) ; GOLD, MERCURY.

B 442, p. 310

TDM 2, p. 18

B 532, p. 105

(Emory Cr.) - (2.7-3.1, 9.1-10.0) approx; GOLD.

B 442, p. 292, 310

B 532, p. 69, 104-105

Eneveloe-(NW $1 / 412,9)$; GOLD, ZINC, SILVER, LEAD, ANTIMONY, COPPER.

B 532, p. 113-115

B 773, p. 262

Engineers' Exp. Syn. - (11-12, 9-10) (?) ; GOLD (?).

B 857-A, p. 37

First Chance. See Eneveloe.

(Garnet Cr.) - (4.05-4.7, 9.3-9.95) ; GOLD.

B 442, p. 292, 310

B 532, p. 69, 104

(Gold Cr.) - (1.5-2.5, 8.55-9.05) ; GOLD, ANTIMONY.

AR 21, p. 486

B 649, p. 64

P 20, p. 99, 101-102, 105

B 662, p. 59

B 345, p. 45

B 844-A, p. 39

B 442, p. 292, 307-308

B 868-A, p. 42

B 532, p. 69, 99-102

B $910-A$, p. 56

B 622, p. 59

B 917-A, p. 55

B 642, p. 64-65

Gold King - (SW 1/4 12,9) ( ?) ; GOLD.

TDM 1, p. 14

B 480 , p. 35

(Gold Myrtle Cr.). See (Myrtle Cr.).

Golden Eagle - (NW $1 / 4$ 12, 9) (?) ; GOLD(?).

B 773, p. 262

Haslem and assoc. See (Myrtle Cr.).

Haynes \& Griffin. See Gold King.

Idaho-Alaska Corp. - (11-12, 9-10) (?) ; GOLD( ?).

B 857-A, p. 37

(Jim Gulch), (Cr.), or (Pup) - (3.4-4.0, 8.0-8.6) (?) ; GOLD.

B 442, p. 292,312

B 868-A, p. 42

B 532, p. 70, 108

B $917-A$, p. 55

B 844-A, p. 39

Kelliher. See (Gold Cr.).

Kelty - (NW 1/4 12, 9) (?) ; GOLD, SILVER, ZINC, LEAD, ANTIMONY, COPPER.

B 532, p. 113-115

(Lake Cr.) - (3.8-3.9, 9.4-9.8) (?) ; GOLD.

B 810 , p. 28

B 824, p. 38

B 836 , p. 39

B 868-A, p. 42

Last Chance. See Eneveloe. 
(Linda Cr.) - $(1.3,9.2)$; GOLD.
B 442 , p. $292,308-310$
B $910-A$, p. 56
B 532 , p. $69,102-104$
B $917-A$; p. 55
B 622, p. 59
BMB 153 , p. 54

B 642 , p. $64-65$

Little Mikado: See Mikado.

Little Squaw - $(12.4,9.9)$; GOLD, ZINC, SILVER, LEAD, ANTIMONY, COPPER.

Includes references to Alaska-Chandalar. Min. Co:
B 480 , p. 35
B 592, p. 68
B 520 , p. 34
B 773 , p. $261-262$
B 532 , p. $113-115$
C 348 , p. 16

(Little Squaw Cr.) - (12.4-12.65, 9.9-10.4) ; GOLD, TUNGSTEN.

Includes references to (Squaw $\mathrm{Cr}$.).
B 622, p. 64
B 844-A, p. 40
B 642 ; p. 67
B $857-$ A, p. 37
B 662 , p. 59
B $864-$ A, p. 41
B 739, p. 41
B $868-$ A, p. 43
B 755 , p. 45
B $880-A$, p. 46
B 773 , p. $256-259,263$
B $897-A$, p. 54-55
B 783 , p. 14
B $910-A$, p. 58
B 797, p. 22
B 813 , p. $33-34$
B $917-$ A, p. 57
B 824, p. 40
B $926-A$, p. 54
B 836 , p. 40
B 933-A, p. 50
C 348, p. 16

(Marion Cr.) - $(0.5,6.0)$ approx ; GOLD (?).

AR 21, p. 485

B 532, p. 69,90

B 442, p. 292

Marr. See (Myrtle Cr.)

Mello. See (Little Squaw Cr.).

Mikado - ( SE 1/4 11, 9) ; GOLD, ZINC, SILVER, LEAD, ANTIMONY, COPPER. Includes reference to Little Mikado.

B 532, p. 112, 114-115

B 773, p. 262

Miller and asso. See (Sheep Cr.) (trib. Middle Fork Koyukuk R.).

(Minnie Cr.) - (0.0-2.5, 7.0-7.45) ; GOLD. NoTE.-Production may have been from part of creek in Wiseman quad.
B 442, p. 292
B 662, p. 59
B 532, p. 69; 94-95
B 836, p. 39

B 542, p. 45

(Mule Cr.) - (4.75, 10.3-11.0) ; GOLD, SILVER, COPPER.

B 442, p. 292, 310

B 532, p. 69, 104-105

(Myrtle Cr.) - $(0.05-0.3,3.9-4.7)$; GOLD. Includes reference to (Gold Myrtle Cr.).

AR 21, p. 483-484

P 20, p. 99, 102

B 345, p. 45

B 442, p. 288, 291-292, 298-300

B 532, p. 69, '86-89

B 622 , p. 59

B 642 , p. 65

B 662, p. 59

B 722, p. 59

B 810, p. 28
B 824, p. 38

B 836, p. 39

B 868-A, p. 43

B 880-A, p. 45

B 897-A, p. 54

B 910-A, p. 56

B 917-A, p. 55

B 926-A, p. 52

B 933-A, p. 47

BMB 153, p. 54 
Newton (\& Yasuda). See (Big Cr:).

Overlook - (NW 1/4 12, 9) (?) ; GOLD (?).

B 773, p. 262

(Phoebe Cr.) - (5.0-6.3, 10.0-10.45) ; GOLD.

B 442, p. $31.0 \quad$ B 532, p. 105

Repo \& Schwaesdall. See (Myrtle Cr.).

(Robert Cr.) - (5.0-7.0, 10.5-13.0) ; GOLD.

B 442, p. $310 \quad$ B 532, p. 105

(St. Marys Cr.) or (Gulch) - (SW 1/4 12,9) ; GOLD.

B 379 , p. 57

B 622, p. 65

B 442, p. 315

B 642, p. 67

B 532, p. 116

(Sheep Cr.) or (Gulch) (trib. Middle Fork Koyukuk R.) - (1.1-1.5, 8.8-8.95) ; GOLD.
B 442, p. 292
B 868-A, p. 42
B 480 , p. 39
B 880-A, p. 46
B 520, p. 38
B 897-A, p. 54
B 532, p. 69,99

(Sheep Cr.) (trib. Robert Cr.) - (6.35-7.0, 11.65-11.75) ; GOLD.

B 442 , p. 310

B 532, p. 105

(Slate Cr.) - $(0.05-2.0,3.85-5.6)$; GOLD. NotE.-Some production may have been from part of creek in Wiseman quad.
AR 21, p. 483-485
B 910-A, p. 56
P 20, p. 99, 102
B 917-A, p. 55
B 442, p. 291-292, 298-299
B $926-\mathrm{A}$, p. 52

B 532 , p. $69,86-88$

Smith. See (Little Squaw Cr.).

(Spruce Cr.) - (5.25-5.35, 11.2-12.0) ; GOLD.

B 442, p. 310

B 532, p. 105

(Squaw Cr.) See (Little Squaw Cr.).

Summit - (NW 1/4 12, 9) ; GOLD, ZINC, SILVER, LEAD, ANTIMONY, COPPER. B 532, p. 113-115

B 773, p. 262

Tobin. See Mikado.

(Tobin Cr.) - $(11,9)$; GOLD, TUNGSTEN, LEAD. NotE.-Includes entire headwater region of creek.
B 836, p. 40
B 844 A, p. 40
B 917-A, p. 57
B 868-A, p. 43
B 926-A, p. 54
B 880-A, p. 46
B 933-A, p. 50
B 897-A, p. 55
C 195 , p. 8,11
C 348, p. 16

B 910-A, p. 58

Tonopah - (NW 1/4 12,9) (?) ; GOLD(?).

B 773, p. 262

(Wakeup Cr.) - $(3.85-4.0,8.65-9.05)$; GOLD.

B 810, p. 28

B $897-A$, p. 54

B 880-A, p. 45

B $917-\mathrm{A}$, p. 55

Woodchuck. See Eneveloe.

CHANDIER LAKE QUADRANGLE

(Anaktuvuk R.) - (9.2-10.4, 5.8) approx; PHOSPHATE.

P 302-A, p. 11, 13, 15

(Chandler L.) - (3.4, 6.2) approx ; PHOSPHATE.

P 302-A, p. 11-12, 15 
(Kiruktagiak R.) - (0.7-0.8, 6.7-6.8) approx; PHOSPHATE, RARE EARTHS, VANADIUM.

P 302-A, p. 1-2, 6, 9-11

(Natvakruak R.) - $(8.9,6.4)$ approx ; PHOSPHATE:

P 302-A, p. 11, 13, 15

(Tiglukpuk Cr.) - (6.9-7.2, 5.2-5.3) approx; PHOSPHATE, RARE EARTHS,

VANADIUM.

P 302-A, p. 2, 6, 9-11

\section{CHARLEY RIVER QUADRANGIE}

Adamic. See (Coal Cr.)

(Alder Cr.) - (E 1/2 2, 5) (?) ; GOLD.

B 917-D, p. 250

(Alice Gulch) - $(4.15,5.5)$; GOLD.
B 314, p. 203
B 538, p. 75
B 520, p. 209-210
B 542, p. 213

Alluvial Gold, Inc. See (Woodchopper Cr.).

Alluvial Placers, Inc. See (Woodchopper Cr.).

Anna May. See (Woodchopper Cr.).

Aztec. See (Woodchopper Cr.).

Bauer. See (Fourth of July Cr.).

(Ben Cr.) - (7, 4) (?) ; GOLD.

B 917-D, p. 250

Bennett. See (Woodchopper Cr.).

Bodacious. See (Woodchopper Cr.).

(Boulder Cr.) - $(5.65,5.25)$ approx ; GOLD.
B 542, p. 213
B 592, p. 360
B 897-C, p. 252
B 917-D, p. 251

Charles. See (Woodchopper Cr.).

(Coal Cr.) - (5.15-6.25, 5.0-6.4) ; GOLD.

B 213, p. 48

B 295, p. 23

B 816 , p. $165-166$

B 314, p. 202-203

B 868-A, p. 39

B 379 , p. 54

B $880-A$, p. $42-43$

B 480 , p. 172

B 897-A, p. 49, 71

B 520, p. 201, 208-209

B 897-C, p. 251-254

B 538 , p. 76

B 910-A, p. 49,76

B 917-A, p. 47

B 542, p. 213

B 917-D, p. 246-251, 254

B 592, p. 360

B 926-A, p. 43, 70

B 622, p. 61

B 642, p. 63

B 933-A, p. 40, 67

Coal Cr. Hyd. Min. Assn. See (Coal Cr.).

(Colorado Cr.) - (5.2, 5.0) approx ; GOLD.

B 314, p. 202-203

B $897-$ C, p. 252

Comet. See (Woodchopper Cr.).

Cordova. See (Woodchopper Cr.).

(Dome Cr.) (trib. Washington Cr.) - (11, 0) (?) ; GOLD.

B 897-C, p. 204

(Dome Cr.) (trib. Woodchopper Cr.) - (4.5-4.65, 5.75-5.95) approx; GOLD.

B $917-D, p .248$

(Drayham Cr.) - (8-9, 2-3) (?) ; GOLD.

B $917-D$, p. 250 
(Eagle Cr.) - (12.25-12.5, 2.3-3.0) approx ; GOLD.
B 314, p. 201
B 897-C, p. 204
B 520, p. 208
B 917-D, p. 250
B 538, p. 76

Ellington. See (Fourth of July Cr.).

Emily Assn. See (Coal Cr.).

(Flat Cr.) - (8.6-9.8, 2.4-3.1) (?) ; GOLD (?).

B 622, p. 64

Florence. See (Woodchopper Cr.).

(Fourth of July Cr.) - (14.2-15.2, 2.15-2.6) ; GOLD, SILVER. Includes reference to Fourth of July Placers Co.
B 213, p. 48
B 816, p. 164-165
B 259, p. 29-30
B 824, p. 39
B 284, p. 126
B 836, p. 38
B 295, p. 23
B $844-$ A, p. 40
B 314, p. 188,200
B 857-A, p. 38
B 345, p. 50
B 864-A, p. 42
B 480 , p. 172
B 868-A, p. 44
B 520, p. 201, 206-208, 216
B $880-A$, p. 47
B 538, p. 77-79
B 897-A, p. 55
B 592, p. 360
B 897-C, p. 201-204
B 642, p. 213
B 910-A, p. 57
B 662 , p. 56
B 917-A, p. 56
B 783, p. 14
B 797, p. 23
B 917-D, p. 246-250, 254,
B 810, p. 29
B 813, p. 34 257-259
B 926-A, p. 54
B 933-A, p. 49

Fourth of July Placers Co. See (Fourth of July Cr.).

Forrest Assn. See (Coal Cr.).

Gladys. See (Woodchopper Cr.).

Gold Medal. See (Woodchopper Cr.).

Gold Placers, Inc. See (Coal Cr.).

Gold Star. See (Woodchopper Cr.).

Golden Eagle Bench Assn. See (Coal Cr.).

(Grouse Cr.) - (E1/2 3, 5) ( ?) ; GOLD ( ?). B 314, p. 203

B 538, p. 75

Herbert. See (Woodchopper Cr.).

Holstrom. See (Woodchopper Cr.).

Hunkey Dory. See (Woodchopper Cr.).

(Irish Gulch) - (8-9, 2-3) ( ?) ; GOLD.

B 917-D, p. 250

(Iron Cr.) - (3.95, 5.55) ; GOLD.

B 538, p. 75

B 897-C, p. 256

B 542, p. 213

B 917-D, p. 250

B 816, p. 165

Joe. See (Woodchopper Cr.).

July Cr. Min. Co. See (Fourth of July Cr.).

July Cr. Placer Co. See (Fourth of July Cr.).

Kelly. See (Woodchopper Cr.).

McDonald. See (Coal Cr.).

Malstrom Assn. See (Coal Cr.). 
Mary Flow. See (Woodchopper Cr.).

(Mineral Cr.) - (4.15-4.2,5.45-5.55) ; GOLD.
B 314, p. 203-204
B 816, p. 165
B 520, p. 201, 209-210
B 897-C, p. 255-257
B 538, p. 74-75
B 917-D, p. 250, 254
B 542, p. 213

(Nugget Cr.) or (Gulch) - (12.2-12.4, 4.0-4.6) approx ; GOLD.
B 314, p. 201
B 520, p. 208
B 897-C, p. 204
B 917-D, p. 250

B 538, p. 76

(Rose Cr.) - $(5,5)($ ?) ;.GOLD.

B 542, p. 213

(Ruby Cr.) - $(14,2)$ (?) ; GOLD.

B 520, p. 208

B 592, p. 360

B 538 , p. 79

(Sam Cr.) - (7, 4) (?) ; GOLD (?).

B 314, p. 202-203

B 542, p. 213

B 480 , p. 172

B 917-D, p. 246, 250

(Sawyer Cr.) - (7, 4) (?) ; GOLD.

B 917-D, p. 250

Seward Assn. See (Woodchopper Cr.).

Sioux. See (Woodchopper Cr.).

Slaven Assn. See (Coal Cr.).

Snowbird. See (Woodchopper Cr.).

State. See (Woodchopper Cr.).

Sunshine. See (Woodchopper Cr.).

(Surprise Cr.) - (12.25-12.5, 2.3-3.0) approx ; GOLD.
B 314, p. 201
B 480 , p. 172
B 538, p. 76
B 520, p. 208
B 897-C, p. 204
B 917-D, p: 250

Valdez. See (Woodchopper Cr.).

(Washington Cr.) - (12.2-12.5, 2.3-4.6) approx ; GOLD. See also (Dome Cr.),

(Eagle Cr.), (Nugget Cr.), (Surprise Cr.).

B 295 , p. 23

(Webber Cr.) - (E 1/2 2, 5) (?) ; GOLD (?).

B 917-D, p. 246-250

(Woodchopper Cr.) - (3.5-4.3, 5.25-5.3) ; GOLD. . See also (Iron Cr.), (Mineral Cr.).

B 213, p. 48

B 225, p. 57

B 263, p. 208

B 284, p. 126

B 295, p. 23

B 314, p. 188, 203

B 917-D, p. 246

B 345 , p. 50

B 379, p. 54

B 442, p. 239

B 480, p. 172

B 520, p. 201, 209-210

B 538, p. 74-75

B 542, p. 213

B 622, p. 61-62

B 642 , p. 63

B 836, p. 37

B 864-A, p. 42

B 868-A, p. 44

B 897-A, p. 49

B 897-C, p. 254-256

B 910-A, p. 49, 76-77

B 917-A, p. 47

B 917-D, p. 246-250, 254, 257-259

B 926-A, p. 43, 70

B 933-A, p. 40, 67

B 592, p. 360

BMB 153, p. 53 


\section{CHIGNIK QUADRANGLE}

(Bear Cr.) - (25.7, 9.15) approx; PUMICE. Note.-Now : called Dry Cr. B 467, p. 75

(Hook Bay) - $(27,9)$; PUMICE.

B 467 , p. 75

(Mallard Duck Bay) - $(24.0,4.8)$; LEAD, ZINC, GOLD (?).

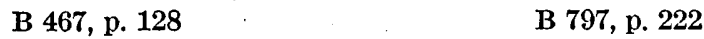

(Prospect Bay) - $(25.35,3.3)$; COPPER, LEAD, ZINC. . Note.-Now called Warner Bay.

B 379, p. 152

B 714, p. 35

B 467, p. 21, 129, 131

B 797, p. 222-223

\section{CIRCLE QUADRANGLE}

Anderson. See (Mastodon Cr.).

(Bachelor Cr.) - $(6.5,8.6-8.9)$; GOLD.
B 379, p. 54-55
B 480 , p. $164-165$
B 442, p. 208-209, 238-239
B 525, p. 149-150

Beaver Dred. Co. See (Nome Cr.).

(Bedrock Cr.) - (13.5, 9.2) ; TUNGSTEN.

\section{348, p. 13}

Berry \& Lamb. See (Mastodon Cr.).

Berry, C. J., Dred. Co. See (Independence Cr.), (Mammoth Cr.), (Mastodon Cr.).

Berry Holding Co. See (Eagle Cr.), (Independence Cr.), (Mastodon Cr.).

(Big Chena R.). See (Chena R.), (Chena R., Middle Fork).

(Birch Cr.) - (16-18, 5-6) (?) ; GOLD.
B 314, p. 192
B 542, p. 213
B 520 , p. 245

(Bonanza Cr.) - (11.95-12.45, 9.45-9.75) ; GOLD.

B 824, p. $36,169 \quad$ B $868-$ A, p. 39

B 836, p. 37 B $880-$ A, p. 43

B 857-A, p. 35 B 897-C, p. 225-228

B 864-A, p. $38 \quad$ B 910-A, p. 49

(Bottom Dollar Cr.) - (16.05-16.15, 7.3-7.55) ; GOLD.

$\begin{array}{ll}\text { B } 480, \text { p. } 160,164 & \text { B } 897-C, \text { p. } 231,235 \\ \text { B } 542, \text { p. } 213 & \text { B } 917-A, \text { p. } 48 \\ \text { B } 897-\text { A, p. } 50 & \text { B } 926-\text { A, p. } 44\end{array}$

(Boulder Cr.) - (13.85, 8.15) approx ; GOLD.

B 662 , p. 56

B 897-C, p. 250

B 824, p. 164

(Buckley Bar) - (17.85, 5.95) approx ; GOLD.

B 480 , p. 160

(Butte Cr.) - (10.3-10.8, 6.45-7.25) ; GOLD.

B 662, p. 56

B $857-A$, p. 35

B 897-C, p. 231

B 910-A, p. 49

Carstens. See (Portage Cr.).

(Charity Cr.) - (5.45-5.5;6.9-7.3) ; GOLD (?).

B442, p. 233 
(Chena R.) - (11-14, 0-1) (?) ; GOLD. Note. Data on tributaries may be included; see also (Chena R., Middle Fork).

B 520, p. 244

B864-A; p. 35-36

B 739, p. 33

B 868-A, p. 36-37

B 810, p. 25

B 880-A, p. 39-41

B 824, p. 33

B 910-A, p. 48

B 836, p. 33

B 917-A, p. 43-44, 46

B 844-A, p. 32, 34

B $857-$ A, p. $30-31$

B 926-A, p. 40, 43

B 933-A, p. 40

(Chena R., Middle Fork) - (11-14, 0-1) (?) ; GOLD. See also (Chena R.).

B 520, p. 244

B 542, p. 208

Clark. See (Mastodon Cr.).

Clayworth Assn. See (Harrison Cr., North Fork).

(Crazy Mts.) - (NE 1/4 quad.) ; GOLD.

B 755, p. 38

(Deadwood Cr.) - (14.5-16.05, 7.5-9.35) ; GOLD, SILVER, TIN, TUNGSTEN;

LEAD, FLUORITE, MERCURY. Includes references to (Discovery Gulch).

AR 18, p. 293, 342-345

B 225, p. 57

B 251, p. 59-61

B 259, p. 39

B 263, p. 14, 208

B 284, p. 126

B 295, p. 21

B 314, p. 188-193

B 345, p. 50

B 379, p. 29, 53-54

B 442, p. 39, 235-236, 246-250

B 480, p. 88, 90, 162-163

B 520, p. 89, 245

B 538, p. 60-62

B 542, p. 211

B 592, p. 360

B 622, p. 61

B 642 , p. 63

B 662, p. 56
B 692, p. 37

B 813, p. 31

B 824, p. 36, 161-164

B 836, p. 37

B 844-A, p. 37

B 857-A, p. 35

B 864-A, p. 38

B 868-A, p. 39

B 880-A, p. 43

B 897-A, p. 49-50

B 897-C, p. 235-245

B 910-A, p. 49, 76-77

B 917-A, p. 47-48

B 926-A, p. 44, 72

B 933-A, p. 40-41

C 248, p. 3

C 335, p. 4

C 348, p. 11, 13

TDM 1, p. 27, 32, 37, 41

Deadwood Min. Co. See (Deadwood Cr.).

(Deep Cr.) - (5.0, 6.5) ; GOLD.

C. $331, \mathrm{p} .8$

Deep Cr. Min. Co. See (Deep Cr.).

(Dempsey Pup) - $(3.9,6.5)(?)$; ANTIMONY.

TDM 2, p. 11

(Discovery Gulch) or (Cr.). See (Deadwood Cr.).

(Dome Cr.) - (10.5-10.65, 9.2-9.65( ?) ; GOLD.

B 755, p. 38

(Eagle Cr.) - (10.6-11.7, 7.65-7.95); GOLD. Includes references to (Eagle Cr., Mastodon Fork).
AR 18, p. 293, 354-355
B 251, p. 59, 61-62, 64-65
B 314, p. 188-189, 191, 197
B 263, p. 14, 52, 87-88, 208
B 345, p. 50
B 284, p. 126
B 379, p. 54
B 295, p. 23
B 442, p. 234, 237-238
B 480 , p. 160, 163-164 
(Eagle Cr.)-Continued
B 520, p. 245
B 824, p. 36, 167-171
B 538, p. 64-65
B 836, p. 37
B 542, p. 212
B 844-A, p. 37
B 592, p. 68
B $857-A, p .35$
B 622, p. 61
B 868-A, p. 39
B 642, p. 63
B 897-A, p. 50
B 662, p. 56
B 897-C, p. 228-231
B 714, p. 87
B 910-A, p. 49
B 739, p. 32
B $917-A$, p. 48
B 797, p. 21
B 926-A, p. 44
B 810, p. 28
B 933-A, p. 41
B 813, p. 31

(Eagle Cr., Mastodon Fork). See (Eagle Cr.).

Eaton. See (Deadwood Cr:).

Elmer. See (Mastodon Cr.).

Erickson. Bee (Mastodon Cr.).

(Faith Cr.) - (4-5, 6-7) ; GOLD. See also (Homestake Cr.), (Hope Cr.).
B 314, p. 37
B 337, p. 30
B 910-A, p. 48
B 442, p. 208
B 917-A, p. 43,46
B 525, p. 149
B 926-A, p. 40,43
B $933-A$, p. 39

(Frying Pan Cr.) - (10.1-10.6, 5.05-6.2) ; GOLD.

B 480, p. 160,164

(Golddust Cr.) - (10.45-12.0, 7.15-7.6) ; GOLD( ?). B 897-C, p. 231

(Greenhorn Cr.) or (Gulch) - (13.9-14.0, 7.95-8.1).
AR 18, p. 239, 345
B 542, p. 213
B 314, p. 188, 193
B 824, p. 164
B 520, p. 245
B $897-$ C, p. 250

B 538, p. 62

(Half Dollar Cr.) - (15-16, 7) ; GOLD, TUNGSTEN, TIN.

B 480, p. $164 \quad$ B $917-A$, p. 48

B 520, p. 245 B 926-A, p. 44

B 542, p. $213 \quad$ B 933-A, p. 41

B 592, p. $68,360 \quad$ C 348, p. 13

B 622, p. $61 \quad$ RI 4174, p. 27

B 880-A, p. 43 TDM 2, p. 19-20

(Harrison Cr.) - (13.65-14.5, 6.35-6.4) ; GOLD. See also (Harrison Cr., North Fork).

AR 18, p. 293, 351-354

B 480, p. 164

B 314, p. 188, 195-197

(Harrison Cr., North Fork) - (12.65-13.8, 6.45-7.5) ; GOLD, TIN. Includes references to (Pitka Bar).

AR 18, p. 351

B 314, p. 195-197

B 442, p. 238

B 538, p. 65-66

B 824, p. 36, 171-172

B 836, p. 37

B 844-A, p. 37

B 864-A, p. 38

B 868-A, p. 39
B 880-A, p. 43

B 897-A, p. 50

B 897-C, p. 231-235

B 910-A, p. 49

B 917-A, p. 48

B 926-A, p. 44

B 933-A, p. 41

TDM 1, p. 32 
(Harrison Cr., South Fork) - (13.2-13.65, 6.4-6.45) ; GOLD.

B 314, p. 195-197

B 897-C, p. 232-233

B 538, p. 65-66

(Holdem Cr.) - (15.9-16.45; 8.25-8.6) ; GOLD.

B 857-A, p. 35

B 868-A, p. 39

B 864-A, p. 38

(Homestake Cr.) - (5.5-5.65, 6.95) ; GOLD.
B 314, p. 37
B 337, p. 30
B 520, p. 242
B 442 , p. 209
B 525, p. 150
B 542, p. 205

B 480 , p. 157

(Hope Cr.) - (4.55-5.1, 6.95-7.1) ; GOLD, LEAD, MOLYBDENUM, ANTIMONY (?).
B 314, p. 37
C.248,p.,3, 5
B 337, p. 30
C 348 , p. 11
B 525, p. 149
TDM 2, p. 12

(Hot Springs Cr.) - (17.1-17.2, 8.55-8.6) ; TUNGSTEN. C 335 , p. 5

(Independence Cr.) - (12.6-13.0, 7.95-8.95) ; GOLD, TIN, LEAD, TUNGSTEN. AR 18, p. 345-346

B 251, p. 59,63

B 824, p. 36, 164-165

B 295, p. 22

B 836, p. 37

B 314 , p. 194

B 844-A, p. 37-38

B 442 , p. 238

B 857-A, p. 35

B 480 , p. 163

B 864-A, p. 38

B 520, p. 245

B 868-A, p. 38

B 542, p. 212

B 880-A, p. 43

B 592 , p. 360

B 897-C, p. 210-211, 218-222

B 538, p. 63

B 910-A, p. 49

B 662, p. 56

B 692 ; p. 37

B 917-A, p. 48

B 813, p. 31

B 926-A, p. 44

B 933-A, p. 41

C 348, p. 11, 13

Independence Min. Co. See (Independence Cr.).

Kelley. See (Miller Cr.).

(Ketchem Cr.) - (16.25-16.65, 7.05-8.85) ; GOLD, TUNGSTEN, TIN.

B 857-A, p. 35

B 864-A, p. 38

B 868-A, p. 39

B 910-A, p. 49

B 917-A, p. 48

B 880-A, p. 43

B 926-A, p. 44

B 897-A, p. 50

B 933-A, p. 41

B 897-C, p. 248-250

C 335 , p. 5

Knutson (\& Larson). See (Deadwood Cr.).

Langlow \& Larson. See (Switch Cr.).

(Lawson Cr.) - (10.1-11.2, 1.1-2.5) ; GOLD.

B 542, p. 213

Lee \& McGregor. See (Dome Cr.).

(Loper Cr.) - (9.0-9.9, 8.7-13.3) ; GOLD.

B 379, p. 54

B 857-A, p. 35

B 442, p. 239

(McLain Cr.) - (13.6-13.7, 4.7-4.9) (?) ; GOLD (?).

B 480 , p. 164 
(Mammoth Cr.) - (12.9-13.9, 8.95-9.7); GOLD, LEAD, MOLYBDENUM, TUNGSTEN.

AR 18, p. $346 \div 347$

B 225, p. 57

B 251, p. 59, 61-62

B 259 , p. 29

B 263, p. 14, 42,208

B 295, p. 21-22

B 314, p. 188, 193

B 379 , p. 54

B 442, p. 234, 236-237

B 480 , p. $160-162$

B 520, p. 245

B 538, p. 62-63

B 542, p. 212
B 622, p. $60-61$

B 642 , p. 63

B 662 , p. 56

B 692 , p. 37

B $897-$ A, p. 50

B 897-C, p. 206-208, 212-213, 218-219

B 910-A, p. 49, 76

B 926-A, p. $43-44,70$

B 917-A, p. 47

B 933-A, p. 40-41, 67

O 348 , p. 13

BMB 153, p. 53

(Mastodon Cr.) - (12.35-12.9, 7.95-8.95) ; GOLD, TIN.

AR 18, p. 347-349

B 225, p. 57

B 251, p. 59, 62-63

B 259, p. 29

B 263, p. 14, 42, 208

B 284 , p. 126

B 295, p. 22

B 314 , p. 188, 191, 194

B 345, p. 50

B 379, p. 54

B 442, p. 235

B 480 , p. 161

B 520, p. 244-245

B 538 , p. 63

B 542, p. 210-211

B 592, p. 68,360

B 622, p. $60-61$

B 642, p. 63

B 662 , p. 56

B 692, p. 37

B 712, p. 45

B 714, p. 87

B 739, p. 9,32

B 755, p. 14, 38

B 773, p. 27

B 783, p. 14,18

B 792, p. 18,25

B 797, p. 21,30

B 810, p. 28

B 813 , p. 31

B 824, p. $36,165-168$

B 836, p. 37

B 844-A, p. 37

B 857-A, p. 35

B 864-A, p. 38

B $868-A$, p. 38

B $880-A$, p. 43

B $897-$ A, p. 50

B 897-C, p. 206-208, 210-218

B 910-A, p. 49

B 917-A, p. 48

B .926-A, p. 44

B 933-A, p. 41

W 342, p. 181-182

C 348, p. 11

BMB 142, p. 25

TDM 1, p. 32

(Mastodon Fork). See (Eagle Cr.).

Mastodon Min. Co. See (Mastodon Cr.).

(Miller Cr.) - (12.15-13.05, 8.85-9.3) ; GOLD.
AR 18, p. 294, 349-350
B 692, p. 37
B 225 , p. 57
B 813, p. 31
B 263, p. 208
B 824, p. 36, 168-169.
B 295, p. 22-23
B 836, p. 37
B 314, p. 188, 194
B 844-A, p. 37
B 442 , p. 238
B 857-A, p. 35
B 480, p. 163
B 864-A, p. 38
B 538, p. 64
B $868-A$, p. 39
B 622, p. 61
B 880-A, p. 43 
(Miller Cr.) -Continued
B $897-$ A, p. 50
B 926-A, p. 41
B 897-C, p. 222-224
B 933-A, p. 41

B 910-A, p. 49

(Miller House) - $(13.1,9.3)$; COPPER, LEAD.

O 335, p. 5

(Nome Cr.) - (0.25-2.8, 5.95-6.35) ; GOLD, TIN. See also (Nome Cr.) under

Livengood quad.

B 480, p. 165

B 525, p. 150

B 844-A, p. 35, 54

B 542, p. 210

B 857-A, p. 34

B 797, p. 19,30

B $880-A$, p. 44

B 810, p. 26,40

B 926-A, p. 40, 43, 70, 72

B 813, p. 30,47

B 933-A, p. 38-39, 67

B 824, p. 35,52

C 331, p. 8

B 836, p. 35, 54

TDM 1, p. 32

Nome Cr. Dred. Co. See (Nome Cr.)

Nome Cr. Min. Co. See (Nome Cr.)

(Palmer Cr.) - (11.3-11.4, 0.1-0.5) (?) ; GOLD, TUNGSTEN.

B 910-A, p. 48

B 926-A, p. 40, 43

B $917-A$, p. 44,46

Peterson \& Co. See (Faith Cr.)

(Pitka Bar). See (Harrison Cr., North Fork).

(Porcupine Cr.) - (10.7-11.4, 9.75-9.95) ; GOLD, TIN:

AR 18, p. 350-351 B 897-A, p. 50

B 314, p. 198 B 897-C, p. 224-226

B 857-A, p. $35 \quad$ B 910-A, p. 49

B 864-A, p. $38 \quad$ B 917-A, p. 48

B 868-A, p. $39 \quad$ B 926-A, p. 44

B 880-A, p. 43

(Porcupine Dome) - $(10.5,9.05)$; GOLD.

B 897-C, p. 225-226

(Portage Cr.) - (17.4-17.55, 8.0-8.4) approx; GOLD, TIN, COPPER, TUNGSTEN, ZINC, BISMUTH, FLUORITE.

B 314, p. 198

B 542, p. 213

B 917-A, p. 48

B 592, p. 360

B 926-A, p. 44

B 864-A, p. 38

B 933-A, p. 41

B 897-A, p. 50

C 335, p. 5

C 348, p. 11, 13-14

B 897-C, p. 250-251

TDM 1, p. 32

B 910-A, p. 49

(Preacher Cr.) - (4-7, 7-9) (?) ; GOLD (?).

B 592, p. 360

(Shamrock Cr.) - (11.35-11.75, 0.1-0.65) (?). GOLD.

B 917-A, p. 46

B $926-A$, p. 43

(Sourdough Cr.) - (3.75-3.8, 6.05-6.4) ; GOLD, TUNGSTEN, ANTIMONY, TIN.

B 857-A, p. 30

B 864-A, p. 35

B 868-A, p. 36

B 926-A, p. 40, 42-43

B 933-A, p. 39

B 880-A, p. 39

C 331, p. 8

B 910-A, p. 48

C 348, p. 11

B 917-A, p. 43-44, 46

TDM 1, p. 14, 32 
(Squaw Cr.) or (Gulch) - (15.25-15.4, 6.7-7.35) ; GOLD."
B 314, p. 195
B 897-C, p. 231

B 538, p. 65

Swanson. See (Hope Cr.).

(Switch Cr.) - (15.5-15.65, 8.4-8.75) ; GOLD, LEAD, TUNGSTEN.
B 251, p. 59-60
B 844-A, p. 37
B 284, p. 126
B 857-A, p. 35
B 314, p. 192
B 864-A, p. 38
B 442 , p. $235,248-250$
B 868-A, p. 39
B 480 , p. 163
B $880-A$, p. 43
B 520, p. 245
B 897-A, p. 50
B 538, p. 60-62
B 897-C, p. 236-238, 245-248
B 542 , p. 212
B 910-A, p. 49
B 622, p. 61
B 917-A, p. 48
B 642, p. 63
B 824, p. 36, 161-164
B 926-A, p. 44
B 836, p. 37
C 335, p. 4
TDM 1, p. 37, 41

(Traverse Cr.) - (16.5-16:7, 6.4-7.5) ; GOLD. Nore.-Traversey Cr. on some maps.

B 897-C, p. 231

(Two Bit Gulch) - (15-16, 17) ; GOLD.

B 480, p. 164

(Xankee Cr.) - (11.3-11.4, 9.8-9.95) ; GOLD.

B 857-A, p. $35 \quad$ B $897-C$, p: 225

Zimmerman. See (Sourdough Cr.).

\section{COLEEN QUADRANGLE}

(Porcupine R.) - (15.85, 3.15) ; NICKEL. NoTE.-Nickeliferous alum.

TDM 1, p. 19

\section{CORDOVA QUADRANGIE}

A. C. Co. See Alaska Comm. Co.

Ajax. See Montezuma.

Alaska. See Steinmetz.

Alaska Comm. Co. - $(3.9,15.0)$; COPPER, ZỊNO, LEAD, GOTD, SILVER.

Includes references to A. C. Co., Alaska Pioneer \& Sourdough, Copper Mtn., Jacobson; see also (Bligh I.).
AR 20, p. 417-418
B 622, p. 133
B 284, p. 83-84
B 963-B, p. 59
B 542, p. 119-121
BMB 142, p. 43
B 592, p. 241
BMB 153, p. 50
B 605 , p. $13,52-54,56,70-71$, RI 5320, p. 2, 6, 18-19 107-108

Alaska Copper Corp. See Schlosser.

Alaska Mines Corp. See Schlosser.

Alaska Pioneer \& Sourdough. See Alaska Comm. Co.

Alaska Standard Copper Min. Co. See Standard Copper Mines Co.

Alice T. See Standard Copper Mines Co.

American Girl. See Galena Bay Min. Co.

Anvil. See Galena Bay Min. Co.

Apex. See Standard Copper Mines Co.

Banta \& Cameron. See Mogul.

593018-61—5 
Banzer - $(3.85,14.35)$; GOLD, COPPER, ZINC, LEAD.

B 542, p. 123-124
B 605, p. 112

B 605, p. 112

Beach. See Reynolds-Alaska Dev. Co. (Landlocked Bay).

Bear Cr. Min. Co. - $(15.65,8.65)$; GOLD.

B 542, p. 79-80

Black Bear. See Dickey Copper Co.

Blakney. See Fidalgo (Min. Co.).

Blakney \& Herren. See Fidalgo (Min. Co.).

(Bligh I.) - $(2.25,14.65)$ ( ?) ; GOLD.

B 542, p. 123

B 605, p. 112

(Bremner R.) - (24.15, 17.9) ; GOLD.

B 520, p. 99-100

B 576, p. 47-48

Buckeye. See Montezuma.

Burke \& Steele. See Standard Copper Mines Co.

Centaur. See Reynolds-Alaska Dev: Co. (Landlocked Bay).

Chisna Consol. Min. Co. - $(4.05,14.8)$; COPPER.

B 605, p. 111-112

Cliff. See Montezuma.

(Cloudman Bay) - $(2.3,14.6)$ ( ?) ; GOLD, COPPER, ZINC. B 605, p. 112

Comet. See Standard Copper Mines Co.

Copper Crown. See Galena Bay Min. Co.

Copper King. See Ellamar.

Copper Mtn. See Alaska Comm. Co.

(Copper Mtn.) - (3.65, 15.15) (?) ; COPPER.

B 284, p. 82

Cordova Copper Co. - $(10.95,10.1)$; COPPER. Includes references to (Fleming Spit).

B 284, p. 84

B 442, p. 165

B 443, p. 54,70

B 692 , p. 149

Cordova-Tacoma Copper Co. See Head-of (-the)-Bay.

Cornet. See Standard Copper Mines Co.

Daisy. See Montezuma.

Dickey. See Threeman.

Dickey Copper Co. - $(5.0,13.5)$; COPPER, GOLD, ZINC. Includes references to Mason \& Gleason.
B 520 , p. 27
B 542, p. 34
B $642 ;$ p. 141
B 592, p. $62,241-243$
B 662, p. 184, 187
B 605, p. 119-122
B 692, p. 144, 149
B 622, p. 133-134
B 714, p. 22
B 963-B, p. 62

Discovery. See Threeman.

Dolan \& Rystrom. See Landlock Bay Copper Min. Co.

Elgin. See Fidalgo (Min. Co.).

Ellamar (Min. Co.) - $(2.55,15.75)$; COPPER, ZINC, LEAD, GOLD, SILVER. Includes references to Gladhaugh.
AR 20, p. 418-419
B 442, p: 32, 39, 164
B 284, p. 82,87
B 443 , p. 52-53, 56-57, 59-61, 78
B 314, p. 27
B 480, p. 31-32, 81
B 345, p. 178
B 520, p. 27
B 379, p. 87-88, 94-95
B 542, p. 97, 100-102, 105-108 
Ellamar (Min. Co.)-Continued

B 592, p. 62, 240-241

B 605 , p. 13-14, 51-55, 57-63, 6668, 71-72, 87-92

B 622, p. 45, 131-133

B 642 , p. 138,140

B 662 , p. $44,184-186$

B 692, p. 31, 144, 147

B 712, p. 35
B 714, p. 22, 69, 77

B 722, p. 20

B 963-B, p. 55-56

B 989-E, p. 228, 296-298, 302

BMB 142, p. 39, 52

BMB 153, p. 44-45

IC 7379, p. 33

Falck - $(3.85,15.15)$; COPPER, zINC.

B 605, p. 72, 103-104

Fidalgo. See Hemple Copper Co., Schlosser. Nore.-Two claims by same name.

Fidalgo (Min. Co.) - $(6.15,14.1)$; COPPER, GOLD. NoTE.-Includes references to McIntosh. This mine is commonly called Fidalgo Mine; for data on other claims named Fidalgo, see Hemple Copper Co., Schlosser.
B 379 , p. 96
B 712 , p. 33
B 443, p. 63
B 714, p. 22, 69, 77
B 542, p. 34
B 592, p. 62, 240-242
B 722, p. 21,40
B 605, p. 113-117
B 622 , p. 134
B 642, p. 138,141
B 739, p. 24
B 755, p. 29
B 963-B, p. 61-62
BMB 142, p. 39, 52
B 662, p. 184, 186
BMB 153, p. 45-46

B 692 , p. $31,144,148$

Fidalgo-Alaska Copper Co. See Schlosser.

Fielder \& Hemple - $(3.2,15.55)$; COPPER.

B 379, p. 94

B 542, p. 114-115

B 605, p. 57,98

(Fleming Spit). See Cordova Copper Co.

Forget-me-not. See Galena Bay Min. Co.

Galena Bay (Min. Co.) - (3.45-3.6, 15.4-15.55) ; COPPER, GOLD, ZINC. Includes references to (Vesuvius Valley).
B 284 , p. 83
B 345, p. 178
B 605 , p. $98-102$
B 622, p. 133
B 379, p. 93
B 642, p. 140
B 442, p. 164
B 963-B, p. 53-54
B 443, p. 59
BMB 142, p. 40, 52
B 542, p. 112-114
BMB 153, p. 46

Glacial I. Copper Co. - $(1,15)(?)$; COPPER.

B 480, p. 31

Gladhaugh (\& Peterson). See Ellamar.

Gladhaugh Bay. See Ellamar.

Gleason. See Dickey Copper Co.

Grizzly. See Reynolds-Alaska Dev. Co. (Landlocked Bay).

Grove. See Hoodoo.

Head-of (-the)-Bay - $(12.45,11.65)$ (?) ; COPPER.

B 442 , p. 165

B 443, p. 70

Hemple. See Hemple Copper Co.

Hemple Copper Co. - $(3.9-3.95,15.0)$; COPPER, GOLD, SILVER, ZINC.

Includes references to Hemple, Hemple Min. Co.

B 379 , p. 95

B 520, p. 27
B 542, p. 118-119

B 605, p. 57-58, 106-107 
Hemple Copper Co.-Continued
B 622 , p. 133
B 783, p. 21
B 662, p. 186
B 963-B, p. 59
B 692, p. 148
RI 5320, p. 6, 18

Hemple Min. Co. See Hemple Copper Co.

Hendrie \& Simenstad. See Galena Bay Min. Co.

Homestake. See Galena Bay Min. Co.

Hoodoo - $(3.85,14.85)$; GOLD, COPPER, ZINC.

B 542, p. 122-123

B 605, p. 111

Hornet's Nest. See Galena Bay Min. Co.

Irish Cove (Copper Co.) - $(4.9,13.45)$; COPPER. Note.-Probably the same property as Dickey Copper Co., which see also.

BMB 142, p. 52

(Jack Bay) - $(4.8,17.5)$ approx ; GOLD.

B 692, p. 173

Jacobson. See Alaska Comm. Co.

Jay. See Threeman.

Keystone. See Threeman.

Landlock Bay Copper Min. Co. - $(3.85,14.85)$; COPPER, ZINC.

B 542, p. 97, 110-112

B 692, p. 148

B 592, p. 62, 241

B 963-B, p. 57-58

B 605, p. 14, 51, 96-97

BMB 142, p. 41, 52

B 622 , p. 133

BMB 153, p. 48

B 642, p. 141

RI 5320, p. 5-6, 17-18

B 662 , p. 186

Last Chance. See Hemple Copper Co.

Lone Hand. See Galena Bay Min. Co.

Lucky Strike Min. Co. - $(15.65,8.35)$; GOWD.
B 542, p. 79
B 824, p. 23
B 797, p. 12
B 836, p. 22

Iucky Strike Syn. See Lucky Strike Min. Co.

McIntosh. See Fidalgo (Min. Co.).

McKinley Lake Min. Co. - $(15.6,8.3)$; GOLD.

B 542, p. 79

Mason (\& Gleason). See Dickey Copper Co.

Minnehaha. See Galena Bay Min. Co.

Minnewaska. See Galena Bay Min. Co.

Mogul - $(3.3,15.65)$; COPPER, ZINC.

B 605, p. 98

Montezuma - $(3.75,15.1)$; COPPER, ZINC, GOLD. Includes references to Buckeye.
B 542, p. 121
B 692, p. 148
B 592, p. 241
B 963-B, p. 59
B 605, p. 108-109
BMB 142, p. 43
B 622, p. 133
BMB 153, p. 50
B 662, p. 184, 186
RI 5320, p. 19

Moonshine. See Landlock Bay Copper Min. Co.

Nero. See Hemple Copper Co.

Odelle D. See Standard Copper Mines.

Panama. See Fidalgo (Min. Co.).

Pioneer. See Steinmetz.

Porcupine. See Reynolds-Alaska Dev. Co. (Landlocked Bay). 
Prince William Sound Min. Co. See Galena Bay Min. Co.

Putz \& Steinmetz. .See Reynolds-Alaska Dev. Co. (Landlocked Bay),

Steinmetz.

Redemption. See Threeman

Reynolds-Alaska Dev. Co. (Boulder Bay) - $(3.2,15.5)$; COPPER.
B 345, p. 178
B 542, p. 115
B 379, p. 94
B 605, p. 57, 61, 102-103
B 443, p. 52,61
B 963-B, p. 54-55

Reynolds-Alaska Dev. Co. (Landlocked Bay) - $(3.8,15.25)$; COPPER.

$\begin{array}{ll}\text { B 284, p. 82-83 } & \text { B 963-B, p. 59 } \\ \text { B 542, p. 115-116 } & \text { RI 5320, p. 5 } \\ \text { B 605, p. 102-103 } & \end{array}$

Schlosser - $(4.95,13.5)$; COPPER, ZINC. Includes references to FidalgoAlaska Copper Co.
B 379, p. 96
B 692, p. 31, 144, 148-149
B 443, p. 63
B 712, p. 33
B 520, p. 27
B 714, p. 22, 69-70
B 542, p. 34
B 722, p. 21, 40
B 592, p. 62, 240-242
B 963-B, p. 60-61
B 605, p. 117-120, 122
B 989-E, p. 298, 302
B 642, p. 141
BMB 142, p. 38, 52
B 662, p. 184, 186-187
BMB 153, p. 43

Scorcher. See Threeman.

Sheep Run. See Galena Bay Min. Co.

Sourdough. See Steinmetz.

Spitz. See Galena Bay Min. Co.

Standard (Copper Mines Co.) - (3.65-3.7, 15.1); COPPER, ZINC, GOLD, SILVER.
B 284, p. 84
B 622, p. 133
B 379, p. 95
B 442, p. 39, 165
B 642, p. 141
B 662, p. 186
B 443, p. 52, 61-63
B 692, p. 148
B 520, p. 27
B 963-B, p. 58-59
B 542, p. 116-118
RI 5320, p. 2, 5-6, 16-17
B 605, p. 57, 61, 104-106
Standard Mines Co. - (S $1 / 2$ 22, 7) ( ?) ; GOLD.
B 576, p. 51

Starvation. See Galena Bay Min. Co.

Steinmetz - $(3.95,14.9)$; COPPER.

B 605 , p. $110-111$

RI 5320, p. 18

Stringer. See Lucky Strike Min. Co.

Summit. See Galena Bay Min. Co.

Sunnyside. Sce Galena Bay Min. Co.

Sylvar. See Hemple Copper Co.

Tacoma-Cordova Mines Co. See Cordova Copper Co.

Threeman - $(4.0,15.0)$; COPPER, LEAD, ZINC, NICKEL, COBALT, SILVER.

Includes references to. Dickey, Keystone, Threeman Min. Co. unless unmistakably to a different prospect.
B 379, p. 95
B 542, p. 97, 101-102, 108-110
B 442 , p. 165
B 592, p. 62,241
B 443, p. $52,54-55,61-62$
B 520, p. 27
B 605 , p. 14, 51, 56-57, 61, 70-72, 92-96 
Threeman-Continued

B 642, p. 138

B 662, p. 184, 186

B 692, p. 144, 148

B 712, p. 33

B 739, p. 24

B 963-B, p. 57
B 989-E, p. 298, 302

BMB 142, p. 43, 52

BMB 153, p. 50

RI 5245, p. 1, 5-6

RI 5320, p. 3, 5-7, 16, 19-21

Threeman Min. Co. See Alaska Comm. Co., Hoodoo, Montezuma, Threeman, Threeman Min. Co. (Billygoat Mtn.), Threeman Min. Co. (south of Landlocked Bay).

Threeman Min. Co. (Billygoat Mtn.) - $(4.25,14.5)$; COPPER. B 542, p. 122 B 605 , p. 109-110

Threeman Min. Co. (south of Landlocked Bay) - (3.95, 14.8) ; COPPER. B 284, p. 84 B 542, p. 121-122 B 605, p. 109

Tiger. See Reynolds-Alaska Dev. Co. (Landlocked Bay).

Tiptop. See Lucky Strike Min. Co.

Vesuvius. See Galena Bay Min. Co.

(Vesuvius Valley). See Galena Bay Min. Co.

Virginia. See Standard Copper Mines.

Whalen \& Nelson - $(7.4,14.3) \cdot(?)$; COPPER. B 379, p. 96 B 443, p. $62-63$

White Hollow. See Galena Bay Min. Co.

(Wilson Pt.) - $(10.6,11.1)$ ( ?) ; GOLD, SILVER. AR 20, p. 421

Winchester. See Fidalgo (Min. Co.).

Wrangle. See Galena Bay Min. Co.

Yellow Dog. See Galena Bay Min. Co.

\section{CRAIG QUADRANGIE}

Alameda - $(23.8,3.3)$ approx ; GOLD. Includes references to Tom Boy ; see also Frisco (Kitkun Bay).
P1, p. 85
B 347, p. 170

Alarm - $(20.15,10.5)$; COPPER, IRON. Includes references to Eagle's Nest; see also It.

P 87, p. 94

B 345, p. 109
B 347 , p. 118-119

B 379 , p. 79

Alaska - (24.5-24.75, 11.65-12.3) (?) ; GOLD. May be in Ketchikan quad.

B 755, p. 23

Alaska British Columbia Gold Mines, Ltd. See Valparaiso.

Alaska (Consol.) Copper Co. See Copper Mtn., Rush \& Brown.

Alaska Consol. Min. \& Smelt. Co. See Copper Mtn.

Alaska Galena Co. See Moonshine (Cholmondeley Sound).

Alaska Gold \& Metals Co. See Rush \& Brown, Salt Chuck.

Alaska Industrial Co. See Green Monster, Jumbo (Hetta Inlet).

Alaska-Kassan Gold Min. Co. See Dawson.

Alaska Metals (Min.) Co. See Corbin.

Alaska Palladium (Min.) Co. See Salt Chuck.

Alaska Shamrock Marble Co. See (Dickman Bay), (Shamrock Inlet).

Alaska Tidewater Co. See. Florence, Teresa.

Alcoa Min. Co. See (Edna Bay). 
Alexander. See Alexandria.

Alexandria - $(24.45,11.35)$; GOLD (?). Includes reference to Alexander.

$$
\text { B 284, p. 44-45 }
$$

B 347, p. 155-156

Allen \& Angleson. See Stumble-On.

Alpha - $(24.6,3.0)$ approx; GOLD, COPPER.

$$
\text { P 1, p. } 82 \quad \text { B 347, p. } 175
$$

Aluminum Corp. of America. See (Edna Bay).

Amazon - (24.6, 3.0) approx ; GOLD.

$\begin{array}{ll}\text { B 259, p. } 64-65 & \text { B 347, p. 174-175 } \\ \text { B 284, p. } 43 & \text { B 379, p. 73 } \\ \text { B 314, p. } 62 & \text { B } 642, \text { p. } 81\end{array}$

American Coral (Marble) Co. See (Dolomi), (North Arm).

Anderson (Cholmondeley Sound) - $(22.65,5.0)$ approx; COPPER (?).

$$
\text { P 1, p. 88, } 95
$$

Anderson (Kasaan Penin.). See Poorman.

Anderson (McKenzie Inlet) - $(21.25,6.25)$; COPPER.

$$
\text { P 1, p. } 96
$$

Andrews. See Mt. Andrew.

(Baker I.) - (9.2-9.3, 5.6-5.85) ; MOLYBDENUM.

$\begin{array}{ll}\text { B 836, p. 81 } & \text { B 926-C, p. 166-167 } \\ \text { B 857-A, p. 75 } & \text { B 947-B, p. 31-36 } \\ \text { B 864-A, p. 79 } & \text { B 1058-A, p. 24, 27 } \\ \text { B 868-A, p. 82 } & \text { C 248, p. 9, 11 }\end{array}$

Beauty - $(24.6,3.0)$ approx ; GOLD, SILVER, COPPER.
P 1, p. 80
B 592, p. 81

B 347, p. 175

Beaver - $(18.8,5.4)$ approx ; GOLD, COPPER:

$$
\text { P 1, p. } 107
$$

Bendigo - (W 1/2 18,9); GOLD(?).

$$
\text { B } 284, \text { p. } 42
$$

Bertha - $(20.95,5.4)$ approx; COPPER.

$$
\text { P 1, p. } 95
$$

Beulah - $(24.6,3.0)$ approx ; GOLD, SILVER (?).

$$
\text { P 1, p. } 82
$$

Big Five - $(20.75,11.6)$ approx ; COPPER.
P 87, p. 101-102
B 347, p. 127
B 345, p. 112

Big Harbor - $(15.2,6.65)$; COPPER. Includes references to Northland Dev. Co.
B 480, p. 102
B 520, p. 26
B 642, p. 91-93
B 542, p. 33
B 662 , p. $69-70$
B 592, p. $60,84-85$
B 692, p. 89
B 622, p. 41-42
B 714,p. 18
B 963-A, p. 15-17

Bix Six - $(22.85,8.85)$ approx ; COPPER.

P 1, p. 103

Black Marble. See (Tokeen) (marble).

Black Point - $(24.2,1.2)$ approx ; GOLD (?).

B 592, p. 82

Black Warrior. See Green Monster.

Blackbird - $(20.5,10.15)$ ( ?) ; COPPER ( ?). B $642, p .86$

Blue Bird. See Westlake. 
Blue Jay (Helm Bay) - (24.5-24.75, 11.65-12.3) (?) ; GOLD. May be in Ketchikan quad.
B $917-$ A, p. 22
B 933-A, p. 19

B 926-A, p. 20

Blue Jay (Kasaan Penin.). See Stevenstown.

Blue Marble. See (Tokeen) (marble).

Bradford. See Niblack.

Bradley. See London.

(Breezy Bay) - $(14.3,2.6)$; MARBLE.

B 682, p. 80

TDM 6 p, p. $28,49,51-52$

Brown. See Stumble-On.

Brown-Alaska Co. See Crackerjack, Mamie, Stevenstown.

Brown \& Metz. See (Noyes I.).

Brown \& Metzdorf - $(20.05,10.6)$; COPPER. Includes references to Brown \& Newell.
P 87, p. 73,97
B 347, p. $120-121$
B 345, p. 113
B 379, p. 79

Brown \& Newell. See Brown \& Metzdorf.

Bruce - $(18-19,2-4)$; COPPER.

B 622, p. 41

Buckhorn - $(18.1,9.55)$; GOLD.

B 284, p. 42

I 232

B 347, p. 165

Buffer. See Rich Hill.

Building. See (Tokeen) (marble).

Burke \& Lang - $(18.4,8.8)$; GOLD (?). B 662, p. 65

Cachelot - $(23.35,8.55)$; COPPER, GOLD, SILVER.

P 1, p. 103-104

Cascade - $(17.85,8.6)$ ( ?) ; GOLD, ZINC, LEAP.
B 259, p. 67
B 347, p. 161-162
B 622, p. 42
Charles - $(20.05,10.85)$; COPPER, GOLD, SILVER. P 87, p. 73, 100

B 347, p. 120

B 345, p. 113

Chicago Kid - (24.6, 3.0) approx; GOLD (?).

B 642, p. 81

Chomley Min. Co. See Moonshine (Cholmondeley Sound).

Cimru. See Cymru.

Clifton. See Minnetonka.

Clipper - (18.15, 9.4) ( ?) ; GOLD. B 347, p. 165

(Coco Harbor) - $(14.3,1.0)$; MARBLE. B 642, p. 102 B 682, p. 82

\section{232}

TDM 6, p. 28, 40, 42-43

Coleman (and assoc.). See Blackbird, Kansas, Poorman.

Commander. See Lucky Nell.

Commonwealth. See Mt. Andrew.

Complex. See Lucky Boy.

Conondrum. See Lookout.

Constitution - (16.5-17.5, 9.0-10.5) approx ; GOLD, COPPER, LEAD, ZINC.

P 1, p. 94

B 347, p. 163 
Cook - (24.6, 3.0) approx; GOLD (?).

B 642, p. 81

Copper (Granite Mtn.) - (W $1 / 2$ 18, 9) ; GOLD (?).

B 284, p. 42

Copper (Lake Bay). See McCullough.

Copper Center - $(19.8,11.05)$; COPPER, IRON.

P 87, p. 94, 100

B 345, p. 112-113

B 379, p. 79

B 347, p. 120

B 520, p. 26

Copper Chief - $(23.65,1.55)$ approx ; COPPER, GOLD. See also Niblack.

P 1, p. 77

Copper City - $(19.0,2.65)$ approx; COPPER, GOLD, SILVER, ZINC. Include's references to Red Wing.

P 87, p. $46-47,55,64$

B 284, p. 51-53

B 480 , p. 101

B 314, p. 71

B. 520, p. 26

B 345, p. 95

B 542, p. 33

B 347 , p. 106-107

B 622 , p. 41

B 379 , p. $81-82$

B 714, p. 18

B 800, p. 318,370

Copper Cliff. See Dama.

Copper Harbor Co. See Copper Mtn.

Copper Hill - (18.05, 8.85); COPPER, GOLD(?). Includes references to Copperplate.

P 1, p. 90

I 231

B 662 , p. $65-66$

Copper King - $(20.55,10.1)(?)$; COPPER, IRON, GOLD.

P. 1. p. $99-100$

Copper Lake - $(24.6,3.0)$ approx ; COPPER, SILVER, GOLD.

P 1, p. 82

Copper Mtn. - (18.8-19.05, 4.05-4.4); COPPER, GOLD, SILVER, GARNET, IRON.

Includes references to Alaska (Consol.) Copper Co., Coppermount, Indiana, New York.
P 1, p. 105-107
P 87, p. 50, 55-58
B 259, p. 64
B 284, p. 51-52
B 314, p. 76
B 345 , p. 94
B 347 , p. $96-98$

B 542, p. 33

B 592, p. 84

B 622, p. 41

B 642 , p. 91

B 714, p. 17

IC 7844, p. 7,11

RI 3952, p. 2

Copper Queen - $(21.0,9.7)$; COPPER.

P 1, p. 100

B 314, p. 68

P 87, p. 94-95

B 345 , p. 108

B 284, p. 48

B 347 , p. $117-118$

Coppermount. See Copper Mtn.

Copperplate. See Copper Hill.

Corbin - $(18.5,4.3)$; COPPER, GOLD, SILVER. Includes references to Corwin.
P 87, p. $43,47,55-56,63-64$
B 347 , p. $105-106$
B 284, p. 51-52
B 379 , p. $81-82$
B 314, p. 71
B 714, p. 18
B 345, p. 95
B 800, p. 318,370 
(Coronation I.) - $(1.7,16.0)$ approx ; LEAD, ZINC.
B 345, p. 97
B $963-A$, p. $38-40$
B 347 , p. $190-191$
B 1058-A, p. 24 .
B 642 , p. $98-99$
C 248 , p. 11

Coronation I. Min. Co. See (Coronation I.):

Corwin. See Corbin.

Crackerjack - $(17.9,8.8)$ approx ; GOLD, SILVER, LEAD, ZINC.
P 1, p. 91
B 259 , p. 66
B 284, p. .42
B 314, p. 62
B 345 , p. 92
B 347 , p. $160-161$
B 480 , p. 70

B 592 , p. 79

B 622, p. 42

B. 642 , p. 80

B 662, p. 65

B 868-A, p. 17

I 231

Croesus - $(23.35,3.2)$ approx ; GOLD.

P 1 , p. $85-86$

B 592 , p. 80

B 347 , p. $170-171$

Cuprite Copper Co. See Houghton.

Cutter - $(18.15,9.5)$ (?) ; GOLD.

B 284 , p. 42

B 347, p. 165

I 232

Cymra. See Cymru.

Cymru (Min. Co.) - $(23.25,2.8)$; COPPER. Includes references to Cimru, Cymra.
B 314 , p. 69
B 622, p. 42
B 345 , p. 96
B 642, p. $83,89-90$
B 347, p. 132-134
B 714 ; p. 18
B 379, p. 82
B 800, p. 317,370

(Dall I.) See (View Cove).

Dama - $(23.95,1.35)$; COPPER. See also Trio.

B 347, p. 131

B 963-A, p. 7-8

Dawson - $(17.85,8.5)$; GOLD, LEAD, ZINC, GRAPHITE. Includes-references to Alaska-Kassan Gold Min. Co., Dunton, Dutton, George, Harris Cr., Hendy, Humboldt, Julia, Kasaan Mines, Kasaan Gold (Min.) Co., Kassan Gold (Min.) Co., Keokuk, Last Chance (Dawson), Ro(d) gers.
P 1, p. 92
B 314, p. 62
B 345, p. 92
B 347, p. 161
B 379 , p. 79
B 442 , p. 139
B 480 , p. 98
B 592, p. $60,78-79$
B 622 , p. 42
B 642, p. 80
B 662 , p. 65
B 692, p. 28,88
B 712, p. 28.
B 714 , p. $127-128$
B 722, p. 35

B 739 , p. 20

B 755, p. 23

B 773 , p. 15

B 783, p. 7,41

B 792 , p. 10

B 797, p. 10

B 800 , p. 321, 371 .

B 810, p. 13,51

B 813 , p. 15-16

B 824, p. 18

B 836, p. 15

B 844-A; p. 16

B 926-A, p. $19-20$

BMB 142, p. 29, 52

Dean. See It. 
Dew Drop - $(16.85,9.25)$ approx ; GOLD, SILVER.
B 259, p. 66
B 314, p. 62
B 284, p. 42
B 347, p. 163

Diamond B. See Green Monster.

(Dickman Bay) - $(22.35,0.7)$ approx ; MARBLE.
B 542, p. 68-70
B 783, p. 61
B 682, p. 86-90
Dolly Varden - $(17.95,6.25)$ approx ; COPPER, GOLD.
P 1, p. 93
B 347, p. 162
(Dolomi) - $(24.7,2.85)$ approx ; MARBLE.
B 284, p. 56-57
B 314 , p. 76
B 347, p. 196-197
B 345, p. 120-121
B 542, p. 67
B 682, p. 85-86

Dolomi Gold Mines, Inc. See Valparaiso.

Dunton. See Dawson.

Dutton. See Dawson.

Eagle's Nest. See Alarm.

Earl No. 1 - (NW 1/8 20,4); commodity, if any, not known. P 1, p. 88

Edith M. - $(23.75,1.5)$ approx ; COPPER, GOLD. P 1, p. 77-78

(Edna Bay) - (7-8, 16-17) ; LIMESTONE.

C 18, p. 5

C 252, p. 12

IC 7379, p. 80

(Egg Harbor) See (Coronation I.)

Ella. See Marion.

Elm City - $(21.2,9.7)$; COPPER, GOLD.

P 1, p. 101

Equator - $(24.5,4.3)$ approx ; GOLD, COPPER. B 347, p. 168

Eureka. See (Hole in the Wall).

Excelsior - (NW 1/8 23,2); COPPER. May be old name for Cymru P 1, p. 79

Fawn. See Kid.

Flagstaff (Min. Co.) - (18.25-18.3, 9.6) ; GOLD, SILVER, COPPER, LEAD, ZINC. Includes references to Last Chance (Flagstaff), Treasure.
B 284, p. 41-42
B 917-A, p. 21-22
B 314, p. 62
B 926-A, p. 19-20
B 347, p. 164-165
B 933-A, p. 18-19
B 642, p. 80
B 963-A, p. 10-13
B 662, p. 65
I 232

Flora (\& Nellie). See Lucky Nell.

Florence - $(18.9,1.5)$ approx ; COPPER (?).

B 662, p. 69

Forest. See Niblack.

Fortuna. See Fortune.

Fortune - $(22.6,30)$ approx; GOLD, SILVER, COPPER. Includes reference to Fortuna.

P 1, p. 80

B 847, p. 175

B 592, p. 81
B 642 , p. 81

B 755, p. 23 
Fowlkes - (22.0, 5.0) (?) ; COPPER.

B 284, p. 50

Free Gold - $(24.75,12.0)(?)$; GOLD. May be in Ketchikan quad.

B 755, p. 23

Friendship - $(21.7,4.05)$ approx ; COPPER, GOLD.

P 1, p. 87

Frisco (Dora Lake). See Lucky Boy.

Frisco (Kitkun Bay) - $(23.8,3.3)$ approx; GOLD. Includes reference to Tom. Boy ; see also Alameda.

P 1, p. 85

B 347, p. 170

George. See Dawson.

Gervis - $(17.85,8.5)(?)$; GOLD.

B 520, p. 26

Gladstone - $(24.5,4.25)$ approx ; GOLD (?).

B 347, p. 166-167

Glory. See Mt. Andrew.

Go-by - $(18.0,9.8)(?)$; GOLD ( ?).

B 347, p. 165

Gold Standard. See Gold Standard under Ketchikan quad. Note.-Property is in both Craig and Ketchikan quads:

Golden Fleece (Min. Co.) - (24.55-24.7, 2.9-3.1) approx ; GOLD, SILVER.

P 1, p. 77, 81-82

B 259, p. 64-66

B 345, p. 92

B 284, p. 43

B 347, p. 175-176

B 314, p. 62

B 642, p. 81

Goodluck. See Mt. Andrew.

Goodro (Min. Co.). See Salt Chuck.

Gopher. See Haida.

Goshen. See Jumbo (Hetta Inlet).

Gould (Hetta Inlet) - $(19.05,4.05)$; COPPER.

P 87, p. 65

B 347, p. 107

B 379, p. 82

Gould (Sukkwan I.) - $(17.8,0.4)$ approx; COPPER. B 692, p. 88-89

(Gould I.) - $(18.85,5.1)$; COPPER, LEAD, ZINC.

P 87, p. 64-65 $\quad$ B 347, p. 107

B 345, p. 95

Granby Consol. Min., Smelt., \& Power Co. See It, Mamie, Rich Hill.

(Green Bay) or (Inlet) - (14.3, 1.2-1.3) ; LIMESTONE.

TDM 6, p. 28, 45-49

Green Monster - (19.55-19.8, 4.45-4.8) ; COPPER, IRON, LEAD, MOLYBDENUM. Includes reference to (Green Monster Mtn.).

P 1, p. 107

P 87, p. 43, 53, 58, 61-62

B 345, p. 95

B 259, p. 64

B 347, p. 102-103

B 284, p. 51-52

B 1058-A, p. 24, 27

C 248, p. 9,11

$\therefore$ B 314, p.71 $\quad \cdots \quad \therefore \quad$ RI 3952, p.4

Green Monster - (19.55-19.8, 4.45-4.8) ; COPPER, IRON, LEAD, MOrYBDE-

Grindall Min. \& Smelt. Co. : See Peacock (west of Mt. Andrew), Tacoma.

Hadley (Consol.) Copper Co: See Stevenstown. 
Haida (Copper Co.) - $(19.95,10.85)$; COPPER, IRON, MOLYBDENUM. Includes references to Hyda, Mammoth (near Kasaan).

$\begin{array}{ll}\text { P 87, p. 94, 100 } & \text { B 347, p. 119-120 } \\ \text { B 284, p. 48-49 } & \text { B 520, p. 26 } \\ \text { B 314, p. 67-68 } & \text { IC 7379, p. 31. }\end{array}$

B 345, p. 109-110

Hal. See Mt. Andrew.

Harris Cr. See Dawson.

Hatchet - $(20.55,7.9)$; GOLD. P 1, p. 96

(Heceta I.) - (9.9-10.1, 10.1-13.85) ; MARBLE.

B 542, p. 66

B 682 , p. 76-77, 113
B 783, p. 61

TDM 6, p. 29, 59-60, 62

Hecla - $(20.95,5.4)$ approx ; COPPER. P 1, p. 95

(Helm Bay) - (24.5-24.75, 11.65-12.3) ; GOLD. Also in Ketchikan quad. B 773, p. 10 B 824, p. 18

B 813, p. 16

Helm Bay Kíng (Min. Co.) - $(24.7,11.95)$; GOLD. B 773, p. 15, 72, 128

Hendy. Sec Dawson.

(Hetta Mtn.) - (19.1-19.25, 3.7-3.8) ; COPPER.

$$
\text { P 87, p. } 56
$$

B 347, p. 108

B 345, p. 95

Hickman \& Co. See Khayyam.

(High Point) - $(15.4,0.55)$; MARBLE.

B 682, p. 82

Hill, Calhoun \& Tucker. See Big Harbor.

Hilma. See (Hole in the Wall).

Hoffman - $(24.4,11.4)$; GOLD.

B 347, p. 155-156

(Hole in the Wall) - (21.75-21.9, 9.5-9.9) approx; COPPER. Note.-Area includes at least 6 prospects.

P 87, p. 97

B 345, p. 114

Hollis - $(17.9,8.7)$ approx ; GOLD.

P 1, p. 91-92

B 347, p. 161
B 347, p. 121-122

B 379, p. 79

B 714, p. 127

I 231

Home - (24.6, 3.0) approx; SILVER( ?).

P 1, 82

Home Camp. See (Tokeen).

Homestake. See Westlake.

Hope (Cholmondeley Sound) - $(21.25,3.45)$ approx; SILVER, LEAD, ZINC. B 284, p. 53-54

B 642, p. 82

B 800, p. $367-368$

Hope (Moira Sound). See Navaho.

Horseshoe. See McCollough.

Hougton - (18.7-18.75, 4.85-4.9); COPPER, IRON. Includes references to Cuprite Copper Co.

P 87, p. $50,56,62$

B 314, p. 71

B 347 , p. 103-104

B 345 , p. 94-95

B 379 , p. 82 
House - (24.5, 3.05) approx; COPPER.

P 1, p. 82

Humboldt. See Dawson.

Hyda. See Haida.

Idaho. See Lucky Boy.

Idela. See Rex.

Independent - (17.0, 9.5) approx; GOLD, LEAD, ZINC.

B 347 , p. $163-164$

Index. See Sultana.

Indiana. See Copper Mtn.

Interval. See Rich Hill.

Iola. See Green Monster.

Iron \& Copper. See Niblack.

Iron Cap - $(20.9,11.6)$ approx; IRON, COPPER. Includes references to (Tolstol Mtn.).
P 1, p. 104
P 87, p. 101
B 347 , p. $126-127$
RI 4373 , p. 1-5

B 345 , p. 111

Iron Cliff. See Rush \& Brown.

Iron Creek. See Venus (Karta Bay).

Iron Crown (Hetta Inlet) - $(19.1,4.2)$ approx; NICKEL, COBALT.

P 87, p. 49

Iron Crown (Tolstoi Mtn.) See Iron Cap.

Iron King. See Poorman.

It (Min. Co.) - $(20.15,10.4)$; COPPER, IRON. Includes references to Taylor ; see also Alarm.

P 87, p. 91, 94-95

B 345, p. 109

B 642 , p. $83-85$

B 347 , p. 119

B 662 , p. 64 .

B 379, p. $78-79$

B 692 , p. $28,85-86$

B 442 , p. 38,141

B 712, p. 28

B 480 , p. 101

B 714, p. 17,19

B 520 , p. 26

B 800 , p. $316-317,369$

B 542, p. 33

BMB 142, p. 30,52

B 622, p. 42

BMB 153, p. 25-26

Jackson. See McCullough.

Jefferson. See Niblack.

Jem. See Mt. Andrew.

Jessie. See Valparaiso.

Jim. See Mt. Andrew.

(Johnson Inlet). See (Dolomi).

Joker. See Salt Chuck.

Judge. See Niblack.

Julia. See Dawson.

Jumbo (Dolomi) - $(24.6,3.0)$ approx ; GOLD, SILVER. P 1, p. 80

B 642, p. 81

Jumbo (Hetta Inlet) - (18.6-19.0, 4.4-4.8) ; COPPER, GOLD, SILVER, IRON, MOLYBDENUM, ZINC.

P 1, p. 107

P 87 , p. $33,36,42,46,49,51,53$, 58-61, 106-107

P 251, p. 1, 3-4, 13-14, 22-23, 27-

$28,31,36-40,42$

B 259, p. 6 t 
Jumbo (Hetta Inlet) - Continued
B 284, p. 51-52
B 662, p. 68
B 314, p. 70-71
B 692, p. 28,88
B 345, p. 94
B 712, p. 28
B 347, p. 99-102
B 714, p. 17, 69
B 379, p. 81-82
B 755, p. 23
B 442 , p. 38,142
B 773, p. 36
B 480, p. 99, 101
B 800 , p. 316-317, 369
B 520, p. 26
B 926-C, p. 165-166
B 542, p. 33
BMB 142, p. 29, 52
B 592, p. 60, 83
BMB 143, p. 26
B 622, p. 41
RI 3952, p. 2-9
B 642, p. 83, 90
(Jumbo Basin). See Jumbo (Hetta Inlet).
Juneau - (18.0, 9.9) approx; GOLD (?).
B 347, p. 165

Kansas - $(20.5,10.15)$ (?) ; COPPER( ?).

B 642, p. 86

Kasaan Bay Min. Co. See Copper Queen.

Kasaan Gold (Min.) Co. See Dawson.

Kasaan Mines. See Dawson.

Kassan (Gold) Min. Co. See Dawson.

(Keete-Inlet) - (NW 1/4 20,1) ; COPPER.

B 642 , p. 90

Kennecott Copper Corp. See (Baker I.).

Keokuk. See Dawson.

Ketchikan Copper Co. - $(21.5,3.85)$ approx ; COPPER, GOLD, SILVER. P 1, p. 87-88

Keystone. See London.

Khayyam - (21.1, 5.55) ; COPPER, GOLD, ZINC, SILVER. Includes references to Kiam.
P 1, p. 94-95
B 347, p. 135-137
B 284, p. 49
B 800, p. 325
B 314, p. 69
RI 3942, p. 1-8

B 345, p. 96

Kiam. See Khayyam.

Kid - $(23.7,3.4)$ approx ; LEAD, ZINC, GOLD, COPPER. Includes reference to Fawn.
P 1, p. 85
B 347, p. 169-170

Kirman. See Poorman.

Knapp. See Moonshine (Cholmondeley Sound).

Koel. See Salt Chuck.

(Kosciusko I.) (northeast of Edna Bay) - $(9.0,17.0)$; MARBLE.

B 542, p. 60

B 682 , p. $65-66$

Lady of the Lake. See Lucky Boy.

Lake Bay. See McCullough.

Lake Vale. See (Tokeen).

Lake View. See Stumble-On.

Lakeside - $(24.3,3.0)$ (?) ; GOLD( ?).

B 314, p. 62

Last Chance (Dawson). See Dawson.

Last Chance (Flagstaff). See Flagstaff. 
Lavina. See Shelton.

Leibrant - (19.2, 11.3) (?) ; COPPER.

B 642, p. 85

Lichtenstadter. See Constitution, Mt. Andrew.

Lide. See Helm Bay King.

(Lime Point) - $(18.75,1.2)$; BARIUM. NoTE.-Not mentioned by name in B 622, 844-A.

B 622 , p. 43

B 800 , p. 333

B 642, p. 104

B 844-A, p. 81

B 662, p. $40,63,73$

B 963-A, p. 17-19

B 714, p. 54

BMB 153, p. 26

B 773, p. 138

IC 7844 , p. 9

Little Annie. See Westlake.

Little Annis. See Westlake.

London - $(24.65,11.2)$ (?) ; GOLD.

P 1, p. 57-58

B 347, p. 157

B 592, p. 86

(Long I.) - (21.1-21.7, 8.85-9.2) ; LIMESTONE, SHALE. Now called Kasaan I. B 345, p. 126

B 347, p. 200 .

Lookout - $(23.65,1.4)$ approx; COPPER, GOLD, SILVER.

P 1, p. 75-77

B 347, p. 131

B 259 , p. $62-63$

Lucky Boy - $(22.7,3.05)$ approx ; ZINC, LEAD, COPPER, GOLD, SILVER. Includes references to Complex, Frisco (Dora L.), Idaho, Lady of the Lake, Oregon (Dora L.).
P 1, p. 79
B 284, p. 53-54
B 692, p. 28
B 347, p. 171-172
B 800, p. 368
B 998-C, p. 73-78

Lucky Find - (18.0,9.5) approx; GOLD(?). B 347, p. 165

I 232

Lucky Jim - (18.1, 9.45) approx ; GOLD(?).
B 347, p. 165
I 232

Lucky Nell - $(16.8,9.15)$; GOLD, SILVER, COPPER, LEAD, ZINC. Includes references to Commander, Flora (\& Nellie), Nellie, President.
P 1, p. 92-93
B 259, p. 66
B 284, p. 42
B 314, p. 62

B 347, p. 162-163

B 592, p. 79

B 692, p. 88

Luella. See Niblack.

McCullough - $(14.75,17.2)$; COPPER, ZINC. Includes references to Lake Bay.

B 622, p. 42

B 642, p. 88-89

B 836, p. 16

B 755, p. 23

B 963-A, p. 13-15

B 783, p. 22, 41

B 1058-A, p. 24, 27

C 248, p. 9, 11

McGilveny. See Independent.

Maggie May. See Oregon (Kitkun Bay), Washington.

Magnet. See Rich Hill.

Mahoney. See Iron Cap.

Mamie - $(22.0,9.5)$; IRON, COPPER, GOLD, SILVER.
P 87, p. 86, 88-89, 92, 94
B 314, p. 67
B 259, p. 63
B 345, p. 103-104
B 284, p. 46
B 347, p. 112-114 
Mamie-Continued
B 379, p. 78
B 714, p. 17, 19
B 442, p. 142
B 800, p. 316-317, 369
B 622, p. 42
BMB 142, p. 30, 52
B 642, p. 83-84
BMB 153, p. 25-26
B 662, p. 64
IC 7379, p. 32
B 692, p. 28, 85
RI 4129, p. 4

B 712, p. 28

Mammoth (McKenzie Inlet). See Stumble-On.

Mammoth (near Kasaan). See Haida.

Mammoth (near Niblack). See Lookout.

Marble Heart - $(\mathbf{1 7 . 5 , 6 . 0 )}$ approx; LEAD.

P 1, p. 93

(Marble I.) - (10.05-10.15, 17.15-17.2) ; MARBLE. See also (Tokeen) (marble). B 542, p. 63-64

B 682, p. 72-74

Marion - $(20.25,2.85)$; COPPER, LEAD. Includes references to Nutqua Gold Min. Co.

B 642 , p. 90-91

B 963-A, p. 19-20

B 844-A, p. 16

Matilda - $(24.6,3.0)$ approx; GOLD.

P 1, p. 81

Mayflower. See Mt. Andrew.

Melville - $(24.4,11.3)$; GOLD (?).

B 347 , p. 155-156

Midnight Sun - $(24.65,11.9)$; GOLD.

B 347, p. 155

Miller - $(14.0,1.2)$ approx ; COPPER.

B 379, p. 83

Miller Bros. - (SE $1 / 4$ 18, 4) (?) ; COPPER, GOLD. P 1, p. 107

Minnetonka - (22.7, 3.05) approx; COPPER, GOLD, LEAD, ZINC, SILViER. Includes reference to Clifton. B 592 , p. 79

B 642, p. 81-82

Mission-Alaska Quarry Co. - $(10.6-10.65,16.85-16.9)$; MARBLE. NoTE.-Property on both sides of Marble Passage; includes references to Mission Marble Co., Mission Marble Works, (Orr I).
B 542, p. 64-65
B 622, p. 73
B 682, p. 74-76
B 783, p. 61

Mission Marble Co. See Mission-Alaska Quarry Co.

Mission Marble Works. See Mission-Alaska Quarry Co.

Moira Copper Co. See Wakefield.

Monday - $(18.6,8.9)$; GOLD, SILVER, LEAD. See also Stella.

P 1, p. 93

Moonshine (Cholmondeley Sound) - (21.3, 3.6) approx : SILVER, LEAD, ZINC, COPPER. Note. - Not mentioned by name in $B \quad 792$; includes reference to Knapp.
P 1, p. 88
B 314, p. 72
B 480 , p. 102
B 345, p. 97
B 520 , p. 26
B 347, p. 187-188
B 542, p. 33-34
B 379, p. 83-84
B 642, p. 82
B 739, p. 20

593018-61-6 
Moonshine (Cholmondeley Sound)-Continued
B 755, p. 16
B 800, p. 327, 367-368

B 792, p. 30

Moonshine (Dall I.) - (NE 1/4 13, 1) ( ?) ; SILVER, LEAD.

B 592, p. 92

Moonshine (near Dolomi) - $(24.6,3.0)$ approx; GOLD, COPPER.

$$
\text { B 592, p. } 81
$$

B 642, p. 81

Morning Star - $(20.5,10.1)$ ( ?) ; IRON, COPPER, GOLD. P 1, p. 100

Morning Vein. See (Tokeen) (marble).

Mt. Andrew - $(21.8,9.35)$; IRON, COPPER, GOLD, SILVER.

Includes references to Jim, North Star.
P 1, p. 102-103
B 642, p. 85
P 87, p. 86, 88-89, 91-94
B 662, p. 64-65
B 259, p. 63-64
B 692 , p. 28
B 284, p. 47
B 714, p. 17, 41
B 314, p. 67
B 345, p. 105-106, 113-114
B 864-A, p. 16-17
B 347, p. 115-117
C 252, p. 10
B 379 , p. 78
B 442, p. 38, 141-142
B 480, p. 76, 100
BMB 142, p. 30,52
BMB 153, p. 26-27
B 520, p. 26
IC 7379, p. 32, 44-46
RI 4129, p. 2, 4-27
B 542, p. 33
RI 5312, p. 2, 14
B 622, p. 42

B 800 , p. 316-317, 326, 329

Mt. Andrew Iron \& Copper Co. See Mt. Andrew.

Mt. Andrew Min. Co. See Mt. Andrew.

(Mt. Burnett) - (24.1-24.2, 13.65-13.8) ; IRON, CHROMITE. See also (Union Bay).
B 947-D, p. 80-83
C 252, p. 11

Mountain Bell - $(17.85,8.6)$ ( ?) ; GOLD.

B 347, p. 162

Myrtle. See Niblack.

Nancy - $(16.85,6.3)$; COPPER.

B 642, p. 93

Navaho - $(23.5,2.35)$ approx; GOLD. Includes references to Hope (Moira Sound).
P 1, p. 78
B 592, p. 82
B 347, p. 176-177

Nellie. See Lucky Nell.

New Era - (24.6, 3.0) approx ; GOLD( ?).

B 642, p. 81

New York. See Copper Mtn.

Niblack (Copper Co.) - $(23.65,1.55)$; COPPER, GOLD, SILVER, ZINC, LEAD.

Includes reference to Judge; See also Copper Chief.

$\begin{array}{ll}\text { B } 259, \text { p. } 62-63 & \text { B } 442, \text { p. } 143 \\ \text { B } 284, \text { p. 50 } & \text { B } 592, \text { p. } 82 \\ \text { B 314, p. 70 } & \text { B } 642, \text { p. } 90 \\ \text { B 345, p. } 95-96 & \text { B } 963-\text { A, p. } 8 \\ \text { B 347, p. 129-131 } & \text { IC } 7844, \text { p. } 11 \\ \text { B 379, p. } 82 & \end{array}$


(North Arm) - (23.3, 2.65) approx ; MARBLE.
B 259 , p. 68
B 347, p. 196-197
B 284, p. 56-57
B 542, p. 67
B 314, p. 76
B 682, p. $85-86$

B 345, p. 120-121

North Star. See Mt. Andrew.

Northland (Dev. Co.). See Big Harbor:

(Noyes I.) - (7-8, 9) ; MOLYBDENUM, COPPER, NICKEL. Includes reference to Brown \& Metz.
B 347, p. 87
B 926-C, p. 167-168

B 800, p. 329

(Nutkwa Lagoon). See Marion.

Nutqua Gold Min. Co. See Marion.

O.K. - $(24.6,4.0)$ approx ; GOLD, COPPER, ZINC, LEAD.

B 347, p. 168

Omar Min. Co. See Khayyam.

Oregon (Dora L.). See Lucky Boy.

Oregon (Hetta Inlet). See Copper Mitn.

Oregon (Kitkun Bay) - $(23.6,3.4)$ approx ; GOLD, SILVER, ZINC, COPPER. P 1, p. 85

B 347, p. 168-169

(Orr I.). See Mission-Alaska Quarry Co.

Ouray. See Rich Hill.

Pacific Coast Cement Co. See (View Cove).

Palladium. See Salt Chuck.

Paris - $(19.05,4.0)$; COPPER, GOLD.

P 87, p. 65

Park View - $(24.45,4.1)$ approx ; COPPER, GOLD.

B 347 , p. 168

Paul. See Valparaiso.

Pauline - $(24.3,3.0)$ approx ; GOLD.

P 1, p. 82-83

B 592, p. 81

Peacock (Mt. Andrew). See Mt. Andrew. Note.-May be confused with Peacock west of Mt. Andrew.

Peacock (west of Mt. Andrew) - (21.55, 9.4) approx; COPPER. NoTE.-May bo confused with Peacock (Mt. Andrew).

P 87, p. 97

B 284, p. 47

B 347, p. 121

Pelaska. See (Hole in the Wall).

Pennsylvania. See (Hole in the Wall).

Pitcher \& Anderson. See Marion.

Plumley. See (Hole in the Wall).

Poorman - $(20.55,10.1)$; IRON, COPPER, GOLD, SILVER.

P 1, p. 100-101

P 87, p. 95-97

B 642, p. 86

B 284, p. 48

B 314, p. 68

B 714, p. 17

B 345 , p. $108-109$

B 800 , p. $316-317,369$

B 347 , p. 118

BMB 153, p. 29

B 379, p. 79

IC 7379 , p. $44-46$

RI 3956, p. 1-8

RI 5312, p. 1, 5-7, 9, 14

(Port Alice) - $(8.8,10.3)$; LIMESTONE.

TDM 6, p. 29, 56, 58-59 
(Port San Antonio) - $(8.85,5.85)$; ZINC, LEAD, GOLD ( ?). B 347, p. 182

B 947-B, p. 35

Portland - (22.7, 3.05) approx ; COPPER, GOLD, LEAD, ZINC, SILVER. B 592, p. 79

B 692, p. 81-82

Portland Superior Cement Co. See (View Cove).

President. See Lucky Nell.

Princeton Min. \& Mil. Co. See Valparaiso.

Puyallup - $(17.9,8.95)$; GOLD
P 1, p. 90
B 259, p. 66
B 642 , p. 79
B 284, p. 42
B 662, p. 65
B 314, p. 62
B 868-A, p. 17
B 347 , p. $159-160$
I 231
B 592 , p. 79
BMB 142, p. 30, 52
BMB 153, p. 28

B 622, p. 42

Puzzler - $(24.5,11.65)$; GOLD (?).

B 347, p. 155

Quartzite Ledge - $(24.25,11.9)$ ( ?) ; GOLD (?).

B 592, p. 87

Ready Bullion. See Puyallup.

Red Jacket - $(16.7,9.05)$; GOLD ( ?).

B 259, p. 66

B 347, p. 163

Red Rose - $(20.95,5.4)$ approx ; COPPER.

P 1, p. 95

Red Snapper. See Rich Hill.

Red Wing. See Copper City.

Reed - $(20.15,10.45)($ ?) ; COPPER. See also Alarm, It. B 379 , p. 79

Rex (SW1/4 NE1/4 19, 4) ; COPPER, IRON. Includes reference to Idela. B 662, p. 68

Reynolds \& Wright. See Jumbo (Hetta Inlet).

Rich Hill - $(21.4,9.55)$; COPPER, GOLD, SILVER. Includes references to Ouray.
P 87, p. 94
B 379, p. 79
B 712, p. 28
B 836, p. 15

B 692, p. 28,87

Rico. See Mt. Andrew.

Rodgers. See Dawson.

Roman - (18.4, 7.9) ( ?) ; COPPER, GOLD, SILVER. B 284, p. 48

Rosalie - $(18.4,7.9)$ ( ?) ; COPPER, GOLD, SILVER. B 284, p. 48

Rose - $(16.85,9.25)$ approx ; GOLD, SILVER.
B 259, p. 66
B 347, p. 163

B 284, p. 42-43

Rush \& Brown - (18.95-19.0;11.2) ; COPPER, IRON, GOLD, SILVER.

P 87, p. 57, 73, 77, 85-86, 90, 98-99 B 442, p. 142

B 284, p. $48-49$

B 314, p. 68

B 480, p. 77-78, 102

B 345 , p. 106-107

B 520, p. 26

B 347, p. 123-125

B 542, p. 33

B 379, p. 78-79

B 592, p. 60,77

B 622, p. 41 
Rush \& Brown-Continued

B 642, p. 83, 86-88

B 662, p. 65

B 692, p. 28,86

B 712, p. 28

B 714, p. 17-18, 69, 76, 119-121

B 722, p. 21, 34-35

B 739, p. 10, 20

B 755, p. 15, 23

B 773, p. 28, 36
B 800, p. $316-318,369$

B. 824, p. 17-18, 59

B 836, p. 62

B 868-A, p. 17

I 230

BMB 142, p. 30-31, 52

BMB 153, p. 28-29

RI 4349, p. 1-7

RI 5312, p. 1-2, 5-6, 8-9, 11-14

Russian Bear - $(19.1,4.2)$ approx ; COPPER.

P 87, p. 65

B 347, p. 108

B 379, p. 82

Saco - $(24.4,4.4)$ approx ; GOLD, SILVER, COPPER.

B 347, p. 168

(St. Ignace I.) - (NE 1/4 10, 6) ; BARIUM.

B 773, p. 138

B 800, p. 333

IO 7844, p. 9

Salmon - $(24.55,2.85)$ approx ; GOLD, LEAD, COPPER. P 1, p. 80

B 347, p. 175

Salt Chuck (Min. Co.) - $(19.25,11.3-11.35)$; GOLD, PLATINUM, . COPPER, SILVER. Includes references to Alaska Gold \& Metals Co., Alaska Palladium Co., Goodro.

P 87, p. 77, 85-86, 98-99

B 345, p. 110-111.

B 347, p. 125-126

B 379 , p. 79

B 442, p. 38, 141

B 480 , p. 80

B 642, p. 83,85

B 662 , p. 22, 65

B 666, p. 96

B 692, p. 28,86

B 712, p. 23, 28

B 714, p. 18-19, 38, 69, 71, 121-127

B 722, p. 21, 23, 34-35

B 739, p. 13, 20

B 755, p. 17, 23

B 773, p. 30-31

B 783, p. 20, 24-25, 41

B 792, p. 10, 27-28, 33

B 797, p. 10, 32-33, 39

B 800, p. 319, 322-323, 351, 370

B 810, p. 13, 51-52

B 813, p. 16, 59

B 824, p. 17, 59, 65

B 836 , p. 15, 61-62, 67-68

B 844-A, p. 66-67.

B 857-A, p. 63-64

B 864-A, p. 69

B 868-A, p. 16-17, 68, 70

B 880-A, p. 17-18, 70, 74

B $897-A$, p. $18,80,83-84$

B 910-A, p. 20-21, 85, 89

B $917-A$, p. 21, 83, 87

B 926-A, p. 19, 77, 80

B 933-A, p. 18-19, 74, 77

I 230

C 252, p. 5

BMB 142, p. 29, 52

IC 7379, p. 30, 73

RI 2731, p. 1, 4

RI 4358, p. 1-16

San Antonio Metals. See (Baker I.).

San Juan. See Croesus.

Saxe - $(15.7,9.4)$ approx; GOLD, SILVER, LEAD, ZINC, COPPER.

B 783, p. 52

Sea I. Copper Min. Co. See Alarm, It.

Seattle - $(22.7,3.05)$ approx; COPPER, GOLD, LEAD, ZINC, SILVER.

B 592, p. 79

B 642, p. 81-82 
(Shamrock Inlet) - $(22.55, \cdot 0.8)$ approx ; MARBLE. Alsó called Dickman Bay, B 682, p. 86-89 ...

Shellhouse - $(14.0,1.2)$ approx; COPPER. B 379, p. 83

Shellhouse, A. See Jumbo (Hetta Inlet).

Shelton - $(18.5,7.8)$ approx; COPPER, GOLD, SILVER. Includes reference to Lavina.

P 1, p. 93

B 963-A, p. 8-10

B 347, p. 128

Silver Star - $(14.4,0.6)$ approx ; GOLD, SILVER, ZINC, LEAD, COPPER. B 662, p. 72

Skookum - $(21.2,9.7)$; COPPER, GOLD.

P 1, p. 101

Sleepy Eye. See Westlake.

Smith \& Fox. See Rex.

Smith, Van Zandt, Roselle \& Runge. See Lucky Boy.

Snowdrift - $(17.4,8.7)$ approx ; GOLD (?). B 642, p. 81

Solar Dev. Co. See Rush \& Brown, Salt Chuck.

Southeastern Alaska Copper Corp. See Big Harbor.

Standby - $(24.5,3.0)$ approx ; GOLD.

B 642, p. 81

Stella - $(18.6,8.9)$ (?) ; LEAD, ZINC, GOLD(?). See also Monday. B 347, p. 162

Stevens - $(19.2-19.4,11.15-11.3)$; COPPER. NoTE.-Two prospects. I 230

Stevens and assoc. See Flagstaff.

Stevenstown - $(21.9,9.4)$; COPPER, IRON, GOLD.

$\begin{array}{ll}\text { P 87, p. 88-89, 91-92, 94 } & \text { B 347, p. 114-115 } \\ \text { B 284, p. 47 } & \text { B 379, p. 78 } \\ \text { B 314, p. 67 } & \text { B 442, p. 142 } \\ \text { B 345, p. 104-105 } & \text { RI 4129, p. 4 }\end{array}$

Stumble-On - $(21.5,5.5)$; COPPER, GOLD, SILVER, ZINC. Includes references to Mammoth (McKenzie Inlet) ; nickel is reported in $P 1$, but not in any later reference.
P 1, p. 95-96
B 347, p. 137

B 284, p. 49

RI 3942 , p. 1, 3-4, 6-8

B 314, p. 69

Sultana - (19.1, 5.35) ; COPPER, NICKEL. NoTE.-Nickel less than 0.2 percent.

P 87, p. 49, 56, 63, 107

B 347, p. 87, 104-105

B 345, p. 95

Sultzer. See Green Monster, Jumbo (Hetta Inlet), (Lime Pt.)

Summit - $(16.85,9.25)$ ( ?) ; GOLD, SILVER(?).

B 259, p. 66

Sunny Day - $(20.7,9.25)$ approx ; COPPER, GOLD, SILVER.

B 284, p. 48

B 347, p. 127

B 314, p. 68

Sunrise. See (Hole in the Wall).

Superior Portland Cement Co. Sec (View Cove): 
Sweet, Sweèt \& Olson. See Big Harbor.

Tacoma - $(21.55,9.4)(?)$; COPPER.

P 87, p. 97

B 284, p. 47
B 345, p. 113-114

B 347, p. 121

Taylor. See It.

Teresa - $(18.9,1.5)$ approx ; COPPER (?). B 662, p. 69

Texas - $(19.1,4.2)$ approx; COPPER.

B 87, p. 65

B 379, p. 82

B 347, p. 108

Texas Gulf Sulphur. See Khayyam.

Thane, B. L., Expl. Co. See Poorman.

(Tokeen) (lead) - $(10.2,17.5)$ approx ; LEAD. B 1058-A, p. 24

(Tokeen) (marble) - $(10.2 ; 17.5)$; MARBLE. Includes references to (Marble

I.), Vermont Marble Co.

\begin{tabular}{|c|c|}
\hline B 314, p. 76 & B 810, p. 80 \\
\hline B 345, p. 121 & B 813, p. 69 \\
\hline B 347, p. 196 & B 824, p. 77 \\
\hline B 542, p. $60-63$. & B 836, p. 80 \\
\hline B 592, p. 74 & B $844-A$, p. 78 \\
\hline B 622, p. 43 & B $857-A$, p. 73 \\
\hline B 662, p. $40,63,70$ & B 864-A, p. 78 \\
\hline B 682, p. $66-72$ & B $868-A$, p. $80-81$ \\
\hline B 692, p. 28 & B $880-A$, p. 85 \\
\hline B 712, p. 29 & B $897-A$, p. 96 \\
\hline B 722, p. 33 & B $910-A$, p. 104 \\
\hline B 739, p. 21 & В 917-A, p. 103-104 \\
\hline B 783, p. $30,59,61$ & B 926-A, p. 95 \\
\hline B 792, p. 38 & В $933-A$, p. $91-92$ \\
\hline B 797, p. 49 & IO 7379 , p. 80 \\
\hline B 800, p. 340 & IO 7844, p. 7 \\
\hline
\end{tabular}

Tolstol - $(21.0,11.25)$ approx; COPPER, IRON.

P 87, p. 101

B 345, p. 112

B 347, p. 127

(Tolstoi Mtn.) See Iron Cap.

Tom Boy. See Alameda, Frisco (Kitkun Bay).

Treasure. See Flagstaff.

Triangle - $(24.6,3.0)$ approx; GOLD.

P 1, p. 80

Trio - $(23.95,1.35)$; COPPER, GOLD. See also Dama.

P 1, p. 77

Tyee Copper Co. See Jumbo (Hetta Inlet).

Uncle Sam - $(21.15,9.7)$; COPPER. Includes references to. White Eagle.

P 1, p. 101

B 314, p. 67

P 87, p. 94-95

B 345, p. 107-108

B 284, p. $47-48$

B 347, p. 117

(Union Bay) - (22.7-22.9, 13.7-14.5) ; IRON. See also (Mt. Burnett).

B 800 , p. 351-352

IC 7844, p. 11 
Valparaiso - (24.25-24.4, 3.0-3.05) ; GOLD, SILVER, LEAD, ZINC, COPPER. Includes references to Dolomi Gold Mines, Inc.; Jessie; Paul ; Princeton Min. \& Mil. Co.

P 1, p. 83-84

B 259, p. 64-65

B 284, p. 43

B 314, p. 62

B 345, p. 91-92

B 347, p. 173-174

B 379, p. 73

B 542, p. 33

B 592, p. 60, 80-81
B 622, p. 42

B 642, p. 81

B 824, p. 18 .

B 844-A, p. 16

B 864-A, p. 17

B 868-A, p. 17

B 880-A, p. 18

BMB 142, p. 30, 52.

BMB 153, p. 27

Venus (Hole in the Wall). See (Hole in the Wall).

Venus (Karta Bay) - $(18.95,10.95)$; IRON, COPPER, ZINC.

$\begin{array}{ll}\text { P } 87, \text { p. 73, 87, 98, 100-101 } & \text { B 347, p. } 125 \\ \text { B 314, p. 68 } & \text { B 379, p. 79 } \\ \text { B 345, p. } 112 & \text { I } 230\end{array}$

Vermont Marble Co. See (Heceta I.), (Kosciusko I.) (northeast of Edna Bay), (Marble I.), (Tokeen) (marble).

Vesta - (NW 1/8 23, 2) ; COPPER. NoTE-May be old name for Cymru, which see also.

P 1, p. 79

(View Cove) - (14.1-14.9, 1.6-1.85) ; LIMESTONE, MARBLE. Includes references to Pacific Coast Cement Co., Superior Portland Cement Co., (Dall I.) unless specifically to some other occurrence of limestone.
B 642, p. 102
B 682, p. 80-81, 113
B $880-A, p .86$
B 800, p. 340, 391
B 897-A, p. 96
B 810, p. 62
B 910-A, p. 103
B 813 , p. 70
B 917-A, p. 102-103
B 824, p. 78-79
B 926-A, p. 94
B 836 , p. $79-80$
B 933-A, p. 90
B 844-A, p. 78
C 18, p. 5
C 252, p. 12
B 857-A, p. 74
IC 7379, p. 80
B 864-A, p. 78
IC 7844, p. 7
B 868-A, p. 81
TDM 6, p. 8, 12-14, 28, 43-45

Vulcan. See Sultana.

(Wadleigh I.) - (13.5, 9.9) ; LIMESTONE.

TDM 6, p. 25, 52, 55-56

Wakefield - $(23.35,1.6)$ approx ; COPPER.
B 284, p. 50
B 642, p. 90
B 347, p. 132

Wakefield Mineral Lands Co. See Lookout, Niblack.

Wallace $\cdot(21.0,11.3)$ approx ; COPPER, IRON.
B 87, p. 101
B 347, p. 127

B 345, p. 111-112

Washington - (23.6, 3.4) approx ; GOLD, SILVER, ZINC, COPPER. P 1, p. 85:

B:347, p. 168-169.

Waters. See (Heceta I.), (Kosciusko I.) (Northeast of Edna. Báy).

Wednesday - $(24.4,2.85)$ approx; GOLD (?).

P 1, p. 83 
Welcome - (24.6, 3.0) approx; GOLD.

P 1, p. 80

Wellfleet - (24.6, 3.0) approx ; GOLD. P 1, p. 82

B 642, p. 81

Westcott - $(23,1)$; COPPER. NOTE.-Near Niblack.

B 642 , p. 90

Westerly. See (Tokeen) (marble).

Westlake - $(23.5,2.25)$ approx; GOLD, LEAD, ZINC. Includes references to Blue Bird, Homestake, Little Annie, Little Annis, Sleepy Eye.

P 1, p. 78

B 347, p. 176

B 712, p. 28

Westlake, J. See Lucky Boy.

White Eagle. See Uncle Sam.

White Ledge. See (Tokeen) (marble).

Yellowstone - (NE1/4 13, 1) (?) ; GOLD, COPPER.

B 442, p. 143

B 480, p. 102

Young - $(18.75,11.0)$ approx; COPPER.

B 692, p. 86-87

Zimmerman. See Big Harbor.

(_..---) (zinc, lead) - $(19.0,5.5)($ ?) ; ZINC, LEAD. NoTE.-On ridge 1.5 miles northeast of Sulzer.

B 662, p. 68-69

\section{DE LONG MOUNTAINS QUADRANGLE}

(Mt. Kelley) - $(8,8)$; GOLD.

B 815, p. 338-339

\section{DIILINGHAM QUADRANGIE}

(Arcana Cr.) See (Marsh Mtn.).

Feeder. See (Marsh Mtn.).

(Feeder Cr.) See (Marsh Mtn.).

(Kakhtul R.) See (Koktalee R.)

(Koktalee R.) - (20.5-22.7, 12.0-15.25) ; GOLD. Called Kakhtul R. on recent maps.

B 442 , p. 202

(Marsh Mtn.) - (4.25-4.35, 4.9-5.1); MERCURY. Includes references to (Arcana Cr.) ; Feeder; Red Top; 3 "W."

RI 4065. p. 54-57

TDM 2, p. 18

(Mklung Hills) - (6-7, 6-7) ( ?) ; GOLD.

B 903, p. 91

(Mulchatna R.) - (NE 1/16 quad.) ; GOLD.

B 442 , p. 202

Red Cap. See (Marsh Mtn.)

Red Top. See (Marsh Mtn.)

Ryan. See (Marsh Mtn.).

3 "W." See (Marsh Mtn.).

Waskey, (Wren \& Wolf). See (Marsh Mtn.)

\section{DIXON ENTRANCE QUADRANTGIE}

Adit - $(19.9,14.0)$; COPPER.
B 592, p. 83
B 662, p. 66 
Alaska Industrial Co. See Mt. Vesta.

Alice - $(19.2,12.8)$; COPPER.

B 662 , p. 67

(American Bay) - $(12.15,14.85)$; MARBLE.

B 682 , p. $82-83$

Anaconda Copper Co. See (McLean Arm).

Apex - $(19.9,14.0)$ approx; COPPER.

$$
\text { B } 592, \text { p. } 83 \quad \text { B } 662 ; \text { p. } 66
$$

Astor - (19.9, 14.0) approx; COPPER.

B 662, p. 66

(Cleva Bay). See (Waters Bay).

(Dakoo Harbor). See (McLeod Bay) (metals).

Daly-West. See (McLean Arm).

Daykoo. See (McLeod Bay) (metals).

Decker \& West - $(20.0,13.8)$ approx ; COPPER.

B 592, p. 83

Delaware. See (McLeod Bay) (metals).

Elk. See (McLeod Bay) (metals).

Elks Pup. See (McLeod Bay (metals).

Eurus. See (Waterfall Bay).

Feickert - (18.8-19.55, 12.55-13.2) ; COPPER.

B 662 , p. 67

Golden Chariot. See (McLeod Bay) (metals).

Goodhope - $(17.3,15.45)$; IRON, COPPER.

B 642, p. 91

(Gotsongni Bay) - $(12.25,16.1)$; MARBLE. Now called Shoe Inlet:

B 642, p. 102-104

B 682, p. 84-85, 114

(Grace Harbor) - (11.1, 16.0) ; MARBLE.

B 682, p. 82

Hanson. See Spik.

Hillside - $(19.9,14.0)$ approx ; COPPER.

B 592, p. 83

(Hunter Bay). See Goodhope.

Johnson \& Gouley. See (McLean Arm).

Lakeside - $(12.6,17.55)$; COPPER.

B 662, p. 69

Lhote \& Ickis. See (Waterfall Bay).

Lily. See (Waters Bay).

Long I. See (Waters Bay).

Lucky Strike - $(10.6,15.9)$ approx ; COPPER.

B 662, p. 72

(McLean Arm) - (19.9-20.0, 13.9-14.05) approx ; COPPER, GOLD, LEAD.

Includes references to Daly-West, Johnson \& Gouley, Thompson. See also

Adit, Apex, Astor, Hillside, Nelson \& Tift, Veta, Wano.
B 379, p. 83
B 897-A, p. 18-19
B 642, p. 90
B 910-A, p. 21
B 714, p. 18

McLeod. See (McLeod Bay) (metals).

(McLeod Bay) (marble) - (12.8, 12.4) approx; MARBLE.

B 682, p. 83 
(McLeod Bay) (metals) - (12.8-13.25, 12.1-12.4) approx; GOLD, COPPER, LEAD. Includes references to (Dakoo Harbor), Daykoo, Delaware, Elk, Elks Pup, Golden Chariot, McLeod, New York, No Name; Virginia, West Virginia.
B 259, p. 67
B 347, p. 181
B 284, p. 43
B 662, p. $70-71$
B 314, p. 62
B 714, p. 18

(Mallard Bay) See (McLean Arm).

Marble Bay. See (Waterfall Bay).

Marble Heart. See (Waterfall Bay).

Mt. Vesta - $(10.55,16.5)$ approx ; COPPER, GOLD, LEAD, ZINC.
P 1, p. 110
B 347, p. 181
B 259, p. 67
B 592, p. $91-92$
B 284, p. $43-44$

Nelson \& Tift - (19.9-20.0, 13.9-14.05) approx; GOLD, COPPER, LEAD. See also (McLean Arm).

B $917-A$, p. 21

B 926-A, p. 19-20.

New York. See (McLeod Bay) (metals).

No Name. See (McLeod Bay) (metals).

Polson \& Ickis. See Adit, Apex, Astor, Veta.

Ranger - $(17.05,14.85)$; IRON, COPPER.

B 662, p. 67-68

St. Augustine. See (Waterfall Bay).

(Shoe Inlet). See (Gotsongni Bay).

Spik - $(19.7,13.75)$; COPPER. Includes reference to Hanson.

B 592, p. 83

B 662, p. 67

B 800, p. 323

(Tah Bay). See Ranger.

Thompson. See (McLean Arm).

Thornton \& Kilpatrick. See Alice.

Tucker \& Gould. See Lakeside.

Veta - $(20.0,13.9)$; COPPER.
B 379, p. 83
B 480, p. 102
B 592, p. 83
B 662 , p. $66-67$

Virginia. See (McLeod Bay) (metals).

Wano - $(19.9,14.0)$; COPPER.

B 592 , p. 83

(Waterfall Bay) - (9.5-9.6, 16.4-16.7); MARBLE. Includes references to

Eurus, Marble Bay, Marble Heart, St. Augustine.
B 542, p. 67
B 642, p. 100
B 682 , p. 77-80
B 662 , p. 73
B 783, p. 61
TDM 6, p. 28, 32-36

(Waters Bay) - (12.4-12.6, 16.1-16.3) ; MARBLE. NoTE.-Now called Cleva

Bay.

B 642, p. 103

B 682, p. 83, 113-114

TDM 6, p. 28, 36, 38-40 .

West Virginia. See (McLeod Bay) (metals).

White Cloud. See (Waters Bay). 


\section{EAGLE QUADRANGLE}

Alaska Consol. Gold Corp. See (Chicken Cr.); (Dome Cr.) (trib. O'Brien Cr.). Alaska Gold Dred Co. See (Mosquito Fork).

(Alder Cr.) - (11.9-11.95, 17.05-17.35) ; GOLD.

B 480 , p. 172

B 520, p. 217

B 538, p. 79

B 542, p. 219

B 662 , p. 56

B 692 , p. 37

B 739 , p. 33

B 755, p. 39

B 783, p. 14

B 824, p. 39

B 836, p. 38

B 797, p. 23

B 844-A, p. 40

B 864 A, p. 42

B 880-A, p. 47

B $897-A$, p. 55

B 897-C, p. 191, 193-191

B 910-A, p. 57

B 917-A, p. 56

B 813 , p. 34

B 816, p. 164

B 917-D, p. 249, 255

B 926-A, p. 54

B $933-A$, p. 50

(American Cr.) - $(19.25-20.55,11.85-13.4)$; GOLD. Includes references to (American Cr., Discovery Fork).

AR 18, p. 294, 339-341

AR 21, p. 377

B 213, p. 48

B 225, p. 57

B 251, p. 53-54

B 259, p. 29-30

B 263, p. 14, 41, 59, 208

B 284, p. 126

B 314, p. 38

B 375, p. 44-45

B 379, p. 53

B 442, p. 244

B 480, p. 172

B 520, p. 217

B 542, p. 220

B 622, p. 63-64

B 642 , p. 63

B 692, p. 37

B 783, p. 14

B 797, p. 23

B 810 , p. 29

B 813, p. 34

B 816, p. 162-163

B 824, p. 39

B 836 , p. 38

B 844-A, p. 40

B 857-A, p. 38

B 864-A, p. 42

B 868-A, p. 44

B $880-A$, p. 47

B $897-A$, p. 55

B 897-C, p. 199-201

B 910-A, p. 57

B 917-A, p. 56

B 917-D, p. 247, 255

B 926-A, p. 54

B 933-A, p. 50

(American Cr., Discovery Fork). See (American Cr.)

Anderson. See (Dennison Fork).

(Arctic Cr.) - (10.5-11.0, 16.4-16.5) ( ?) ; GOLD.

B 542, p. 219

Atwater. See (Fortymile R.).

(Atwater Bar). See (Fortymile R.).

Auburn Gold Min. Co. See (Dome Cr.) (trib. O’Brien Cr.).

(Barney Cr.) - (14.9-15.0, 17:45-17.75) ; GOLD.
B 251, p. 56
B 375, p. 46
B 622, p. 64
B 379 , p. 53
B 662, p. 56
B 442, p. 244
B 797, p. 23
B 480 , p. 171
B 813 , p. 34
B 520, p. 217-218
B 816 , p. 164
B 542, p. 219
B 824, p. 39
B 836, p. 39 
(Barney Cr.)-Continued
B 864-A, p. 42
B 910-A, p. 57
B $868-A$, p. 44
B 917-A, p. 56
B $880-A$, p. 47
B 917-D, p. 246-247, 249, 254
B $897-A$, p. 55
B 926-A, p. 54
B $897-$ C, p. 191, 195
B 933-A, p. 50

(Berkshire Cr.) - (SE 1/8 quad.) (?) ; GOLD.

B 379, p. 53

(Bonanza Bar). See (Fortymile R.).

(Boulder Cr.). See (Colorado Cr.). Called Colorado Cr. on recent maps.

(Boundary Cr.) - (21.0-22.5, 12.0-12.4) ; GOLD.

B 213, p. $47-48$

B 917-D, p. 249, 255

B 897-C, p. 201

Boundary Dred. Co. See (Canyon Cr.) (Fortymile dist.).

(Broken Neck Cr.) - $(16.85,16.55)$; GOLD.
B 251, p. 54-55
B 375, p. 45
B 480, p. 172
B 542, p. 220
B 813 , p. 34 :
B 816, p. 163
B 824, p. 39
B 836, p. 38
B 868-A, p. 44
B $880-A$, p. 47
B $897-A$, p. 55
B 897-C, p. 191, 195-196
B 910-A, p. 57
B 917-D, p. 246-247, 249, 254
B 933-A, p. 50

Bryant and assoc. See (Alder Cr.).

Bryant \& Parsons. See (Alder Cr.).

(Buckskin Cr.) - (15.0-17.0, 3.3-3.65) ; GOLD.
B 284 , p. 126
B 622, p. 63
B $880-A$, p. 43
B $897-$ C, p. 185

(Camp Cr.) - (22.2, 2.3) approx ; GOLD.

B 375 , p. 42

B 642, p. 62

Canadian Securities Co. (Ltd.). See (Fortymile R.).

(Canyon Cr.) (Fortymile dist.) - (21.7-22.1, 2.4-4.8) ; GOLD. NotE.-Most of production from Canyon Cr. basin was from (Camp Cr.), (Squaw Gulch), and (Woods Cr.), which see also.
AR 21 , p. 376
B 345 , p. 184,197
B 810, p. 29
B 375, p. 35,41
B 844-A, p. 36
B 442, p. 244
B 868-A, p. 42
B 480, p 169
B 880-A, p. 43
B 520, p. 215-216
B $897-C$, p. 187
B 542 , p. 216
B 917-A, p. 53
B 797, p. 22
B $926-$ A, p. 48,71
B $933-A$, p. 44,67

(Canyon Cr.) (Seventymile dist.) - (15-18, 15-16) (?) ; GOLD, MERCURY.

B 542, p. 220

B 872 , p. 245

TDM 1, p. 27

Caribou - (11.4, 17.25) approx ; GOLD.

B 622, p. 64

Central Der. Syn. See (Chicken Cr.).

(Cherry Cr.) - (22.1, 1.0) ; GOLD (?).
B 251, p. 43-44
B 480 , p. 169
B 520, p. 215 
(Chicken Cr.) - (15.8-16.2, 1.4-2.2) ; GOLD, ANTIMONY, TUNGSTEN.

AR 18, p. 331-332

AR 21, p. 376

B 225 , p. 57

B 251 , p. $46-51$

B 259 , p. 30

B 263, p. 54,208

B 284 , p. $125-126$

B 314, p. 38

B 345 , p. 184,197

B 375, p. $35,37-38,40$

B 379, p. 53

B 442 , p. 244

B 480 , p. 169

B 520 , p. 214

B 542, p. 214-215

B 622, p. 63

B 642, p. 62

B 722 , p. 24
B 783, p. 14

B 797, p. 22

B 810, p. 29

B 813, p. 32, 136-137

B 824, p. 37

B 836, p. 38

B 844-A, p. 36

B 857-A, p. 37

B 864-A, p. 42

B 868-A, p. 42

B 897-C, p. $170-175$

B $910-A$, p. 54

B 917-A, p. 54

B $926-$ A, p. 48

B 933-A, p. 45,85

RI 4174, p. 28

TDM 2, p. 20

(Claghorn Bar). See (Fortymile R.).

(Coldfoot Cr.). See (Confederate Cr.).

(Colorado Cr.) - $(19.0-19.15,13.8-14.35)$; GOLD. Includes reference to (Boulder Cr.).

AR 18, p. 339

B $897-C$, p. 201

B 213, p. 48

(Confederate Cr.) - (12.35-12.8, 5.0-5.25) ; GOLD. Includes references to (Coldfoot Cr.).
B 251, p. 52
B 480 , p. 170
B 538 , p. 80
B 542, p. 217

(Copper Cr.) - $(5.3,14.85)$; COPPER, LEAD, GOLD, SILVER, TUNGSTEN. C 335, p. 7, 9, 22

(Crooked Cr.) - $(17.3,16.45-16.6)$; GOLD.

B 442, p. 244

B 480 , p. 171

B 836 , p. 38

B 520, p. 217-218

B 844-A, p. 40

B 542, p. 219

B 857-A, p. 38

B 622 , p. 64

B 864-A, p. 42

B 662 , p. 56

B 868-A, p. 44

B 739, p. 33

B $880-A$, p. 47

B 755, p. 39

B $897-$ A, p. 55

B 783, p. 14

B $897-$ C, p. 191, 196-198

B 797, p. 23

B 813, p. 34

B 816, p. $163-164$

B 824 , p. 39

B 910-A, p. 57

B $917-$ A, p. 56

B $917-$ D, p. 246-247, 249-250, 254

B 926-A, p. 54

B 933-A, p. 50

(Curtis Bar). See (Seventymile R.).

(Curtis Cr.) - $(17.3,16.1)$; GOLD(?). Note.-Assumed to be the same as Curtis Bar Cr. B 622 , p. 64 
(Davis Cr.) - (22.65-22.75, 1.6-1.7) ; GOLD.
AR 18, p. 330-331
B 592 , p. 361
B 345 , p. 197
B 857-A, p. 37
B 375, p. 35
B 864-A, p. 42
B 480 , p. 169
B 868-A, p. 42
B 520, p. 215
B 897-C, p. 158-159
B 542, p. 215

(Dennison Fork) - $(16.2,1.3)$ approx ; GOLD.
B 520, p. 214
B 714, p. 88
B 542, p. 215
B 722, p. 50

B 712, p. 46

(Discovery Bar). See (Fortymile R.).

(Discovery Fork). See (American Cr.).

Dome - (SE1/4 NE1/4 quad.) (?) ; GOLD (?).

B 792, p. 18

(Dome Cr.) (trib. American Cr.) - (19.2-19.55, 11.2-11.75) approx ;

GOLD.

B 797, p. $23 \quad$ B 836, p. 38-39

B 824, p. 39

(Dome Cr.) (trib. O'Brien Cr.) - (20.75-21.2, 7.2-7.35) ; GOLD,

MERCURY, LEAD.

B 345, p. 197

B 520, p. 211, 216

B 542, p. 217

B 836, p. 38

B 642 , p. 62

B 844-A, p. 36

B 714, p. 88

B $880-A$, p. 43

B 739, p. 34

B $897-$ C, p. $188-190$

B 813, p. 32,130

B 910-A, p. 54, 57

B 824, p. 37

B $917-A$, p. 54

B 926-A, p. 48

B 933-A, p. 45

Dome Cr. Gold Corp. See (Dome Cr.) (trib. O'Brien Cr.).

(Eagle Bluff) - (21.0, 14.5) ; GOLD, NICKEL, COBALT. C 316, p. 3

(Eagle Cr.) - (15.1, 2.1) approx; GOLD. See also (Ingle Cr.) ; probably the same stream.
B 225, p. 57
B 520, p. 214
B 284, p. 126
B $857-$ A, p. 37

B 345 , p. 197

(Engle Cr.). See (Ingle Cr.).

(Excelsior Cr.). See (Twelvemile Cr.). Note.-Small stream; Excelsior is new name.

(Falls). See (Seventymile R.).

(Falls Cr.) - (11-18, 16-17) (?) ; GOLD.

B $880-A$, p. 47

B 926-A, p. 54

B 910-A, p. 57

B 933-A, p. 50

B 917-A, p. 56

(Fish Cr.) - (9.0-12.0, 4.5-7.1) ; GOLD (?).

B 542, p. 217

(Flat Cr.) - (SE1/8 quad.) (?) ; GOLD.

B 379, p. 53

B 542, p. 217

B 520, p. 217

B 642, p. 62 
(Flume Cr.) - (11.4, 17.25) (approx) ; GOLD.

B 251, p. 57

B 295, p. 24

B 538 , p. 79

B 345, p. 184

B 375, p. 46

B 379, p. 53

B 480, p. 172

B 520, p. 217

B 542, p. 219

B 622 , p. 64

B $880-$ A, p. 47

B 897-C, p. 191, 193-194

B 917-D, p. 249

(Fortyfive Gulch), (Pup), or (Pass) - (13.9-15.1, 3.5-4.1) ; GOLD, TUNGSTEN, TIN.

B 480 , p. 170

B 520, p. 217

B $897-$ C, p. 185

RI 4174, p. 28

B 542, p. 216

TDM 2, p. 19-20, 28

(Fortymile R.) - (16.2-22.75, 1.2-6.05) ; GOLD, TUNGSTEN, TIN. Includes references to (Fortymile R., South Fork).

AR 18, p. 295, 317

B 251, p. 51

B 263, p. 41, 44, 117, 208

B 284, p. 126

B 314, p. 39

B 345, p. 194-195, 197

B 375, p. 35, 42-43

B 379, p. 53

B 442, p. 244

B 480 , p. 170-171

B 520, p. 211, 216-217

B 542, p. 216-218

B 592, p. 68

B 622, p. 63

B 642, p. 63

B 662 , p. 56

B 692, p. 36

B 755, p. 39
B 783, p. 14

B 797, p. 22

B 810 , p. 29

B 813, p. 32

B 836 , p. 38

B 844-A, p. 36

B 857-A, p. 37

B 864-A, p. 42

B 868-A, p. 42

B 880-A, p. 43

B 897-A, p. 71

B 897-C, p. 185-187

B 910-A, p. 54

B 917-A, p. 54

B 926-A, p. 48

B 933-A, p. 45

C 335, p. 10-11

(Fortymile R., Middle Fork) - (5.0, 4.2) (?) ; ANTIMONY. See also (Mt. Veta), (My Cr.).

B 692, p. 36

(Fortymile R., North Fork) - (14.6-15.0, 6.25-6.55) ; GOLD, TIN.

B 250, p. 57

B 251, p. 51-52

B 284, p. 126

B 259, p. 30

B 722 , p. 50

TDM 2, p. 19

(Fortymile R., South Fork). See (Fortymile R.).

(Fourth of July Cr.) - $(10.6,10.55)$; LEAD.

C 335, p. 19

(Fox Cr.) - (17.95-18.2, 16.25-16.8) ; GOLD, TIN.

B 520, p. 218

B 542, p. 219

B 868-A, p. 44

B 622 , p. 64

B 897-A, p. 55

B 642, p. 63

B 897-C, p. 191, 198-199

B 783, p. 14

B 917-A, p. 56

B 797, p. 23

B 917-D, p. 246-250, 254

B 824, p. 39

B 926-A, p. 54

TDM 1, p. 32 
(Franklin Cr.) or (Gulch) - (16.3-16.85, 3.0-3.05) ; GOLD, MEROURY.

$\begin{array}{ll}\text { AR 18, p. 332-334 } & \text { B 797, p. 22 } \\ \text { AR 21, p. 376 } & \text { B 810, p. 29 } \\ \text { B 225, p. 57 } & \text { B 813, p. 135-136 } \\ \text { B 251, p. 44-45, 51 } & \text { B 827, p. 36 } \\ \text { B 284, p. 126 } & \text { B 824, p. 37 } \\ \text { B 345, p. 194, 197 } & \text { B 836, p. 38 } \\ \text { B 375, p. 35, 41 } & \text { B 844-A, p. 36 } \\ \text { B 442, p. 244 } & \text { B 857-A, p. 37 } \\ \text { B 480, p. } 170 & \text { B 864-A, p. 42 } \\ \text { B 520, p. 215 } & \text { B 868-A, p. 42 } \\ \text { B 542, p. 216 } & \text { B 880-A, p. 43 } \\ \text { B 622, p. 63 } & \text { B 897-C, p. 181-183 } \\ \text { B 642, p. 62 } & \text { TDM 2, p. 18 }\end{array}$

Fritsch. See (American Cr.).

Fritz. See (American Cr.).

Froelich, Kummer, Ott \& Scheele. See (Crooked Cr.).

(Gilliland Cr.) - (E 1/2 19,3) ( ?) ; GOLD.

B 897-C, p. 168

Glasgow. See (My Cr.).

(Gold Run) or (Cr.) - (8.9-9.2, 10.45-10.55) ; GOLD.

B 259, p. 30

B 538, p. 80

B 295, p. 24-25

C 335, p. 19

(Green I.). See (Fortymile R.).

Grey Wolf. See (My Cr.).

Hagen. See (Crooked Cr.).

(Healy R.) - $(0.35,3.95)$; MOLYBDENUM.

B 692, p. 23, 329

TDM 1, p. 29

B 926-C, p. 194

Hudson Bros. See (Copper Cr.).

(Hutchinson Cr.) - (12.75-13.0, 5.0-6.2) ; GOLD. See also (Montana Cr.). B 480 , p. 170

B 538, p. 80

(Ingle Cr.) - (15.1, 2.1) approx; GOLD. Includes references to (Engle Cr.) ; see also (Eagle Cr.), (Iilliwig Cr.).

B 345, p. 197

B 375, p. 40

B 797, p. 22

B 379, p. 53

B 810, p. 29

B 442, p. 244

B 813, p. 139

B 480 , p. 170

B 844-A, p. 36

B 542, p. 214

B 864-A; p. 42

B 592, p. 361

B 868-A, p. 42

B 622, p. 63

B 880-A, p. 43

B 642, p. 62

B $897-$ C, p. 171, 173, 179-180

Ingle Cr. Gold Co. See Dome, (Mosquito Fork).

(Irene Gulch) - $(16.0,2.2)$ approx ; GOLD.

B 251, p. 50-51

B 375 , p. $39-40$

B 263, p. 208

(Jack Wade Cr.). See (Wade Cr.).

Jack Wade Dred. Co. See (Wade Or.).

(Jennie Bench). See (Lost Ohicken Cr.).

(Joe Wilson Cr.) - (16.0, 5.0) approx ; GOLD.

B 880-A, p. 43

593018-61-7 
Johnson. See (Healy R.), (Seventymile R.). NoTE.-Two Johnsons.

(Ketchumstuk Cr.) - (7.0-7.3, 1.3-1.5) (?) ; COPPER.

$$
\text { B 520, p. } 213
$$

B 542, p. 214

King. See (Walker Fork).

(Kink). See (Fortymile R., North Fork).

(Liberty Cr.) - (21-22, 9) ; GOLD.

B 642, p. 62

(Lilling Gulch). See (Lilliwig Cr.).

(Lilliwig Cr.) - $(15.1,2.1$ ) approx ; GOLD, SILVER, COPPER. Includes reference to (Lilling Gulch); see also (Ingle Cr.).

B 442, p. 244

B 480, p. 170

B 813, p. 141

(Little Miller Cr.). See (Dome Cr.) (trib. O'Brien Cr.).

(Long Cr.) - (NE 1/4 quad.) ; GOLD( ?). B 692, p. 37

(Lost Chicken Cr.) - (16.05-16.35, 1.35-1.6) ; GOLD.

B 251, p. 41, 48, 50-51

B 284, p. 125-126

B 314, p. 38

B 345, p. 197

B 375, p. 35, 38

B 379, p. 53

B 480, p. 170

B 622, p. 63

B 642, p. 62

\author{
B 813, p. 138-139 \\ B 864-A, p. 42 \\ B 880-A, p. 43 \\ B $897-$ C, p. 171, 173, 177-179 \\ B 910-A, p. 54 \\ B 917-A, p. 54 \\ B 926-A, p. 48 \\ B 933-A, p. 45
}

(Lost Chicken Hill). See (Lost Chicken Cr.).

Lowery's Ledge - $(22.75,1.7)$; GOLD.

AR 18, p. 292

(Lucky Gulch) - (17.85-18.0, 16.8-17.1) (?) ; GOLD, PLATINUM.

B 542, p. 219

B 797, p. 23

B 917-A, p. 56

B $897-$ C, p. 198

B 917-D, p. 250

B 910-A, p. 57

B 926-A, p. 54

TDM 1, p. 20

McDowell-Allen Co. See (Fortymile R.).

Machette. See (My Cr.).

Manske \& Purdy. See (My Cr.).

Martin (and assoc.). See (Wade Cr.).

(Miller Cr.) - (SW 1/4 21, 7) (?) ; GOLD. NoTE.-May be the same as Little Miller Cr.; see also (Dome Cr.) (trib. O'Brien Cr.).
B 251, p. 53
B 375, p. 44

(Mission Cr.) - (18.15-20.9, 12.0-14.9) ; GOLD.

AR 18, p. 338-339

B $897-$ C, p. 201

B 263, p. 208

B 917-D, p. 246

B 897-A, p. 55

(Mogul Cr.) - (17.0, 16.4) approx; MERCURY.

TDM 1, p. 27

(Montana Cr.) - (12.0-12.8, 5.6-5.8) ; GOLD.
B 251, p. 52
B 480, p. 170
B 284, p. 126
B 538, p. 80
B 295, p. 24
B 542, p. 217

B 345, p. 197 
(Mosquito Fork) or (Cr.) - (15.3-16.5, 1.32-1.6) ; GOLD. Note.-A fork of South Fork of Fortymile R. ; see also (Lost Chicken Cr.).

$\begin{array}{ll}\text { B } 251, \text { p. 48-49 } & \text { B 897-C, p. 180-181 } \\ \text { B 284, p. 126 } & \text { B 910-A, p. 54, 76 } \\ \text { B 345, p. } 184 & \text { B 917-A, p. 53 } \\ \text { B 792, p. } 18 & \text { B 926-C, p. 48 }\end{array}$

B 797, p. 22

(Mt. Veta) - $(7,3)$; ANTIMONY. See also (My Cr.). B 880-A, p. 87

Mulvane. See (Fortymile R.) ; (Walker Fork).

(My Cr.) - $(5.65,1.7)$; ANTIMONY. Includes references to Glasgow ; see also (Mt. Veta).

$\begin{array}{ll}\text { C 335, p. } 18 & \text { TDM 1, p. } 13 \\ \text { RI 4173, p. 4, 28-30 } & \text { TDM 2, p. 15-16 }\end{array}$

(Myers Fork) - (15.9-15.95, 1.8-1.95) ; GOLD, TUNGSTEN.

$\begin{array}{ll}\text { B } 251, \text { p. 46, 50-51 } & \text { B 897-C, p. 170, 173, 175-177 } \\ \text { B 345, p. 197 } & \text { B 910-A, p. 54 } \\ \text { B 375, p. 40 } & \text { B 917-A, p. } 54 \\ \text { B 379, p. 53 } & \text { B 926-A, p. 48 } \\ \text { B 480, p. 169-170 } & \text { B 933-A, p. 45 } \\ \text { B 797, p. 22 } & \text { RI 4174, p. } 28 \\ \text { B } 813, \text { p. 137-138 } & \text { TDM 2, p. } 20 \\ \text { B } 880-\text { A, p. } 43 & \end{array}$

(Napoleon Cr.) - (17.3-17.45, 2.1) ; GOLD.

\begin{tabular}{|c|c|}
\hline AR 18, p. $335-337,365$ & B 797, p. 22 \\
\hline AR 21, p. 376 & B 813, p. 32 \\
\hline B 251, p. 52 & B 824, p. 37 \\
\hline B 284, p. 126 & B 836, p. 38 \\
\hline B 345, p. 194, 197 & B $844-A$, p. 36 \\
\hline B 375, p. $35,40-41$ & B $857-A$, p. 37 \\
\hline B 379, p. 63 & B $864-A$, p. 42 \\
\hline B 442, p. 244 & B $868-A$, p. 42 \\
\hline B 480, p. 170 & B $880-\vec{A}$, p. 43 \\
\hline B 520, p. 215 & В $897-A$, p. 183 \\
\hline
\end{tabular}

B 542, p. 216

North American Mines, Inc. See (Wade Cr.)

Norvill. See (Chicken Cr.)

(Nugget Cr.) - $(12.7,17.3)$; GOLD
B 251, p. 56-57
B 813, p. 34
B 295, p. 24
B 824, p. 39
B 375, p. 45-46
B 836, p. 38
B 379, p. 53
B $880-A$, p. 47
B 480, p. 172
B 897-A, p. 55
B 542, p. 220
B 897-C, p. 191, 193-194
B 622, p. 64
B 917-D, p. 249, 255

B 797, p. 23

(Nugget Gulch) - $(21.25,5.05)$; GOLD.

AR 21, p. 376

B 275, p. 44

B 251, p. 52

Olson \& Johnson. See (American Cr.).

Patterson \& Olson. See (Wade Cr.). 
(Pleasant Cr.) - $(14.7,17.25)$ approx; GOLD.

B 480 , p. 171-172

B 542, p. 219-220

B 622, p. 64

(Poker Cr.) - (22.8-22.95, 1.45-1.5) ; GOLD.

AR 18, p. 326-330

B 345 , p. 197

B 375, p. $35-36$

B 480 , p. 169

B 520, p. 215

B 542, p. 215

B 592, p. 361

B 857-A, p. 37

B 864-A, p. 42

B 868-A, p. 42

B 897-C, p. 158-159

(Pump Bar). See (Fortymile R.).

Purdy, Manske \& Taft. See (My Cr.).

Robinson. See (Wade Cr.).

(Rock Cr.) - $(18.0,15.35)$ approx ; GOLD.

B 480, p. 171

Ruby Silver - $(6.0,1.9)$; LEAD.

C 335, p. 18-19

(Seventymile R.) - (16.0-18.5, 15.9-16.5) ; GOLD, MERCURY. Includes references to (Curtis Bar), (Falls).

B 251, p. 55

B 284, p. 126

B 295, p. 24

B 442, p. 244

B 480 , p. 171

B 520, p. 218

B 542, p. 219-220

B 622, p. 63-64

B 662 , p. 56

B 692, p. 37

B 755, p. 39

B 783, p. 14

B 810 , p. 29

B 813, p. 34

B 816, p. 163-164

(Smith Cr.) - (22,3-4) ; GOLD.

B 857-A, p. 37

(Sonickson Cr.) - $(15.85,16.5)$; GOLD.

B 251, p. 55-56

B 375, p. 45

(Squaw Gulch) or (Cr.) - (21.4-21.9, 2.85-3.3) ; GOLD.

B 345, p. 197

B 375, p. 41-42

B 480 , p. 169

B 520, p. 215

B 542, p. 216-217

B 592, p. 361

(Star Gulch) - $(20.3,12.1)$; GOLD.

B 897-C, p. 201

Steele. See (Fox Cr.).
B 622, p. 63
B 642, p. $62-63$
B $857-A$, p. 37
B $868-$ A, p. 42
B $897-$ C, p. 187
B 824, p. 39

B 836 , p. 38

B 844-A, p. 40

B 857-A, p. 38

B 864-A, p. 42

B 868-A, p. 44 .

B $880-$ A, p. 47

B 897-C, p. 191-192, 194-195

B 910-A, p. 57

B 917-A, p. 56

B 917-D, p. 246-247, 254, 257-258

B 926-A, p. 54

B 933-A, p. 50

TDM 1, p. 27 
(Stonehouse Cr.) or (Fork) - $(16.0-16.15,2.05-2.2)$; GOLD, MERCURY, TUNGSTEN.

B 251, p. 46-47, 50-51

B 263, p. 208

B 345, p. 197

B 375, p. 39-40

B 480, p. 169

B 813, p. 138
B 827, p. 36

B 864-A, p. 42

B 897-C, p. 170, 173, 177

RI 4174, p. 28

TDM 2, p. 20

(Texas Cr.) - (9-11, 4-6) (?) ; GOLD.

B 622, p. 63

(Troublesome Pt.) See (Fortymile R.).

Tweeden - $(12.5,1.95)$ approx.

B 520, p. 213-214

(Twelvemile Cr.) - $(17-19,14)$; GOLD (?). Now called Excelsior Cr.

AR 18, p. 339

(Twin Cr.) - (20.8-21.0, 5.05-5.8) ; GOLD.

B 345, p. 197

B 542, p. 217

U.S. Smelt., Ref. \& Min. Co. See (Mosquito Fork).

(Wade Cr.) - (18.4-19.5, 1.9-3.0) ; GOLD, MERCURY, TIN, TUNGSTEN, FLUORITE. Includes references to (Jack Wade Cr.).

AR 21, p. 376-877

B 225, p. 57

B 251, p. 39-42, 52

B 259, p. 30

B 263, p. 54, 208

B 284, p. 125-126

B 314, p. 38

B 345, p. 194, 197

B 375, p. 35-37

B 379, p. 53

B 442, p. 244

B 480, p. 169

B 520, p. 215

B 542, p. 215-216

B 592, p. 361

B 622, p. 62-63

B 642, p. 62-63

B 692 , p. 7, 21

B 714, p. 88

B 722, p. 50

B 755, p. 39
B 783, p. 14

B 797, p. 22

B 810, p. 29

B 813, p. 32, 133-135

B 827, p. 36

B 844-A, p. 36

B 857-A, p. 37

B 864-A, p. 41-42

B 868-A, p. 42

B 880-A, p. 43

B 897-A, p. 71

B 897-C, p. 163-170

B 910-A, p. 54, 76

B 917-A, p. 53

B 926-A, p. 48, 71

B 933-A, p. 44-45, 67

C 348, p. 19

RI 4174, p. 28

TDM 1, p. 27, 32

TDM 2, p. 20

Wade Cr. Dred. Co see (Wade Cr.).

(Walker Fork) or (Cr.) - (20.55-22.7, 1.45-1.65) ; GOLD, FLUORITE.
B 225, p. 57
B 251, p. 42-44
B 259, p. 30
B 263, p. 54, 208
B 284, p. 126
B 314, p. 39
B 345, p. 194, 197
B 375, p. 35-36

B 379, p. 53

B 442, p. 244

B 480, p. 168-169

B 520, p. 215

B 542, p. 215

B 592, p. 361

B 622 , p. 63

B 714 , p. 88 
(Walker Fork) or (Cr.) - Continued

B 792, p. 18

B 797 , p. 22

B 810 , p. 29

B 868-A, p. 41-42, 59

B 813, p. 32, 131-132

B 880-A, p. 43, 61

B 824, p. 37

B 836 , p. 38

B 897-A, p. 71

B 897-C, p. 159-163

B $844-$ A, p. 36

B 910-A, p. 54, 76

B 864-A, p. 42

B 917-A, p. 53

B 926-A, p. 48

C 348, p. 19

Walker Fork Dred. Co. See (Walker Fork).

Walker Fork Gold Corp. See (Walker Fork).

Walker Fork Min. Co. See (Walker Fork).

(Washington Cr.) - $(16.0,16.55)$; GOLD.

B 520, p. 218

B 622, p. 64

B 542, p. 219

(Wolf Cr.) - (19.1-20.0, 12.9-14.6) ; GOLD, CHROMITE.

B 520, p. 217

B 926-A, p. 54

(Woods Cr.) - (22.0, 2.2) approx; GOLD.

B 345, p. 197

B 375, p. 42

\section{FAIRBANRS QUADRANGLE}

Alaska Metals Min. Co. See Yellow Pup.

Alaska Min. \& Dev. Co. See Ryan.

Alaska Tungsten Mines Co. See Stepovich (tungsten), White Assn.

(Alder Cr.) - (13.7-14.9, 14.2-14.7) ; GOLD.

B 337, p. $45-46$

B 480, p. 158

B 345, p. 41-42

(Allen Cr.) - (12.85-13.55, 14.4-15.0) ; GOLD.

B 520, p. 241

American - $(\mathbf{1 9 . 9 5}, \mathbf{1 7 . 5 5})$; GOLD. Includes reference to Perrault.
B 525, p. 166
B 592, p. 329-330
B 542, p. 151
B 849-B, p. 154

American Eagle - $(\mathbf{1 9 . 9 5}, \mathbf{1 7 . 5})$; GOLD. See also American.
B 525, p. 166
B 849-B, p. 154

B 542, p. 151

Anderson - (18.3, 17.0) approx; TUNGSTEN, GOLD. NotE.-Reference in C 196 may not be to this prospect.
B 662, p. 424
RI 4174, p. 26

C 196, p. 9

Aurora. See Stepovich (tungsten).

Barker \& McQueen - $(14.4,15.4)$; GOLD. Note.-May be same as St. Jude.

B 525, p. 209

B 592, p. 352-353

B 542, p. 195

Barlow \& Koska. See Rambler.

Bartholomae Oil Corp. See Ready Bullion, (Rex Cr.), Ryan, Stay.

Bearpaw. See Liberty Bell.

Berry (\& Hamil Co.). See (Gold King Cr.).

Berton. See (Cripple Cr.).

Big Blue - $(14.8,15.55)$ approx ; GOLD.

B 849-B, p. 148

Big Chief. See Colbert. 
(Big Eldorado Cr.) - (16.9-16.95, 17.4-17.45) ; GOLD.
B 337, p. 39, 41
B 525, p. 106, 111, 113
B 345, p. 42
B 642 , p. 59
B 379, p. 191
BMB 153, p. 51

(Big Moose Cr.). See (Moose Cr.).

Bigelow. See McDonald, Merian.

Billy Sunday - $(15.0,15.4)$; GOLD, ANTIMONY. Includes references to Bill Sunday Fraction, Leah, Lean Fraction, Smith \& McGonnigle, Smith Bros.
B 662, p. $412-413$
B 739, p. 30
B 692, p. 323
B 755, p. 35
B 712, p. 40
B 773, p. 15
B 714, p. 81
B 849-B, p. 139-142
B 722, p. 45

Bill Sunday Fraction. See Billy Sunday.

Black Bear. See Blossom.

Black Eagle. See Scrafford.

Blossom - $(18.35,16.9-16.95)$; TUNGSTEN. Includes reference to Black Bear. B 662, p. 422, 424

B 1024-I, p. 179-180, 201, 203-204

B 692, p. 327

RI 4174, p. 24-26

B 849-B, p. 157-158

Blue Bird (McDonald). See McDonald.

Blue Bird (Miller \& O'Conner) - $(14.95,15.35)$; GOLD. B 849-B, p. 139

Blue Bird Min. Co. - $(15.0,15.1)(?)$; GOLD. Note.-May be the same as Blue Bird (McDonald) ; see also McDonald.

B 910-A, p. 25

Blue Bonanza - $(14.15,15.75)$; GOLD, SILVER, ANTIMONY, LEAD. Includes references to Grant (near Nugget Cr.), Midnight Sun.

B 525, p. 197

B 592, p. 353

B 542, p. 183

B 849-B, p. 122

Bondholder. See Mohawk.

(Bonnifield Cr.) - (15-16, 0.5) ; GOLD.

B 824, p. 40

B 880-A, p. 46

Borovich (\& Stevens). See Ready Bullion.

Bunker Hill - $(17.1,17.7)$; GOLD. See also Goodwin (gold). B 592, p. 345

B 849-B, p. 154

(California Cr.) (lode) - $(9.7,1.2)$; ANTIMONY, BISMUTH, COPPER, GOLD, SILVER. See also Prospect Min. Co.
C196, p. 9
TDM 2, p. 13-14

(California Cr.) (placer) - $(9.7-9.75,0.95-1.45)$; GOLD, MERCURY, PLATINUM.

B 480, p. $224 \quad$ B 662, p. 380, 383

B 501, p. 44, 46 TDM 1, p. 20, 27

Camp. See Ready Bullion.

(Caribou Cr.) (trib. California Cr.) - (9.65-10.1, 1.15-1.4) (?) ; ANTIMONY, TUNGSTEN.

TDM 1, p. 12, 41

(Caribou Cr.) (trib. Dry. Cr.) - (20.6-21.2, 1.25-1.85) approx; GOLD. B 480, p. 229

Caucasian. See Stepovich (tungsten).

Chief. See Scrafford. 
Chipperwan. See Stepovich (tungsten).

Christenson. See Yellow Pup.

Cleary Hill Mines Co. See Colbert, Stepovich (tungsten).

Clipper - $(14.85,15.4)$; GOLD.

B 849-B, p. 152

(Cody Cr.) - $(8.05,1.15)$ approx; GOLD, ANTIMONY. See also Rambler. B 662, p. 368

Colbert-- (19.45-19.75, 17.45-17.55) ; TUNGSTEN.

B 1024-I, p. 179, 189, 199-200

TDM 1, p. 40

RI 4174, p. 4, 6, 8-11, 13-15

TDM 2, p. 22-23

Columbia - $(18.5,17.0)$; TUNGSTEN.

B 662, p. 422-423

B 692 , p. 326

B 1024-I, p. 179-180, 201, 205-206

B 849-B, p. 157-158

(Columbia Cr.) - (18.2, 16.15) ; GOLD. B 525, p. 198

RI 4174, p. 24-25

B 542, p. 196

Columbia Min. Co. See Columbia, Spruce Hen.

Combination. See McDonald.

Comet. See Stay.

Comstock. See Crown Pt.

Cottonblossom - $(14.4,15.3)$; GOLD, ANTIMONY.

B 525, p. 208-209

B 542, p. 194-195

B 592, p. 352

B 649, p. 40

(Cripple Cr.) - (14.9-15.45, 14.65-15.15) approx; GOLD, TIN. Many references include data for Ester Cr. and other tributaries.

B 314, p. 36

B 337, p. 29, 44-45

B 345, p. 42

B 442, p. 234

B 525, p. 110, 112-113

B 542, p. 208

B 592, p. 360

Crown Pt. - (15.95-16.05, 15.15-15.25) ( ?) ; GOLD.

B 592, p. 353

Curlew. See Stay.

Daly Bench. See (Eva Cr.) (Fairbanks dist.).

(Daniels Cr.) - (11.1-11.2, 1.8-1.85) ; GOLD.

B 662 , p. $389-391$

B 712, p. 44

B 722, p. 51
B 897-A, p. 46-48

B 910-A, p. 46-47

B 917-A, p. 44-45

B 926-A, p. 40-41

B 933-A, p. 38-40

TDM 1, p. 32

Danzinger. See (California Cr.) (lode).

Diebold. See (Caribou Cr.) (trib. California Cr.).

Dorothy \& Dorice - $(14: 85,15.55)$; GOLD. Includes some references to McGlone $\&$ Smith.

B 525, p. 209

B 542, p. 195
B 739, p. 35

B 813, p. 35

B 824, p. 40

Eagle. See Goodwin.

(Eagle Cr.) (Bonnifield dist.) - $(10.35,1.2)$; ANTIMONY. TDM 1, p. 12 B 592, p. 354-355 B 642 , p. 61

(Eagle Cr.) (Fairbanks dist.). See Goodwin.

Edna. See Ryan. 
Elmes (Gold Min. Co.) - $(15.15,15.7)$; GOLD. Includes references to Nickaloff.

B 797, p. 13

B 810, p. 15

B 813, p. 17

B 824, p. 20

B 836, p. 19
B 849-B, p. 150

B 864-A, p. 20

B 897-A, p. 22

B 910-A, p. 25

B 926-A, p. 23

(Emma Cr.) - (14.2-14.25, 14.6) ; GOLD.

B 480, p. 158

Engineer - $(17.7,16.5)$; GOLD.

B 849-B, p. 153

(Engineer Cr.) - (17.4-17.85, 16.45-16.8) ; GOLD.

B 337, p. 39, 41

B 379, p. 190-191

B 442, p. 230, 232

B 480, p. 155

B 520, p. 241

B 525, p. 105-106, 110-111, 113

(Ester Cr.) - (14.35-15.05, 14.95-15.1) ; GOLD, ANTIMONY, TUNGSTEN.

P 70, p. 182

B 284, p. 119-120

B 314, p. 30,36

B 337, p. 29, 44-46

B 345, p. 41-42

B 379 , p. $190,192,195,197,199-200$

B 442, p. 233-234

B 480, p. 157-158

B 520, p. 243

B 525, p. 103-105, 110, 112-113

B 542, p. 209

B 592, p. 68, 359

B 622, p. 54, 234-235

B 642, p. 58-59

B 662, p. 51, 54

B 692, p. 35

B 712,p. 39

B 714, p. 81

B 722, p. 45

B 739, p. 29

B 755, p. 35,146

B 773, p. 45
B 542, p. 206

B 592, p. 68, 359

B 622, p. 234

B 844-A, p. 33

BMB 153, p. 51
B 783, p. 13

B 792, p. 17

B 797, p. 20

B 810, p. 25

B 813, p. 28

B 824, p. 33

B 836, p. 33

B 844-A, p. 32

B 857-A, p. 30

B 864-A, p. 35

B 868-A, p. 36

B 880-A, p. 39-40

B 897-A, p. 46-47

B 910-A, p. 46-47, 76

B 917-A, p. 43-45

B 926-A, p. 40-41, 70

B 933-A, p. 38

B 1024-I, p. 188

C 331, p. 6

BMB 142, p. 23

BMB 153, p. 51

TDM 1, p. 39

Eva (Fairbanks dist.). See Ryan.

Eva (Min. Co.) (Bonnifleld dist.) - See Liberty Bell.

(Eva Cr.) (lode) (Bonnifield dist.). See Liberty Bell.

(Eva Cr.) (placer) (Bonnifield dist.) - (9.0-9.45, 0.95-1.05) ; GOLD, TUNGSTEN.

B 662, p. $381,385-386$

B 722, p. 51

B 739, p. 35

B 783 , p. 26

B 864-A, p. 42

B 868-A, p. 44

B $880-A$, p. 46
B 897-A, p. 56

B 910-A, p. 55

B 917-A, p. 54

B $933-A$, p. 47

RI 4174, p. 27

TDM 1, p. 39, 41

TDM 2, p. 20 
(Eva Cr.) (placer) (Fairbanks dist.) - (14.9-15.05, 15.0-15.2) ; GOLD

B 520, p. 243

B 622 , p. 235

B 542 , p. 209

B 642 , p. 59

B 592 , p. 359

B 662 , p. 54

Eva Cr. Min. Co. (Bonnifield dist.). See Liberty Bell.

Eva Cr. Min. Co. (Ester Dome) - (14.95, 15.1) (?) ; GOLD(?). See also Stay. B 525, p. 204

Eva No. 2. See Stay.

Eva Quartz Co. - $(14,15)$ (?) ; GOLD. See also Ryan, Stay. B 813, p. 17

Eva Quartz Min. Co. (Bonnifleld dist.). See Liberty Bell.

Eva Quartz Min. Co. (Fairbanks dist.) See Ready Bullion.

Ewers. See Stepovich (tungsten).

Excelsior. See Ryan.

Fair Chance - (14.95-15.0, 15.4-15.45) ; GOLD.

B 592 , p. 353-354

B 692 , p. 323

B 622 , p. 237

B $849-$ B, p. 139

Fairbanks Exp. Co. See U.S. Smelt., Ref., \& Min. Co.

Farmer - $(14.55,15.7)$; GOLD.

B 525, p. 198

B 592 , p. 352

B 542, p. 184

B $849-$ B, p. $122-123$

First Chance - $(14.9,15.5)$; GOLD.

B 783, p. 8-9

B 813, p. 17

B 792, p. 12

B 849-B, p. 147-148

(First Chance Cr.) (Fish Cr. basin). See (Last Chance Cr.).

(First Chance Cr.) (Goldstream basin) - (18.0-18.25, 16.95-17.15) ; GOLD, TUNGSTEN, TIN.

B 379, p. 194

B 917-A, p. 43

B 520, p. 241

B 926-A, p. 40

B 525, p. 113

B 933-A, p. 39

B 542, p. 206

B 1024-I, p. 188, 211

B 592, p. 358

BMB 142, p. 23

B 622 , p. 234

TDM 1, p. 32, 39-41

Flagler - $(14.55,15.65)$; GOLD. Nore.-Not mentioned by name in B 525. B 525, p. 197-198

B 592, p. 352

Flower - $(14.65,15.25)$; GOLD (?).

B 849-B, p. 152

(Fourth of July Cr.) (lode) - $(11.0,1.1)$; ANTIMONY, LEAD, SILVER. TDM 2, p. 13-14

(Fourth of July Cr.) (placer) - (11.1, 0.75-0.85) ; GOLD.

B 662, p. 351, 388, 393-394

(Fox) - $(17.55,17.0)$ approx ; LIMESTONE.

C 18, p. 6, 8-9

RI 4932 , p. $120-121$

(Fox Cr.) - (17.6-17.7, 17.35-17.8) ; GOLD, TUNGSTEN.

B 337, p. 39, 41

B 379, p. 191, 193

B 797, p. 20

B 442, p. 232

B 1024-I, p. 188, 210

B 525, p. 105-106, 111-113, 198

RI 4174, p. 28-29

TDM 2, p. 20, 24, 28

Franklin - $(19.5,17.5)$ approx ; TUNGSTEN.

B 692, p. 327 
Freeman \& Scharf - $(17,17)(?)$; GOLD, SILVER, LEAD. May be in Livengood quad.

B 520, p. 32

B 542, p. 184

B 525, p. 198

Frisco. See Fair Chance.

Garnet. See Yellow Pup.

Gem. See Ryan.

General Jaffre. See Tungsten Hill.

Geneva. See Ready Bullion.

(Gilmore Cr.) - (18.25-19.2, 17.4-17.6) ; GOLD, TUNGSTEN, BISMUTH.

B 251, p. 70

B 849-B, p. 71

B 284, p. 115, 118-119

B 897-A, p. 46

B 337, p. 39-40

B 910-A, p. 46

B 379, p. 192, 194

B 917-A, p. 43, 46

B 442, p. 232

B 926-A, p. 40, 42

B 480, p. 154-155

B 933-A, p. 39

B 520, p. 241

B 1024-I, p. 88, 211

B 525, p. 105-106, 109, 111, 113

C 331, p. 6

B 542, p. 206

C 335, p. 1

B 592, p. 359

BMB 142, p. 23

B 622, p. 234

BMB 153, p. 51

B 642 , p. 59

TDM 1, p. $39-40$

B 662, p. 54

(Gold Hill). See (Cripple Cr.), (Ester, Cr.).

(Gold King Cr.) - (14.8-15.1, 0.7-2.2) ; GOLD, TUNGSTEN.
P 70, p. 174
B 314, p. 212
B 836, p. 345
B 442 , p. 44
B 857-A, p. 38
B 480 , p. 226-228
B $864-A$, p. 42
B 501, p. 44, 49-51
B 868-A, p. 44
B 662, p. 400-401
B $880-A$, p. 46
B 755, p. 138
B 933-A, p. 49
TDM 1, p. 39

Gold King Hyd. Min. Co. See (Gold King Cr.).

Golden Champion. See (Cripple Cr.).

(Goldstream Cr.) - (17.15-18.25, 16.7-17.45) ; GOLD, TUNGSTEN, TIN.
P 70, p. 182
B 251, p. 67
B 284, p. 118
B 314, p. 36-37
B 337, p. 29, 38-41
B 345, p. 41-43
B 379, p. 190-191, 195
B 442, p. 232
B 480 , p. 154-155
B 520, p. 240-241
B 525, p. 105, 109-111, 113
B 542, p. 205-206
B 592, p. 68
B 622, p. 54-55, 234
B 642, p. 58-59
B 662, p. 51,54
B 692, p. 35

B 712, p. 39

B 714, p. $80-81$

B 722, p. 45

B 739, p. 29

B 755, p. 35,146

B 773, p. 45

B 783, p. 13

B 792, p. 14, 17

B 797 , p. 19

B 810, p. 25

B 813, p. 28,47

B 824, p. 32-33, 52

B 836, p. 32-33, 54

B S44-A, p. 32-33, 54

B 857-A, p. 30-31, 51

B 864-A, p. 34-35, 56

B $868-A$, p. $35-36,58$ 
(Goldstream Cr.) -Continued
B $880-A$, p. $39-40,61$
B 1024-I, p. 188, 211
B 897-A, p. 46-47, 71
C 331, p. 6
B 910-A, p. 46-47, 76
BMB 142, p. 23
B 917-A, p. 43-46
B 926-A, p. $40-42,70$
BMB 153, p. 51
RI 2192, p. 1-3
B 933-A, p. 38-39
TDM 1, p. 32, 39

Goldstream Min. Co. See (Goldstream Cr.).

Goodwin (antimony) - $(16.6,17.75)$; ANTIMONY. Includes references to Eagle, (Eagle Cr.) (Fairbanks dist.).

B 849-B, p. 157

TDM 2, p. 10

BMB 153, p. 52

Goodwin (gold) - $(17.15,17.7)$; GOLD. See also Bunker Hill.

B 849-B, p. 153-154

Grand Duke Nikolas. See Tungsten Hill.

Granite Hill. See Voght.

Grant (near Happy Cr.) - $(15.25,15.65)$; GOLD. Includes references to Irishman.
B 836 , p. 19
B $910-$ A, p. 25
B $849-$ B, p. 150

Grant (near Nugget Cr.). See Blue Bonanza.

Grant \& Hirschberger. See Tanana.

Green Mtn. - (18.6, 17.2) ; GOLD.

B 592 , p. $345-346$

(Grubstake Cr.) - (13.7-14.75, 0.35-0.8) ; GOLD, MERCURY, TUNGSTEN.
P 70, p. 173-174
B 813 , p. 35
B 314, p. 210-211
B 824, p. 40
B 442, p. 44
B 836, p. 41,345
B 480 , p. 225
B $880-$ A, p. 46
B 501, p. 44,48
B $926-$ A, p. 51
B 662 , p. $399-400$
B 933-A, p. 48-49
B 739, p. 35
TDM 1, p. 27, 39

Hanot Bros. See (Pedro Cr.).

Hansen. See (First Chance Cr.) (Goldstream Basin).

Happy Cr. $(15.15,15.7)$ (?) ; GOLD. See also Elmes; may be same mine. B $933-$ A, p. 23

(Happy Cr.) - (14.95-15.95, 15.2-15.8) ; GOLD ANTIMONY. See also Happy Cr.
B 592, p. 68,360
B 933-A, p. 39
B 622, p. 235
BMB 142, p. 23
B 642, p. 59
BMB 153, p. 51
B $917-A$, p. 43,45
TDM 1, p. 11

B 926-A, p. 40

Happy Home. See (Eva Cr.) (Fairbanks dist.)

Harrais. See Tungsten Hill.

(Hearst Cr.) - $(\mathbf{1 3 . 8 , 1 . 7 5 )}$ approx ; GOLD.
P 70, p. 173-174
B 501, p. 49
B 314, p. 211
B 662 , p. $399-400$
B 480 , p. 226

Hegan \& Lefebre - $(14.95,15.1)$; GOLD.

B 525, p. 207

B 542, p. 193

Henderson (\& McGinn). See Mohawk. 
Hess. See Prometheus.

Hess \& Thomas - $(\mathbf{1 4 . 7 5}, \mathbf{1 5 . 2 5 )}$; GOLD (?). See also Prometheus. B 525, p. 208

B 542, p. 194

Hightower. See Mohawk.

(Hill Cr.) - $(19.05,17.3)$; GOLD.

B 337 , p. 40

B 480 , p. 69

B 773, p. 19

(Homestake Cr.) - (11.1-11.15, 0.1-0.2) ; GOLD. See also (Homestake Cr.) under Healy quad.

P 70, p. 172-173

B 662, p. $388,395-397$

B 314, p. 209

B 480, p. 223-224

B $880-A$, p. 46

B 501 , p. $44-46$

B $897-$ A, p. 56

B $926-A$, p. 51

Horseshoe. See Ready Bullion.

Hosanna. See Ready Bullion.

Hudson. See Ready Bullion.

Hudson Bros. See Farmer.

Ijim. See Ryan.

(Iowa Cr.) - (20.55-21.0, 16.1-17.7) ; GOLD.

B 525, p. 113

Irene. Sce Liberty Bell.

Irishman. See Grant (near Happy Cr.).

Isaacson. See Ridge.

Janiksela - $(17.9,17.35)$; TIN ( ?).

B $849-$ B, p. 154

Jennie C. - $(14.35,15.5)$; ANTIMONY. Includes references to McQueen.

B 649, p. $18,40-41$

B $849-$ B, p. 157

B 692, p. 323

RI 4173, p. 38

Johnson. See Stepovich (tungsten).

Johnson, Norberg \& Ericson. See Liberty Bell.

Jolly Roger. See Prometheus.

(July Cr. ). See (Fourth of July Cr.) (placer).

Keys. See (Moose Cr.).

Koegley - $(14.4,15.15)$; GOLD.
B 525, p. 206
B 542, p. 192

Krutsch \& Cosgrove. See (Happy Cr.).

(Last Chance Cr.) - $(20.65,17.8)$; GOLD.
B 520, p. 243
B 592 , p. 359
B 542, p. 208
B 622, p. 233

Leah. See Billy Sunday.

Lean Fraction. See Billy Sunday.

Leidy - $(18.85,17.6)$; BARREN. Note.-Gold reported was not from lode. B $849-$ B, p. 155

Lepsoe - $(14.2,16.0)$ approx ; GOLD (?).

B 849-B, p. 152

Liberty. See Mohawk.

Liberty Bell - $(8.75,1.0)$; GOLD, BISMUTH, COPPER. Includes references to Eva (Min. Co.) (Bonnifield dist.), (Eva Cr.) (lode) (Bonnifield dist.), Eva Cr. Min. Co. (Bonnifield dist.), Eva Quartz Min. Co. (Bonnifield dist.); not mentioned by name in $B \mathbf{7 7 3}$.
B 662, p. 351, 355-356, 360
B 714, p. 41
B 666, p. 98
B 755, p. 40,139 
Liberty Bell-Continued
B 773, p. 31
B 868-A, p. 24
B 836 , p. $340-345$
B 880-A, p. 28-29
B 844-A, p. $19,80-81$
B $897-A$, p. $35-36$
B $857-A$, p. 19,76
C 196, p. 9

B 864-A, p. 23,80

Little Eva. See Stay.

(Little Moose Cr.) - (8.1-8.15, 0.65) ; GOLD, TUNGSTEN.
B 662 , p. $365-368$
RI 4174, p. 27
B 910-A, p. 55
TDM 2, p. 20

Lounsbury. See Clipper.

Lucky. See Stepovich (tungsten).

Lundbled \& Anderson. See Blossom.

M. B. Min. Co. See (Goldstream Cr.).

(McAdams Cr.) - (9.85-10.25, 0.6-0.85) approx; GOLD.

B 836, p. 41, 345

McCann \& Olsen. See Flower.

McCann, Thomas, Mickley \& Hagel. See St. Paul.

McDonald (\& Morton) - (14.95-15.), 15.1-15.2) ; GOLD, ANTIMONY. See also Blue Bird Min. Co.
B 692 , p. 323
B $849-$ B, p. 133-135

B 792 , p. 12

TDM 1, p. 11

McDonald, J. - See Wandering Jew.

McDonald, Michley, Hess, Thomas \& McCann. See Clipper.

McGlone. See Fair Chance.

McGlone \& Smith. See Billy Sunday, Dorothy \& Dorice, Fair Chance.

McLaughlin, Franklin \& Stay. See First Chance.

Macomb - $(15.25,15.65)$ approx ; GOLD (?).

B 849-B, p. 152

McQueen. See Jennie C.

Makaich. See Silver Dollar.

Maloney - $(14.25,15.2)$; GOLD, ANTIMONY.

B 849-B, p. 123

Marie. See Yellow Pup.

Mary Stay. See Ready Bullion.

Meier (, Hoffman \& Wallace). See Columbia.

Melba. See Yellow Pup.

(Melba Cr.) - (19.7, 17.7-17.9) ; GOLD, BISMUTH.
B 592 , p. 325
B $849-$ B, p. 71
B 666 , p. 98
C 335 , p. 1-2
B 714, p. 41

Merian - (15.1, 15.25) approx; GOLD (?).

B $849-B$, p. 152

Michley - $(14.7,15.7)$; GOLD.

B 849-B, p. 149.

Midnight Sun. See Blue Bonanza.

Mihalcik Bench. See (Ready Bullion Cr.).

(Mile 414.5, ARR) - $(6.8,10.05)$; LIMESTONE.

C 18, p. 6,8

Miller \& O'Conner. See Blue Bird (Miller \& O'Conner).

Minnesota. See Liberty Bell. 
Mohawk (Min. Co.) - (14.9-15.05, 15.5-15.55) ; GOLD, ANTIMONY, LEAD, ZINC. Includes references to Bondholder, Henderson (\& McGinn), Tyndall \& Finn ( St. Patricia Cr.), Tyndall \& Flynn.

B 525, p. 209

B 542, p. 195

B 592, p. 355

B 622, p. 237-238

B 642, p. 61

B 662, p. 413-414

B 712, p. 40

B 714, p. 81

B 755, p. 35

B 773, p. 15

B 783, p. 8

B 792, p. 11-12

B 797, p. 13

B 810, p. 15
B 813, p. 17

B 824 , p. 17

B 836, p. 18-19

B 844-A, p. 18

B 849-B, p. 142-147

B 864-A, p. 20

B 880-A, p. 21

B 897-A, p. 22

B 910-A, p. 25

B 926-A, p. 23

B 933-A, p. 23

BMB 142, p. 24, 52

TDM 2, p. 11

Monte. See Ryan.

Monte Cristo - $(19.7,17.8)$; GOLD, BISMUTH, TUNGSTEN. B 662, p. 412

(Monte Cristo Pup) - (19.55-19.7, 17.7-17.9) ; GOLD. B 622, p. 233

Montie. See Ryan.

(Moose Cr.) - (7.7-85, 0.8-0.9) ; GOLD, PLATINUM, MERCURY, TIN, TUNGSTEN. Includes references to (Big Moose $\mathrm{Cr}$.).

B 480, p. 221-222

B 501, p. 44

B 813, p. 35

B 520, p. 38

B 824, p. 40

B 662 , p. $351,355,360,365-368$

B 844-A, p. 41

B 712, p. 44

B 910-A, p. 55

B 722, p. 51

B 917-A, p. 54

B 739, p. 35

B 755, p. 138

B 926-A, p. 50-51

B 933-A, p. 47

TDM 1, p. 20, 27, 34, 39

Mother - $(19.95,15.8)$; GOLD.

B 842-B, p. 120-122

Murphy (lode). See Mother.

Murphy (lode and placer). See White Assn.

Murphy, J. See (Fourth of July Cr.) (lode).

Murphy \& Perrault. See American, American Eagle.

Murray \& Savage. See Yellow Pup.

Native Daughter. See Ready Bullion.

Neimi. See Yellow Pup.

(New Years Pup) - $(18,17)$; GOLD.

B 592, p. 359

Nickaloff. See Elmes.

North Pole. See Ready Bullion.

(Nugget Cr.) - (13.95-14.05, 15.9-15.95) ; GOLD.

B 917-A, p. 43

B 933-A, p. 39

B 926-A, p. 40

(O'Connor Cr.) - (15.95-16.05, 17.25-17.3) ; GOLD.

B 337, p. 39, 41

B 525, p. 106, 111, 113 
Ogram - $(18,17)$; GOLD (?). Note.-On Rose Cr.

B 525, p. 198

B 542, p. 184

Old Granite. See Stay.

Olsen. See St. Jude.

Orion. See Stepovich (tungsten).

(Our Cr.). See (Our Cr.) under Livengood quad.

Owl. See (Engineer Cr.).

Pearl. See Colbert.

(Pearl Cr.) - (19.95-20.0, 17.55-17.75) ; GOLD, TUNGSTEN, BISMUTH. Includes references to (Yellow Pup Cr.) ; see also White Assn.

B 520, p. 243 B 926-A, p. 40

B 542, p. $208 \quad$ B 933-A, p. 39

B 592, p. 359 B 1024-I, p. 188, 211

B 622, p. 233 C 335, p. 1

B 849-B, p. $71 \quad$ TDM 1, p. 39-40

B 917-A, p. 44 TDM 2, p. 27

(Pedro Cr.) - (18.2-18.4, 17.45-17.85) ; GOLD, TIN. See also (Pedro Cr.) under Livengood quad.

B 251, p. 67, 70-71, 75-77, 83-84

B 810, p. 25

B 259, p. 39-40

B 824, p. 33

B 263, p. 32-33, 42, 53-54, 208

B 836, p. 32-33

B 284, p. 111, 118-119

B 844-A, p. 32

B 314, p. 36

B 337, p. 29, 38

B 857-A, p. 30

B 379, p. 190, 192-193

B 864-A, p. 34-35

B 442, p. 232

B 868-A, p. 35-36

B 480, p. 154-155

B 880-A, p. 39

B 520, p. 241

B 897-A, p. 46

B 525, p. 105-106, 109, 111, 113

B 910-A, p. 46

B 542, p. 206

B 917-A, p. 43-44

B 592, p. 68, 359

B 926-A, p. 40, 70

B 622, p. 234

B 933-A, p. 38

B 642, p. 59

C 331, p. 6

B 662, p. 54

B 797, p. 20

BMB 142, p. 22-23

BMB 153, p. 51

TDM 1, p. 32

Pegleg. See Mohawk.

Perrault. See American.

Perrault \& Murphy. See American, American Eagle.

Peterson - $(17.9,16.75)$; GOLD (?).

B 849-B, p. 153

Polaris. See Stepovich (tungsten).

Prometheus - $(14.8,15.45)$; GOLD, SILVER, ANTIMONY, COPPER. Inciudes references to Hess, Jolly Roger.

B 525, p. 208

B 542, p. 194

B 649, p. 39

B $849-$ B, p. 148

B 592, p. 355

Prospect Min. Co. - $(9.7,1.3)$, SILVER, GOLD, COPPER, LEAD. Note.-Not mentioned by name in $\mathrm{C} 196$; see also (California Cr.) (lode).

B 868-A, p. 24, 63, 66-67

B $880-A$, p. $28,65,70$

Ptarmigan - (19.5, 17.5) (approx) ; TUNGSTEN. See also Franklin.

B 692, p. 327 
Quinn. See Scrafford.

Radovich. See Silver Dollar.

Rambler - $(8.05,1.15)$ approx; ANTIMONY, GOLD, SILVER, LEAD. Includes reference to lode antimony deposit on Cody $\mathrm{Cr}$.

B 662, p. 351, 357, 360-361, 368

TDM 1, p. 12

Ready Bullion - (14.3-14.35, 15.0-15.2) ; GOLD, ANTIMONY. Includes references to Borovich \& Stevens, Hudson, Stevens \& Borovich.

B 525, p. 203-206

B 542, p. 189-192

B 592, p. 325, 350-352

B 649 , p. $18,40-41$

B 824, p. 19

B 836, p. 18

(Ready Bullion C

B 337, p. 45

B 442, p. 234

B 480, p. 157-158

B 520, p. 243
B 844-A, p. 18

B 849-B, p. 123-127

B 864-A, p. 20

B 897-A, p. 22

B 910-A, p. 24

(Rex Cr.) - (8.85-8.9, 1.95) ; GOLD, ANTIMONY.
B 480 , p. 224
B 501, p. 44, 47
B 662 , p. $380-383$
B 926-A, p. 51
C 331, p. 7
TDM 1, p. 12

B 917-A, p. 54

B 525, p. 110, 112-113

B 542, p. 209

B 592, p. 359

B 622 , p. 235

Ridge - (18.1, 16.35) ; GOLD.

B 849-B, p. 153

(Roosevelt Cr.) - $(13.9,1.25)$ approx ; GOLD.

P 70, p. 173-174

B 314, p. 211

B 480 , p. 225-226

B 501, p. 44, 48-49

B 662 , p. $399-400$

Rose (Bonnifield dist.). See Liberty Bell.

Rose (Fairbanks dist.). See Stay.

(Rose Cr.) (lode) - $(18.5,17.35)$; ANTIMONY.

B 592, p. 346

B 649, p. 24, 41

(Rose Cr.) (placer) - (18.4-18.45, 17.35-17.42) ; GOLD, TUNGSTEN.

B 525, p. 113

B 1024-I, p. 188, 211

Royal Flush - (15.0, 15.6) approx; GOLD.

B 910-A, p. 25

Kyan - (14.9-15.1, 15.2-15.4) ; GOLD, SILVER, ANTIMONY. Includes references to Gem.

B 520, p. 33

B 525, p. 207

B 836, p. 19

B 542, p. 193

B 844-A, p. 18-19

B 662, p. 413

B 849-B, p. 135-138

B 692 , p. 323

B 868-A, p. 20

B 792, p. 12

B 917-A, p. 26

B 810 , p. 15

B 813, p. 17

B 824, p. 20

B $926-A$, p. 23

B 933-A, p. 23

TDM 1, p. 11

St. Jude - $(14.4,15.4)$; GOLD. May be same as Barker \& McQueen, which see also.

B 849-B, p. 123

593018-61-8 
(St. Patrick Cr.) - (15.05-15.55, 15.55) ; GOLD.

B 442, p. 234
B 480, p. 158
B 520, p. 243
B 525, p. 112-113

B 622, p. 235

B 880-A, p. 21

BMB 142, p. 23

BMB 153, p. 51

St. Paul - $(14.65,15.3)$; GOLD, ANTIMONY.

B 662, p. 409-410

B 692, p. 323

B 714, p. 81

B 712, p. 40

Sanford - $(14.85,15.85)$ GOLD.

B 849-B, p. 149

Scheelite. See Stepovich (tungsten).

Schubert - $(19.4,17.4)$; TUNGSTEN.

B 1024-I, p. 179, 189, 201

Scrafford - $(16.55,17.75)$; ANTIMONY, GOLD. NOTE.-Not mentioned by name in B 525 ; includes reference to Black Eagle.

B 525, p. 196

B 642 , p. 29

B 649, p. 17, 28-29

B 662, p. 415

B 849-B, p. 128-129

September 1st. See Colbert.

September 2nd. See Colbert.

Short. See Liberty Bell.

Silver Dollar - $(14.35,15.25)$ GOLD.

B 849-B, p. 127-128

Slav. See Stepovich (tungsten).

Smallwood. See Colbert.

(Smallwood Cr.) - (19.65-19.75, 16.6-17.0) ; GOLD.
B 337, p. 46-47
B 542, p. 208
B 345, p. 41-43
B 592, p. 68,361
B 379, p. 191
B 810, p. 25
B 520, p. 244
BMB 142, p. 23
B 525, p. 103, 110, 112-113
BMB 153, p. 51

Smith \& McGlone. See Billy Sunday.

Smith \& McGonnigle: See Billy Sunday.

Smith Bros. See Billy Sunday.

South Pole. See Ready Bullion.

Spite Fraction. See Mohawk.

(Spruce Cr.) - (8.7, 1.5) (?) ; ANTIMONY, SILVER, LEAD, GOLD. TDM 2, p. 14

Spruce Hen - (18.45-18.5, 17.1-17.15) ; TUNGSTEN, MOLYBDENUM, FLUORITE.
B 662, p. $422-423$
B 692 , p. $326-327$
B 926-C, p. 196
B 712 , p. 40
B $849-$ B, p. $157-158$
B 1024-I, p. 179-180, 188, 201-203
RI 4174, p. 24-25
TDM 1, p. 29

Standard Mines, Inc. See (Eva Cr.) (placer) (Bonnifield dist.).

Star Crystal. See Fair Chance. 
Stay - $(14.95,15.1)$; GOLD. NoTE.-Not mentioned by name in B 525, B 542 ; includes references to Little Eva.
B 520, p. 32-33
B 864-A, p. 20
B 525, p. 206-207
B $897-$ A, p. 22
B 542, p. 192-193
B 910-A, p. 24

B 849-B, p. 129-133

Stay, Sam, \& Co. See First Chance.

Stepovich (gold) - $(19.93,17.5)$; GOLD (?).

B 592, p. 330

Stepovich (tungsten) - (19.45-19.85, 17.45-17.7) ; TUNGSTEN. Includes references to Tungsten (Stepovich).

B 525, p. 166

B 542, p. 151

B 1024-I, p. 179, 183-184, 188-198

B 622 , p. $419-421$

B 692, p. 325-326

IC 7379, p. 68

B 712, p. 40

RI 4174, p. 4, 6-13, 15-23

B 755, p. 148

TDM 1, p. 40

TDM 2, p. 22-23

B 849-B, p. 157-158

Stevens \& Borovich. See Ready Bullion.

Stibnite (Eva Cr.) - $(14.9,15.4)$ ANTIMONY.

B 642, p. 29

RI 4173 , p. 38

B 649, p. 38-39

TDM 1, p. 8

Stibnite (Ready Bullion). See Ready Bullion.

Stipp, Logan \& Murphy. See Mother.

Stahl. See Yellow Pup.

Stahl, Birklid \& Anderson. See Yellow Pup.

Strand \& Diebold. See (Eagle Cr.) (Bonnifield dist.).

Sunflower. See Ready Bullion.

Sunrise. See Scrafford.

Swanson \& Montaine. See Liberty Bell.

Tanana - $(18.3,17.0)$; TUNGSTEN, GOLD.

B 662, p. $422-423$

B 755 , p. 148

B 1024-I, p. 179-180, 201,

B 849-B, p. 157-158

204-205

RI 4174, p. 23-24

Thomas \& Hess - $(15.05,15.35)(?) ; \operatorname{GOLD}(?)$

B 525, p. 209

B 542, p. 195

Titanic. See Stepovich (tungsten).

(Totatlanika Cr.) or (R.) - (11.05-11.15, 0.5-2.4) ; GOLD.

B 314, p. 208-209 B 836, p. 345

B 480, p. 222-223 B $917-$ A, p. 54

B 501, p. 44-45 B 926-A, p. 51

B 662, p. $388,391-394 \quad$ B $933-A$, p. 48

B 755, p. 40

Triangle. See Colbert.

Triple $\mathrm{X}$ Placers. See (Moose Cr.).

(Trixey Cr.) - $(6-15,0-4)(?)$; GOLD. May be in Healy quad.

B 910-A, p. 55

Tungsten (Stepovich). See Stepovich (tungsten).

Tungsten (Tungsten Hill). See Tungsten Hill. 
Tungsten Hill - (18.25, 17.0) ; TUNGSTEN, GOLD.
B 662 , p. $422-424$
B 1024-I, p. 179-180, 201, 205
B 692 , p. 327
B $849-$ B, p. $157-158$
RI 4174 , p. $24-26$
Tyndall \& Finn (Ready Bullion Cr.) - $(14.7,15.12)$ approx; GOLD.
B 525, p. 208
B 542, p. 194

Tyndall \& Finn (St. Patrick Cr.). See Mohawk.

Tyndall \& Flynn. See Mohawk.

Tyndall, Finn \& McLoughlin. See Mohawk.

Tyndall, Henderson \& McLaughlin. See Mohawk.

U.S. Smelt., Ref. \& Min. Co. - (NE 1/8 quad.). Includes references to Fairbanks

Exp. Co. ; see also U.S. Smelt., Ref. \& Min. Co. under Livengood quad.
B 792 , p. 14,17
B 868-A, p. 35-37, 58
B 797, p. $19-20$
B $880-A$, p. $39-40,61$
B 810 , p. 25
B 813, p. $28-29,47$
B $897-A$, p. $46-47,71$
B 824, p. $32-33,52$
B $910-A$, p. 46
B 836, p. 24, 32-33, 54
B 917-A, p. 43-45
B 844-A, p. 32-33, 54
B $926-A$, p. $40-42,70$
B 857-A, p. 30-31, 51
B 933-A, p. 38-39, 66-67
B 864-A, p. 34-35, 56
GQ 110
IC 7379 , p. $26-28$

Venus. See Stepovich (tungsten).

Verdin. See (Fox Cr.).

Voght - $(19.7,17.8)$; GOLD, BISMUTH. Includes references to Granite Hill, bismuth east of Monte Cristo $\mathrm{Cr}$.
B 592, p. $330-331$
C 331, p. 7
B 662 , p. 412

Vuyovich (Ester Cr.) - $(15.0,15.1)$; GOLD, ANTIMONY.

B 849-B, p. 128

Vuyovich (Ready Bullion Cr.) - $(14.6,15.4)$; GOLD.

B $849-$ B, p. 128

Wandering Jew - $(14.7,15.35)$; GOLD.

B $849-B$, p. 147

White Assn. - $(20.0,17.75)$; TUNGSTEN. Includes reference to Murphy (lode and placer).

B 662, p. 421

B 692 , p. 326

Wild Goose (Bonnifield dist.). See Liberty Bell.

Wild Goose (Fairbanks dist.). See (Engineer Cr.).

Wood (s). See Scrafford.

Woodpecker - $(\mathbf{1 8 . 7}, 17.2)$ approx ; GOLD.

B 592, p. 346

Yellow Pup - (19.75-19.85, 17.5-17.6) ; TUNGSTEN.

B 1024-I, p. 179, 189, 200-201

RI 4174, p. 4, 6, 8-9, 11, 14-16

IC 7379, p. 68

(Yellow Pup Cr.) See (Pearl Cr.).

Yellow Jacket. See Mohawk.

Zimmerman. See Franklin, Ptarmigan, Spruce Hen.

\section{FORT YUKON QUADRANGLE}

(_-..-) (potash) - (N 1/2 quad.) ; POTASH.

B 714, p. 55

B 797, p. 123 


\section{GOODNEWS QUADRANGLE}

(Aloric R.). See (Arolik R.).

(Arolik R.) - (4-5, 7-10) ; GOLD, PLATINUM. See also (Butte Cr.), (Kowkow Cr.).
B 692, p. 7, 21-22
B 844-A, p. 44
B 714, p. 38
B 857-A, p. 42
B 739, p. 158
B 864-A, p. 47
B 810, p. 32
B 868-A, p. 47
B 813, p. 37
B 880-A, p. 50
B 824, p. 43
B 897-A, p. 59
B 836, p. 44
B 910-A, p. 62
(Bear Cr.) - (7.8-7.9, 7.1-7.4) ; GOLD.
B 662, p. 61
B 813, p. 37
B 714, p. 222-223, 226-227
B 824, p. 43
B 722, p. 61
B 836, p. 44
B 797, p. 25
B 844-A, p. 44
B 810, p. 32
B 868-A, p. 47

Bradford and assoc. See (Arolik R.).

Bristol Bay Min. Co. See (Wattamuse Cr.).

(Butte Cr.) - (4.75-4.95, 8.65-8.7) ; GOLD, PLATINUM.

B 520, p. 41

B 813, p. 37

B 592, p. 71

B 880-A, p. 50

B 622 , p. 67, 304, 357-358

B 897-A, p. 59

B 662, p. 61

B 910-A, p. 62

B 714, p. 222, 227

B 917-A, p. 62

B 792, p. 20

B $926-A$, p. 58

B 797, p. 25

B $933-A$, p. 54

B 810, p. 32, 52-53

(Cascade Cr.) - (6.95-7.05, 5.6-5.85) ; GOLD.

B 844-A, p. 44

(Chowchow Cr.). See (Kowkow Cr.)

(Cow Cow Cr.). See (Kowkow Cr.)

Discovery Min. Co. See (Wattamuse Cr.)

(Fox Gulch) - (7.85-7.95, 6.25-6.3) ; GOLD.

B 897-A, p. 59

Goodnews Bay Min. Co. See (Arolik R.), (Snow Gulch).

(Goodnews R.) - (SW 1/4 quad.) ; GOLD. NoTE.-Report is probably of production from a tributary, rather than from main stream.

B 897-A, p. 59

Huff. See (Butte Cr.)

Kow Kow Min. Co. See (Kowkow Cr.).

(Kowkow Cr.) - (4.9-5.25, 8.15-8.4) ; GOLD, PLATINUM. Includes references to (Chowchow Cr.), (Cow Cow Cr.).

B 592, p. 71

B 622, p. 357-358

B 662, p. 61

B 714, p. 222-223, 227

B 722, p. 61

B 797, p. 25, 40

B 810, p. 32, 52-53

(Malaria Cr.) - (W 1/2 6,5) ; GOLD.

B 897-A, p. 59
B 864-A, p. 47

B 868-A, p. 47

B 880-A, p. 50

B 910-A, p. 62

B 917-A, p. 61-62

B 926-A, p. 58

B 933-A, p. 54 
Mosness. See (Snow Gulch).

(Olympic Cr. - (7.1-7.2, 5.8-6.8) ; GOLD.
B 797, p. 25
B 824, p. 43
B 810, p. 32
B 836, p. 44
B 813, p. 37
B 844-A, p. 44

Pioneer Gold Dred. Co. See (Arolik R.).

(Rainy Cr.). See (Rainy Cr.) under Bethel quad.

(Slate Cr.) - (6.95-7.05, 5.45-5.6) ; GOLD.

B 844-A, p. 44

B 910-A, p. 62

B 864-A, p. 46

B 917-A, p. 61

B 897-A, p. 59

(Snow Gulch) - (5.15-5.3, 9.55-9.95) ; GOLD.

B 592, p. 71

B 926-A, p. 58

B 917-A, p. 62

B 933-A, p. 54

(Wahmus Cr.) See (Wattamuse Cr.).

(Watermouse Cr.). See (Wattamuse Cr.).

(Watermuse Cr.). See (Wattamuse Cr.).

(Wattamuse Cr.) - $(6.55-6.95,5.85-6.1)$; GOLD. Includes references to (Wahmus Cr.), (Watermouse Cr.), (Watermuse Cr.).

B 692 , p. 40

B 714, p. 222-223, 225-226 · B 857-A, p. 42

B 722, p. 61

B 783, p. 15

B 797, p. 25

B 810, p. 32

B 813, p. 37

B 824, p. 42-43

B 844-A, p. 44

B 836, p. 44

B 864-A, p. 46

B 868-A, p. 47

B 880-A, p. 50

B 910-A, p. 62

B 917-A, p. 61

B 926-A, p. 58, 71

B 933-A, p. 54, 68

GULKANA QUADRANGLE

(Ahtell Cr.) - (23.45-24.1, 13.55-15.5) approx; GOLD. See also (Grubstake Cr.).
P 15, p. 47
B 926-A, p. 34
B 904, p. 48, 51
B 989-D, p. 190

B 917-A, p. 38

(Ahtell Cr., West Fork). See Neversweat.

Ahtell Min. Co. See (Grubstake Cr.).

Blue Ridge. See Indian.

Bronnicke. See (Porcupine Cr.).

Burns - $(21.5,15.35)$; LEAD, COPPER. Mentioned by name in RI 3940 only.

B 824, p. 124

RI 3940, p. 5-6

B 904, p. 46

(Eagle Cr.) - (20.55, 17.7-18.85) ; GOLD, COPPER, PLATINUM.

B 379, p. 157

B 989-D, p. 191, 194-195

B 943-B, p. 40-42

(Excelsior Cr.) - $(14-15,16)$ (?) ; GOLD (?).

P 15, p. 49

(Granite Cr.) - (22-23, 16-17) ; GOLD.
B 904, p. 51
B $989-$ D, p. 196 
(Grubstake Cr.) or (Gulch) - $(23.7,14.25)$; GOLD, SILVER, COPPER.

B 868-A, p. 32

B 868-C, p. 139-141

B 880-B, p. 106-107

B 897-A, p. 41

B 904, p. 48-50

B 910-A, p. 40

(Hidden Cr.) - (22.75, 13.65-14.35) ; GOLD.

B 904, p. 51

Indian - $(22.4,14.9)$; SILVER, LEAD, COPPER. Includes references to (Indian Cr.).

B 824, p. 122-124

B 904, p. 45-46

B 989-D, p. 211

(Indian Cr.). See Indian.

(Indian Cr., West Fork). See Burns.

Johnson. See (Grubstake Cr.).

Lyons and assoc. See (Ahtell Cr.), (Grubstake Cr.).

(Nadina R) - $(17.9,0.3-0.5)$; PLATINUM (very doubtful). May be in Valdez quad.

P 15, p. 63-64

Neversweat - $(23.3,14.85)$; LEAD, COPPER.

B 989-D, p. 211

(Porcupine Cr.) - $(24.05,14.35)$, GOLD, SILVER, COPPER, BISMUTH.

B 904 , p. 50-51

B 943-B, p. 43-44

Routsen \& Wallace. See Neversweat.

(Shnu) - (NW $1 / 4$ NE $1 / 4$ quad.) (?) ; GOLD(?). Note.-Probably the same as Sinona Cr.

P 15, p. 49

Silver Circle. See Burns.

(Silver Cr.) - $(23.6,13.35)$; SILVER, COPPER, LEAD, ZINC, GOLD. B 904, p. 46-47

B 989-D, p. 210-211

C 331, p. 16, 18

C 348, p. 3

C 248, p. 8

RI 3940, p. 7-8

Swanson, Olson, Johnson \& DeWitt. See (Grubstake Cr.).

White Bros. See (Eagle Cr.).

Whitham. See Burns, Indian.

Whitman. See Indian. Note.-Misspelling of Whitham.

\section{HAGEMEISTER ISLAND QUADRANGLE}

Chanie \& St. Clair. See (Dry Gulch).

(Clara Cr.) - (7.1-7.35, 16.8-17.0) ; PLATINUM, GOLD, CHROMITE. Includes references to Clara Cr. Min. Co.
B 813, p. 37-38, 60
B 880-A, p. 73
B 824, p. 66
B 836, p. 68
B 844-A, p. 66
B 857-A, p. 62
B 864-A, p. 67
B 868-A, p. 70

\author{
B 897-A, p. 83 \\ B $910-B$, p. $128-131$ \\ B 917-A, p. 82-83 \\ B 918 , p. 57-59, 67-70 \\ B 926-A, p. 76-77 \\ B 933-A, p. 73-74
}

Clara Cr. Min Co. See (Clara Cr.) 
Corrigal. See (Fox Gulch).

(Dowry Cr.) - (7.1-7.3, 16.7-16.8) ; PLATINUM.
B $880-A$, p. 73
B 933-A, p. 74

(Dry Gulch) - (6.9-6.95, 16.2-16.25) ; PLATINUM.

B 844-A, p. 66

B 918 , p. 58

B $857-A$, p. 62

B 933-A, p. 73-74

(Fox Gulch) or (Cr.) - (6.75-6.85, 16.2-16.3) ; PLATINUM, GOLD.

B 824 , p. 66

B 836, p. 68

B 844-A, p. 66

B 880-A, p. 73

B $897-$ A, p. 83

B 857-A, p. 62

B 910-B, p. 120-125

B 864-A, p. 67

B $917-$ A, p. $82-83$

B 868-A, p. 70

B 918 , p. 57-64

Garthe. See (Clara Cr.).

Goodnews Bay Min. Co. See (Dry Gulch,), (Fox Gulch), (Platinum Cr.), (Salmon R.), (Squirrel Cr.).

Haroldsen \& Wicklund. See (Clara Cr.).

Moeck \& Wolters. See (Squirrel Cr.).

Olson and assoc. See (Clara Cr.), (Fox Gulch), (Platinum Cr.), (Salmon R.).

(Platinum Cr.) - (6.9-7.2, 16.05-16.2) ; PLATINUM, GOLD, CHROMITE.
B 813, p. 60
B 824, p. 66
B 836 , p. 68
B $844-$ A, p. 66
B $857-A$, p. 62
B 864-A, p. 67

B $868-A$, p. 70

B $897-$ A, p. 83

B $910-B$, p. $120-125$

B $917-$ A, p. $82-83$

B 918 , p. $57-64$

B $933-$ A, p. 73-74

St. Clair. See (Squirrel Cr.).

(Salmon R.) - (7.05-7.35, 15.7-16.7) ; PLATINUM, GOLD. Includes references to Goodnews Bay Min. Co. if no other locality is specified.
B 864-A, p. 67-68
B $880-A$, p. 73
B 917-A, p. 61, 82-83
B $897-$ A, p. 83
B 918 , p. $65-67$
B $910-A$, p. 62,89
B 926-A, p. 76-77
B $933-$ A, p. $73-74$

B 910-B, p. 127-128

Sampson \& Garthe. See (Clara Cr.).

(SIug R.) - (7-8, 11-12) (?) ; GOLD.

B $910-A$, p. $63 \quad$ B $917-A$, p. 62

(Squirrel Cr.) - (7.0-7.05, 16.2-16.45) ; PLATINUM, GOLD, CHROMITE.
B 813, p. 60
B 868-A, p. 70
B 824, p. 66
B $880-A$, p. 73
B 836 , p. 68
B $897-$ A, p. 83
B $844-A$, p. 66
B $910-B$, p. 125-126
B $857-A$, p. 62
B 864-A, p. 67
B 918 , p. 57-58, 64-65
B 933-A, p. 73-74

Strandberg \& Sons. See (Dowry Cr.), (Dry Gulch), (Platinum Cr.), (Salmon

R.), (Squirrel Cr.).

Thompson \& St. Clair. See (Squirrel Cr.)

Thorsen. See (Clara Cr.), (Fox Gulch), (Platinum Cr.).

Thorsen \& Olson. See (Clara Cr.).

(Togiak Bay) - (9.0-10.0, 11.1-11.6) approx; GOLD.

B 622, p. 358

B $917-$ A, p. 62

B 910-A, p. 63 
Tonietzko \& Bennett. See (Platinum . Cr.)

Wickert. See (Platinum Cr.)

\section{HEALY QUADRANGLE}

Accident - $(21.6,4.0)$; GOLD.

B 498, p. 57

B 897-B, p. 119

Alaska Central Min. \& Exp. Co. See (White Cr.).

(Alaska Cr.) - $(9.3,15.2)$; GOLD.

B 622, p. 65

B 662, p. 376,378

Alaska Exp. \& Min. Co. - $(20.45,3.7)$; GOLD, SILVER. Includes references to

Timberline.
B 849-H, p. 461-463
B 868-A, p. 25-26
B 897-A, p. 34
B 880-A, p. 29
B 897-B, p. 114-117
B $910-\mathrm{A}$, p. 30-31

Alaska Range Min. \& Dev. Co. - (20.45, 3.7) ( ?) ; GOLD.

B 810, p. 17-18

Anaconda Copper Co. See Golden Zone.

(Antimony Cr.) See North Carolina.

Babel and assoc. See (Lucky Gulch).

Babel \& McDonald. See (Lucky Gulch).

Babel, McDonald, Johnson \& Johnson. See Lucky Top.

(Bain Cr.) - (8.45-8.7, 8.1-8.15) ; LIMESTONE.

RI 4932, p. 116-117

Beaudin. See Windy Cr. Deposit No.1.

Boedecker \& McGahan. See Yellowhorn.

(Bryn Mawr Cr.) - (2.95, 3.85) ; GOLD.

B 692, p. 221, 231

B 836, p. 295

Bucke. See (Valdez Cr.).

(Butte Cr.) - (16-19, 0-1) (?) ; GOLD, COPPER. B 592, p. 305

B 608, p. 76

Campbell \& Boedeker - $(20.8,3.55)$; GOLD, SILVER.

B 897-B, p. 117-118

(Cantwell) - $(8.35,7.0)$; CLAY.

C 18, p. 10

RI 4932, p. 39-40, 62-63

Carlson and assoc. See (Valdez Cr.).

Carlson, Fairfield \& Ohman. See (Valdez Cr.).

Center Star - $(3.55,4.6)(?)$; GOLD ( ?).

B 692, p. 230

(Chulitna R., W. Fork) - (0-2, 4-5) ( ?) ; CHROMITE, MANGANESE ( ?). B 692, p. 230

B 712, p. 23

(Chute Cr.) - $(\mathbf{1 7 . 4 5}, \mathbf{1 6 . 7 5 )}$ approx ; GOLD.

B 442, p. 36-37

B 480, p. 230

B 501, p. 53

(Cody Cr.) - $(13-15,14)$ (?) ; ANTIMONY. Reference may be to the Cody Cr. ir

Fairbanks quad.; see also (Rambler) under Fairbanks quad.

B 910-A, p. 103

Coffield and assoc. See Alaska Exp. \& Min. Co.

Coffield, Duff \& Olsen. See Alaska Exp. \& Min. Co.

(Colorado Cr.) - (2.8-3.5, 4.45-5.0) ; HAYDITE.

RI 4932 , p. $66-68$

(Copeland Cr.) - (2.45-2.75, 2.85-3.3) ; LIMESTONE.

RI 4932, p. 119-120 
Copper King - $(2.8,3.45)$; GOLD, SILVER, COPPER. Includes references to Hector.
B 692, p. 227-228
B 755, p. 135
B 849-E, p. 320-321
C 196, p. 7

Cora. See (Valdez Cr.).

Denali. See Alaska Exp. \& Min. Co.

(Dry Cr.) - (20.4-21.1, 17.0-17.6) approx ; GOLD.

B 480, p. 228-229

B 501, p. 51

Dunkle. See Golden Zone.

Eagle - $(3.75,4.7)$; GOLD, SILVER, COPPER, ANTIMONY, ZINC. Includes references to Northern Light.

B 692, p. 221, 223-224

B 849-E, p. 329-330

B 755, p. 133

Fairfield and assoc. See (Valdez Cr.).

Fairfield \& Ohman. See (Valdez Cr.).

Flaurier - $(3.1,4.2)$; GOLD, SILVER.

B 692, p. 230

(Foggy Pass) - (5.8-6.7, 7.8-8.0) ; LIMESTONE.

RI 4932, p. 117-118, 123

Folk. See (Valdez Cr.).

(Fox Gulch) - (11.05-11.1, 17.3) ; GOLD.

B 662, p. 396-397

Frates. See Yellowhorn.

(French Gulch) - $(9.55,15.25)$; GOLD.

B 662 , p. 376, 378

(Gagnon Cr.) - (9.75-9.85, 15.6-15.7) ; GOLD. Includes references to (Home Cr.).

B 622, p. 65

B 662 , p. 376-378

Giske. See (Portage Cr.).

(Glory Cr.) - (19.3, 15.2) approx; ANTIMONY, GOLD, LEAD, SILVER, ZINC.

TDM 2, p. 13

Gold Hill. See Accident, Yellow Horn.

(Gold Run) - (10.0-10.9, 15.55-15.75) ; GOLD.

B 813 , p. 35

B 836, p. 41

B 824, p. 40

B 844-A, p. 41

Golden Zone Extension - $(2.9,3.8)$; GOLD.(?).

B 692, p. 230

Golden Zone (Mine, Inc.) - (2.96, 3.83); GOLD, SILVER, ZINC, LEAD, COPPER ANTIMONY(?). Includes references to Dunkle.
B 692 , p. $221,226-227$
B 910-A, p. 30
B 755 , p. 135
B $917-$ A, p. 29
B 836 , p. $298-300$
B 926-A, p. 27
B 849-E, p. 321-325
B 933-A, p. 27
B $880-A$, p. 29
C 196, p. 7
B 897-A, p. 34
RI 5245, p. 1, 6-8

(Grizzly Cr.) - (14.5-15.5, 12.8-13.3) ; GOLD.

B 897-A, p. 56

(Healy Cr.) - (8.4-10.55, 14.95-15.6) ; GOLD, CLAY.

B 712, p. 44

RI 4932, p. $41-43,47,63-64$

B 963-E, p. 165-168

Hector. See Copper King. 
Hill Top. See Lindfors.

Holt. See (Grizzly Cr.).

(Home Cr.). See (Gagnon Cr.).

(Homestake Cr.) - (11.05-11.15, 17.3-17.65) ; GOLD. Includes references to (Platte Cr.) ; see also (Homestake Cr.) under Fairbanks quad.

P 70, p. 172-173

B 314, p. 209

B 442, p. 44

B 480, p. 223-224

B 501, p. 44-46

B 662, p. 388, 395-397

B 755, p. 40

B 813, p. 35

Jerome - See (Chute Cr.).

Joplin. See (Valdez Cr.).

Jumbo - $(3.05,4.1)$; GOLD(?), SILVER(?).

B 692 , p. 230

(Kansas Cr.) (antimony) - $(18.4,15.35)$; ANTIMONY, LEAD, ZINC.

RI 4173 , p. 5, 36-37

TDM 1, p. 11

(Kansas Cr.) (gold) - $(17.05,15.65)$ approx; GOLD.

B 480 , p. $230-231$
B 844-A, p. 41

B $857-$ A, p. 38

B 868-A, p. 44

B $880-$ A, p. 46

B $897-$ A, p. 56

B 926-A, p. 51

B 933-A, p. 48

Kirk, M. P., \& Sons, Inc. See (Cody Cr.).

Kroenung. See Windy Cr. Deposit No. 1.

Liberty - $(3.55,4.65)$; GOLD, SILVER.

B $849-\mathrm{E}$, p. 328

(Lignite Cr.) - (7.95-10.4, 15.9-16.5) approx ; CLAY, GOLD(?), ANTIMONY(?). B 662 , p. 369

TDM 1, p. 12

C 18, p. 9

Lindfors - $(2.85,3.7)$; GOLD, ZINC, COPPER.
B 692 , p. 226
B 849-E, p. 321

B 755, p. 134-135

C 196 , p. 7

(Little Windy Cr.) (clay) - (8.3, 7.8) ; CLAY.

RI 4932 , p. $39-40,63$

(Little Windy Cr.) (limestone) - (8.0-8.2, 8.0) ; LIMESTONE. RI 4932, p. 115-117

(Long Cr.) - (2.3-2.5, 3.3-3.75) ; LIMESTONE. RI 4932, p. 120

(Lucky Gulch) or (Cr.) - (21.35-21.55, 3.95-4.2) ; GOLD.

P 70, p. 168-169

B 379, p. 159-160

B 480, p. 119,122

B 498, p. 55,63

B 592, p. 307-308

B 662, p. 49

B 755, p. 137

B 783, p. 12

B 797, p. 18

B 813, p. 26
B 824, p. 31

B 836, p. 29

B 849-H, p. 454-455

B 868-A, p. 34

B 880-A, p. 37

B $897-$ B, p. 129

B 910-A, p. 42

B 917-A, p. 40

B 926-A, p. 36

Lucky Strike. See Lindfors.

Lucky Top - $(21.65,3.85)$; GOLD.

B 897-B, p. 119-120 
Lucrata - $(3.65,4.7)$; GOLD, SILVER. Includes reference to Lucrative.

B 692, p. 224

B 849-E, p. 328-329

B 755, p. 133-134

Lucrative. See Lucrata.

McCallie. See Ready Cash.

(McCuen Gulch) - (11.9, 17.35-17.45) ; GOLD.

B 662 , p. 351, 355, 387-388, 397-398

McKinley Gold Placers Co. See (Valdez Cr.).

McKinley Placer Min. Co. See (Valdez Cr.).

(Marguerite Cr.) - (10.7-10.75, 17.05-17.1) ; GOLD.
B 813, p. 35
B 880-A, p. 46
B 824, p. 40
B $897-$ A, p. 56
B 836, p. 41, 345
B $910-A$, p. 55
B 844-A, p. 41
B $917-$ A, p. 54
B 857-A, p. 38
B 926-A, p. 51
B 864-A, p. 42
B $933-$ A, p. 48

B 868-A, p. 44

Mayflower - $(3.05,3.85)$; SILVER, GOLD, COPPER.

B 849-E, p. 325-326

Monahan. See (Valdez Cr.).

(Moose Cr.) - (13.7-13.85, 17.6-17.7) ; GOLD.

$\begin{array}{ll}\text { B } 836, \text { p. } 41,345 & \text { B } 868-A, \text { p. } 44 \\ \text { B } 844-A, \text { p. } 41 & \text { B } 880-A, \text { p. } 46 \\ \text { B } 857-A, \text { p. } 38 & \text { B } 897-A, \text { p. } 56\end{array}$

B 864-A, p. 42

Morning Glory. See Lindfors.

Muir. See (Marguerite Cr.).

(Newman Cr.) - (21.05-21.3, 17.1-17.6) ( ?) ; GOLD.

B 480, p. 229

B 501, p. 52

Nicola. See (Valdez Cr.).

North Carolina - $(4.3-4.45,1.75-1.8)$; ANTIMONY, GOLD.

B 692 , p. $229-230$

TDM 1, p. 12

B 755 , p. $135-136$

TDM 2, p. 14-15

Northern Light. See Eagle.

Pardners' Mines. See Golden Zone.

(Platte Cr.) See (Homestake Cr.).

(Popovich Cr.) - (9.0-9.1, 16.2-16.5) ; GOLD( ?).

B 662 , p. $369-370$

(Portage Cr.) - $(22.35,16.4)$ approx.

B 480 , p. 229

B 501, p. 44, 52

B 910-A, p. 55

B $864-A$, p. 42

B $917-A$, p. 54

B $897-A$, p. 55

B $926-$ A, p. 51

B 933-A, p. 49

Powless. See Alaska Exp. \& Min. Co.

Ready Cash - $(1.0,2.7)$; SILVER, GOLD, LEAD, COPPER, ZINC.

B 692 , p. 228-229

B 849-E, p. 311-313, 316, 318-320

B 755, p. 135

C 196, p. 7

Ridge. See (Kansas Cr.) (antimony).

Riverside - $(3.15,4.15)$; GOLD, SILVER, ZINC, LEAD, COPPER.

B 692, p. $225-226$

B $\$ 36$, p. $297-298$

B 755, p. 134

B $849-\mathrm{E}$, p. $326-327$

(Rock Cr.) - $(16.95,16.05)$ approx ; ANTIMONY.

TDM 2, p. 13 
Rogers. See (Chute Cr.).

(Rusty Cr.) - (21.0, 3.45-4.0) ; GOLD.

B 480, p. 119, 122-123

B 755, p. 137

B 498, p. 64-65

(Sanderson Cr.) - (10.4-10.65, 16.05-16.1) ; GOLD( ?).

B 662 , p. $369-371$

(Shotgun Cr.) - (1.85-2.0, 0.85-0.9) ; GOLD.

B 692, p. 231

Silver King - $(3.5,4.6)$; ANTIMONY, GOLD, COPPER.

B 692 , p. 224-225

B 849-E, p. 327-328

B 755 , p. 134

Smith \& Wickersham. See Yellowhorn.

Sparks. See (Kansas Cr.) (antimony), North Carolina.

Stevens. See Mayflower.

Stevens \& Colvin. See Liberty.

Stibnite. See Silver King.

(Sugarloaf Mtn.) - (9.1-9.25, 13.8-14.0) ; PERLITE.

P 293-A, p. 15

RI 4932 , p. $79-81$

(Suntrana) - $(8.9,15.1)$; CLAX.

B 963-E, p. 165-168

RI 4932, p. $41-43,47,63-64,125$

C 18, p. 9

Tammany (Channel). See (Valdez Cr.).

Tammy. See (Valdez Cr.).

Tangel, Frisley, Murry \& Miller. See Ready Cash.

Timberline. See Alaska Exp. \& Min. Co.

(Timberline Cr.) - (20.1-20.6, 3.6-3.7) ; GOLD.

B 498 , p. 55

B 797 , p. $17-18$

Tommy. See (Valdez Cr.).

(Trixey Cr. ) - (7-15, 14-17) (?) ; GOLD. May be in Fairbanks quad.

B 910-A, p. 55

Tunnell Min. Co. See (Valdez Cr.).

(Valdez Cr.) - (19.85-20.1, 3.5-3.75) ; GOLD, PLATINUM(?). Includes references to Carlson and assoc., Folk, Tammany (Channel), Valdez Cr. Placer Min Co.; unconflrmed report of platinum in $\mathrm{B} 692$.

P 41, p. 117-118

P 70, p. $165,167-169$

B 345, p. 37-38

B 810, p. 23

B 379, p. $52,159-160$

B 813 , p. 26

B 442 , p. $42-43$

B 824, p. 31

B 480 , p. $119-121$

B 836 , p. 29

B 498, p. 53-54, 56-62

B 844-A, p. $29-30$

B 542, p. 44

B $849-\mathrm{H}$, p. $427-428,437,444-453$

B 592, p. 67, 307-308

B 857-A, p. 28

B 622, p. 49

B 864-A, p. 32

B 642 , p. 56

B 868-A, p. 34

B 662, p. 49

B $880-A$, p. 37

B 692 , p. 22

B $897-$ B, p. $125-127$

B 714, p. 78

B $910-A$, p. 42

B 722 , p. 39

B 755, p. 32,137

B 917-A, p. 40

B 926-A, p. 36

B 797 , p. 18

B 933-A, p. 36

BMB 153, p. 42-43 
Valdez Cr. Placer Mines Co. See (Valdez Cr.).

Valdez Cr. Placer Min. Co. See (Valdez Cr.).

Wagner and assoc. - $(21.8,3.75)$; GOLD.

B 897-B, p. 120-121

Wells. See Golden Zone, Riverside.

Wells Bros. See Golden Zone.

(White Cr.) - (21.05-21.15, 3.75-3.9) ; GOLD.
B 480 , p. 119,123
B $857-A$, p. 28
B 498, p. $55,64-65$
B $864-A$, p. 32
B 662, p. 49
B 868-A, p. 34
B 755 , p. 137
B $897-$ B, p. 128-129
B 783, p. 12
B 910-A, p. 42
B 797 , p. 18
B 917-A, p. 40
B 844-A, p. 29-30
B 926-A, p. 36
B $849-\mathrm{H}$, p. $453-454$
B 933-A, p. 36

Wickersham Bros. See (Lucky Gulch), (White Cr.).

(Wickersham Cr.) - (18, 1-2) ; GOLD.

B 592, p. 305

B 608, p. 76

Windy Cr. Deposit No. 1 - (8.3-8.4, 7.9-8.0) ; LIMESTONE. RI 4932, p. 95-115, 123

Windy Cr. Deposit No. 2 - $(6.75-7.05,7.5-7.65)$; LIMESTONE. RI 4932, p. 116-117

Windy Cr. Deposit No. 3 - (7.4-7.8, 7.9-8.1) ; LIMESTONE, CLAY. RI 4932, p. 116-117, 125

(Windy Ridge) - (8.2-8.75, 8.0-8.1) ; LIMESTONE.

C 18, p. $5-6,8$ RI 4932 , p. $116-117$

Yellowhorn - $(21.4,4.0)$; GOLD, SILVER.

B 498 , p. 56

B 910-A, p. 30-31

B 897-B, p. 118-119

\section{HOLY CROSS QUADRANGLE}

(Anvik R.) - (10, 12-17), (11, 11-12) ; GOLD, PLATINUM(?). May be in Unalakleet quad.

B 683 , p. $62-63$

B 692, p. 22,40

(Flat Cr.) - $(8.5,1.35)$; GOLD, MERCURY. Includes references to (Stuyahok

Cr.), (R.), or (Valley).
B 712, p. 50
B 739, p. 7,40
B 783, p. 15
B 797, p. 24
B 810, p. 30
B 813, p. $35-36$
B 824, p. 41
B 836, p. 41
B 844-A, p. 41
B 857-A, p. 38

B 864-A, p. 43-44

B 868-A, p. 43

B 880-A, p. 45

B 897-A, p. 53

B 910-A, p. 54-55

B 917-A, p. 52

B 926-A, p. 47

B 933-A, p. 44

TDM 1, p. 27

Hitt. See (Flat Cr.).

(Stuyahok Cr.), (R), or (Valley). See (Flat Cr.). 


\section{HOWARD PASS QUADRANGLE}

(Mt. Bupto) - $(9.5,8.8)$ approx ; PHOSPHATE.

P 302-A, p. 11-12, 15

(-----) (phosphate) - $(14.5,11.0)$ approx ; PHOSPHATE.

P 302-A, p. 11-12, 15

\section{HUGHES QUADRANGLE}

(Alatna R.) - (17.5-18.0, 16.8-17.9) ; GOLD.

P 10, p. 50

B 536, p. 143-144

B 910-A, p. 56

B 815, p. 334-335

B 926-A, p. 52

(Bear Cr.) - $(2.0,4.0)(?)$; GOLD.

B 739, p. 41

(Black Cr.) - $(\mathbf{1 5 . 3 5}, \mathbf{1 . 6})$; GOLD.

$\begin{array}{ll}\text { B } 592, \text { p. } 383 & \text { B } 642, \text { p. } 65 \\ \text { B } 631, \text { p. } 83-84 & \text { B } 692, \text { p. } 39\end{array}$

(Felix Cr.) or (Fork) - $(15,1)(?)$; GOLD.

B 520, p. $38 \quad$ B 692, p. 39

B 536, p. 143

(Helpmejack Cr.) - (16.75-17.5, 16.8-17.9) ; GOLD.

P 10, p. 50

(Hughes Bar) - (12, 0-1) (?) ; GOLD. May be in Melozitna quad.

AR 21, p. 483

B 442, p. 297

P 20, p. 98

B 536, p. 142

(Indian R.) - (15.7-15.9, 1.05-1.35) ; GOLD, MOLYBDENUM.

B 536, p. $143 \quad$ B 642, p. 65

B 592, p. $383 \quad$ B 692, p. 39

B 631, p. 82 TDM 1, p. 29

McGee. See (Utopia Cr.).

(Pocahontas Cr.) - $(14.25,0.3)$; GOLD.

B 631, p. 83

(Red Mtn.) - (S1/2 14,5) ; GOLD.

B 592, p. 383

B 631, p. $82-83$

(Reed R.) - (7-8, 14-17) ; GOLD(?).

B 824 , p. 50

B 836, p. 51

(Rocky Bottom Cr.) - (17.3-17.55, 17.6-17.9) ( ?). P 10, p. 50

(Snyder Cr.) - $(15,1)(?)$; GOLD.

B 520, p. 38 B 536, p. 143

(Utopia Cr.) - (15.7-16.1, 0.4-0.6) ; GOLD.

B 631, p. 83

B 917-A, p. 55

B 642 , p. 65

B 926-A, p. 52

B $897-$ A, p. 54

B 933-A, p. 47

B $910-A$, p. 56 


\section{IDITAROD Q QUADRANGLE}

Alaska. See (Moore Cr.).

AIpha Min. Co. See (Flat Cr.).

Awe. See (Chicken Cr.).

Awe \& Durant. See (Flat Cr.).

B \& C. See (Moore Cr.).

Bauquier \& Hatten. See (Willow Cr.).

Beatson \& Donnelly. See (Otter Cr.).

(Beaver Cr.) - (SE 1/2 quad.) ( ?) ; GOLD.

B 520, p. 40

Becker. See (Chicken Cr.).

Belanger, Thibault \& LaChance. See (Willow Cr.).

Black Bear Min. Co. See (Chicken Cr.).

(Black Cr.) or (Gulch) - (8.45-8.7, 7.75-7.95) ; GOLD, MERCURY, ANTIMONY, TIN, TUNGSTEN.

B 480, p. 267

B 662, p. 59

B 520 , p. 40

B 754, p. 112

B 542, p. $300,302-303$

B 836, p. 43

B 578, p. $31-35$

B 933-A, p. 43

B 592, p. 69

B 622, p. $56-57,286$

B 642, p. 253, 255, 266

C 255, p. 9

B 649 , p. $48-49$

BMB 142, p. 26

B 655, p. 145

BMB 153, p. 54

TDM 1, p. 26, 35

Bonanza. See (Flat Cr.).

(Bonanza Cr.) - (8.0-9.0, 5.8-5.9) (?) ; GOLD (?).

B 520 , p. 40

Brink. See DeCourcy.

Chicago. See (Flat Cr.).

(Chicken Cr.) - (8.25-8.45, 5.85-6.75) ; GOLD, SILVER, MERCURY, ANTI-

MONY. Includes references to Chicken Cr. Min. Co.

P 268, p. 107

B 520 , p. 40

B 542, p. 300, 302-303

B 578, p. 31, 33, 35

B 592, p. 69

B 622 , p. $56-57,257$

B 642 , p. $66,253,257,259,266$

B 649, p. 49

B 662, p. 59

B 722, p. 56

B 739, p. 40

B 754, p. 114, 116

B 773, p. 14, 19

B 783, p. 14

B 797, p. 21
B 813, p. 29

B 824, p. 34

B 836, p. 34

B 844-A, p. 34

B 857-A, p. 32

B 864-A, p. 37

B 864-C, p. 213-215

B 868-A, p. 37

B 880-A, p. 41

B $910-$ A, p. 50

B 933-A, p. 43

C 255, p. 1, 9

BMB 142, p. 26

BMB 153, p. 54

Chicken Cr. Min. Co. See (Chicken Cr.).

(Crooked Cr.) See (Donlin Cr.). 
DeCourcy - $(4.3,1.15)$; MERCURY. Includes references to (DeCoursey Mtn.), (Montana Cr.), Thrift; not mentioned by name in B 933-A.
B 722 , p. 24,57
B 739 , p. 13
B 773, p. 31,47
B 783 , p. $25-26$
B $864-$ C, p. 243

P 268, p. 2, 105, 108, 111-113

B 933-A, p. 91

IC 7379 , p. $63-64$

RI 4065 , p. 3, 9, 29-43

TDM 1, p. 24-26

TDM 2, p. 17

(DeCoursey Mtn.). See DeCourcy.

DeCoursey Mtn. Min. Co. See DeCourcy.

(Donlin Cr.) - (6.0-6.8, 0.5-1.6) ; GOLD, ANTIMONY, TIN, TUNGSTEN, MERCURY. Includes references to (Crooked Cr.), (Lewis Gulch), (Quartz Gulch), (Queen Gulch), (Ruby Cr.), (Snow Gulch).

$\begin{array}{ll}\text { P } 268, \text { p. } 4,69,116,118 & \text { B } 844-A, \text { p. } 43 \\ \text { B } 480, \text { p. } 267,270 & \text { B } 857-A, \text { p. } 41 \\ \text { B } 520, \text { p. } 40 & \text { B } 864-\text { A, p. } 45 \\ \text { B } 622, \text { p. } 257,261,304,351-353 & \text { B } 868-A, \text { p. } 46 \\ \text { B } 722, \text { p. } 60 & \text { B } 880-A, \text { p. } 49 \\ \text { B } 783, \text { p. } 15 & \text { B } 897-A, \text { p. } 58 \\ \text { B } 797, \text { p. } 24 & \text { B } 910-A, \text { p. } 60 \\ \text { B } 810, \text { p. } 31-32 & \text { B } 917-A, \text { p. } 59 \\ \text { B } 813, \text { p. } 37 & \text { B } 926-A, \text { p. } 56 \\ \text { B } 824, \text { p. } 42 & \text { B } 933-A, \text { p. } 52\end{array}$

B 836 , p. 43

Duffy \& Co. See (Chicken Cr.).

Durand \& Awe Bros. S'ee (Flat Cr.).

Erickson. Sce (Ganes Cr.) under Ophir quad.

Felder-Gale \& Co. See (Yankee Cr.) under Ophir quad.

Felder, Gale \& Higgins. See (Ganes Cr.) under Ophir quad.

Fidelity-Kuskokwim (Quicksilver Co.). See DeCourcy.

Fine Gold. See (Willow Cr.).

(Flat) - (7.8-8.85, 6.85-8.05) ; MERCURY. NotE.-Reference is to "Iditarod Cr.," but probably should be to either Flat or Otter Cr.

RI 4065 , p. 5

(Flat Cr.) - (7.95-8.15, 6.85-8.0) ; GOLD, SILVER, MERCURY, ANTIMONY.

B 480 , p. $255-258,262-267$

B 783, p. 14

B 520 , p. 40

B 797, p. 21

B 542 , p. $299,302-303$

B 813 , p. 29

B 578 , p. $31-35$

B 824, p. $34-35$

B 592, p. 69

B 622, p. $56-57,257,260$

B 836, p. 34

B $844-A$, p. 34

B 642 , p. $23-24,26,253,255-\quad$ B $857-A$, p. 33,51

258,266

B 655, p. 152

B 662, p. 59

B $864-A$, p. $36-37,56$

B 864-C, p. 201-208, 243

B 712 , p. 48

B $868-A$, p. 37,59

B 722, p. 56

B 739 , p. 40

B 754 , p. $112-117$

B 755 , p. 44

B 773 , p. $14, .19$

B $880-A$, p. 41,61

B $897-$ A, p. $48-49,71$

B 910-A, p. 50

B 926-A, p. 45, 71

B 933-A, p. 43,67

C 255, p. 1, 4-5, 9

$593018-61-9$ 
(Flat Cr.)-Continued BMB 142, p. 26

BMB 153, p. 54

TDM 1, p. 26

Flume Dred. Co. See (Yankee Cr.) under Ophir quad.

(Fourth of July Cr.) - $(15,11)(?)$; GOLD.

B 520, p. 40

B 910-A, p. 59

French Hill. See (Ganes Cr.) under Ophir quad.

Gane, Spencer, Roke \& Maki. See (Ganes Cr.) under Ophir quad.

(Ganes Cr.) - (18.7-19.8, 16.1-17.9). See (Ganes Cr.) under Ophir quad.

NoTE.-Mining on Ganes Cr. has been in both Iditarod and Ophir quads.

Ganes Cr. Dred. Co. See (Ganes Cr.) under Ophir quad.

Ganes Cr. Min. Co. See (Ganes Cr.) under Ophir quad.

(George Cr.) - (SE1/4 quad.) (?) ; GOLD. May be in Bethel, Russian Mission, or Sleetmute quad.

B 722, p. 60

(George R.) - (SE1/2 quad.) ( ?) ; GOLD. May be in Sleetmute quad. B 722, p. 60

Garnet - $(8.15,6.8)(?)$; GOLD.

B 824, p. 23

Gibbs \& Eison. See (Ganes Cr.) under Ophir quad.

(Glacier Gulch) - (NE1/4 19,17) ( ?) ; GOLD. B 379, p. 261

B 410 , p. 70

Glen. See (Flat Cr.).

(Glen Gulch) or (Cr.) - (8.75, 7.9) ; GOLD, ANTIMONY, MERCURY, TUNGSTEN. See also Golden Horn.

B 520, p. 40

B 542, p. 300, 302-303

B 578, p. 31-32, 35

B 592, p. 69

B 622, p. 56,287

B 642, p. 253, 255, 266

B 649, p. $48-49$

(Gold Cr.) or (Run) - $(7.35,6.45)$ approx ; GOLD.

B 520, p. 40

Golden Horn (Min. Co.) - $(8.8,7.8)$; GOLD, ANTIMONY, MERCURY, LEAD. Includes references to lode at head of Glen Gulch, Mohawk (lode); see also (Glen Gulch).

B 622, p. 266-267, 287

B 642 , p. 259

B 649, p. 47-48

B 655, p. 145, 152

B 754, p. 116-117

(Granite Cr.) - (8.7-8.85, 8.05-8.35) ; GOLD. Includes references to Salen.

B 480 , p. 254

B 783, p. 14

B 797 , p. 21

B 824 , p. 35

B 836 , p. 35

B 844-A, p. 34

B $864-A$, p. 37
B 655 , p. 145

B 755, p. 44

B 864-C, p. 218-219, 242

C 255, p. 9

BMB 142, p. 26

TDM 1, p. 26
B 864-C, p. 209

B 864-C, p. 242

B 868-A, p. 25

B $880-A$, p. 27,72

B 910-A, p. 32-33

TDM 1, p. 26

B 864-C, p. 220-221

B 868-A, p. 37

B $880-$ A, p. 41

B 910-A, p. 50

B 933-A, p. 43

C 255, p. 9,12

Guinan \& Ames Dred. Co. See (Ganes Cr. ) under Ophir quad.

Gum Boot. See (Otter Cr.). 
Haggerty. See (Willow Cr.).

(Happy Cr.) or (Gulch) - (7.6-8.15, 6.65-6.8) ; GOLD, SILVER, MERCURY, ANTIMONY, TUNGSTEN.

B 480 , p. $256,258-259$

B 844-A, p. 34

B 542, p. $300,302-303$

B $857-$ A, p. 32

B 578, p. 31, 33, 35

B 864-A, p. 37

B 592, p. 69

B 622 , p. $56,257,286$

B 864-C, p. 211-213

B 642 , p. $66,253,257,266$

B 868-A, p. 37

B 655, p. 145

B 880-A, p. 41

B 662 , p. 59

B 897-A, p. 49

B 722, p. 56

B 910-A, p. 50

B 754, p. 114

B 926-A, p. 45

B 755, p. 44

B 933-A, p. 43

B 773, p. 14, 17

C 255, p. 1, 9

B 783, p. 14

BMB 142, p. 26

B 797, p. 21

BMB 153, p. 54

B 824, p. 34

RI 4065, p. 5

B 836, p. 34-35

TDM 1, p. 25-26

Higgins. See (Yankee Cr.) under Ophir quad.

Hillside. See (Flat Cr.).

Hilltop. See (Flat Cr.).

Holky \& Shonbeck. See (Ganes Cr.) under Ophir quad.

Holky Dred. Co. See (Ganes Cr.) under Ophir quad.

Idaho. See (Flat Cr.).

Iditarod Min. Co. See (Willow Cr.).

Innoko Dred. Co. See (Ganes Cr.) under Ophir quad.

Jensen. See (Willow Cr.).

Joaquin. See (Ganes Cr.) under Ophir quad.

Johnson \& Martin. See Golden Horn.

(Jualin Cr.). See (Julian Cr.).

(Julian Cr.) - (13.15-13.4, 3.65-4.0) ; GOLD, MERCURY. Includes references to (Jualin Cr.).

P 268, p. 71, 116, 119

B 480, p. 267,270

B 520, p. 40

B 622, p. 261

B 844-A, p. 43

B 857-A, p. 41

B $868-$ A, p. 46

B $880-A$, p. 49

B 897-A, p. 58

B 910-A, p. 60

B $864-A, 0.45$

B 917-A, p. 59

B 926-A, p. 56

C 255, p. 16,18

Kaatz - $(19,17)(?)$; ANTIMONY. May be in Ophir quad.

B 642, p. 259

B 754 , p. 116

B 649, p. $49-50$

B 864-C, p. 228

Kaskinen. See (Moore Cr.).

Keturi. See (Moore Cr.).

K.P.M. See (Otter Cr.).

La Chance \& Thibault. See (Willow Cr.).

Last Chance. See DeCourcy.

(Last Chance Gulch) - (NE 1/4 19, 17) (?). See (Last Chance Gulch) under Ophir quad.

(Lewis Gulch). See (Donlin Cr.).

Lindfors and assoc. See DeCourcy. 
(Little Cr.) - (2.4-2.6, 0.2-0.8) ; GOLD, MERCURY.

P 268, p. $120 \quad$ B 622, p. 304

B 480, p. 267,270

Loranger (\& Jensen). See (Willow Cr.).

Lusher. See (Malamute Cr.).

Lyman (\& Rocheleau). See DeCourcy.

(Mackie Cr.) - (NE $1 / 419,17)(?)$; GOLD. May be in Ophir quad. B 864-C, p. 188

(Malamute Cr.), (Gulch), or (Pup) - $(8.8,8.05)$; GOLD. Includes reference to Lusher.

B 797, p. 21

B 864-A, p. 37

B 824, p. 35

B 864-C, p. 220-221, 242-243

B 836 , p. 35

B 868-A, p. 37

B $844-A$, p. 34

Manley (\& Loranger). See (Willow Cr.).

Marietta. See (Flat Cr.).

(Marietta Cr.) - (SE 1/2 quad.) (?) ; GOLD.

B 520 , p. 40

Miscovich. See (Otter Cr.).

Miscovich \& Rodman. See (Julian Cr.).

Miscovich \& Roslund. See (Otter Cr.).

Mohawk (lode). See Golden Horn.

Mohawk (placer). See (Flat Cr.), (Otter Cr.).

(Montana Cr.). See DeCourey.

(Moore Cr.) - $(14.9,10.7)$; GOLD, MERCURY, CHROMITE, TUNGSTEN. Includes references to Moore Cr. Min. Co.

B 480 , p. 267,270

B 520, p. 40

B 542 , p. $300,302-303$

B 578, p. 34-35

B 592, p. 69

B 622, p. $67,259,261$

B 642, p. $252-253,265$

B 692, p. 40

B 722, p. 60

B 754, p. 108-109

B 783, p. 15

B 836, p. 43

B 844-A, p. 43
B 857-A, p. 40

B 864-A, p. 45

B 864-C, p. 223-224

B 868-A, p. 46

B $880-$ A, p. 48

B 897-A, p. 57

B $910-A$, p. 59

B 917-A, p. 58 .

B 926-A, p. 55-56

B 933-A, p. 51-52

C 255, p. 16, 18

BMB 153, p. 54

TDM 1, p. 26

Moore Cr. Min. Co. See (Moore Cr.).

Moore Cr. Placers. See (Moore Cr.).

(Moose Cr.) See DeCourcy. Old name for Montana Cr.

Moss \& Larson Min. Co. See (Ganes Cr.) under Ophir quad.

Myrtle. See (Flat Cr.).

North American Dred. Co. See (Flat Cr.), (Otter Cr.):

Northern Alaska Dred. Co. See (Otter Cr.).

Northland Dev. Co. See (Flat Cr.), (Willow Cr.).

Ogriz. Sce (Slate Cr.).

OK. See (Moore Cr.).

Olson \& Co. See (Happy Cr.).

Otter. See (Otter Cr.). 
(Otter Cr.) - (7.8-8.85, 7.95-8.05) ; GOLD, SILVER, MERCURY, TUNGSTEN, ANTIMONY.

B 410, p. 63

B 442 , p. 45

B 480 , p. $238,240,253-255,262,267$

B 520, p. 40

B 542, p. 299, 302-303

B 578, p. 31-32, 34

B 592, p. 69

B 622, p. 56-57, 257, 260

B 642, p. $66,253-255,266$

B 649 , p. $47-48$

B 662, p. 59

B 692, p. 38

B 722, p. 56

B 739, p. $9,40,157$

B 754, p. 83, 111-112

B 755, p. $14,43-44$

B 773, p. 27

B 783 , p. 18

B 792, p. 18,25

B 797, p. 21
B 810, p. 27,40

B 813, p. $29-30,47$

B 824, p. 34,52

B 836, p. 34-35, 54

B 844-A, p. 34, 54

B 857-A, p. 32,51

B 864-A, p. 36-37, 56

B 864-C, p. $216-220$

B 868-A, p. 37, 59

B 880-A, p. 41,61

B 897-A, p. 48,71

B $910-A$, p. 50, 76

B 917-A, p. 48-49

B 926-A, p. 44, 71

B 933-A, p. 42-43, 67

C 255 , p. 4,9

BMB 142, p. 26

BMB 153, p. 54

TDM 1, p. 26, 40

Otter Cr. Dred. Co. See (Black Cr.).

Pelky. See (Ganes Cr.) under Ophir quad.

(Prince Cr.) - (8.5-8.6, 6.4-6.5) ; GOLD.

B 864-C, p. 216

B 910-A, p. 50

Prospector. See (Otter Cr.).

Puntila. See (Ganes Cr.) under Ophir quad.

(Quartz Gulch). See (Donlin Cr.).

(Queen Gulch). See (Donlin Cr.).

Ray. See (Happy Cr.).

Riley \& Marston. "See (Otter Cr.).

Riley, J. E., Inv. Co. See (Otter Cr.).

Roslund. See (Otter Cr.).

(Ruby Cr.) or (Gulch). See (Donlin Cr.).

Sacco \& Scott. See (Flat Cr.).

Sakow \& Co. See (Flat Cr.).

Salen. See (Granite Cr.).

Savage. See (Flat Cr.).

Savage \& Matheson. See (Ganes Cr.) under Ophir quad.

Shonbeck. See (Ganes Cr.) under Ophir quad.

(Slate Cr.) - (8.7-8.95, 7.3-7.7) ; GOLD, ANTIMONY.

B 642 , p. 253, 255, 266

B 897-A, p. 49

B 649 , p. 49

B 754, p. 112

B $864-$ A, p. 37

B $910-$ A, p. 50

B 864-C, p. 222-223

B 926-A, p. 45

B 868-A, p. 37

B 933-A, p. 43

C 255 , p. 15

(Snow Gulch). See (Donlin Cr.).

Snowbird. See DeCourcy.

Speljack. See (Ganes Cr.) under Ophir quad. 
Speljack, Messick, Mynttic \& Repo. See (Ganes Cr.) under Ophir quad.

S.S.S. See (Slate Cr.).

Star. See (Slate Cr.).

Steen (\& Sternberg). See (Julian Cr.).

Strandberg (\& Son). See (Flat Cr.).

Stuver (Bros.). See (Flat Cr.), (Happy Cr.).

Summit. See (Happy Cr.).

Thrift (Min. Co.) See DeCourcy.

U.S. Smelt., Ref., \& Min. Co. See (Fourth of July Cr.), (Moore Cr.).

Uotila (\& Ogriz). See (Slate Cr.).

Uotila \& Scott. See (Flat Cr.).

Upgrade. See (Flat Cr.).

Utilla \& Ogris. See (Slate Cr.).

Virgin. See (Otter Cr.).

White Star. See (Willow Cr.).

Wildcat. See (Flat Cr.), (Willow Cr.).

(Willow Cr.) - (7.3-7.6, 6.4-6.75) ; GOLD.

B 480, p. 256, 258-262, 267

B 836, p. 34

B 542, p. 300, 302-303

B 844-A, p. 34

B 578, p. 31-32, 35

B 857-A, p. 32

B 592, p. 69

B 864-A, p. 37

B 622, p. 57

B 864-C, p. 208-211

B 642, p. $66,253,256,266$

B 868-A, p. 37-38

B 722, p. 56

B $880-A$, p. 41

B 739, p. 40

B $897-$ A, p. $48-49$

B 754, p. 113-114

B 755, p. 44

B 783, p. 14

B 910-A, p. 50

B 797, p. 21

B $926-$ A, p. 45,56

B 813, p. 29

B 933-A, p. 43

B 824, p. 34

C 255, p. 1

BMB 142, p. 26

(Yankee Cr.) - (20.55-20.8, 17.4-17.95). See (Yankee Cr.) under Ophir quad.

Note.-Mining on Yankee Cr. has been in both Iditarod and Ophir quads.

Yost \& Nash. See (Flat Cr.).

Yukon Gold (Dred.) Co. See (Flat Cr.).

\section{ILIAMNA QUADRANGLE}

Alaska Katmalite Corp. See (Augustine I.).

$$
\text { B 1039-C, p. } 34
$$

RI 4932 , p. $72-74,81$

(Augustine I.) - (23.0, 6.45), PUMICE.

Aukney - $(6.7,7.3)$; GOLD(?).

B 442 , p. 198

B 485 , p. $125-126$

Black Prince. See Copper King.

Cook \& Bornland. See McNeil.

Copper King - $(20.75,12.3)$; COPPER, IRON. Includes reference to Keyes.

B 442 , p. 194

B 862, p. 91

B 485 , p. 121

C 207 , p. 2

B 655 , p. 150

IC 7379, p. 33 
Copper King Ledge. See Millet.

(Diamond Pt.) - (21.05, 11.65) ; GOLD(?).

B 442 , p. $194 \quad$ B 485, p. 125

Durand - $(17.65,12.7)$; COPPER.
B 442 , p. 197
B 862 , p. 91-92
B 485 , p. 123
IC 7379 , p. 33

Duryea - $(18.25,12.3)$; SILVER, GOLD, LEAD, ZINC. Includes reference to Duryea \& McNeil, which is probably the same property.
B 442, p. 196-197
B 739, p. 27
B 485 , p. 124-125
B 755, p. 33
B 542, p. 38-39
B 862 , p. $93-94$
B 592, p. 64
C 207 , p. $3-4$
B 622, p. 47
IO $7379, \mathrm{p} .33$

Duryea \& McNeil. See Duryea.

Dutton (Min. \& Dev. Co.) - $(18.05,12.15)$; COPPER, MOLYBDENUM, GOLD, SILVER, LEAD.
B 442, p. 194-196
B 862 , p. 91
B 485 , p. 117-121
B 926-C, p. 191-192
B 592, p. 64
C 207, p. 2-3
B 655, p. 150
IC 7379, p. 33

Dutton, Goodro \& Thomas. See Dutton.

Dutton Min. (\& Mil.) Co. See Dutton.

Ida G. See Duryea.

Keyes. See Copper King.

Knudsen. See Knutson.

Knutson - (15.15, 14.85) ; COPPER, GOLD, SILVER. Includes reference to Knudsen.
B 442 , p. 197
B 542, p. 39
B 485 , p. $123-124$

McNeil (-Cook) - $(12.25,2.5)$; COPPER, GOLD, SILVER. Includes references to Cook \& Bornland, (Okchiak Cr.).
B 542, p. 39
B 714, p. 78
B 592, p. 64
B 773, p. 39, 173-174
B 622, p. 47
C 207, p. 2-3
B 662, p. 49
IC 7379, p. 33

B 712, p. 35

McNeil, Holly \& others. See McNeil.

Millet - $(13.3,13.9)$; COPPER, GOLD, SILVER.
B 442, p. 197-198
B 655, p. 150
B 485, p. 122-123
B 862, p. 92
B 542, p. 39
C 207, p. 2-3
B 592, p. 64
RI 4890, p. 1-22

(Okchiak Cr.). See McNeil.

Reward-Ridgeway. See McNeil.

St. Eugene Min. Corp., Ltd. See Millet.

Silver Bell. See Duryea.

Success. See Durand.

War Eagle. See Duryea. 


\section{JUNEAU QUADRANGIE}

AB. See Aurora Borealis.

Admiralty. See Admiralty Alaska.

Admiralty Alaska (Gold Min. Co.) - (10.25-10.75, 3.85-4.55) ; GOLD, NICKEL, COPPER, COBALT, LEAD, ZINC, MOLYBDENUM. Includes references to (Funter Bay), Mertie Lode, Pekovich, Tellurium.
AR 18, p. 62-63, 77-78
B 864-A, p. 17
B 259, p. 55
B 868-A, p. 16, 82-83
B 287 , p. 149
B 880-A, p. 17
B 642 , p. 76
B 897-A, p. 17
B 662, p. $86-92$
B 897-D, p. 264-268
B 712, p. 30
B $910-$ A, p. 19
B 714, p. 113-116
B $917-$ A, p. 19,105
B 722, p. 36-37
B 926-A, p. 18
B 739, p. 22
B 926-C, p. 174
B 773 , p. $12,72,95,109$
B 933-A, p. 17
B 783 , p. $7,41-46$
B 936-O, p. 349-361
B 800 , p. 348
B 1024-B, p. 51-52
B 813, p. 14
C 252, p. 7
B 824, p. 16
BMB 153, p. 16
B 836 , p. 16
IC 7379, p. 70-71
B 844-A, p. 15-16
RI 3950, p. 1-15

B 857-A, p. 15

Alaska. See Admiralty Alaska.

Alaska Attin Min. Co. - (15, 3-4) (?) ; GOLD (?).

B 314, p. 54

Alaska Chief - See Ground Hog.

Alaska Consol. See Perseverance.

Alaska Consol. Mines Co. See Peterson.

Alaska Consol. Min. Co. See Alaska Treasure.

Alaska Dano (Mines Co.) - (10.15-10.5, 3.65-4.25) ; GOLD, SILVER, LEAD, COPPER. Includes references to Nowell-Otterson, Otterson, War Horse.
AR 18, p. 62-63, 77
B 739, p. 22
B 259 , p. 54
B 773, p. 12
B 287, p. 149
B 662, p. 85,92
B 783, p. $7,41-44$
B 714, p. 113, 116-117
B 797, p. 11
B 824, p. 16

B 722, p. 37

Alaska Ebner. See Ebner.

Alaska Empire Gold Min. Co. - (10.75-11.45, 3.05-4.15) ; GOLD. See also Hawk Inlet Min. Co.
B 864-A, p. 17
B $868-A$, p. 16
B 880-A, p. 16-17
B 897-A, p. 17
B 910-A, p. 19
B 917-A, p. 19
B 926-A, p. 18
B $933-$ A, p. 17

Alaska Endicott (Min. \& Mil. Co.) - $(6.85,12.15)$; GOLD, SILVER, COPPER. Includes references to Endicott-Alaska Min. \& Mil. Co., Endicott (Min. \& Mil. Co.), (William Henry Bay) (copper).
B 642, p. 76
B 739, p. 22
B 712, p. 30
B 755, p. 25
B 714, p. 19, 109-112
B 773, p. 36
B 722, p. 37
B 963-A, p. 28-30 
Alaska Gastineau (Min. Co.). See Perseverence.

Alaska Gold Belt (Min.) Co. See Nelson-Lott.

Alaska Gold Mines Co. See Perseverance.

Alaska Gold Min. Co. See Admiralty Alaska, Indiana.

Alaska-Juneau - (15.25-15.3, 5.55); GOLD, SILVER, LEAD, ZINC, COPPER. Note.-Includes references to Bennet, Lane \& Hayward; after 1923 one or more other mines were operated by Alaska-Juneau Gold Min. Co., which see also.

AR 18, p. 62-63, 72-73

B 592, p. 59

B 225, p. 29-30, 35

B 259, p. 53

B 622 , p. $41,98,100$

B 284, p. 37

B 287, p. 58, 69-73

B 642, p. 73,75

B 314, p. 55

B 662, p. $80-81$

B 345 , p. $87-88$

B 692 , p. 29

B 712, p. 29

B 379, p. 70

B 714, p. 106-107

B 442 , p. 135

B 722, p. 14,36

B 480 , p. 96

B 739, p. 21

B 520, p. 25

B 542, p. 32

B 755, p. 8, 24

B 773, p. $14-15,37$

Alaska-Juneau Gold Min. Co. - (14.8-15.35, 4.75-5.7) ; GOLD, SILVER, LEAD.

NoTe.-In 1923 began operation of Ebner; in 1928 bought properties of Alaska

Mexican Gold Min. Co., Alaska Treadwell Gold Min. Co., and Alaska United Gold Min. Co.; in 1931 held option on Dora and Hallam claims ; in 1933 bought Perseverance mine and connected it with Alaska-Juneau workings.

B 783, p. 6-7, 22

B 792 , p. $9,29-30$

B 797, p. 9-10, 38-39

B 810, p. $10-12,48,51,63$

B 813, p. $12-13,55,58$

B 824, p. 13-15, 61, 64

B 836, p. 12-14, 63, 66-67

B 844-A, p. 13-14, 62, 65

B $857-$ A, p. 12-14, 58, 61

B 864-A, p. 13-14, 63, 66

B 868-A, p. 13-15, 61, 68

B 880-A, p. 13-16, 64, 71-72

B 897-A, p. 14-16, 74, 81
B 910-A, p. 16-18, 79, 86-87

B 917-A, p. 17-19, 78, 89

B 926-A, p. 16-18, 72, 81-82

B 933-A, p. 15-17, 69, 78-79

B 943-A, p. 8-9, 14-15

B 1024-B, p. 53

BMB 142, p. 32-33, 52

BMB 153, p. 18-19

IC 6186, p. 2-18

IC 6236 , p. 1-16

IC 7379, p. $14-20,34$

IC 7844 , p. 7

Alaska Mexican (Gold Min. Co.). See Alaska Treadwell Gold Min. Co.

Alaska Min. \& Power Co. See Perserverance.

Alaska Perserverance (Min. Co.). See Perserverance.

Alaska Taku - (16.0-16.4, 4.6-5.0) (?) ; GOLD(?).

B 642, p. 76

Alaska Treadwell. See Alaska Treadwell Gold Min. Co.

Alaska Treadwell Gold Min. Co. - (14.95-15.2, 4.75-4.9); GOLD, SILVER, LEAD, ZINC, MOLYBDENUM, COPPER, TUNGSTEN. Includes references to Alaska Mexican (Gold Min. Co.), Alaska Treadwell, Alaska United (Gold Min. Co.), Mexican, Ready Bullion, Seven Hundred Foot, Treadwell, Treadwell Group ; see also Alaska-Juneau Gold Min. Co.

AR 18, p. $62-70$

B 225, p. 29, 39-40

B 259, p. 52-53, 69-87
B 263, p. 13

B 284, p. 6,39

B 287, p. 3-4, 36, 90-116 
Alaska Treadwell Gold Min. Co.-Continued

B 314, p. $50-53$

B 345, p. 35, 86-87

B 379, p. $28-29,69$

B 394, p. 194-195, 199

B 442, p. 33, 134

B 480, p. 28, 60, 67-76, 93, 95-96

B 520, p. 25

B 542 , p. 31-32

B 592, p. 58

B 622, p. 41, 96-97

B 642 , p. 73-74

B 662 , p. 77-79

B 692, p. 29

B 712, p. $14,29-30$

B 714, p. 112-113

B 722, p. 14, 36
B 739, p. 21

B 755, p. 8, 23-24

B 792, p. 10

B 800 , p. $319,329,331,347,355,364-$ $365,377,388$

B 810, p. 12

B 813 , p. 13

B 880-A, p. 15

B 897-A, p. 18

B 926-C, p. 172-174

B 1024-B, p. 52-53

BMB 142, p. 33-35, 52

BMB 153, p. 20-22

IC 7379, p. 12-14

IC 7844 , p. 7

Alaska Treasure Consol. Mines Co. See Alaska Treasure.

Alaska Treasure (Gold Min. Co.) - $(15.45,4.1)$; GOLD, LEAD, ZINC, COPPER. Includes references to Alaska Consol. Min. Co., Alaska Treasure Consol. Mines Co., Nevada Cr., (Nevada Cr.).
B 225 , p. $40-41$
B 284 , p. $39-40$
B 622 , p. 97
B 287 , p. $92-93$
B 642 , p. 75
B 314, p. $53-54$
B 662 , p. 86
B 442, p. 135
B 880-A, p. 17
B 480 , p. 96
BMB 142, p. 35, 62
BMB 153, p. 22

B 520, p. 25

Alaska United (Gold Min. Co.). See Alaska Treadwell Gold Min. Co.

Alaska-Washington (Gold Min. Co.) - (10.45-10.55, 10.3-10.33) ; GOLD.
B 259, p. 54
B 284, p. 35
B 287, p. 133
B 502, p. 48-49

Alaska Willoughby Min. Co. See Admiralty Alaska.

Algunican Dev. Co. See Jualin.

Alice Miller. See Admiralty Alaska.

Allan. See Hawk Inlet Min. Co.

Alma. See Hawk Inlet Min. Co.

Alta. See Preseverance.

Amalga. See Eagle $\mathbf{R}$.

Amelia. See Hawk Inlet Min. Co.

American Gold Min. Co. See (Gold Cr.), Silver Queen.

Anderson - $(15.8,5.2)$; GOLD, SILVER, LEAD, ZINC.

B 287, p. 55

Armor. See Hawk Inlet Min. Co.

Ascension. See Silver Queen.

(Auk Bay) - (12.5-12.6, 6.75-7.15) (?) ; GOLD(?). See also Dull \& Stephens, Winn.
B 592 , p. 59
B 712, p. 30

B 642, p. 78

Aurora Borealis - $(10.45,10.5)$; GOLD. Includes references to $\mathrm{AB}$, Morning

Star.

B 284, p. 35

B 502, p. $47-48$

B 642 , p. 77 
Bandy. See Hawk Inlet Min. Co.

Batella. See Hawk Inlet Min. Co.

B.C. See Black Chief.

Beach. See Admiralty-Alaska.

Bear - $(8.3,15.15)$; GOLD.

AR 18, p. 62-63, 76-77

B 284 , p. 32

B 446 , p. $39-40$

B 642, p. 77

(Bear Cr.) - $(11.05,4.6)$; ASBESTOS.

B 813, p. 71-72

B 844-A, p. 81

B 824, p. 80

B 963-A, p. 34-37

B 836, p. 82

Bear Valley. See Hawk Inlet Min. Co.

Bear's Nest - $(14-15,4)$ (?) ; GOLD (?).

B 520, p, 25

Bengley. See Hawk Inlet Min. Co.

Bennet. See Alaska-Juneau.

Berners Bay - $(9,11-12)$; GOLD(?).

B 284, p. 34

Berners Bay Min. \& Mil. Co. See Bear, Comet, Kensington, Ophir, Seward.

Bessie (Gold Min. Co.) - (10.35-10.4, 10.45) ; GOLD.

B 284, p. 34

B 502, p. 48

B 287, p. 133

Black Chief - $(11.05,10.6)$; GOLD.

B 345, p. 89

B 502, p. 51

B 379, p. 71

Blue Jay (Admiralty I.). See Admiralty Alaska.

Blue Jay (Eagle R. dist.) - $(10.85,10.75)$; GOLD.

B 345, p. 89

B 502, p. 51

Blue Lead. See Alaska Dano.

Bonanza. See Alaska Endicott.

Boston (Admiralty I.). See Hawk Inlet Min. Co.

Boston (near Juneau) - $(14.65,5.55)$; GOLD.

B 284, p. 38

B 314, p. 55

B 502, p. 27-28, 59

B 622, p. 102

Boston \& Alaska Co. - $(10.25-10.75,3.85-4.55)$; GOLD. See also Admiralty Alaska.

AR 18, p. 78

Brown. See Hawk Inlet Min. Co.

Bull Consol. - (15.4-15.7, 5.35-5.6) ; GOLD.

B 284, p. 38

California (Admiralty I.). See Hawk Inlet Min. Co.

California (Eagle R. dist.) - $(10.2,11.6)$; GOLD.

B 284, p. 34

B 502, p. 46-47

California \& Nevada Copper Co. See Ebner, Hallam.

Cannonball. See Peterson.

Cardiff. See Hawk Inlet Min. Co.

Carrie Nation. See Admiralty Alaska.

Cascade - $(11.05,10.3)$; GOLD.

B 284, p. 34-35

B 345, p. 89

B 314, p. 57

B 502, p. 50

Chatham. See Alaska Dano.

Cheechako. See Clark (Carlson Cr.). 


\section{INDEX OF METALLIC AND NONMETALLIC MINERAL DEPOSITS}

Clark (Carlson Cr.) - (16.15, 6.1) approx ; GOLD, ANTIMONY, LEAD, ZINC. Includes reference to (Sunset Cove).
B 783 , p. 50-52
B $857-A$, p. 15
B 800 , p. 331,363
IC 7844, p. 9

Clark (Lemon Cr.) - $(14.5,6.8)(?)$; GOLD (?).

B 287, p. 118-119

Cleveland. See Admiralty Alaska.

Cliff. See Hawk Inlet Min. Co.

Cobalt. See Hawk Inlet Min. Co.

Columbia. See Admiralty Alaska.

Comet - $(8.45,15.0)$; GOLD.

AR 18, p. $62-63,76-77$

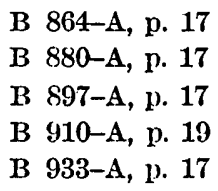

B 225, p. 38

B 284, p. 32

B 446, p. $42-43$

B $933-\mathrm{A}, \mathrm{p} .17$

B 800, p. 317,345

Cottrell-Spalding - $(11.0,10.6)(?)$; GOLD. See also E Pluribus Unum.

B 345, p. 89

Creek. See Treasury Hill.

Davies \& Pond. See California (Eagle $\cdot$ R. dist.).

Deer Meadow. See Hawk Inlet Min. Co.

De Roux. See (Bear Cr.).

Detroit-Alaska Min. Co. See (Windfall Cr.) (placer).

Devil Club. See Admiralty Alaska.

Dividend - $(10.95 .10 .3)$; GOLD.
B 284, p. 34-35
B 379, p. 71
B 314, p. 57
I 502, p. $49-50$
B 345, p. 89

Dora. See Hallam.

Doran - $(14.1,6.2)$; GOLD.

B 502, p. 60

Dorothy. See Hawk Inlet Min. Co.

Douglas Min. Co. - $(14.55,5.0)$ (?) ; GOLD.

B 783 , p. 50

Dublin. See Hawk Inlet Min. Co.

Dull \& Stephens - $(12.5,7.15)$; GOLD.

B 480 , p. 97

B 502, p. 58

Dunkle. See Alaska Dano.

Eagle. See Hawk Inlet Min. Co.

Eagle R. (Min. Co.) - (11.2-11.3, 10.1-10.2) ; GOLD, COPPER, LEAD.

Includes references to Amalga.
B 259, p. 54
B 542, p. 32
B 284 , p. 35
B 592, p. 59
B 287 , p. $130-131$
B 622, p. 41,101
B 314, p. 57
B 642, p. $76-77$
B 345, p. 89
B 662, p. 77
B 379, p. $70-71$
B 712, p. 30
B 442 , p. 138
B 864-A, p. 17
B 480 , p. 97,110
BMB 142, p. 35, 52
B 502 , p. $44-46$
BMB 153, p. 23
B 520, p. 26 
Eastern Alaska Min. \& Mil. Co. See Perseverance.

Ebner (Gold Min. Co.) - $(15.05,5.65)$; GOLD. Includes references to AlaskaEbner, Taku Min. \& Mil. Co.; see also Alaska-Juneau Gold Min. Co.

$\begin{array}{ll}\text { A R 18, p. 62-63, 73 } & \text { B 592, p. } 59 \\ \text { B 225, p. 29-30, 35 } & \text { B 622, p. 41, 98, 100 } \\ \text { B 259, p. 54 } & \text { B 642, p. 76 } \\ \text { B 284, p. 37 } & \text { B 692, p. } 29 \\ \text { B 287, p. 66-69 } & \text { B 712, p. 30 } \\ \text { B 314, p. 54 } & \text { B 714, p. } 107 \\ \text { B 345, p. 87-88 } & \text { B 722, p. 36 } \\ \text { B 379, p. 70 } & \text { B 783, p. 7 } \\ \text { B 442, p. } 135 & \text { B 792, p. } 9 \\ \text { B 480, p. } 96 & \text { BMB 142, p. 35, 52 } \\ \text { B 520, p. } 25 & \text { BMB 153, p. 23 }\end{array}$

B 542, p. 32

Echo Cove Min. Co. - $(10.2,11.7)$ (?) ; GOLD. See also Cailfornia (Eagle R. dist.), Gold Standard.

B 933-A, p. 17

Endicott-Alaska Min. \& Mil. Co. See Alaska Endicott.

Endicott (Min. \& Mil. Co.) See Alaska Endicott.

E Pluribus Unum - $(11.0,10.6)$; GOLD. See also Cottrell-Spalding. B 442 , p. 138

B 502, p. 50-51

Eureka. See Kensington.

Fairview. See Hawk Inlet Min. Co.

Falls - $(8.8,14.75)$ approx ; GOLD (?).

B 284, p. 34

Florence. See Admiralty Alaska.

Francis. See Alaska Dano.

Fremming - $(8.85,14.6)$ approx ; GOLD, LEAD, ZINC, COPPER. B 284, p. 34

B 446, p. 47

Funter. See Admiralty Alaska.

(Funter Bay). See Admiralty Alaska.

Funter Bay Min. Co. See Admiralty Alaska.

Galveston. See Hawk Inlet Min. Co.

George. See Hawk Inlet Min. Co.

Geyser. See Admiralty Alaska.

Gilbert. See Perseverance.

Glacier. See Silver Queen.

Golconda. See Silver Queen.

Gold Belt. See Nelson-Lott.

(Gold Cr.) - (14.8-15.35, 5.55-5.75) ; GOLD. Includes references to Jualpa Min. Co., (Last Chance Basin), (Little Basin), (Middle Flat), Nowell, (Silver Bow Basin), Silver Bow (Hyd.) Mines Co.
AR 18, p. 71
B 442, p. 41,135
B 225, p. $28,35,46$
B 480 , p. 37
B 259, p. 31, 53
B 520, p. 36
B 263, p. $13,52,54,92,110,143,207$
B 755, p. 24
B 284 , p. 38
B 773, p. 37
B 287 , p. 2-4, 57-60, 77-85
B 792, p. 14
B 314, p. 51,55
B 797, p. 16
B 345, p. 88
B 933-A, p. 32
B 379, p. 51
IC 7844, p. 11 
Gold King - $(8.65,15.2)$ approx ; GOLD (?).

B 284, p. 38

Gold Knob. See Treasury Hill.

Gold Standard - $(10.2,11.75)$; GOLD.

B 284, p. 34

B 379, p. 71

B 345, p. 89

B 502, p. 46-47

Golden Bear. See Hawk Inlet Min. Co.

Golden Treasure - $(15.7,4.9)$ approx ; GOLD (?). B 284, p. 38

Gould \& Curry - $(16.0,5.15)$; GOLD.
AR 18, p. 62-63, 73-75
B 287, p. 49-50

B 284, p. 38

Greek Boy (Min. Co.) - (9.1, 15.15) ; GOLD.

B 225, p. 38

B 287, p. 134

B 284, p. 32-33

B 446, p. 47-48

Ground Hog - $(15.35,5.4)$; GOLD.

B 287, p. 73-74

Hallam - $(14.8,5.7)$; GOLD. Includes references to Dora.
B 284, p. 38
B 442, p. 135
B 287, p. 63-66
B 844-A, p. 14
B 379, p. 70

Happy Days. See Alaska Dano.

Harris. See Ground Hog.

Harris \& Juneau. See (Gold Cr.).

Hartford. See Silver Queen.

Hawk Inlet Min. Co. - (10.75-11.45, 3.05-4.15) ; GOLD. Includes references to Williams (Min. Co.) ; see also Alaska Empire Gold Min. Co.

B 773, p. 12, 72

B 783, p. 7, 41-44, 47-50

B 824, p. 16

Hayden-Stone. See Bear, Comet, Johnson, Kensington.

Heckler. See Admiralty Alaska.

Herbert R. See Holland Alaska Gold Co.

Hidden Rock. See Admiralty Alaska.

Holland Alaska Gold Co. - (11.3, 9.3) (?) ; GOLD (?).
B 868-A, p. 16
B 897-A, p. 17

B $880-A$, p. 17

Holman - $(14.6,5.0)(?)$; GOLD (?).

B 662, p. 77

Horrible - $(8.25,15.25)$; GOLD. Includes reference to Portland-Alaska Gold Min. Co.

B 284, p. 33

B 287 , p. $135-136$

B 446, p. 39

Horse Fly. See Admiralty Alaska.

(Howard Bay) - $(8.55,5.4)$; SILVER, LEAD, ZINC.

B 739 , p. 21

Hulda A. See Clark (Carlson Cr.).

Hull. See Hawk Inlet Min. Co.

Humboldt - $(14.95,5.7)$; GOLD.

B 225 , p. 35

B 259 , p. 53

B 284, p. 38

B 287 , p. $68-69$

Hunter. See Admiralty Alaska.

Husky. See Hawk Inlet Min. Co. 
Ibex. See Silver Queen.

Indiana - $(8.65,14.85)$; GOLD.
B 284, p. 34
B 446, p. 44

Isaiah R. See Clark (Carlson Cr.).

Ish Nik. See Admiralty Alaska.

Ivanhoe - $(8.15,15.45)$; GOLD. Includes references to Mellen Min. \& Mfg. Co. B 284, p. 33 B 446, p. 38-39

B 287, p. 135-136

Jersey - $(14.8,4.85)($ ?) ; GOLD( ?). See also Jersey City. B 662, p. 77

Jersey City - $(14.8,4.85)$; GOLD. See also Jersey. B 622, p. 98

John W. See Clark (Carlson Cr.).

Johnson - $(8.5,15.2)$; GOLD.

B 284, p. 33

B 287 , p. 137

B 622, p. 101

B 446, p. $43-44$

B 642 , p. 77

B 662, p. 82-83

Joyce-Jensen (-Johnson) - $(10.85,10.75)$; GOLD. Includes references to Yankee Boy.

B 345, p. 89

Jualin - $(8.7,14.75)$; GOLD.

B 225, p. 38

B 259, p. 54

B 284, p. 33

B 287, p. 134-135

B 314 , p. $57-58$

B 345 , p. 90

B 379, p. 71

B 442 , p. 138

B 446 , p. $44-47$

B 520, p. 26

B 542, p. 32

B 592, p. 59

B 622, p. 41, 101-102

Jualin (Alaska) Mines Co. See Jualin.

Jualin Berners Min. Co. See Jualin.

Jualpa Min. Co. See (Gold Cr.).

Julia - (10.95-11.05, 10.3) ; GOLD.

B 284, p. 34-35

B 345, p. 89

Jumbo. See Admiralty Alaska.

Kensington (Min. Co.) - (8.3-8.4, 15.2-15.25) ; GOLD. Includes references to Eureka ; see also Sherman Cr.

B 225, p. 38

B 259, p. 54

B 284, p. 32-33

B 287, p. 137

B 446, p. 40-42

B 480 , p. 67-68

B 520, p. 26

B 542, p. 32

B 592, p. 59
B 502, p. 51

B 642, p. 77

B 662, p. 77,81

B 692, p. 29

B 712, p. 30

B 714, p. 107-108

B 722, p. 36

B 755, p. 24-25

B 800, p. 317

B 813, p. 14

B 824, p. 16

BMB 142, p. 35, 52

BMB 153, p. 23-24
B 502, p. 50

B 622, p. 41,101

B 642, p. 77-78

B 662, p. 77, 82-83

B 712, p. 30

B 800 , p. 317-318, 345-346

B 813, p. 14

B 824, p. 16

B 864-A, p. 17

B 868-A, p. 16 
Kensington (Min. Co.)-Continued
B $880-A$, p. 17
B 933-A, p. 17
B 897-A, p. 17
BMB 142, p. 36, 52
B $910-A$, p. 19
BMB 153, p. 24

Keystone (Gold Min. Co.). See Alaska Dano.

King Bee. See Admiralty Alaska.

Lake. See Hawk Inlet Min. Co.

Lakeview. See Alaska Dano.

Lane \& Hayward. See Alaska-Juneau.

Last Chance (Hyd. Min. Co.). See (Gold Cr.).

(Lemon Cr.) - (14.2-14.7, 6.7-7.2) approx ; GOLD.

B 225, p. 46

B 284, p. 37

B 263, p. 207

B 287, p. 119- -120

Lemon Cr. Co. See (Lemon Cr.).

(Little Basin). See (Gold Cr.).

Little Johnson - $(8.6,15.2)$ approx ; GOLD (?).

B 284, p. 34

Little Pet. See Alaska Dano.

London. See Silver Queen.

Lone Duck. See Alaska Dano.

Lone Star. See Admiralty Alaska.

Lost Cabin. See Hawk Inlet Min. Co.

Low Hill. See Admiralty Alaska.

Lowhee. See Admiralty Alaska.

Lucky Strike. See Hawk Inlet Min. Co.

Lurvey - (15.4, 5.45) ; GOLD.

B 287, p. $79-80$

(McGinnis Cr.) - (12.75; 8.15) approx ; GOLD.
B 225, p. 37,46
B 259, p. 54
B 287, p. 123-124
B 284, p. 36
B 314 , p. 56
B 345, p. 88

Mammoth (Admiralty I.) - (12.45-12.8, 2.0-2.2) ; GOLD, LEAD, ZINC.

B 259, p. 55-56

B 314, p. 59

B 284 , p. 40

B 345 , p. 90

B 287, p. 150

B 379 , p. 72

Mammoth (Douglas I.) - $(\mathbf{1 5 . 4 , 4 . 0 )}$ approx ; GOLD. B 284, p. 40

B 314, p. 54

Mansfield Gold Min. Co. (Admiralty I.) - $(10.7,4.7)$ approx ; COPPER, LEAD, ZINC.

B 314, p. 59

B 379 , p. 72

Mansfield Gold Min. Co. (McGinnis Cr.). See (McGinnis Cr.).

Mary. See Hawk Inlet Min. Co.

Maude S. - $(10.85,10.75)$; GOLD.

B 345, p. 89

B 502, p. 51

Medecine Bird - $(8.65,15.2)$ approx ; GOLD (?).

B 284, p. 34

Mellen Min. (\& Mfg.) Co. See Ivanhoe.

Mendenhall (Admiralty I.). See Hawk Inlet Min. Co.

Mendenhall (near Juneau) - $(12.95,7.6)$; GoLD.

B 502, p. 59-60

Mertie (Lode). See Admiralty Alaska.

Mexican. See Alaska Treadwell Gold Min. Co. 
(Middle Flat). See (Gold Cr.).

Minnie. See Hawk Inlet Min. Co.

Mitchell \& McPherson - (11.7,9.75) ; GOLD.

B 480, p. $97 \quad$ B 622, p. 102

B 502, p. 53

Montana. See Hawk Inlet Min. Co.

(Montana Basin) - (12.3-12.35, 8.25-8.4) ; GOLD.

B 284, p. 36

B 480 , p. 109

B 314, p. 56

B 502, p. 55

B 345, p. 88

(Montana Cr.) - (12.35-12.4, 8.35) approx ; GOLD.

$\begin{array}{ll}\text { B } 225, \text { p. } 37 & \text { B } 813, \text { p. } 24 \\ \text { B 287, p. 124-125 } & \text { B } 824, \text { p. } 28 \\ \text { B } 480, \text { p. } 109 & \text { B } 933-\text { A, p. } 32 \\ \text { B 542, p. } 43 & \end{array}$

Morning Star. See Aurora Borealis.

Mother Lode (Treasury Hill). See Treasury Hill.

Mother Lode (Yankee Bay) - $(10.6,10.15)$; GOLD (?). B 502, p. 49

Mountain King. See Admiralty Alaska.

Mountain Kink. See Admiralty Alaska.

Mountain Queen. See Admiralty Alaska.

Mykor. See Hawk Inlet Min. Co.

Nelson-Lott - $(16.0-16.05,4.9)$; GOLD. Includes references to Alaska Gold Belt Co., Gold Belt.

B 642, p. 76

B 662 , p. 77

BMB 142, p. 32, 52

BMB 153, p. 18

Nevada Cr. See Alaska Treasure.

(Nevada Cr.) See Alaska Treasure.

New York. See Hawk Inlet Min. Co.

Noonday - (10.95-11.05, 10.3) ; GOLD.

B 284, p. 34-35

B 345, p. 89

B 502, p. 50

Northern Bell (Gold Min. Co.) - $(8.45,15.15)$; GOLD.

B 284, p. 32

Northern Light. See Johnson.

Nowell. See.(Gold Cr.).

Nowell Gold Min. Co. See (Gold Cr.), Silver Queen.

Nowell Min. \& Mil. Co. See Johnson.

Nowell-Otterson. See Alaska Dano.

Ocean Swell. See Admiralty Alaska.

O.K. Sce Alaska Dano.

Olds. See (Montana Cr.).

Oleson - $(11.25,9.85)$; GOLD (?).

B 502, p. 51-52

Ophir - $(8.2,15.25)$; GOLD.

B 284, p. 33

B 800, p. 317

B 446 , p. 39

Oregon. See Admiralty Alaska.

Otter. See Admiralty Alaska.

Otterson. See Alaska Dano.

Patterson. See Admiralty Alaska.

593018-61-10 
Patton. See (Montana Basin).

Pekovich. See Admiralty Alaska.

Penn-Alaska (Min. Co.) - $(16.95,3.95)$; GOLD (?).

B 622, p. 102

B 642 , p. 76

Perseverance (Min. Co.) - (15.35, 5.45); GOLD, SILVER, LEAD, ZINC. Includes references to Alaska Gastineau (Min. Co.), Alaska Perseverance Min. Co., Alaska Min. \& Power Co.; see also Alaska-Juneau Gold Min. Co.

B 225, p. 35

B 284, p. 37-38

B $662 ;$ p. 80

B 287, p. 74-76

B 692, p. 29

B 314, p. 55

B 712, p. 29

B 345, p. 87-88

B 714, p. 106

B 379 , p. 70

B 722, p. 14, 36

B 442 , p. 135

B 739, p. 21

B 480 , p. 96

B 755, p. 8

B 520, p. 25

B 800, p. 355

B 542, p. 32

B 880-A, p. 15

B 592, p. 58-59

B 897-A, p. 15-16

B 622, p. $41,98-100$

BMB 142, p. 31-32, 52

B 642, p. 75-76, 98

BHB 153, p. 17-18

Peterson - (11.65-11.75, 7.55-8.05); GOLD. Includes references to (Peterson Cr.), Prairie (Eagle R. dist.).
B 259, p. 54
B 592 , p. 59
B 284, p. 36
B 622 , p. 102
B 287 , p. 126
B 642, p. 77
B 314, p. 66
B 712, p. $29-30$
B 345 , p. 88
B 714, p. 109
B 442 , p. 138
B 722, p. 36
B 480 , p. 97
B 739, p. 21
B 502, p. 53-54
B 755 , p. 24
B 542, p. 32
GQ 100

(Peterson Cr.). See Peterson.

Point. See Admiralty Alaska.

Portage - $(10.6,5.05)$ approx; GOLD, COPPER, LEAD.
B 284 , p. 40
B 379, p. 72
B 287, p. 148,150
B 662, p. $85-86,88$
B 314, p. 59
GQ 100

Portland-Alaska (Gold) Min. Co. See Horrible.

Prairie (Admiralty I.). See Hawk Inlet Min. Co.

Prairie (Eagle R. dist.). See Peterson.

Premier. See Hawk Inlet Min. Co.

Premier Gold Min. Co. See Clark (Carlson Cr.).

Pungle Down. See Admiralty Alaska.

Pungle Up. See Admiralty Alaska.

Puzzler - $(10.95-11.05,10.3)$; GOLD.

B 284, p. 34-35

B 502, p. 50

B 345 , p. 89

Pyrate. See Admiralty Alaska.

Queen. See Silver Queen.

Queen Bee. See Admiralty Alaska.

Raisin Valley. See Hawk Inlet Min. Co.

Ready Bullion. See Alaska Treadwell Gold Min. Co. 
Reagan - $(15.85,5.0)$; GOLD, SILVER.
B 225, p. 36
B 287, p. 55-57
B 284 , p. 38
B 800, p. 327
Red Diamond - $(15.4,4.0)$; GOLD.
B 284 , p. $39-40$
B 314, p. 54
Rex - (11.1, 10.2); GOLD.
B 284, p. 35
B 287, p. 132

Riverside. See Hawk Inlet Min. Co.

Saddle Rock. See Hawk Inlet Min. Co.

St. Louis - (12.15, 9.4) ; GOLD.

B 502, p. 52

(Salmon Cr.) - (14.2, 6.0) approx; GOLD(?).

B 287 , p. 116

Salmon Cr. (Gold Min. Co.). See Wagner.

Sandstone \& Ward. See Eagle R.

(Sandy Cove) - $(0.3,12.35)$; MARBLE.

B 592, p. 98-99

B 783, p. 61

B 682, p. 41

Seattle - $(10.8,4.6)$ approx; GOLD.

B 662, p. 86

Seven Hundred Foot. See Alaska Treadwell Gold Min. Co.

Seward - $(8.3,15.2)(?)$; GOLD.

B 284, p. 33

Sherman Cr. - $(18.35,15.2)$ approx ; GOLD. See also Kensington.

B 314, p. 57

(Silver Bow Basin.) See (Gold Cr.).

Silver Bow Basin Min. Co. See (Gold Cr.).

Silver Bow Hyd. (Mines) Co. See (Gold Cr.).

Silver Cord. See Silver Queen.

Silver Queen (Min. Co.) - $(15.5-15.75,5.2-5.25)$; SILVER, GOUD, LEAD, ZINC,

ANTIMONY. Includes references to Ascension, Glacier, Ibex, Queen.

AR 18, p. 62-63, 73-75

B 542, p. 32

B 225 , p. 29,36

B 649, p. 62

B 287, p. 4, 36, 50-55

B 800, p. 327,331

B 480 , p. 61

Skyscraper. See Hawk Inlet Min. Co.

Smith \& Heid - $(12.25,8.5)$; GOLD.

$\begin{array}{ll}\text { B } 284, \text { p. } 36 & \text { B } 502, \text { p. } 55 \\ \text { B } 287, \text { p. } 129 & \text { GQ } 100 \\ \text { B } 314, \text { p. } 56 & \end{array}$

Spaulding. See Treasury. Hill.

Star. See Admiralty Alaska.

Summit (Admiralty I.). See Alaska Dano.

Summit (Eagle R. dist.) - $(12.25,9.45)$ approx ; GOLD.

B 502, p. 52

Summit (Gold Cr.). See Ground Hog.

Sunburst. See Alaska Dano.

Sunrise. See Hawk Inlet Min. Co.

Sunset. See Hawk Inlet Min. Co.

(Sunset Cove.) See Clark (Carlson Cr.).

Sutherland. See Perseverance.

Swamp Lily. See Admiralty Alaska. 
Sylvia. See Hawk Inlet Min. Co.

Tacoma - $(10.0,12.5)$ approx; GOLD(?).

B 642 , p. 78

Taku (Min. \& Mil. Co.). See Ebner.

Tellurium (Min. \& Mil. Co.). See Admiralty Alaska.

Texas. See Hawk Inlet Min. Co.

Tingwalla. See Admiralty Alaska.

Treadwell. See Alaska Treadwell Gold Min. Co.

Treadwell Group. See Alaska Treadwell Gold Min. Co.

Treasury Hill - (12.25-12.3, 7.4-7.45) ; GOLD.

B 480, p. 97

GQ 100

B 502, p. 55-58

Tremont. See Ground Hog.

Two Shaft. See Alaska Dano.

Tyee- $(14.6,5.1)$ ( ?) ; GOLD (?).

B 662, p. 77

Uncle Sam. See Admiralty Alaska.

Uncow. See Admiralty Alaska.

U.S. Smelt. \& Ref. Co. See Ebner.

Valley. See Admiralty Alaska.

Wagner - $(14.2,6.0)$; GOLD. Includes references to Salmon Cr.

B 284, p. 37

B 287, p. 117

B 314, p. 56

Walla Walla. See Hawk Inlet Min. Co.

War Eagle. See Admiralty Alaska.

War Horse. See Alaska Dano.

Washington. See Admiralty Alaska.

Wasp. See Alaska Dano.

Water Witch. See Treasury Hill.

Wild Cat. See Hawk Inlet Min. Co.

Wiley (\& Spaulding). See Treasury Hill.

(William Henry Bay) (copper). See Alaska Endicott.

(William Henry Bay) (rare earths) - $(6.65,13.35)$; RARE EARTHS.

I 303

William N. See Clark (Carlson Cr.).

Williams (Min. Co.). See Hawk Inlet Min. Co.

Willoughby \& Weir. See Admiralty Alaska.

(Windfall Cr.) (lode) - $(12.25,8.5)$; GOLD, LEAD, ZINC, COPPER. See also

Swith \& Heid.
B 287, p. 128-129
B 502, p. 55
GQ 100

B 345, p. 88

(Windfall Cr.) (placer) - (11.75-11.8, 8.75-8.8) ; GOLD.

B 225, p. 37,46

B 259 , p. 54

B 263, p. 207

B 284 , p. 36

B 287, p. 127-128

B 314, p. 51,56

B 345, p. 88

B 480 , p. 109

Winn - $(12.6,6.8)$; GOLD.

B 502, p. 59

Winona. See Alaska Dano. 
Yakima (Min. Co.) - $(15.0,4.65)$; GOLD (?).
B 225, p. 41
B 314, p. 54

B 284, p. 40

Yankee Boy. See Joyce-Jensen.

Yanke Girl. See Joyce-Jensen.

Yellow Hornet. See Clark (Carlson Cr.).

(-.---) (chromite, rear earths) - $(2.8,16.7)$; CHROMITE, RARE EARTHS. I 303

(_-.--) (cobalt, copper) - $(4.05,17.0)$; COBALT, COPPER.

I 303

(----) (copper, nickel, zinc) - $(11.1,0.6)$; COPPER, NICKEL, ZINC.

I 303

$(-\ldots-\ldots)$ (copper, zinc) - $(3.0,14.75)$; COPPER, ZINC.

I 303

(----) (copper, zinc) - $(3.2,16.4)$; COPPER, ZINC.

I 303

(-.---) (copper, zinc) - $(3.7,14.85)$; COPPER, ZINC.

I 303

(-.--) (copper, zinc) - $(16.1,4.8)$; COPPER, ZINC.

I 303

(----) (marble) - $(0.45-0.7,12.05-12.2) ;$ MARBLE.

B 592, p. $99 \quad$ B 682, p. $41-43$

(....-) (rare earths, zinc) - $(14.3,1.4)$; RARE EARTHS, ZINC.

I 303

\section{KANTISHNA RIVER QUADRANGLE}

(Cosna R.) - (6-10, 6-10) ; TIN (?). Note-Very indefinite.

TDM 2, p. 19

\section{RARIUK QUADRANGLE}

(Ayakulik R.) - (13.9, 3.6) ; GOLD, PLATINUM. Includes references to (Red R.), (Red R. Beach).
AR 18, p. 86
B 836, p. 31
B 542, p. 134-135
B $880-$ C, p. 172
B 662, p. 23
B $926-A$, p. 37
B 666, p. 96
B $933-A$, p. 36

(Canvas Pt.) - $(13.6,4.2)$ approx ; PLATINUM.

B 692 , p. 316

B 714, p. 38

(Cape Kubugakli) - $(8.3,15.2)$; GOLD, ANTIMONY, MOLYBDENUM, COPPER, LEAD.

B 773, p. 39, 206-207

Mason. See (Cape Kubugakli).

(Portage R.) - (14.1, 2.25) ; GOLD.

AR 18, p. 86

(Red R.). See (Ayakulik R.).

(Red R. Beach). See (Ayakulik R.).

(Sevenmile Beach) - (17.1-18.2, 11.65-11.75) ; GOLD. Note.-Not mentioned by name in $B 520$.
B 520, p. 37
B 880-C, p. 172
B 542, p. 134

(Tigidik Beach) - (13.9-14.2, 0.2-3.5) (?) ; GOLD.

B 836, p. 31 


\section{RENAI QUADRANGLE}

(Indian Cr.) - (20.7-21.3, 2.4-2.6) ; GOLD. Note.-Not referred to by name in B 225.
B 225, p. 48
B 587, p. 111
B 277, p. 44

(Kenai R.) - (20.3-24.8, 8.8-9.2) ; GOLD. See also (Kenai R.) under Seward quad.
B 277, p. 43-44
B 587, p. 197
B 520, p. 141, 163
B 592, p. 63

(Nikishka) - (13.8-13.9, 12.15-12.25) ; DIATOMITE.

B 1039-B, p. 25-30

Trachsel. See (Tuxedni Bay).

(Tustumena L.). See (Indian Cr.).

(Tuxedni Bay) - (1.2-1.3, 4.3) ; IRON.
B 712, p. 35
B 789 , p. $55-56$
B 714, p. 42
B 1024-D, p. 95, 99-106

\section{KETCHIKAN QUADRANGLE}

Adanac. See Victoria.

Alaska - $(0.0-0.5,10.35-11.35)($ ?) ; GOLD. May be in Craig quad.

\section{B 755, p. 23}

Alaska Gold Standard Min. Co. See Gold Standard.

Alaska Venture Syn. See Old Glory.

Algonquin - $(2.75,3.25)$ approx ; COPPER.
B 347, p. 140
B 642, p. 93-94

Ambrose - $(19.4,17.6)$; commodity, if any, not known.

B 1024-B, p. 33, 44

American Eagle. See Old Glory.

American Min. \& Mil. Co. - (19.15-19.35, 17.55-17.75) (?) ; LEAD(?). See also

Fish Cr. (mine).

B 824 , p. 17

(Annette I.) - (SW 1/4 6, 1) ; GOLD, SILVER, COPPER.

B 347 , p. 180

Annex. See Gold Mtn.

Annie. See Gold Mtn.

Anthony - $(2.6,3.4)$ approx ; COPPER, GOLD.

B 642 , p. 94

Apex. See Concord.

Asche's. See Mahoney.

Ash. See Mahoney.

Baby George - (8.1, 6.5) ; GOLD (?).

B 347, p. 147

Baltic - $(8.05,6.75)$ approx ; GOLD, ZINC.

$$
\begin{array}{ll}
P 1, \text { p. } 68 & \text { B } 347, \text { p. } 148
\end{array}
$$

Baltic Star - $(8.05,6.75)$ approx ; GOLD, LEAD, ZINC.

P 1, p. 68

B 347, p. 148

Bawden - $(5.1,8.9)$; MARBLE.
B 284, p. 57
B 345, p. 121-122
B 542, p. 71
B 682 , p. 96

B 347 , p. 197 
Bay View (Helm Baý) - (0.0-0.5, 10.35-11.35) (?) ; GOLD (?).

B 642, p. 82

Bay View (Seal Cove) - $(2.65,3.35)$ approx ; COPPER.

P 1, p. 70

B 347, p. 140

B 314, p. 72

Bear. See Lucky Boy Extension.

Beaver. See Tyee (Thorne Arm).

Bell. See Goldstream.

Big Four Min. Co. See Mahoney.

Big Joe - $(2.6,3.4)$ approx; COPPER.

$$
\text { P 1, p. } 70
$$

B 347, p. 140

Big Three. See Plutyas.

Birdseye - $(4.35,5.4)$; GOLD, LEAD, ZINC.

P 1, p. 62

B 347 , p. 152

B 800, p. 337

Bishop - $(19.15,17.6)$; SILVER, GOLD, COPPER, LEAD.

B 807, p. 67

Black Warrior - $(2.6,3.4)(?)$; COPPER(?).

B 347, p. 140

Blackhand. See (Caamańo Pt.).

Blue Bucket - $(0.4,10.35)$; GOLD (?).

B 347 , p. $156-157$

Blue Jay (Helm Bay) - $(0.0-0.5,10.35-11.35)(?)$; GOLD. May be in Craig quad.

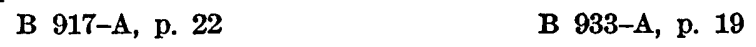

B 926-A, p. 20

Blue Jay (Seal Cove). See Concord.

Bonney. See Fish Cr. (mine).

British American Holding \& Dev. Co. See Fish Cr. (mine).

Buck - $(2.65,3.35)$ approx ; GOLD, COPPER.

B 347 , p. $139-140$

Bugge. See Gold Nugget.

(Caamano Pt.) - $(0.25,9.0)$; ANTIMONY.
B 662, p. 72-73
B 800, p. 317,331
B $864-$ A, p. 80
B 836 , p. 80
B $844-A$, p. 80
B $857-A$, p. $75-76$
B 1024-H, p. 163-166, 169-177
IC 7844 , p. 9
RI 4173 , p. 5, $39-40$

Carita - (2.25-2.3, 2.55-2.6) ; COPPER. Includes references to Erhart, Starlight; not mentioned by name in $B 642$.
P 1, p. 73
B 642, p. 94

B 347, p. 140

(Carroll Inlet). See Dickinson \& Bell.

(Cascade Inlet) - (SW 1/4 6, 3) ( ?) ; GOLD.

B 592 , p. 92

(Chickamin R.). See Gnat.

Coughlan. See Lucky Boy Extension.

Commonwealth - $(17.6,13.4)$ approx ; GOLD, MOLYBDENUM, ZINC, COPPER. B 792, p. 31

B 807 , p. 111-112

Concord - (2.5-2.6, 3.0-3.15) approx; COPPER, ZINC, GOLD, SILVER. Includes reference to Apex.

P 1, p. 72-73

B 347, p. 139 
(Crab Bay) - (SW 1/4 6, 2) or (SE $1 / 45,2)$; COPPER, LEAD, BARIUM.

B 592 , p. $92-93$

Curio. See Dickinson \& Bell.

Dall - $(2.75,2.95)$; COPPER, GOLD, SILVER.

P 1, p. 71-72

Damon - (2.6, 3.4) approx; COPPER (?). Includes reference to O'Brien. P 1, p. 70

Davis \& Elvigion. See Sixmile.

Deer Lodge - $(2.6,3.4)$ approx ; COPPER, GOLD.

B 642 , p. 94

Dickinson \& Bell - $(\mathbf{7 . 0 5}, \mathbf{8 . 9})$ approx; MARBLE. Includes references to (Carroll Inlet).
B 314, p. 77
B 345 , p. 122
B 662 , p. 73
B 642, p. 104
B 682 , p. 97,114

Doe - (2.6, 3.4) approx ; COPPER. Includes reference to Trio. P 1, p. 70 B 347, p. 139

Dyer \& Johnson. See Gold Standard.

Easter - $(1.9,7.1)$; GOLD

P 1, p. 62-63

Elliot. See Gold Standard.

Erhart. See Carita.

Family Lode. See Baby George.

Fannie. See Gold Mountain.

Fehring. See Last Shot.

Fish Cr. (claims). See Mountain View.

Fish Cr. (mine) - (19.15-19.35, 17.55-17.75) ; SILVER, LEAD, GOLD, COPPER, ZINC, TUNGSTEN. Note.-Includes references to Fish Cr. (Bradfield Canal quad.), Fish Cr. Min. Co., Murphy \& Stevenson; property is in both Bradfield Canal and Ketchikan quads., but most development has been in Ketchikan quad.

$\begin{array}{ll}\text { B } 259, \text { p. } 66 & \text { B } 800, \text { p. } 317,324, \\ \text { B } 642, \text { p. } 98 & \text { B } 807, \text { p. } 43,68-71 \\ \text { B } 722, \text { p. } 128,131,134-138 & \text { B } 1024-\text { B, p. } 30 \\ \text { B } 739, \text { p. } 21 & \text { B } 1024-\text { F, p. } 138 \\ \text { B } 783, \text { p. } 42 & \text { IC } 7379, \text { p. } 42\end{array}$

Fish Cr. Min. Co. See Fish Cr. (mine).

Fitzgerald - $(19,17)(?)$; SILVER(?), LEAD(?). May be in Bradfield Canal quad.

B 739, p. 21

Folwarzny. See Gold Standard.

Free Gold - $(0.0-0.5,10.35-11.35)(?)$; GOLD. May be in Craig quad.

B 755, p. 23

Freeburn and assoc. See (Moth Bay).

(George Inlet) - (5.15-5.3, 7.3-7.95) ; MARBLE

B. 314, p. 77

B 682, p. 96

B 542, p. 71

Gertrude. Sce Gold Mtn.

Glory. See Old Glory. 
Gnat - $(10.85,14.6)$ approx; MOLYBDENUM. Includes reference to (Chickamin R.) ; not mentioned by name in B 926-C.

B 807 , p. 120

B 926-C, p. 171-172

IC 7844, p. 12

Gold Banner. See Golden Banner.

Gold Dollar. See Gold Mtn.

Gold Helm Min. Co. See Gold Standard.

Gold Mtn. - $(0.1,10.8)$ approx ; GOLD, COPPER, LEAD.

$\begin{array}{ll}\text { P 1, p. 58-59 } & \text { B } 347, \text { p. } 156 \\ \text { B 284, p. 44-45 } & \text { B } 622, \text { p. } 42\end{array}$

Gold Nuggett - $(0.0-0.5,10.4-10.7)$; GOLD. Includes references to Bugge, Martin-Bugge.

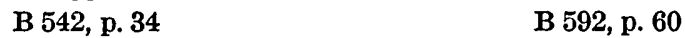

Gold Standard - $(0.0,11.35)$ approx; GOLD. Note.-Includes references to Gold Standard (Craig quad.) ; part of property in Craig quad.

$\begin{array}{ll}\text { P 1, p. 59-60 } & \text { B 813, p. } 16 \\ \text { B 284, p. 44-45 } & \text { B 857-A, p. 16 } \\ \text { B 314, p. 63 } & \text { B 864-A, p. 16 } \\ \text { B 345, p. 92 } & \text { B 868-A, p. 16-17 } \\ \text { B 347, p. 153-155 } & \text { B 880-A, p. 17 } \\ \text { B 592, p. 86 } & \text { B 897-A, p. 18 } \\ \text { B 622, p. 42 } & \text { B 917-A, p. 22 } \\ \text { B 642, p. 82 } & \text { B 926-A, p. 20 } \\ \text { B 722, p. 35-36 } & \text { B 933-A, p. } 19\end{array}$

Gold Standard Min. Co. See (Moth Bay).

Golden Banner - $(8.15,6.5)$; GOLD, LEAD, ZINC. Includes reference to Golden Tree.
P 1, p. 67-68
B 347, p. 147

Golden Dream. See Goo Goo.

Golden Link. See Baby George.

Golden Rod - $(8.15,6.65)$; GOLD (?).

B 347, p. 146

Golden Rule. See Goo Goo.

Golden Tree. See Golden Banner.

Goldstream - $(3.6,5.35)$; GOLD, LEAD, ZINC, COPPER. Includes references: to Bell.
P 1 , p. 62
B 480 , p. 98
B 284, p. 44
B 520, p. 27
B 314, p. $50,62-63$
B 542, p. 33
B 345, p. 92
B 592, p. 93
B 347, p. $177-178$
B 642 , p. 83

B 379 , p. 74

Gonheath. See Fish Cr. (mine).

Goo Goo - (8.05-8.2, 6.5-6.55); GOLD, ZINC, LEAD. Includes reference to Golden Dream.
P 1, p. 67
B 520, p. 26
B 347, p. 147
B 642 , p. 82

Grenadier - $(2.5,2.95)$ approx ; COPPER (?)

P 1, p. 73

Griswold, McGuire \& Dodge. See (Moth Bay). 
Grotto - $(2.6,3.4)$ approx ; COPPER.
P 1, p. 70-71
B 347, p. 140

Heather. See Fish Cr. (mine).

Heckman - $(3.75,5.1)$; GOLD.
P 1, p. 62
B 347 , p. 179

Helen. See Howard.

(Helm Bay) - (0.0-0.5, 10.35-11.35) ; GOLD Also in Craig quad.
B 773 , p. 10
B 824 , p. 18
B 813 , p. 16

Helm Bay Min. Co. See Gold Standard.

High Horse - $(8.15,6.5)$; GOLD, ZINC. Includes reference to Monster.
P 1, p. 68
B 347 , p. 147-148

Highland. See Fish Cr. (mine).

Hoadley (Bros.) - $(3.3,6.25)$; GOLD, BISMUTH.
B 259 , p. 65
B 800 , p. 332
B 347, p. 151
B 1024-B, p. $46-47$

Hobo - $(2.6,3.4)$ approx ; COPPER.

P 1, p. 70

B 347, p. 140

Hot Air. See (Caamano Pt.).

Hoveland. See Mtn. View.

Howard - $(19.0,17.7)$; LEAD, ZINC, BARIUM.

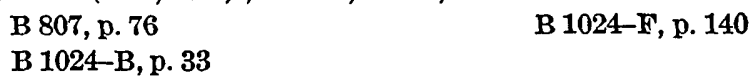

Irving Consol. Min. Co. See Goldstream.

Jeff. See Dickinson \& Bell.

Jewel (Gravina I.) - $(2.7,3.5)$ approx; COPPER. Includes reference to Jumbo. P 1, p. 71

B 347, p. 139

Jewel (Helm Bay). See Gold Mtn.

Jumbo. See Jewel (Gravina I.).

Ketchikan Gold Co. See Massachusetts.

Keystone. See Massachusetts.

King. See Gold Standard.

Kingston - $(0.3,10.7)(?)$, GOLD. P 1, p. 58

Klemm. See (Caamano Pt.).

Lake - $(8.05,7.2)$; GOLD, LEAD, ZINC.

B 347, p. 149

Laskawonda - $(3.6,6.1)$; GOLD (?). B 347, p. 152

Last Chance - $(0.15,10.55)$; GOLD, COPPER.

P 1, p. 57

B 347, p. 157

B 284, p. 44-45

Last Shot - $(19.1,17.65)$; SILVER, GOLD, COPPER, LEAD, ZINC, TUNGSTEN. B 807, p. 75-76

B 1024-B, p. 33

B 1024-F, p. 123, 125, 128, 136

Little Maumee - $(0.5,10.4)$; GOLD.

P 1, p. 58

Lizzie L. - (2.6, 3.4) approx ; COPPER, GOLD.

B 642, p. 94

Ion de Van Min. \& Mil. Co. See Londevan. 
Londevan - (5.1-5.15, 6.95-7.0) ; GOLD, SILVER, LEAD, ZINC, COPPER. Includes references to Lon de Van Min. \& Mil. Co., Telegraph.
P 1, p. 63
B 284, p. 44
B 480 , p. 98
B 345 , p. 92
B 542, p. 33
B 347 , p. 150
B 592, p. 60,88
B 800, p. 34,337

Low. See Bishop.

Lucky Bay. See Lucky Boy Extension.

Lucky Boy Extension - $(19.2,17.4)$; LEAD, ZINC, COPPER, TUNGSTEN. Includes references to Bear, Lucky Bay.
B 739, p. 21
B 1024-F, p. 140

B 807, p. 67

Mahoney - $(4.85,7.5)$; ZINC, LEAD, SILVER, GOLD. Includes references to Asche's, Ash, (Mahoney Cr.).
P 1, p. 63-64
B 998-C, p. 79-82
B 347 , p. $150-151$
C 252 , p. 6
B 592 , p. $88-90$

(Mahoney Cr.) See Mahoney.

Majestic. See Goo Goo.

Mammoth. See Victoria.

Martin-Bugge. See Gold Nugget.

Mary T. - $(0.3,10.45)$; GOLD, COPPER. P 1, p. 57

B 347, p. 157

Massachusetts - $(8.25,6.85)$; GOLD, LEAD, ZINC. Includes reference to Keystone.

P 1, p. 68

B 347 , p. 148-149

Miller. See Goldstream.

Moa, A.O., and assoc. See Mountain View.

Monster. See High Horse.

Montana. See Fish Cr. (mine).

Montana Lead \& Zinc, Inc. See Mahoney.

Moonshine - $(3.7,5.05)$ approx; GOLD (?).

B 347 , p. 179

Morning. See Mountain View.

(Moth Bay) or (Cove) - $(6.6,5.2)$; ZINC, COPPER, LEAD, SILVER, GOLD.

B 592 , p. $90-91$

C 252, p. 6

B 824 , p. 18

IC 7844, p. 12

B 836 , p. 15-16

RI 5245, p. 1, 3-5

B 998-C, 59-71

Mother Lode. See Goo Goo.

Mountain. See Goo Goo.

Mountain Top. See Gold Mtn.

Mountain View - (19.1-19.25, 17.3-17.6) ; SILVER, GOLD, LEAD, ZINC, TUNGSTEN, ANTIMONY, MOLYBDENUM, COPPER. Includes references to Fish Cr. (claims), Hoveland, Morning, Mountainview Gold Min. Co.
B 722, p. 138-139
B 739, p. 21
B 807, p. 43, 63-67
B 773, p. $74,76-77$
B 813, p. 15
B 824, p. 17
B 783, p. $41-42,54-55$
B $926-$ C, p. 171
B 792, p. 31
B 1024-B, p. 25-27, 30-32, 34-44
B 800, p. $317,324,327,330,358$
B 1024-F, p. 123, 125-128, 137-138 
Mountain View-Continued
B $1058-A$, p. $27-29$
IC 7844, p. 13
C 248 , p. 6, 12
IC 7379 , p. 42, 68-69
RI 3944, p. 1-10
RI 4174, p. 4-5, 36, 38, 45-49

Mountainview Gold Min. Co. See Mountain View.

Murphy \& Stevenson. See Fish Cr. (mine).

Mutt. See Dickinson \& Bell.

Nevada. See Fish Cr. (mine).

O'Brien. See Damon.

Old Glory - $(0.1,10.7)$; GOLD. Includes references to Glory.
P 1, p. 57
B 379, p. 74
B 284, p. 44-45
B 520 , p. 26
B 314, p. 63
B 347, p. 157-158
B 592, p. 85-86
B 622, p. 42

Old Man. See Concord.

Olympia. See Fish Cr. (mine).

Patterson Co. See Big Joe, Hobo, War Eagle.

Peerless (Consol. Min. Co.) - $(8.0,6.5)(?)$; GOLD. Note.-May be the same as Sealevel, which see also.
B 810, p. 13
B 813, p. 16

Perkins. See Mahoney.

Peterson - $(5.3,6.5)$; GOLD, SILVER, LEAD, ZINC. Includes reference to Surprise.
B 284, p. 44
B 347, p. 150
B 592, p. 88, 90

Phityas. See Plutyas.

Plutyas - (2.6, 3.4) approx ; COPPER( ?). Includes reference to Big Three.

$$
\text { P 1, p. } 70
$$

B 347, p. 140

Portal. See Londevan.

Queen - $(8.0,6.7)$; GOLD, ZINC.

B 347, p .148

Rainy Day - $(0.25,10.85)(?)$; GOLD

P 1, p. 58

B 347, p. 156

Riverside - $(19.0,17.75)$. See Riverside under Bradfield Canal quad.

(Roe Pt.) - $(10.3,4.65)$; GOLD, SILVER, COPPER.

B 347, p. 185

Rossland \& Deer Park Min. Co. See Dall.

Russel. See Salve.

Salve - $(8.0,6.6)$; GOLD.

B 347, p. 148

Sanford - $(2.75,3.25)$ approx ; COPPER.
B 347, p. 139
B 642, p. 93
B 542, p. 33

Sanford \& Lhote. See Algonquin, Sanford.

Sea Breeze - $(8.1,6.55)$; GOLD, LEAD, ZINC.

P 1, p. 67

BMB 153, p. 29

B 347 , p. 146

(Seal Cove) or (Bay) $\cdot(2.45-2.75,2.75-3.5)$; COPPER, GOLD.
P 1, p. 70
B 379, p. 83
B 592, p. 93
B 480 , p. 102
IC $7844, \mathrm{p} .11$ 
Sea Level Dev. Co. See Sea Breeze, Sealevel.

Sealevel - $(8.05,6.5)$; GOLD, SILVER, LEAD, ZINC.

P 1, p. 66-67
B 259, p. 66-68
B 284, p. 44
B 314, p. 63
B 345, p. 93
B 347, p. 144-146
B 480, p. $69-70$
B 592, p. 91
B 622, p. $42-43$

B 642, p. 82

B 836, p. 16

B 857-A, p. 16

B 864-A, p. 16

B 868-A, p. 17

B $880-A$, p. 18

B 897-A, p. 18

BMB 153, p. 29

Silver.Falls. See Mountain View.

Six Point - $(1.45,6.6)$; COPPER.

$$
\text { P 1, p. 73-74 }
$$

B 347, p. 140

Sixmile - $(19.0,17.65)$; SILVER, GOLD, COPPER, LEAD, ZINC.

B 807, p. 76-77

B 1024-F, p. 140

B 1024-B, p. 33-34, 36, 44

Skookum. See Mountain View.

(Smugglers Cove) - (0.0-0.5, 10.4-10.7) ; GOLD.

B 836, p. 16

South Lakeview - $(0.0,11.35)$ approx ; GOLD, COPPER.

B 642, p. 82

Southern. See Fish Cr. (mine).

Starboard. See Fish Cr. (mine).

Starlight. See Carita.

Starry Banner. See Gold Mtn.

Sunrise. See Concord.

Surprise. See Peterson.

Telegraph. See Londevan.

Thorne Arm. See Salve.

(Thorne Arm) - $(8,6)$; GOLD. See also Sealevel.

B $926-A$, p. 20

Thorne Arm Consol. See Sealevel.

Tide Water. Sce Wild West.

Tiernan \& Lhote. See Anthony, Deer Lodge, Lizzie L.

Tillicum Min. Co. See (Caamano Pt.).

Tongass - $(1.95,7.1)$; GOLD (?).

P 1, p. 61

Trio. See Doe.

Tyee (Annette I.). See (Crab Bay).

Tyee (Thorne Arm) - $(8.15,6.85)$; GOLD, LEAD, ZINC.

B 347, p. 148

Typhoon - $(1.9,7.15)$; GOLD (?).

P 1, p. 61

U.S. - $(0.3,10.45)$; GOLD.

P 1, p. 57

B 347, p. 157

Utah. See Fish Cr. (mine).

(Vallenar Bay) - $(1.65,6.55)$; CLAY.

B 345, p. 126

Victor Copper Min. Co. See Bay View, War Eagle. 
Victoria - (19.4, 17.4); commodity, if any, not known. Includes references to Adanac.

B 807, p. 67-68

Victory - (2.6-2.65, 3.35-3.4) approx ; COPPER.

B 1024-B, p. 33, 44 B 520, p. 26

Victory (Copper) Min. Co. See Bay View (Seal Cove), Damon, Doe, Plutyas, Victory, War Eagle.

War Eagle - $(2.6,3.4)$ approx ; COPPER, GOLD.
P 1, p. 70
B 347, p. 140
B 314, p. 72
B 442 , p. 143

Washington - $(2.45,2.75)$ approx ; COPPER.

P 1, p. 72

Watson \& Bain. See Mountain View.

Weir. See Fish Cr. (mine).

West Bay View - $(0.0-0.5,10.35-11.35)(?)$; GOLD ( ?). B 642, p. 82

White Knight - $(1.4,6.0)(?)$; COPPER. NOTE.-Not mentioned by name in $\mathrm{P} 1$. P 1, p. 74

B 347 , p. 140

White Rose. See Dickinson \& Bell.

Wild West - $(8.1,6.45)$; GOLD. Includes reference to Tidewater.
P 1, p. 68
B 347, p. 147

Wildcat - $(3.4,6.2)$; GOLD, ANTIMONY (?), BISMUTH(?).

P 1, p. 61

B 1024-B, p. 46-47

B 347, p. 151-152

\section{KODIAK QUADRANGLE}

Amok (Gold Min. Co.) - $(1.75,8.1)$; GOLD. Includes reference to Aniak Gold Min. Co.; see also Katmai Min. Co.

B 542, p. 132-133

B 622 , p. 47

B 592, p. 64

B 880-C, p. 175-176

Aniak Gold Min. Co. See Amok.

(Barling Bay) - $(5.8,3.85)$ approx ; GOLD, SILVER.

B 880-C, p. 181-182

Baumann \& Strickler - $(7.55,14.9)$; GOLD.

B 868-B, p. 132-133

B 880-C, p. 177

Bear (Uyak Bay) - $(0.7,8.95)$; GOLD.

AR 18, p. 62-63, 80-81

B $880-$ C, p. 175

B 542, p. 132

Bear (Womens Bay). See (Womens Bay).

Brennan. See Brenneman.

Brenneman - $(7.2,16.6)$ approx ; GOLD. Includes reference to Brennan.

B 622, p. 47

B 880-C, p. 177

B 868-B, p. 133

Brown Bear. See (Barling Bay).

Calaveras - $(0.6,9.4)$; GOLD, LEAD.

AR 18, p. 62-63, 80-81

B $880-$ C, p. 175

B 542, p. 132

(Chiachi Pt.) - See Friedland and assoc.

Dan - $(0.7,9.05)$; GOLD.

AR 18, p. 62-63, 80-81

B 542, p. 132 
(Dry Spruce I.) - $(9.05,16.75)$; GOLD.

B 868-B, p. 132

B $880-$ C, p. 178

Fields. See (Dry Spruce I.).

Friedland and assoc. - $(10.8,17.05)$; GOLD(?).

B $868-B$, p. 132

B $880-$ C, p. 178

Hinton, Krauter, Cook \& Christianson. See (Barling Bay).

Katmai Min. Co. - $(1.75,8.1)$ ( ?) ; GOLD (?). See also Amok. B 797, p. 12

Kizhuyak - (12.7-12.75, 15.35-15.5) approx; GOLD, SILVER. Includes reference to (Kizhuyak Bay).

B 542, p. 133

B 880-C, p. 180-181

B 868-B, p. 130-132

(Kizhuyak Bay). See Kizhuyak.

(Kizhuyak Pt.). See (Womens Bay).

Lake - $(1.5,10.1)$; GOLD (?).

AR 18, p. 62-63, 80-81

B $880-$ C, p. 175

B 542, p. 132

(Larson I.). See Kizhuyak.

(Matson's Ledge) - $(1.75,8.0)$; GOLD (?).

B 542, p. 133

B $880-$ C, p. 176

Mayle. See Moyle.

(Miners Pt. Beach) - (2.65-2.95, 15.45-15.7) ; GOLD. B 880-C, p. 172

Moyle - $(6.85,14.25)$; GOLD, SILVER. Includes reference to Mayle. B 868-B, p. 133

B $880-$ C, p. $\mathbf{1 7 6 - 1 7 7}$

Old Harbor. See (Barling Bay).

Silver Queen. See (Barling Bay).

(Uganik Beach) - (4.45-5.0, 16.45-17.0) ; GOLD. B 542, p. 135

B 880-C, p. 172

Wanberg - $(1.75,8.1)$ approx ; GOLD.

B 542 , p. 133

B $880-$ C, p. 176

Wanberg \& Boyer - $(1.3,11.35)$; GOLD.

AR 18, p. 62-63, 80-81

B $880-$ C, p. 175

B 542, p. 132

(Whale I.). See Friedland and assoc.

(Womens Bay) - $(12.9,16.15)$; GOLD, SILVER, ZINC, LEAD, COPPER.

Note.-Local name; not the same as Womens Bay on published maps.

B 542, p. 133

B $880-$ C, p. 178-180

B 868-B, p. 129-130

\section{KOTZEBUE QUADRANGLE}

(Alder Cr.) - (19.8-20.0, 0.85-1.45) ; GOLD.
P 10, p. 51
B 247, p. 65
B 225, p. 78-79
B 810, p. 34

(Sullivan Cr.) - (13.2-13.95, 0.15-1.35) ; GOLD.

P 10, p. 50

\section{LAKE CLARK QUADRANGLE}

Barnes. See (Kasna Cr.).

Belle. See (Kasna Cr.).

(Big Bonanza Cr.). See (Bonanza Cr.). 


\section{INDEX OF METALLIC AND NONMETALLIC MINERAL DEPOSITS}

(Bonanza Cr.) - (7.4-9.5, 11.1-11.6) ; GOLD. Includes reference to (Big Bonanza $\mathrm{Cr}$.).
B 542, p. 44
B 824, p. 154
B 622, p. $47,257,263$
B 862 , p. 94-95

B 655, p. 136-137

Bowman. See (Portage Cr.).

Brooks \& von Hardenberg. See (Kasna Cr.).

(Caribou Cr.). See (Koksetna R.).

Cook. See (Kasna Cr.).

Cyanide. See (Kasna Cr.).

Edwards. See (Kasna Cr.).

(Franklin Gulch) - (16-17, 8-9) (?) ; GOLD (?). See also (Kijik R.).
B 442, p. 200
B 622 , p. 263-264
B 485, p. 126
B 655, p. 137

Gilt Edge. See (Kasna Cr.).

Gleason. See (Kijik R.).

Hardenberg. See (Kasna Cr.).

(Ingersoll Gulch). See (Franklin Gulch).

(Kasna Cr.) - (17.05-17.1, 2.9-3.0) ; COPPER, IRON. Includes reference to Hardenberg.

B 442, p. 198-199

B 485, p. 121-122

C 207, p. 3-4

IC 7379 , p. 33

B 655 , p. 150-151

RI 4828, p. 3-10

B 862 , p. $92-93$

(Keejik R.) See (Kijik R.).

(Kellet Cr.). See (Franklin Gulch).

Kendall. See (Kasna Cr.).

(Kijik R.) - (14-17, 6-9) (?) ; SILVER, LEAD, GOLD, MOLYBDENUM, ZINC, COPPER. Includes references to Gleason, (Keejik R.), Thompson; see also (Franklin Gulch), (Ingersoll Gulch), (Kellet Cr.), (Lincoln Gulch).

B 542, p. 39

B 655, p. 137, 153

B 592, p. 64

B 926-C, p. 190-191

B 622, p. 257,268

C 207 , p. 3-4

King. See (Kasna Cr.).

(Koksetna R.) - (7.6-11.5, 4.0-8.0) ; GOLD. Includes references to (Caribou Cr.).
B 442, p. 200
B 622, p. 257
B 485 , p. 126
B 655, p. 137

(Lincoln Gulch) - (16-17, 8-9) (?) ; GOLD(?). See also (Kijik R.).

B 442, p. 200

B 622, p. 263-264

B 485, p. 126

B 655, p. 137

(Mulchatna R.) - (0.0-17.0, 4.5-15.75) (?) ; GOLD. See also (Bonanza Cr.).

B 225, p. 48

B 442, p. 202

Peary. See (Kasna Cr.).

Platsburg. See (Kasna Cr.).

(Portage Cr.) - (17.05-17.2, 6.45-6.65) ; GOLD.

B 442, p. 200

B 485, p. 126

B 542, p. 44

B 592, p. 64

B 622, p. 264

B 655, p. 137
B 692 , p. 33

B 712, p. 35

B 824, p. 154

B 862, p. 94

B 926-A, p. 37 
(Ptarmigan Cr.) - (NW $1 / 2$ quad.) (?); GOLD(?). NoTE-Domewhere in Mulchatna drainage.

B 542, p. 44

St. Eugene Min. Corp., Ltd. See (Kasna Cr.).

Shamrock Ledge. See (Kasna Cr.).

Thompson. See (Kijik R.). NoTE.-Silver, lead, and molybdenum claims.

\section{LIVENGOOD QUADRANGLE}

(Alabam Cr.) - (11.65-12.0, 10.25-10.5) ; GOLD.

B 642, p. 207-208

B 662, p. 273

Alabama (lode). See Cleary Hill.

Alabama (placer). See (Vault Cr.).

Alaska - $(19.25,1.65)$ approx; GOLD. Includes all references to Gladstone and most references to Jupiter-Mars; see also Genki.

B 480, p. 34

B 542, p. 161

B 520, p. 31

B 592, p. 337

B 525, p. 175

B 849-B, p. 75, 99-100

Alaska Exp. Co. See (Fairbanks Cr.) (placer).

Alaska Flyer - $(17.95,0.85)$; GOLD (?). B 525, p. 194

B 542, p. 180

Alaska Min. \& Dev. Co. See Wackwitz (Wyoming).

(Alder Cr.) - (20.4-20.6, 1.7-2.0) ; GOLD.
B 542, p. 208
B 933-A, p. 39

B 592, p. 68,359

BMB 142, p. 22

Alpha - $(18.1,0.95)$ approx; GOLD (?).

B 849-B, p. 74, 77

American Eagle. See McCarty (Henry Ford).

(Army Cr.) - (11.2-11.4, 9.45-9.6) ; GOLD, CHROMITE, ANTIMONY.

B 662, p. 268-269

B 864-A, p. 39

B 712, p. 41, 181

B 868-A, p. 39-40

B 755, p. 37

B 783, p. 14

B $880-$ A, p. 44

B 797, p. 21

B 897-A, p. 52

B 813, p. 30

B 910-A, p. 53

B 824, p. 36

B 917-A, p. 53

B 836, p. 36

B 926-A, p. 49

B 844-A, p. 35

B 933-A, p. 46

B 857-A, p. 34

BMB 153, p. 52

TDM 1, p. 14, 17

Anchor. See Chechako No. 1.

Anderson \& Birch. See Hoover.

Anna - $(19.15,1.5)$; GOLD (?).

B 849-B, p. 100

Apex. See David.

Aroostook. See Perrault.

Baltic. See (Livengood Cr.).

Balzimer. See Homestake.

Bank. See (Vault Cr.).

Banner - $(19.45,1.65)$; GOLD.

B 525, p. 171

B 542, p. 157

Barry. See (Livengood Cr.).

$693018-61-11$ 
(Bear Cr.) . (19.5-21.0, 1.1-1.2) ; GOLD (?).

B 442, p. 233

(Beaver Cr.) - (15-21, 5-15) (?) ; GOLD (?). May be in Circle quad. B 714, p. 82

(Bedrock Cr.) - (18.85-18.9, 1.4-1.6) ; GOLD, TUNGSTEN, SILVER, TIN. B 739, p. 30 B 1024-I, p. 188, 210

Big Jim. See Jackson.

Big Lead. See North Star (Skoogy Gulch).

Bill Taft. See Mazeppa.

Billy - (SE 1/4 21, 2) ; GOLD (?).

B 849-B, p. 155-156

Birch (\&) Anderson. See Hoover.

Bishop. See Whitehorse.

Black Joe. See Mizpah.

Black Warrior. See McCarty (Pioneer).

Blue Bell. See Pioneer (Chatham Cr.).

Blue Moon - (17-20, 0-1) ( ?) ; GOLD (?).

B 849-B, p. 75

Bob - $(18.65,1.4)$; GOLD (?).

B 849-B, p. 75

Bobbie - $(19.05,1.5)$; SILVER, LEAD, ANTIMONY.
B 525, p. 177
B 649, p. 35

B 542, p. 163

Bostrum \& Wickstrom. See (Wilbur Cr.).

B.P. See Butler \& Petree.

Bradley - (18.75-18.8, 0.95-1.0); GOLD(?). NotE.-See also North Star (Skoogy Gulch); not mentioned by name in text, but descriptions fit prospects named on maps.

B 525, p. 200, 202

B 542, p. 186, 188

Branholm-Jenkins - $(19.7,1.75)$; ANTIMONY, LEAD. Includes references to

Leindecker, McNeil. B 662, p. 415

B 849-B, p. 104

Burnet - (18.65-18.85, 0.8-1.05) ; GOLD, SILVER, LEAD. See also Burnet

Galena; not mentioned by name in B 525, 542.
B 525, p. 200
B 592, p. 349-350

B 542, p. 186

Burnet Galena - $(18.65,0.9)$; LEAD, ANTIMONY.

B $849-$ B, p. 118

Burns. See Chatham.

Busch \& Anderson. See Hoover.

Butler (\& Petree) - $(19.05,1.6)$; GOLD, LEAD, ZINC, ANTIMONY. Includes references to B.P., Rex (Min. Co.) ; see also Reese.

B 442, p. 226-227

B 480, p. 34

B 520, p. 31

California. See Cleary Hill.

Caribou. See McCarty (Henry Ford).

Carlisle. See Cleary Hill.

Carnation. See Soo.

Center Star. See North Star Extension.

Central Star. See North Star Extension.
B 525, p. 176-177

B 542, p. 162-163

B 849-B, p. 98 
Charles - $(20.85,2.05)$; GOLD.

B 520, p. 31

B 542, p. 141

B 525, p. 156

(Chatanika R.) - (16.7-18.5, 1.5-2.4) ; GOLD.

B 379, p. 190-191

B 480 , p. 156

B 525, p. 107, 112-113

B 592, p. 68

B 810, p. 25

B 813, p. 28,47

B 824, p. 32-33, 52

B 836, p. $32-33$

B 844-A, p. 32-33

B 857-A, p. 30-31

Chatham (Cr.) - $(19.3,1.55)$; GOLD, ANTIMONY. Includes references to Chatham Min. Co.

B 525, p. 172-173

B 542, p. 158-159

B 592 , p. $335-336$

B 622, p. 238

B 642 , p. 29

B 649, p. $35-36$

B 662 , p. 415

B 692, p. 322

(Chatham Cr.) - (18.95-19.15, 1.5-1.65) ; GOLD, ANTIMONY, TUNGSTEN, TIN.

B 225, p. 68, 70-71.

B 251, p. $67,72,78-81,84$

B 259, p. 27,39

B 263, p. 32-33, 208

B 284, p. 119

B 314, p. 30

B 337 , p. $41-42$

B 442, p. 226, 233

B 520, p. 241

B 525, p. 98, 112-113

B 542, p. 205

B 592, p. 358

B 622 , p. 232
B 864-A, p. 34-35

B 868-A, p. 35-36

B 880-A, p. 39

B $897-A$, p. $46-47$

B $910-A$, p. $46-48$

B $917-$ A, p. $43-44,46$

B 926-A, p. 40

B 933-A, p. 39

BMB 153, p. 51
B 849-B, p. 75, 100-101

B 868-A, p. 20

B 897-A, p. 22

BMB 142, p. 23-24, 53

BMB 153, p. 52

RI 4173, p. 38

TDM 1, p. 8

TDM 2, p. 8-9

B 642, p. 59

B 797, p. 20

B 810, p. 25,40

B 813, p. 28,47

B 824, p. 32-33, 52

B 836, p. 32, 54

B 844-A, p. 32, 54

B 864-A, p. 56

B 868-A, p. 58

B $880-A$, p. 62

B 1024-I, p. 188, 210

TDM 1, p. 10-11, 32, 37

TDM 2, p. 9

Chatham Cr. Dred. Co. See (Chatham Cr.).

Chatham Gold Dred. Co. See (Chatham Cr.), (Cleary Cr.).

Chatham Min. Co. See Chatham.

Chechako No. 1 - $(18.75,1.5)$; SILVER, LEAD, ANTIMONY, GOLD, ZINC, COPPER(?). Includes references to Anchor, Eldorado (lode), Eldorado Cr. Min. Co., Eldorado Min (\& Mil.) Co., Franklin, Westonvich; see also Tolovana.

B 525, p. 186-187

B 542 , p. 175

B 662, p. 416

B 649, p. $31-32$

B 692, p. 324

B 849-B, p. 72-73, 75, 89-90

Cheyenne - $(18.85,1.2)$; GOLD. Note.-Not mentioned by name in texts, but descriptions fit prospect named on maps.

B 525, p. 182-183

B 542, p. 169

Chief. See Soo. 
Christmas. Bee (Dome Cr.).

Cleary. See Cleary Hill.

(Cleary Cr.) (near Falrbanks) - (18.2-19.05, 1.5-2.3) ; GOLD, TUNGSTEN, ANTIMONY TIN.

P 70, p. 182

B 225, p. $68,70-71$

B 251, p. 67, 71-72, 77-81, 83-84

B 259, p. 26-28, 127

B 260, p. 181

B 263, p. 41-42, 208

B 284, p. 111, 119

B 314, p. 30,36

B 337, p. 29, 41-42

B 345, p. 39, 41-42

B 379 , p. 188, 190-191, 193, 195

B 442 , p. 232-233

B 480 , p. 155-156

B 520, p. 89, 241

B 525, p. 98-99, 107, 112-113

B 542, p. 204-205

B 592, p. 68,358

B 622, p. 54-55, 230-232

B 642 , p. 58-59

B 662 , p. 51,54

B 739, p. 29

B 755, p. 35, 146

B 783, p. 13, 18

B 792, p. 17,25

B 797 , p. $19-20$

B 836, p. 32-33, 54

B 844-A, p. 32-33, 54

B 857-A, p. 30-31, 51

B 864-A, p. 34-35, 56

B 868-A, p. 35-36, 58

B 880-A, p. 39-40, 61

B 897-A, p. 46-47, 71

B 910-A, p. 46-47, 76

B 917-A, p. 43-45

B 926-A, p. $40-41,70$

B 933-A, p. 38-39

B 1024-I, p. 188, 210

C 331, p. 6-7

BMB 142, p. 22

BMB 153, p. 51

RI 4174, p. 27

TDM 1, p. 8, 10, 32, 37

TDM 2, p. 20, 28

(Cleary Cr.) (near Livengood) - (11.85-12.05, 8.25-8.85) ; GOLD( ?).

B 642, p. 208

Cleary Extension. Bee Newsboy.

Cleary Hill Alaska Gold Mines Co. See Cleary Hill, Wackwitz (Wyoming).

Cleary Hill Alaska Min. Co. See Cleary Hill.

Cleary Hill (Mines, Inc.). - (18.85-19.1, 1.4-1.55) ; GOLD, ANTIMONY, TUNGSTEN, ZINC, TIN, LEAD, COPPER. Includes references to Cleary, Cleary Hill (Alaska) Min. Co., Rhoads (\&) Hall.

B 442 , p. 225

B 480 , p. $33-35$

B 520 , p. 31

B 525, p. 177-182

B 542 , p. $163-168$

B 592, p. $337-338$

B 622 , p. $236-237$

B 642 , p. 60

B 649, p. 34-35

B 662 , p. 411

B 692, p. 322

B 739, p. 30

B 783, p. 9

B 792, p. 12

B 797 , p. 13

B 810, p. 15

B 813 , p. 17

B 824, p. 20
B 836, p. 19

B 844-A, p. 19

B $849-$ B, p. $30,49,52,62,69-71,75$, $84,93-96$

B 857-A, p. 17

B $864-A$, p. $19-20$

B 868-A, p. 20

B 880-A, p. 20

B 897-A, p. 21

B 910-A, p. 23

B 917-A, p. 25

B $926-A$, p. $22-23$

B 933-A, p. 22-23

B 1024-I, p. 180, 206, 208-209

C 335, p. 2

BMB 142, p. 23-24

TDM 1, p. 8

TDM 2, p. 9 
Colby. See Chatham.

Colorado. See Cleary Hill.

Connors \& Stevens. See Gilmore, Ohio (Fairbanks Cr.).

Cornell - $(18.75,1.3)$; GOLD(?).

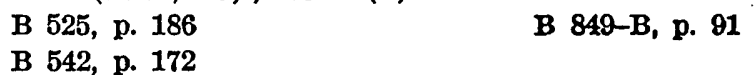

Crane. See (Olive Cr.)

(Crane Cr.) or (Gulch) - $(20.2-20.4,1.65-2.0)$; GOLD.

B 251, p. 73

B 525, p. $112-113$

Creighton \& Heilig. See Mohawk.

Crites \& Feldman. See Hi-Yu.

Cunningham - $(18.95,1.65)$; GOLD, ANTIMONY. NoTE-Not mentioned by name in texts, but descriptions fit prospect named on maps; see also Sunrise (Cleary Cr.).
B 525, p. 182
B 542, p. 168

Dahl \& Co. See (Gertrude Cr.).

David - $(18.8,0.95)$; GOLD. Includes references to Apex.
B 525, p. 201
B 712 , p. 40
B 542 , p. 187
B 849-B, p. 74, 115-116
B 692 , p. 322

(Dawson Cr.) - (0.05-0.2, 7.75-8.4) ; GOLD.
B 442 , p. 240
B 592, p. 362
B 520, p. 282-283
B 844-D, p. 180

B 542 , p. 222

Day Dawn. See (Dome Cr.).

Deep Channel. See (Livengood Cr.).

(Deep Cr.) - (21.1, 1.5) approx; GOLD.

B 592, p. 359

Diamond. See (Cleary Cr.).

(Dome Cr.) - (17.05-18.0, 0.8-1.6) ; GOLD, TUNGSTEN, TIN.

$\begin{array}{ll}\text { B 284, p. 11, 119 } & \text { B 773, p. 45 } \\ \text { B 314, p. 36 } & \text { B 783, p. 13 } \\ \text { B 337, p. 29, 43 } & \text { B 792, p. 17 } \\ \text { B 345, p. 41-42 } & \text { B 810, p. 25 } \\ \text { B 379, p. 190-191, 195 } & \text { B 824, p. 33 } \\ \text { B 442, p. 232-233 } & \text { B 857-A, p. 30 } \\ \text { B 480, p. 156 } & \text { B 864-A, p. 35 } \\ \text { B 520, p. 241-242 } & \text { B 868-A, p. 36 } \\ \text { B 525, p. 100-101, 108, 112-113 } & \text { B 880-A, p. 39 } \\ \text { B 542, p. 204 } & \text { B 897-A, p. 46 } \\ \text { B 592, p. 68, 358 } & \text { B 910-A, p. 46 } \\ \text { B 622, p. 54, 232 } & \text { B 917-A, p. 43 } \\ \text { B 642, p. 58-59 } & \text { B 926-A, p. 40 } \\ \text { B 662, p. 51, 54 } & \text { B 933-A, p. 39 } \\ \text { B 692, p. 35 } & \text { B 1024-I, p. 188, 210 } \\ \text { B 712, p. 39 } & \text { BMB 142, p. 22 } \\ \text { B 714, p. } 80-81 & \text { BMB 153, p. 51 } \\ \text { B 722, p. 45 } & \text { RI 4174, p. 28 } \\ \text { B 739, p. 29 } & \text { TDM 1, p. 32, 37 } \\ \text { B 755, p. } 35,146 & \text { TDM 2, p. 20, 28 }\end{array}$

Dome View - (18.55-18.65, 1.1-1.15) ; GOLD.

B 849-B, p. 75, 83-84 
(Dominion Cr.) - (18-21, 4-7) (?) ; GOLD(?). May be in Circle quad. B 480 , p. 165 B 525, p. 151

Dorando. See Hi-Yu.

Dorothy. See McCarty (Pioneer).

Duncan. See (Livengood Cr.).

Eagan and assoc. See Egan.

Eagle. See (Livengood Cr.).

Early Bird. See Ohio (Fairbanks Cr.).

Egan - $(18.65,0.75)$; TUNGSTEN. Includes reference to Eagan and assoc.
B 1024-I, p. 180, 206, 210
RI 4174, p. 29
C 331, p. 7, 22
TDM 2, p. 23

Egan \& Egan - $(20.85,2.35)$; GOLD.

B $849-$ B, p. 155

El Toro No.3. See McCarty (Henry Ford).

Eldorado (lode). See Chechako No.1

Eldorado (placer). See (Little Eldorado Cr.) (placer), (Livengood Cr.). Note.-Two different claims.

(Eldorado Cr.). See (Little Eldorado Cr.) (placer).

Eldorado Cr. Min. Co. See Chechako No. 1.

Eldorado Min. (\& Mil.) Co. See Chechako No. 1.

Emma - $(18.85,1.3)$; GOLD. Includes references to Overgaard.
B 442, p. 225
B 622, p. 238
B 525, p. 185-186
B 692 , p. 322
B 542, p. 171-172
B 849-B, p. 90-91

B 592, p. 340

Empire - (19.1-19.25, 1.45-1.65) (?); GOLD. See also Alaska, Pioneer (Chatham Cr.).

B 592, p. 337

B $849-B$, p. 75

Equity Assn. See Soo.

(Ester Cr.) - $(11.05,8.65)$; GOLD, MERCURY.

B 642 , p. 208

B 824, p. 36

B 662 , p. 271-272

BMB 142, p. 24

B 813, p. 30

Estorffe \& Radak. See (Ruth Cr.).

Etna. See (Livengood Cr.).

Eureka - $(20.7,2.05)$ approx; GOLD.

B 520, p. 31

B 525, p. 156

B 542, p. 142

Excelsior - $(19.8,1.7)$, SILVER, GOLD, ANTIMONY, LEAD.

B 480 , p. 34

B 542, p. 146-147

B 525, p. 161-162

B 649, p. 38

Fairbanks. See Homestake.

(Fairbanks Cr.) (lode) - $(19.9,1.55)$ approx ; SILVER, GOLD. B 525, p. 163

B 542, p. 148-149

(Fairbanks Cr.) (placer) - (20.0-21.2, 1.35-1.7) ; GOLD, TUNGSTEN, TIN, ANTIMONY.

P 70, p. 182

B 225, p. 68, 70-71

B 251, p. $67,81-83$

B 259 , p. 26-28

B 263, p. 14, 53-54, 92, 194, 208

B 284, p. 111, 118-119
B 314 , p. 36

B 337, p. 29,38

B 345, p. 39, 41-42

B 379 , p. 190-191, 194

B 442, p. 233

B 480, p. 158-159 
(Fairbanks Cr.) (placer)-Continued

B 520, p. 89, 240, 242-243

B 525, p. 102, 108-109, 112-113

B 542, p. 206-207

B 592, p. 68, 359

B 622, p. 54, 233

B 642 , p. 58-59

B 662 , p. 51, 54

B 692 , p. 35

B 712, p. 38-39

B 714, p. $80-81$

B 722, p. 44-45

B 739, p. 9, 29

B 755, p. 14, 35, 146

B 773, p. 27, 45

B 783, p. 13,18

B 792, p. 17,25

B 797, p. 20,30

B 810, p. 25,40

B 813, p. 28,47
B 824, p. $32-33,52$

B 836, p. 32-33, 54

B 844-A, p. 32, 54

B 857-A, p. 30, 51

B 864-A, p. 35, 56

B 868-A, p. 35-36, 58

B 880-A, p. 39, 61

B 897-A, p. 46, 71

B 910-A, p. 46, 77

B 917-A, p. 43-45

B 926-A, p. 40

B 933-A, p. 39

B 1024-I, p. 188, 210-211

C 331, p. 6

BMB 142, p. 22

BMB 153, p. 51

RI 2158, p. 1-4

TDM 1, p. 11, 32, 37, 40

Fairbanks Exp. Co. See U.S. Smelt., Ref. \& Min. Co.

Fairbanks Gold Dred. Co. See (Fairbanks Cr.) (placer).

Fairbanks Gold Min. Co. See (Fairbanks Cr.) (placer), (Twin Cr.).

Fairhaven \& Foss. S.ee McCarty (Henry Ford).

Fall. See (Lillian Cr.), (Ruth Cr.), (Wilbur Cr.).

Falls \& Barker. See (Lillian Cr.).

Faulkner. See North Star (Skoogy Gulch), North Star Extension, White Elephant.

Fay. See Chatham.

Feldman. See Hi-Yu.

Fish. See (Livengood Cr.).

(Fish Cr.) - (19.7-20.1, 0.4-1.05) ; GOLD, TUNGSTEN, BISMUTH, ANTIMONY, TIN.

B 379, p. 191

B 442, p. 233

B 480 , p. 159

B 520, p. 242-243

B 525, p. 102-103, 112-113.

B 542, p. 208

B 592, p. 68

B 622, p. 233

B 797 , p. 20,30

B 810, p. 25,40

B 813, p. 28,47

B 824, p. $32-33,52$

B 836, p. 32-33, 54

B 844-A, p. 33

B 864-A, p. 56

B 868-A, p. 58

B 880-A, p. 61

B 897-A, p. 46

B $910-A$, p. 46,48

B 917-A, p. 43-45

B 926-A, p. $40-41$

B 933-A, p. 38-39

B 1024-I, p. 188, 211

C 331, p. 6-7

C 335, p. 1, 3

BMB 142, p. 23

BMB 153, p. 51

TDM 1, p. 11, 32

Fish Cr. Min. Co. See (Fish Cr.).

Fiske Bros. \& Wickstrom. Seen (Amy Cr.).

(Flume Cr.) - $(18.2,0.35)$ (?) ; GOLD. Note--Mining may have been in Fairbanks quad.

B 642 , p. 59 
Foster \& Hungerford. See Alaska, Empire.

Franklin. See Chechako No. 1.

Franklin and assoc. See (Seattle Cr.).

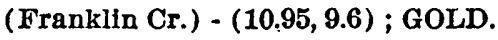

B 642, p. 208

Franklin No. 2. See (Livengood Cr.).

Frederichs. Bee Gilmer.

Fredericks - $(17.25-17.3,0.65)$; GOLD, ANTIMONY. Includes references to Friederich.
B 480, p. 34-35
B 649, p. 17-18, 29-31
B 520, p. 32
B 849-B, p. 80-81
B 525, p. 194-196
BMB 153, p. 52
B 542, p. 181-182
RI 4173, p. 38
B 592, p. 345
$\operatorname{TDM} 1$, p. 8

Free Gold. See Cleary Hill, McCarty (Pioneer). Note.-Two different claims.

Friederich. See Fredericks.

Fursteneau - $(19.25,1.65)$ approx; GOLD (?).

B 525, p. 169

Gan. See (Livengood Cr.).

Genki - $(19.25,1.65)$ approx ; GOLD (?). See also Alaska.

B $849-$ B, p. 100

Georgia. See (Our Cr.).

(Gertrude Cr.) - (10.8-10.95, 9.2-9.5) ; GOLD. See also (Glen Gulch).

B 642, p. 208

B 662, p. 56,269

B 868-A, p. 39-40

B 712, p. 41, 181

B 880-A, p. 44

B 836 , p. 36

B $897-$ A, p. 52

B 844-A, p. 35

B 910-A, p. 53

B 857-A, p. 34

B 917-A, p. 53

B 864-A, p. 39

B 926-A, p. 49

B 933-A, p. 46

Gibbs. See Hi-Yu.

Gilmer - $(17.3,0.55)$; ANTIMONY, GOLD, SILVER.

B 642, p. 29

B 649, p. 17, 29-30

RI 4173, p. 38

TDM 1, p. 8, 10

Gilmore-(19.9, 1.55) ; GOLD. See also Mizpah, Ohio (Fairbanks Cr.); not mentioned by name in $B \mathbf{7 1 2}$.
B 712, p. 39
B $849-$ B, p. 108

B 714, p. 81

Gilmore \& Stevens. See Gilmore, Ohio (Fairbanks Cr.).

Gilmore \& Stevenson. See Gilmore, Ohlo (Fairbanks Cr.).

Gladstone. Bee Alaska.

(Glen Gulch) - $(\mathbf{1 0 . 9 5 , 9 . 2 ) ~ ; ~ G O L D . ~ S e e ~ a l s o ~ ( G e r t r u d e ~ C r . ) . ~}$
B 662, p. 269
B 864-A, p. 39

B 844-A, p. 35

B 868-A, p. 39

B 857-A, p. 34

Glen Gulch Min. Co. See (Gertrude Cr.).

Goepfert - (18.7-18.75, 0.95-1.0) ; GOLD, LEAD.

B 525, p. 201-202 $\quad$ B 542, p. 187-188

Goessmann. See Cleary Hill, Wackwitz (Wyoming).

Gold Dollar. See (Livengood Cr.).

(Gold Mtn. Cr.) - (18-21, 4-7) (?) ; GOLD(?). May be in Circle quad.

B 480, p. 165

B 525, p. 151 
Golden Eagle. See McCarty (Henry Ford).

Goldstone. See Alaska.

(Goldstream Cr.). See (Hess Cr., S. Fork).

(Goodluck Cr.) - (11.45-11.65, 9.5-9.8) ; GOLD, CHROMITE, MERCURY, TIN,

RARE EARTHS. Includes references to (Lucky Cr.).

B 662, p. 268

B 926-A, p. 49

B 712, p. 41

C 335, p. 3

B 868-A, p. 39-40

Governor - $(19.95,1.65)$; GOLD.
B 520, p. 31
B 542, p. 145

B 525, p. 160

Goyett. See Hidden Treasure (lode), Mohawk.

Gray Eagle. See Ohio (Fairbanks Cr.).

Grimsoe- (19.1, 1.5) approx; GOLD(?).

B 849-B, p. 75

(Grouse Cr.) - (14.05-16.0, 12.8-14.85) ; GOLD( ?).

B 642, p. 209

(Gunnison Cr.) - (2.25-3.4, 6.65-8.0) ; TUNGSTEN, GOLD.
B 280, p. 48
B 337, p. 96
B 844-D, p. 192, 235-236
B 712, p. 41,182
TDM 1, p. 39

Gustafson Bros. See Cleary Hill, Wackwitz (Wyoming).

Haley \& Magnussen. See (Wilbur Cr.).

H \& K. See Soo.

Hanot Bros. See (Pedro Cr.).

Hard Lack. See (Vault Cr.).

Hard Scramble. See (Chatanika R.).

Harrais. See Independence, Moonlight.

Harrietta. See McCarty (Pioneer).

Harris. See Independence.

Harris \& Brown - $(19.3,1.6)$; ANTIMONY, GOLD. B 525, p. 175-176

B 542, p. 162

Hawkins (and assoc.). See Soo.

Healy. See (Goodluck Cr.).

Heath \& Kearns. See Soo.

Heilig \& Creighton. See Mohawk.

Helen S. See HI-Yu.

Helen W. See Gilmer.

Henry Clay. See McCarty (Pioneer).

Henry Ford. See McCarty (Henry Ford).

Herschberger, Beall \& Phipps - $(18.8,1.45)$ approx ; GOLD.

B 480, p. 34

Hess \& Barnet. See Jackson.

(Hess Cr.) - (N 1/2 quad.) ; GOLD (?). Includes reference to (Mike Hess Cr.) ; see also (Hess Cr., South Fork).

B 642, p. 208

B 714, p. 82

(Hess Cr., South Fork) - (11.3-11.6, 10.4-11.2) ; GOLD. See also (Alabam Cr.), (Moose Cr.).

B 642, p. 63, 207

Hess, Douglas \& Lowe. See (Gertrude Cr.). 
Hidden Treasure (lode) - $(18.5,1.3)$; GOLD.
B 480, p. 35
B 592, p. 342-343
B 849-B, p. 82

Hidden Treasure (placer). See (Livengood Cr.).

Hindenburg. See Markovich.

Hinton. See McCarty (Pioneer).

Hirschberger \& Zimmerman - $(18.7,1.0)$ approx; GOLD. See also Zimmerman (near Twin Cr.).

B 520, p. 32

Hi-Yu (Min. Co.) - (19.85-20.05, 1.7-1.75) ; GOLD, SILVER, ANTIMONY, LEAD, ZINC. Includes references to Crites \& Feldman, Dorando, Feldman, Gibbs, Helen S., Insurgent, Nars (, Anderson \& Gibbs), Summit, Sunnyside (lode), Teddy R., Yankee Doodle.

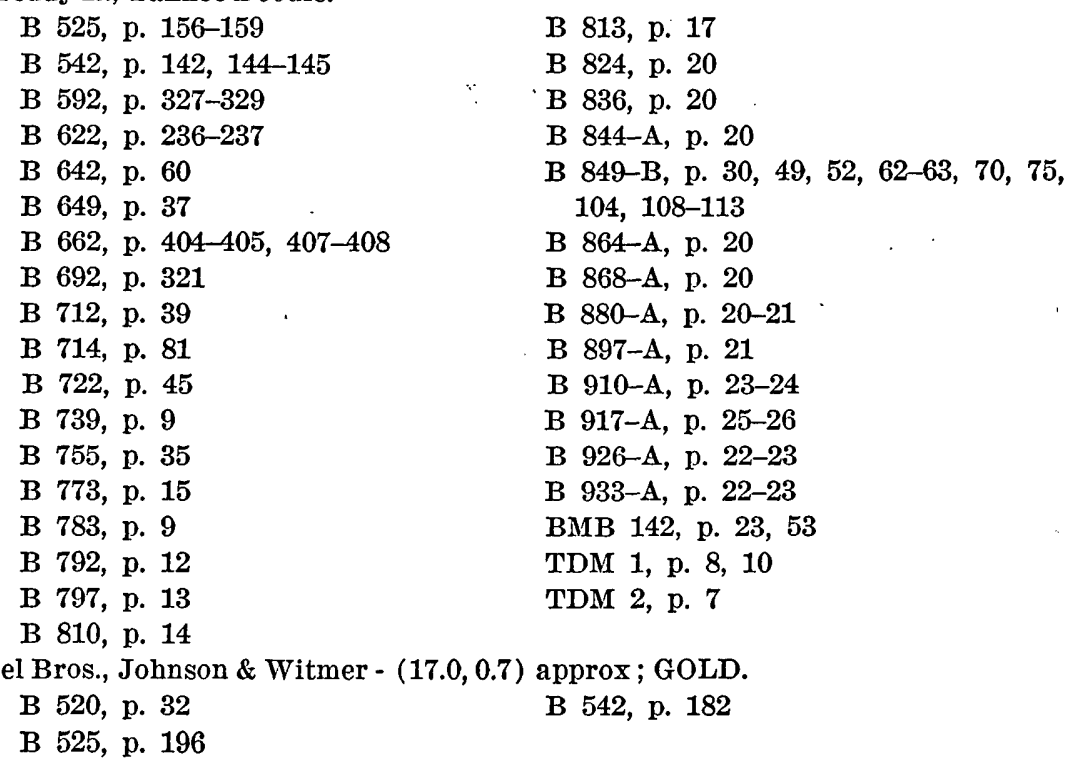

Homestake (Min. Co.) - $(19.45,1.65)$; GOLD, SILVER, ANTIMONY, COPPER. Includes references to Horton \& Solomon, Rexall.
B 480 , p. 34
B 649 , p. $36-37$
B 520, p. 31
B 662 , p. $406-407$
B 525 , p. $168,170-171$
B 849-B, p. 75, 101-102
B 542 , p. $153,155,157$
BMB 142, p. 23, 53
B 592, p. 325, 331, 333-335
B 622 , p. $237-238$
TDM 1, p. 8
B 642 , p. 60
TDM 2, p. 8

Homestead. See (Vault Cr.).

(Hoosier Cr.) - (18-21, 4-7) (?) ; GOLD (?). May be in Circle quad.
B 480 , p. 165
B 525, p. 151

Hoover - $(18.5,0.7)$; GOLD, ANIIMONY. Includes references to Anderson \& Birch, Birch \& Anderson, Busch \& Anderson, Robinson \& Lieman.
B 520 , p. 32
B 592 , p. $347-348$
B 525 , p. 198
B $849-$ B, p. $119-120$

B 542 , p. 184

Hope (lode). See Homestake. 
Hope (placer). See (Chatanika R.).

.Horton \& Solomon. See Homestake.

(Hosiana Cr.) - (SW 1/8 quad.) ( ?) ; GOLD( ?). May be in Tanana quad. B 480 , p. 167

Hudson. See (Olive Cr.).

(Hunter Cr.) - (0.0-0.2, 8.3-8.4) ; GOLD, MERCURY, TIN, COPPER. See also (Hunter Cr.) under Tanana quad.

$\begin{array}{ll}\text { AR } 18, \text { p. } 294,358-359 & \text { B } 712, \text { p. } 42 \\ \text { B } 213, \text { p. } 55 & \text { B 714, p. } 83 \\ \text { B } 225, \text { p. } 58 & \text { B } 739, \text { p. } 32 \\ \text { B } 259, \text { p. } 112 & \text { B } 783, \text { p. } 14 \\ \text { B } 263, \text { p. } 52,54,208 & \text { B } 797, \text { p. } 23 \\ \text { B } 280, \text { p. } 31-33,48-50 & \text { B } 813, \text { p. } 35 \\ \text { B } 314, \text { p. } 37 & \text { B } 824, \text { p. } 40 \\ \text { B } 337, \text { p. } 65,72-75,96-98 & \text { B } 836, \text { p. } 42 \\ \text { B } 345, \text { p. } 49 & \text { B } 844-A, \text { p. } 41 \\ \text { B } 379, \text { p. } 55 & \text { B } 844-D, \text { p. } 165,177-181,232 \\ \text { B } 442, \text { p. } 240 & \text { B } 857-A, \text { p. } 39 \\ \text { B } 480, \text { p. } 167 & \text { B } 864-A, \text { p. } 43 \\ \text { B } 520, \text { p. } 278-279,282 & \text { B } 868-A, \text { p. } 44 \\ \text { B } 535, \text { p. } 28,30-31,35 & \text { B } 880-A, \text { p. } 47 \\ \text { B } 542, \text { p. } 222 & \text { B } 910-A, \text { p. } 57 \\ \text { B } 592, \text { p. } 362 & \text { B } 917-A, \text { p. } 56-57 \\ \text { B } 622, \text { p. } 64 & \text { B } 926-A, \text { p. } 53 \\ \text { B } 642, \text { p. } 64 & \text { B } 933-A, \text { p. } 49 \\ \text { B } 662, \text { p. } 57 & \text { BMB 153, p. } 53 \\ \text { B } 692, \text { p. } 37 & \end{array}$

I.B. See McCarty (Pioneer).

Idaho (lode). See Cleary Hill.

Idaho (placer). See (Little Eldorado Cr.) (placer).

Idaho Min. \& Leasing Co. See (Little Eldorado Cr.) (placer).

Independence - $(18.8,0.85)$; GOLD, LEAD.

B 642, p. $60-61$

B 849-B, p. 114-115

B 662 , p. $410-411$

Inspiration. See Soo.

Insurgent. See Hi-Yu.

Iron Mask. See McCarty (Pioneer).

Isabel. See (Vault Cr.).

Isabella. See (Vault Cr.).

Italy. See (Livengood Cr.).

I.X.L. - (19.1, 1.45) ; GOLD (?).

B $849-$ B, p. 75

Jack - $(18.65,1.4)$; GOLD (?).

B 849-B, p. 75

Jackson - (18.9-19.0, 1.3-1.35) ; GOLD, ANTIMONY, SILVER, LEAD. Includes references to Hess \& Barnet, Silver King; not mentioned by name in $B$ 525, 542.

B 525, p. 182

B 662, p. $416-417$

B 542, p. 168-169

B 849-B, p. 92-93

B 592, p. 338-339

Jackson and assoc. - $(19.05,1.1)$; GOLD (?).

B 525, p. 201

B 542, p. 187 
Johnson - $(18.8,1.55)$; ANTIMONY, TUNGSTEN, TIN, GOLD(?). Includes references to (Willow Cr.) (near Fairbanks) ; see also Tolovana; not mentioned by name in RI 4174 .
B 1024-I, p. 180, 210
TDM 2, p. 7, 19-20

RI 4174, p. 28

Jupiter-Goldstone. See Alaska.

Jupiter-Mars (Consol. Min. Co.). See Alaska, Genki.

Jurich. See (Lillian Cr.).

Katherine. See Emma.

Kawilita. See Homestake.

Kellen - $(19.75,1.55)$; GOLD, ANTIMONY.
B 525, p. 163-164
B 649, p. 38

B 542, p. 149

Keystone. See Homestake.

(Kokomo Cr.) - (20.0-21.3, 2.4-3.7) ; GOLD.
B 739, p. 6, 29
B 926-A, p. 40, 42
B 910-A, p. 48
B 933-A, p. 39
B 917-A, p. 43,46

La Rose. See Soo.

Laughing Water. See McCarty (Pioneer).

Le Boyteau. See (Goodluck Cr.).

Leindecker. See Branholm-Jenkins.

Lemor. See McCarty (Alder Cr.).

Leroy. See McCarty (Pioneer).

Leslie \& Hawks. Bee Old Glory.

Lietrim. See (Livengood Cr.).

(Lillian Cr.) - $(10.4-10.55,9.0)$; GOLD, MERCURY, TUNGSTEN, ANTIMONY, CHROMITE, NICKEL.

B 642, p. 208

B 662, p. 56, 270-271

B 857-A, p. 34

B 712, p. 41, 181-183

B 864-A, p. 39

B 739, p. 157

B 868-A, p. 39

B 792, p. 19

B 880-A, p. 44

B 797, p. 21

B 897-A, p. 52

B 783, p. 14

B 910-A, p. 53

B 813, p. 30

B 917-A, p. 53

B 824, p. 36

B 926-A, p. 49

B 836, p. 36

B 844-A, p. 35

B 933-A, p. 46

BMB 153, p. 52

TDM 1, p. 14, 18, 26, 39

Lime. See McCarty (Alder Cr.).

(Little Eldorado Cr.) (lode) - $(18.4,1.15)$; GOLD ( ?).

B 525, p. 190

B 542, p. 176

(Little Eldorado Cr.) (placer) - (17.85-18.2, 1.55-1.9) ; GOLD, TIN, TUNGSTEN. Includes references to (Eldorado Cr.).

B 337, p. $42-43$

B 345, p. 42

B 379, p. 188, 190-191

B 442, p. 233

B 480, p. 156-157

B 520, p. 242
B 525, p. 100, 107, 112-113

B 542, p. 205

B 592, p. 68,358

B 622, p. 54,232

B 642 , p. $58-59$

B 662, p. 51, 54 
(Little Eldorado Cr.) (placer)-Continued
B 692, p. 35
B 712, p. 39
B 714, p. 81
B 722, p. 45
B 739 , p. 29
B 755 , p. 35,146
B 773, p. 45
B 783, p. 11, 13
B 792, p. 17
B 797, p. 20

B 810, p. 25

B 836, p. 33

B 844-A, p. 32

B 917-A, p. 43,45

B 926-A, p. $40-41$

B 933-A, p. 39

B 1024-I, p. 188, 210

BMB 142, p. 22

BMB 153, p. 51

TDM 1, p. 32, 37

Little Jim. See Jackson.

Livengood \& Hudson. See (Gertrude Cr.), (Lillian Cr.), (Livengood Cr.), (Olive Cr.), (Ruth Cr.).

(Livengood Cr.) - (10.45-11.35, 9.45-9.75) ; GOLD, ANTIMONY, CHROMITE, TIN, TUNGSTEN.

B 622, p. 51

B 642 , p. 63, 205-208

B 836, p. 36

B 662, p. 56, 262-268

B $857-$ A, p. 34

B 712, p. 178-181

B $864-A$, p. 39

B 714, p. 82

B 868-A, p. 39-40

B 722, p. 47

B 880-A, p. 44

B 739, p. 31

B 897-A, p. 52

B 755, p. 37

B 910-A, p. 53-54

B 773, p. 21

B 917-A, p. 52-53

B 783 , p. 14

B 926-A, p. 48-49

B 792, p. 19

B 933-A, p. 45-46, 68

B 797, p. 21

C 331, p. 11

BMB 142, p. 24-25

B 810, p. 27

BMB 153, p. 2, 52

B 813, p. 30

TDM 1, p. 14, 17, 34, 39

B 824 , p. 36

Livengood Placers, Inc. See (Livengood Cr.).

(Lucille Cr.) or (Gulch) - (11.1-11.15, 9.2-9.55) ; GOLD, CHROMITE.

B 662, p. 269

B 868-A, p. 40

TDM 1, p. 17

Luckman \& Co. See (Amy Cr.).

Luckman \& West. See (Amy Cr.).

(Lucky Cr.). See (Goodluck Cr.).

(Lucky Gulch) - (N 1/2 11, 8) ( ?) ; GOLD, CHROMITE.
B 844-A, p. 35
B 857-A, p. 34
B 868-A, p. 39
TDM 1, p. 17

B 864-A, p. 39

Lucky Lad. See Newsboy.

McCarthy. See McCarty.

McCarty (Alder Cr.) - $(20.45,2.1)$; GOLD. Includes references to North Star (Alder Cr.).

B 442, p. 227

B 592, p. 326

B 525, p. 156

B 642, p. 60

B 542, p. 141-142 
McCarty (Henry Ford) - $(19.65,1.55)$; GOLD, ANTIMONY. Includes references to American Eagle, Caribou, El Toro No. 3, Golden Eagle, Henry Ford, McCarty \& Ewers (Gold Min. Co.), McCarty, L. J., and assoc., McCarty Min. Co., Schreiber.
B 520, p. 31
B 525, p. 164
B 542, p. $149-150$
B 622, p. 238
B 642 , p. 60
B 692 , p. 322
B 810 , p. 14
B 813, p. 17
B 824, p. 20
B 836 , p. $19-20$

\author{
B 844-A, p. 19 \\ B 849-B, p. 52, 75, 104-106. \\ B 864-A, p. 20 \\ B 897-A, p. 22 \\ B 910-A, p. 23-24 \\ B 917-A, p. 25-26 \\ B 926-A, p. 22 \\ B 933-A, p. 22 \\ BMB 142, p. 23, 53
}

McCarty (Pioneer) - (19.5-19.65, 1.6-1.65) ; GOLD, ANTIMONY, LEAD, ZINC. Includes references to Black Warrior, Dorothy, Free Gold (Fairbanks Cr.), Harrietta, Henry Clay, Hinton, I. B., Iron Mask, Laughing Water, Leroy, McCarty \& Lawson, McDougall, Marigold, Minnie Ha-Ha, Pennsylvania, Pioneer (Fairbanks Cr.), Russsian Kid, War Eagle, Willie.

B 442, p. 227
B 480, p. 34
B 520, p. 31
B 525, p. $164-167$
B 542, p. $150-153$
B 592, p. 331

B 622, p. 237

B 642 , p. 60

B 662, p. 411-412

B 849-B, p. 75, 102-103

TDM 1, p. 10

TDM 2, p. 7-8

McCarty, L. J., and assoc. See McCarty (Henry Ford).

McCarty \& Ewers (Gold Min. Co.). See McCarty (Henry Ford).

McCarty \& Lawson. See McCarty (Pioneer).

McCarty Min. Co. See McCarty (Henry Ford).

McDougall. See McCarty (Pioneer).

McGillvray \& Ellis. See Soo.

McNeil. See Branholm-Jenkins.

McNeil \& Huddleson - $(19.7,1.75)$ (?) ; GOLD (?). See also Branholm-Jenkins. BMB 142, p. 23, 53

Mahan. See (Livengood Cr.).

Mandich (\& Jurich). See (Lillian Cr.).

Marietta. See (Livengood Cr.).

Marigold. See McCarty (Pioneer).

Markovich - $(17.95,1.25)$; GOLD, ANTIMONY. Includes references to Hindenburg, Ohio (Little Eldorado Cr.).
B 662, p. 415
RI 4173, p. 38
B 712, p. 40
TDM 1 , p. 10
B 849-B, p. 83
TDM 2, p. 9 .

Mars-Emerald. See Alaska.

Martin. See Johnson.

Mary - $(19.15,1.5)$; SILVER, LEAD, GOLD (?).

B 849-B, p. 100

May Florence - $(18.35,0.6)$; GOLD (?).

B 592, p. 346

Mayflower. See Ohio (Fairbanks Cr.).

Mazeppa - $(19.1,1.45)$; GOLD (?). See also Butler \& Petree.

B $849-$ B, p. 75 
(Mike Hess Cr.). See (Hess Cr.).

Million Dollar Corp. See Thompson \& Burns.

Mines Dev. Syn. See (Livengood Cr.).

Minnie. See Perrault.

Minnie Ha-Ha. See McCarty (Pioneer).

Mizpah - $(19.85,1.61)$; GOLD, ANTIMONY, TUNGSTEN, LEAD, MANGA:

. NESE. Includes references to Black Joe; see also Gilmore, Ohio (Fairbanks Cr.).
B 525, p. 162
B 692, p. 321-322
B 542 , p. 17-19, 38
B 712, p. 39
B 592, p. 329
B 755 , p. 148
B 622 , p. 237-238
B $849-$ B, p. 107
B 642, p. 60
B 1024-I, p. 180, 208
B 662, p. $405-406,421$
BMB 142, p. 23, 53

Mohawk - $(18.65,1.2)$; GOLD, ANTIMONY. Includes references to Heilig \& Creighton, Rose.
B 525, p. 190
B 692, p. 322
B 542, p. 176
B 712, p. 40
B 592, p. 342
B $849-$ B, p. 82
B 662, p. 407

Moonlight - (18.8-18.9, 0.9) ; GOLD. Includes references to Moonshine, Sunlight, Sunshine, Twilight.
B 525, p. 201
B 622 , p. 238
B 542, p. 187
B $849-$ B, p. 114

B 592, p. 349

Moonshine. See Moonlight.

(Moose Cr.) - (11.9-13.15, 10.35-11.95) ; GOLD.

B 642, p. 209

B 662, p. 273

Mother Lode (Dome Cr.) - $(17.6,1.0)$; GOLD (?). B 525, p. 194

B 542, p. 180

Mother Lode (IVillow Cr.) - $(\mathbf{1 8 . 8}, \mathbf{1 . 2 5})$ approx; ANTIMONY, GOLD. Note.Not mentioned by name in $B \quad 442$.

B 442, p. 221

B 649, p. 32-33

Muchano. See Gilmer.

Murphy. See (Fairbanks Cr.) (placer).

Myntti. See Markovich.

(Myrtle Cr.) - $(10.45,9.5)(?)$; GOLD.

B 662, p. 56

Nars (, Anderson \& Gibbs). See Hi-Yu.

Nelson. See (Livengood Cr.).

Nerich, Jackson \& Faulkner. See White Elephant.

Nevada. See (Vault Cr.).

New York. See Cleary Hill:

Newsboy - $(18.65,1.4)$; GOLD, ANTIMONY, ZINC, COPPER. Includes references to Newsboy Extension.
B 520, p. 31-32
B 836, p. 19
B 525, p. $187-190$
B 844-A, p. 19
B 542, p. $172-175$
B 849-B, p. $30,49,63,75,85-89$
B 592 , p. $340-341$
B 864-A, p. 20
B 622 , p. 238
B 868-A, p. 20
B 649, p. 32
B $880-$ A, p. 21

Newsboy Extension. See Newsboy.

Newsboy Min. Co. See Emma, Newsboy. 
Nickaloff. See Robinson (Pedro Dome).

Niggerhead. See (Dome Cr.).

Nightingale, G. See Homestake.

Nightingale, J. - $(18.3,0.55)$; LEAD, ANTIMONY.

B 525, p. 198

B 542, p. 184

Nirige \& Hershberger. See Rainbow.

(Nome Cr.) - (20.9-21.9, 6.0-7.25) ; GOLD. See also (Nome Cr.) (Circle quad.).

B 480 , p. 165

B 520, p. 243-244

B 542, p. 209-210

B 525, p. 140

B $880-$ A, p. 44

B 933-A, p. 39

Nordale. See Homestake.

North Star (Alder Cr.). See McCarty (Adler Cr.).

North Star (Chatham Cr.). Bee Pioneer (Chatham Cr.).

North Star (Skoogy Gulch) - $(18.75,0.95)$; GOLD, ANTIMONY.

B 442, p. 223

B 712, p. 40

B 525, p. 202-203

B 849-B, p. 74, 116-117

B 542, p. 188-189

North Star Extension - (18.16-18.7, 0.95-1.0) ; GOLD. Includes references to Center Star, Central Star.

B 442, p. 223

B 662 , p. 409

B 525, p. 202-203

B 849-B, p. 74, 116-118

B 542, p. 188-189

Northern Comm. Co. See David.

Ohio (Fairbanks Cr.) - $(19.85,1.65)$; GOLD, ANTIMONY, LEAD, SILVER. See also Gilmore, Mizpah.

B 525, p. 162-163

B 649 , p. 38

B 542, p. 148

B 662, p. 408-409

B 592, p. 329

B 692, p. 322

B 622, p. 237-238

B 849-B, p. 107-108

B 642 , p. 60

BMB 142, p. 23, 53

Ohio (Little Eldorado Cr.). See Markovich.

Oklahoma. See Wackwitz (Wyoming).

Old Glory - $(17.9,0.75)$; TUNGSTEN. Includes reference to Leslie (\& Hawks). B 1024-I, p. 180, 206, 209-210

RI 4174, p. 5, 26-27, 29

(Olive Cr.) - $(10.8,8.55-8.65)$; GOLD, MERCURY, TUNGSTEN, CHROMITE.

B 622 , p. 51

B 642, p. 63, 208-209

B 662, p. $56,271-272,274$

B 712, p. 41, 182-183

B 755, p. 37

B 792, p. 19

B 797, p. 21

B 813, p. 30

B 824 , p. 36

B 836, p. 36,81

B 844-A, p. 35

Omega - (18.1, 0.95) approx; GOLD (?).

B 849-B, p. 74, 77

(Ophlr Cr.) - (20.15-21.2, 5.6-6.9) ; GOLD.

B 480 , p. 165

B 520, p. 243

B 525, p. 150
B 857-A, p. 34

B 864-A, p. 39

B 868-A, p. 39

B 897-A, p. 52

B 910-A, p. 53-54

B 917-A, p. 53

B 926-A, p. 49

B 933-A, p. 46

BMB 142, p. 24

BMB 153, p. 52

TDM 1, p. 17, 26, 39
B 542, p. 210

B 712, p. 38 
Oregon. See (Vault Cr.).

Oro Fino - $(18.65,1.4)$; GOLD (?).

B $849-B$, p. 75

Oro Grande - $(18.65,1.4)$; GOLD (?).

B 849-B, D. 75

Ott \& McGowon. See Mizpah.

(Our Cr.) - (15.5-15.8, 0.35-0.5) ; GOLD.

B 337, p. 44

B 345 , p. $41-42$

B 379 , p. 191

B 525, p. 101-102, 108, 112-113

Our Jim. See Jackson.

Overgaard. See Emma.

Parker. See (Olive Cr.).

Paupers Dream. See Cleary Hill.

(Pedro Cr.) - (18.3-18.65, 0.35-0.65) ; GOLD, TIN. See also (Pedro Cr.) under Fairbanks quad.

B 225, p. 68

B 251, p. 67, 70-71, 75-77, 82-84

B 259, p. 26-28, 39-40

B 263, p. 32-33, 42, 53-54, 208

B 284, p. 111, 118-119

B 314, p. 36

B 337, p. 29, 39

B 379, p. 190, 192-193

B 442 , p. 232

B 480 , p. $154-155$

B 520, p. 244

B 525, p. 105-106, 109, 111, 113

B 542, p. 206

B 592, p. 68,359

B 622, p. 234

B 642 , p. 59

B 662 , p. 54
B 797, p. 20

B 810, p. 25

B 824 , p. 33

B 836, p. 32-33

B 844-A, p. 32

B 857-A, p. 30

B 864-A, p. 34-35

B 868-A, p. 35-36

B 880-A, p. 39

B 910-A, p. 46,48

B 917-A, p. 43-44

B 926-A, p. 40, 70

B 933-A, p. 38

C 331, p. 6

BMB 142, p. 22-23

BMB 153, p. 51

TDM 1, p. 32

Pennsylvania. See McCarty (Pioneer).

Perrault - $(19.75,1.65)$; GOLD, SILVER, ANTIMONY. Includes references to Aroostook, Minnie.

B 592, p. 329

Pilgrim. See Newsboy.

Pioneer (Chatham Cr.) - $(19.1,1.45)$; GOLD, ANTIMONY, ZINC.

B 442, p. 226

B 592, p. 336

B 480, p. 34

B 649, p. 35

B 525, p. 173-174

B 542, p. 159-161

B 849-B, p. 75, 99

Pioneer (Fairbanks Cr.). See McCarty (Pioneer).

Pioneer Cr. See Pioneer (Chatham Cr.).

Pioneer Discovery. See Pioneer (Chatham Cr.).

Pioneer Quartz Min. Co. See Pioneer (Chatham Cr.).

Plumbum - $(19.9,1.6)$; GOLD (?).

B 525, p. 160-161

B 849-B, p. 103-104

B 542, p. 146

Poz \& Contardi. See Markovich. 
(Quail Cr.) - (0.9-1.8, 6.1-6.3) ; GOLD, SILVER, TIN, TUNGSTEN, MERCURY.

P 70, p. 183

B 259, p. 114-115

B 280, p. 47-50

B 337 , p. $65,95-98$

B 379, p. 55

B 442, p. 241

B 480 , p. 167

B 520, p. 277, 283

B 525, p. 146-147

B 535, p. 29, 35

B 542, p. 221

B 592, p. 362

B 622 , p. 64
B 642 , p. 64

B 662, p. 57

B.712, p. 41, 182

B 813, p. 35

B 824, p. 40

B 844-D, p. 189-191, 235

B 864-A, p. 43

B 868-A, p. 44

B 880-A, p. 47

B 926-A, p. 53

B 933-A, p. 49

TDM 1, p. 27, 34

Queen - $(20.4,1.9)$; GOLD.

B 592 , p. 326

Quemboe Bros. - $(19.45,1.55)$; GOLD, ANTIMONY.

B 525, p. 171-172

B 649 , p. 36

B 542 , p. 158

Radak. See (Ruth Cr.).

Rainbow - $(18.85,0.95)$; GOLD, LEAD, ZINC, TUNGSTEN. See also Whitman \& Murray.

B 520, p. 32

B 622 , p. 238

B 525, p. 198-200

B 692 , p. 322

B 542, p. 184-186

B 849-B, p. 74, 115

B 592, p. 348

B 1024-I, p. 180, 210

Ready Bullion. See (Livengood Cr.).

Recorder. See (Dome Cr.).

Red. See (Livengood Cr.).

Reese - $(19.05,1.6)$; GOLD ( ?). See also Butler \& Petree.

B 849-B, p. 98-99

Reliance (Min. Cr.). See Soo.

Rex (Min. Cr.). See Butler \& Petree.

Rexall. See Homestake.

Rhoades-Hall. See Cleary Hill.

Rhoads (\&) Hall. See Cleary Hill.

Rob (\& Roy) - $(19.8,1.8)$ approx; GOLD, ANTIMONY. Includes references to Rob-Rye, Roy ; see also Hi-Yu.
B 622, p. 237
B 649, p. 37-38
B 642, p. 60
B 712 , p. 39

Robinson (Little Eldorado Cr.). See Mohawk.

Robinson (Pedro Dome) - $(18.3,0.9)$ approx ; GOLD (?).

B 849-B, p. 81-82

Robinson \& Drouin. See David.

Robinson \& Lieman. See Hoover.

Rob-Rye. See Rob \& Roy.

Rock Run. See Dome View.

Rogach. See Markovich.

Rose (claim). See Mohawk.

Rose, J. C. See Pioneer (Chatham Cr.).

Roth \& Maddocks. See David.

Roy. See Rob \& Roy. 
(Ruby Cr.). See (Ruth Cr.).

Russian Kid. See McCarty (Pioneer).

(Ruth Cr.) - (10.6-10.7, 9.0-9.35) ; GOLD, CHROMITE, TUNGSTEN, MERCURY, NICKEL, ANTIMONY. Includes reference to (Ruby Cr.).

B 662, p. 22, 56, 269-271, 273-274 B 868-A, p. 39

B 712, p. 41, 181, $183 \quad$ B 880-A, p. 44

B 783, p. 14

B 792, p. 19

B 797, p. 21

B 813 , p. 30

B 824, p. 36

B 836, p. 36

B 844-A, p. 35

B 857-A, p. 34

B 897-A, p. 52

B 910-A, p. 53

B 917-A, p. 53

B 926-A, p. 49

B 933-A, p. 46

C 331, p. 11

B 864-A, p. 39

BMB 153, p. 52

TDM 1, p. 16-17, 26, 39

TDM 2, p. 16

Saucy - $(19.8,1.8)$ approx ; GOLD, ANTIMONY.

B 649, p. 37-38

(Sawtooth Mtn.) - $(3.45,6.6)$; ANTIMONY.

TDM 2, p. 16

Schaefer - $(19.8,1.55)$; GOLD ( ?).

B 525, p. 163

B 542, p. 149

Scheuyemere. See Tolovana.

Scheuyemeress. See Tolovana.

Schreiber. See McCarty (Henry Ford).

(Seattle Cr.) - $(18.0,0.7)$; TUNGSTEN.

TDM 2, p. 23

Shakespeare. See (Dome Cr.).

Sierra. See (Vault Cr.).

Silva (\& Co.). See (Livengood Cr.).

Silver Dollar - $(18.35,0.55)$; GOLD (?).

B 592 , p. 346

Silver King. See Jackson.

Sky High - $(19.2,1.7)$; GOLD.

B 525, p. 175

B 542, p. 161-162

Snow Drift. See Cleary Hill.

Solomon - $(19.4,1.7)$; ANTIMONY. NoTE.-Not mentioned by name in texts, but descriptions fit prospect named on maps.

B 525, p. 171

B 542, p. 157

Somerville. See (Livengood Cr.).

Soo (Min. Co.) - (18.1-18.3, 0.95-1.05) ; GOLD, SILVER, ANTIMONY. Includes references to $\mathbf{H} \& \mathbf{K}$, Hawkins, Heath \& Kearns, McGillvray \& Ellis, Reliance (Min. Co.), Spaulding (\& Brumbaugh), Spaulding-Roman-Cunningham, Stevens \& Martin.
B 480 , p. 34
B 810 , p. 15
B 520, p. 32
B 813, p. 17
B 525, p. 190-194
B 824, p. 20
B 542, p. 176-180
B 836, p. 19
B 592, p. 343-345
B 844-A, p. 19
B 622 , p. 236
B 849-B, p. $62,65,68-70,74,77-80$
B 642 , p. 60
B 857-A, p. 18
B 649, p. 17-19, 31
B 864-A, p. 20
B 797, p. 13
B 868-A, p. 20 


\section{INDEX OF METALLIC AND NONMETALLIC MINERAL DEPOSITS}

Soo (Min. Co.)-Continued

B $880-$ A, p. 21

RI 4173, p. 38

B 897-A, p. 22

TDM 1, p. 10

BMB 142, p. 24,53

TDM 2, p. 9

Spall \& Livengood. See (Livengood Cr.).

Spaulding (\& Brumbaugh). See Soo.

Spaulding-Roman-Cunningham. See Soo.

(Spruce Cr.) - (17.7, 1.3) (?) ; GOLD.

B 525, p. 190

B 542, p. 176

S.S. - $(18.7,0.95)$ approx ; GOLD. See also North Star Extension.

B 442, p. 223

B 542, p. 188-189

B 525, p. 202-203

Stadelman. See (Amy Cr.), (Livengood Cr.).

(Steamboat Pup) or (Cr.) - $(18.4,0.5)$ approx; GOLD.

B 525, p. 113

B 642, p. 59

Steel. See Steil.

(Steel Cr.) - (11.0-11.45, 7.8-8.2) ; TUNGSTEN.

TDM 2, p. 20

Steil - $(18.75,1.5)$; GOLD, ANTIMONY. Includes reference to Steel.

B 525, p. 187

B 649 , p. 32

B 542, p. 175-176

Stevens. See Alpha, Omega.

Stevens \& Martin. See Soo.

Stibnite. See Johnson.

Stier. See (Chatanika R.).

Summit. See Hi-Yu.

Sunlight. See Moonlight.

Sunny. See (Livengood Cr.).

Sunnyside (lode). See Hi-Yu.

Sunnyside (placer). See (Livengood Cr.).

Sunrise (Cleary Cr.) - $(18.95,1.65)$; ANTIMONY, GOLD(?). See also Cunningham.

B 592, p. 337

Sunrise (Last Chance Cr.) - $(18.6,1.35)$; GOLD ( ?).

B 592, p. 342

Sunshine. See Moonlight.

Tanana Quartz (\&) Hyd. Min. Co. See Chatham, Wackwitz (Wyoming).

Tanana Quartz Min. Co. - $(19.3,1.55)$ (?) ; GOLD. See also Chatham.

B 520, p. 31

Tanana Valley Gold Dred. Co. See (Fish Cr.).

Teddy R. See Hi-Yu.

Texas. See Cleary Hill.

Thompson- $(18.55,1.2)$; GOLD. NotE.-Not mentioned by name in B 525, B 542 .

B 542, p. 176

B 525, p. 180

B 592, p. 342

B $849-$ B, p. 82

Thompson, C. See Mizpah.

Thompson \& Burns - $(18.6,1.0)$; GOLD(?).

B 849-B, p. 118

Thrift - (17.3, 0.9) approx; GOLD.

B 525, p. 196

B 542, p. 182 
Tolovana (Min. \& Mil. Co.) - (18.75-18.85, 1.5-1.55) ; GOLD, ANTIMONY, TUNGSTEN, SILVER. Includes references to Scheuyemere, Tolovana-Stibnite, Willow Cr.-Tolovana Min. Co.; see also Chechako No. 1, Johnson.
B 442, p. 225
B 836, p. 19
B 480 , p. 34
B 844-A, p. 19
B 525, p. $183-185$
B $849-$ B, p. $30,52,68,75,91-92$
B 542, p. 169-171, 175
B 1024-I, p. 180, 210
B 592 , p. $339-340$
C 335, p. 2
B 649, p. 33-34
TDM 1, p. 8
B 792, p. 12

(Tolovana R.) - (10-11, 8-9) ; GOLD. NoTe-Most production was from tributaries, of which Ester, Livengood, Olive, and Wilbur Creeks were the most important.

$\begin{array}{ll}\text { B } 712, \text { p. } 41 & \text { B } 844-A, \text { p. } 35 \\ \text { B } 813, \text { p. } 30 & \text { B } 857-\text { A, p. } 34 \\ \text { B } 824, \text { p. } 36 & \text { BMB 142, p. } 24 \\ \text { B } 836, \text { p. } 36 & \end{array}$

Tolovana-Stibnite. See Tolovana.

Tonaskate. See (Treasure Cr.) (placer).

(Trail Cr.) - (17.9-19.5, 4.5-7.15) ; GOLD.
B 480, p. 165
B 525, p. 150-151

(Treasure Cr.) (lode) - $(16.6,0.45)$; GOLD.
B 525, p. 196
B 542, p. 182

(Treasure Cr.) (placer) - (16.8-16.9, 0.6-0.8) ; GOLD.
B 314, p. 36
B 520, p. 242
B 337, p. 29
B 525, p. 101, 107, 112-113
B 379 , p. 191
B 542, p. 205
B 442, p. 232
B 592, p. 68,358

Triangle. See (Livengood Cr.).

Trojan - (17-20, 0-1) (?) ; GOLD (?). NoTE.-In Pedro Dome area.

B $849-$ B, p. 75

(Troublesome Cr.) - (1.8-2.9, 6.3-8.9) ; GOLD, MERCURY, TIN. see also (Quail Cr.).

B 525, p. 146

TDM 1, p. 27

B $844-\mathrm{D}$, p. 192,236

Twilight. See Moonlight.

(Twin Cr.) - (18.6-18.75, 0.6-0.9) ; GOLD, TIN.
B 225, p. 68-69
B 251, p. $67,71-72,75$
B 592, p. 359
B 622, p. 233
B 259, p. 26-28, 39-40
B 642, p. 59
B 263, p. 32,208
B 810 , p. 25
B 379, p. 188, 192, 194
BMB 153, p. 51
B 525, p. 105-106, 109, 111, 113
TDM 1, p. 32

B 542, p. 206

Union. See I.X.L.

U.S. Smelt., Ref. \& Min. Co. - (16.7-21.2, 0.35-2.4) ; GOLD. Includes references to Fairbanks Exp. Co.; see also (Chatanika R.), (Cleary Cr.), (Dome Cr.), (Fairbanks Cr.) (placer), (Fish Cr.), (Little Eldorado Cr.) (placer), MeCarty (Henry Ford), (Pedro Cr.).

B 792, p. 17

B 797, p. $19-20$

B 810, p. 25

B 813, p. 28-29, 47

B 824, p. 32-33, 51-52
B 836, p. 24, 32-33, 53-54

B 844-A, p. 32-33, 53-54

B 857-A, p. 30-31, 51

B 864-A, p. 34-35, 56

B 868-A, p. 35-36, 58 
U.S. Smelt., Ref. \& Min. Co.-Continued B 880-A, p. 39-40, 61

B $897-$ A, p. $46-47,71$

B 926-A, p. 40-42, 70

B 910-A, p. 24, 45-47

B 933-A, p. 22, 38-40, 66-67

B $917-A$, p. $43-46$

IC 7379 , p. $26-28$

V. See Cleary Hill.

(Vault Cr.) - (16.8-17.15, 0.65-1.35) ; GOLD.
P 70, p. 182
B 314, p. 36
B 755, p. 35,146
B 337, p. 29, 43-44
B 773, p. 45
B 345, p. 41-42
B 783, p. 13
B 379, p. 190-191, 195
B 792, p. 17
B 442, p. 232-233
B 797, p. 20
B 480 , p. 156
B 836, p. 33
B 520, p. 242
B 844-A, p. 32
B 525, p. 101, 108, 112-113
B 857-A, p. 30
B 542, p. 205
B 864-A, p. 35
B 592, p. 68, 358
B 868-A, p. 36
B 622, p. 54,233
B $880-$ A, p. 39
B 642 , p. 58-59
B 897-A, p. 46
B 662 , p. 51, 54
B 910-A, p. 46
B 692 , p. 35
B 917-A, p. 43
B 712, p. 39
B 714, p. $80-81$
B 722, p. 45
B 926-A, p. 40
B 933-A, p. 39
BMB 142, p. 22
BMB 153, p. 51

B 739, p. 29

Vergil. See Jackson.

Victor. See (Vault Cr.).

Victoria. See (Treasure Cr.) (placer).

Virginia. See (Livengood Cr.).

Wackowitz Bros. (\& Nelson). See Wackwitz (Wyoming).

Wackwitz (Wyoming) - $(18.9,1.5)$; GOLD, ANTIMONY, TUNGSTEN. Includes references to Alaska Min. \& Dev. Co., Goessman, Wyoming Quartz Min. Co.

B 442, p. 225

B 797, p. 13

B 480 , p. 33

B 810 , p. 15

B 520, p. 31

B 813 , p. 17

B 525, p. 181

B 824 , p. 20

B 542, p. 167

B 836 , p. 19

B 592, p. 338

B $849-$ B, p. 30, 52, 96-98

B 622, p. 237

B 868-A, p. 20

B 662 , p. 411

B $880-$ A, p. 21

B 692 , p. 322

B $897-$ A, p. 22

B 739 , p. 30

B 1024-I, p. 180, 206-208

B 783, p. 9

BMB 142, p. 24, 53

B 792, p. 12

Wackwitz, F. M. See Alaska.

Wackwitz Bros. See Dome View, Wackwitz (Wyoming).

Wall. See (Amy Cr.).

(Walnut Cr.) - (20.7-20.8, 1.7-1.95) ; GOLD.
B 251, p. 73
B 525, p. 102, 112-113
B 480, p. 159
B 542 , p. $207-208$ 
War Eagle. See McCarty (Pioneer).

Warren. See Anna, Mary.

Washington, See (Our Cr.).

(Washington Cr.) - (10.0-17.5, 1.25-4.1) ; GOLD(?): B 442, p. 208,234

Waterbury. See Soo.

Waverly. See Soo.

Westonvich. See Chechako No. 1.

White Elephant - $(18.95,1.1)$; LEAD, SILVER. NoTE.-Not mentioned by name in $B 542$.
B 542, p. 188
B $849-$ B, p. 114

B 592, p. 348

Whitehorse - $(19.9,1.6)$; GOLD, ANTIMONY, LEAD. NotE.-Not mentioned by name in $B 525,542$ :
B 525 , p. 160
B $849-$ B, p. 104
B 542, p. $145-146$
BMB 142, p. 23

Whitman \& Murray - $(18.85,0.95)$ ( ?) ; GOLD. See also Rainbow.

B 480 , p. 35

(Wilbur Cr.) - (11.0-11.85, 7.1-8.25) ; GOLD.
B 642 , p. 208
B $864-A$, p. 39
B 739, p. 6,31
B 868-A, p. $39-40$
B 755, p. 37
B $880-$ A, p. 44
B 797, p. 21
B $897-$ A, p. 52
B 813, p. 30
B $910-A$, p. 53
B 824, p. 36
B 917-A, p. 53
B 836 , p. 36
B $926-$ A, p. 49
B 844-A, p. 35
B $933-\mathrm{A}$, p. 46

B 857-A, p. 34

Wilcox. See Gilmer.

Wild Rose. See Soo.

(Wildcat Cr.) - (16.85-16.9, 0.55-0.65) ; GOLD.
B 525, p. 101
B 592, p. 68,358
B 542 , p. 205
B 642, p. 59

Willie. See McCarty (Pioneer).

(Willow Cr.) (near Fairbanks). See Johnson.

(Willow Cr.) (near Livengood) - $(11.3,11.2)$ approx ; GOLD.

B 783, p. 14

Willow Cr.-Tolovana Min. Co. See Tolovana.

Wolf (Too Much Gold Cr.) - $(19.8,1.8)$ approx; GOLD, ANTIMONY.

B 649, p. $37-38$

Wolf (Wolf Cr.). See Homestake.

(Wolf Cr.) - (19.05-19.45, 1.75-1.9) ; GOLD.
B 225, p. 68, 70-71
B 592, p. 358
B 251, p. $67,72,78-81,84$
B 622, p. 232
B 263, p. 53
B 642, p. 59
B 284, p. 119
B $910-$ A, p. 46
B 337, p. 41-42
B $917-\mathrm{A}$, p. 43
B 442, p. 233, 241
B $926-$ A, p. 40
B 480 , p. $155-156$
B $933-A$, p. 39
B 525, p. 112-113
BMB 142, p. 22

B 542, p. 205 
Wolverine. See Jackson.

Wyoming (Quartz Min. Co.). See Wackwitz (Wyoming).

Yankee Doodle. See Hi-Yu.

Yellowjacket - $(19.9,1.7)$ (?) ; GOLD.

BMB 142, p. 23

Your Jim. See Jackson.

Zimmerman (Kokomo Cr.). See (Kokomo Cr.).

Zimmerman (near Twin Cr.) - $(18.9,1.0)$; SILVER, GOLD. See also Hirschberger \& Zimmerman. B 525, p. 201

B 542, p. 187

Zimmerman (Twin Cr.) - (18.7, 0.7) ; GOLD. See also (Twin Cr.). B 849-B, p. 63, 70, 118-119

\section{MCCARTHY QUADRANGLE}

Acme Min. Co. See Wiley.

Alaska (Consol.) Copper Co. See Copper Queen, (Nugget Cr.).

Alaska Copper Mines, Inc. See Radovan.

Alaska Kotsina Copper Co. See (Peacock Cr.), (Surprise Cr.).

Alaska United (Copper) Exp. Co. See Bear Paw, Big Horn; (Contact Gulch), Golden Eagle, (Porcupine Cr.), Snow Bird, Westover.

Alaska Westover Copper Co. See Westover.

(Ames Cr.). See (Amy Cr.) (trib. Kotsina R.).

Ammann. See (Fall Cr.), Good Enough, Lost Cabin, (Roaring Cr.), Skyscraper.

(Amy Cr.) (trib. Bremner R.) - (3,0) (?) ; GOLD (?).

P 41, p. 120

(Amy Cr.) (trib. Kotsina R.) - (1.2-2.0, 12.25-12.3) ; COPPER(?).

B 345, p. 137-138

B 379, p. 156

B 374, p. 55

B 745, p. 104

Andrus. See (Chititu Cr.), (Rex Cr.).

Baldwin. See Yellow Band.

Barrett (copper). See Green Butte.

Barrett (gold). See (Williams Peak).

Barrett, Young \& Nafsted. See (Mineral Cr.), (Porcupine Cr.).

Baultoff. See Tjosevig.

Bear Paw - $(12.45,6.85)$; COPPER.

B 947-F, p. 120

(Beaver Cr.). See Wiley.

Bekka. See Woodin \& Herman.

Berg. See (Berg Cr.).

(Berg Cr.) - (1.6-1.7, 9.5-9.65) ; GOLD, SILVER, COPPER. Includes references to Berg, Midas, North Midas, North Midas Copper Co.

B 542, p. 83

B 592, p. 61

B 622, p. 114

B 642, p. 54

B 662, p. 160

B 712, p. $15,31-32$

B 714 , p. 30, 191-192

Big Ben. See Tjosevig.
B 722, p. 38

B 745, p. 140-141, 143-146

B 755, p. $26,65,68-71$

B 773, p. 15,37

B 783, p. 7-8

B 947-G, p. 140-141

BMB 153, p. 33 
Big Horn - $(3.1,11.35)$; COPPER. Includes references to Finch and to Alaska United Copper Exp. Co. if definitely applicable to this prospect.
B 622 , p. 113-114
B 745, p. $90-91,133-135$
B 662 , p. 158-159
B 947-G, p. 137-138

(Bigfoot Cr.). See Calcite, War Eagle.

Binocular. See Radovan.

Birch. See (Dan Cr.).

Bird Larsen. See (Lime Cr.) (trib. Rock Cr.).

Blackburn. See (Porcupine Cr.).

Blackstone. See (Porcupine Cr.).

Bonanza. See Kennecott.

Bonanza Gold Min. Co. - $(9,7)(?)$; GOLD.

B 592, p. 61

Bremner Min. Co. See (Golconda Cr.).

Broken Leg. See (Chokosna R.).

Brooks. See (Rex Cr.).

(Calamity Gulch) - (12.4-12.9, 4.05-4.6) ; GOLD.
P 15, p. 61
B 448 , p. 108

Calcite - $(2.2,9.4)$; COPPER. Includes reference to (MacDougall Cr.).
B 714, p. 192
B 755, p. 65-66
B 745, p. 137-139
B 947-G, p. 140

California-Alaska Min. \& Dev. Co. See (Roaring Cr.).

(Canyon Cr.) (gold) - $(15.1,2.5)$ approx; GOLD.

B 642, p. 135

(Canyon Cr.) (molybdenum) - $(16.6,5.35)$; MOLYBDENUM.

$\begin{array}{ll}\text { B } 642, \text { p. } 54,135 & \text { B } 714, \text { p. } 41 \\ \text { B } 662, \text { p. } 25 & \text { B } 926-\text { C, p. } 182-183 \\ \text { B } 666, \text { p. } 98 & \text { TDM 1, p. } 30\end{array}$

B 675 , p. 79

Carvey. See (Rex Cr.).

Cascade Quartz. See Nelson (copper).

Castle. See Skyscraper.

Chiti. See Erickson.

Chitina-Kuskulana Copper Co. See Calcite, War Eagle.

(Chititu Cr.) - (11.4-11.9, 5.2-5.6) ; GOLD, COPPER, SILVER, ANTIMONY, LEAD.

P 15, p. 59-60, 63

P 41, p. 118-119

B 755, p. 27-28, 69-71

B 263, p. 207

B 345, p. 172-173

B 783, p. 12

B 792, p. 15, 29

B 374, p. 45, 95-97

B 797 , p. $16-17$

B 379, p. 156

B 442, p. 158, 162-163

B 810 , p. 21

B 813, p. 24

B 824, p. 28

B 448 , p. 76, 80, 98-100, 103-107

B 836, p. 27

B 520, p. 107

B 542, p. 84-85

B 592, p. 61-62

B 622, p. 105, 115-117

B 649, p. 62-63

B 662, p. 43

B 714, p. 196

B 722, p. 39

B 844 A, p. 28

B 857-A p. 26

B 864-A, p. 30

B 868-A, p. 31

B $880-A$, p. 34

B 880-B, p. 98

B 897-A, p. $40-41$

B $910-A$, p. 38 
(Chititu Cr.) -Continued
B 917-A, p. 36
B 926-A, p. 34
B 947-F, p. 98
BMB 153, p. 33

Chititu Mines. See (Chititu Cr.), (Rex Cr.).

Chittyna Exp. Co. See Nikolai.

(Chokosna R.) - $(2,9)($ ?) ; COPPER(?).
B 442 , p. 161
B 714, p. 193

(Clear Cr.) - (1.15-1.4, 10.7-11.0) ; COPPER. Includes references to Copper

Mtn. and to Great Northern Dev. Co. if definitely applicable to these prospects.

P 15, p. 18

B 379 , p. 156

B 662 ; p. 157-158

B 442 , p. 161

B 714, p. 29-30

B 520, p. 106

B 745, p. 94, 126-128

B 542, p. $82-83$

B 755, p. 66

B 592, p. 61

B 947-G, p. 132-136

B 622, p. 113

BMB 142, p. 37, 52

B 642, p. 54

BMB 153, p. 30

(Contact Gulch) - $(13.6,8.65)$; COPPER.
B 345, p. 167
B 947-F; p. 120
B 374, p. 90

(Copper Cr.) - (12.85-13.6, 5.9-6.6) ; GOLD. See also (Dan Cr.):
B 442 , p. 162
B 542, p. 84
B 448 , p. $100-103$
B 622, p. 115-116

B 520, p. 107

Copper King (Kotsina R.) - (NW 1/4 2, 12) ; COPPER. P 15, p. 19 B 213 , p. 146-147

P 41, 95

Copper King (White R.) - $(16.6,13.6)$; COPPER.

B 379 , p. 174

B 417 , p. $55-56$

B 480, p. $86-87$

B 630 , p. 121-122

Copper Mtn. See (Clear Cr.).

Copper Queen - $(1.9,9.75)$; COPPER, IRON. Includes references to Rarus.

B 542 , p. 83

B 622, p. 44,113

B 714, p. 193

B 662 , p. 160

B 745, p. 71, 95, 129, 139

B $947-$ G, p. 139

Dalton. See (Kletsan Cr.).

(Dan Cr.) - (12.1-12.85, 6.6-6.7) ; GOLD, COPPER, SILVER. See also (Copper Cr.), (Idaho Gulch), (Rader Gulch), (Seattle Gulch).
P 15, p. 59-61
B 722, p. 39
B 259, p. 44-46
B 263, p. 37, 39, 207
B 755, p. $27,69-71$
B 345, p. $37,168-169$
B 783 , p. 12
B 792, p. 15, 29
B 374, p. 45, 91-92, 97-99
B 797, p. 16-17
B 379 , p. 156
B 810, p. 21
B 442 , p. $158,162-163$
B 813 , p. 24
B 448 , p. $76,80,98-103$
B 824, p. 28
B 836, p. 27
B 520 , p. 107
B 844-A, p. 28
B 542, p. 84
B 857-A, p. 26
B 592, p. 61
B 864-A, p. 30
B 622 , p. $105,115-116$
B 868-A, p. 31
B 714, p. 196 
(Dan Cr.)-Continued
B $880-A$, p. 34
B 926-A, p. 34
B $880-B$, p. 98
B 933-A, p. 37
B $897-$ A, p. 40
B $947-$ F, p. $98,119-120$
B 910-A, p. 38-39
C 184 , p. 3
B $917-A$, p. 37
BMB 153, p. 33

Dan Cr. Gold \& Copper Co. - (N 1/2 12, 6) ( ?) ; COPPER.

B 947-F, p. 120

Dan Cr. Min. Co. See (Dan Cr.).

Davy - (1.55-1.65, 12.1-12.25) ; COPPER, SILVER. See also Skyscraper.

P 15, p. 19-20

B 213, p. $145-146$

P 41, p. $95-96$

Dillman. See Westover.

Donohoe - (10.2-10.55, 8.4-8.85) (?); COPPER. See also Green Butte. B 448, p. 97

Drake. See (Surprise Cr.).

Drake \& Grenig. See (Shower Gulch).

Eleanor - $(1.55-1.65,12.1-12.25)$; COPPER, SILVER. See also Skyscraper.
P 15, p. 19-20
B 213 , p. 145-146
P 41, p. 95-96

Eli. Sce Woodin \& Herman.

Engineer Syn. See (Berg Cr.).

Erickson (\& Madden) - $(14.65,7.35)$; COPPER. Includes references to Chiti.

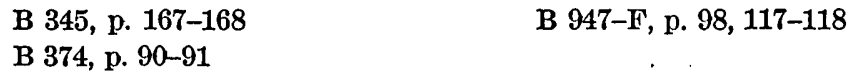

Erie. See Kennecott.

Esterly \& Andrus. See (Chititu Cr.), (Rex Cr.).

(Fall Cr.) - $(0.45-0.85,13.5-13.85)$; COPPER. Includes references to (Kluvesna Cr.).
P 15, p. 21
B 442 , p. 161
P 41, p. 97
B 622, p. 109
B 345 , p. $142-144$
B 745, p. 113-114

B 374, p. $60-61$

Fennesand Bros. See Silver Star.

Finch. See Big Horn.

(Fourth of July Cr.) (arsenic) - (7.1, 9.1) approx; ARSENIC.

B 345, p. 161

B 374, p. 99

(Fourth of July Cr.) (copper). See Woodin \& Herman.

G. \& B. See (Lime Cr.) (trib. Rock Cr.).

George M. See (Surprise Cr.).

Gilleneau, Bell \& Barrett. See (Lime Cr.) (trib. Rock Cr.).

Glacier. See Kennecott.

(Glacier Cr.) (trib. Chitistone R.) - (13.4-14.7, 7.0-8.3) ; COPPER.

See also Erickson, Nelson (copper), Radovan.

B 813, p. 54

(Glacier Cr.) (trib. Hidden Cr.). See (Hidden Cr.).

Golconda (Min. Co.). See (Golconda Cr.).

(Golconda Cr.) - $(4.35-4.8,0.05-0.9)$; GOLD. Includes references to Bremner

Mitn. Co., Ramos Bros.

P 15, p. 62

P 41, p. 120

B 576, p. 43-47, 49-50

B 520, p. 94-99

B 844-A, p. 22, 28

B 857-A, p. 21, 26 
(Golconda Cr.)-Continued

B 864-A, p. 24, 30

B 868-A, p. 24, 31

B 880-A, p. 28, 34-38

Golden Eagle - $(12.5,6.75)$; COPPER.

B 662, p. 176

Good Enough - $(0.5,13.4) ;$ COPPER.

B 745, p. 112-113

Grand Prize See (Golconda Cr.).

Gray's Copper. See (Clear Cr.).

Great Northern Dev. Co. See (Amy Cr.) (trib. Kotsina R.), (Clear Cr.), (Hidden Cr.), (Kotsina R.), Regal, (Roaring Cr.), Woodin \& Herman.

Green. See Green Butte.

Green, Joshua, Assn. See (Dan Cr.).

Green Butte (Copper Co.) - (10.2-10.55, 8.4-8.85) ; COPPER, SILVER.

Includes references to Green; see also Donohoe.

B 712, p. 31

B 739, p. 23

B 755, p. 27

B 773, p. 28, 37

B 783, p. 20

B 792, p. 27-28

Greenstone. Bee Radovan.

B 797, p. 35-36

B 810, p. 47

B 813 , p. 54

B 824, p. 60

B 836, p. 63

B 947-F, p. 98, 103-104

Grenig. See (Surpise Cr.).

Hammon. See (Chititu Cr.).

Happell, Hawes, Gillis \& Wheaton. See (Golconda Cr.).

Hero. See Kennecott.

(Hidden Cr.) - (6.75-7.1, 9.25-9.7) ; COPPER. See also Woodin \& Herman.

$$
\begin{aligned}
& \text { B 345, p. 158-160 } \\
& \text { B 374, p. 77-79 } \\
& \text { B 442, p. } 161
\end{aligned}
$$

B 622, p. 115

B 662 , p. 162-163

B 947-F, p. 98, 118-119

Hidden Treasure. See (Fall Cr.).

Homestake. See (Fall Cr.).

Houghton Alaska Exp. Co. (Chitistone R.) - (13.3, 8.35) ; COPPER. See also Peavine.

B 345, p. 166-167

B 947-F, p. 120

B 374, p. $89-90$

Houghton Alaska Exp. Co. (McCarthy Cr.) - (9.9-10.2, 9.1-9.4) approx; COPPER.

B 379, p. 155

B 448, p. 92-93

B 442 , p. $160-161$

Hubbard. See (Surprise Cr.).

(Idaho Gulch) - $(12.95,6.45)$; GOLD.

B 442 , p. 162

B 448, p. 100-101

Independence. See Kennecott.

Iowa. See Erickson.

Jackson. See Tjosevig.

Joe Dandy. See (Surprise Cr.).

(Jolly Gulch). See (White Cr.).

Josevig-Kennecott Corp. See (Hidden Cr.).

Jumbo. See Kennecott. 
Kennecott (Copper Corp.) - (9.2-9.8, 9.0-9.35) ; COPPER, SILVER. Includes references to Bonanza, Erie, Independence, Jumbo, Kennecott-Bonanza, Kennecott Mines Co., Marvelous, Mother Lode (Coalition Mines Co.), Mother Lode Copper Mines Co., Old Hero, Old Independence; see also Nelson (copper).

P 15, p. 16-18, 27-28

P 41, p. 92, 103-104

B 213, p. 144-145

B 314, p. 28

B 345, p. 161-165

B 374, p. $80-88$

B 379, p. 153-154

B 442, p. 34,160

B 448, p. 76, 83-93

B 480, p. 27-28, 84-86

B 520, p. 105-106

B 542, p. 82,84

B 592, p. 60-61

B 622, p. 44, 104-105, 114-115

B 642, p. 54

B 662, p. 16, 43, 163-175

B 692, p. 17-18, 30

B 712, p. 30-31

B 714, p. 12-13, 25, 27-28, 69, 194-195

B 722, p. 19, 21, 38-39

B 739, p. 13, 23

B 755 , p. $15,26-27,62-65,68,70-72$

Kennecott-Bonanza. See Kennecott.

Kennecott Mines Co. See Kennecott.

Kernan. See (Rex Cr.).

Keystone - (NW 1/4 2, 12); COPPER.

P 15, p. 19

P 41, p. 94-95
B 773, p. 28, 37

B 783, p. 20

B 792, p. 27-28

B 797, p. 32, 34-35

B 810, p. $45-46,48$

B 813, p. 52-53

B 824, p. 58-59

B 836, p. 60-61

B 844-A, p. 59-60

B 857-A, p. 56-57

B 864-A, p. 59

B 868-A, p. 67

B 880-A, p. $69-70$

B 880-B, p. 98

B 897-A, p. 78-80

B 910-A, p. 84-85

B 917-A, p. 85-87

B 947-F, p. 98-101

C 252, p. 5

BMB 142, p. 32-38, 52

BMB 153, p. 30-32

BMB 405, p. 25, 205-206, 209-211, 275

IC 7379, p. 32-33

Killian. See Yellow Band.

Kinney-Golden - $(2.8,8.95)$; COPPER. B 662, p. 160-161

(Kletsan Cr.) - $(24.9,11.0-11.5)$ approx; COPPER.
AR 20, p. 488-489
AR 21, p. 379-381
B 379, p. 175-176
P 15, p. 40-42
B 417 , p. 57
B 213, p. 148
B 622, p. 226
B. 630 , p. $90,120,124-125$

B 314, p. 28

(Kluvesna Cr.). See (Fall Cr.).

Kotsina Min. Co. See (Fall Cr.), Skyscraper.

(Kotsina R.) - (0.6-1.2, 12.45-12.5) ; COPPER.
B 345, p. 137
B 442 , p. 161
B 374, p. 54-55
B 622 , p. 109
B 379, p. 156
B 745, p. 100

Laddie. See (Surprise Or.).

Lakina Copper Co. See (Lakina R.). 
(Lakina R.) - (5.0-5.4, 9.7-9.9); COPPER. Includes references to Lakina Copper Co.
B 345, p. 155-158
B 622, p. 115
B 374, p. 75-77
B 642 , p. 54
B 442, p. 161
B 662, p. 161-162

Larson - $(0.95,12.2)$; COPPER (?).

B 745, p. 100

Last. See Nikolai.

Le Tendre - $(4.95,0.6)$; GOLD.

B 880-B, p. 102

(Lime Cr.) (trib. Rock Cr.). - (0.45-0.5, 12.0) ; COPPER. Includes references to $\mathrm{G} \& \mathrm{~B}$, United Verde.

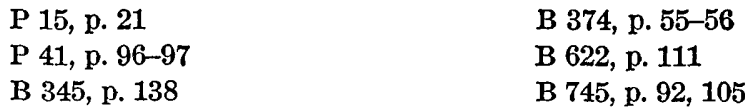

(Lime Cr.) (trib. White R.) - (17.35-18.2, 13.2-14.0) ; GOLD.
B 622, p. 223
B 630 , p. 116

London \& Cape Co. - $(2.3,9.9)$; COPPER. B 662 , p. $159-160$

B 745, p. 136-137

Lost Cabin - $(0.05,12.75)$; COPPER.

B 745, p. 112

Low Contact. See Radovan.

Lucky Girl. See (Golconda Cr.).

Lucky Six - (S 1/2 quad.) (?) ; GOLD (?).

B 810, p. 17

McCarthy. See (Nugget Cr.).

McConnell \& Johnson. See Mayflower.

MacDougall. See Calcite, War Eagle.

(MacDougall Cr.). See Calcite, War Eagle.

McGavock \& Erickson. See Erickson.

Mahar. See Peavine.

Mammoth. See (Golconda Cr.).

Marvelous. See Kennecott.

Mayflower - $(3.0,11.0)$; COPPER.

B 662, p. 159

B 745, p. $135-136$

Meloy (\& O'Hara). See Yellow Band.

Midas. See (Berg Cr.).

(Mineral Cr.) - $(0.7-0.85,13.05-13.1)$; COPPER, GOLD, SILVER. Includes references to Valdez (Mineral Cr.).

B 622 , p. $109-110$

B 745, p. $114-115$

Mineral King. See (Chokosna R.).

Mint. See (Peacock Cr.).

Monahan, Banty \& Anderson. See (Golconda Cr.).

(Monahan Cr.) - (5.45-6.0, 0.05-1.95) ; GOLD. See also. (Golconda Cr.).

B 576, p. 44

B 864-A, p. 30

B $844-$ A, p. 28

B 868-A, p. 31

B $857-$ A, p. 26

B $880-A$, p. $34-35$

(Moraine Cr.) - $(18.45,11.7)$; COPPER.

B 379, p. 174-175

B 630 , p. 123-124

B 417 , p. 56

Morning Star. See Skyscraper.

Mother Lode (Coalition Mines Co.) See Kennecott. 
Mother Lode Copper Mines Co. See Kennecott.

Mountain. See (Peacock Cr.).

Mt. Wrangell Copper Co. See Copper Queen.

Murie. Sce (Chititu Cr.).

Nebraska. See (Hidden Cr.).

Nelson (copper) - $(13.6,8.0)$; COPPER.

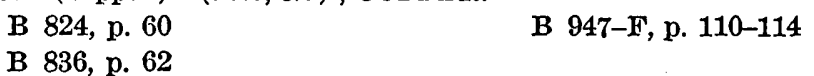

Nelson (gold) - $(5.1,0.6)$; GOLD.

B 880-B, p. 102

Newhome. See (Fall Cr.).

Nikolai - (10.85-11.05, 8.0-8.15) ; COPPER.
AR 21, p. 437
B 448, p. 75, 93-95
P 15, p. 16-18, 28-29
B 480 , p. $84-85$
P 41, p. 92, 104-105
B 520, p. 106
B 213, p. 144
B 542, p. 84
B 345, p. $165-166$
B 714, p. 28
B 374, p. $88-89$
B $947-$ F, p. $98,106-108$

Nikolai Butte Copper Co. - (N 1/2 12, 6) ( ?) ; COPPER.

B 947-F, p. 120

Nikolai Placer Co. See (Dan Cr.).

Nikolai Placer Mines. See (Dan Cr.).

Nizina Min. Co. See (Chititu Cr.), (Rex Cr.).

North Midas. See (Berg Cr.).

North Midas Copper Co. See (Berg Cr.).

Northern Comm. Co. See (White R., Middle Fork).

(Nugget Cr.) - (2.15-2.4, 11.2-11.25) ; COPPER, GOLD, SILVER.
P 15, p. 27
P 41, p. 103
B 345, p. 153-155
B 374, p. 45, 72-74
B 379, p. 155
B 442 , p. 161
B 542, p. 83
B 592, p. 61
B 622, p. 44,113

B 642, p. 54

B 662 , p. 158

B 712, p. 31

B 714, p. 29, 190-191

B 745, p. $82-83,85,90,93,129-133$

B 947-G, p. 136-137

BMB 142, p. 37, 52

BMB 153, p. 30

Old Hero. See Kennecott.

old Independent. See Kennecott.

One Girl. See (Nugget Cr.).

O'Neill. See Westover.

Pardners Mines Corp. See (Dan Cr.).

(Peacock Cr.) - (SE 1/4 1, 12) ; COPPER. P 15, p. $19-20$

B 374, p. 57-58

B 345, p. 140-141

B 745, p. 107

Peavine - $(12.85,8.0)$; COPPER. See also Houghton Alaska Exp. Co. (Chit. istone R.).

B 345, p. 166-167

B $947-$ F, p. 120

B 374, p. $89-90$

Pierson - $(2.55,10.55)$; GOLD, COPPER.

B 745, p. 136

B $947-$ G, p. 138 
(Porcupine Cr.) - (1.25-1.7, 10.75-11.05); COPPER, GOLD. See also (Clear Cr.).

B 542, p. 83

B 745, p. 128

B 662 , p. 158

Powell. Bee (Rex Cr.).

(Ptarmigan Cr.) - (24, 16-17) (?) ; GOLD (?).

B 622, p. 223

B 630, p. 116

(Rabbit Cr.) - (24.0, 14.6) approx; COPPER.

B 379, p. 175

B 417, p. 57

(Rader Gulch) - $(13.5,6.1)$; GOLD.

B 442 , p. 162

B 448, p. 100-101

Radovan - (13.65-13.85, 7.2-7.6); COPPER, ANTIMONY.

B 947-F, p. 98, 114-117

Ramos Bros. See (Golconda Cr.).

Rarus. See Copper Queen.

Red Rover. See Nikolai.

Regal (Min. Co.) - $(8.5,9.6)$; COPPER. Includes references to Great Northern Der. Co. if definitely applicable to this prospect.

B 622, p. 44, 115

B 947-F, p. 101-103

B 662, p. 163

(Rex Cr.) or (Gulch) - (11.2-12.1, 5.2-5.7) ; GOLD, SILVER, MOLYBDENUM.

P 15, p. 60

B 442 , p. 162

B 448, p. $98-100,103-107$

B 520, p. 107

B 542 , p. 85

B 592 , p. 62

B 622, p. 115-117

B 662, p. 43

B 714, p. 196

B 722, p. 39

B 755, p. 27, 69-70

B 783, p. 12

B 810, p. 21

B 813, p. 24
B 824 , p. 28

B 836, p. 27 .

B 844-A, p. 28

B 857-A, p. 26

B 864-A, p. 30

B 868-A, p. 31

B 880-A, p. 34

B $897-$ A, p. $40-41$

B 910-A, p. 38-39

B 917-A, p. 37

B 926-A, p. 34

B 933-A, p. 33

C 184, p. 3

BMB 153, p. 33

Rex Cr. Min. Co. See (Rex Cr.).

(Roaring Cr.) - (1.15-1.4, 11.8-12.3) ; COPPER. See also Skyscraper.

B 345, p. 139

B 374, p. 56-57
B 622, p. 111

B 745, p. 107

Rose. See (Peacock Cr.).

Satterfields. See Green Butte.

(Seattle Gulch) - $(\mathbf{1 3 . 6 , 5 . 9 ) ; ~ G O L D . ~}$

B 442 , p. 162

B 448 , p. $100-101$

Sheehan. See (Surprise Cr.).

(Sheep Cr.) - (19.6, 12.15) approx ; COPPER.

B 630, p. 124

Sheriff. See Yellow Band.

(Shower Gulch) - $(2.2,12.65)$ approx ; COPPER.

B 345 , p. 141

B 374, p. 58-59

Side Partner. Sce Nikolai.
B 745, p. $107-108$

B 755 , p. 67 
Silver Star - $(0.75,12.85)$; SILVER, COPPER, BISMUTH, LEAD. NoTE-Not mentioned by name in $\mathrm{B} 622$.
B 622 , p. 110
B 745, p. $87,110-112$

Simms Co. See Wiley.

Siwash Jack. See Nikolai.

Skolai Min. Co. See (White R., headwaters), (Wiley Cr.).

Skyscraper - $(1.55-1.65,12.1-12.25)$; COPPER. See also Davy, Eleanor, (Roaring $\mathrm{Cr}$.).
P 15, p. 20
B 374, p. 57
P. 41 , p. 96
B. 622 , p. $110-111$
B 345 , p. 139
B 745 , p. $88-89,106-107$

Slide. See Houghton Alaska Exp. Co. (McCarthy Cr.).

Snow Bird - $(12.45,7.05)$; COPPER.
B 662 , p. $176-177$
B $947-F$; p. 120

Snowshoe. See Skyscraper.

Spruce. See Nelson (copper).

(Standard Cr.) - (4.55-5.1, 0.05-0.1) ; GOLD (?).

P 41, p. 120

(Strelna Cr.) - (0.0, 11.0) approx ; COPPER.
P 15, p. 27
B 345 , p. 155
P 41, p. 103

(Summit Cr.) - (NE1/4 4, 0) (?) ; GOLD(?).

P 15, p. 62

P 41, p. 120

Sunrise. See (Fall Cr.).

Sunset. See (Fall Cr.).

(Sunshine Cr.). See (Surprise Cr.).

Surprise. See Nikolai.

(Surprise Cr.) - (1.5, 12.9) approx ; COPPER, TIN(?). Includes references to Hubbard, Laddie, Sheehan.
P 41, p. 123
B 345 , p. $141-142$
B 374, p. $59-60$

B 442 , p. 161

B 622 , p. 110

B 745, p. $89,108-110$

Tjosevig - $(10.3,8.85)$; COPPER.

B 947-F, p. 104-105

Tjosevig Bros. See (Hidden Cr.).

Triassic. See Radovan.

True Blue. See (Surprise Cr.).

United (Alaska) Copper Exp. Co. See Bear Paw, Big Horn, (Contact Gulch), Golden Eagle, (Porcupine Cr.), Snow Bird, Westover.

United Verde. See (Lime Cr.) (trib. Rock Cr.).

Valdez. See (Mineral Cr.), (Nugget Cr.). Notz.-Two separate groups of claims.

Valdez Exp. Co. See (Hidden Cr.).

War Eagle - $(1.95,9.75)$; IRON, COPPER. Includes references to (MacDougall Cr.).
B 622 , p. 114
B 662 , p. 160
B 714 , p. 192

B 745, p. 137-139

B 755 , p. $65-66$

B 947-G, p. 139-140

Warner - $(0.25,12.3)$; COPPER.

P 15, p. 18, 20

B 374, p. 55

P 41, p. 94, 96

B 745, p. 104-105

B 345, p. 138

$593018-61-13$ 
Warner \& Kain. See (Dan Cr.).

West Skyscraper. See Skyscraper.

Westover - $(12.5 ; 7.0)$; COPPER.
B 345, p. 169
B 542, p. 84
B 374, p. 92
B 622, p. 44,115
B 442, p. 161
B 662, p. 175-177
B 448, p. $83,95-97$
B 712, p. 31
B 520, p. 106
B $947-F$, p. $98,108-110,119-120$

Whistler. See Big Horn.

(White Cr.) - (11.9-12.45, 4.9-5.2); GOLD, SILVER, COPPER. See also

(Chititu Cr.).
B 225, p. 47
B 448, p. 103-107
B 622, p. 115, 117
B 714, p. 196

White Dog. See (Peacock Cr.).

(White R., headwaters) - (17.9-17.95, 12.4-12.55) ; COPPER. B 417, p. 57

B 630, p. 122-123

(White R., Middle Fork) - $(17.25,12.95)$ approx ; COPPER( ?).

B 542, p. 40

B 630, p. 122

Wiley - $(24.3,17.45)$; GOLD. Includes references to (Beaver Cr.).

B 379, p. 177

B 630 , p. 90,118

B 417 , p. 59

B 933-B, p. 163-164

B 622, p. 224

(Wiley Cr.) - $(18.8,12.15)$; COPPER.

B 630, p. 123

(Williams Peak) - $(12.55,6.35)$; GOLD, ANTIMONY.

B $880-A$, p. 87

B $880-$ B, p. 98

Wonder. See Nikolai.

Woodin \& Herman - (7.05, 9.1) approx; COPPER. Includes references to Bekka, Eli, (Fourth of July Cr.) (copper).

B 345, p. 160-161

B 374, p. 79-80

B 662, p. 163

B 947-F, p. 98, 118-119

Yellow Band (Gold Min. Co.) - $(4.9,0.5)$; GOLD. Includes references to Sheriff. B 880-B, p. 101-102

B 926-A, p. 27

B 910-A, p. 32

B 933-A, p. 25-26

B 917-A, p. 30-31

Young \& Pierson. See Pierson.

(Young Cr.) - (11.45-13.2, 4.0-4.35) ; GOLD, COPPER.

P 15, p. 59-61

B 448, p. 98, 107-108

B 622, p. 115

B 520, p. 107

B 755, p. 27-28

B 542, p. 84

B 947-F, p. 98

\section{MCGRATH QUADRANGLE}

Blackburn \& Eldridge. See (Candle Cr.).

(Candle Cr.) - (1.15-1.7, 15.1-15.8) ; GOLD, MERCURY, TUNGSTEN.

B 592, p. 70

B 622, p. 67, 257, 261

B 642 , p. 238, 251-252, 265

B 714, p. 39, 67, 93

B 655, p. 131

B 722, p. 11, 60

B 739, p. 9, 42, 157

B 662, p. 60

B 754, p. 107-108

B 692, p. 40

B 755, p. 14, 47

B 712, p. 50

B 773, p. 27, 48

B 783, p. 15, 18 
(Candle Cr.)-Continued
B 792, p. 20, 25
B 880-A, p. 48
B 797, p. 24-25, 30
B 897-A, p. 57
B 810, p. 31
B 910-A, p. 60
B 813, p. 36-37
B 917-A, p. 58-59
B 824, p. 42
B 836, p. 43
B 926-A, p. 56
B 844-A, p. 43
B 933-A, p. 52
B 857-A, p. 40-41
C 255, p. 16, 18
B 864-A, p. 45
BMB 153, p. 55
RI 4065, p. 5
B 864-C, p. 197-198
TDM 1, p. 25-26

B 868-A, p. 46

Kuskokwim Dred. Co. See (Candle Cr.).

Red Devil. See (Candle Cr.).

(Roundabout Mtn.) - $(2-3,15)$; COPPER, NICKEL.

B 714, p. 93

Strandberg \& Sons, Inc. See (Candle Cr.).

(Vinasale) - (1-2, 11-12); GOLD.

B 864-A, p. 45

\section{MEDFRA QUADRANGLE}

Alaska Treadwell (Gold Min. Co.) See Nixon Fork, Whalen.

(Adler Cr.) or (Gulch) - $(9,3)$; GOLD.

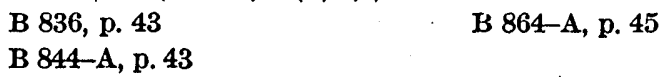

B 836, p. 43

B 844-A, p. 43

B 864-A, p. 45

(Birch Gulch) or (Cr.) - (9.75-9.8, 3.85-4.0) ; GOLD BISMUTH.

B 783, p. 135, 137 B 864-A, p. 34

B 797, p. 25 B 864-C, p. 195-196

B 810, p. 31

(Boulder Cr.) - (10.55-10.9, 6.6-6.85) approx ; GOLD(?). B 783, p. 141

(Canyon Cr.) - (12.7-13.4, 7.6-7.85) ; GOLD(?).

B 783, p. 141

(Clearwater Cr.) - (8.8-9.0); GOLD(?).

B 783, p. 141

Clow \& Strand. See (Crooked Cr.).

(Colorado Cr.) $\because(0.9-10.0)$; GOLD.
B 542, p. 296
B 836, p. 35
B 578, p. 35, 38
B 864-C, p. 172-173
B 592, p. 70
B 880-A, p. 42
B 642, p. 249,265
B 926-A, p. 46
B 754, p. 101
B 933-A, p. 42

Cripple Cr. Min. Co. See (Colorado Cr.).

(Crooked Cr.) - $(8,2)$; GOLD, ASBESTOS ( ?).
B 622, p. 67
B 783, p. 139

Crystal. Sce Nixon Fork.

(Crystal Gulch) - $(9.6,4.25)$; GOLD, BISMUTH.
B 783, p. 138
B 864-C, p. 196

(Eagle Cr.) - (S 1/2 8, 2) ; GOLD, TUNGSTEN.
B 797, p. 25
B 844-A, p. 43
B 810, p. 31
B 864-A, p. 45
B 813, p. $36-37$
B 864-C, p. 197
B 824, p. 42
C 279, p. 16
B 836, p. 43 
Eakin. See Nixon Fork.

Garnet. See Nixon Fork.

Griffin \& Whalen. See (Holmes Gulch).

Groble \& Blackburn. See (Hidden Cr.).

Groshong. See (Birch Gulch).

Hard, Uotila \& Hanson. See (Colorado Cr.).

(Hidden Cr.) - $(9.8-9.9,3.85-4.0)$; GOLD, BISMUTH, TUNGSTEN. Includes references to Matthews \& Blackburn.

$\begin{array}{ll}\text { B } 722, \text { p. 60, 159, 161 } & \text { B 864-A, p. 45 } \\ \text { B 783, p. 15, 127, 135-137, 139 } & \text { B 864-C, p. 193-195, 235 } \\ \text { B 797, p. 25 } & \text { B 868-A, p. 46 } \\ \text { B 810, p. 31 } & \text { B 880-A, p. 48-49 } \\ \text { B 813, p. 36-37 } & \text { B 897-A, p. 57 } \\ \text { B 824, p. 42 } & \text { B 910-A, p. 60 } \\ \text { B } 836, \text { p. 43 } & \text { B 917-A, p. 59 } \\ \text { B 844-A, p. 43 } & \text { C 279, p. } 12\end{array}$

High Grade. See Nixon Fork.

(Holmes Gulch) or (Cr.) - (9.6-9.7, 3.9-4.0) ; GOLD, BISMUTH.

B 722, p. 161

B 783, p. 15, 128, 137

B 864-C, p. 196

B 813, p. 37

B 880-A, p. 49

B 824, p. 42

B 897-A, p. 57

B 836, p. 43

B 844-A, p. 43

B 910-A, p. 60

B 917-A, p. 59

B 926-A, p. 56

Jensen \& Matson. See (Holmes Gulch).

(Jones Cr.) - (9.8-10.2, 6.15-6.35) approx ; GOLD (?).

B 783, p. 141

Keen. See Nixon Fork.

McGowan \& Lind. See Nixon Fork.

McGowan \& Mespelt. See Nixon Fork.

Matthews (\& Blackburn). See (Hidden Cr.).

Mespelt \& Co. See Nixon Fork.

Mespelt Bros. See Nixon Fork.

Nixon. See Nixon Fork.

Nixon Fork (Min. Co.) - (9.65-9.7, 4.35-4.45) ; GOLD, SILVER, COPPER, BISMUTH. Includes references to Crystal, Garnet, High Grade, Keen, McGowan \& Lind, McGowan \& Mespelt, Mespelt \& Co., Mespelt Bros., Nixon, Recreation, Southern Cross, Texas, Twin, Winan \& McGowan and references to lodes owned or operated by Pearson \& Strand, Strand.

B 722, p. 59-60, 158-161

B 739, p. 42

B 754, p. 116

B 755, p. 47

B 773, p. 15

B 783, p. 127-128, 130-134

B 810 , p. $15-16$

B 813, p. 18

B 824, p. 20-22

B 836, p. 21

.B 844-A, p. 22
B 857-A, p. 19

B 864-A, p. 23

B 864-C, p. 229-241

B 868-A, p. 21-22

B 880-A, p. 27

B 897-A, p. 33

B 910-A, p. 28

B $917-A$, p. 29

B $926-$ A, p. 26

B 983-A, p. 26

C 279 , p. $10,12,16,18$ 
(Nixon Fork, headwaters) - (12-13, 8-9); GOLD(?). Nore-Near Von Frank Mtn.

B 783, p. 141

O'Malley \& Walden. See (Ruby Cr.).

(Our Cr.) - $(12.65,16.55)$; GOLD. B 642, p. 220

B 667, p. 51

Paulson (and assoc.). See (Colorado Cr.).

Pearson \& Strand. See (Crystal Gulch), Nixon Fork, (Ruby Cr.).

Recreation. See Nixon Fork.

(Riddle Gulch) - (9.8-9.9,4.0-4.2) ; GOLD.
B 824, p. 42
B 864-C, p. 194
B 836, p. 43
B 910-A, p. 60
B 844-A, p. 43
B 917-A, p. 59

(Ruby Cr.) - (9.5-9.7, 4.2-4.25) ; GOLD, TIN, TUNGSTEN, COPPER.

B 722, p. 60,161

B 836, p. 43

B 783, p. 127,138

B 844-A, p. 43

B 797, p. 25

B $864-C$, p. 196-197, 235

B 810, p. 31

B 897-A, p. 57

B 813, p. $36-37$

B 910-A, p. 60

B 824, p. 42

C 279, p. 12, 16, 19

Southern Cross. See Nixon Fork.

Stone - (S 1/2 8,2) ; GOLD. . See also (Eagle Cr.).

C 279 , p. 10,18

Strand. See Nixon Fork, (Ruby Cr.).

(Sunshine Mtns.) - (7-8, 8-9) ; GOLD(?).

B 783, p. 141

Texas. See Nixon Fork.

Treadwell Yukon Co. (Ltd.). See Nixon Fork, Whalen.

Twin. See Nixon Fork.

Whalen (\& Griffin) - $(9.7,4.1)$; GOLD, SILVER, BISMUTH, COPPER, TUNG-

ISTEN, RARE EARTHS, NICKEL(?). See also (Holmes Gulch).

B 714, p. 93

B 722, p. 59-60, 159-160

B 739, p. 42

B 755, p. 47

B 783, p. 9, 127-130, 134

B 792, p. 13

B 810, p. 16

B 824, p. 22
B 836, p. 21

B 844-A, p. 22

B 857-A, p. 19

B 864-A p. 23

B 864-C, p. 230-235, 237-238, 241-242

B $910-$ A, p. 28

C 279 , p. $10,12,18-19$

Whelan. See Whalen.

(Whirlwind Cr.) - (12.0-14.0, 7.6-8.7) ; GOLD(?).

B 783, p. 141

Winan \& McGowan. See Nixon Fork.

(Wyoming Cr.) - (0, 8-9) (?) ; MERCURY, ANTIMONY. NotE.-In Cripple

Cr. Mts.

B 642, p. 258

B 649 , p. 50

B $864-$ C, p. 228

B.754, p. 116

TDM 1, p. 26

(_-_- ) (gold) - (13.1, 14.12) ; GOLD. NoTE.-Unnamed trib. of Sulukna R. B 642, p. 220 B 667 , p. 51 
(Florence Bar) - (10, 15-16) (?) ; GOLD.
AR 21, p. 483
B 442, p. 297 .
P 20, p. 98
B 536, p. 142

(Illinois Cr.) - (21.0, 4.9) approx ; GOLD.
P 70, p. 184
B 535, p. 34

B 520, p. 281

(Mason Cr.) - (20.05-20.3, 4.1-4.7) ; GOLD, TIN.
P 70, p. 184
B 379, p. 237
B 824 , p. 40
B 410, p. 83
B 836 , p. 42
B 442, p. 44
B $844-$ A, p. 41
B 692 , p. 39
B $857-A$, p. 39
B 712, p. $22,47-48$
B $864-$ A, p. 43
TDM 1, p. 34

B 797, p. 23

(Moraine Cr.) - (19-22, 3-5) (?) ; GOLD. May be in Tanana quad. B 824, p. 40

(Moran Cr.) or (Gulch) - (18-19, 6) ( ?) ; GOLD, TIN.
B 712, p. 22,48
B 868-A, p. 45
B 836, p. 42,70
B 880-A; p. 47
B $844-$ A, p. 41
TDM 1, p. 34

\section{MIDDLETON ISLAND QUADRANGLE}

(Middleton I.) - (5.7-5.9, 7.15-7.2) ; GOLD.
B 542, p. 43
B 773, p. 23

\section{MISHEGUK MOUNTAIN QUADRANGLE}

(Kugururok R.) - (SW 1/4 SW1/4 quad.) ; CHROMITE. TDM 5-R, p. 18

\section{MOUNT FAIRWEATHER QUADRANGLE}

(Adams Inlet) - $(22.25,15.35)$; MÓLYBDENUM.

B 800, p. 330

IC 7844, p. 12

B 926-C, p. 178

Alaska Independence Min. Co. - $(18.6,6.15)($ ) ; GOLD ( ?). B 857-A, p. 15

B 864-A, p. 15

(Brady Glacier) - (NW $1 / 217,6)$; MOLYBDENUM. NoTE.-Float on glacier. B 800, p. 329-330 B 926-C, p. 177-178

Christmas. See (Lemesurier I.) (molybdenum).

Dodson. See Monarch.

Enterprise. See (Lemesurier I.) (molybdenum).

(Francis I.) - $(21.2,11.35)$; GOLD, SILVER, COPPER. B 783, p. 56

B 800, p. 323

Galena - $(15.2,15.15)$; GOLD, SILVER, ZINC, LEAD. B 963-A, p. 31, 33

B 1058-B, p. 37-38

(Geikie Inlet) - $(18,10)$; MOLXBDENUM. Includes references to Yehring. B 722, p. 24

B 926-C, p. 178

B 800, p. $329-330$

Highland Chief - $(15.0,14.9)$; GOLD.

B $1058-B$, p. $36-38,44,54-55,57$ 
Hopalong - (15.6, 15.2) approx ; GOLD.

B $1058-B$, p. 38,56

Ibach. See Galena, Incas, (Lemesurier I.) (asbestos), Monarch, Rainbow, Sentinel.

Ibach \& Beach. See Highland Chief.

Ibach \& Smith. See Galena, Rainbow.

Incas - $(15.2,15.3)$; GOLD.

B $1058-B$, p. $35,37-39,46-48$

Johnson \& Smith. See (Nunatak).

Koby. See (Geikie Inlet).

(Lemesurier I.) (asbestos) - $(22.5,8.2-8.45)$; ASBESTOS. IC 7313, p. 1-5

(Lemesurier I.) (molybdenum) - (22.15-22.25, 4.9-5.0); MOLYBDENUM. B 662, p. 25

B 666, p. 98

B 800 , p. $329-330,340$

B 714, p. 41

B 926-C, p. 176-177

B 783, p. 26, 55-56

IC 7844, p. 12

LeRoy - $(14.7,15.5)$; GOLD, ZINC, LEAD.

B 933-A, p. 17

B $1058-B$, p. $37-39,42,45-46$

B 963-A, p. 32-34

LeRoy Min. Co. See Incas, LeRoy, Rainbow.

Lincoln. See LeRoy.

(Lituya Bay) - (5.1-9.7, 9.5-13.9) ; GOLD, PLATINUM.

$\begin{array}{ll}\text { B 225, p. 46 } & \text { B 836, p. 133-136 } \\ \text { B 314, p. 64-65 } & \text { B 864-A, p. 29 } \\ \text { B 662, p. 23, 41-42 } & \text { B 868-A, p. 30 } \\ \text { B 666, p. 96 } & \text { B 880-A, p. 34 } \\ \text { B 712, p. 28 } & \text { B 897-A, p. 40 } \\ \text { B 714, p. 76 } & \text { B 910-A, p. 38 } \\ \text { B 722, p. 34 } & \text { B 917-A, p. 36-37 } \\ \text { B 739, p. 23 } & \text { B 926-A. p. 33 } \\ \text { B 755, p. 25 } & \text { B 933-A, p. 32-33 } \\ \text { B 773, p. 23 } & \text { B 947-D, p. 71 }\end{array}$

Lituya Bay Gold Min. Co. See (Lituya Bay).

Manville \& Smith. See (Francis I.).

(Miner I.) - $(20.1,0.5)$; GOLD.

B 800 , p. 378

Monarch - (15.1, 15.2) ; GOLD, LEAD.

B 1058-B, p. 37, 39, 48, 50-52

Mt. Fairweather Min. Co. See Monarch.

Mt. Parker Min. Co. See LeRoy.

(Muir Inlet). See (Nunatak).

Newmont Min. Co. See Highland Chief, Rambler.

(North Marble I.) - $(22.25,12.2)$; MARBLE.

B 592 , p. 100

B 682, p. 43-44, 114

(Nunatak) - (21.65-21.8, 17.55-17.7); MOLYBDENUM, COPPER. Includes reference to (Muir Inlet).
B 926-C, p. 178-180
IC 7844, p. 12

B 947-B, p. 9-18

RI 4421, p. 1-6

O.K. See (Nunatak).

Parker, A.F. - $(14.6,15.62)$; GOLD.

I 963-A, p. 33-34 
Parker, A. L. - See LeRoy.

(Ptarmigan Cr.) - $(14.8,15.3)$; ZINC. B 1058-B, p. 55-56

Rainbow - (15.05-15.15, 15.3-15.55) ; GOLD, SILVER, ZINC.

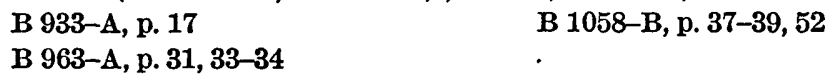

Rambler - $(14.7,14.9)$; GOLD, LEAD, ZINC. B 1058-B, p. 35-36, 38, 44, 55

Sentinel - $(15.2,15.5)$; GOLD, LEAD. B 1058-B, p. 37-38, 54

(South Marble I.) - $(12.45,11.7)$; MARBLE. B 592, p. 100 B 682, p. $43-44,114$

Sunrise - $(15.4,15.2)$ approx ; GOLD (?). B 1058-B, p. 38

(Surge Bay) - $(18.2,0.55)$ approx ; COPPER, NICKEL. B 773, p. 95 B 800 , p. 373

Triton. See (Nunatak).

Vevelstad, C. See (Nunatak).

Vevelstad, S. H. P. See (Surge Bay).

Vevelstad \& Comstock. See (Nunatak).

Whirlaway - (15.6, 15.2) approx; GOLD. B 1058-B, p. 38, 56

Whitney. See (Lemesurier I.) (molybdenum). (Willoughby Cove). See (Lemesurier I.) (molybdenum). (Willoughby I.) - $(22.0,10.7)$; MARBLE. B 592, p. 100

(Yakobi I.) - $(20.05,0.55)$; GOLD. B 692, p. 121 B 682, p. $43-45,114$

Yehring. See (Geikie Inlet).

(_-_--) (gold, tungsten) - (15.5, 15.3) approx; GOLD, TUNGSTEN(?). B 1058-B, p. 56

\section{MOUNT HAYFS QUADRANGLE}

Acme Min. Co. See (Chisna R.).

Ahlgren. See (Miller Gulch).

Akerman \& Todd. See (Miller Gulch).

Alaska Middle Fork Min. Co. See (Chistochina R., Middle Fork).

Albertson \& Pettijohn. See Kathleen-Margaret.

Algren and assoc. See (Miller Gulch), (Slate Cr.).

(Bedrock Cr.). See (Chistochina R., Middle Fork).

Big Four. See (Big Four Cr.).

(Big Four Cr.) - (17.5-17.65, 3.6-3.75) ; GOLD. Includes references to Big Four.

P 15, p. 54

P 41, p. 112

B 213, p. 74

B 498, p. 70, 74-75

(Black Rapids) - $(9.05,9.05)$; ANTIMONY.

B 989-D, p. 207-208 , $\quad$ RI 4173, p. 5, 35-36

Blix \& Torgerson. See (Rainy Cr.).

California. See (Slate Cr.).
B 692, p. 140-141

B 943-B, p. 34

B 989-D, p. 193-194 
(Chisna R.) - (17.45-17.95, 1.45-2.9) ; GOLD. .

P 15, p. 49, 56

P 41, p. 113-114

B 213, p. 71-74

B 379, p. 157

B 442, p. 42

B 480 , p. $126-127$

B 498 , p. 56, 70, 76-77
B 520, p. 37

B 542, p. 43

B 692, p. 137

B 943-B, p. 29-31

B 989-D, p. 191-192

BMB 153, p. 33-34

(Chistochina R., Middle Fork) - (18.75, 2.7) ; GOLD, PLATINUM, ISILVER, COPPER, CHROMITE, LEAD. ' Includes references to (Bedrock Cr.), (Kraemer Cr.), (Lake Cr.), (Lime Cr.), (Limestone Cr.).

B 379, p. 157 .

B 480 , p. 126

B 498 , p. 78

B 692, p. 137

B 755, p. 28

B 810, p. 22

B 857-A, p. 26-27
B:864-A, p. $30 \cdots$

B 880-B, p. 105-106

B 897-A, p. 41

B 910-A, p. 39

B 917-A, p. 38

B $943-B$, p. $29,34-40$

B 989-D, p. 191, 194

(Chistochina R., W. Fork). See (Slate Cr.). Note.-By context, reference (B 662, p. 43) is actually to (Slate Cr.).

Cleveland. See (Chistochina R., Middle Fork).

Coles, Jacobson, Kramer \& Levell. See (Miller Gulch), (Slate Cr.).

Conradt and assoc. See (Ptarmigan Cr.).

(Daisy Cr.) - (16.6-17.4, 0.7-1.4) ; GOLD.

B 379 , p. 157

(Delta R.) - $(9.35,3.8$ ) approx; GOLD. Note, -3 miles above Eureka Cr.

B 498, p. 65

Dempsey. See (Chisna R.).

(Dempsey) - $(17.25,1.3)$; GOLD.

B 480, p. 127

B 498 , p. 77

DeWitt. See (Ober Cr.).

(Dry Delta R.). See (Ptarmigan Cr.).

Elmer. See (Slate Cr.).

Elmer, Walker \& Watkins. See (Big Four Cr.), (Slate Cr.).

(Eureka Cr.) - (6.0-9.4, 4.45-5.2) ; GOLD.

B 498 , p. $65-66$

Gillespie. See (Ptarmigan Cr.).

Goldfleld Consol. Min. Co. See (Slate Cr.).

Gull. See (Ptarmigan Cr.).

(Gunnysack Cr.) - (9.1, 9.25) ; GOLD, ANTIMONY.

B 501, p. 54

B 926-B, p. 144

TDM 1, p. 12

Hazelet (\& Meals). See (Chisna R.).

(Hidden Treasure Cr.) - (17:0-19.0, 2.7-3.6) (?) ; GOLD ( ?). B 622, p. 45

Holmes \& Brail. See (Chisna R.).

Horn. See (Chisna R.).

Jackpot. See (Ruby Gulch).

(Jackpot Cr.). See (Ruby Gulch).

(Jarvis Cr.) - (9.5-11.3, 12.1-12.25) (?) ; GOLD(?). See also (McCumber Cr.)

(placer), (Morningstar Cr.), (Ober Cr.).

B 797, p. 20

C 331, p. 18 
Jasper. See (Chistochina R., Middle Fork).

Kathleen-Margaret - $(3.3,4.9)$; COPPER, GOLD, SILVER. C 332, p. 1-5

RI 5245, p. 1, 9

(Kenyon Cr.) - (17-18, 16-17).(?) ; GOLD. May be in Big Delta quad. B 622, p. 50-51 B 642, p. 67

(Kraemer Cr.). See (Chistochina R., Middle Fork).

Kreamer, Leavell \& Hemple. . See (Slate Cr.).

(Lake Cr.). See (Chistochina R., Middle Fork).

(Lime Cr.). See (Chistochina R., Middle Fork).

Limestone. See (Chistochina R., Middle Fork).

(Limestone Cr.). See (Chistochina R., Middle Fork).

(McCumber Cr.) (lode) - (11.5-13.0, 11.8-12.5) approx; LEAD. B 926-B, p. 144

(McCumber Cr.) (placer) - (11.0-11.3, 12.4-12.55) approx; GOLD. B 542, p. 45

B 824, p. 34 B 926-B, p. 143-144

B 836, p. 34

C 331, p. 18

McDowell. See (Slate Cr.).

(McLaren R.) - (NE1/4 3,4) ; COPPER. B 712, p. 20 B 714, p. 35

(Macomber Cr.). See (McCumber Cr.). Old spelling. M. E. W. Gold Min. Co. See (Big Four Cr.), (Slate Cr.). Meyer \& Prolig. See (Slate Cr.).

Middle Fork Min. Co. See (Chistochina R., Middle Fork).

Miller. See (Ober Cr.), (Slate Cr.). Note May be two Millers.

(Miller Gulch) or (Cr.) - (17.4-17.5, 3.3-3.5) ; GOLD, PLATINUM, MERCURY, COPPER. See also (Slate Cr.).

P 15, p. 49, 52-53

P 41, p. 110-111, 123

B 213, p. 71-73

B 263, p. 207

B 379, p. 157

B 442, p. 42

B 480, p. 124-125, 127

B 662, p. 22

B 692, p. 138,140

B 714, p. 38

B 857-A, p. 27

B 917-A, p. 38

B 926-A, p. 34

B 498, p. 56, 70-73

B 943-B, p. 27, 29, 33

B 520, p. 37

B 989-D, p. 191-193.

B 642, p. 54

BMB 153, p. 33

(Morningstar Cr.) - (11.2-11.3, 12.1-12.4) approx ; GOLD. B 824, p. 34

B 836, p. 34

(Ober Cr.) - (9.55-9.6, 12.7) ; GOLD.

B 926-B, p. 143

C 331, p. 18

B 989-D, p. 190, 196

(Paxson's) - (11.55, 0.9) approx; COPPER.

B 712, p. 20

(Pegmatite Cr.) - (15.6-16.3, 10.55-11.55) ; GOLD.

B 926-B, p. 143-144

Petrokov. See (Black Rapids).

(Ptarmigan Cr.) - (3.5-3.8, 13.85-14.1) ; MOLYBDENUM, GOL1). Includes references to (Dry Delta R.).
B 692, p. 23
B 712, p. 24, 44
B 714, p. 41 
(Rainy Cr.) - (8.0-9.5; 4.9-6.0); GOLD, COPPER. Includes references to (Wilder Cr.).

P 15, p. 59

B 712, p. 20, 44

P 41, p. 117

B 810, p. 26

B 498 , p. $65-66$

B 824, p. 34

B 662, p. $43-44$

(Redemption Cr.) - (N $1 / 2$ quad) (?) ; GOLD(?). May be in Big Delta quad. B 797, p. 20

(Ruby Gulch) or (Cr.) - (17.85-17.95, 3.1-3.25); GOLD. Includes reference to (Jackpot Cr.).

P 15, p. 55

P 41, p. 113

B 622 , p. 50

B 213 , p. 73-74

B 662, p. 43

B 480 , p. $125-126$

B 692 , p. 137

B 498, p. 70,75

B 989-D, p. 191-192

(Savage Cr.) or (Gulch) - (9-11, 10-13) (?) ; GOLD.
B 755, p. 38
B 824, p. 34
B 797, p. 20
B 836, p. 34

B 810, p. 26

Schroeder. See (Slate Cr.).

(Slate Cr.) - (17.05-17.6, 3.3-3.45) ; GOLD, PLATINUM. Includes references to Slate Cr. Placers, Inc. ; see also (Miller Gulch).
P 15, p. 49, 53-54
B 783, p. 12, 25
P 41, p. 111-112
B 792, p. 33
B 213, p. 71-73
B 225, p. 47
B 263, p. 207
B 797, p. 16, 40
B 379, p. 157
B 810, p. 22
B 813, p. 24
B 442, p. 42
B 824, p. 28
B 480, p. 124-125
B 836, p. 28
B 498 , p. 56, 70-74
B 844-A, p. 28
B 520, p. 37
B 857-A, p. 26-27
B 542, p. 43
B 864-A, p. 30
B 622, p. 45
B 868-A, p. 31
B 642, p. 54
B $880-A$, p. 35
B 662, p. 21-22, 43
B $880-$ B, p. 105
B 666, p. 96
B 897-A, p. 41
B 692, p. 21, 30-31, 137-141
B 910-A, p. 39-40
B 917-A, p. 38
B 712, p. 23
B 714, p. 38, 71
B 926-A, p. 34
B 933-A, p. 33-34
B 722, p. 23, 39
B 943-B, p. 29, 31-33
B 739, p. 13,23
B 989-D, p. 191-193
B 755, p. 28
BMB 153, p. 33
B 773, p. 30
TDM 1, p. 20

Slate Cr. Min. Co. See (Slate Cr.).

Slate Or. Placers, Inc. See (Slate Cr.).

Sundt. See (Slate Cr.).

Tacoma. See (Slate Cr.).

(Treasure Gulch) - (17.0-19.0, 2.7-3.6) (?); GOLD, PLATINUM. See also

(Hidden Treasure $\mathrm{Cr}$.).

B 933-A, p. 75 
Yukon Corp. - $(9.85,5.85)$; GOLD.

C 331, p. 18

(Wilder Cr.). See (Rainy Cr.).

\section{MOUNT. KATMAI QUADRANGLE}

(Geographic Harbor) - (12.6-13.3, 1.8-2.45) ; PUMICE, CEMENT MATERIALS. B 1039-C, p. 34 RI 4932, p. 76-77, 84-85

(Hidden Harbor). See (Kinak Bay).

(Kinak Bay) - (13.7-14.6, 3.3-3.8) ; PUMICE.

RI 4932 , p. $75-76$

(Kukak Bay) - (14.4-18.0, 4.4-6.55) ; COPPER, PUMICE.
B 712, p. 35
RI 4932 , p. 78

Pumice Building Block Co. See (Geographic Harbor).

Shelikof Min. Co. See (Kukak Bay).

Stock \& Grove. See (Geographic Harbor).

(Takli I.) - (13.7-14.3, 1.1-1.5) ; PUMICE.

RI 4932 , p. 77-78

\section{MOUNT MCKINLEY QUADRANGLE}

Aitken. See (Mt. Eielson), (Quigley Hill).

Alice. See (Quigley Hill).

Alpha - $(15.55,9.4)$; SILVER, LEAD, GOLD, ZINC, ANTIMONY.

B 773, p. 29

B 849-F, p. 354, 358, 375-376

B 792, p. 30

Anderson. See (Mt. Eielson), (Thorofare Cr.).

Antimony. See Taylor (antimony).

Apex. See (Quigley Hill).

Arkansas - $(17.35,10.1)$ approx ; SILVER, LEAD, ANTIMONY, ZINC.

B 849-F, p. 374

Azurite. See (Quigley Hill).

Banjo. See (Quigley Hill).

Bear. See (Mt. Eielson).

(Bearpaw R.) or (Cr.) - (15-17, 9-11) ; GOLD. See also (Caribou Cr.),

(Crevice Cr.), (Friday Cr.), (Eureka Cr.), (Glacier Cr.), (Twenty-two Pup), (Yellow Cr.).

B 284, p. 125

B 520, p. 38

Biglow \& Perry. See (Mt. Eielson).

Blue Bell. See (Quigley Hill).

Bosart. See (Quigley Hill).

Bright Light - $(15.6,9.6)$; LEAD ( ?).

B 836, p. 332

Busia. See Neversweat.

Caribou (Caribou Cr.) - $(17.15,10.95)$; ANTIMONY, SILVER, GOLD.

Notz.-Not mentioned by name in P 70, B 314, 649 .

P 70, p. 178-179

B 314, p. 219

B 662 , p. 326-327

B 649, p. 42

B 687, p. 108-109

B $849-$ F, p. $353,377-378$

Caribou (Mt. Eielson). See (Mt. Eielson).

Caribou (Quigley Hill). See (Quigley Hill). 
(Caribou Cr.) - (17.1-17.4, 10.9-10.95) ; GOLD, TUNGSTEN, ANTIMONY.
P 70, p. 178-180
B 797, p. 23
B 314, p. 30, 218-219
B 864-A, p. 43
B 649, p. 42
B 880-A, p. 46
B 662, p. 309-310
B 910-A, p. 58
B 687 , p. 92-93
B $917-\mathrm{A}$, p. 57
B 739 , p. 37
B 755, p. 41-42, 141-142
B 926-A, p. 51-52
B 933-A, p. 49

Caribou Mines Co. See (Caribou Cr.).

Carlson \& Averil - (20.0, 6.0) approx.; LEAD, SILVER, COPPER. B 792, p. 108

Carrie. See (Mt. Eielson).

Christenia. See (Mt. Eielson).

(Clearwater Cr.) - (16-19, 6) (?) ; GOLD (?). B 933-A, p. 49

Cleary. See Highway, (Mt. Eielson).

Copper Base. See (Mt. Eielson).

Copper Lode - $(16.95,5.3)$ approx ; COPPER.

B 836, p. 322

(Copper Mtn.). See (Mt. Eielson).

(Crevice Cr.) - (17.35, 10.95) ; GOLD.

B 314, p. 218

(Crooked Cr.) - (19.0-21.2, 13.9-15.55) ; GOLD.
B 797, p. 23
B $910-A$, p. 58
B 864-A, p. 43
B 917-A, p. :
B $897-A$, p. 56
B 933-A, p: 49

Dalton. See (Quigley Hill).

Dalton \& Stiles. See (Eureka Cr).

Damon \& Pythias. See (Quigley Hill).

Darling. See (Quigley Hill).

Dee. See (Mt. Eielson).

Denver. See (Mt. Eielson) .

Doherty. See (Quigley Hill).

Drayton \& Trundy. See Stampede.

Dunkel. See (Caribou Cr.), (Slippery Cr.).

(Fldorado Cr.) - (15.55-15.95, 9.1-9.5) ; GOLD.

B 662 , p. 305-306

B 687, p. 88

Eureka (lode). See (Quigley Hill).

Eureka (placer). See (Eureka Cr.).

(Eureka Cr.) - (16.0-16.65, 9.5-9.9) ; GOLD, ANTIMONY, LEAD.
P 70, p. 176-177, 179
B 314, p. 30, 215-217, 220
B 520, p. 38
B 649, p. $42-43$
B 662, p. 302-304
B 687, p. $85-87$
B 722, p. 52
B 739, p. 36
B 755 , p. $41,141-142$
B 797, p. 23
B 813, p. 35
B 824 , p. 40

B 836, p. 41, 334-335

B 844-A, p. 41

B $849-F$, p. 353,376

B 857-A, p. 39

B 864-A, p. 43

B 868-A, p. 44

B $880-A$, p. 29,46

B $897-A$, p. 56

B $910-$ A, p. 58

B 917-A, p. 57

B 926-A, p. 52

B 933-A, p. 48-49 
Ferrara. See Alpha.

Florence Lode. See (Quigley Hill).

Francis. See (Quigley Hill).

Fransen \& Hawkins. See (Quigley Hill).

Friday. See (Quigley Hill).

(Friday Cr.) - (15.8-16.05, 9.7-10.0) ; GOLD, LEAD, ANTIMONY.
P 70, p. 178-180
B 314, p. 30, 217
B 687, p. $87-88$
B 649, p. $42-43$
B 755 , p. 141-142
B 662, p. 304-305
B 792, p. 106
B $880-$ A, p. 29

Galena. See (Quigley Hill).

Galena Lode - $(16.95,5.3)$ approx ; LEAD, ZINC.

B 836 , p. $322-323$

Georgia. See (Mt. Eielson).

(Glacier Cr.) - (15.9-16.9, 10.3-11.3) ; GOLD, LEAD.
P 70, p. 178-180
B 813 , p. 35
B 314, p. 218, 220
B 836, p. 335
B 662 , p. $307-309$
B $849-$ F, p. 353,371
B 687 , p. $90-92$
B 868-A, p. 44
B 739 , p. 37
B 755 , p. $41,141-142$
B $910-A$, p. 58

B 797, p. 23

Glen - $(17.35,10.2)$; GOLD, LEAD, ZINC, ANTIMONY.

B 662 , p. 318

B 836, p. 334

B 687, p. 100

B $849-F$, p. 373

(Glen Cr.) - (17.6-18.05, 9.7-10.1) ; GOLD, LEAD, MANGANESE.

P 70, p. 176

B 824 , p. 40

B 314, p. 215

B 836, p. 41,335

B 520, p. 38

B 844-A, p. 41

B 662 , p. $300-302$

B 857-A, p. 39

B 687 , p. $83-85$

B 864-A, p. 43

B 722 , p. 52

B $868-A$, p. 44

B 755 , p. $41-42,141-142$

B 910-A, p. 58

B 797, p. 23

B 917-A, p. 57

B 813 , p. 35

(Glen Cr. divide) - (17.25-17.3, 10.0-10.1) approx ; LEAD.

B $849-$ F, p. 373

Glen Ridge No. 1 - $(17.5,10.25)$; GOLD, LEAD. Includes references to Skookona.

B 662 , p. $317-318$

B 687 , p. 100

B 836 , p. $333-334$

B $849-$ F, p. 373

Gold Dollar. See (Quigley Hill).

Gold King. See (Quigley Hill).

Golden Eagle. See (Quigley Hill).

Grant. See (Mt. Eielson), (Quigley Hill).

Grant \& Henderson. See (Mt. Eielson).

Grant \& Jiles. See (Mt. Eielson).

Grant \& McGarvey. See (Mt. Eielson).

Grant, Anderson \& McGarvey. See (Mt. Eielson).

Greenback - $(14.9,3.8)$ approx.; ZINC, COPPER, LEAD.

B 836 , p. 319-320.

Greiss. See (Quigley Hịll). 
Guggenheim. See (Mt. Eielson).

Haney. See (Quigley Hill).

Highlander. See (Mt. Eielson).

Highway - $(21.7,7.2)$; ZINC.

B 849-D, p. 284

Home Lode - $(17.55,10.85)$; ANTIMONY.

B 849-F, p. 377

Hugh John. See (Mt. Eielson).

Humboldt - $(17.6,10.4)$; GOLD, LEAD ZINC. Includes reference to Stendall.

B 662, p. 317

B 836, p. 333

B 687, p. 99

B 849-F, p. 374

Isobel. See (Mt. Eielson).

Jiles. See (Mt. Eielson).

Julian. See (Home Lode).

Jumbo. See (Quigley Hill).

Kantishna Hyd. Min. Co. See (Moose Cr.).

Kantishna Min. Co. See (Clearwater Cr.).

Kelly. See (Mt. Eielson).

Keystone. See (Quigley Hill).

Kirk, Morris P., \& Sons., Inc. See Stampede.

Lena - $(17.9,10.5)$; SILVER, GOLD, LEAD.

B 836, p. 333

B 849-F, p. 375

Leposky. See Stampede.

Lillian. See (Mt. Eielson).

Little Annie. See (Quigley Hill).

Little Maud. See (Quigley Hill).

(Little Moose Cr.) - (19.95-20.2, 13.65-13.95) ; GOLD, TUNGSTEN.

B 662, p. 310-311

B 687 , p. 93

B 722, p. 52

B 824, p. 40

B 739, p. 37

B 836, p. 41, 335

B 755, p. 41

B 797, p. 23

B 813, p. 35

Lloyd - $(17.7,10.1)$ approx ; GOLD, ZINC, COPPER.

B 662, p. 317

B 849-F, p. 353

B 687, p. 99

Lloyd and assoc. See Glen, Lloyd.

Lucky Strike. See (Quigley Hill).

McGarvey. See (Mt. Eielson).

McGonogill - $(17.2,10.2)$; GOLD, SILVER, LEAD, ANTIMONY, COPPER.

B 662, p. 318

B 849-F, p. 372

B 687, p. 100

McGonogill, C. See McGonogill, (Quigley Hill).

MacKenzie. See (Mt. Dielson).

(McKinley Fork) - (15-19, 5-7) (?) ; GOLD.

B 520, p. 38

Magnet - (15.0, 3.9) approx; ZINC, LEAD.

B 836, p. 320

Malachite. See (Quigley Hill). 
Mammoth. See North Star.

Marjorie. See (Mt. Eielson).

(Marten Cr.) - (19.5-20.05, 14.6-15.0) ; GOLD. Includes reference to (Martin Cr.).

B 824, p. 40

B 910-A, p. 58

Martha Q. See (Quigley Hill).

(Martin Cr.) See (Marten Cr.)

Mary. See (Mt. Eielson).

Matheson. See (Mt. Eielson).

Maurer. See Taylor (antimony).

Mehling \& Maurer. See (Caribou Cr.).

Merinser - (14.5, 3.5) approx; ANTIMONY, MERCURY. See also (Slippery Cr.).

B 836 , p. $80-81,313-314,321-322$

Merry Widow. See (Quigley Hill).

(Moose Cr.) - (15.35-16.0, 9.5-10.1) ; GOLD.

B 284, p. 125

B 314, p. 217

B 520, p. 38

B 662 , p. 306

B 687 , p. 88-89

B 722, p. 52

B 739, p. 37

B 755, p. 41, 141-142

B 797, p. 23

B 910-A, p. 58

B 917-A, p. 57

B 926-A, p. 52

(Mt. Eielson) - (20.85-21.35, 7.3-7.55) ; ZINC, LEAD, SILVER, COPPER, GOLD. Includes references to (Copper Mtn.).
B 739, p. 36-37
B 755, p. $42-43$
B 849-D, p. 270-284
B 792, p. 107
C 252, p. 6
B 836, p. 314-319
RI 4121, p. 1-13

Mt. McKinley Gold Placers, Inc. See (Caribou Cr.), (Glacier Cr.).

Neversweat - $(15.55,9.1)$; SILVER, GOLD, LEAD, COPPER.

B 849-F, p. 376

North Star - $(17.9,10.6)$ approx ; LEAD, ZINC. Includes reference to Mammoth.

B 662, p. 316

B 849-F, p. 375

B 687 , p. 99

Old Sourdough - (15.1, 3.95) approx ; ZINC, COPPER, LEAD.

B 836, p. $320-321$

Owen. See (Mt. Eielson).

Pennsylvania. See (Quigley Hill).

Pension - (17.35, 10.1) approx; SILVER(?).

B 849-F, p. 374

Pilgrim. See (Little Moose Cr.), Stampede.

Pioneer. See Caribou (Caribou Cr.).

Pittsburgh. See (Quigley Hill).

Polly Wonder. See (Quigley Hill).

Question Mark - $(14.75,3.7)$ approx. ; COPPER

B 836, p. 321

Quigley. See (Mt. Eielson), (Quigley Hill).

Quigley \& Dalton. See (Quigley Hill).

Quigley \& Horn. See (Glacier Cr.). 
(Quigley Hill) - (15.75-16.95, 9.5-10.3) ; SILVER, LEAD, GOLD, COPPER, ZINC, ANTIMONY, TUNGSTEN. Includes references to Alice, Apex, Banjo, Blue Bell, Bosart, Caribou (Quigley Hill), Damon \& Pythias, Darling, Doherty, Eureka (lode), Florence Lode, Francis, Friday, Galena, Gold Dollar, Golden Eagle, Gold King, Gneiss, Jumbo, Keystone, Little Annie, Little Maud, Lucky Strike, Martha Q., Merry Widow, Pennsylvania, Pittsburgh, Polly Wonder, Red Top, Silver Pick, Star, Sulphide, Water Level, White Hawk.
B 622, p. 318-319
B 662, p. 319-324
B 687, p. 101-106
B 722, p. $52-53$
B 739, p. 36
B 755, p. 16, 42
B.773, p. 29
B 836, p. 326-332

B 849-F, p. 353-372

B 864-A, p. 25

B 910-A, p. 31

B 917-A, p. 30

B 926-A, p. 24

B 933-A, p. 24-25

Red Top (Min. Co.). See (Quigley Hill).

Shannon. See Copper Lode, Galena Lode, Greenback, Magnet, Merinser, (Mt.

Eielson), Old Sourdough, Question Mark, Stibner, (Straightaway Glacier),

Terminus, Twin Hills.

Silver Pick. See (Quigley Hill).

Silver Wire - $(17.9,10.5)$; SILVER, GOLD, LEAD.

B 849-F, p. 375

Skookona. See Glen Ridge No. 1

(Slate Cr.). See Taylor (antimony).

(Slippery Cr.) - (14-15, 3) ; GOLD. See also Merinser.
B 897-A, p. 35
- B 910-A, p. 31

Snowdrift. See (Mt. Eielson).

Sour Dough. See (Mt. Eielson).

(Spruce Cr.) - (18.1, 10.25) ; GOLD.

P 70, p. 176

B 755, p. 41

B 314, p. 214-215

Spruce Cr. No. 1 - $(17.85,10.55)$; GOLD (?).

B 849-F, p. 374-375

Stampede - $(20.45,13.3)$; ANTIMONY, GOLD, SILVER, LEAD.

B 662 , p. 327

B 687, p. 109

B 755, p. 144

B 813, p. 70-71

B 824 , p. 79

B 836, p. $80,311-313$

B 917-A, p. 102

B 926-A, p. 93

B 933-A, p. 89

B 936-N, p. 332-348

RI 4173, p. 3-20

B 897-A, p. 35, 97-98 * ～～: TDM 1, p. 3

B 910-A, p. 102-103

(Stampede Cr.) - (20.1-20.5, 13.3-13.35) ; GOLD, TUNGSTEN.

B 936-N, p. 335

TDM 1, p. 39

Standard Mines, Inc. See (Eureka Cr.).

Stanford. See (Mt. Eielson).

Star. See (Quigley Hill).

Stendall. See Humboldt.

Stibner - $(14.05,3.15)$ approx; ANTIMONY.

B 836, p. 314

(Straightaway Glacier) - $(13.7,2.4)$ approx; ANTIMONY.

B 836, p. 314

$593018-61-14$ 
Sulphide. See (Quigley Hill).

Swisher. See Glen, (Stampede Cr.).

Taylor (antimony) - $(15.15,8.7)$; ANTIMONY. Includes references to (Slate Cr.).

B 649, p. 43
B 662, p. $325-326$
B 687, p. $107-108$
B 755 , p. 144
B 836, p. 313

B 849-F, p. 376-377

IC 7379, p. 67

RI 4173, p. 4-5, 20-28

B 836, p. 313

Taylor (Eureka Cr.). See (Quigley Hill).

Taylor (Glacier Cr. divide) - (16.8-16.9, 9.95-10.05) approx; SILVER(?), LEAD (?).

B 849-F, p. 371

Taylor, W. (and assoc.). See (Quigley Hill), Stampede, Taylor (antimony), Taylor (Glacier Cr. divide).

Tennessee. See (Mt. Eielson).

Terminus - (14.85, 3.75) approx; ZINC, COPPER.

B 836, p. 320

(Thorofare Cr.) or (R.) - (21.5-21.8, 7.0-7.4) approx ; ZINC, LEAD. B 792, p. 108

Trundy. See Glen Ridge No. 1, Lena, North Star, Silver Wire, Spruce Cr. No. 1.

(Twenty-two Gulch) or (Pup) - (16.5, 10.3-10.35) ; GOLD.

B 849-F, p. 371

B 910-A, p. 58

B 917-A, p. 57

B 933-A, p. 49

Twin Hills - $(19.8,6.2)$ ( ?) ; GOLD, SILVER, LEAD, ZINC, IRON, COPPER. B 836, p. 323-324

Venora. See (Mt. Eielson).

Virginia. See (Mt. Eielson).

Water Level. See (Quigley Hill).

Whistler - $(15.6,9.6)$; GOLD ( ?).

B 836, p. 332

White Hawk. See (Quigley Hill).

(Wickersham Cr.) - (NE 1/4 quad.) ( ?) ; GOLD.

B 722, p. 52

(Willow Cr.) - (18.35-18.55, 9.8-10.35) ; GOLD.

B 868-A, p. 44

Wolf. See Snowdrift.

(Yellow Cr.) - (16.9-17.0, 10.1-10.3) ; GOLD.

B 314, p. 218

B 662, p. 309

B 797, p. 23

B 836, p. 41

B 844-A, p. 41

B $849-$ F, p. 371
B 857-A, p. 39

B 864-A, p. 43

B 880-A, p. 46

B $897-$ A, p. 56

B 917-A, p. 57

\section{MOUNT MICHELSON QUADRANGLE}

(Canning R.) - (W 1/2 quad.) (?) ; MERCURY (?).

TDM 5-R, p. 34

(Hulahula R.) - $(16.1,8.9)$ approx ; PHOSPHATE.

P. 302-A, p. 12-13, 15 
(Katakturuk R.) - $(8.8,10.5)$ approx ; PHOSPHATE. P 302-A, p. 12, 14-15

(Okpilak R.) - (17.5, 7.4) approx; PHOSPHATE. P 302-A, p. 12, 14

(Sadlerochit R.) - (11.2-15.8, 8.3-11.4) approx ; PHOSPHATE. P 302-A, p. 12-15

(Shublik Mtns.) - $(4.8,9.8)$ approx ; PHOSPHATE. P 302-A, p. 12, 14-15

NABESNA QUADRANGLE

Adams \& Cotter. See (Cheslina Cr.).

(Ahtell Cr.) - (0.0-0.25, 12.95) ; GOLD(?). See also (Ahtell Cr.) under Gulkana quad.

P 15, p. 47

B 904, p. 51

Alaska Nabesna Corp. See (Orange Hill).

(Big Eldorado Cr.) - (17.1-17.25, 2.1-2.15) ; GOLD.

B 622, p. 204-205, 211, 220-221

B 868-A, p. 44

B 630 , p. $93,95,104,113-114,117$

B 910-A, p. 56

B 642, p. 62

B 917-A, p. 55

B 662, p. 55

B 926-A, p. 53

B 692, p. 36

B 989-D, p. 199

B 864-A, p. 42

Big Seven. See (Chathenda Cr.).

(Bonanza Cr.) - (17.6-18.2, 1.7-2.45) ; GOLD, LEAD, SILVER, MERCURY, MOLYBDENUM, COPPER. See also Erie.

B 592, p. 316

B 622, p. 60, 204, 208-216, 222

B 630, p. 90, 92-95, 99-109, 115

B 642 , p. 62

B 662, p. 55

B 692 , p. 36

B 714, p. 84

B 783, p. 14

B 797, p. 23

B 813, p. 35

B 864-A, p. 42

B 868-A, p. 44

B 880-A, p. 47
B 880-B, p. 105

B 897-A, p. 55

B 910-A, p. 56

B 917-A, p. 55

B 926-A, p. 53

B 926-C, p. 193

B 933-A, p. 50

B 933-B, p. 170-173

B 989-D, p. 196-200, 203

C 248, p. 7

C 348, p. 4-5

BMB 142, p. 26

Bronniche. See (Mineral Pt.).

(Bryan Cr.) - (16.65-16.8, 0.9-0.95) ; GOLD, COPPER.

B 622, p. 223

B 630 , p. 115-116

B 989-D, p. 200

California. See (Orange Hill).

(California Gulch). See (Orange Hill).

Camp Bird. See (Orange Hill).

(Camp Cr.) - $(9.8,6.15)$ approx; COPPER. P 15, p. 39

B 314, p. 28

B 213, p. 148

(Canyon Cr.) - (17.6, 2.05) ; GOLD(?).

B 622, p. 225

B 630, p. 119 
(Carden Cr.) - (22.1, 4.5) approx; GOLD. B 933-B, p. 164

Carroll. See (Discovery Pup), (Glacier Cr.).

(Cathenda Cr.). See (Chathenda Cr.).

(Chathenda Cr.) - (16.8-17.6, 1.4-1.75) ; GOLD, COPPER. See also Erie. B 622, p. 221-222, 225 B 933-B, p. 165 B 630, p. 92-94, 114, 118-119

(Chavolda Cr.). See (Wilson Cr.).

(Cheslina Cr.) or (R.) - (11.35-11.85, 11.45-11.5) ; GOLD. B 917-B, p. 153-154 B 989-D, p. 190, 200-201

Chisana Mines, Inc. See Reynolds, Sulzer.

(Coarse Money Cr.) - (18.0, 2.3-2.5) ; GOLD. B 642, p. 62 B 933-B, p. 173

Copper King. See (Orange Hill).

(Cross Cr.) - (11.6-11.9, 1.5-1.8) approx; COPPER, LEAD, ZINC. See also (Tinast Gulch).

B 379, p. 173

B 933-B, p. 174

B 417 , p. 55

B 989-D, p. 210

B 630, p. 121

Davis. See (Little Eldorado Cr.).

Deadman. See (Bonanza Cr.).

(Discovery Gulch) or (Pup) - (17.35-17.55, 2.3-2.45) approx; GOLD. B 897-A, p. 55

B 933-B, p. 173

(Dry Gulch) - (17.0, 1.65) approx ; GOLD.

B 622, p. 222-223, 225

BMB 142, p. 26

B 630, p. 115, 119

Erie - $(17.5,1.8)$; GOLD, SILVER, LEAD. NoTE.-Not mentioned by name in B 622,630 .
B 622, p. 224-225
B 933-B, p. 164-165

B 630, p. 118-119

Eureka. See (Eureka Cr.).

(Eureka Cr.) - (22-24, 0-3) ; GOLD, SILVER, LEAD, ZINC.

$\begin{array}{ll}\text { B 379, p. 177-178 } & \text { B 622, p. 224 } \\ \text { B 417, p. } 59 & \text { B 630, p. } 118\end{array}$

Fjeld \& Paulson. See Nabesna.

(Fourmile Cr.) - (22.6-24.0, 2.8-3.3) approx; GOLD.
B 379, p. 178
B 622, p. 224

B 417, p. 60

B 630, p. 118

Fram, Horn \& Todd. See (Rock Cr.).

Glacier. See (Orange Hill).

(Glacier Cr.) - (17.3-17.55, 2.3-2.5) ; GOLD.

B 630, p. 93-95

B 933-A, p. 50

(Gold Run) or (Cr.) - (17.35-17.55, 2.3-2.45) ; GOLD.

P 15, p. 61

B 622, p. 218-219

B 630 , p. 111-112, 116

B 642 , p. 62

B 662 , p. 55

B 692, p. 36

B 824, p. 39-40

B 836, p. 41

B 868-A, p. 44

B 880-A, p. 47

B 910-A, p. 56

B 917-A, p. 55

B 926-A, p. 53

B 933-A, p. 50

B 933-B, p. 173

B 989-D, p. 199 
Golden Eagle. See Nabesna.

Green. See (Bonanza Cr.).

Hamshaw. See (Big Eldorado Cr.), (Bonanza Cr.), (Little Eldorado Cr.).

Hirst. See (Bonanza Cr.).

Hirst \& Gamblin. See Erie.

Husky. See (Fourmile Cr.).

(Jacksina R.). See Nabesna.

James \& Nelson. See (Big Eldorado Cr.), (Bonanza Cr.), (Little Eldorado Cr.).

J'ames, Nelson \& Wales. See (Bonanza Cr.).

(Johnson Cr.). See (Chathenda Cr.).

Jumbo. See (Fourmile Cr.).

Kennecott Copper Corp. See (Rock Cr.).

Lemon. See (Orange Hill).

Lewis. See (Little Eldorado Cr.).

(Little Eldorado Cr.) - (17.8-17.85, 2.1-2.3) ; GOLD.

B 592, p. 67, 316

$\therefore$ B 622, p. $60,211-213,216-217, \quad$ B $844-A$, p. 42 222

B 857-A, p. 39

B 630 , p. $92-93,95,104-106,109$ $110,113-115$

B 642, p. 62

B 864-A, p. 42

B 868-A, p. 44

B 662, p. 55

B $880-A$, p. 47

B 692, p. 36

B $897-$ A, p. 55

B 783, p. 14

B 910-A, p. 56

B 792, p. 23

B 917-A, p. 55

B 824, p. 39

B 926-A, p. 53

B 836, p. 40

B 933-A, p. 50

B 989-D, p. 196-199

McGettigan. See (Bonanza Cr.)

McKinney. See (Gold Run).

(Mentasta Pass) - $(2,16)$; GOLD.

P 15, p. 47

(Mineral Pt.) - $(4.6,16.65)$; SILVER, COPPER, GOLD, NICKEL ( ?).

C 248, p. 8

RI 3940, p. 8-9

C 348, p. 2

(Monte Cristo Cr.) - $(\mathrm{N} 1 / 4,8,3)$; GOLD.

P 15, p. 43-45

B 417 , p. 58

(Moose Cr.) - (8.3-8.6, 15.3-16.0) ; GOLD. B $989-\mathrm{D}$, p. 190

Nabesna (Min. Corp.) - (7.65-7.95, 6.25-6.85) ; GOLD, SILVER; COPPER, LEAD, ZINC, ANTIMONY. Includes references to (Jacksina Cr.), Royal Dev. Co.

B 379, p. 176-177

B 417, p. 58

B 480 , p. 65

B 622 , p. 224

B 630, p. 90,118

B 824, p. 22-23

B 836, p. 22

B 844-A, p. 21

B 844-C, p. 159-162

B 868-A, p. 21, 66

B 868-C, p. 141-142

B 880-A, p. 21-22, 70, 72

B 880-B, p. 103-104

B 897-A, p. 23-24, 80

B 910-A, p. 25-26, 85

B 917-A, p. 24, 87

B 926-A, p. 23-24, 80

B 857-A, p. 18-19

B 933-A, p. 24, 76-77

B 864-A, p. 21

B $933-B$, p. 168, 175-195

B 943-B, p. 45-46 
Nabesna (Min. Corp.) -Continued B 989-D, p. 66, 189-190, 201-203

C 248, p. 7

C 348, p. 3-4

IC 7379, p. 30

Nabesna Claim. See (Orange Hill).

Nelson \& LaBell. See (Trail Cr.).

Nelson Min. Co. See (Bonanza Cr.).

(Nikonda Cr.). See (Orange Hill).

North Star. See (Orange Hill).

(Notch Cr.) - (12.0-15.0, 3.0-4.4) ; GOLD. B 692, p. 36

O'Hara. See Sulzer.

Orange Hill. See (Orange Hill).

(Orange Hill) - (9.3-9.8, 3.2-3.6) ; COPPER, GOLD, MOLYBDENUM, SILVER.

Includes references to Alaska Nabesna Corp., (California Gulch), Shamrock.

P 15, p. 37-38, 43-45

B 379, p. 173

B $880-$ B, p. 103

B 417, p. 54-55, 58

B 622, p. 227

B 926-C, p. 193

B 797, p. 36

B 933-B, p. 166-168

B 810, p. 47

B 989-D, p. 189, 201, 203, 205-207, 209

B 813, p. 54

C 248, p. 6-7

B 824, p. 60

C 252, p. 5-6

B 836, p. 63

C 348, p. 4

IC 7784, p. 6, 9-10

Peterson \& Hawkins. See (Coarse Money Cr.).

(Poorman Cr.) - $(\mathbf{1 7 . 4}, 2.4)$; GOLD.
B 622, p. 219-220
B 989-D, p. 199

B 630, p. 112, 116

Price \& Ives. See (Big Eldorado Cr.), (Bonanza Cr.), (Little Eldorado Cr.).

Rams Horn. See Nabesna.

Reynolds - $(21.1,3.7)$; COPPER.

B 933-B, p. 169

B 989-D, p. 204-205

(Rock Cr.) - $(5.05,10.5)$; MOLYBDENUM.
B 910-A; p. 105
B 989-D, p. 190, 201, 209-210
B 917-A, p. 104
C 248 , p. 7
B 917-B, p. 150-153
C 348, p. 3
B 926-C, p. 184-185
TDM 1, p. 30

Royal Dev. Co. See Nabesna.

Shamrock. See (Orange Hill).

(Skookum Cr.) - (17.75-17.8, 2.25) ; GOLD.
B 622, p. 60, 217-218
B 662 , p. 55
B 630, p. 93, 95, 110-111
B 989-D, p. 199

B 642, p. 62

(Snag R.). See Reynolds, Sulzer.

(Snow Gulch) - (17.8-17.9, 2.25-2.45) ; GOLD.

B 622 , p. 222

B 630, p. 115

Stonehead. See Nabesna.

Sulzer - $(20.75,4.05)$; COPPER. See also Reynolds. B 933-B, p. 169-170 B 989-D, p. 204-205

(Tinast Gulch) - (11.6-11.9, 1.5-1.8) (?) ; COPPER. See also (Cross Cr.). P 15, p. 39-40

Todd (, Frame, Horne \& DeWitt). See (Rock Cr.). 
(Trail Cr.) - (5.9-6.0, 10.7-10.8) ; GOLD.

B $917-B$, p. 155

Violet. See (Eureka Cr.).

White Mtn. See Nabesna.

Whitham. See (Cheslina Cr.), (Little Eldorado Cr.), Nabesna.

(Wilson Cr.) - (16.3-17.15, 2.6-2.7) ; GOLD.

B 622, p. 223

B 630, p. 116

Wright \& Hundell. See (Gold Run).

(_-..-) (gold) - $(4.15,11.0)$; GOLD.

- B 917-B, p. 155

B $989-D$, p. 203

\section{NARNER QUADRANGLE}

(Keeler's Bar) - $(1.2,15.95)$ approx ; GOLD.

B 903 , p. 91

\section{NOME QUADRANGLE}

Alaska Mines Corp. See (Flat Cr.), (Snake R.).

Alaska Sunset Mines Co. See (Sunset Cr.).

(Albion Cr.) - $(19.4,11.1)$ approx ; GOLD.

B 722, p. 247

(Alpha Cr.) - (18.7, 11.3) ; GOLD.

B 722, p. 249

American - $(16.15,13.8)$; IRON. Note.-Not mentioned by name in B 714 .

B 622, p. 361, $365 \quad$ B 714, p. 42

B 662, p. 446

B 722, p. 261

American Gold Dred. Co. See (Peluk Cr.).

Ames \& Guinan. See (Glacier Cr.).

(Anvil Cr.) - (19.35-19.85, 10.1-10.75) ; GOLD, ANTIMONY, SILVER, COPPER. TUNGSTEN, LEAD. Includes references to Bangor Dred. Co., Hendrickson. (, Kotovic \& Stipek), McIntosh, Olsen, Widstedt, Winsted; see also (Dexter Hill).
B 213, p. 45
B 225, p. 52-53
B 662, p. 430-432, 454-455
B 259, p. 21
B 263, p. 14, 56, 86-87, 191, 209
B 712, p. 189
B 722, p. 184, 225, 238-240
B 284, p. 134-135
B 314, p. 140
B 328, p. 169-170, 186-192
B 755, p. 14
B 773, p. 21,27
B 783, p. 16, 18
B 810, p. 34,40
B 345, p. 216, 232
B 379, p. 279-280, 282-283
B 813, p. 39,48
B 824, p. 44, 53
B 442 , p. 359
B 864-A, p. 48
B 533, p. 79-83
B 880-A, p. 52-53
B 592 , p. 389, 401-402
BMB 142, p. 27
B 622, p. 369
RI 4174, p. 32
B 642, p. 71
TDM 5-R, p. 11-12, 27
B 649 , p. 50, 56-57
(Arctic Cr.) - (16.4-17.0, 10.85-11.45) ; GOLD.
B 284, p. 136
B 722, p. 63
B 622, p. 367
B 755, p. 14
B 714, p. 233 
Arctic Cr. Dred Co. See (Arctic Cr.).

Arctic Gold Dred. Co. See (Hobson Cr.), (Sanders Cr.).

Aretic Placer Min. \& Mil. Co. See (Bangor Cr.).

(Balto Cr.) - (19.1-19.2, 11.45-11.55) ; GOLD, TUNGSTEN.

B 328, p. 196

B 592, p. 389

B 533, p. 98

RI 4174, p. 33-34

(Bangor Cr.) - (18.85-19.0, 12.4-12.45) ; GOLD, TUNGSTEN.

B 480, p. 42

B 712, p. 187-188

B 533, p. 87

BMB 142, p. 27

B 622, p. 367

RI 4174, p. 33

B 662, p. 452,455

Bangor Cr. Dred. Co. See (Bangor Cr.).

Bangor Dred. Co. See (Anvil Cr.).

(Banner Cr.) - (20.1-20.3, 11.7-11.8) ; GOLD.
B 328, p. 181
B 533, p. 99

B 345, p. 234

(Basin Cr.) - (20.4-20.6, 12.4-12.45) ; GOLD, TUNGSTEN.

B 263, p. 150, 209

B 533, p. 99-100

B 328, p. 174-175

(Beaches, old) - (19.1-21.95, 8.5-10.0) ; GOLD. Includes references to (Intermediate Beach), (Monroeville Beach), (Second Beach), (Submarine Beach), (Third Beach).
B 284 , p. 133-134
B 712 , p. $188-189$
B 314, p. 134-137, 141-144
B 783, p. 16
B 379 , p. 271-279
B 844-A, p. 46, 55
B 442 , p. $356-357$
B 857-A, p. 43, 51
B 533, p. $40-44,109-123$
BMB 153, p. 56

B 622, p. 454

(Beaches, present) - (14.9-22.35, 8.25-10.0) ; GOLD.

B 533, p. 110-111

B 662, p. $452,454-455$

(Bear Cr.) or (Gulch) - $(20.0,10.3)$ approx; GOLD.

B 712 , p. 189

Bessie (Gold Dred. Co.). See (Holyoke Cr.).

Big Four. See New Era.

(Boer Cr.) - $(20.15,15.6-15.7)$; GOLD. B 328 , p. 182

B 533, p. 76, 100

(Bonanza Gulch) - $(19.5,10.8)$; GOLD.

B 263, p. 209

B 533, p. 86

B 328, p. 194-195

(Bonita Cr.) - $(20,12)$; ANTIMONY, GOLD.

B 345, p. 245

B 722, p. 185

B 649 , p. 58-59

TDM 5-R, p. 11

B 662, p. 440

Borasco. See Jorgensen.

Boulder - $(18.7,11.7)$; ANTIMONY, GOLD, TUNGSTEN. See also (Boulder Cr.) (trib. Snake R.).

B 662, p. 427-429, 440

B 722, p. 184, 244, 248-252

(Boulder Cr.) (trib. Sinuk R.) - (14.75-15.05, 12.35-12.55) ; GOLD.

B 328, p. 216-217 
(Boulder Cr.) (trib. Snake R.) - (18.4-18.8, 11.6-11.9) ; GOLD, ANTIMONY, TUNGSTEN, BISMUTH. See also Boulder, Dakota.
B 328, p. 196
B 712, p. 188
B 480, p. 42
B 722, p. 250-251
B 533, p. 87, 131
B 783, p. 16
B 592, p. 389
BMB 142, p. 27
B 622, p. 370
RI 4174, p. 33
B 642, p. 71
TDM 5-R, p. 12, 42-43
B 662, p. $427,429,455$
(Bourbon Cr.) - (19.5-19.9, 9.25-9.7) ; GOLD.
B 263, p. 165
B 442, p. 357-358
B 284, p. 135
B 520, p. 342
B 314, p. 141
B 533, p. $77,89-90,111,122-123$
B 328, p. 166-168
B 592, p. 387, 389

Breen - (19.9-20.2, 13.7-13.8); ANTIMONY. Includes references to antimony lode between Hobston and Manila Creeks.
B 722, p. 184, 228, 230-231
TDM 5-R, p. 10

Bunker Hill. See Christophosen (Waterfall Cr.).

Bursik \& Kern - $(20.0,10.7)$ approx ; GOLD.
B 662 , p. 429
B 722, p. 238

(Buster Cr.) - (20.7-21.15, 10.8-11.25) ; GOLD.
B 314, p. 141
B 622, p. 370
B 328, p. 172
B 662, p. 455
B 345, p. 216
B 712, p. 189
B 533, p. 93, 96-97

(Butterfield Cr.) - (18.7-18.95, 12.4-12.45) ; GOLD, TUNGSTEN.

RI 4174, p. 33

California - $(19.05,13.9)$; GOLD, MOLYBDENUM, TUNGSTEN, ANTIMONY.

Includes references to Connolly \& Jensen, Jannsen, lode near head of Goldbottom $\mathrm{Cr}$.

$\begin{array}{lll}\text { B 345, p. 233, 245 } & & \text { B 722, p. 185, 244, 253-255 } \\ \text { B 379, p. 280-282 } & \text { B 857-A, p. 22 } \\ \text { B 533, p. 131-132, 134 } & & \text { B 917-A, p. 31 } \\ \text { B 592, p. 402 } & \text { B 926-C, p. 202 } \\ \text { B 649, p. 59 } & \text { TDM 5-R, p. 12, 35 } \\ \text { B 662, } 426-427,440 & \quad\end{array}$

B 662, p. $426-427,440$

(Canyon Cr.) - (14.6-16.6, 16.1-17.75) (?) ; GOLD. May be in Teller quad. B 810, p. 36

(Cape Nome) - $(22.65,8.5)$; CHROMITH.

$$
\text { C 244, p. } 7
$$

Caribou. See (Dexter Hill).

Caribou Bill. See (Dexter Hill).

Casa De Paga Gold Co. See (Monument Cr.).

(Center Cr.) - (19.5-19.6, 9.55-9.8) ; GOLD.
B 328, p. 170
B 442, p. 356
B 533, p. 88-89, 117
B 622, p. 369
B 662, p. 452,459
B 712, p. 187-188
B 722, p. 63

B 836, p. 45

B 844-A, p. 46

B 857-A, p. 43

B 864-A, p. 48

B 868-A, p. 59

BMB 142, p. 27 
Center Cr. Dred. Co. See (Center.Cr.), (Snake R.).

Charles. See (Cooper Gulch), (Dry Cr.).

(Charley Cr.) - (18.7-18.85, 15.2-15.4) ; BISMUTH, GOLD.
B 314, p. 138
B 328, p. 216
B 662, p. $447-448$
B 345, p. 247
B 666, p. 98
B 480 , p. 93
B 714, p. 41
B 533, p. 133
B 722 , p. $185,223-224$
B 592, p. 404-405
C 196 , p. 4
TDM 5-R, p. 17

Christophosen (Anvil Cr.). See (Anvil Cr.).

Christophosen (Last Chance Cr.) - $(18.65,13.1)$; SILVER, LEAD, GOLD, ANTIMONY. NOTE.-Reported in B 722 as being on Waterfall $\mathrm{Cr}$.

B 622 , p. 446

B 722, p. 183

Christophosen (Waterfall Cr.) - (18.55, 13.25) ; ANTIMONY, GOLD, SILVER, COPPER, LEAD. See also. (Waterfall Cr.).

B 662, p. $438-440,442$

TDM 5-R, p. 11, 19, 27

B 722, p. 182, 184, 231-232, 253

Christophosen (zinc) - $(18.05,12.7)$; ZINC, GOLD. Note.-Not mentioned by name in B 722, p. 183 ; TDM 5-R.

B 662, p. 447

B 722, p. 183, 232-233

TDM 5-R, p. 27

(Coal Cr.) - (15.4-15.65, 13.05-13.35) ; GOLD.

B 328, p. 216-217

Cochrane \& Rodine. See (Alpha Cr.), Boulder, (Boulder Cr.) (trib. Snake R.), Dakota, (Sledge Cr.), (Twin Mtn. Cr.).

Connelly \& Janssen. See California.

Connelly \& Listen. See (Monument Cr.).

Connolly \& Jensen. See California.

(Cooper Cr.). See (Copper Mtn.).

(Copper Gulch) - (19.75-19.85, 10.1-10.3) ; GOLD.
B 328 , p. 165-166, 186
B 592, p. $389,402-403$
B 533, p. 89,124
B 662, p. 431, 454

(Copper Mtn.) - (20.8-21.05, 15.7-16.05) ; COPPER, LEAD, SILVER, GOLD. Includes references to (Cooper Cr.), (Dickens Cr.).
B 345, p. 240-242
B 533, p. 134-135
B 722, p. 181-182, 217-221
TDM 5-R, p. 18-19, 27

(Cripple R.) - (15.75-16.8, 9.7-12.05) ; GOLD.
B 225, p. 53
B 520, p. 342
B 259 , p. 21
B 592, p. 387,390

B 328, p. 210-211

Cripple R. Dred. Co. See (Cripple R.).

Cub Bear - $(16.9,12.9)$; IRON. NOTE.-Not mentioned by name in B 714 .
B 622, p. 361, 365
B 662, p. 446
B 714, p. 42
B 722, p. $183,237,261$

(Daisy Gulch) - (19-20, 10-11) ( ?) ; GOLD.

B 622 , p. 370

Dakota - $(18.7,11.7)$; GOLD. See also (Boulder Cr.) (trib. Snake R.). B 662, p. 429

B 722, p. 250-251

Dakota-Alaska Min. Co. See (Alpha Cr.), Boulder, (Boulder Cr.) (trib.

Snake R.), Dakota, (Sledge Cr.), (Twin Mtn. Cr.).

(Deer Gulch). See (Dexter Hill). 
(Derby Cr.) - $(21.65,8.65)$ approx ; GOLD.

B 662, p. 455

(Dewey Cr.) - (20.4-20.7, 11.5-11.7) ; GOLD, TUNGSTEN. B 328, p. 174

(Dexter Cr.) - (20.0-20.4, 10.3-10.65) ; GOLD, COPPER, TIN(?). Includes references to (Dexter Cr., Left Fork); see also (Dexter Hill), (King Mtn.). B 225, p. 52-53

B 259, p. 21, 127

B 263, p. 14, 209

B 714, p. 232-235

B 284, p. 135

B 328, p. 176-181, 207-209

B 722, p. 63, 181, 233

B 739, p. 9

B 345, p. 216, 240

B 755, p. 14

B 773, p. 27

B 379, p. 279

B 783, p. 18

B 533, p. 77, 93-95, 107-109, 135

B 792, p. 24

B 592, p. 389,403

B 797, p. 30

B 622, p. $369-370$

B 897-A, p. 61

B 662, p. 442-443, 454-455

BMB 142, p. 27

B 712, p. 187-188

TDM 5-R, p. 18

(Dexter Cr., Left Fork). See (Dexter Cr.).

Dexter Cr. Dred. Co. See (Dexter Cr.).

(Dexter Hill) - (19.7-20.15, 10.5-10.75) ; GOLD, LEAD. Includes references to to Caribou Bill, (Deer Gulch), Gambrinus, (Grass Gulch), (Grouse Gulch), Honey, Lenà, Madeline, Mattie, (Nekula Gulch), (Nicola Gulch), Snowflake, (Specimen Gulch), Sugar, Summit; see also (Anvil Cr.), (Dexter Cr.), (Dry Cr.).
B 263, p. 209
B 622, p. 370
B 284, p. 135
B 662, p. 454-455
B 314, p. 140
B 712, p. 188-189
B 328, p. 178-181, 191, 199-207
B 722, p. 238
B 442 , p. 359
B 773, p. 76, 83-84
B 533, p. 76-77, 81, 83-84, 94-96,
B 926-A, p. 60 101-109
B 933-A, p. 57

B 592, p. 389

(Dexter Station). See (Dexter Hill).

(Dickens Cr.). See (Copper Mtn.).

(Divide Cr.) - $(20.25,15.1)$ approx ; GOLD. B 328, p. 182

B 533, p. 100

(Dorothy Cr.) - (20.6-20.7, 14.85-14.9) ; GOLD, ANTIMONY.
B 259, p. 21
B 933-A, p. 57
B 328 , p. $181-182$
TDM 5-R, p. 11

B 533, p. $77,93,98$

(Dry Cr.) - (19.8-20.15, 9.35-10.3) ; GOLD, TUNGSTEN. See also (Dexter Hill).
B 225, p. 52-53
B 263, p. 14, 209
B 712, p. $188-189$
B 284, p. 135
B 722, p. 63,237
B 314, p. 141
B 739, p. 9
B 328 , p. $168-170,184-186$
B 792, p. 24
B 797, p. 30
B 345, p. 216, 231
B 810, p. 34,40
B 442 , p. 357
B 813, p. 39,48
B 533 , p. 77, 90-92, 101, 117-118,
B 864-A, p. 44,53
123,132
B $868-A$, p. 49,59
B 662, p. 431, 454
B $880-A$, p. 52,62 
(Dry Cr.)-Continued
B 897-A, p. 61, 71
B 926-A, p. 72
B 910-A, p. 64-65, 77
BMB 142, p. 27
B 917-A, p. 63-64

Dry Cr. Dred. Co. See (Dry Cr.), (Newton Gulch).

Ernst Alaska Gold Dred. Co. See (Rocker Gulch).

Eureka. See Jorgensen.

(Extra Dry Cr.) - (20.4-20.55, 10.3-10.4) ; GOLD.
B 328, p. 175-176
B 533, p. 98-99

(Fairview Cr.) - (12.9ら-14.0, 13.0-14.4) ; GOLD.

B 328, p. 218-219

(Flambeau R.) - (21.5-23.55, 11.5-12.5) (?) ; GOLD. May be in Solomon quad. B 379, p. 280

(Flat Cr.) - (19.7-19.75, 10.0-10.1) ; GOLD.
B 533, p. 88
B 722, p. 63
B 714, p. 232-233

Fox. See Galena.

(Fox Cr.) - (21.5-22.45, 13.85-14.1) ; GOLD.

B 328, p. 221

(Fred Gulch) - (19.2-19.25, 14.15-14.25) approx; GOLD, TIN.
B 284, p. 157
B 533, p. 101
B 692, p. 353

Galena - $(16.25,12.95)$; IRON, LEAD, ZINC, GOLD. Nore.-Not mentioned by name in B 714, TDM 5-R.
B 622, p. 361, 364-365
B 722, p. 260
B 662, p. 445
TDM 5-R, p. 26-27

B 714, p. 42

Gambrinus. See (Dexter Hill).

Gillette. See (Dexter Hill).

(Glacier Cr.) - (19.45-20.0, 10.85-11.15) ; GOLD, TUNGSTEN, ANTIMONY.

TIN. See also (Hot Air Bench), (Snow Gulch).
B 225, p. 53
B 259, p. 21
B 622, p. 370
B 263, p. 14, 209
B 662 , p. $437,452,455,457$
B 284, p. 134-135
B 712, p. 187
B 714, p. 233
B 314, p. 140
B 722, p. 182, 184, 233-234
B 328 , p. 170, 192-194
B 739, p. 9
B 345, p. 216, 232
B 755, p. 14
B 379, p. 279-280
B 442, p. 359
B 533, p. 77, 84-85, 130-131
BMB 153, p. 55
RI 4174, p. 31-33
TDM 5-R, p. 12, 40, 42-43

Golconda. See (Twin Mtn. Cr.).

Gold Beach Dred. Co. See (Osborn Cr.).

Gold Beach Placers, Inc. See (Peluk Cr.).

Gold Bug. See West.

(Gold Hill) - (19.05, 10.95-11.05) ; GOLD.

B 722, p. 247

(Goldbottom Cr.) - (19.1-19.6, 13.05-13.65) ; GOLJ), TIN. See also California. B 229, p. 36

B 259, p. 127

B 520, p. 89

B 284, p. 157

B 533, p. 25-26, 87

B 328, p. 197

B 692 , p. 353

B 712, p. 188 
Golden Cow - $(20.3,9.5)$ approx ; GOLD.

$$
\text { B 592, p. } 389
$$

Golden Eagle. See West.

(Grace Gulch) - (21.05-21.1, 11.1-11.15) ; GOLD.
B 328, p. 173-174
B 533, p. 97

(Grand Central R.). See (Windy Cr.).

(Grass Gulch). See (Dexter Hill).

Greenstone Mines, Inc. See (Osborn Cr.), (St. Michael Cr.).

(Grouse Cr.) - (19.55-19.75, 13.1-13.6) ; GOLD, LEAD.

B 328, p. 197-198 B 533, p. 88

B 379, p. 280

TDM 5-R, p. 27

(Grouse Gulch). See (Dexter Hill).

(Grub Gulch) - (19.5-19.65, 13.0) ; GOLD.

B 328, p. 197

B 533, p. 88

Guinan \& Ames. See (Glacier Cr.).

Gustafson \& Swedman. See (Oregon Cr.).

Hall. See (Arctic Cr.).

Hammon Consol. Gold Fields. . See. D.S. Smelt., Ref. \& Min. Co.

(Hastings Cr.) - (21.85-21.95, 8.4-8.65) ; GOLD.
B 225, p. 53
B 259, p. 20, 22
B 662, p. 452
B 263, p. 209
B 712, p. 187
B 284 , p. 133
B 813, p. 39
B 314, p. 144
B 824, p. 44
B 328, p. 167
B 836, p. 45,54
B 533, p. 77, 100-101, 111
BMB 142, p. 27

Hastings Cr. Dred. Co. See (Hastings Cr).

(Hazel Cr.) or (Gulch) - (21.65-21.8, 12.85-12.9) ; GOLD. B 533, p. 101

B 662, p. 455

Hed \& Strand - $(20.15,14.35)$; ANTIMONY. Includes references to Hed \& Strom.
B 642, p. 30,71
B 649 , p. 54-56
B 836, p. 23
B 662, p. $62,437-438$
B $857-$ A, p. 22
B 722, p. 184, 224, 226-230
C 196, p. 4
BMB 142, p. 27
B 813 , p. 19
B 824, p. 23
TDM 5-R, p. 9-10

Hed \& Strom. See Hed \& Strand.

Hendrickson (, Kotovic \& Stipek). See (Anvil Cr.).

Hines (\& McLaughlin). See (King Mtn.).

(Hobson Cr.) - (20.35-20.4, 13.2-13.5) ; GOLD.

B 328, p. 181

B 592, p. 390

(Holyoke Cr.) - (19.8, 9.6-9.8) ; GOLD.

B 314, p. 136, 142

B 328, p. 165, 167

B 442 , p. 356

Homburger. See (Newton Gulch).

Homburger \& Shoemaker. See (Charley Cr.).

Honey. See (Dexter Hill).
B 662 , p. 452

BMB 142,p. 21

Includes references to Bessie.

B 533, p. 89, 117, 119

B 592, p. 390 
(Hot Air Bench ) - (19.5-19.6, 10.9) ; GOLD.
B 328, p. 194
B 533, p. $85-86$
B 722, p. 243

(Hume Cr.) - (13.0, 16.15) approx ; GOLD.

B 328, p. 220

(Hungry Cr.) - (17.2-17.4, 11.9-12.1) ; GOLD, BISMUTH.
B 328, p. 214
TDM 5-R, p. 17

(Igloo Cr.) - $(12,13)$; GOLD.

B 328, p. 218

(Independence Cr.) - (14.5-15.3, 12.6-13.2) ; GOLD.

B 328, p. 216-217

(Intermediate Beach). See (Beaches, old).

(Irene Cr.) - (21.0-21.1, 9.0-9.05) ; GOLD.
B 284, p. 135

Isabel. See Christophosen (Waterfall Cr.).

Jannsen. See California.

(Jess Cr.) - (17.0, 9.8) approx; GOLD.
B 284, p. 133-134
B 712, p. 189
B 622 , p. 370

(Johnston Cr.) - (13.7-15.5, 14.7-15.1) ; GOLD.

B 328, p. 219

Jorgensen - $(19.5,10.6)$; GOLD, TUNGSTEN, LEAD.
B 662 , p. $434-435$
B 722, p. 240-241

Julian. See (Osborn Cr.).

Julien (Gold Min. \&) Dred. Co. See (Osborn Cr.).

Kentucky. See Galena.

(King Mtn.) - (20.05-20.15, 10.55-10.75) ; GOLD, MOLYBDENUM. Includes references to Hines \& McLaughlin, Royal, molybdenite on divide between Anvil and Glacier Creeks; see.also Bursik \& Kern, (Dexter Cr.).
B 533 , p. $130-131$
B 722 , p. 237
B 592, p. 397
B 926-C, p. 202-203
B 662 , p. 430
TDM 5-R, p. 35

(Lake Cr.) - (19.3-19.85, 9.2-10.05) ; GOLD.

B 868-A, p. 59

Lane, Charles D., Co. See (Dexter Cr.).

(Last Chance Cr.) - (18.65-18.95, 12.95-13.8) ; GOLD, ANTIMONY, TUNGSTEN.

B 328, p. $197 \quad$ B 622, p. 370

B 345, p. $245 \quad$ B 722, p. 253

B 533, p. $87,134 \quad$ B 783, p. 16

Lee \& Swanberg. See (Osborn Cr.).

Lena. See (Dexter Hill).

(Lillian Cr.) - $(20.7-20.8,10.95-11.4)$; GOLD. Includes references to (Tug Gulch).

B 328, p. 172-173. $\quad$ B 533, p. 96

Lilly - $(18.9,12.2)$; GOLD.

B 722, p. 252

(Lindblom Cr.) - (19.1-19.3,11.2-11.3) ; GOLD, TUNGSTEN.

B 328 , p. 196

B 533, p. $86-87$

RI 4174, p. 33-34

TDM 5-R, p. 42-43 
(Little Cr.) - (19.4-19.8, 9.75-10.0) ; GOLD. See also (Beaches, old).

B 284, p. 133, 135

B 314, p. 141-142

B 328, p. 170

B 442 , p. 357,359

B 592, p. 389

(Livingston Cr.) - (13.7-15.2, 13.7-14.8) ; GOLD.

B 328, p. 219

Lynx. See (Glacier Cr.).

Mabel. See Christophosen (Waterfall Cr.).

McAllister - $(20,15)(?)$; GOLD.

B 442, p. 38

McCarthy \& Panos. See (Beaches, old).

(McDougall Cr.) - $(20.65,9.5)(?)$; GOLD.

B 662, p. 455

McDuffee - $(19.6,13.6)$ approx ; ANTIMONY.

TDM 5-R, p. 10-11

McIntosh. See (Anvil Cr.).

Madeline. See (Dexter Hill).

(Manila Cr.) - (20.1-20.3, 13.5-13.8) ; GOLD, COPPER. See also Sliscovich.

B 592, p. 389

B 662, p. 454

B 722, p. 182

TDM 5-R, p. 19

Mattie. See (Dexter Hill).

(May Gulch) - $(17.5,11.8)$ approx ; GOLD. B 328, p. 215

Miocene (Ditch) Co. See (Dexter Hill), (Glacier Cr.), (Snow Gulch).

Monarch - $(16.8,13.75)$; IRON, MANGANESE. NOTE.-Not mentioned by name in $B 714$.

B 592 , p. $406-407$

B 714, p. 42

B 622, p. 361-364

B 722, p. 258-260

B 662, p. 444-445

Mogul - $(17.1,13.7)$; IRON. Note.-Not mentioned by name in B 714 .

B 622, p. 361, 364

B 662, p. 446

B 714, p. 42

B 722, p. 183, 261

Monitor. See Christophosen (Waterfall Cr.).

(Monroeville Beach). See (Beaches, old).

(Monument Cr.) - (18.65-19.05, 10.65-10.9) ; GOLD, TIN.
B 712, p. 51, 188, 190
B 868-A, p: 49-50
B 813, p. 39
B 824, p. 44
B 880-A, p. 52
B 836, p. 46
B 917-A, p. 63
B. $926-A$, p. $59-60,71$
B 844-A, p. 46
B.933-A, p. 56
B 857-A, p. 43
TDM 5-R, p. 40

B 864-A, p. 48

Monument Cr. Min. Co. See (Monument Cr.).

Moore. See (Osborn Cr.).

(Moss Gulch) or (Cr.) - (21.1-21.9, 9.4) ; GOLD.

B 520, p. 342

B 662, p. 455

B 592, p. 387,390

(Mountain Cr.) - $(17.6,12.6)$ approx ; GOLD, LEAD, TUNGSTEN.

B 328, p. 214

TDM 5-R, p. 27, 43 
(Nekula Gulch). See (Dexter Hill).

Nelson - $(19.55,13.8)$; LEAD, ZINC. See also (Bonita Cr.). B 662 , p. 447

B 755 , p. $182-183,232$

TDM 5-R, p. 27

New Era (Min. Co.) - $(19.65,10.75)$; GOLD. Includes references to Big Four; see also (Snow Gulch).

B 542 , p. 41

B 662 , p. $433-434$

B 592 , p. $400-401$

B 722 , p. $243-244$

(Newton Gulch) - (20.1-20.2, 9.6-9.9) ; GOLD.

B 284, p. 135

B 328 , p. $168-169,183-184$

B 379, p. 282

B 442, p. 359

B 533, p. 77, 90-92, 113, 132
B 662 , p. 431

B 722, p. 237

B 836, p. 45, 54

: $844-A$, p. 46,55

B $857-A$, p. 43,51

(Nicola Gulch). See (Dexter Hill).

Noble. See (Beaches, old).

Nome Consol. Dred. Co. See (Bourbon Cr.), (Wonder Cr.).

Nome Consol. Min. \& Dev. Co. - (19.3-19.85, 9.2-10.05) (?) ; GOLD. BMB 153, p. 56

Nome Gold Gravel Co. See (Cripple R.).

(Nome R.) - (20.4-21.15, 8.9-11.1) ; GOLD. Note.-Nearly all production has been from tributaries.
B 225 , p. 49
B 379 , p. $279-280$
B 263, p. 46,53
B 284, p. 133, 135.
B 533, p. 77, 93
B 712 , p. 189

B 328 , p. 165,170

(North Fork) - $(19.0,13.5)$ approx; COPPER.
B 722 , p. 182
TDM 5-R, p. 19

Nugent - $(19.5,11.25)$; GOLD. See also (Rock Cr.).

B 662 , p. 433

(Nugget Cr.) or (Gulch) - $(17.5-17.9,12.25-12.35)$; GOLD, TUNGSTEN, BISMUTH.
B 284 , p. 136
B 622 , p. 370

B 328 , p. 213-214

Olsen. See (Anvil Cr.).

(Oregon Cr.) - (17.25-17.65, 12.2-12.65) ; GOLD, TUNGSTEN, BISMUTH.
B 284, p. 136
B 328, p. 211-213
B 622 , p. 370
B 662 , p. 455

B 712 , p. 189

B 933-A, p. 57

TDM 5-R, p. 17

Osborn (e) Min. Co. See (Osborn Cr.).

(Osborn Cr.) - (21.4-21.85, 10.0-11.55) ; GOLD, ANTIMONY, COPPER.
B 259, p. 22
B 263 , p. 53
B 328 , p. 171-172
B 345 , p. 233-234
B 379 , p. 279
B 520 , p. 342
B 533, p. $77,93,97-98,132,134$
B 712, p. 187-188
B 714, p. 233
B 722, p: 63
B 739, p. 9
B 755, p. 14
B 773, p. 27
B 592, p. 387, 389-390
B 783, p. 16, 18
B 622 , p. 370
B 662, p. 452,455
B 813, p. 39 .
B 824, p. 44, 53
B 836, p. 45,54 
(Osborn Cr.)-Continued

B 844-A, p. 46

B 910-A, p. 64-65, 77

B 868-A, p. 49

B 917-A, p. 63-64

B 880-A, p. 52, 62

B 926-A, p. 60, 71

B 897-A, p. 61, 71

B 933-A, p. 56, 68

Osborne Cr. Dred. Co. See (Osborn Cr.), (St. Michael Cr.).

Osborne Dred. Co. See (Osborn Cr.).

Oso. See Galena.

(Otter Cr.) - (20.5, 9.0) ; GOLD.

B 592, p. 387, 390

(Peluk Cr.) - (20.05-20.1, 9.0-9.35) ; GOLD.

B 263, p. 14

B 284, p. 133, 135

B 328, p. 165

B 592, p. 387, 389-390

B 622, p. 367, 372-373

B 933-A, p. 57

(Penny R.) - (17.1-18.2, 9.7-12.6) ; GOLD, PLATINUM(?). Note.-Platinum not confirmed.

B 712, p. 189

B 773, p. 30

Peterson \& Lamoreaux - $(\mathbf{1 9 . 4 5}, \mathbf{1 0 . 4 5})$; GOLD, LEAD, ANTIMONY.

B 662, p. 432,439

B 722, p. 240

Pioneer Co. See U.S. Smelt., Ref. \& Min. Co.

(Pioneer Gulch) - $(19.4-19.45,12.6)$; GOLD.

B 328, p. 183

B 533, p. 76

B 345, p. 233

B 722, p. 253

Pioneer Min. Co. See U.S. Smelt., Ref. \& Min. Co.

Plein Min. \& Dred. Co. See (Otter Cr.).

Poorman. See (Monument Cr.).

(Prospect Cr.) - (19.1-19.3, 11.4-11.5) ; GOLD, TUNGSTEN, LEAD, BISMUTH, COPPER (?).

RI 4174, p. 33-34

TDM 5-R, p. 17, 19, 27, 42

(Quartz Cr.) - $(14.95,10.25)$ approx ; GOLD.

B 328, p. 215

(Quartz Gulch) - $(19.65,10.6)$; ANTIMONY.

B 722, p. 239

Red - $(20.1,10.25)$; GOLD. See also (Dry Cr.).

B 722, p. 236-237

Reinisch - (19.45, 11.15) ; GOLD. See also (Rock Cr.).

B 722, p. 245

Rex - $(20.0,10.25)$; GOLD. See also (Dry Cr.).

B 722, p. 236

(Rock Cr.) - (19.35-19.5, 11.0-11.25) ; GOLD, TUNGSTEN, LEAD, ANTIMONY.

See also Stipek \& Kotovic, Reinisch.

B 263, p. 53

B 284, p. 135

B 722, p. 244, 246-247

B 328, p. 195-196

B 533, p. 75-76, 86

B 773, p. 31

B 783, p. 16

B 662, p. 455,457

RI 4174, p. 32-33

B 712, p. 188

TDM 5-R, p. 42

(Rocker Gulch) - (19.95, 9.05) ; GOLD.

B 592, p. 387, 389

(Rocky Mtn. Cr.) - (20.8-21.05, 13.8-14.1) ; TUNGSTEN, TIN.

TDM 5-R, p. 40, 42

Rowe. See (Snake R.).

593018-61-15 
Royal. See (King Mtn.).

Ruby. See (Twin Mtn. Cr.).

(Rulby Cr.) - (16.5-16.75, 13.9-13.95) ; GOLD.

B 328, p. 216

(St. Michael Cr.) - (21.45-21.6, 9.95-10.0) ; GOLD.

B 533, p. 98

B 880-A, p. 52

B 897-A, p. 61

B 917-A, p. 63

(Saturday Cr.) - $(19.7,9.9)$ approx ; GOLD, TUNGSTEN.

B 328, p. 166-168

B 533, p. 88-89

(Saunders Cr.) - (21.85-21.9, 8.7-9.0) ; GOLD.

B 520, p. 342

B 592, p. 387, 390

Saunders (Cr.) Dred. Co. See (Saunders Cr.).

(Second Beach). See (Beaches, old).

Sioux-Alaska Min. Co. See (Moss Gulch).

(Sledge Cr.) - $(18.75,11.5)$; GOLD, TUNGSTEN.

B 662, p. 454

B 722, p. 249

RI 4174, p. 33

Slisco. See Sliscovich.

Sliscovich - $(20.1,13.7)$; ANTIMONY, GOLD, SILVER. Includes reference to Slisco.

B 314, p. 30, 139
B 345, p. 244-245
B 442, p. 38
B 520, p. 344
B 533, p. 133
B 592, p. 403-404

(Snake R.) - $(19,9)$; GOLD.

B 263, p. 53

B 284, p. 135

B 328, p. 170

B 345, p. 214

B 533, p. 79, 111, 123
B 642, p. 29-30, 71

B 649, p. 50-51, 53-54

B 662 , p. $425-426,437$

B 722, p. 184, 224, 226-227, 229-230

BMB 142, p. 27

TDM 5-R, p. 10

B 714, p. 232-233

B 739, p. 9

B 755, p. 14

B 773, p. 27

B 783, p. 18

(Snow Gulch) - $(19.55-19.65,10.75-10.85)$; GOLD, TUNGSTEN. See also New Era.
B 284, p. 135
B 328, p. 195
B 533, p. 77, 85, 131
RI 4174, p. 33

Snowflake. See (Dexter Hill).

(Sonora Cr.) - (15.5-15.65, 9.9-10.2) ; GOLD.

B 328, p. 215

Sophie. See (Sophie Gulch).

(Sophie Gulch) - $(19.45-19.5,11.15)$; TUNGSTEN, LEAD. Includes references to Sophie.

B 662, p. 433, 436, 457

B 692, p. 21,41

B 739, p. 161

B 722, p. 182, 233-234, 245-246

RI 4174, p. 30-32

(Specimen Gulch). See (Dexter Hill).

(Steep Cr.) - (19.5-19.55, 13.4-13.55) ; GOLD. See albo Nelson. B 533, p. 88

Steiner - $(17.4,10.45)$; GOLD.

B 662, p. 437

B 722, p. 256-258

(Stewart R.) - (17.1-20.0, 14.15-15.05) ; GOLD(?).

B 345, p. 234

B 379, p. 280 
Stipek \& Kotovic - $(19: 4-19.5,11.0-11.1) ;$ GOLD, TUNGSTEN. NotE.-Not mentioned by name in $B$ 662, p. 437.

B 662, p. $432-433,437,457$

RI 4174, p. 31

B 722, p. 244-245

(Submarine Beach). See (Beaches, old).

Sugar. See (Dexter Hill).

Summit. See (Dexter Hill).

Sunrise. See Galena.

(Sunset Cr.) - (SE 1/4 18,10) ; GOLD.

B 783, p. 16

B 864-A, p. 48

B 868-A, p. 49, 59

B 880-A, p. 52, 62
B $897-A$, p. 61,71

B 910-A, p. 64-65, 77

B 917-A, p. 63-64

B 926-A, p. 59, 71

Sunset Mines Corp. See (Sunset Cr.).

(Third Beach). See (Beaches, old).

(Tomboy Cr.) - (13.0-13.35, 14:3-14.35) ; GOLD.

B 328, p. 219

(Tribly Cr.) - $(17.3,12.0)$ approx ; GOLD.

B 328, p. 215

(Tub Mtn.) - (16.9, 13.9) approx ; IRON.

B 662, p. 446

(Tug Gulch). Seee (Lillian Cr.).

(Twin Mtn. Cr.) - (18.7-18.9, 11.7-12.2) ; GOLD, TUNGSTEN, COPPER. Bee also Lilly.

B 328, p. 197

B 722, p. 182, 233, 248-249, 251

B 662, p. 437, 442, 455, 457

TDM 5-R, p. 19, 27

(Union Gulch) - $(20.95,11.1)$; GOLD.

B 328, p. 173

B 533, p. 96

U.S. Smelt., Ref. \& Min. Co. - (18.7-20.0, 9.2-12.18) ; GOLD.

Includes references to Hammon Consol. Gold Fields, Pioneer Min. Co.; see also (Anvil Cr.), (Glacier Cr.), (Rock Cr.,), (Sophie Gulch), (Specimen Gulch), (Twin Mtn. Cr.).

B 755, p. 48

B 773, p. 27, 49

B 783, p. 24

B 792, p. 21, 24

B 797, p. 25, 29-30

B 810, p. 33-34, 39-40

B 813, p. 38-39, 47-48

B 824, p. 44, 52-53

B 836, p. 45, 53-54

B 844-A, p. 46, 53-55
B 857-A, p. 42-43, 50-51

B 864-A, p. 47-48, 56-57

B 868-A, p. 49, 58-59

B 880-A, p. 51-52, 60, 62

B 897-A, p. 60-61, 70-71

B 910-A, p. 64, 75, 77

B 917-A, p. 63, 74-75

B 926-A, p. 59, 70-71

B 933-A, p. 55-56, 67-68

Warren. See (Rock Cr.).

(Washington Cr.) - (15.55-15.65, 12.3-12.4) ; GOLD.

B 328, p. 216

(Washington Gulch) - (21.05-21.15, 9.5-9.7) approx; GOLD. B 533, p. 98

(Waterfall Cr.) - $(18.75,13.1)$; GOLD. See also Christophosen (Waterfall Cr.). B 662, p. 442 B 722, p. 253 
West - $(19.5,10.7)$; GOLD. Includes reference to Golden Eagle.

B 592, p. 401

B 722, p. 241

B 662, p. 434

Widstedt. See (Anvil Cr.), (Quartz Gulch).

Wild Goose Co. See (Anvil Cr.).

Wild Goose Min. \& Trading Co. See (Cooper Gulch).

Williams. See Galena.

(Willow Cr.) - (17.3-17.6, 10.65-11.2) ; GOLD, TIN.

B 622, p. 370

B 692, p. 7, 20

B 662, p. 455

(Windy Cr.) - $(19.55,17.2)$; GRAPHITE.

B 533, p. 136 B 692, p. 363-364

B 662, p. 448-449 B 722, p. 185, 223

Winsted. See (Anvil Cr.).

(Wonder Cr.) - (19.65-19.75, 9.8), approx ; GOLD.

B 442, p. 357-358 $\quad$ B 592, p. 389

B 520, p. 342-343 B 662, p. 454-455

B 533, p. 88

NORTON BAY QUADRANGLE

(Bonanza Cr.) - (9.3-9.4, 9.65-9.85) ; GOLD, ANTIMONY, TUNGSTEN.

B 284, p. 7

B 379, p. 58

B 442, p. 46, 331-333

B 712, p. 22, 50

B 449, p. 105-108

B 480, p. 52, 62

B 910-A, p. 70-71

B 926-A, p. 64, 71

B 649, p. 64

B 933-A, p. 60-61, 68

I-286

B 692, p. 7, 21

(Christmas Cr.) - (10-11, 11-12) (?) ; GOLD(?).

B 442, p. 334

TDM 1, p. 40

(Christmas Mtn.) - (10.6-11.2, 9.7-10.2) ; ANTIMONY, GOLD(?).

B 442 , p. 334

B 449 , p. 108

B 649, p. 64

TDM 5-R, p. 13

(Garryowen Cr.) - (10-11, 14-15) (?) ; GOLD. May be in Candle quad. B 442, p. 334

B 449, p. 108-109

(Hopeful Gulch) - $(9,9)$; BISMUTH, TUNGSTEN.

TDM 5-R, p. 18, 45

(June Cr.) - $(0,15)($ ?) ; GOLD (?). May be in Solomon quad.

B 813, p. $41 \quad$ B 824, p. 46

(Kenwood Cr.) - (3.5-5.0, 16.7-17.55) (?) ; GOLD(?). May be in Candle quad.

B 442, p. 339

B 622, p. 371

B 449 , p. 113

Moon - (12, 12) approx; ANTIMONY.

TDM 5-R, p. 13

Moon and partner. See (Bonanza Cr.).

(Negromoon Cr.) - $(10,15-17)(?)$; GOLD. May be in Candle quad.

B 442, p. 334

B 449, p. 108-109

Nelson. See (Bonanza Cr.).

Shaw and assoc. See (Ungalik R.).

Shaw \& Cook. See (Ungalik R.). 
(Ungalik R.) - (8.8-9.5, 9.15-9.66) ; GOLD.

B 442, p. 333-334

B 926-A, p. 64, 71-72

B 449 , p. 108

B 933-A, p. $60-61,68$

B 917-A, p. 68

Ungalik Syn. See (Bonanza Cr.), (Ungalik R.).

\section{NULATO QUADRANGLE}

(Bishop Cr.). See Perseverance, Valley.

(Kluklaklatna R.) - (7-9, 3-4) (?) ; GOLD. B 410, p. 23

B 868-D, p. 173

Perseverance - $(14.1,8.9)$; SILVER, LEAD.

B 739, p. 39

B 755, p. 16, 43

B 868-D, p. 174-175

B 864-C, p. 227

I-291

(Tlatskokot) - (11.2, 6.85) ; GOLD. I-291

Valley - $(14.1,8.9)$; SILVER, LEAD.

B 739, p. 39

B 864-C, p. 227

\section{B 868-D, p. 175 \\ I-291 \\ OPHIR QUADRANGLE}

Anderson, Pontella, Utilla \& Larson. See (Little Cr.).

(Anvil Cr.) - (19.8-19.9, 2.25-2.35) ; GOLD, MERCURY.

B 755, p. $43 \quad$ B 868-A, p. 38

B 824, p. $35 \quad$ B 897-A, p. 50

B 864-C, p. 190-191

(Bear Cr.) - (22.0-22.4, 9.85-10.S) ; GOLD.

B 755, p. 44

B 933-A, p. 42

B $910-A$, p. 51

C 255, p. 16, 18

(Beaver Cr.) - $(22,10)$ (?) ; GOLD.

B 933-A, p. 42

(Bedrock Gulch) - (SE $1 / 4$ quad.) ( ?) ; GOLD.

B 880-A, p. 42

Bonanza. See (Little Cr.).

(Boob Cr.) - (15.6-15.8, 5.8-6.15) ; GOLD, PLATINUM, MERCURY, TIN.

B 642, p. 251

B 662, p. 23-24, 58

B 797, p. 40

B 666, p. 96

B 810 , p. 53

B 692 , p. 7, 20-21, 349-350

B 813 , p. 60

B 712, p. 23

B 824, p. 67

B 714, p. 71

B 836, p. 69

B 739, p. 158

B 844-A, p. 67

B 754, p. 106,118

B $897-A$, p. 84

B 755, p. 44

B $910-A$, p. 90

B 773, p. 30

BMB 153, p. 2, 54

B 792, p. 33

TDM 1, p. 26,35

(Butte Cr.) - (SW1/4 23,9) ; GOLD.

B 578, p. 38

B 754, p. 101

B 642, p. 249

(Carter Gulch). See Independence.

Collins \& Hard. See (Ophir Cr.). 
(Cripple Cr.) - (22.5-23.2, 10.0-10.6) ; GOLD.

B 542, p. 294, 296

B 578, p. 35, 38

B 622 , p. 62

B 642 , p. 249

B 783, p. 14

B 813, p. 31

B 824, p. 35

B 836, p. 35

B 844-A, p. 36

B 857-A, p. 33
B 864-A, p. 38

B 864-C, p. 170-172

B 868-A, p. 38

B 880-A, p. 42

B 897-A, p. 50

B 910-A, p. 51

B 917-A, p. 49

B 926-A, p. 46

B 933-A, p. 42

C 255, p. 16, 18

Cripple Cr. Min. Co. See (Cripple Cr.).

(Dodge Cr.) - (19.0-19.45, 1.85-3.0) ; GOLD (?).

B 642, p. 65

Erickson. See (Ganes Cr.).

(Esperanto Cr.) - (17.15, 7.9) approx ; GOLD.

$\begin{array}{ll}\text { B } 692, \text { p. } 350 & \text { B } 897-A, \text { p. } 50 \\ \text { B } 754, \text { p. } 107 & \text { B } 910-A, \text { p. 51 } \\ \text { B 755, p. } 44 & \text { B } 917-A, \text { p. } 49 \\ \text { B } 864-\text { C, p. } 193 & \text { B } 926-A, \text { p. } 46 \\ \text { B } 880-\text { A, p. } 42 & \text { B } 933-A, \text { p. } 42\end{array}$

(Ester Cr.) - (19.25-19.3, 0.8-0.9) ; GOLD.

B 864-C, p. 192

Felder \& Gale. See (Yankee Cr.).

Felder-Gale \& Co. See (Yankee Cr.).

Felder, Gale \& Higgins. See (Ganes Cr.), (Little Cr.), (Yankee Cr.).

Flume Dred. Co. See (Little Cr.), (Yankee Cr.).

Fothergill. See (Little Cr.).

(Fox Cr.) or (Gulch) - (22.5-23.2, 10.0-10.6) ; GOLD.

B 578, p. 35, 38

B 592, p. 70
B 642, p. 249

C 255, p. 16, 18

French Hill. See (Ganes Cr.).

Gane, Spencer, Roke \& Maki. See (Ganes Cr.).

(Ganes Cr.) - (19.7-20.45, 0.4-1.7) ; GOLD. Includes references to (Ganes Cr.) under Iditarod quad.

B 345, p. 48

B 379, p. 238-239, 258-263, 265-266

B 410 , p. 21-23, 64, 67-72, 74

B 442, p. 45

B 480 , p. 236, 246-247

B $52^{\circ}$, p. 40

B 542, p. 296-299

B 578, p. 35, 37-38, 40

B 592 , p. 70

B 622 , p. 62

B 642, p. 65, 249-251, 265

B 662, p. 58

B 692 , p. 38

B 714, p. 92

B 722, p. 55

B 739, p. 39

B 754, p. 105-106
B 755, p. 43

B 773, p. 27

B 783, p. 18

B 792, p. 25

B 797, p. 20, 30

B 810, p. 26, 40

B 813, p. 30-31

B 824, p. 35, 52

B 836, p. 35, 54

B 844-A, p. 35, 54

B 864-A, p. 37, 57

B 864-C, p. 184-188

B 868-A, p. 38, 59

B 880-A, p. 41, 61

B 897-A, p. 50, 71

B $910-A$, p. 51,76

B 917-A, p. 49 
(Ganes Cr.)-Continued

B 926-A, p. 45, 71

BMB 142, p. 25

B 933-A, p. 41, 68

BMB 153, p. 54

Ganes Cr. Dred. Co. See (Ganes Cr.).

Ganes Cr. Min. Co. See (Ganes Cr.).

Gibbs \& Eison. See (Ganes Cr.).

Gold. See (Little Cr.).

Gold Run. See (Little Cr.).

Goodluck. See (Cripple Cr.).

(Graham Cr.) - (22.4-22.5, 11.8-12.8) ; GOLD.

B 933-A, p. 42

Greenberg \& Jones. See (Cripple Cr.).

Hard. See (Bear Cr.), (Cripple Cr.), (Ophir Cr.).

Hard and assoc. See (Bear Cr.).

Hard \& Johnson. See (Ophir Cr.).

Hard, Votila \& Hanson. See (Bear Cr.).

Higgins. See (Yankee Cr.).

Holky \& Shonbeck. See (Ganes Cr.).

Holky Dred. Co. See (Ganes Cr.).

Horseshoe. See (Cripple Cr.).

Independence - $(20.4,0.5)$; GOLD. Includes references to (Carter Gulch).

B 379, p. 255

B 578, p. 38

B 410, p. 64,70

B 754, p. 115

B 542, p. 294, 298-299

B $864-$ C, p. 229

(Iron Cr.) - (16.3-16.55, 7.85-8.0) ; GOLD.

B 692 , p. 350

B 754, p. 107

Joaquin. See (Ganes Cr.).

Kaatz. See Kaatz under Iditarod quad.

(Keating Gulch) - $(\mathbf{1 7}, \mathbf{7})(?)$; GOLD. B $917-A$, p. 49

(Last Chance Gulch) - $(19,0)(?)$; GOLD. Note.-Now called Six Gulch. B 379, p. 261

B 410, p. 70

(Little Cr.) - (19.8-20.3, 1.0-1.75) ; GOLD, TUNGSTEN: Includes references to (No. 6 Pup).

B 379, p. 239, 256, 263-265

B 410 , p. $22-23,64,72-74$

B 813, p. 31

B 480, p. 247-249

B 520, p. 40

B 542, p. 296-299

B 578, p. 35-37, 39

B 592 , p. 70

B 622, p. 62

B 642, p. 65, 249-250, 265

B 662, p. 58

B 692, p. 38

B 739, p. 39

B 754, p. 105

B 755, p. 43-44

B 792, p. 18,25

B 797, p. 20,30

B 824, p. 35, 52

B 836, p. 35, 54

B 844-A, p. 35-36

B 857-A, p. 32-33, 51

B 864-A, p. 37-38, 57

B 864-C, p. 181-184

B 868-A, p. 38, 59

B 880-A, p. 41-42, 61

B 897-A, p. 50, 71

B 910-A, p. 51-76

B 917-A, p. 49

B 926-A, p. 45, 71

B 933-A, p. 41,68

BMB 142, p. 25

B 810, p. 26,40

TDM 1, p. 39, 42 
(Little Spruce Cr.). See (Little Cr.), (Spruce Cr.).

McFarland. See (Little Cr.).

(Mackie Cr.) - $(19,0)($ ?) ; GOLD. May be in Iditarod quad. B 864-C, p. 188

(Madison Cr.) - (17.0, 7.8) approx ; GOLD. B 345, p. 48

B 692, p. 350-351

B 755 , p. 44

B 754, p. 107

B 910-A, p. 51

Matheson \& Savage. See (Spruce Cr.).

Meier (\& Berg). See (Ophir Cr.).

Moss \& Larson Min. Co. See (Ganes Cr.).

(No. 6 Pup). See (Little Cr.).

(Ophir Cr.) - (19.3-19.7, 1.65-2.57) ; GOLD.

\begin{tabular}{|c|c|}
\hline B 379, p. $239-240,256,264-265$ & B 797, p. 20 \\
\hline В 410, p. $22-23,64,73-74$ & B 810, p. 26 \\
\hline B 442, p. 45 & B 813, p. 31 \\
\hline B 480, p. $250-252$ & B 824, p. 35 \\
\hline B 520, p. 40 & B 836, p. 35 \\
\hline B 542, p. 296-299 & B $844-A$, p. 36 \\
\hline В 578, p. $35-36,39$ & B $857-A$, p. 33 \\
\hline В 592, p. 70 & B $864-A$, p. 38 \\
\hline B 622, p. 62 & B 864-C, p. 176-179 \\
\hline B 642, p. $65,249-250,265$ & B $868-A$, p. 38 \\
\hline B 662, p. 58 & B $880-A$, p. $41-42$ \\
\hline B 692, p. 38 & B $910-A$, p. 51 \\
\hline B 714, p. 92 & B $917-A$, p. 49 \\
\hline B 722, p. 55 & B $926-A$, p. 46 \\
\hline В 739, p. 39 & B $933-A$, p. 41 \\
\hline B 754, p. 104-105 & BMB 142, p. 25 \\
\hline B 755, p. 43 & BMB 153, p. 54 \\
\hline
\end{tabular}

Paulson. See (Cripple Cr.).

Pelky. See (Ganes Cr.).

Puntila. See (Ganes Cr.), (Little Cr.).

Savage \& Matheson. See (Ganes Cr.).

Schwaesdall \& Vibe. See (Spaulding Cr.).

Shonbeck. See (Ganes Cr.).

(Spaulding Cr.) - (SE 1/4 quad.) (?) ; GOLD.

B 880-A, p. 42

B 917-A, p. 49

B 897-A, p. 50

B 926-A, p. 46

B 910-A, p. 51

(Spaulding Gulch) - (SE 1/4 quad.) (?) ; GOLD.

B 880-A, p. 42

Speljack. See (Ganes Cr.), (Little Cr.).

(Spruce Cr.) - (19.7-20.0, 1.7-2.25) ; GOLD.

B 379, p. 239, 264

B 410, p. 22, 72-73

B 642, p. $65,249-250,265$

B 480 , p. 249-250

B 662, p. 58

B 520, p. 40

B 692, p. 38

B 542, p. 296-297, 299

B 722 , p. 55

B 578, p. 35-36, 39

B 754, p. 105

B 592, p. 70

B 797, p. 20

B 622 , p. 62

B 810 , p. 26

B 813, p. 31 
(Spruce Cr.)-Continued

$\begin{array}{ll}\text { B } 824, \text { p. } 35 & \text { B } 897-A, \text { p. } 50 \\ \text { B } 857-A, \text { p. } 33 & \text { B } 926-A, \text { p. } 46 \\ \text { B } 864-A, \text { p. } 38 & \text { B } 933-A, \text { p. } 41 \\ \text { B } 864-\text { C, p. } 179-181 & \text { BMB 142, p. 25 } \\ \text { B } 868-\text { A, p. } 38 & \text { BMB 153, p. } 54\end{array}$

B $880-A$, p. 42

Stayton. See (Ophir Cr.).

Strandberg \& Co. See (Cripple Cr.).

Strandberg \& Ohlson. See (Cripple Cr.).

Strandberg \& Sons. See (Fox Gulch.)

(Tamarack Cr.) - $(19.85,1.95)$ approx ; GOLD.

B $864-C$, p. 180

Three Miners, Inc. See (Esperanto Cr.), (Keating Gulch).

(Tolstoi Cr.) - (14-15, 0-6) ; GOLD( ?).

B 642, p. 251

Uotila \& Hard. See (Ophir Cr.).

Vibe. See (Spruce Cr.), (Yankee Cr.).

Vibe and assoc. See (Little Cr.).

Vibe \& Schwaesdall. See (Spaulding Cr.).

(Victor Gulch) or (Cr.) - $(19.95,2.35)$; GOLD, MERCURY.
B 722, p. 55
B 739, p. 39
B 797, p. 20
B 810, p. 26
B 824 , p. 35
B 857-A, p. 33

B 864-A, p. 38

B 864-C, p. 176, 191

B 868-A, p. 38

B 880-A, p. 42

B $897-A$, p. 50

Warner and assoc. See (Cripple Cr.).

White Mule. See (Cripple Cr.).

Wilson \& Hard. See (Cripple Cr.).

(Yankee Cr.) - (20.75-21.0, 0.4-0.7) ; GOLD. Includes references to (Yankee Cr.) under Iditarod quad.

\begin{tabular}{|c|c|}
\hline B 410, p. 74 & B 810, p. 26,40 \\
\hline B 480, p. 252-253 & B 813, p. $30-31,47$ \\
\hline B 520, p. 40 & B 824, p. 35,52 \\
\hline В 542, p. $296,298-299$ & B 836, p. 35,54 \\
\hline B 578, p. $35,38-40$ & B $844-A$, p. 54 \\
\hline B 592, p. 70 & B $857-A$, p. $32-33$ \\
\hline B 622, p. 62 & B $864-$ A, p. $37-38,57$ \\
\hline B 642, p. $65,249,251,265$ & B $864-$ C, p. $188-190$ \\
\hline B 662, p. 58 & B $868-$ A, p. 38,59 \\
\hline B 692, p. 38 & B $880-A$, p. 41,61 \\
\hline B 722, p. 55 & B $897-A$, p. 50,71 \\
\hline B 739, p. 9,39 & B $910-A$, p. 51,76 \\
\hline B 754, p. 106 & B $917-A$, p. 49 \\
\hline В 755 , p. $14,43-44$ & B $926-A$, p. 45,71 \\
\hline В 773, p. 27,31 & B $933-A$, p. 41,68 \\
\hline B 783, p. 14,18 & BMB 142, p. 25 \\
\hline B 792, p. 25 & BMB 153, p. 54 \\
\hline B 797, p. 20,30 & \\
\hline
\end{tabular}




\section{PETERSBURG QUADRANGIE}

Alaska Chief. See (Shakan).

Alaska Garnet (Min. \& Mfg.) Co. - (15.85, 10.35) ; GARNET. Includes references to unnamed garnet occurrences in area.
B 347, p. 92
B 714, p. 55
B 480, p. 42
B 739, p. 73-74
B 542, p. 51
B 800, p. 316-317, 333, 363
B 622 , p. 44
B 642, p. 104
B 936-C, p. 81-83, 88-91
IC 7844, p. 11

Alaska Juneau Gold Min. Co. See (Shakan).

Alaska Marble Co. See (Calder), (Dry Pass).

Alaska Treadwell Gold Min. Co. See (Castle Is.), (Shakan).

Basin. See (Groundhog Basin).

Beckett. See (Taylor Cr.).

Berg (, Berg \& Wedow). See (Berg Basin).

(Berg Basin) - (19.3, 8.05) ; SILVER, LEAD, ZiNC, GOLD, COPPER.
B 739, p. 57-58, 67
B 800, p. 361
B 998-B, p. 47, 49, 51-55
C 252, p. 6

(Blake Channel) - (19.2, 6.65) ; MARBLE.
B 542, p. 73
B 682, p. 90, 92-93, 114

(Blashke Is.) - (SE 1/4 10, 2) ; PLATINUM, COPPER.

B 947-D, p. 78

Bon Alaska ( Min.) Co. See (Groundhog Basin), Lake.

Browne. See Alaska Garnet Min. \& Mfg. Co.

(Buck Bar) - (16-19,12) ( ?) ; GOLD.

B 773, p. 20

Buckhorn. See Northern Copper Co.

(Calder) - (5.3, 3.1) ; MARBLE. Includes references to Alaska Marble Co., (Marble Cove), (Marble Cr.), (Shakun), marble near Shakan; see also (Dry Pass).
P 1, p. 111
B 542, p. 56-58
B 284, p. 56
B 642 , p. 100
B 314, p. 75-76
B. 682 , p. 59-62
B 345, p. 117-119
B 783, p. 59, 61
B 347, p. 192-195
IC 7379, p. 80
B 379, p. 84
IC 7844, p. 7
B 480, p. 42
TDM 6, p. 29, 65-66

(Calder Bay) - $(4.8,3.5)$; LIMESTONE.

TDM 6, p. 29, 67-69

Castle \& Co. - $(5.35,2.35)$ approx ; GOLD.

P 1, p. 111

(Castle Is.) - (8.1, 11.4) ; BARIUM, ZINC, SILVER, LEAD. Includes references to (Duncan Canal) (barium).

B 592, p. 109-113, 116-117

B 714 , p. 54

B 739, p. 72-73

B 755, p. 24

B 773, p. 138

B 800, p. 318,333

B 844-A, p. 81

IC 7379, p. 77

IC 7844, p. 9

Copper: Bell. See Northern Copper Co.

Crystal Lead. See (Taylor Cr.). 
(Dry Pass) - (5.3-5.45, 2.75-3.0) ; MARBLE. See also (Calder).
B 542 , p. 58
B 682 , p. $62-63$
B 642, p. 100

(Duncan Canal) (barium). See (Castle Is.).

(Duncan Canal) (marble) - $(8.6,10.2)$ approx ; MARBLE.

B 682 , p. 56

(Duncan Canal) (pyrite) - (7.1, 11.75) approx; PYRITE.

B 739, p. 70

East Side. See (Red Bay).

(El Capitan). See El Capitan Marble Co.

El Capitan Marble Co. - $(6.4-6.45,2.75-3.05)$; MARBLE. Includes reference to (El Capitan).
B 284, p. 56
B 542, p. $58-59$
B 314 , p. 76
B 682, p. $63-64$
B 345, p. $119-120$
B 783, p. 59
B 347, p. 195-196

(El Capitan Passage) - (6.5-6.8, 2.7-2.8) ; MARBLE.
B 542, p. 59
B 682 , p. 64-65

Exchange - (14.25, 7.45) ; GOLD.

B 347, p. 185

(Exchange Cove) - $(9.0,3.8)$; LIMESTONE.

TDM 6, p. 29, 69, 71-72

Galvin. See Lake.

Garnet. See Alaska Garnet Min. \& Mfg. Co.

(Garnet Cr.). See Alaska Garnet Min. \& Mfg. Co.

General Grant. See (Groundhog Basin).

General Lee. See (Groundhog Basin).

General Logan. See (Groundhog Basin).

General Sherman. See (Groundhog Basin).

Georgia. See (Glacier Basin).

(Glacier Basin)-(19.1, 8.5) ; LEAD, ZINC, IRON, COPPER, FLUORITE, SILVER(?), GOLD (?).
B 284, p. 53
B 314, p. 72
B 345 , p. 97
B 347, p. 189
B 739 , p. 57-58, 66-67

B 800, p. 327, 337, 361-362

B $998-$ B, p. $29-40$

C 252, p. 6

IC 7844, p. 12

Grant and assoc. See (Groundhog Basin).

Grant, Blackburn, Grant \& Sinclair. See (Groundhog Basin).

(Groundhog Basin) - (18.65-18.75, 9.15-9.25) ; ZINC, LEAD, SILVER, GOLD, MOLYBDENUM, COPPER.
B 259 , p. 61
B 284, p. 53
B 314, p. 72
B 347, p. 189
B 642 , p. $78,98-99$
B 662, p. $74-75$
B 739, p. 57-63
B 836, p. 81 .
B $926-$ C, p. 172
B 947-B, p. 37-38
B $998-$ B, p. 15, 17-29, 33, 37, 40
C 252, p. 6
IC 7379, p. 41
IC 7844, p. 12
B 800 , p. $318,328,361$
Harvey - (9.7-10.3, 10.7-14.2) (?) ; COPPER.
B 622, p. 44 
Hattie - $(9.2,9.45)$; GOLD, SILVER, COPPER, LEAD, ZINC.
B 259 , p. 59
B 379, p. 73
B 347, p. 182-184

Heid. See (Taylor Cr.).

Helen S. - (9.0-9.1, 9.9-10.25) ; GOLD, LEAD, ZINC. Includes references to Smith.
B 259, p. 59
B 379, p. 73
B 314, p. 63,72
B 642 , p. 78
B 345, p. 91
B 739, p. 67
B 347, p. 184

Idaho. See Northern Copper Co.

Johnson \& Oleson. See (Groundhog Basin).

Kupreanof (Min. Co.). See Northern Copper Co.

Lake - $(18.45,8.5)$; LEAD, ZINC, SILVER, COPPER. Includes references to Lake Virginia Min. Co., Margery.
B 259, p. 61
B 792, p. 30
B 347, p. $189-190$
B 800, p. 327,362
B 739, p. 57-58, 63-65
B 810 , p. 14
B 783, p. 23,41
B $998-B$, p. $41-46$

(Lake Virginia) - (17.9-18.0, 8.4-8.5) ; MARBLE.
B 682, p. 91-92
B 783, p. 61

Lake Virginia Min. Co. See Lake.

Lead. See (Taylor Cr.).

McGill. See (Pt. St. Albans).

Maid of Mexico (Min. Co.) - (9.3, 9.8) approx; SILVER, xCLD, ZINC, LEAD, COPPER.
B 642, p. 78
B 844-A, p. 16
B 662 , p. $73-74$
B 864-A, p. 17
B 739, p. $63,67-68$
B 926-A, p. 20

B 824 , p. 18

Maid of Texas - (9.3, 9.8) approx; GOLD(?), SILVER(?).

B 662 , p. 74

(Marble Cove). See (Calder).

(Marble Cr.). See (Calder).

Margery. See Lake.

Montana. See Northern Copper Co.

Mt. Berg - $(18,7)$; SILVER, LEAD.

B 379, p. 84

Mt. Wedar - $(18,7)$; SILVER, LEAD.

B 379, p. 84

(Mud Bay) - $(9.8,4.3)$; LIMESTONE.

TDM 6, p. 29, 72, 74-75

Nelson \& Smith. See (Glacier Basin).

Northern Copper Co. - $(6.3,15.5)$ approx; COPPER, GOLD, SILVER, ZINC,

IRON. Includes reference to Kupreanof; not mentioned by name in B 345 .

B 314, p. 72

B 739, p. 21, 70-72

B 345, p. 91

B 963-A, p. 37-38

B 347, p. 141-142

Olympic Min. Co. See Hattie, Helen S.

Oregon. See Northern Copper Co.

Paystreak. See (Salmon Bay). 
(Piledriver Bay) - $(10.4,4.0)$; LIMESTONE.

TDM 6, p. 29, 75-76

(Pitcher I.) See (Salmon Bay).

(Pt. Colpoys) - (7.25-7.75, 5.8-5.9) ; MARBLE. B 682, p. 56-57

(Pt. St. Albans) - $(0.4,1.8)$ approx; ZINC. Includes reference to McGill. B 1058-A, p. 24, 27

(Port Protection) - $(4.0,4.95)$ approx ; LIMESTONE. B 682, p. 59

(Portage Mtn.) - (7.15, 14.9) ; SILVER, GOLD, COPPER, PLATINUM. Includes references to Silver Star.
B 259, p. 60
B 314, p. 72
B 379, p. 73
B 739, p. 69
B 347, p. 141

Portage Mtn. Min. Co. See Northern Copper Co. ; (Portage Mtn.).

(Pyramid Peak) - $(6.2,0.8)$ approx; MARBLE. B 542, p. 59

(Red Bay) - (6.35-6.8, 4.2-5.7) ; MARBLE.

$\begin{array}{ll}\text { B 542, p. 55 } & \text { B 682, p. 57-59, } 113 \\ \text { B 642, p. } 100 & \text { B 783, p. 59, 61 }\end{array}$

Ruby. See Alaska Garnet Min. \& Mfg. Co.

(St. John Harbor) - (10.0, 7.5) approx; PYRITE. B 739, p. 69-70

(Salmon Bay) - (8.1-8.65, 4.6-5.6) ; RARE EARTHS, COPPER. B 1058-A, p. 6-12, 19-23 C 248, p. 9-10

Schoonover. See (Taylor Cr:).

(Shakan) - $(5.3,2.35)$; MOLYBDENUM, COPPER, ZINC.
B 692, p. 23, 28, 85-89
B 712, p. 23, 29
B 926-C, p. 168-171
B 714, p. 41, 118-119
B 947-B, p. $19-30$
B 722, p. 24
B 1058-A, p. 5, 24, 27
B 739, p. 21
B 761, p. 14-15
B 800, p. 317, 330, 345, 348
C 248, p. 9-10
IC 7379, p. 69
IC 7784, p. 10
IC 7844, p. 12

Shakun. See (Calder).

Silver King. See (Berg Basin), (Duncan Canal), (Groundhog Basin).

Note.-Three different claims.

Silver Star. See (Portage Mtn.).

Skyrus. See (Red Bay).

Smith. See Helen S.

Smith, Pitcher \& Co. See (Salmon Bay).

Spaulding. See (Blake Channel).

(Taylor Cr.) - $(6.1,13.95)$; LEAD, ZINC, SILVER, Cr,PPER.

B 347, p. 142

RI $4669 ;$ p. $2-13$

IC 7844, p. 12

(Thomas Bay) - (11.6, 17.4) ; GOLD, SILVER, LEAD, COPPER. B 739, p. 63, 68-69.

('Towers Arm) or (Bay) (limestone) - $(6.3,14.3)$; LIMESTONE. TDM 6, p. 29, 81, 83-84

(Towers Bay) (metals). See Northern Copper Co.

Treasure Box. See Northern Copper Co. 
Tuscarora. See Northern Copper Co.

Ventures, Ltd. See (Groundhog Basin).

Vermont Marble Co. See (Pyramid Peak), (Red Bay).

Walker \& Kiss. See (Taylor Cr.).

Wandve. See (Salmon Bay).

Washington. See Northern Copper Co.

Woodbridge \& Lowery. See (Pt. Colpoys), (Red Bay).

(Zarembo I.) - $(10.3,5.0)$ approx ; FLUORITE.

B 739, p. 75

\section{PORT ALEXANDER QUADRANGLE}

Barrows. See (Cornwallis Peninsula).

Bauer - $(8.75,16.9)$ approx ; GOLD. Includes references to Haley \& Rogers.
AR 18, p. 63, 79
B 504, p. 27-28

B 259, p. 58

Bullion - (8-9, 16-17) (?) ; GOLD(?). May be in Sitka quad.
B 259, p. 58
B 504, p. 29

Cache - $(8.9,17.0)$ approx; GOLD, SILVER. Includes reference to Stewart.
AR 18, p. 62-63, 79-80
B 314, p. 60
B 259, p. 57-58
B 504, p. 27
B 284, p. 45
IC 7844, p. 7

Comstock. See (Cornwallis Peninsula), (Kuiu I.).

(Cornwallis Peninsula) or (Bay) - (17.2-17.4, 16.2-17.45) ; BARIUM. See also (Kuiu I.).

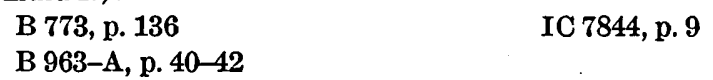

Eureka - $(8.1,17.0)$; COPPER, GOLD (?).

AR 18, p. 62, 79

Free Gold - $(9.25,16.75)$ approx ; GOLD (?).

$$
\text { B } 259 \text {, p. } 58
$$

Haley \& Rogers. See Bauer.

B 504, p. 29

Hanlon \& Haley. See (Redfish Bay).

Hanlon \& Morgan. See (Redflsh Bay).

Hill - (9.8-10.55, 15.85-16.9) ; CHROMITE. B 936-G, p. 173, 179-180

Hofstad. See (Snipe Bay).

Hungerford. See (Cornwallis Peninsula), (Keku Islet) (barium).

(Keku Islet) (barium) - (18.35-18.45, 15.9-15.95) ; BARITE. See also (Kuiu I.).

B 773, p. 136

B 963-A, p. 40-41, 43-44

(Keku Islet ) (metals) - $(\mathbf{1 7 . 9}, \mathbf{1 6 . 5})$; ZINC, SILVER.

B 773, p. 138-139

(Keku Strait) - $(16.05,15.95)$; LIMESTONE.

B 783, p. 60-61

(Kuiu I.) - (16.4-18.45, 15.4-16.45) ; BARIUM. Includes reference to barite near Keku Strait; see also (Cornwallis Peninsula), (Keku Islet) (barium), (Saginaw Bay) (barium).

B 773, p. 72, 138

B 844-A, p. 81-82

B 800, p. 317

Lower Ledge - $(8.85,17.0)$ approx ; GOLD.

B 259, p. 58

B 504, p. 29 
Lucky Chance - $(9.25,16.7)$ approx ; GOLD, SILVER.
AR 18 , p. $62-63,79-80$
B 314, p. 60
B 259 , p. 58
B 504, p. 29

B 284, p. 45

(Port Conclusion) - (12.8-13.2, 4.4-5.0) ; GOLD ( ?)

B 314, p. 60

(Port Lucy) - (12.1-13.2, 5.1-6.3) ; GOLD(?)

B 314, p. 60

(Red Bluff Bay) - (12.25-12.5, 14.85-15.3) ; CHROMITE.
B $880-A$, p. 88
B 947-D, p. 73-75

B $897-$ A, p. 98

C 252 , p. 11

B $936-G$, p. $173,178-187$

(Redfish Bay) - $(10.9,6.35)$; FELDSPAR, QUARTZ, MICA, GOLD( ?), TIN (?). B 1024-G, p. 141-152

(Saginaw Bay) (barium) - (16.4-17.1, 15.4-16.05) ; BARIUM. See also (Kuiu I.) B 773 , p. $72,136-138$ IC 7844, p. 9

(Saginaw Bay) (limestone) - (16.9-17.6, 15.6-15.8) ; LIMESTONE.

B 783 , p. 61

TDM 6, p. 29, 79-81

Silver Bay - $(8.25,17.4)$; GOLD ( ?)

B 259 , p. 58

B 314, p. 60

B 284, p. 45

B 504, p. 29

(Silver Bay) - (8-9, 16-17) ; GOLD(?), SILVER(?). See also (Silver Bay) under Sitka quad.

B 345 , p. 91

B 379 , p. 73

(Snipe Bay) - $(10.2,7.55)$; NICKEL, COPPER.

B 773, p. $31,37,72,95,106-107$, B 936-M, p. 321-330 110,113

B 800, p. $337,348-351$

C 252 ; p. 7

B $897-$ A, p. 98

Stewart. See Cache.

Vevelstad. See (Snipe Bay).

PORT MOLLER QUADRANGLE

Apollo (Consol.) - (14.35-14.4, 3.45) ; GOLD, LEAD, ZINC, COPPER. See also (Unga I.).
AR 18, p. 62-63, 83-85
B 520, p. 28
B 259, p. 100-101
B 542, p. 38
B 284, p. 6
B 622, p. 47
B 345, p. 28-29
B 379 , p. $149-150$
B 662, p. 49-50
B 394, p. 196, 199
B 755, p. 33
B 442, p. 34
B 844-A, p. 24
B $926-A$, p. 28
B 467, p. $21,125-126$
IC 7379, p. 22

B 480 , p. 33, 66

(Balboa Bay) - (14.1, 10.45) ; COPPER.

B 379, p. 152

B 714, p. 35

B 467, p. 21, 129

Herman (\& Duchen). See (Popof I.).

King. See Sitka.

(Mt. Hague) - $(0.4,6.5)$; SULFUR.

B 1028-A, p. 15 
(Popof I.) - (14.85-15.05, 5.5-5.6) ; GOLD.
B 259, p. 101
B. 542, p. 43
B 379, p. 52, 149
B 622, p. 47
B 467, p. 20,125
B 773, p. 23
B 520, p. 37
B 824, p. 23

(Port Moller) - $(17.2,13.8)$; GOLD.

B 467, p. 128

(Sand Pt.). See (Popof I.).

Shumagin - $(14.2,4.15)$; GOLD.

B 259, p. 101

B 467, p. 21, 125, 127

B 379, p. 149,151

Sitka - $(14.5,3.65)$; GOLD, LEAD, ZINC. Includes references to King.

B 259, p. 101

B 467, p. 21, 125-127

B 379, p. $149-151$

B 926-A, p. 28

(Unga I.) - (12-14, 2-5) ; GOLD. See also Apollo.

B 520, p. 37

B 824, p. 23

\section{PRINCE RUPERT QUADRANGLE}

B. C. Mica Mines, Ltd. See (Sitklan I.), (Sitklan Passage).

Blasher, Ater, Pearson \& O'Leary. See (Sitklan I.), (Sitklan Passage).

Hyder Mica No. 1. See (Sitklan I.).

Last Chance. See (Sitklan Passage).

(Sitklan I.) - $(13.45,13.5)$; MICA, FELDSPAR.

B 1024-G, p. 141, 143, 154-161

(Sitklan Passage) - (13.2, 13.6) ; MICA, FELDSPAR.

B 1024-G, p. 141, 143, 154-156,

159-161.

\section{RAT ISLANDS QUADRANGLE}

(Little Sitkin I.) - $(5.9,12.25)$; SULFUR.

B 1028-H, p. 205-206

\section{RUBY QUADRANGLE}

(American Cr.) - (18.25, 2.7) ; GOLD.
B 642, p. 220
B 667, p. 51

(Baker Cr.) : $(22.8,4.85)$; GOLD.
B 642 , p. 220
B 667, p. 51

(Banner Cr.) - (3.0-3.5, 3.45-4.0) ; GOLD.
B 642 , p. 244
B 754, p. 95

(Basin Cr.) - (3.0-3.35, 6.95-7.3) ; GOLD. See also (Swift Cr.), (Willow Cr.). B 642, p. 243,265

(Bear Gulch) or (Pup) - (3.95-4.15, 7.0-7.25) ; GOLD, SILVER, TIN.

B 520 , p. 291

B 797, p. 23

B 542, p. 289, 291-292

B 824, p. 37

B $578, \mathrm{p} .41,44$

B 836 , p. 38

B 592, p. $365-366$

B 844-A, p. 38

B 622 , p. 57

B 857-A, p. 34

B 642, p. 241,265

B 864-C, p. 146, 148-151

B 662 , p. 58

BMB 153, p. 53 
(Beaver Cr.) (lode) - $(5.2,10.45)$; SILVER, LEAD, GOLD. Includes reference to (New York Cr.).
B 722, p. 54-55
B $868-D$, p. $175-176$
B 783, p. $145-150$
I 289
B 864-C, p. 226-227
C 279, p. 1, 3-4

(Beaver Cr.) (placer) - $(2.1,1.55)$ approx; GOLD.
B 836, p. 38
B 857-A, p. 34
B $844-A$, p. 38
B 864-C, p. 169

(Big Cr.) - $(4-5,12)$; GOLD, TIN.
B 379, p. 232-233
B 836, p. 38,70
B 410, p. 79
B 864-C, p. 144-145
B 520 , p. 294
I 289
B 813, p. 34,62
TDM 1, p. 34
B 824, p. 37

(Birch Cr.) - (4.85-5.05, 7.8-8.3) ; GOLD, SILVER, TIN, BISMUTH.

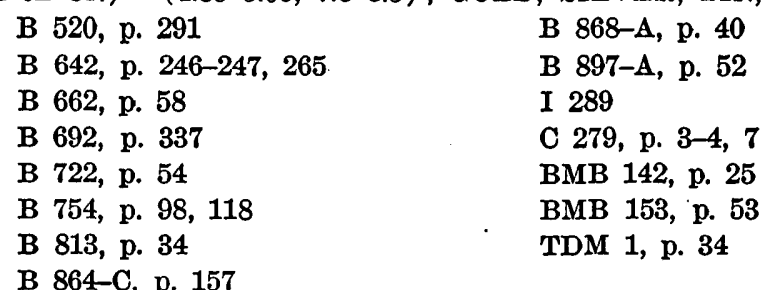

Bluebell. See (Poorman Cr.).

(Boston Cr.) - $(3,11)$; GOLD(?).

B 410 , p. 79

(Boston Gulch) - $(3.7,11.0)$ approx; GOLD(?).

B 410 , p. 79

Buckeye. See (Long Cr.).

Burke. See (Long Cr.).

Collins. See (Tamarack Cr.).

Coyle (\& Jensen). See (Poorman Cr.).

(Cox Gulch) or (Pup) - $(3-5,11-12)$; GOLD, TIN. Note.-Trib. Big Cr. B 810, p. 29,54 B 836, p. 70

B 824, p. $68-69$ TDM 1, p. 34

(Crooked Cr.) - (5.0, 7.9) approx; GOLD(?).

B 864-C, p. 157-158

Dakota. See (Long Cr.).

Deacon. See (Long Cr.).

(Duncan Cr.) - $(3.8,1.6)$; GOLD.
B 592, p. 68, 368-369
B 910-A, p. 52
B 622, p. 58
B 810, p. 29
B 917-A, p. 51
B 864-C, p. 163
BMB 142, p. 25
BMB 153, p. 53

Eagle. See (Monument Cr.).

(Easy Money Cr.) - $(6.8,7.95)$ approx; GOLD(?).

B '864-C, p. 158

(Eldorado Cr.) (trib. Flint Cr.) - $(5.2,6.9)(?)$; GOLD(?).

B 864-C, p. 158

(Eldorado Cr.) (trib. Poorman Cr.) - (2.15-2.7, 1.2-2.0) ; GOLD.

B 836, p. 38

$593018-61-16$ 
(Elk Cr.) - (0-6, 0-12) ; GOLD.

B 662 , p. 58

Emil. See (Long Cr.).

Fernander \& Johnson. See (Long Cr.).

Ferres. See (Poorman Cr.).

(Flat Cr.) (trib. Long Cr.) - (3.35, 5.8) ; GOLD (?).

B 864-C, p. 146

(Flat Cr.) (trib. Timber Cr.) - (3.25-3.5, 0.95-1.2) ; GOLD, SILVER, TIN.

B 642, p. 248, 260, 265

B 662, p. 57

B 692 , p. 337

B 714, p. 91

B 739, p. 38

B 754, p. $99-100,118$

B 755, p. 43

B 797, p. 23

B 810, p. 29

B 824, p. 37

B 836, p. 38

B 844-A, p. 38

B 857-A, p. 34

B 864-A, p. 40

B 864-C, p. 166-167

B 868-A, p. 40

B 897-A, p. 53

B 910-A, p. 51-52

B 917-A, p. 50-51

B 926-A, p. 49

B 933-A, p. 46

BMB 142, p. 25

BMB 153, p. 53

TDM 1, p. 35

(Flint Cr.) - (5.25-6.55, 7.45-8.45) ; GOLD.
B 520, p. 291, 295
B 868-D, p. 173
B 754, p. 97-98
C 279, p. 4, 8

B 864-C, p. 148-149, 157-158

Forno (\& Coyle). See (Poorman Cr.).

(Fourth of July Cr.) - (3.9-3.95, 6.75-6.8) ; GOLD.

B 642, p. 244

B 662, p. 57

(Glacier Cr.) - (4.5-5.6, 11.4-12.5) ; GOLD, BISMUTH. B 864-C, p. 145

C 279, p. 3

(Glen Gulch) - (4.75-5.15, 7.1-7.15) ; GOLD.

B 263, p. 53, 208

B 520, p. 291, 295

B 592, p. 366

B 542, p. 288, 290-292

B 622, p. 57-58

B 578, p. 40-42, 44-45

B 642, p. 246, 265

B 864-C, p. 158

Gold. See (Poorman Cr.).

(Gold Run) (trib. Flint Cr.) - (5.15, 7.0(?) ; GOLD( ?). B 864-C, p. 158

(Gold Run) (trib. Sulatna R.) - (2.0-3.4, 2.4-4.2) ; GOLD(?). B 642, p. 244

B 754, p. 95

(Granite (Cr.) - $(4.8,6.0)$ approx ; GOLD, PLATINUM.

B 783, p. $25 \quad$ B 836, p. 69

B 792, p. 33 B $844-$ A, p. 67

B 797, p. 40 B 864-C, p. 158

B 810, p. 53 B $897-$ A, p. 84

B 813, p. 60 B 910-A, p. 90

B 824, p. $67 \quad$ TDM 1, p. 20

Greenstone. See (Greenstone Cr.).

(Greenstone Cr.) - (3.7-3.95, 5.1-5.25) ; GOLD, SILVER, TIN. Includes references to Greenstone.

B 592, p. 367

B 622, p. 57

B 662, p. 57

B 642, p. $65,243,260$

B 692, p. 38, 337

B 712, p. 48-49 
(Greenstone Cr.)-Continued

$\begin{array}{ll}\text { B 754, p. 94, 117-118 } & \text { B 897-A, p. 52 } \\ \text { B 797, p. 23 } & \text { B 910-A, p. 51-52 } \\ \text { B 810, p. 29 } & \text { B 917-A, p. 50-51 } \\ \text { B 813, p. 34 } & \text { B 926-A, p. 49 } \\ \text { B 824, p. 37 } & \text { B 933-A, p. 46 } \\ \text { B 836, p. 38 } & \text { I-289 } \\ \text { B 844-A, p. 38 } & \text { C 279, p. 4 } \\ \text { B 857-A, p. 34 } & \text { BMB 142, p. 25 } \\ \text { B 864-A, p. 40 } & \text { BMB 153, p. 2, 53 } \\ \text { B 864-C, p. 146, 152-153 } & \text { TDM 1, p. 34 } \\ \text { B 868-A, p. 40 } & \\ \text { reenstone Gulch) - (3.8-3.9, 5.25-5.3) ; GOLD. } \\ \text { B 714, p. } 91\end{array}$

Hard Times. See (Solomon Cr.).

Hitt. See (Moose Cr.), (Poorman Cr.).

Jensen and assoc. See (Poorman Cr.).

(Ketchum Cr.). See (Meketchum Cr.).

Keystone. See (Long Cr.).

Knoll. See (Poorman Cr.).

(Little Pup) or (Gulch) - (3.55-3.6, 1.5-1.6) ; GOLD.

B 622, p. 58

B 754, p. 246

B 642, p. 247

B 864-C, p. 163

(Logger Gulch) - $(4.0,11.0)$ approx ; GOLD (?).

B 410 , p. 79

Lone Pine. See (Long Cr.).

(Long Cr.) - (3.5-4.05, 6.5-7.4) ; GOLD, SILVER, TIN.

B 520, p. 291, 296

B 542, p. 283, 288-291

B 578, p. 40-42, 44-45

B 592, p. 364-365

B 622, p. 57

B 642, p. 65, 239-242, 265

B 662, p. 57

B 692, p. 38, 337

B 722, p. 54

B 739, p. 38

B 754, p. 91-95

B 797, p. 23

B 813, p. 34

B 836, p. 38

B 844-A, p. 38
B 857-A, p. 34

B 864-A, p. 40

B 864-C, p. 146-151

B 868-A, p. 40

B 868-D, p. 173

B 880-A, p. 44

B 897-A, p. 52-53

B 910-A,p. 51-52

B 917-A, p. 50-51

B 926-A, p. 49

B 933-A, p. 46

I-289

BMB 142, p. 25

BMB 153, p. 53

TDM 1, p. 34

Long Cr. Min. Co. See (Long Cr.).

Lucky. See (Solomon Cr.).

(Lucky Cr.) - (4.7-4.95, 7.4-7.85) ; GOLD. Also called Crooked Cr. B 592, p. 367 B 864-C, p. 158

Mascot. See (Long Cr.).

(Meketchum Cr.) - $(4.4-4.5,3.4-3.45)$; GOLD. Includes reference to (Ketchum Cr.).
B 692, p. 38
B 824, p. 37
B 857-A, p. 34
B 836 , p. 38
B 864-A, p. 40
B 844-A, p. 38
B 864-C, p. 155
B $868-\mathrm{A}$, p. 40 
(Midnight Cr.) - (3.5-3.95, 5.35-5.7) ; GOLD, TIN, TUNGSTEN.
B 520, p. 291, 296
B 692, p. $20,38,337$
B 542, p. 287-292
B 754, p. $94,117-118$
B 578, p. $41-42,44-45$
B 864-C, p. 146, 152
B 592, p. 366
BMB 153, p. 53
B 622, p. 57
RI 4174, p. 28
B 642, p. 242-243, 260, 265
TDM 1, p. 34
B 662, p. $57-58$
TDM 2, p. 20

Monaghan. See (Poorman Cr.).

Monaghan, Stevens \& Lohr. See (Timber Cr.).

(Monument Cr.) - (4.45-4.7, 4.5-4.7) ; GOLD, SILVER, TIN.
B 592, p. 367
B 810, p. 29
B 622, p. 57
B 864-A, p. 40
B 642, p. 243-244, 260, 265
B $864-$ C, p. 153-154
B 692 , p. 337
C 279, p. 4
B 754, p. 117-118
TDM 1, p. 34

(Moose Cr.) - (1.55-1.7, 0.65-0.85) ; GOLD, SILVER.
B 824, p. 37
B $868-\mathrm{A}$, p. 40
B $836, p .38$
B 880-A, p. 44-45
B 844-A, p. 38
B $897-$ A, p. 53
B $857-A$, p. 34
B 910-A, p. 52
B 864-A, p. 40
B 917-A, p. 51
B 864-C, p. 168-169
B 926-A, p. 50

(Nevada Cr.) - (2.4-2.9, 1.4-2.3) ; GOLD.

B 836, p. 38

(New York Cr.). See (Beaver Cr.) (lode).

Novikikat. See (Long Cr.).

Nuahmah. See (Flint Cr.).

Odergaard \& Ross. See (Moose Cr.).

(Ophir Cr.) - (3.95-5.25, 3.65-4.9) ; GOLD.

B 592, p. 367-368

I 289

(Poorman Cr.) - $(2.85-3.65,1.25-1.85)$; GOLD, TIN.
B 592, p. 68, 368-369
B 844-A, p. 38
B 622, p. 57-58
B 857-A, p. 34
B 642, p. $65,247-248,265$
B 864-A, p. 40
B 662, p. 57-58
B 864-C, p. 160-162, 165-166
B 692, p. 38,337
B 868-A, p. 40
B 714, p. 91
B 880-A, p. 44
B 722, p. 54
B 897-A, p. 53
B 739, p. 38
B 910-A, p. 52
B 754, p. 98-99, 118
B 917-A, p. 51
B 755, p. 43
B $926-A$, p. 50
B 797, p. 22
B 933-A, p. 47
B 810, p. 29
I 289
B 813, p. 34
BMB 142, p. 25
B 824, p. 37
BMB 153, p. 53
B 836, p. 38
TDM 1, p. 35
(Quartz Cr.) - (5.8, 5.35) approx ; GOLD (?).
B 542, p. 291
B 578, p. 43

Red Dog. See (Meketchum Cr.).

(Reeley Cr.). See (Ruby Cr.). 
(Root Cr.) - (5.0, 6.45) (?) ; GOLD (?).

B 864-C, p. 158

(Ruby Cr.) - $(3.8,12.9)$; GOLD, TIN. Includes reference to (Reeley Cr.)

B 263, p. 52

B 345 , p. $46-47$

B 379, p. 229, 232-233

B 410 , p. $77-80$

B 480 , p. 237

B 520, p. 287, 294-295

B 542, p. 283, 288

(Short Cr.) - $(3.3,6.0)$; GOLD, TIN.

B 642, p. 242, 260, 265

B 692, p. 337

B 712, p. 22
B 578, p. 40, 43

B 642, p. 239, 260, 265

B 692, p. 337

B 754, p. $90-91,118,122$

B 864-C, p. 144

TDM 1, p. 34

B 754, p. 118

B 864-C, p. 151

TDM 1, p. 34

(Shovel Cr.) - (6.8-7.3, 15.4-16.6) ; GOLD(?).

B 520, p. 296

Shropshire. See (Moose Cr.), (Poorman Cr.).

Shropshire (, Gragan) \& Pedretti. See (Moose Cr.).

(Solomon Cr.) - (2.9-3.35, 1.2-1.3) ; GOLD, SILVER.

B 739, p. 38

B 864-A, p. 40

B 755, p. 43

B 864-C, p. 164-165

B 810, p. 29

B 868-A, p. 40

B 824, p. 37

(Spangle Cr.) - $(3.5,3.15)$; GOLD.

B 642, p. 244

B 910-A, p. 52

(Spruce Cr.) - $(4.2-4.35,2.55-2.85)$; GOLD, TIN.

B 592, p. 68

B 642, p. 244, 265

B 880-A, p. 44

B 662, p. 57

B $897-A$, p. 53

B 692, p. 38,337

B 754, p. 95-96, 118

B $910-$ A, p. 52

B 755, p. 43

B $917-A$, p. 50-51

B 813, p. 34

B $926-A$, p. 49

B $864-C$, p. 167

B 868-A, p. 40

B $933-A$, p. 46

BMB 142, p. 25

BMB 153, p. 53

TDM 1, p. 35

(Star Cr.) - (4.65-4.95, 3.05-3.5) ; GOLD (?).

B 642, p. $244 \quad$ B 754, p. 95

Stevens \& Lohr. See (Poorman Cr.).

(Straight Cr.) - (4.4-4.85, 8.05-8.1) ; GOLD, TIN.

BMB 153 , p. 53

(Swift Cr.) - $(3.0,7.3)$; GOLD.

B 642, p. $243 \quad$ B $864-A$, p. 40

B 662, p. $58 \quad$ B $864-$ C, p. 145,155

(Tamarack Cr.) - (4.6-4.75, 1.9-2.4) ; GOLD, TIN.

B 592, p. $68,369 \quad$ B 836, p. 38

B 622 , p. $57-58$

B $864-$ C, p. 167

B 642, p. 244-245, 265

B 662, p. 58

B 692, p. 38,337

B 754, p. 96,118

B 824, p. 37

B 880-A, p. 44

B 926-A, p. 50

BMB 142, p. 25

BMB 153, p. 53

TDM 1, p. 35 
(Tenderfoot Cr.) - (3.4-3.55, 1.75-1.82) ; GOLD. See also (Poorman Cr.).
B 592, p. $68,368-369$
B $917-A$, p. 51
B 662, p. 58
B 910-A, p. 52
BMB 142, p. 25
BMB 153, p. 53

(Timber Cr.) - $(2.9,1.2)$ approx ; GOLD.
B 622, p. 58
B $864-A$, p. 40
B 662, p. 58
B $868-$ A, p. 40
B 797, p. 23
B $910-A$, p. 52
B 810, p. 29
B $917-$ A, p. 51
B 844-A, p. 38
B $926-A$, p. 50

B $857-$ A, p. 34

Timber Cr. Min. Co. See (Timber Cr.).

(Tip Cr.). - $(6.9,7.7)$ approx ; GOLD.
B 542, p. 291
B 864-C, p. 158
B 578, p. 43

(Trail Cr.) - (5.45-5.8, 6.25-6.85) ; GOLD, TIN, SILVER, TUNGSTEN.
B 520 , p. 291,296
B 868-A, p. 40
B 542, p. $288,290-292$
B $910-A$, p. 52
B 578, p. 40,42
B 917-A, p. 50-51
B 592 , p. 366
B 926-A, p. 49-50
B 622, p. 58
B 933-A, p. 46
B 642, p. $245,260,265$
I 289
B 692 , p. 337
BMB 142, p. 25
B 754 , p. $96-97,117-118$
BMB 153, p. 53
B 755, p. 43
RI 4174, p. 28
B 813, p. 34
TDM 1, p. 34
B 864-A, p. 40
TDM 2, p. 20
B 864-C, p. 148-149, 156-157

Trail Cr. Min. Co. See (Trail Cr.).

Vik \& Sutro. See (Spruce Cr.).

Vuicich. See (Flat Cr.) (trib. Timber Cr.).

Washington. See (Poorman Cr.).

(White Channel Cr.). - $(6.75,6.7)$ approx; GOLD (?).

B 864-C, p. 157

(Willow Cr.) - (3.1-3.35, 6.95-7.25) ; GOLD.

B 622, p. 58

B 864-C, p. 145, 154-155

B 642, p. 243

Windy. See (Long Cr.).

Yukon Gold Co. See (Greenstone Cr.).

RUSSIAN MISSION QUADRANGLE

Arnold - $(0.85,14.6)$; GOLD, LEAD, MOLYBDENUM, COPPER, TUNGSTEN.

B 662, p. 345-346

B 683, p. 63-64

B 926-C, p. 198-199

B 773, p. 46

C 328, p. 8-9

(Bear Cr.) - (18.45-19.05, 0.9-1.45) ; GOLD.
B 410, p. 63
B 722, p. 60
B 480, p. 40
B 783, p. 15
B 542, p. 46
B 792, p. 20
B 592, p. 70-71
B 797, p. 24,30
B 622 , p. $67,303,309-319,324-325$,
B 810, p. $31-32,40$
327-330
B 642 , p. 68
B 813, p. 36, 47
B 824, p. 52 
(Bear Cr.)-Continued

B 836, p. 43

B 897-A, p. 58, 71

B 844-A, p. 44, 54

B 910-A, p. 61, 76-77

B 857-A, p. 41, 51

B 917-A, p. 60

B 864-A, p. 46, 57

B 926-A, p. 56-57, 71

B 868-A, p. 46-47, 59

B 933-A, p. 53,68

B 880-A, p. 49,61

Bering Alaska Placers - $(17.8,0.4)(?)$; GOLD. See also (Tiny Gulch).

B 910-A, p. 61

Bettles. See (Cobalt Cr.).

(Black Mtn.) - $(22.25,14.15)$ approx; ANTIMONY. Includes reference to antimony on Owhat R.; not mentioned by name in $P 268$.

P 268, p. 122

RI 4173, p. 5,40

(Bobtail Cr.) - (NW 1/4 5, 15) (?) ; GOLD, MERCURY. See also (Kako Cr.).

B $917-A$, p. 52

B $926-A$, p. $46-47$

B 933-A, p. 44

TDM 1, p. 27 .

(Bogus Cr.) - $(16.15,2.7)$; GOLD.

B 622 , p. 331-332

(Bonanza Cr.) - $(18.75,1.15-1.25)$; GOLD.
B 592, p. 71
B 642, p. 68
B 622 , p. 311-312, 327-329

Brink (gold). See (Bear Cr.).

Brink (molybdenum) - $(22.75,14.55)$ approx ; MOLYBDENUM. NoTE-Not mentioned by name in text, but descriptions fits prospect named on fig. 23, RI 4173. P 268, p. 122

Bumblebee. See (Willow Cr.).

(Buster Cr.) - $(5,15)$; GOLD.
B 783, p. 15
B 810, p. 30
B $864-A$, p. 43

(California C.). See (Tuluksak R.).

(Cobalt Cr.) - (24.1-24.15, 12.2-12.4) ; COPPER, GOLD, SILVER, ANTIMONY, TIN, LEAD, ZINC, NICKEL(?). Includes references to copper lode in Russian Mtns.
P 268 , p. 5-7
B 622, p. $67,304,359-360$
B 722, p. 60
C 328, p. $5-7$

B 714, p. 35

(Disappointment Cr.) - $(0.85-0.9,15.3-15.45)$; GOLD, PLATINUM. See also (Wilson Cr.).
B 622 , p. $65-66$
B 783, p. 15
B 642 , p. 67
B $910-A$, p. 54
B 662, p. $342-343$
B $926-A$, p. 46
B 683, p. 59
C 328, p. 8
B 692 , p. 39
BMB 142, p. 26
B 755, p. 45
BMB 153, p. 53

(Edgar Cr.) - (1.7, 15.75) ; GOLD.

C 328, p. 8

(Elephant Cr.) - (1.1, 15.2) ; GOLD, TUNGSTEN. See also (Wilson Cr.).

B 622, p. 66

B 642, p. 67

B 662, p. 342

B $910-A$, p. 54

B 683, p. 59

B 692, p. 39

B $868-$ A, p. 43

B $933-A$, p. 44

C 328, p. 8

BMB 153, p. 53

TDM 1, p. 40 
February. See (Cobalt Cr.).

(Happy Cr.) - (1.1-1.2, 15.4-15.75) ; GOLD. B 622 , p. 66

Johnson \& Ostnes. See (Willow Cr.).

(Kako Cr.) - $(5,15)$; GOLD. Includes reference to (Kato Cr.) ; see also (Bobtail Cr.).

B 722, p. 57

B 797, p. 23

(Kato Cr.). See (Kako Cr.).

Konechney. See (Mission Cr.).

March. See Tuluksak R.).

(Mary Cr.) - (15.7-15.95, 0.2-0.5) (?) ; GOLD. May be the same as Mary Lou Gulch.

B 722, p. 60

(Mission Cr.) - (23.8-24.1, 11.9-12.0) ; COPPER, GOLD, SILVER, LEAD, TUNGSTEN, ANTIMONY. Includes references to Konechney, (Russian Mts.), if definitely to this locality.
P 268, p. 122
B 622, p. 304, 359
C 248, p. 2, 4
C 328, p. 5-7

B 649, p. 47

(Montezuma Cr.) - $(5,15)$; GOLD.
B 783, p. 15
B 824, p. 41
B 864-A, p. 43
B 868-A, p. 43
B 836, p. 41
B 880-A, p. 45
B 844-A, p. 40
B 897-A, p. 53

B 857-A, p. 38

New York-Alaska Gold Dred. Corp. See (Bear Cr.).

(Ophir Cr.) - (17.55-18.2, 4.25-4.95) ; GOLD.

B 622, p. 332-336

(Owhat R.). See (Black Mtn.), Brink (molybdenum).

Plunkett. See Arnold.

Rhode \& Edgar. See (Wilson Cr.).

(Russian Mtns.). See (Cobalt Cr.), (Mission Cr.).

(Spruce Cr.) - (18.4-18.5, 0.9-1.15) ; GOLD.
B 622, p. 311-312, 321-327, 330
B 722, p. 60

B 642, p. 68

(Stuyak Cr.) - (NE 1/4 5, 15) ; GOLD.

B 722, p. 57

(Tiny Gulch) - $(17.8,0.4)$; GOLD. See also Bering Alaska Placers.

B 622, p. 312, 328-329

B 722, p. 60

(Tuluksak R.) - (17.4-17.6, 0.4-0.9) ; GOLD, ASBESTOS, GRAPHITE. Includes references to (California Cr.).
B 379, p. 58
B 622, p. 331
B 410, p. 63
B 897-A, p. 58
B 442, p. 46
B 910-A, p. 61
B 520, p. 41
TDM 1, p. 44

.Willow Cr.) - (0.8-0.85, 14.2-14.55) ; GOLD, SILVER, PLATINUM(?).

B 622, p. 66

B 783, p. 15

B 642, p. 67

B 797, p. 24

B 662, p. 59, 343-345

B 810, p. 30

B 683, p. 60-62

B 813, p. 35

B 692, p. 39

B 824, p. 40-41

B 739, p. 40

B 836, p. 41 
(Willow Cr.)-Continued

B 844-A, p. 40

B 857-A, p. 38

B 864-A, p. 43

B 868-A, p. 43

B 880-A, p. 45

B 897-A, p. 53

B 910-A, p. 54
B 917-A, p. 52

B 926-A, p. 46

B 933-A, p. 44

C 328, p. 8

BMB 142, p. 26

BMB 153, p. 53

TDM 1, p. 20

(Wilson Cr.) - (0.85-1.1, 15.2-15.45) ; GOLD, PLATINUM. See also (Disappointment Cr.), (Elephant Cr.).
B 622, p. 65-66
B 917-A, p. 52
B 642, p. 67
C 328, p. 8
B 662, p. 342-343
B 683, p. 59
BMB 142, p. 26
TDM 1, p. 20

Wilson Cr. Min. Co. See (Disappointment Cr.), (Elephant Cr.), (Wilson Cr.). Yukon Min. Co. See (Bobtail Cr.).

(-----) (molybdenum) - $(22.75,14.55)(?)$; MOLYBDENUM. See also Brink (molybdenum).

B 926-C, p. 200-201

\section{ST. LAWRENCE QUADRANGLE}

(Boxer Bay) - (SW 1/4 3, 8) ; TIN.

TDM 5-R, p. 41-42

(St. Lawrence I.) (iron) - ( ?) ; IRON.

TDM 5-R, p. 26

(St. Lawrence I.) (lead) - (W 1/8 quad.) ; LEAD.

TDM 5-R, p. 33.

(West Cape) - (1.5, 9.75) approx ; MOLYBDENUM, COPPER.

TDM 5-R, p. 24, 35-36

\section{SELDOVIA QUADRANGLE}

Alaska Cement Corp. See (Gray Cliff).

Alaska Comm. Co. See (Port Dick).

Alaska Hills (Mines Corp.) - $(20.95,10.55)$; GOLD. Includes reference to Alaska Hills Min. Co., Paystreak.
B 783, p. 8
B 864-A, p. 21
B 792,p. 11
B $868-A$, p. 23
B 797, p. 12
B 880-A, p. 26
B 810, p. 16
B $897-$ A, p. $29-30$
B 813, p. 17
B $910-A$, p. 29
B 824, p. 21
B $926-$ A, p. 25

Alaska Hills Min. Co. See Alaska Hills.

Alley - (11.1-11.2, 5.95-6.25) ; GOLD.
B 442, p. 176-177
B 587, p. 232

(Anchor Pt.) - (10.05-10.15, 13.25-13.5) ; GOLD, PLATINUM(?).
P 70, p. 156, 163
B 277, p. 44
B 379, p. 147-148
B 442 , p. 171
B 520, p. 37, 132, 141, 161

B 587, p. 110-111

B 742, p. 49

B 773, p. 23

B 1058-F, p. 227

Anderson. See (Red Mtn.). 
Anderson \& Anderson. See (Claim Pt.).

(Aurora) - $(17.0,12.2)$; GOLD (?).
B 277, p. $47-48$
B 587, p. 232-233

B 442, p. 170

Aurora Gold Mines Co. See (Aurora).

Babcock \& Downey. See Sonny Fox.

Babcock \& Martin. See (Red Mtn.).

Barrister Lode. See (Red Mtn.).

Bessie B. See (Red Mtn.).

Big Bend. See (Red Mtn.).

Big Chrome. See (Red Mtn.).

Big Meadow. See (Red Mtn.).

Big Parade. See (Red Mtn.).

Blair. See Nukalaska Min. Co.

Blodgett. See (Red Mtr.).

Bluff. See (Claim Pt.).

Cawing Crow. See (Red Mtn.).

Charles Francis. See (Red Mtn.).

Cherokee Chief. See (Red Mtn.).

Chrome Queen. See (Red Mtn.).

Chrome Queen Min. Co. See (Red Mtn.).

(Claim Pt.) - (10.45-10.7, 3.6-3.9) ; CHROMITE. Includes references to Bluff,

(Port Chatham), Reef; not mentioned by name in B 714 .
B 442, p. 169
B 742, p. 1-2, 13, 16-29, 44-45
B 587, p. 237-238
B 931-G, p. 139-141, 148-152, 156-162
B 662, p. 22
C 252, p. 10-11
B 692 , p. 265-267
B 712, p. 23, 34, 100-111, 124-125
IC 7379, p. 69
B 714, p. 40
RI 4419 , p. 2=11

Clarberg. See (Red Mtn.).

Cliffside Lode. See (Red Mtn.).

Cramer \& Martin. See (Red Mtn.).

Double R. See (Red Mtn.).

Edith. See (Red Mtn.).

Goliath. See (Red Mtn.).

Goyne - $(22.35,9.35)$; GOLD, SILVER.

B 897-A, p. 30-31

Gray Cliff. See (Gray Cliff).

(Gray Cliff) - $(11.5,8.1)$; LIMESTONE.

B 587, p. 42, 111-112

RI 4932, p. 122-123

Hatcher. See Nukalaska Min. Co.

Horseshoe. See (Red Mtn.).

Johnston \& Degan - $(22.4,9.5)$; GOLD.

B 897-A, p. 28

Juneau. See (Red Mtn.).

Kearns \& Cooper. See (Red Mtn.).

Kusturin \& Johanson - (25.2-25.35, 11.5-11.6) ; GOLD (?).

B 442, p. 173-174

B 587, p. 229

Lass (\& Whitney). See (Red Mtn.).

Letha. See (Red Mtn.).

Linder \& Ballan. See (Red Mtn.). 
(Little Cr.) - (20.75, 9.95) ; GOLD (?).

$$
\text { B 897-A, p. } 32
$$

Lyle \& Abbott. See (Gray Cliff).

McCray. See (Claim Pt.).

Markle. See (Red Mtn.).

Mammoth. See (Red Mtn.).

Merle. See (Red Mtn.).

(Miller's Landing) - $(13.9,11.6)$; CLAY, HAYDITE.

RI 4932 , p. $45-47,70-71,80-81,125-126$

Mills \& Trimble - $(13.0,4.7)$; GOLD, SILVER, COPPER, NICKEL.

$$
\text { B 442, p. 175-176 }
$$

B 587, p. 231

Morris, Sheridan, Kuppler \& Lee - (21.5-23.35, 10.3-10.65) ; GOLD (?). B 442, p. 174

B 587, p. 229-230

Mount. See (Little Cr.).

Nuka Bay Mines Co. - $(21.15,10.2)$; GOLD (?). B 824, p. 21 B 897-A, p. 30

Nukalaska Min. Co. - $(20.95,9.35)$; GOLD, LEAD, COPPER. B 897-A, p. 27-29 B 917-A, p. 28 B 910-A, p. 29 B 926-A, p. 25

Omega. See (Red Mtn.).

Patterson \& Ogle. See Goyne.

Paystreak. See Alaska Hills.

(Port Chatham). See (Claim Pt.).

(Port Dick) - (15.4-16.2, 5.55-5.8) ; COPPER, ZINC, GOLD( ?).
B 379, p. 30
B 587, p. 230-231
B 442, p. 174-175

Port Dick Min. \& Power Co. See (Port Dick).

Question Mark. See (Red Mtn.).

Rapp. See (Claim Pt.), (Red Mtn.).

Ray. See (Red Mtn.).

(Red Mtn.) - (13.2-13.75, 6.4-7.2) ; CHROMITE.

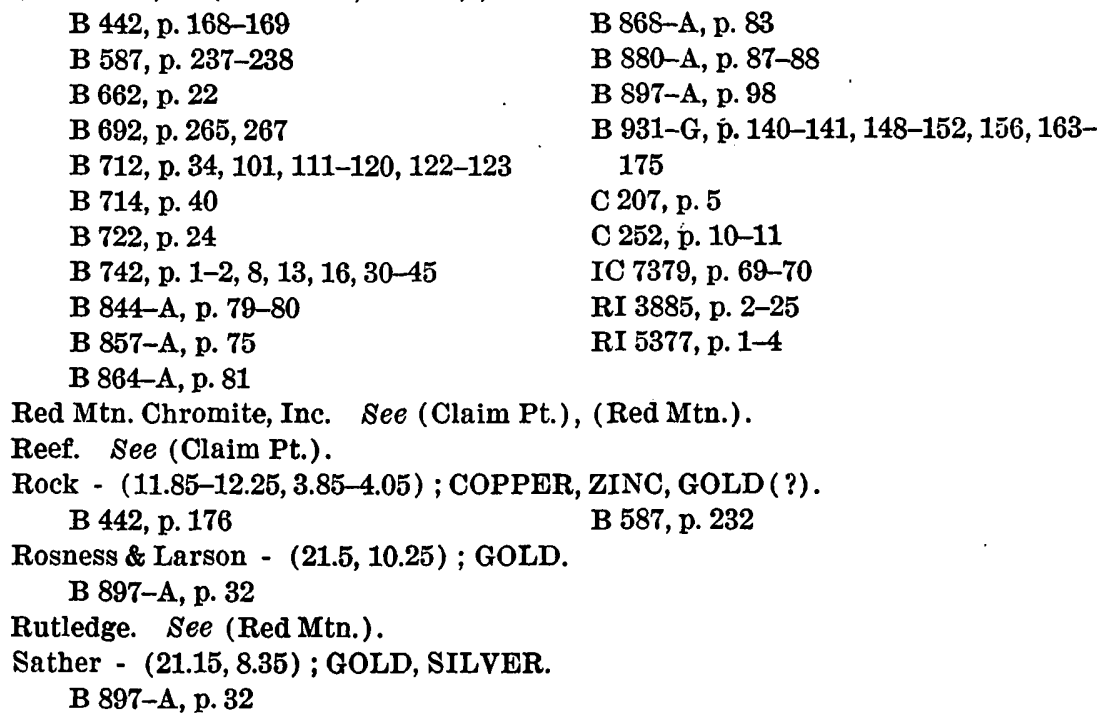

Rosness \& Larson - (21.5, 10.25) ; GOLD.

B 897-A, p. 32

Rutledge. See (Red Mtn.).

Sather - $(21.15,8.35)$; GOLD, SILVER.

B 897-A, p. 32 
( Seldovia) - (11.55, 7.9) ; GRAPHITE.

B 712, p. 34

Skeen. See (Red Mtn.).

Skinner - $(21.25,9.3)$; GOLD, LEAD, ZINC, COPPER.

B 897-A, p. 31-32

Sonny Fox - $(22.5,9.85)$; GOLD, SILVER, COPPER, ZINC, LEAD. Includes references to Babcock \& Downey, Sonny Fox Min. Co. if definitely to this mine.

B 813, p. 17

B 824 , p. 20-21

B 836, p. 20

B 844-A, p. 20

B 857-A, p. 20

B 864-A, p. 21
B 868-A, p. 23

B 880-A, p. 26

B 897-A, p. 26-28

B 910-A, p. 29

B 926-A, p. 25

Sonny Fox Min. Co. See Johnston \& Degan, Sonny Fox.

Star Chrome. See (Red Mtn.).

Star Four. See (Red Mtn.).

(Two Arm Bay). See Kusturin \& Johanson.

Whitney \& Lass. See (Claim Pt.), (Red Mtn.).

Widow Maker. See (Red Mtn.).

\section{SEWARD QUADRANGLE}

Alameda. See Latouche I. Copper Min. Co. (Ltd.).

Alaska Crow Cr. Min. Co. See (Crow Cr.).

Alaska Free Gold Co. See Alaska Homestake.

Alaska Glaciers Min. Co. - $(11-18,12-17)$ (?) ; GOLD (?). May be in Anchorage quad.

B 797, p. 12

Alaska Homestake (Min. Co.) - (13.1, 15.6) (?) ; GOLD. Includes references to Homestake Min. Co.
B 662, p. 189
B 712 , p. 33

B 692 , p. 150

I 273

Alaska Minerals Co. See Lucky Strike (Palmer Cr.).

Alaska Hyd. Syn. See (Kenai R.).

Alaska Oracle (Min. Co.) - $(3.85,10.9)$; GOLD, LEAD, ZINC, MOLYBDENUM, COPPER. Includes references to Oracle.
B 836 , p. 20
B $880-$ A, p. 26-27
B $849-\mathrm{I}$, p. 507-510
B $864-$ A, p. 22
B $910-$ A, p. 29
B $868-$ A, p. 23
B $926-$ A, p. 25
B $926-\mathrm{C}, \mathrm{p} .186$

Alaska Securities Corp. See (Canyon Cr.), (Lynx Cr.).

Alaska-Washington Gold Min. Co. See Eldorado.

Alhambra - (18-19, 5-6) ; COPPER (?).

B 662, p. 218

Allison. See (Canyon Cr.).

Anderson - $(20,16-17)$ (?) ; COPPER (?). May be in Anchorage quad.

B 662, p. 185

Anderson \& Little. See Golden Eagle, Golden Wonder No. 1, Golden Wonder No. 9, Lucky Swede.

Anderson \& Yannes - $(15.4,17.35)$; GOLD.

I 273

Anthony. See (Sixmile Cr.). 
Arrowhead - $(16.95,17.1)$; GOLD. Includes references to Johnson \& Wagner, Wagner (\& Johnson).
B 592, p. 223
B 692, p. 150
B 642, p. 142
I 273
B 662, p. 189

Bald Eagle. See Jonesy.

Ballaine \& Nelson. See Skeen-Lechner.

Banner - $(14.5,16.55)$ approx ; GOLD, LEAD, ZINC.
B 662 , p. 187-189
BMB 153, p. 43
B 692, p. 150
I 273

Banta \& Sullivan - (11-12, 13-15) ; GOLD.

B 622, p. 138

Barrack. See (Latouche).

Barrack-Girdwood. See (Latouche).

Baughman \& Swetmann. See Swetmann.

Bazard. See (Wilson Bay).

Beady, Walcott \& Riley. See (Bear Cr. ).

Bear. See California-Alaska Min. Co., Mile Seven. Note.-Two different claims.

(Bear Cr.) - (3.7-4.1, 15.3-15.85) ; GOLD, SILVER, LEAD, ZINC, ANTIMONY. See also Coon, Gold Stamp, Taylor.

AR 18, p. 81-82

AR 20, p. 318, 320

P 70, p. 163

B 259, p. 91-92, 95, 98

B 277, p. 8, 36, 46-47

B 442 , p. 42

B 520, p. 141, 160-161

B 587, p. 172, 182-185, 196-197

B 592 , p. 63

B 622, p. 46

B 642, p. 55

B 649, p. 60
B 662, p. 44-45

B 755, p. 29

B 783, p. 12

B 797, p. 17

B 836, p. 30

B 844-A, p. 30

B 849-I, p. 505-506, 521, 526

B 857-A, p. 28

B 910-A, p. 43

B 926-A, p. 37

C 196, p. 10

Beatrice - (NW 1/8 quad.) (?) ; GOLD (?). B 692, p. 175

Beatson (Copper Co.). See (Latouche).

Beatson-Bonanza. See (Latouche).

Belmont. See (Bear Cr.).

Bennett, Bailey \& Heinz - (14.0-14.7, 15.5-16.5) ; GOLD (?). B 642 , p. 142

Bettles. See (Snug Harbor).

Bettles Bay. See Hermann \& Eaton.

Betty. See California-Alaska Min. Co.

Betty No. 1. See Skeen-Lechner.

Big Bonanza. See (Latouche).

Big Passage Copper Min. Co. - $(20.15,7.95)$; COPPER. I 273

(Bird Pt.) - $(5.45,16.3)$; GOLD, LEAD, COPPER. Includes reference to Conway.

B 542, p. 38

B 642 , p. 191-192

Black Bear. See Dunklee \& Reilly.

Black Butte. See Kenai-Alaska. 
Blackbird. See (Latouche).

Blue Fiord - (14.9-15.3, 7.9-9.0) approx ; GOLD(?).

B 592, p. 237

Blue Fox. See Reynolds Alaska Dev. Co.

(Blue Gulch) - $(4,10)$ (?) ; GOLD.

B 824, p. 30

Bluebell. See Conley \& McChesney, Primrose. Note.-Two different claims.

Bonanza. See Downing, (Latouche). NoTe.-Two separate claims.

Brereton, Oome \& Howell. See Yakima.

Brewer Alaska Syn. See Mile Seven.

Brewster - $(6.15,11.65)$; GOLD.

B 849-I, p. 519

Brown Bear - $(5.8,5.95)$; GOLD, LEAD, ZINC.

B 587, p. 148

Bruhn \& Ray. See (Canyon Cr.).

Bugaboo. See Thomas-Culross Min. Co.

Bullion (Passage Canal) - $(12.0,14.8)$; GOLD.

B 592, p. 233

I 273

Bullion (Rua Cove). See Copper Bullion.

Buster. See (Quartz Cr.) (Kenai L.).

Byers - $(21.95,15.75)$; COPPER.

I 273

California-Alaska Min. Co. - $(6.35,7.55)$; GOLD, LEAD, ZINC. See also Skeen-

Lechner.

B 379, p. 107

B 442, p. 173

B 520, p. 138, 141-142, 146-147

B 480, p. 32

B 587, p. 151-154

(California Cr.) - (7.3-7.45, 17.0-17.5) ; GOLD.
B 277, p. 9, 40, 43
B $849-$ G, p. 406
B 587, p. 192
B 857-A, p. 29
B 642, p. 186
B 864-A, p. 33
B 824, p. 30
B 868-A, p. 34
B 836 , p. 30-31
B 897-A, p. 44

B 844-A, p. 30

(Canyon Cr.) - (4.55-5.0, 11.7-13.7) ; GOLD. Includes reference to Canyon Cr. Placers.

AR 18, p. 82

AR 20, p. 313-320

B 259, p. 96

B 277, p. 37-38, 45

B 314, p. 119, 122-123

B 379, p. 52

B 442 , p. 42

B 520, p. $141,160-161,168$

B 587, p. 203-204

B 642, p. 55

B 662, p. 44

B 692, p. 32, 176

B 722, p. 40

B 755, p. 29, 118

B 783, p. 12

B 813, p. 26

B 824, p. 30

B 836, p. 30

B 844-A, p. 30

B 849-I, p. 521-522, 527

B 857-A, p. 28

B 864-A, p. 32

B 868-A, p. 33

B 880-A, p. 36-37

B 910-A, p. 43

B 917-A, p. 40

B 926-A, p. 37

B 773, p. 38

BMB 153, p. 34

Canyon Cr. Dev. Co. See (Canyon Cr.). 
Canyon Cr. Placers. See (Canyon Cr.).

Carson. See (Resurrection Cr.).

Carter - $(17.3,16.9)$; GOLD.

B 592, p. 224-225

I 273

Case - $(6.0,8.8)$; GOLD. Includes references to Grant L., (Grant L.).
B 622, p. 46
B $880-A$, p. 26
B 662, p. 45
B $897-A$, p. 33
B 692 , p. 176
B $910-A$, p. 29
B 755 , p. 30
$\mathrm{BMB} 142$, p. 43

B $849-$ I, p. 520

(Cathead Bay) - $(18.7,5.25)$; COPPER.
B $963-B$, p. 73
I 273

(Cedar Bay) - $(22.3,17.25)$; ZINC, COPPER. Includes reference to Lenora; see also Peterson.

B 692 , p. 146

I 273

B $963-$ B, p. 76-77

Chamberlain - $(24.05,15.7)$; COPPER.

I 273

Champion. See Ronan.

Chelan. See Thomas-Culross Min. Co.

Chenega. See (Latouche).

Chicago-Latouche Min. \& Power Co. See (Wilson Bay).

(Chickaloon R.) - $(0.1,10.11)$ (?) ; MOLYBDENUM.
B 587, p. 137
B $926-C$, p. $186-187$

Chinega. See (Latouche).

Christiansen, Thomas \& Thomas. See Thomas-Culross Min. Co.

Christopher. See Banner.

Clara. See Gilpatrick:

Clark \& Beiswanger. See (Resurrection Cr.).

Collins, Fish \& Barry. See Golden Giant.

Collins, Fish \& Stewart. See Fish, Collins \& Stewart.

Colorado - $(3.85,11.05-11.3)$; GOLD, SILVER. B 587, p. 167

B 849-I, p. 510-511, 516

Columbia. See California-Alaska Min. Co., McMillan. NotE.-Two separate claims.

Colwell \& Robertson - (4.3-4.35, 13.0-13.1) ; GOLD ( ?).

B 587, p. 168

B $849-$ I, p. 517

Conley \& McChesney - $(17.85,17.6)$; GOLD, LEAD, ZINC, COPPER.

B 592 , p. 219

I 273

Connelly. See (Sixmile Cr.).

Consolidated - $(17.65,17.55)$; GOLD.

B 542, p. 37

B 662, p. 189

B 592 , p. 220

I 273

B 622 , p. 135

Consol. Min. \& Smelt. Co. See Copper Bullion.

Conway. See (Bird Pt.).

Coon (\& Plowman) - $(3.8,15.8)$; SILVER, GOLD, LEAD.

B 587, p. 178-179

B 849-I, p. 506-507

(Cooper Cr.) - (1.05-1.55, 7.6-8.5) ; GOLD.

AR 20, p. 320

B 259, p. 92,98

B 277 , p. 44
B 314, p. 123

B 520, p. 141, 160-162, 165-167

B 587, p. 199-201 
(Cooper Cr.)-Continued
B 622, p. 46
B 692 , p. 176
B 642, p. 55
B 849-I, p. 521

(Copper Bay) - (18.6-18.75, 5.0-5.15) ; COPPER, ZINC. Includes references to

Erricksen \& Allen, Larson.

B 662, p. 218

I 273

Copper Bullion - (20.3-20.55, 6.4-6.7); COPPER, ZINC. Includes references to Consol. Min. \& Smelt. Co., (Rhea Cove), Rua, (Rua Cove), Solar Dev. Co.

B 379, p. 92

B 442 , p. 165

B 773, p. 28, 38

B 443 , p. 69

B 783, p. 21

B 662 , p. 213-214

B 813, p. 54-55

B 692, p. 31, 145

B 824, p. 22,60

B 714, p. 22-23

B 836, p. 21-22

B 722, p. 40

B 947-E, p. 85-92

B 739 , p. 24

B 989-E, p. 300-301

B 755, p. 28-29

Copper Chief - $(6.45,0.4)$; COPPER.

B 379, p. 103-104

I 273

RI 4986, p. 1-6 .

Copper Coin - $(19.3,5.6)$; COPPER. Includes references to Russell Ball Copper Co.
B 379, p. 90-91
B 692, p. 146
B 443 , p. 68
B 662 , p. 184, 216-217
B $963-$ B, p. 72-73
I 273

Copper Queen - $(19.6,3.55)$; COPPER. See also Happy Jack Min. \& Dev. Co. B 692, p. 146

Cordova Min. \& Dev. Co. See Golden Wonder No. 9, Nugget.

(Crow Cr.) - (7.85-7.9, 17.5-17.6) ; GOLD, SILVER, COPPER. Includes references to Erickson, Holmgren (-Erickson) ; see also (Crow Cr.) (Anchorage quad.).
AR 20, p. 278, 320
B 259, p. 92,97
B 277 , p. $9,40-43,46$
B 792, p. 16
B 797, p. 17
B 810, p. 23
B 314, p. 119-121
B 813 , p. 26
B 480 , p. 38
B 520, p. 141, 160-161, 171-173
B 824, p. 30
B 542, p. 44
B 836, p. 30
B 587, p. 183, 185, 187-192
B 844-A, p. 30
B 592 , p. 63
B 622 , p. 46
B 642, p. 55, 181-186
B 849-G, p. 398-404
B 857-A, p. 28-29
B 864-A, p. 33
B 868-A, p. 33
B 662, p. 44
B 880-A, p. 37
B 692 , p. 176
B 897-A, p. 44
B 739, p. 24
B 910-A, p. 43
B 755, p. 29,118
B 917-A, p. 41
B 773, p. 38
B 926-A, p. 37
B 783, p. 12
BMB 153, p. 34

Crow Cr. Consol. Min. Co. See (Crow Cr.).

Crown Copper Co. - $(19.85,7.8)$; COPPER, ZINC.

B 379, p. 93

B 662, p. 212

B 963-B, p. 69

I-273 
Crown Pt. Min. Co. - (6.2-6.25, 7.6) ; GOLD. See also California-Alaska Min. Co.
B 880-A, p. 26
B 910-A, p. 29

B 897-A, p. 32-33

Culross. See Thomas-Culross Min. Co.

Culross I. Min. Co. See Thomas-Culross Min. Co.

Culross No. 1. See Sells.

Dahl. See (Canyon Cr.).

Dark \& Leach. See (Bear Cr.).

Dawson and assoc. See (California Cr.).

(Day Harbor) - $(7.4,0.6)$ approx ; COPPER ( ?).

B 442, p. 170

B 587, p. 233

Devil Club - $(5.85,5.85)$; GOLD(?).

B 587, p. 148

(Devils Cr.) - $(2.95,10.35)$; GOLD.

B 587, p. 163-164

B 849-I, p. 519

Dickey. See Copper Bullion, (Latouche).

(Disk I.) - $(20.3,8.85)$; COPPER.

B 662, p. 211

I-273

Dominick. See Olsen \& Viette.

(Donaldson Cr.) - $(4.4,13.5)$; GOLD. B 587, p. 168-169

Downing - (4.2-4.35, 14.4-14.45) ; GOLD, LEAD.

B 849-I, p: 500-501

Drennan \& Sweitzer. See Kenai-Alaska.

Duchess. See Reynolds Alaska Dev. Co.

Duke. See Reynolds Alaska Dev. Co.

Dunfranwald Gold Mines. See (Gulch Cr.).

Dunklee \& Reilly - $(13.6,15.5)$; GOLD, COPPER, LEAD, ZINC.
B 592, p. 233
I-273

B 662, p. 188

(East Fork - (5.0-5.15, 13.6-13.7) ; GOLD.
B 277, p. 39
B 379, p. 52
B 314, p. 119
B 587, p. 205

Edwards \& Plowman. See (Mills Cr.).

El Primero Min. \& Mil. Co. See Granite.

Eldorado $(19.15,15.05)$; GOLD.

B 592, p. 235

B 622, p. 139

(Elrington I.) - $(17.4,0.6)$; COPPER. I-273

Elwood \& Reuef. See Sweepstake Min. Co. (Avery R.).

Erickson. See (Crow Cr.).

Erricksen \& Allen. See (Copper Bay).

Evans, Cooper \& Matson. See (Jackpot Bay).

Everson, Gauthier \& Cooper. See Reed, Gauthier \& Cooper.

Everson, Harris \& Parker. See Hummer.

Fairman \& Madsen. See (Quartz Cr.) (Kenai L.), (Slate Cr.) (trib. Quartz Cr.).

Falls. See California-Alaska Min. Co. 
(Falls Cr.) - $(5.45-6.0,7.55-7.65)$; GOLD. Includes references to (False Cr.), (Trail Cr.).

B 277, pp. 44

B 587, p. 202

B 520, p. 141, 168

(False Cr.). See (Falls Cr.).

Finch \& Campbell. See (Canyon Cr.).

Fish, Collins \& Stewart - $(16.05,14.45)$; GOLD.

B 592, p. 234-235

I-273

Francisco. See Downing.

Frederick. See Graystone, Homestake (Porcupine Cr.), Porcupine.

(Frenchy Cr.). See Colwell \& Robertson.

(Fresno Cr.) - (4.2-4.6, 11.85-12.1) ; GOLD (?).

B 587, p. 168

B 849-I, p. 517

Frodenburg \& Bloom - $(17.25,17.2) ;$ GOLD.

B 592, p. 221-222

I 273

Gallagher. See (Bear Cr.).

Gaydon. See (Slate Cr.) (trib. Quartz Cr.).

George \& McFarland - $(14.55,16.65)$; GOLD, LEAD.

B 592, p. 232

I 273

Getchell. See (Gulch Cr.).

Gilnow - $(23.3,17.15)$; COPPER.

I-273

Gilpatrick - (3.8, 10.7-10.85) ; GOLD, SILVER, LEAD, ZINC. Includes references to Wanowski Gold Min. Co.
B 442, p. 171-172
B 480, p. 32
B 692, p. 175
B 587, p. 164-167
B 849-I, p. 512-515
B 592, p. 63
B $880-A$, p. 26
B 622, p. 46
B 926-A, p. 25
B 642, p. 56
BMB 142, p. 43,52
B 662, p. 34
BMB 153, p. 35

Gilpatrick, Sprague \& Byers. See Gilpatrick.

Girdwood. See (Latouche).

(Glacier Bay). See Jensen.

(Glacier Cr.) - (7.1-8.2, 16.5-17.6) ; GOLD. See also (California Cr.), (Crow Cr.), (Winner Cr.).
P 70, p. 162-163
B 642, p. 186
B 259, p. 91-92

Gladiator. See Ronan.

Glendenning - $(21.5,17.95)$ ( ?) ; COPPER. May be in Anchorage quad. B 662, p. 185

Gold Queen - (16.8-17.9, 16.8-17.7) ; GOLD.

B 622, p. 136

Gold Stamp (Min. Co.) - $(4.0,15.45)$;GOLD.

B 592, p. 63

B 849-I, p. 506

B 622, p. 46

Gold Wonder. See Golden Wonder No. 9.

Golden Eagle - $(17.2,17.0)$; GOLD.

B 592, p. 224

B 622, p. 45, 135-136

BMB 153, p. 46

B 642, p. 142 
Golden Giant - $(12.15,15.0)$; GOLD, ZINC, LEAD. Includes reference to Collins, Fish \& Barry.
B 592, p. 234
I-273

B 662, p. 188

Golden Wonder No. 1 - $(17.4,17.15)$; GOLD, LEAD.
B 592, p. 222-223
I-273

Golden Wonder No. 9 - $(17.45,17.25)$; GOLD, ZINC, COPPER. Includes reference to Gold Wonder.
B 542, p. 37
B 592, p. 221
B 642, p. 142
B 622, p. 136
I-273

Gould. See Seward Bonanza Gold Mines Co.

Gould, Hewitt \& Connor. See Northern Light.

Graham \& Harrison - $(19.55-19.65,4.95-5.15)$; COPPER. B 379, p. 92

I-273

Granite (Gold Min. Co.) - $(15.35,17.2)$; GOLD, ZINC, ANTIMONY, LEAD, COPPER: Includes references to El Primero Min. \& Mil. Co.
B 592, p: 230
B 622, p. $45,135-138$
B 642, p. 141-142
B 649, p. 61
B 662, p. $187-188$
B 692, p. 149
B 712, p. 33
B 722, p. 14
B 813, p. 18
B $864-A$, p. 22

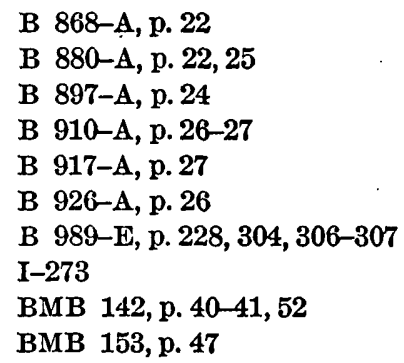

(Granite Cr.) - $(6.4,13.2)$; GOLD.
B 277, p. 40
.B 587, p. 206

Grant $\mathrm{L}$. See Case.

(Grant L.). See Case.

Gray Bros. - $(18.4,15.3)$; GOLD (?).

\section{273}

(fraystone - NW 1/4 5, 5) ; GOLD(?).

$$
\text { B 587, p. } 147
$$

Griset- $(16.85,16.95)$; GOLD. Includes reference to Keynote.
B 592, p. 223-224
B 642, p. 142

Groth, Malone \& others. See Consolidated, Morning Star, North Star (Avery R.).

(Gulch Cr.) - (5.15-5.3, 13.6-13.75) ; GOLD. Includes reference to Dunfranwald Gold Mines.
B 277, p. 37, 39-40
B 314, p. 122
B 379, p. 52
B 587, p. 206-207
B 520, p. 141, 160-161, 169
B 622, p. 46
B 642 , p. 55
B 692, p. 176

Hamilton - $(13.9,15.5)$ approx; GOLD ( ?). See also Lone Star. B 642, p. 142

Hammer. See (Sixmile Cr.).

Hansen \& Young. See Hillside.

Hanson, Gustafson \& Berklund. See Gold Queen. 
Happy Jack (Copper) Min. \& Dev. Co. - $(19.6,3.55)$; COPPER, ZINO. Includes reference to (Hogan Bay); see also Copper Queen.

B 379, p. 91

B 963-B, p. 74

B 443, p. $68-69$

I-273

B 662 , p. $219-220$

Harper and assoc. See (Resurrection Cr.).

Harris, J. - (15.0, 17.45) ; GOLD. $\mathrm{I}-273$

Harris, W. See Sells.

Harvey - $(19.2,4.5-4.75)$; COPPER. B 379, p. 91

B 443, p. 56

Hatcher \& McPherson. See Seward Gold.

Hattie. See Gilpatrick.

Hawkins, Brown, Hayden \& Adams. See Kenai-Alaska.

Heaston. See Alaska Oracle.

Herman-Everman. See Hermann (\& Eaton).

Hermann (\& Eaton) - $(14.15,16.85)$; GOLD, ZINC, LEAD, COPPER. Includes references to Bettles Bay, Herman-Everman, Merrill (Min. Co.), Mineral King (Min. Co.).
B 542, p. 37
B 592, p. 231-232
B 880-A, p. 25
B 622, p. 138
B 897-A, p. 24
B 662, p. 189
B 910-A, p. 26-27
B 692, p. 150
B 917-A, p. 27
B 792, p. 11
B 926-A, p. 26
B 797, p. 12
I-273
B 810, p. 17
BMB 142, p. 41-42, 52
BMB 153, p. 48-49

B 836, p. 22

Eerron (Dred. Co.). See (Sixmile Cr.).

Hershey. See Lucky Strike (Palmer Cr.).

Hickey. See Seward Bonanza Gold Mines Co.

Hillside - $(11.4,14.4)$; GOLD, ZINC, LEAD, COPPER. B 592, p. 234 I-273

Hirshey. See (Bear Cr.), Lucky Strike (Palmer Cr.), Teddy Bear.

Hirshey \& Carlson - $(4.2,14.6)$ approx; GOLD, LEAD, ZINO, COPPER. B 587, p. 171-172

Hoben \& Davis. See (Bear Cr.).

(Hogan Bay). See Happy Jack Min. \& Dev. Co.

Hogan Bay Copper Co. See Happy Jack Min. \& Dev. Co.

Hogan, Hample \& Egan - $(19.5,3.9)$; COPPER.

B 379, p. 92

I 273

Hogg. See (Hogg Bay).

(Hogg Bay) - (15.5-15.8, 1.6-1.8) ; COPPER.

B 783, p. 21

Holmgren (-Erickson). See (Crow Cr.).

Home. See Mile Four Min. Co.

Home Camp - $(19.25,4.5)$; COPPER, ZINC. Includes reference to Schultz.

B 379, p. 91

B 662, p. 219

B 963-B, p. 75

I 273

Homestake (Lost Cr.). See Mile Seven.

Homestake (Pigot Bay) - $(13,15)($ ?) ; GOLD ( ?).

B 662, p. 188 
Homestake (Porcupine Cr.) - (NW 1/4 5,5) ; GOLD( ?).

B 587, p. 147

Homestake Min. Co. See Alaska Homestake.

Hope Min. Co. See (Resurrection Cr.).

(Horseshoe Bay). See Reynolds Alaska Dev. Co.

Houston Dred. Co. See (Falls Cr.).

Hubbard \& Elliott - $(19.1,5.5)$; COPPER.

I 273

Hubbard-Elliott Co. See Jonesy, Monarch.

Hummer - $(14.3,16.05)$; GOLD, LEAD, COPPER. Includes references to Everson, Harris \& Parker.

B 592, p. 232

I 273

B 622, p. 138

Imhoff (, Weidlich \& Saulsbury), See McMillan.

Imp. See Sweepstake Min. Co. (Harriman Fiord).

Independence - $(3.95,11.55) ;$ GOLD ( ?).

B 587, p. 168

B $849-I$, p. 516

(Indian Cr.) - (3.85-4.25, 17.3-17.45) ; GOLD. See also Strong.

B 642, p. 187, 192

Ingalls. See California-Alaska Min. Co.

(Ingram Cr.). See (Quartz Cr.) (Turnagain Arm).

Iron Bullion. See Copper Bullion.

Iron Cap $(6.6,0.2)$; COPPER.

B 379, p. 103-104

B 587, p. 234

Iron Mask - $(3.7,11.7)$; GOLD.

B 587, p. 168

B $849-$ I, p. 516

Iron Mtn. See Reynolds Alaska Dev. Co.

(Jackpot Bay) - (15.2, 5.85) ; GOLD, SILVER, LEAD, ZINC.
B 379, p. 97
B 443, p. 76,78
B 592, p. 237
I-273

Jacobs. See (Lynx Cr.).

James \& Ronan. See Ronan.

Jensen- $(24.85,16.05)$; COPPER. Includes reference to (Glacier Bay).

B 662, p. 185

B 692 , p. 146-147

B 963-B, p. 75-76

I-273

Jensen, Wallace, Kilbourne - $(24.8-24.85,16.15-16.2)$; COPPER. I-273

Johnson. See Colorado.

Johnson \& Johnson. See Arrowhead.

Johnson \& Skeen - $(2.15,11.4)$; GOLD.

B 849-I, p. 519

Johnson \& Wagner. See Arrowhead.

Jonsey - $(19.5,6.35)$; COPPER. Includes references to Bald Eagle; see also Monarch.
B 345, p. 178
B 662, p. 215
B 379, p. $89-90$
B 963-B, p. 70-71

(Juneau Cr.) - $(4.75-4.9,11.85)$; GOLD (?). See also (Mills Cr.).

B 587, p. 204-205

Kacerosky \& Hargood. See Robin Red Breast.

Kaffir. See (Quartz Cr.) (Kenai L.).

Kavanaugh \& Boon - $(16.15,15.3)$; GOLD, LEAD, COPPER.

B 592, p. 234 
Kenai-Alaska (Gold Co.) -. (6.1,7.9) ; GOLD, LEAD, ZINC. Includes references to Stephenson and Stevenson if definitely to these mines.
B 379, p. 107
B 592, p. 63
B 442, p. 173
B 642, p. 56
B 480, p. 32
B 662, p. 45
B 520, p. $138,142,147-150,160$
B 692, p. 175
B 542, p. 37-38
BMB 142, p. 43, 50
B 587, p. 157-163
BMB 153, p. 35

Kenai Dred. Co. See (Kenai R.), (Sixmile Cr.).

Kenai.Gold, Inc. See (Sixmile Cr.).

(Kenai L.). See Lyengholm, Hargood \& Larson.

Kenai Min. \& Mil. Co. See (Cooper Cr.).

Kenai Peninsula Placer Mines. See (Canyon Cr.).

(Kenai R.) - (0.0-1.45, 8.4-8.6) ; GOLD. See also (Kenai R.) under Kenai quad.
AR 20, p. 320-321
B 592, p. 63
B 277, p. $43-44$
B 622, p. 46
B 520, p. 141, 160-165
B 642, p. 55
B 542, p. 44
B $926-\mathrm{A}, \mathrm{p} .37$
B 587, p. 181-183, 185, 187, 197-199

Kenai Star - $(4.15,14.8)$; GOLD, LEAD, ZINC, COPPER. Includes reference to Logman.

B 542, p. 38

B 755, p. 30

B 587, p. 171

B 773, p. 38

B 592, p. 63

B 849-I, p. 501-503

Kennecott Copper Corp. See (Latouche).

Kennedy (-Pullen-Davis). See Mizpah.

(Kern Cr.) - (7.8-8.45, 16.0-16.4) ; GOLD.

B 642, p. 186-187

Keynote. See Griset.

King - (11.1, 14.1) ; GOLD (?).

B 592, p. 234

I-273

(King R.). See (Kings Bay).

(Kings Bay) - (10-11, 7-8) ; GOLD.

B 379, p. 97

B 592, p. 237

Knights I. Alaska Copper Co. - (19.8, 6.0) ; COPPER, ZINC.

B 379, p. 90

B 662, p. 215-216

B 963-B, p. 71-72

I-273

Knights I. Consol. Copper Co. See Jonesy, Monarch.

Knights I. Copper (Min.) Co. - $(19.55,5.75)$; COPPER. NoTE-Not mentioned by name in $\mathrm{B} 284$.

\begin{tabular}{|c|c|}
\hline B 284, p. 85 & B 622, p. 133 \\
\hline В 379, р. 90 & B 642, p. 139 \\
\hline В 443, p. 56 & B 963-B, p. 73 \\
\hline B 520, p. 28 & I-273 \\
\hline
\end{tabular}

Knights I. Min. \& Dev. Co. - $(20.15,8.0)$; COPPER, ZINC. Note. Not mentioned by name in $\mathrm{B} 662$.
B 379 , p. 93
B 443, p. 69-70
B 963-B, p. 68-69.
B 662, p. 212
I-273

Kopovich. See Robinson \& Bowman, Teddy Bear.

Kopovich \& Sobel. See Sunshine. 
Ladysmith (Smelt. Corp.). See (Latouche).

Lakeside - $(5.8,5.95)$; GOLD ( ?).

B 587, p. 148

Lakeview Min. Corp. - $(5.9,6.55)(?)$; GOLD. See also Seward Bonanza Gold Mines Co.

B 824, p. 21

Lane. See California-Alaska Min. Co.

Lansing - $(13.8,15.45)$; GOLD, LEAD, ZINC. I-273

Larson. See (Copper Bay).

Last Dollar. See Kenai-Alaska.

(Latouche) - (18.4-18.45, 1.1-1.2) ; COPPER, GOLD, SILVER, ZINC, LEAD, NICKEL. Includes references to Barrack, Barrack-Girdwood, BeatsonBonanza, Beatson (Copper Co.), Big Bonanza, Blackbird, Bonanza (Latouche), Chenega, Chinega, Girdwood, Kennecott Copper Corp., Ladysmith (Smelt. Corp.), Latouche Copper Min. Co., Whitewing.

$\begin{array}{ll}\text { AR 20, p. 419-420 } & \text { B 755, p. 15, 28, } 119 \\ \text { B 284, p. 82, 85-87 } & \text { B 773, p. 28, 38 } \\ \text { B 314, p. 27 } & \text { B 783, p. 20 } \\ \text { B 345, p. 178 } & \text { B 792, p. 27-28 } \\ \text { B 379, p. 88 } & \text { B 797, p. 32, 35 } \\ \text { B 442, p. 39, 164-165 } & \text { B 810, p. 45-46 } \\ \text { B 443, p. 52-54, 56-58, 63-67 } & \text { B 813, p. 53-54 } \\ \text { B 480, p. 31, 81 } & \text { B 824, p. 59 } \\ \text { B 520, p. 27-28 } & \text { B 836, p. 61 } \\ \text { B 542, p. 34 } & \text { B 844-A, p. 60-61 } \\ \text { B 592, p. 62, 240, 243 } & \text { B 857-A, p. 57 } \\ \text { B 605, p. 13-14, 60-61 } & \text { B 963-B, p. 63-65 } \\ \text { B 622, p. 45, 131-133 } & \text { B 989-E, p. 228, 262, 266, 281, } \\ \text { B 642, p. 138-139 } & 298-300,302 \\ \text { B 662, p. 44, 184, 201-202, 204-206, } & \text { B 1024-E, p. 110, 118 } \\ 208-209 & \text { I-273 } \\ \text { B 692, p. 18, 31, 144-145 } & \text { BMB 142, p. 41, 52 } \\ \text { B 712, p. 32-33 } & \text { BMB 153, p. 47-48 } \\ \text { B 714, p. 12-14, 21-24, 69, 77 } & \text { BMB 405, p. 25-26, 205-206, } \\ \text { B 722, p. 19, 21, 39-40 } & 219-220,269 \\ \text { B 739, p. 10, 23-24 } & \text { IC 7379, p. 33 }\end{array}$

Latouche Copper Min. Co. See (Latouche).

Latouche I. Copper Min. Co. (Ltd.) - (18.7-19.05, 0.3-1.05) ; COPPER, ZINC.

B 379, p. 89

B 642, p. 139

B 662, p. 210-211

I-273

(Lawing) - $(5.4,7.7)$; HAYTITE.

B 1039-C, p. 57-58, 60, 62-63

Lechner. See Skeen-Lechner.

Lenora. See (Cedar Bay).

Likes \& Frazer. See Copper Chief, Iron Cap, Real Thing.

Liljegren. See Copper Bullion.

Lindblad. See (Winner Cr.).

Lisbon. See Gilpatrick.

Logman. See Kenai Star.

Lone Star - $(13.9,15.5)$ approx ; GOLD (?). See also Hamilton.

B 662, p. 188 
Losness. See Mile Four Min. Co.

(Louis Bay) - (20.1-20.15, 8.35-8.5) ; COPPER. See also (Disk I.), Knights I. Min. \& Dev. Co.

B 662, p. 211-212 I-273

B $963-B$, p. 69

(Lower Herring Bay) - $(19.3,7.25)$; COPPER.

B 662, p. 213

Lucky Strike (Falls Cr.). See Kenai-Alaska.

Lucky Strike (Palmer Cr.) - $(3.95,13.65)$; GOLD, LEAD, ZINC. Includes references to Alaska Minerals Co., Hershey.

$\begin{array}{ll}\text { B 587, p. 171 } & \text { B 813, p. 18 } \\ \text { B 622, p. 46 } & \text { B 824, p. 21 } \\ \text { B 642, p. 56 } & \text { B 836, p. 20 } \\ \text { B 662, p. 45 } & \text { B 844-A, p. 20-21 } \\ \text { B 692, p. 176 } & \text { B 849-I, p. 494-498 } \\ \text { B 722, p. 40 } & \text { B 857-A, p. 20 } \\ \text { B 739, p. 24 } & \text { B 864-A, p. 22 } \\ \text { B 755, p. 29-30 } & \text { B 880-A, p. 26 } \\ \text { B 773, p. 15, 38 } & \text { B 897-A, p. 33 } \\ \text { B 792, p. 11 } & \text { B 910-A, p. 29 } \\ \text { B 797, p. 12 } & \text { B 926-A, p. 25 } \\ \text { B 810, p. 17 } & \text { BMB 153, p. 35-36 }\end{array}$

Lucky Swede - $(17.5,17.15)$; GOLD.

B 592, p. 222

I-273

Lyengholm, Hargood \& Larson - $(2.65,8.5)$ approx; ANTIMONY. Includes references to antimony near (Kenai L.) and near (Quartz Cr.) (Kenai L.).

$\begin{array}{ll}\text { B 442, p. } 178 & \text { B } 642, \text { p. } 55 \\ \text { B 587, p. } 179 & \text { B } 649, \text { p. } 62\end{array}$

(Lynx Cr.) - (6.0-6.05, 12.15-12.35) ; GOLD, COPPER. See also Ready Bullion Copper Co.

$\begin{array}{ll}\text { B 277, p. 9, 37, 40 } & \text { B 810, p. 23 } \\ \text { B 314, p. 123-124 } & \text { B 813, p. 26 } \\ \text { B 379, p. 62 } & \text { B 824, p. 30 } \\ \text { B 520, p. 141, 161 } & \text { B 836, p. 30 } \\ \text { B 587, p. 207-208 } & \text { B 844-A, 30 } \\ \text { B 642, p. 55 } & \text { B 849-I, p. 522, 527 } \\ \text { B 662, p. 44 } & \text { B 857-A, p. 28 } \\ \text { B 682, p. 176 } & \text { B 864-A, p. 32 } \\ \text { B 755, p. 29 } & \text { B 868-A, p. 33 } \\ \text { B 773, p. 38 } & \text { B 880-A, p. 37 } \\ \text { B 783, p. 12 } & \text { B 910-A, p. 43 } \\ \text { B 797, p. } 17 & \text { BMB 153, p. 34 }\end{array}$

(McClure Bay) - $(15,9)(?)$; GOLD (?).

B 592, p. 237

McMillan - (3.7-3.75, 10.45-10.5) ; GOLD. Includes references to Columbia,

Imhoff (, Weidlich \& Saulsbury), Ophir.

B 542, p. 38

B 692 , p. 175

B 587, p. 164

B 849-I, p. 518-519

B 622, p. 46

Mallard - $(18.95,5.05)$; COPPER, GOLD.

B 662, p. 217

$I-273$

B 963-B, p. 73 
Martin. See (Sixmile Cr.).

Mary. See California-Alaska Min. Co.

Mascot - (3.4, 11.75) ; GOLD.

B 587, p. 168

B $849-I$, p. 516

Mathison (Min. Co.). See (Resurrection Cr.).

Matz (\& Schmesar). See (Mills Cr.).

Mayflower - (17.4, 17.15) ; GOLD.

B 592, p. 221

I-273

Meat-in-the-pot. See Gilpatrick.

Merrill Min. Co. See Hermann \& Eaton.

Metz. See (Mills Cr.).

Michaelson. See (Blue Gulch), (Mills Cr.).

Michaelson \& McCash. See (Sixmile Cr.).

Mile 4. See Mile Four Min. Co.

Mile Four Min. Co. - $(5.05,2.95)$; GOLD. Includes reference to Mile 4. B 520, p. 155

B 587, p. 144

Mile Seven - $(5.2,3.95)$; GOLD, LEAD, ZINC. Includes references to Brewer Alaska Syn., Sevenmile.

B 542, p. $37-38$

B 592, p. 63

B 587, p. 144-145

Millard. See Granite.

Miller. See (Resurrection Cr.).

(Miller Cr.). See (Mills Cr.).

Miller, Tolson \& Plowman. See (Mills Cr.).

(Mills Cr.) - (4.55-5.05, 11.45-11.85) ; GOLD. Includes reference to (Miller Cr.).

AR 18, p. 82

AR 20, p. 277, 319, 321

B 259 , p. $96-97$

B 277, p. 9, 37-39

B 314, p. 119, 123

B 379, p. 62

B 520, p. 141, 160-161, 168

B 587, p. 204-205

B 622 , p. 46
B 642 , p. 55

B 692 , p. 176

B 755, p. 29

B 783, p. 12

B 813, p. 26

B 849-I, p. 521-522, 527

B 910-A, p. 43

B 917-A, p. 40

B 926-A, p. 36-37

Mineral King (Min. Co.). See Hermann \& Eaton.

Minnie - $(19.45,4.3)$; COPPER, ZINC.

B 662, p. 219

B 963-B, p. 74-75

I-273

Mizpah - $(5.05,4.75)$; GOLD, LEAD, ZINC, COPPER. Includes reference to Pullen-Davis.
B 520, p. 155
B 587, p. 145-146

Monarch - $(19.25,6.65)$; COPPER. See also Jonesy.
B 345, p. 178
B 379, p. 89-90
B 963-B, p. 125
B 662, p. 125
I-273

Moon Anchor. See Kenai-Alaska.

Moore - $(18.9,8.3)$; COPPER.

B 692 , p. 144, 146

Moose Cr. Placers. See (Mills Cr.). 
Moose Pass (Min. Co.) - $(3,10)(?)$; GOLD.

B 592 , p. 63

BMB 142, p. 43

B 622, p. 46

Moose Pass Placers, Inc. See (Mills Cr.).

Morning Star - $(17.65,17.55)$; GOLD.

B 592, p. 220

Mountain - $(17.25,17.3)$; GOLD.

B 592, p. 222

$\mathrm{I}-273$

Mullins, James, Coal Co. See Wilcox.

(Mummy Bay) - (18.75-19.4, 4.3-4.65) ; COPPER. See also Home Camp, Minnie. B 284, p. 85

Nearhouse (\& Smith) - $(4.25,15.2)$; GOLD, SILVER.
B 849-I, p. 503-505
B 910-A, p. 29
B 880-A, p. 26
B 926-A, p. 25
B 897-A, p. 33

Nellie - $(19.6,6.2)$; COPPER.

B 662, p. 217-218

(Nellie Juan R.) - (8.5-11.2, 4.5-7.7) ( ?) ; GOLD.

B 592, p. 237

Nelson \& Rystrom - $(24.7,16.15)$; COPPER. I-273

New Hope - $(3.95,13.65)$ ( ?) ; GOLD.

B 910-A, p. 29

B 926-A, p. 25

New York. See Carter.

Nordstrom, Carlson \& Furman. See Bullion (Passage Canal).

Nordstrom, Carlson, Christiansen \& Others. See Thomas-Culross Min. Co.

North Star (Avery R.) - $(17.65,17.55)$; GOLD.

$$
\text { B 592, p. } 220
$$

B 642, p. 142

North Star (Falls Cr.). See California-Alaska Min. Co.

Northern Light - $(4.85,1.6)$; GOLD. Includes references to unnamed lodes near Seward.

B 277, p. 47

B 587, p. 142-143

B 379, p. 107

Nugget - $(17.4,17.3)$; GOLD, SILVER, LEAD, COPPER.

B 542, p. 37

B 592, p. 220-221

B 642, p. 142

B 622, p. 135

B 662, p. 189

Nutter \& Dawson. See (Crow Cr.).

Nutter-Dawson Co. See (Crow Cr.).

OK. See Carter.

Olson \& Viette - $(15.1,17.75)$; GOLD, ZINC, COPPER.

B 592, p. 229-230

Olympia. See Gilpatrick.

Ophir. See McMillan.

Oracle. See Alaska Oracle.

Oregon. See Skeen-Lechner.

Pacific Securities Co. See Seward Gold.

(Palmer Cr.) - (3.15-3.4, 14.9-15.1) ; GOLD, SILVER.

B 259, p. 95-96

B 277, p. 8, 35-36

B 520, p. 141

B 587, p. 195-196
B 755, p. 118

B 849-I, p. 526

B 917-A, p. 40

B 926-A, p. 37 
Palmer Cr. Min. Co. See (Resurrection Or.).

Pandora - $(19.9,6.55)$; COPPER, ZINC.

B 379, p. 92-93

B 692, p. 146

B 520, p. 28

B 963-B, p. 69-70

B 642, p. 139

I-273

B 662, p. 213

(Pass Cr.) See (Tributary Cr.).

(Passage Canal) - $(11.05,13.7)$; GOLD (?).

B 926-D, p. 233

Pearson Bros. Bee (Resurrection Cr.).

Pedersen - $(13,15)(?)$; GOLD (?).

B 692 , p. 150

Pedersen \& Hanson. See Sweepstake Min. Co. (Harriman Fiord).

Peel \& Iverson. See Independence.

Perseverance. See Conley \& McChesney.

Peterson \& Mayfield. See (Crow Cr.).

(Peterson Cr.) - (NW 1/4 8, 15) ; GOLD.

B 642, p. 186, 192

Plowman, Tolson, Miller \& Davies. See (Canyon Cr.).

Polly Min: Co. See (Mills Cr.).

Porcupine (Gold Min. Co.) - (NW 1/4 5, 5) ; GOLD, LEAD, ZINC.

B 587, p. 148

BMB 142, p. 52

Port Wells (Granite Mines). See Granite.

Portage - $(12.5,15.25)$; GOLD.

I-273

(Portage Bay) - $(12.55,15.25)$ (?) ; GOLD(?). See also Portage Bay Min. Co. B 824, p. 22

Portage Bay Min. Co. - $(12.55,15.25)$; GOLD. Includes references to Portage Gold Mines (Ltd.) ; not mentioned by name in B 926-D.

B 910-A, p. 27

B 917-A, p. 27

B $926-\mathrm{D}$, p. 233

B $926-A$, p. 26

B $933-A$, p. $26-27$

Portage Gold Mines (Ltd.). See Portage Bay Min. Co.

(Portage Pass) - $(10,13)$; COOPER ( ?)

B 642, p. 139

B 926-D, p. 233

Portland. See Skeen-Lechner.

Portsmouth. See Jensen.

Primrose (Min. Co.) - $(5.05,5.75)$; GOLD, ZINO, LEAD, COPPER.

B 542, p. 37-38

B 587, p. 146-147

B 592, p. 63

B 622, p. 46

B 642 , p. 56

B 662 , p. 45

B 692, p. 175

B $880-$ A, p. 26

B 897-A, p. 32

BMB 142, p. 43, 52

BMB 153, p. 34-35

Pullen-Davis. See Mizpah.

(Quartz Cr.) (Kenai L.) - (3.15-3.45, 9.25-9.65) ; GOLD, LEAD, ZINC, COPPER.

See also Lyengholm, Hargood \& Larson.

B 442, p. 172

B 520, p. 141, 160-161, 167-168

B 622, p. 46

B 587, p. 163, 201-202

B 642 , p. 55

B $849-$ I, p. 518 
(Quartz Cr.) (Turnagain Arm) - (7.25-8.1, 13.5-14.9) ; GOLD.

B 277, p. 43

Queirolo. See (Canyon Cr.), (Donaldson Cr.).

RADCO, Inc. See Reynolds Alaska Der. Co.

Ready Bullion Copper Co. - $(5.85,11.75)$; COPPER. See also (Lynx Cr.).
P 70, p. 164
B 314, p. 124-125
B 259, p. 99
B 587, p. 177-178
B 277, p. 48
B $849-$ I, p. 520-521

Reagan - $(15.35,13.15)$ ( ?) ; GOLD. See also Sells.

B 662, p. $187-188$

Real Thing - $(6.55,0.5)$; IRON, COPPER.

B 379, p. 103-104

B 587, p. 234

Redman \& Guyot - $(6.4,1.95)$; COPPER. B 379, p. 103

B 587, p. 233

Reed, Gauthier \& Cooper - $(15.1,16.85)$; GOLD, ZINC, COPPER. Includes reference to Everson, Gauthier \& Cooper.

B 592, p. 230-231

I-273

B 622, p. 138

Renner. See (Mills Cr.).

Resurrection Bay Min. Co. - $(4.85,1.7)$; GOLD, ZINC, LEAD, COPPER. Includes reference to unnamed lodes near Seward.

B 379, p. 107

B 587, p. 142

(Resurrection Cr.) or (R.) - (3.1-3.2, 14.95-16.0) ; GOLD. Includes references to Mathison, St. Louis.
AR 18, p. 81-82
B 783, p. 12
P 70, p. 156, 162-163
B 797, p. 17
B 259, p. 91-92, 95
B 810, p. 23
B 277, p. 8-9, 34-35
B 813, p. 26
B 442, p. 42
B 824, p. 30
B 520 , p. 141, 160-162, 170-171
B 836, p. 30
B 587, p. 193-195
B 844-A, p. 30
B 592, p. 63
B $849-$ I, p. 521, 525-526
B 622, p. 46
B 857-A, p. 28
B 642, p. 55
B 864-A, p. 32
B 662, p. 44
B 692, p. 32,176
B 868-A, p. 33
B 722, p. 40
B 755, p. 29, 118
B $880-A$, p. 36
B 917-A, p. 40
B 773, p. 38
B 926-A, p. 36-37
BMB 153, p. 36-37

Reynolds Alaska Dev. Co. - (18.1-18.3, 0.45-0.7) ; COPPER, GOLD, SILVER, ZINO, LEAD. Includes references to Duchess, Duke, (Horseshoe Bay), Iron Mtn.
B 284, p. 85
B 345, p. 178
B 379, p. 88-89
B 443, p. 52-53, 56, 66-67
B 520, p. 28
B 662 , p. 201-202, 206-208
B 692, p. 145

B 714, p. 22-23

B 963-B, p. 65-66

B 989-E, p. 262, 299-300

B 1024-E, p. 107, 109-110, 113-122

I-273

BMB 153, p. 49

(Rhea Cove). See Copper Bullion.

Richards, Mathieson and assoc. See (Resurrection Cr.). 
Robin Red Breast - $(4.2,14.8)$; GOLD.

B 773, p. $38 \quad$ B 849-I, p. 503

Robinson \& Bowman - $(3.3,14.9)$; GOLD, SILVER, LEAD.

B 773, p. 38-39

B 849-I, p. 505

Roe. See Nugget.

Ronan (\& James) - $(3.65,10.85)$; GOLD. Includes references to Champion, Gladiator, James \& Ronan, Ronan \& Jones.

B 662, p. 45

B 849-I, p. 510

B 692, p. 32,175

BMB 153, p. 36

Ronan \& Jones. See Ronan.

Rua \& Bettels. See Copper Bullion.

(Rua Cove). See Copper Bullion.

Russell Ball Copper Co. See Copper Coin.

Russian-American Co. See (Kenai R.).

St. Louis (Min. Co.). See (Resurrection Cr.).

St. Louis Min. \& Mil. Co. See (Resurrection Cr.).

St. Louis Min. \& Trad. Co. See (Resurrection Cr.).

Sampson - (NW 1/8 quad.) ; GOLD (?).

B 692, p. 175

(Sawmill Cr.) - (6.3, 15.45) ; GOLD, ZINC, LEAD. See also (Slate Cr.) (Turnagain Arm).
AR 20, p. 306
B 277, p. 43, 47
P 70, p. 163

B 587, p. 172-173

B 259, p. 98

Scheen-Lechner. See Skeen-Lechner.

Schoonover - (5.0, 5.0) (?) ; GOLD.

B 520, p. 155

Schultz. See Home Camp.

Scotia Bell. See Jensen.

(Seattle Cr.) - (6.5-7.2, 14.4-15.5) ; GOLD.

B 277, p. 43

Sells - $(15.35,13.15)$; GOLD ( ?). See also Reagan.

B 592, p. 236

I-273

Sevenmile. See Mile Seven.

(Seward). See Northern Light, Resurrection Bay Min. Co.

Seward Bonanza Gold Mines Co. - $(5.9,6.55)$; GOLD, LEAD, ZINC, COPPER.

Includes references to Gould, Hickey; see also Lakeview Min. Corp.

B 442, p. 173

B 520, p. 142, 144-146, 160
B 542, p. 38

B 587, p. 149-151

Seward Gold (.Co.) - $(6.2,11.4)$; GOLD, LEAD, ZINC.

B 520, p. 151-152

B 587, p. 169-171

B 849-I, p. 519

Seward Min. Co. See (Resurrection Cr.).

Seward Placer Co. See (Mills Cr.).

Seward Placers, Inc. See (Canyon Cr.).

Shaw. See Skeen-Lechner.

Shaw, Deubruel \& Bouchaert - $(6.25,2.0)$; COPPER, GOLD.

B 379, p. 103

B 587, p. 233

Shell \& Richards. See (Bear Cr.).

Silverman. See Seward Gold. 
(Silvertip Cr.) - $(5.5,12.85)$; GOLD.
B 277 , p. 40
B 520 , p. $141,160,169-170$
B 587 , p. 206

Singletary. See (Louis Bay).

Singletary-O'Neil - $(13,17)(?)$; GOLD. May be in Anchorage quad.

B 542, p. 37

(Siwash Bay) - $(20.25,17.3)$; COPPER. Includes reference to Wagner.

B 662, p. 185

I-273

B $963-B$, p. 77

(Sixmile Cr.) or (R.) - (4.8-5.0, 13.7-15.6) ; GOLD.
AR 18, p. 81-82
B 755, p. 118
AR 20, p. 318,320
B 783, p. 12
P 70, p. 162-163
B 813 , p. 26
B 259 , p. $91-92,96$
B 824 , p. 30
B 263, p. 207
B 844 -A, p. 30
B 277, p. 9, 37
B. $849-$ I, p. 521,526
B 314, p. 119, 122
B 868-A, p. 33
B 379, p. 52
B 520, p. $141,160-162,168$
B $880-A$, p. 36-37
B 587, p. 202-203
B $897-A$, p. 43
B 622, p. 46
B 642 , p. 22-23, 55
B 722, p. 40
B 910-A, p. 43
B 926-A, p. 37
BMB 142, p. 43
BMB 143, p. 35

(Sixmile Cr., East Fork). See (East Fork).

Skee \& Lechner. See California-Alaska Min. Co.

Skeen. See Skeen-Lechner.

Skeen \& Lechner. See California-Alaska Min. Co., Skeen-Lechner.

Skeen-Lechner (Min. Co.) - (6.3, 7.7); GOLD, LEAD. Includes references to

Lechner, Scheen-Lechner, Skeena-Lechner; see also California-Alaska Min. Co.
B 442 , p. 173
B 520, p. $142,150-151$
B 622 , p. 46
B 542 , p. $37-38$
B 662 , p. 45
B 587 , p. $154-157$
BM.B 142, p. 43
B 592 , p. 63
BMB 153, p. 36

Skeen-Stevenson - (5.9-6.3, 6.55-7.9) (?) ; GOLD. Reference may be to KenaiAlaska, Seward Bonanza Gold Mines Co., Skeen-Lechner.

B 642, p. 56

Skeena-Lechner. See Skeen-Lechner.

Skypilot - $(12.95,17.2)$; GOLD.

B 592, p. 229

(Slate Cr.) (trib. Quartz Cr.) - $(3.8,10.6)$; GOLD, SILVER.

B 849-I, p. 490-491, 516

(Slate Cr.) (Turnagain Arm) - (6.45-6.6, 15.5-15.6) ; GOLD. Includes an occurrence on shore of Turnagain Arm east of Slate Cr.; see also (Sawmill Cr.). B 259, p. 98

B 277, p. 47

B 587, p. 172

Slator. See (Fresno Cr.).

(Slide Cr.). See (Slate Cr.) (Turnagain Arm).

Smith \& Powers. See (Lynx Cr.).

(Snipers Pt.) - (NW 1/4 5, 15) ; GOLD.

B 520, p. 161

Snow \& Watson. See Gilpatrick. 
Snowstone - $(20.05,7.5)$; COPPER. Includes reference to Wallace, McPherson \& Valentine.
B 379, p. 92
I-273

(Snug Harbor) - $(19.8,4.9)$; COPPER, ZINC. Includes references to Bettles. B 379, p. 92

B 442, p. 165 B 963-B, p. 74

B 662, p. 218-219

Snyder. See Copper Bullion.

Solar Dev. Co. See Copper Bullion.

Sollars - $(6.45,8.4)$; GOLD (?).

B $849-1$, p. 520

(Squaw Bay) - $(19.0-19.05 ; 14.9-15.15)$; GOLD.

$$
\text { I-273 }
$$

Stephenson. See Kenai-Alaska, Seward Bonanza Gold Mines Co.

(Stetson Cr.) - (1.1-1.3, 7.1-7.8) ; GOLD.
AR 20, p. 320
B 259, p. 98
B 587, p. 200
B 692, p. 176

B 277, p. 44

Stevenson. See Kenai-Alaska, Mile Seven.

Stines. See (Canyon Cr.), (Kenai R.), (Sixmile Cr.).

Strong - $(4.1,17.3)$; GOLD. See also (Indian Cr.).

B 739, p. 24

B 755, p. 30

Summit. See Gilpatrick.

Sunshine - $(3.8,14.2)$; GOLD.

B 849-I, p. 498

Sweepstake Min. Co. (Avery R.) - $(17.8,17.65)$; GOLD. B 592, p. 219-220

B 622, p. 135

Sweepstake Min. Co. (Harriman Fiord) - (13.8, 17.2) ; GOLD, ZINO, LEAD, ANTIMONY, COPPER. Includes references to Sweepstakes.
B 592, p. 228-229
B 622, p. 139
B 692, p. 150
I-273
B 662, p. 189
BMB 153, p. 50

Sweepstakes. See Sweepstake Min. Co. (Harriman Fiord).

Swetmann - (3.75, 10.8) ; GOLD; SILVER, LEAD, COPPER. Includes reference to Baughman \& Swetmann; see also New Hope.

B 824, p. 21

B 849-I, p. 515

Tatum (\& Erving). See Granite.

Taylor - $(3.9,15.6)$; GOLD, LEAD.

B $849-\mathrm{I}$, p. 506

Teddy Bear - $(4.05,13.85)$; GOLD, LEAD, ZINC, COPPER.

B 849-I, p. 499-500

Telluride. See Seward Gold.

Tenderfoot - (W 1/4 quad.) ; GOLD( ?).

B 592, p. 63

Texas-Oklahoma Consolidation. See (Resurrection Cr.).

(Thomas Bay). See Thomas-Culross Min. Co.

Thomas-Culross Min. Co. - $(15.7,13.25)$; GOLD, LEAD, ZINC, COPPER. Includes references to Bugaboo, Chelan, Culross, Culross I. Min. Co.
B 592, p. 235-236
B 622, p. 139
B 642, p. 142
B 692, p. 149
B 755, p. 29
B 662 , p. 188
B 792, p. 10-11
B 797, p. 12 
Thomas-Culross Min. Co.-Continued
B 926-A, p. 26
BMB 142, p. 42, 52
B 989-E, p. 307
BMB 153, p. 50

I-273

Tolsen, Plowman, Miller \& Davies. See (Canyon Cr.).

Tolson \& Stanton - $(\mathbf{1 7 . 3 5}, \mathbf{1 6 . 8 5})$; GOLD, ZINC.

B 592, p. 225

I-273

B 622, p. 136

Tomboy - $(13.9,15.7)$; GOLD, LEAD.

B 592, p. 233

B 692 , p. 150

B 622, p. 138

I-273

Totten \& Gaydon. See McMillan.

Tozier. See Northern Light.

(Trail Cr.). Bee (Falls Cr.).

Triangle. See (Mills Cr.).

(Tributary Cr.) - $(4.1,12.3)$; GOLD (?).

B 587, p. 168

B 849-I, p. 517

Twentieth Century Knight I. Copper Min. Co. - $(19.65,5.8)$; COPPER(?).

B 379, p. 90

I-273

B 963-B, p. 73

(Twentymile R.) - $(9.2,15.2)$ approx, GOLD ( ?).

B 642, p. 186, 192

U \& I - (18-19, 5-6) ; COPPER( ?). See also Una.

B 662, p. 218

Una - $(18-19,5-6)(?)$; COPPER. See also U \& I.

B 622, p. 133

Viette - $(12.55,15.25)$ ( ?) ; GOLD. See also Portage Bay Min. Co.

B 880-A, p. 25-26

Virginia - (NW 1/8 quad) ( ?) ; GOLD.

B 722, p. 40

Von Greunther. See (Louis Bay).

Wagner. See (Siwash Bay).

Wagner (\& Johnson). See Arrowhead.

Wallace, McPherson \& Valentine. See Pandora, Snowstone.

Wanowsky Gold Mines Co. See Gilpatrick.

Wanowsky Gold Min. Co. See Gilpatrick.

Watson \& Snow. See Gilpatrick.

Wells Bay Gold \& Copper Min. Co. - $(21,16)(?)$; COPPER(?). May be in Anchorage quad.

B 662, p. 185

Westberg \& Domenzet. See Tomboy.

Wheatley \& McDonald. See Brewster.

Whistler. See Conley \& McChesney, Downing, Kenai-Alaska. Note.-Three separate claims.

White. See (Lynx Cr.).

White \& Jones. See Skypilot.

White \& Pedersen. See Eldorado.

White Wing. See (Latouche).

Whittlesey. See Ronan.

Wible. See (Canyon Cr.). 
Wilcox - (19.45-19.6, 4.1-4.2) ; COPPER.

B 379, p. 91

$\mathrm{I}-273$

Wilson. See (Canyon Cr.).

(Wilson Bay) - (18.3-18.4, 0.9-0.95) ; COPPER. Includes reference to Chicago-

Latouche Min. \& Power Co.
B 379, p. 88
I-273

B 963-B, p. 66

(Winner Cr.) - $(7.9,17.35)$; GOLD.
B 277, p. 40,43
B 642, p. 55,186
B 520, p. 160
B 692 , p. 176
B 587, p. 192-193
B $849-$ G, p. $405-406$

B 622, p. 46

Yakima - $(14.6,16.95)$; GOLD, ZINC, LEAD, COPPER.

B 592, p. 231

B 622, p. 138

B 662, p. 189

I-273

Yannes - $(15.4,17.7)$; GOLD (?).

I-273

Yellow Horse. See Dunklee \& Reilly.

Yellow Jacket. See (Quartz Cr.) (Kenai L.).

Young. See (East Fork), (Gulch Cr.).

( ....-) (lead) - $(14.15,17.25)$ approx ; LEAD.

B 662, p. 189

\section{SHUNGNAK QUADRANGLE}

(California Cr.) - (15.95-16.6, 17.25-17.75) ; GOLD, ASBESTOS, JADE, QUARTZ.

B 712, p. 52, 197-198

B 836, p. 51

B 755, p. 49

B 844-A, p. 51-52

B 773, p. 52

B 792, p. 23

B 857-A, p. 48

B 797, p. 28

B $880-$ A, p. 58

B 810, p. 37

B 897-A, p. 68

B 813, p. 44

B $933-$ A, p. 64

B 815, p. 325

TDM 3-R, p. 13, 25

B 824, p. 49

TDM 5-R, p. 14, 47

(Dahl Cr.) - (14.5-14.65, 17.0-17.75). See (Dahl Cr.) under Ambler R. quad.

(Dall Cr.). See (Dahl Cr.) under Ambler R. quad.

Ferguson \& Son. See (California Cr.).

Lammers and assoc. See (California Cr.).

(Lynx Cr.) - (15.7-16.2, 17.25-17.5) ; GOLD.
B 542, p. 50
B 857-A, p. 48
B 592, p. 72
B 864-A, p. 53
B 642, p. 71
B 868-A, p. 55-56
B 692, p. 42
B 880-A, p. 58
B 792, p. 23
B $897-A, 68$
B 813 , p. 44
B 910-A, p. 73
B 824, p. 49
B 917-A, p. 71
B 836 , p. 51
B 926-A, p. 67
B 844-A, p. 51
B 933-A, p. 64

$593018-61-18$ 


\section{SITRA QUADRANGLE}

Alaska. See (Mirror Harbor).

Alaska-Chichagoff Min. Co. - $(8.4,11.55)$; GOLD, SILVER. Includes references to McKallick Chichagoff Gold Mines, Inc.
B 844-A, p. 15
B 864-A, p. 15
B 857-A, p. 15
B 929 , p. $130-132$

Alaska Gypsum Co. See Gypsum-Camel, (Gypsum Cr.).

Alaska Nickel Co. See (Mirror Harbor).

Alaska Nickel Mines. See (Mirror Harbor).

American Gold Co. (8.25, 11.45) ; GOLD.

B 929 , p. $125-126$

Anderson - $(9.2,11.2)$; GOLD(?).

B 929, p. 124

Andy. See Handy-Andy Min. Co.

Anna. See Hirst-Chichagof.

Apex. See Apex-El Nido.

Apex-El Nido (Min. Co.) - $(6.55-6.65,16.65-16.7)$; GOLD, TUNGSTEN, SILVER, ZINC, COPPER. Includes references to Apex, El Nido.
B 722 , p. $37-38$
B 739 , p. 22
B 755, p. 25
B 773, p. $12,31,114-121$
B 783, p. 7,41
B 792 , p. 10
B 797 , p. 10
B 857-A, p. 15
B 864-A, p. 15
B 868-A, p. 15
B 880-A, p. 16
B $897-A$, p. 17
B $910-$ A, p. 19
B 800 , p. 317-319, 330-331, 346,
B 917-A, p. 20
372,378
B 810, p. 12
B 813 , p. 14
B 824 , p. 16
B 926-A, p. 19
B 929 , p. 143-145
B 963-A, p. 20-23
IC 7844, p. 13
RI 4174, p. 5, 48-51

B 836, p. 14

Armstrong \& Nyland. See Golden Hand.

Aurora. See (Mirror Harbor).

Bach, Gamble \& Kelly. See Chichagoff.

Bahrt. See Hirst-Chichagof.

(Baker Peak) - (7.07-7.15, 14.3-14.4) ; COPPER, GOLD, SILVER, LEAD. Includes references to Gold-Copper, Golden Copper.

B 692 , p. 121-123

B 773, p. 121

B 755, p. 25

Baker, Toby \& Bolyan. See (Baker. Peak), Snow Slide.

Baldy Lode - $(18.4,14.4)$ approx; probably barren. Note.-Staked as nickel prospect.

B 773 , p. 108-109

Baney - $(8.4,10.9)$; GOLD. See also Gloria B.

B 929 , p. $120-121$

(Basket Bay) - (19.35-20.0, 11.3-12.0) ; MARBLE.

B 592, p. 102, 107

B 783, p. 61

B 682 , p. $46-48,113$

Bauer. See Radio.

Bauer \& Soni - $(8.15,12.0)$ approx ; GOLD (?).

B 929, p. 135 
Bear. See Hirst-Chichagof.

Bear Extension. See Tillson.

Bear in All. See Hirst-Chichagof.

Bell Boy. See (Gypsum Cr.).

Bergstrom \& Gustafson. See Falcon Arm.

Bernard. See Hirst-Chichagof.

Bertha. See Hirst-Chichagof.

(Bertha Bay) - $(6.05,14.15)$; COPPER.

B 692, p. 124

Betty. See (Bohemia Basin).

Bez. See Alaska-Chichagoff Min. Co.

Big Four. See Chichagoff.

Big Ledge - $(18.75,14.25)$ approx ; NICKEL, COPPER, ZINC. Includes reference to (Tenakee Inlet) (nickel).

B 773, p. 107-108 B 800, p. 348-349

(Billy Basin) - (NE 1/4 16, 1); GOLD, SILVER, LEAD. Includes reference to Thetis.

AR 18, p. 62-63, 80

Bohemia. See (Bohemia Basin).

(Bohemia Basin) - (5.25-5.45, 17.05-17.3) ; NICKEL, COPPER.

B 773, p. 95, 98-105, 113

B 800 , p. $348-351,373,389$

B 947-C, p. 42-43, 45-56

B 917-A, p. 104

C 252, p. 7

B 931-F, p. 118-125, 127-130,

RI 4182, p. 1-28

132-138

Boston - (16.75, 0.9) ; GOLD.

B 259, p. 58

B 504, p. 29

Brown. See (Gambier Bay) (metals).

Brown Bear. See Hirst-Chichagof, New Chichagof Min. Syn. Note. Two separate claims.

Bullion - (16-17, 0-1) (?) ; GOLD (?). May be in Port Alexander quad.

B 259, p. 58

B 504, p. 29

Camel Gypsum Co. See Gypsum-Camel.

Cann, J. H. See Apex-El Nido, Etna.

Cann, J. R. See Apex-El Nido.

Canyon. See (Bohemia Basin).

Cascade - $(16.45,1.45)$; GOLD (?).

B 504, p. 28-29

Casey. See Chichagoff.

(Chaik Bay) - (23, 5-6) ; MARBLE. Includes reference to (Chiak Bay).

B 314, p. 77

B 345, p. 122

B 682, p. 55

(Chiak Bay). See (Chaik Bay).

Chichagof Extension - (8.6, 11.55) ; GOLD( ?)

B 929, p. 128-129

Chichagof Extension Min. Co. See Chichagof Extension, Handy-Andy Min. Co.

Chichagof Prosperity Min. Co. - $(8.2,12.1)$; $\operatorname{GOLD}(?)$. Includes reference to Monte Cristo.

B 504, p. 25

B 929, 133-134 
Chichagoff (Min. Co.) - $(8.35-8.45,11.65-11.8)$; GOLD, SILVER, LEAD. Includes references to Big Four, Chichagoff Dev. Co., Chichagoff Mines, Ltd., DeGroff, Golden Gate, Golden Horn, Mills, Sitka ; see also Alaska-Chichagoff Min. Co., Apex-El Nido.

B 314, p. 61

B 345, p. 91

B 379, p. 73

B 442 , p. 139

B 480, p. 98

B 504, p. 18, 22-25

B 520, p. 24

B 542, p. 34

B 592, p. 60

B 622, p. 43

B 642, p. 78

B 662, p. 41

B 692 , p. $30,110-111,113-116$, 119

B 712, p. 30

B 714, p. 76

B 722, p. 37

B 739, p. 22

B 755, p. 25

B 773, p. 11-12, 15, 87

B 783, p. 7, 41

B 792, p. 10
B 797, p. 10

B 800, p. $317,319,343,372$

B 810, p. 12, 46

B 813, p. 14

B 824-A, p. 15-16

B 836-A, p. 14

B 844-A, p. 15

B 857-A, p. 14

B 864-A, p. 14-15

B 868-A, p. 15

B 880-A, p. 16, 72

B 897-A, p. 16

B 910-A, p. 18

B 917-A, p. 20

B 926-A, p. 18-19

B 929, p. 86-101

B 933-A, p. 18

B 1024-B, p. 49-50

BMB 142, p. 36, 52

BMB 153, p. 29

IC 7844, p. 7

Chichagoff Dev. Co. See Chichagoff.

Chichagoff Gold Min. Co. See Chichagoff.

Chichagoff Mines, Ltd. See Chichagoff.

Clyde. See (Mineral Hill).

Cobol - $(10.65,8.8)$; GOLD, LEAD. Includes references to (Slocum Arm).

B 797, p. 11

B 910-A, p. 19

B 929, p. 139-140

Condor Min. Co. See Apex-El Nido.

Congress - $(6.7,12.9)$; COPPER.

B 692, p. 123-124

B 929, p. 140-141

Cook. See (Gambier Bay) (metals).

Cox \& Bolyan. See Cobol.

Cox, Bolyan \& Loberg - $(7.4,15.0)$; GOWD, ZINC, LEAD, COPPER. Includes applicable references to (Pinta Bay), Pinta Bay Min. Co., West Coast Dev. Co.

B 755, p. 25

B 773, p. 72, 114, 121-123

B 810, p. 12

B 797, p. 10

B 929, p. 142-143

B 800, p. 346, 378

B 963-A, p. 23

(Davison Bay). See (Mirror Harbor).

(Deep Bay). See New Chichagof Min. Syn.

Degroff. See Chichagoff.

Deining. See (Magoun Is.).

Duluth - $(8.4,11.5)$; GOLD (?).

B 929, p. 126 
East Tripod. See (Bohemia Basin).

El Nido. See Apex-El Nido.

Elbow Passage - $(8.6,10.8)$; GOLD.

B 929, p. 119-120

Elsinore. See Hirst-Chichagof.

Etna - $(6.4,16.05)$; GOLD.

B 773, p. 124

(Falcon Arm). See Falcon Arm.

Falcon Arm - $(10.1,9.85)$; GOLD, SILVER, LEAD, ZINC. Includes references to (Falcon Arm), Falcon Bay Co.

B 692, p. 120

B 929, p. 118-119

B 755, p. 25

Falcon Bay Co. See Falcon Arm.

Falcon Min. Co. See Falcon Arm.

Fir-Tex Insulating Board Co. See Gypsum-Camel.

Fleming. See (Mirror Harbor).

(Fleming I.). See (Mirror Harbor).

Flora - $(8.35,11.65)$; GOLD.

B 504, p. 25

B 929, p. 132

B 692 , p. 119

Freeburn \& Rust. See Chichagoff.

Fries. See Hirst-Chichagof.

(Gambier Bay) (marble) - (27-28, 8-9) ; MARBLE. B 682, p. 55

(Gambier Bay) (metals) - (27.1-27.2, 8.9-9.4) approx; COPPER, GOLD. Includes references to Brown, Cook. B 259, p. 56

B 287, p. 151

Gloria B. - $(8.25,12.0)$; GOLD (?). See also Baney. B 929, p. 133

Gold Bug. See Hirst-Chichagof.

Gold-Copper. See (Baker Peak).

Gold Reef - (8.3-8.35, 11.55-11.6) ; GOLD( ?). B 929, p. 126, 128

Golden Copper. See (Baker Peak).

Golden Gate (Min. Co.). See Chichagoff.

Golden Hand - (7.65-7.75, 13.6-13.7) ; GOLD. B 929, p. 136-137

Golden Horn. See Chichagoff.

Goldwan - (6.15-6.2, 17.25-17.4) ; GOLD. Includes reference to Paramount. B 773, p. 114, 123-124 B 929, p. 145

Gypsum. See Gypsum-Camel, (Gypsum Cr.).

(Gypsum). See (Gypsum Cr.).

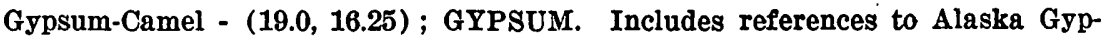
sum Co., (Iyoukeen Cove) if definitely to this prospect.

B 824, p. 80-81, 174-176

B 963-A, p. 25-28

B 880-A, p. 88

B $926-A$, p. 95-96

B 989-B, p. 27-29, 32-36

B 933-A, p. 92

IO 7844, p. 11

RI 4852, p. 1-5 
(Gypsum Cr.) - $(18.75,16.15)$; GYPSUM. Includes references to Alaska Gypsum Co., Gypsum, (Gypsum) (Iyoukeen Cove) if definitely to this mine, Pacific Coast Gypsum Co., Standard Gypsum Co.; not mentioned by name in B 622 , 712.
B 259, p. 58
B 314, p. 79-80
B 345, p. 124-125
B 379, p. 85
B 504, p. 30-31
B 542, p. 50-51
B 622, p. 43
B 642 , p. 104
B 692, p. 30
B 697 , p. $47-48$
B 712, p. 30
B 714, p. 54
B 739, p. 22

\author{
B 755, p. 25 \\ B 783, p. 30 \\ B 792, p. 38 \\ B 800, p. 332, 342 \\ B 813, p. 32 \\ B 824, p. 80-81, 173-174 \\ B 880-A, p. 88 \\ B 963-A, p. 24-26 \\ B 989-B, p. 27-29, 31-32, 34-36 \\ BMB 142, p. 36, 52 \\ BMB 153, p. 30 \\ IC 7844, p. 11
}

Haley \& Hanlon - $(17.15,1.15)$ approx ; COPPER, NICKEL, COBALT. B $947-C$, p. $63-64$

Handy. See Handy-Andy Min. Co.

Handy-Andy Min. Co. - $(8.55,11.6)$; GOLD(?). Includes reference to Handy. B 692, p. 119-120

B 929, p. 129

Hanlon - $(7.8,11.7)$; GOLD (?).

B 929 , p. 132-133

Hansen \& Bolshan. See Elbow Passage.

Henrietta. See Hirst-Chichagof.

Hill \& Berkland - (8.55, 11.5-11.55) ; GOLD (?).

B 929, p. 128

Hirst. See Hirst-Chichagof.

Hirst-Chichagof (Min. Co.) - (8.4-8.55, 11.85-12.1) ; GOLD, SILVER, ZINC, LEAD, COPPER. Includes references to Bahrt, Bear, Hirst, Hirst Cove Min. Co., Hodson, Hurst, Kay; see also Chichagof Prosperity Min. Co., (Krestof I.).
B 314, p. 61
B 810 , p. 12
B 379 , p. 73
B 813 , p. 14
B 504, p. 25
B 824, p. 15
B 592, p. 60
B 836 , p. 14
B 622 , p. 43
B 844-A, p. 14-15
B 662, p. 41
B 857-A, p. 14-15
B 692 , p. 116-119
B 864-A, p. 14-15
B 712, p. 30
B 868-A, p. 15
B 722, p. 37
B $880-A$, p. 16,72
B 739 , p. 22
B $897-A$, p. 16
B 755 , p. 9
B $910-A$, p. 18
B 773 , p. $11,15,124-125$
B 917-A, p. 20
B 783, p. 7,41
B 926-A, p. 18-19
B 792 , p. 10
B 929, p. 101-118
B 797 , p. 10
B 933-A, p. 18
B 800, p. $317,343-344,372$
B 1024-B, p. 50

Hirst Cove Min. Co. See Hirst-Chichagof.

Hodson (\& Berkland). See Hirst-Chichagof. 
Hofstad \& Johnson - $(8,11-12)$ (?) ; GOLD.

B 622 , p. 43

(Hood Bay) - (23.75-23.85, 7.6-7.65) ; MARBLE.
B 314, p. 77
B 592, p. 105-106
B 345, p. 122
B 682 , p. 54-55

Housel. See Gypsum-Camel.

Hurst. See Hirst-Chichagof.

(Iyoukeen Cove). See Gypsum-Camel, (Gypsum Cr.).

Jackson - $(8,11-12)$; GOLD (?).

B 844-A, p. 15

Jumbo- $(8.4,11.5)$; GOLD, LEAD, ZINC.
B 504, p. 25
B 739 , p. 22
B 692 , p. $118-119$
B 929 , p. $126-128$

Juneau Sea Level Copper Mines. See (Mirror Harbor).

Juneau Sea Level Copper Min. Co. See (Mirror Harbor).

Kaiser Industries, Inc. See (Gypsum Cr.).

Kay. See Hirst-Chichagof.

Klag Bay Min. Co. See Duluth, Gold Reef, Jumbo, Minnesota.

Knutson \& Bergland. See Tillson.

Koby \& Shepard - $(9.55,14.4)$; GOLD. B 929, p. 141

(Krestof I.) - (13-14, 2-4) ; GOLD.

B 910-A, p. 19

B 917-A, p. 21

Lagergren. See Big Ledge.

Lagergren \& Winerman. See Baldy Lode.

(Lake Anna) - $(8.65,11.0)$ approx ; GOLD, ZINC, LEAD. B 682, p. 120

Larson, Anderson and assoc. See Gypsum-Camel.

Lena. See Tillson.

Liberty - $(17.45,0.35)$; GOLD.
AR 18, p. 62-63, 78-80
B 259 , p. 58
B 314, p. 60
B 504, p. 29

Lillian. See Lillian \& Princela.

Lillian \& Princela - $(8.4,11.65)$; GOLD, LEAD.

B 929, p. 132

(Little Bay) - $(6.65,13.75)$; COPPER, SILVER, GOLD, NICKEL.

B 692, p. 123

Lucky Shot - (8.8-8.95, 11.1-11.35) ; GOLD, LEAD, ZINC.

B 929, p. 121-123

McKallick (lode) - $(8.65,12.1)$; GOLD ( ?). See also Alaska-Chichagoff Min. Ca. B 929, p. 135

McKallick (placer) - $(8.05,11.25)$; GOLD.

B 929, p. 124-125

McKallick Chichagoff Gold Mines, Inc. See Alaska-Chichagoff Min. Co.

McKallick Chichagof Mines, Inc. See Lillian \& Princela.

(Mogoun A.) - (13.35-13.8, 2.9-3.45) ; MOLYBDENUM, COPPER.

B 926-C, p. 174-175

(Marble Bluffs) - (21.25-21.3, 13.1-13.45) ; nothing. Nore.-Quartz monzonite rather than marble as first suspected.

B 314, p. 77

B 682 , p. $48-49$

B 345, p. 122 
(Marble Cove) - (21.4-21.7, 12.3-12.8) ; MARBLE.

B 592, p. 104-105, 107

B 682, p. 25, 50-54, 113-114

B 783, p. 61

Marinovich - $(8.45,12.1)$; GOLD (?).

B 929, p. 134

Mayflower. See (Bohemia Basin).

Mills ,\& Simons). See Chichagoff.

(Mineral Hill) - $(8,11-12)$; GOLD.

B 868-A, p. 15

B 897-A, p. 17

B 880-A, p. 16

Minnesota - $(8.4,11.5)$; GOLD.

B 929, p. 126-128

(Mirror Harbor) - (6.4-6.5, 13.8-13.95) ; NICKEL, COPPER, GOLD, SILVER. Includes references to (Davison Bay), Fleming, (Fleming I.), (Nickel), (Pinta Bay) if applicable.

B 642, p. 78

B 662, p. 25,41

B 666, p. 97

B 692, p. 22-23, 30, 125-133

B 773, p. 95-98, 110-111

B 800, p. 348-351
B 936-I, p. 222, 229-236, 238-241

B 947-C, p. 56-59, 62-63

C 252, p. 7

IC 7844, p. 12

RI 4168, p. 2-12

Monte Cristo. See Chicagof Prosperity Min. Co.

Mosquito Ledge. See Big Ledge.

Mother Lode. See Hirst-Chicagof.

New Chicagof Min. Syn. - (7.6, 13.7) ; GOLD. Includes references to Brown Bear (Pinta Bay), (Deep Bay).
B 739, p. 22
B 864-A, p. 15
B 755, p. 25
B 929, p. 137-139

B 773, p. 121

Newell \& Young. See Chicagoff.

(Nickel). See (Mirror Harbor).

Nickel Corp. of America. See (Bohemia Basin).

Nilsen - $(6.45,17.2)$ approx ; GOLD (?).

B 773, p. 124

North Muskeg. See (Bohemia Basin).

North Takanis. See (Bohemia Basin).

Pacific Coast Gypsum Co. See (Gypsum Cr.).

Paramount. See Goldwan.

Pihl, Simmons \& Mills. See Chicagoff.

(Pinta Bay). See Cox, Bolyan \& Loberg, (Mirror Harbor).

Pinta Bay Min. Co. See (Baker Peak), Cox, Bolyan \& Loberg, New Chicagof Min. Syn., South-Side.

(Pt. Hepburn) - (20.85-21.0, 16.9-17.1) ; MARBLE.

B 592, p. 103

B 682, p. $49-50,114$

Portia. See (Bohemia Basin).

Portlock Harbor Copper Min. Co. See (Baker Peak).

President - $(21.6,14.2)$ approx ; COPPER, ZINC, LEAD.

B 287, p. 151

Princela. See Lillian \& Princela.

Princess Pinder - $(7,13)$ (?) ; GOLD, COPPER.

B 504, p. 26

(Pybus Bay) - (26-27, 5-7) ; MARBLE.

B 682 , p. 55 
Radio - $(8.05,12.15)$; GOLD ( ?).

B 929, p. 135-136

(Rodman Bay) - $(15.8,6.75)$ approx; GOLD (?).
B 259, p. 58-59
B 314, p. 60

Romanof, Dixon \& Hirst. See Hirst-Chichagof.

Romanof, Dixon, Hirst \& Archangelsky. See Hirst-Chichagof.

Ronning, Repik, Chopp, Thompson \& Schotter. See Goldwan.

Rose, Bee Hirst-Chichagof.

Rust \& Wallace. See Chicagoff.

Rust, Wallace, Bratnober \& Jarvis. See Chichagoff.

Schaffer. See Hirst-Chichagof.

Schetter, Dodge \& Borland. See Goldwan.

(Seymour Canal) - (25.0, 17.3), approx ; COPPER, GOLD.

B 287, p. 150-151

Sholin. See Hirst-Chichagof.

Side Hill. See (Bohemia Basin).

(Silver Bay) - (17, 0-1) (?) ; GOLD(?), SILVER(?). See also (Silver Bay)

(Port Alexander quad.).

B 345, p. 91

B 379, p. 73

Sitka. See Chichagoff.

(Slocum Arm). See Cobol.

Smith. See Duluth, Gold Reef, Jumbo, Minnesota.

Smith \& Martinson. See Hirst-Chichagof.

Snow Slide - $(7.0,13.95)$; COPPER.

B 692, p. 123

South Muskeg. See (Bohemia Basin).

South-Side - $(7.4,14.75)$; GOLD.

B 773, p. 123

South Takanis. See (Bohemia Basin).

(Square Cove) - (NE 1/4 20,17) ; MARBLE.

B 314, p. 77 B 345, p. 122

(Stag Bay) - (6.1-6.5, 16.0-16.1) ; GOLD, IRON.

B 692, p. $121 \quad$ B 963-A, p. 23-24

B 773, p. 114

Standard Gypsum Co. See (Gypsum Cr.).

Stannard \& Martin. See $3 \mathrm{~J}$.

Submarine - $(8.5,11.65)$; GOLD (?).

B 692, p. 120

Sunday Alliance. See Hirst-Chichagof.

Sunday Queen. See Hirst-Chichagof.

Surprise. See Hirst-Chichagof.

(Takanis Bay) - (4.9, 15.35) approx; NICKEL(?), COPPER(?).

B 773, p. 95

B 800, p. 373

Tasmania. See (Bohemia Basin).

(Tenakee Inlet) (Marble) - (17.0-17.4, 13.85-13.95) ; MARBLE. B 592, p. 101

B 682, p. 45-46.

(Tenakee Inlet) (nickel). See Big Ledge.

Thetis. See (Billy Basin).

$3 \mathrm{~J}-(18.15,13.95)$ approx ; MOLYBDENUM, COPPER.

B $926-$ C, p. $175-176$

Tillson - $(8.5,11.9)$; GOLD (?)

B 929, p. 133 
Topscott, Simons \& Mills. See Chichagoff.

Tripod. See (Bohemia Basin).

Tunnel. See (Bohemia Basin).

Turner. See Liberty.

Vevelstad. See (Bohemia Basin), (Takanis Bay).

Vevelstad and assoc: See (Mirror Harbor).

Vevelstad, Sholtz and assoc. See (Bohemia Basin).

Wakefield - $(6.55,16.7)$ approx; GOLD(?).

B 773, p. 124

West Coast Dev. Co. See Cox, Bolyan \& Loberg.

West Tripod. See (Bohemia Basin).

Woll. See Lucky Shot.

Yakobi. See (Bohemia Basin).

Young. See Chichagoff.

\section{SKAGWAY QUADRANGLE}

Alaska Corp. See (Porcupine Cr.).

Alaska Iron \& Steel Co. See (Haines).

Alaska Juneau Gold Min. Co. See Stampede.

Alaska-Sunshine Min. Co. See (Porcupine Cr.).

Anway. See (Cottonwood Cr.), (Nugget Cr.).

(Bear Cr.) - (16.4-17.5, 9.5-10.1) ; GOLD, COPPER, ZINC. Bear Cr. is now called Kelsall $\mathbf{R}$.
B 225, p. 63
B 662 , p. $93-98$
B 236, p. 13
B 699 , p. $15,18-19$

B 259, p. 52

(Cahoon Cr.) - (15.65-15.75, 6.8-6.95) ; GOLD. Includes references to Cahoon Cr. Placer Min. Co., (Calhoun Cr.).
B 480 , p. 37
B 520 , p. 36
B 662 , p. $97-98$
B 699 , p. $22,24-25$
B 542 , p. 43
B 755, p. 25
B 592 , p. 59

Cahoon Cr. Gold Min. Co. See (Cahoon Cr.), (McKinley Cr.).

Cahoon Cr. Placer Min. Co. See (Cahoon Cr.).

(Calhoun Cr.). See (Cahoon Cr.).

Chisolm (\& Clark). See (McKinley Cr.).

(Clifton) - $(24.75,9.75$; MOLYBDENUM. Includes references to Combination and to an unnamed molybdenite prospect north of Skagway.
B 642 , p. $99-100$
B 662 , p. 25
B 714, p. 41
B 800 , p. 330
B 692 , p. 23,30
B $926-C$, p. $180-181$

Combination. See (Clifton).

(Cottonwood Cr.) - (16.2, 5.05) ( ?) ; GOLD.

B 236, p. 13

Cranston. See (Porcupine Cr:).

Delta. See (Porcupine Cr.).

Finley. See (Porcupine Cr.).

(Glacier Cr.) - (14.75-15.2, 7.2-7.5) ; GOLD.
AR 21, p. 375
B 542, p. 43
B 225, p. 61
B 592, p. 61
B 236, p. 12, 19
B 662, p. 93-97, 99
B 259, p. 52
B 699, p. 19, 21-22, 25 
Gold Nugget Min. Co. See (Porcupine Cr.).

(Grizzly Cr.) - (15, 6-7) ; GOLD ( ?).

B 236, p. 12

Guyot \& Cartwright. See (Clifton).

(Haines) - $(22.45,4.65)$ approx ; IRON.

B 379, p. 86

B 714, p. 42

B 442, p. 145-146

B 800, p. 319, 352

B 699, p. 27-29

Inspiration Pt. Min. Co. - (25.4-25.5, 11.4-11.5) ( ?) ; LEAD, SILVER, GOLD.

B 797, p. 37

B 810, p. 14

B 813, p. 57

B 836, p. 16, 65

B 844-A, p. 64

B 824, p. 63

B 857-A, p. 60

B 864-A, p. 65

(Kelsall R.) See (Bear Cr.).

(Klehini R.) - (15.9, 7.7) ( ?) ; GOLD.

B 662 , p. 93-97, $99 \quad$ B 868-A, p. 30

B 699 , p. 19

B 880-A, p. 33-34

B 824, p. 27

B $897-$ A, p. 40

B 844-A, p. 27

(Klukwan) - (18.4-19.2, 7.3-8.3) ; IRON, TITANIUM.

IC 7844, p. 11

RI 4984, p. 1-4

Legal Tender. See (Porcupine Cr.).

(McKinley Cr.) - (15.6-15.8, 6.9-7.15) ; GOJ,D.

AR 21, p. 375

B 263, p. 207

B 225, p. 62-63

B 662, p. 95, 99-100

B 236, p. 19-20, 24-26, 29, 31

B 699, p. 21-25

B 259 , p. 52

B 755, p. 25

McKinley Cr. Min. Co. See (McKinley Cr.).

(Marble Cr.) - (15, 6-7) ; GOLD (?).

B 236, p. 12

B 824, p. 27

Mix. See (Porcupine Cr.).

(Nugget Or.) - (16.4-16.7, 5.25-5.5) ; GOLD.

B 225, p. 61, 63

B 236, p. 13, 19-20, 26-27, 31

B 259 , p. 52

B 284, p. 45

B 314, p. 64

B 520, p. 36

B 592, p. 59-60

B 662, p. 98

B 699, p. 19, 23

B 824, p. 27

Porcupine (Gold) Min. Co. See (Porcupine Cr.).

(Porcupine Cr.) or (R.) - (15.55-15.9, 7.05-7.65) ; GOLD, LEAD, COPPER.

Includes references to Porcupine (Gold) Min. Co.

AR 21, p. 375

B 213, p. 48

B 225 , p. $60-63$

B 236, p. 12, 19-24, 28-29, 31

B 259, p. 31, 51-52

B 263, p. $29,41,207$

B 284, p. 45

B 314, p. 64

B 345, p. 37

B 379, p. 51

B 442, p. 41

B 480, p. 37
B 520, p. 36

B 542, p. 43

B 592, p. 59

B 662, p. 93, 98-100

B 699, p. 19, 22-25

B 755, p. 25

B 797, p. 16

B 810, p. 21

B 813, p. 23

B 824, p. 27

B 880-A, p. 34 
(Salmon R.) or (Cr.) - (16.7, 5.35) approx; GOLD. Includes references to (Solomon R.) ; Salmon R. is now called Tsirku R.
B 225, p. 63
B 236, p. 13, 27, 31
B 284, p. 45
B 259, p. 52
B 662, p. 93
B 699, p. 18-19

(Solomon R.). See (Salmon R.).

Stampede - $(13.45,7.9)(?)$; GOLD. May be in British Columbia. B 824, p. 15

(Summit Cr.) - (17.15; 6.4) ; GOLD, SILVER, LEAD, COPPER. NOTE :-Not mentioned by name in $B 662$.

B 662 p. $97-98$

B 699 , p. 14-15, 18

(Takhin R.) - (16.9-17.4, 4.95) approx ; GOLD.

B 662, p. 98

B 699, p. 23

(Tsirku R.). See (Salmon R.).

United Gold Min. Co. See (McKinley Cr.).

Woodin. See (McKinley Cr.).

(----) (copper) - (21.0, 6.0) approx ; COPPER.

B 800, p. 323

\section{SLEETMUTE QUADRANGLE}

Alice. See Alice \& Bessie.

Alice \& Bessie - $(\mathbf{1 3 . 7 5}, \mathbf{1 4 . 0})$ MERCURY, ANTIMONY. Includes references to Parks unless specifically to Barometer.

$\begin{array}{ll}\text { P 268, p. 4, 65, 105-107, 109-110 } & \text { B 824, p. 79-80 } \\ \text { B 622, p. 67, 266, 272, 274-280, 288 } & \text { B 836, p. 81 } \\ \text { B 642, p. 259 } & \text { B 844-A, p. 79 } \\ \text { B 649, p. 45 } & \text { B 857-A. p. 74 } \\ \text { B 655, p. 139-147 } & \text { B 864-A, p. 79 } \\ \text { B 662, p. 25, 60 } & \text { B 864-C, p. 243-244 } \\ \text { B 666, p. 97 } & \text { B 868-A p. 86 } \\ \text { B 714, p. 39-40, 93-94 } & \text { B 880-A, p. 86 } \\ \text { B 722, p. 23, 60 } & \text { B 897-A, p. 97 } \\ \text { B 739, p. 13, 159 } & \text { B 933-A, p. 90-91 } \\ \text { B 754, p. 117 } & \text { IC 7379, p. 63 } \\ \text { B 773, p. 47 } & \text { RI 4065, p. 5, 8-9, 19-23 } \\ \text { B 797, p. 41 } & \text { TDM 1, p. 24 }\end{array}$

Ammeline - $(13.75,14.25)$; MERCURY.

P 268, p. 70, 111

Barometer - $(13.85,13.6)$; MERCURY, ANTIMONY. See also Alice \& Bessie,

(Napamute); not mentioned by name in B 722.

P 268, p. 110

RI 4065, p. 9, 24-27

B 722, p. 60-61

TDM 1, p. 24

IC 7379, p. 63

Bessie. See Alice \& Bessie

Bettles. See Cinnabar Chief.

(California Cr.) - (11.1-12.0, 15.5-16.1) ; GOLD.

P 268, p. 69, 120

(Central Cr.) - (8.5-8.9, 15.4-16.2) ; GOLD.

P 268, p. 120 
Cinnabar Chief - $(13,13-14)$ (?) ; MERCURY.

B 797, p. 41

TDM 1, p. 24

Decoursey Mtn. Min. Co., Inc. See Red Devil.

Dunkle. See Alice \& Bessie.

(Eightmile Cr.) - (10.8-11.1, 13.1-14.5) ; GOLD. P 268, p. 120

Eurica. See Red Devil.

Fairview - $(13.6,13.55)$; MERCURY, ANTIMONY.
P 268, p. 70, 111
RI 4065, p. 27-28

IC 7379, p. 63

(Forty seven Cr.) - $(7.25,0.85)$; GOLD, TUNGSTEN, ANTIMONY, SILVER,

LEAD.

P 268, p. 119-121

Fuller \& Willis. See Willis.

(Fuller Cr.) - (12.2-13.3, 11.6-14.0) ; GOLD. P 268, p. 120

(Girl Cr.) - (4.0-6.0, 3.6-5.0) ; GOLdD $P 268$, p. 120

(Gold Run) - (14.4-14.6, 7.0-7.5) ; GOLD. P 268, p. 120

Halvorson. See Barometer, Red Devil.

Halvorson \& Mellick. See Red Devil.

(Holitna R.) - (10-16, 0-5) (?) ; GOLD. May be in Taylor Mtns. quad.

B 692, p. 40

B 880-A, p. 49

B 722, p. 60

B 897-A, p. 58

B 864-A, p. $45-46$

B $910-A$, p. $60-61$

B 868-A, p. 46

B 917-A, p. 59-60

(Horn Mtns.) - $(3,12-13)$; TUNGSTEN.

P 268, p. 121

(Kay Cr.) - $(2,5)$; ANTIMONY. P 268, p. 122

(Kolmakof) - $(0.2,10.45)$; MERCURY. AR 20, p. 261-262

P 268, p. 4, 66, 109, 116

B 622, p. 272-274, 280-286

B 480, p. 93

RI 4065, p. 49-50

TDM 1, p. 24

Kusko. See Red Devil.

Kuskokwim Mercury Co. See Cinnabar Chief.

Kuskokwim Min. Co. See Red Devil.

Landru - $(15.1,12.5)$ approx ; MERCURY. TDM 1, p. 23-24

(Little Cr.) - (2.6-2.7, 16.3-17.55) ; GOLD. Occurrence may be in part of creek in Iditarod quad. B 480, p. 267, 270

(McCally Cr.) - $(13.7,12.9)$ approx; MERCURY. P 268, p. 111

(Mary Cr.) - (3.3-3.45, 9.95-10.0) (?) ; GOLD. See also (Murray Gulch). B 662, p. 61

Mellick \& Halverson. See Red Devil.

(Mellick's) - $(15.05,12.15)$; MERCURY. P 268, p. 111

Mercury - $(\mathbf{1 3 . 9 5}, \mathbf{1 3 . 5})$; MERCURY. P 268, p. 111 
(Murray Gulch) - (3.3-3.45, 9.95-10.0) ; GOLD. See also (Mary Cr.), (New York Cr.).

P 268, p. 119

B 622, p. 353-355

(Napamute) - $(13.8,13.55)$ ( ?) ; MERCURY. See also Barometer.

B 792, p. 33-34

TDM 1, p. 24

New Idria-Alaska Quicksilver Min. Co. See Red Devil.

(New York Cr.) - (3.4-3.5, 9.8-10.1) ; GOLD. See also (Murray Gulch).

P 268, p. 4, 119

B 622, p. 304, 353

B 642, p. 68

(Oskawalik R.) - $(10,9)$; GOLD.

P 268, p. 120

Parks. See Alice \& Bessie, Barometer.

Rabidoux. See (Kolmakof).

Red Devil - $(14.05,13.4)$; MERCURY, ANTIMONY.

P 268, p. 2, 65, 106, 108-110

B 933-A, p. 90

IC 7379, p. 63

RI 4065, p. 3 9-18

Schaefer. See (Fortyseven Cr.).

Skidmore. See Barometer.

(Timber Cr.) - $(0,0)$; GOLD.

P 268, p. 120

Two Genevieves - $(13.8,14.45)$; MERCURY.

P 268, p. 65, 111

Vermillion - $(13.85,13.55)$; MERCURY.

P 268, p. 111

Willis - $(13.55,14.4)$; MERCURY, ANTIMONY. Includes references to Fuller

\& Willis, Willis \& Fuller.

$P$ 268, p. 63, 65, 111

B 622, p. 286

B 739, p. 13

B 797, p. 41
RI 5243, p. 1-2, 4-10

RI 5433, p. 1-6, 8, 11-19

TDM 1, p. 21, 23-24

TDM 2, p. 17

Willis \& Fuller. See Willis.

(----) (antimony, mercury) - $(\mathbf{1 3 . 2 5}, \mathbf{1 3 . 5 5 )}$; MERCURY, ANTIMONY.

P 268, p. 111

\section{SOLOMON QUADRANGLE}

Adams \& Edlin. See (Goose Cr.).

Adams \& Wik. See (Goose Cr.).

(Adams Cr.) - (3.85-4.05, 12.35-12.4) ; GOLD.

B 433, p. 178

B 622 , p. 370

(Adventuress Cr.) - (3.2-3.3, 15.1-15.15) ; GOLD.

B 379, p. 331

(Aggie Cr.) - (13.2-13.8, 15.55-16.0) ; GOLD.

B 824, p. 46

B 836, p. 47

B 897-A, p. 65

B 857-A, p. 45

B 910-A, p. 69

B 864-A, p. 50

B 917-A, p. 67-68

B 868-A, p. 52

B 926-A, p. 63, 71

B 880-A, p. 53 
Alaska Gold Dred. Co. See (Goldbottom Cr.), (Lower Willow Cr.), (Warm Cr.). Alaska Homestake Gold Min. Co. See (Daniels Cr.).

Alaska Mercury Corp. See (Swede Cr.).

Alaska Placer Co. See (Niukluk R.), (Ophir Cr.).

Alden. See (West Cr.).

(Allgold Cr.) - (4.05-4.1, 15.1-15.2) ; GOLD.

B 379, p. 337

(American Cr.) - (3.4-4.35, 15.6-16.55) ; GOLD.

B 379, p. 333

B 864-A, p. 51

B 433, p. 204

B 868-A, p. 53

B 792, p. 21

B 880-A, p. 55

B 813, p. 42

B 897-A, p. 63-64

B 824, p. 47

B 917-A, p. 65

B 836, p. 49

B $926-A$, p. 61

B 844-A, p. 49

B 933-A, p. 58, 68

B 857-A, p. 46

American Cr. Dred. Co. See (American Cr.).

(Auburn Ravine) - (3.5-3.85, 15.8-16.2) ; GOLD, MERCURY. B 379, p. 333-335

B 433, p. 204-206

(Banner Cr.) - (5.0-5.3, 14.35-14.5) ; GOLD.

B 314, p. 153

B 345, p. 224-225

B 328, p. 259, 265

B 433, p. 156, 188, 199

(Barney Cr.) - (2.1-2.2, 16.3) ; GOLD.

B 314, p. 163

B 379, p. 327

(Basin Cr.) - (10.05-10.65, 15.9-16.0) ; GOLD.

B 345, p. 217

B 810 , p. 40

B 722, p. 63

B 813, p. 41

(Bear Gulch) - $(4.9,11.95)$; GOLD (?).

B 433, p. 179

(Beaton Cr.) - $(6.45,10.45-10.55)$; GOLD.

B 433, p. 204

(Beaver Cr.) - $(0.85-1.65,10.0-11.6)$; GOLD.

B 933-A, p. 56-57, 68

(Ben Gulch) - $(6.05,16.75)$; GOLD.

B 433, p. 190

(Benson Cr.) - (2.3-2.7, 15.95-16.0) ; GOLD, COPPER.
B 314 , p. 163
B 379 , p. $328-329$
B 868-A, p. 53
B 622 , p. 372
B $880-A$, p. 55
B 722, p. 210,215
B 897-A, p. 64
B 864-A, p. 51
B 910-A, p. 66
B 917-A, p. 65

(Benson Gulch) - $(10,16)$; GOLD.

B 824, p. 46

B $857-A$, p. 45

B 836 , p. 47

B $864-A$, p. 50

B 844-A, p. 49

(Big Four Cr.) - $(6.15-6.9,14.65-15.95)$; GOLD.
B 314, p. 153
B 328 , p. 264
B 345 , p. 223
B 433, p. $191-192$

Big Hurrah - $(5.75,11.45)$; GOLD, SILVER, TUNGSTEN. Includes reference to Hurrah; see also (Big Hurrah Cr.).

B 259 , p. 22

B 284 , p. 6,137
B 314, p. $146-147,155$

B 328 , p. $223,228-232$ 
Big Hurrah-Continued

B 345, p. 234-237

B 836 , p. 23

B 379 , p. 292

B 844-A, p. 24

B 433 , p. $59,93,139,143-147$

B $857-A$, p. 22

B 442 , p. 38,360

B $910-$ A, p. 33

B 480 , p. 73

B $917-A$, p. 31

B 642 , p. 69

C 196, p. 1-2, 4

B 722 , p. 163, 173-174,

BMB 153, p. 55

179, 198, 200-204

TDM 5-R, p. 43

B 824, p. 23

(Big Hurrah Cr.) - (5.15-6.05, 11.3-11.5) ; GOLD, SILVER, TUNGSTEN, ANTIMONY. See also Big Hurrah.
B 259 , p. 22
B 739, p. 9
B 263, p. 14, 209
B 755, p. 14
B 328, p. $223,227-232$
B 773 , p. 27
B 345 , p. 222
B 783 , p. 18
B 379, p. 291
B 792, p. 22,25
B 433 , p. $139,156,180-182$
B 797, p. 31
B 442 , p. 360
B $857-A$, p. 45
B 520 , p. 342
B 864-A, p. 50
B 592 , p. 390
B 868-A, p. 52
B 662, p. 455
B $880-A$, p. 56
B 712 , p. 187,189
B 933-A, p. 62
B 714, p. 233
TDM 5-R, p. 11

B 722, p. 63

(Birch Cr.) - $(6.45,14.6)$; GOLD.

B 314, p. 153

B 328, p. 264

Black Chief Min. Co. See (Daniels Cr.).

Blue Goose (Min. Co.). See (Ophir Cr.).

(Bluff). See (Daniels Cr.).

(Bobs Cr.) - (1.3-2.0, 16.1) ; GOLD.

B 314, p. 163

B 379 , p. $327-328$

(Bonanza Cr.) - (5.7-6.5, 16.5-16.85) ; GOLD.

B 314, p. 152

B 328, p. 264

(Boulder Cr.) - (4.45-4.55, 15.05-15.2) ; GOLD.

B 328, p. 258-259

B 433 , p. 198

Brookins - (NE 1/4 7, 15) (?) ; ANTIMONY.

TDM 5-R, p. 12

Bunker Hill - $(9.4,10.6)$; GOLD, COPPER. NoTE.-Not mentioned by name on p. 182, B 722, or TDM 5-R.

B 722, p. 182,188

TDM 5-R, p. 19

(Bunny Cr.). See (Barney Cr.).

Burness-Iverson-Johnson. See (Big Hurrah Cr.).

(Butte Cr.) - (4.95-5.1, 12.5-12.55) ; GOLD.

B 345 , p. 222

B 433, p. 183

B 379 , p. 291

(Butte Cr., South Fork) - (4.95-5.0, 12.4-12.5) ; GOLD.

B 433, p. 183

(Cache Cr.). See (Spruce Cr.) (East of Solomon).

(Cahill Cr.) - (4.1, 14.35-14.45) ; GOLD.

B 379, p. 337

B 433 , p. 202 
(California Cr.) - (8.05-8.35, 10.9-11.1);-GOLD.
B 813, .p. 41
B 864-A, p. 51
B 824, p. 47
B 926-A, p. 66, 71-72
B 836 , p. 49
B $933-A$, p. 61,68

(Camp Cr.) - (7.5-8.0, 15.0-16.45) ; GOLD.

B 284 , p. 138

B 314, p. 151, 155

B 592 , p. 392

B 328, p. 256

B 662 , p. 452

B 345, p. 217

B $7: 12$, p. 187 ,

B 379 , p. 295

B 926-A, p. $63,71-72$

B 442 , p. 343

B $933-$ A, p. 60,68

B 449 , p. 117, 121-122

BMB 142 , p. 28

Camp Cr. Dred. Co. See (Camp Cr.).

(Canyon Cr.) (trib. Casadepaga R.) - (4.1-5.05, 15.0-15.05) ; GOLD.
B 314, p. 153
B 328, p. $: 259,265$
B 442 , p. 360
B 345, p. 224
B 662 , p. 452
B 379, p. 337
B 712 , p. 187
B 433 , p. $188-189,197-199$
BMB 153, p. 55

(Canyon Cr.) (trib. Iron Cr.) - (1.3-2.15, 14.85-15.7) ; GOLD.

B 379, p. 329-330

Canyon Cr. Gold Min. Co. See (Canyon Cr.) (trib. Casadepaga R.).

(Cape Darby) - $(16.7,7.25)$; TUNGSTEN.

C 300, p. $4-5$

(Carson Cr.) - (18.5, 9.4) approx ; COPPER.
B 449, p. 134-135
B 722, p. 181
C 300, p. 3
TDM 5-R, p. 20

Casa De Paga Gold Co. See (Beaver Cr.), (Casadepaga R.).

Casadepaga Gold Dred. Co. See (Lower Willow Cr.).

Casa Gold Mines Co. See (Casadepaga R.).

Casadepaga Min. Co. See (Casadepaga R.).

(Casadepaga R.) - (3.55-6.4, 13.35-16.25) ; GOLD.

B 259, p. 23

B 314, p. 147, 152-154

B 328, p. 236, 257-258, 264

B 345, p. 223

B 433, p. 184-185, 187

B 592, p. 387, 392

B 622, p. 371

B 662, p. 455

B 712, p. 189
B 792, p. 24 .

B 797, p. 30

B 810, p. $34-35,40$

B 813, p. 41,47

B 824, p. $46-47,53$

B 868-A, p. 52

B $880-A$, p. 56,61

B 897-A, p. 65-66, 71

B 910-A, p. 69-70, 76

(Casadepaga R., Left Fork). See (Lower Willow Cr.).

(Chickamin Gulch) - $(2.5-2.55,15.35-15.45)$; GOLD.

B 379, p. 341-342

(Chukajak Cr.) - (21.3-22.25, 16.5-17.9) ; GOLD.

B 442, p. 341

B. 449, p. 115

(Clear Cr.) - $(20.7,15.7)$; TIN, TUNGSTEN.

C 300, p. 6-7

Coal Cr. Dred. See (Solomon R.).

(Coca Cola Cr.) - $(8,11)$ (?) ; GOLD.

B 864-A, p. 51

$593018-61 \longrightarrow 19$ 
Connor, Erichinger \& Hanot. See (Casadepaga R.).

Consolidated - (9.35-9.6, 10.0-10.3) (?) ; GOLD. See also (Daniels Cr.). B 542, p. 41

Council Dred. Co. See (Aggie Cr.), (Camp Cr.), (Niukluk R.).

(Dane Cr.). - (0.6-0.65, 15.75-15.95) ; GOLD.

B 379, p. 320

(Daniels Cr.) - (9.35-9.6, 10.0-10.3) ; GOLD, MERCURY, TUNGSTEN. Includes references to (Bluff), Eskimo, Idaho, Labay \& Meegan, Sea Gull; see also Consolidated:

B 259, p. 22-23

B 284, p. 139

B 328, p. 283, 286-293

B 345, p. 247

B 442, p. 343; 360

B 449, p. 123-125, 128-129

B 480, p. 93

B 662, p. 435,455

B 714, p. 39, 236

B 722, p. $65,182,185-187,189-$ 197,235

B 797, p. 26

B 810, p. 35 .

B 813, p. 41

(Darby Mts.) - (17-18, 14-17) ; TIN (?). B 259, p. 120, 125

(Dawson Cr.) or (Gulch) - (6:05-6.15, 16.05-16.1) ; GOLD. B 328, p. 258

B 345, p. 223

(Discovery Cr.) - (2.2-2.25, 15.2-15.6) ; GOLD. B 379, p. 330

(Dividend Cr.) - (3.2-3.25, 14.9-15.05) ; GOLD. B 379, p. 331

(Dixon Cr.) - (5.75-5.85, 15.65-15.9); GOLD.

B 314, p. 153

B 328, p. 258, 264

B 433, p. 186, 192

(Dome Cr.). See (Iron Cr.).

(Dome Cr., Left Fork) - (2.4-2.75, 15.5-15.65) ; GOLD, COPPER. B 379, p. 330-331

(Dry Cr.) - (5.75-5.8, 15.55-15.6) ; GOLD. B 328, p. 258

B 722, p. 216

B 433, p. 193

(Dutch Cr.) or (Gulch) - (10.05-10.7, 17.1-17.55) ; GOLD. B 284 , p. 138

B 314, p. 150-151

B 328, p. 244, 251, 262

B 449 , p. 120

B 592, p. 392

B 622, p. 371

B 345, p. 217

(Easy Cr.) - (2.3-2.45, 16.15) ; GOLD.

B 314, p. 163

B 379, p. 328, 340-341

(El Patron Cr.) - (1.7-1.8, 15.1-15.15) ; GOLD.

B 314, p. 163

B 379, p. 330 
(Eldorado Cr.) - (9.0-9.3, 10.1-10.4) ; GOLD, MERCURY.

$\begin{array}{ll}\text { B } 328, \text { p. 283, 288-289, } 293 & \text { B } 824, \text { p. } 47 \\ \text { B 442, p. } 343 & \text { B } 836, \text { p. } 49 \\ \text { B 449, p. } 123,125 & \text { B } 844-\text { A, p. } 50 \\ \text { B 722, p. } 196 & \text { B 864-A, p. } 51\end{array}$

B 813, p. 41

(Eldorado R.) - $(0.15,13.8)$; GOLD. See also (Venetia Cr.). B 379, p. 337

Elkhorn. See (Elkhorn Cr.), (Niukluk R.).

(Elkhorn Cr.) - (7.3-7.9, 16.15-16.45) ; GOLD.

\begin{tabular}{|c|c|c|}
\hline B 284, p. 138 & : & B 592, p. 392 \\
\hline B 314, p. 152 & & B 622, p. 371 \\
\hline В 328, p. $236,256-257,263$ & & B 662, p. 452 \\
\hline B 345, p. 217 & $\therefore$ & B 712, p. 187 \\
\hline B 442, p. 343 & . & BMB 142, p. 28 \\
\hline
\end{tabular}

B 449, p. 117, 122-123

Eskimo. See (Daniels Cr.).

Eskimo (Gold) Dred. Co. See (Solomon R.).

Eskimo Gold Min. Co. See (Solomon R.).

Esquimeaux. See (Daniels Cr.).

Esquimo Dred. Co. See (Solomon R.).

Esterbrook. See (Solomon R.).

(Etchepuk R.) - (NE 1/4 quad.) ; MICA.

TDM 5-R, p. 46

(Fish R.) (lode) - $(12.6,15.5)$ approx; SILVER, LEAD, MERCURY.

B 662, p. 446

TDM 5-R, p. 28, 34

B 722, p. 183

(Fish R.) (placer) - (13.2, 16.0) approx ; GOLD. See also (Aggie Or.). B 910-A, p. 69

Flodin. See (Canyon Cr.) (trib. Casadepaga R.).

Flodin (Gold) Min. \& Dred. Co. See (Big Hurrah Cr.), (Solomon R.).

Flower. See (Solomon R.).

Flume Dred Co. See (Basin Cr.), (Melsing Cr.), (Ophir Cr.).

Flynn - $(5.4,11.65)$ approx ; GOLD.

B 722, p. 198, 204

(Fox Cr.) - $(5.3,12.85)$; GOLD.

B 345, p. 222-223

B 433, p. 184

(Fox R.) - $(8.9,13.65)$ approx ; GOLD.

B 314, p. 147-148

B 328, p. 236-237

B 442, p. 343

B 449 , p. 117

(Game Cr.) - (4.35-4.4, 16.4-16.5) ; GOLD. Includes reference to (Gem Or.).

B 433, p. 206

B 933-A, p. 58

B 926-A, p. 61

G \& O (Dred. Co.) See (Elkhorn Cr.).

Garrod \& Overbaugh. See (Warm Cr.).

Garrod \& Pfaffle. See (Warm Cr.).

(Gem. Cr.). See (Game Cr.).

Glass Dred. Co. See (Melsing Cr.).

(Gold Moon Gulch) - (5.15-5.3, 15.7-15.85) ; GOLD.

B 433, p. 197 
(Goldbottom Cr.) - (7.9-8.9, 16.5-17.3) ; GOLD, TUNGSTEN.

B 259, p. 23

B 314, p. 147,151

B 328, p. 236, 254-255
B 442, p. 343, 363

B 449, p. 117, 122, 128

B 520, p. 342

Goldbottom Dred. Co. See (Goose Cr.).

Goldsmith Dred. Co. See (Solomon R.).

(Golornin Bay) - $(15.7,9.55$ ) ; TUNGSTEN. C 300, p. 4.

Goose Cr. See (Goose Cr.).

(Goose Cr.) - (4.75-5.45, 15.3-15.5) ; GOLD.

B 314, p. 153

B 328, p. 259, 265

B 592, p. 387, 393

B 345, p. 223-224

B 622, p. 370-371

B 433, p. 156, 188-189, 194-197

B 662, p. 452

B 442 , p. 360

B 712, p. 187

B 520, p. 342

B 714, p. 323-325

Gray Eagle. See Quiggley.

(Guy Cr.) - (9.55-9.9, 17.45-17.55) ; GOLD ( ?).

B 722, p. 207-208

Haney Min. Co. See (Big Hurrah Cr.).

Hanson. See (Koyana Cr.).

(Hardluck Cr.) - (2.65-2.8, 15.4-15.5) ; GOLD.

B 379, p. 331

B 442, p. 364

Hill. See (Koyana Cr.).

(Hilliard .Cr.) - (NW 1/4 2, 15) ; GOLD.

B 379, p. 329

(Hobo Gulch) - $(2.8,15.5)$; GOLD.

B 379, p. 331

Hunter \& Walker. See (Daniels Cr.).

Hurrah. See Big Hurrah.

Idaho. See (Daniels Cr.).

(Independence Cr.) - (2.55-2.65, 15.1-15.2) GOLD.

B 379, p. 331

Inland Dred Co. See (Aggie Cr.).

(Iron Cr.) - (2.15-3.2, 15.05-17.05) ; GOLD, COPPER, MERCURY. Includes references to (Dome $\mathrm{Cr}$ ), (Telegram $\mathrm{Cr}$.).
B 225 , p. 53
B 622, p. 372
B 259, p. 23
B 722 , p. 217
B 284 , p. 136
B 910-A, p. 66
B 314, p. 162-163
B 917-A, p. 65
B 379 ; p.: $322-327,345$
B 926-A, p. 61, 71-72
B 442, p. $363-364$
B $933-A$, p. 58,68
B 592, p. 393

(Iron Cr., Left Fork). See (Dome Cr., Left Fork).

Iverson (\& Johnson). See (Big Hurrah Cr:),

(IXL Gulch) - $(8.9,13.75)$; GOLD.

B 314, p. 148

B 592, p. 392

B 328, p. 237

(Jerome Cr.) - (4.15-4.7, 10.55-10.95) ; .GOLD.
B 328 , p. $225-226$
B 857-A, p. 45
B 433 , p. 171
B $864-A$, p. 50
B 622 , p. 370
B 868-A, p. 52 
(Johns Cr.) - (4.5-5.1, 12.95-13.0) ; GOLD.

B 433 , p. 184

(Kasson Cr.) - (4.35-4.55, 12.1-12.2) ; GOLD.
B 328 , p. 227
B 433 , p. $177-178$
B 345, p. 222
B 592, p. 390
B 379 , p. 291
B 868-A, p. 52

Kimball. See (Adams Cr.), (Melsing Cr.), (Shovel Cr.), (Solomon R.).

Kimball \& Saupe. See (Melsing Cr.), (Ophir Cr.).

(Koyana Cr.) - (10.05-10.1, 10.0-10.05) ; GOLD, MERCURY.
B 328, p. 283,289
B 722 , p. $185-188,235$
B 824, p. 47
B 836, p. 49

B 813 , p. 41

TDM 5-R, p. 33

(Kwiniuk R.) - (SE 1/4 NE 1/4 quad.) ; COPPER, TUNGSTEN, MERCURY(?). Copper and tungsten at $(19.85,13.2)$.

C 300, p. 6

TDM 5-R, p. 34

Labay \& Meegan. See (Daniels Cr.).

Lane Inv. Co. See Big Hurrah.

(Last Chance Cr.) (trib. Ameriean Cr.) - (3.25-3.4, 15.4-15.6) ; GOLD, COPPER (?).

B 379 , p. 333

B 722, p. 217

(Last Chance Cr.) (trib. Big Hurrah Cr.). See Quiggley.

Lee Bros. (Dred Co.). See (Solomon R.).

Libby, Melsing, Mordaunt \& Blake. See (Melsing Cr.).

(Lightning Cr.) - $(5.76,15.5)$; GOLD.

B 433, p. 193

(Linda Vista Cr.) - (5.5, 11.45) ; GOLD.

B 345, p. 237

(Lion Cr.) - $(6.05,11.15-11.3)$; GOLD. B 328, p. 232

B 433, p. 182

(Little Anvil Cr.) - (8.55-8.7, 10.05-10.55) ; GOLD. Includes reference to (Silver Bow Cr.).
B 328, p. 283, 293
B 662, p. 455
B 449 , p. 125
B 836, p. 49

Lomen Reindeer \& Trad. Co. See (Solomon R.).

(Lower Willow Cr.) - $(3.4-4.95,14.2-14.65)$; GOLD. Includes references to (Willow Cr.) (trib. Casadepaga R.).
B 284, p. 137
B 433, p. 201-203
B 314, p. 153-154
B 442, p. 360
B 328, p. 259,265
B 520, p. 342
B 345, p. 225
B 592, p. 387, 392-393
B 379, p. 336
B 622, p. 371

Lubbe. See (Mystery Cr.) (trib. Shovel Cr.).

(Lulu Cr.). See (Benson Cr.).

Lylles. See (Koyana Cr.).

Mahan \& Slack. See (California Cr.).

(Manila Cr.) - $(4.5,10.25)$; GOLD.
B 328, p. 225-226
B 433, p. $157-158,170,214$

Margraf \& Kowalski. See (Big Hurrah Cr.).

(Meddler Gulch) - $(4.85,11.9)$; GOLD (?).

B 433, p. $179-180$

Megan, Megan \& Somerville. See (Daniels Cr.). 
(Melsing Cr.) - (10.0-10.5, 15.75-16.7), ; GOLD.

B 284, p. 139

B 314, p. 147, 149-150

B 328, p. 235-236, 240-242, 260-261

B 345 , p. 217

B 379, p. 294-295

B 442 , p. 343,363

B 449, p. 117-118

B 520, p. 342

B 592, p: 388,392

B 622 , p. 371

B 662 , p. 452

B 714, p. 232-235
B 722, p. 63

B 813, p. 41

B 824 , p. 46

B 836, p. 47

B $868-A$, p. 52

B 880-A, p. 53, 61

B 897-A, p. 65; 71

B 910-A, p. 69,76

B 917-A, p. 67

B 926-A, p. 72

BMB 142, p. 28

Melsing Cr. Dredge. See (Basin Cr.).

(Minnesota Cr.) - (4.7-4.8, 11.6-11.85) ; GOLD.

B 433, p. 179

Moody Min. Co. See (Canyon Cr.) (trib. Casadepaga R.).

(Moonlight Cr.) - (4.1-4.15, 13.65-13.7) ; GOLD.

B 662 , p. 455

(Moonlight Divide) - $(4.15,14.0)$; COPPER.

B 345, p. 243

TDM 5-R, p. 19-20

(Moran Gulch) - $(4.7,10.8)$; GOLD.

B 379, p. 289

B $857-$ A, p. 45

B 433, p. 161

B $864-$ A, p. 50

B 592, p. 390

(Mt. Dixon) - $(5.7,16.15)$; COPPER.

B 345, p. 243

TDM 5-R, p. 19-20

(Mud Cr.) - (NE 1/4 10, 15) ; GOLD.

B 328, p. 240

Mulligan. See (West Cr.).

(Mulligan Cr.) -(0.75-1.35, 12.0-12.45) ; GOLD.

B 328 , p. 221

(Mystery Cr.) (trib. Niukluk R.) - (10.65-11.0, 15.5-15.7) ; GOLD.

B 263, p. 14, 36, 42, 194, $209 \quad$ B 379, p. 295

B 314, p. 148

B 328 , p. $236,240-242$

B 449 , p. 117

BMB 142, p. 28

(Mystery Cr.) (trib. Shovel Cr.) - (4.0-4.45, 11.3-11.5) ; GOLD.
B 328 , p. 226
B 442 , p. 343,360
B 345, p. 221
B 520 , p. 342
B 379 , p. 291
B 592, p. 388,392
B 433 , p. 172-174
B 622, p. 371

Newburg \& Flower. See (Solomon R.).

(Niukluk R.) - (7.8-10.55, 15.1-16.45) ; GOLD.
B 328, p. 236, 238-239, 263
B $897-A$, p. 65,71
B 345 , p. $217-218$
B $910-A$, p. 69,76
B 714, p. 232-233
B $917-A$, p. $67-68$
B $868-$ A, p. 52
B 926-A p. 63-64, 71
B $880-A$, p. 53, 61
B $933-A$, p. 60,68

(No Man Cr.) - $(6.15,16.05)$; GOLD.

B 433 , p. 191

Nome, Montana \& New Mexico (Consol. Min. Co.). See (Goose Cr.). (Solomon R.). 
North (ern) Star Dred. Co. See (Niukluk R.), (Ophir Cr.).

Northern Light Min. Co. See (Niukluk R.), (Ophir Cr.).

(Nugget Cr.) (trib. American Cr.) - (3.1-3.4, 15.7-15.95) ; GOLD. B 379 , p. 333

(Nugget Cr.) or (Gulch) (trib. Solomon R.) - (5.3-5.4, 13.5-13.6).; GOLD.

B 433, p. 184

Nylen, Hultberg \& others. See (Shovel Cr.).

(Ophir Cr.) - (9.35-10.05, 16.15-17.6) ; GOLD. Includes reference to Wild Goose

Min. \& Trad. Co. ; see also (Ophir Cr.) (Bendeleben quad.).
B 213 , p. 46
B 755, p. 14
B 225, p. 49,54
B 773, p. 19, 27
B 259 , p. $20-23$
B 783, p. 18
B 284 , p. 138
B 792, p. 21,24
B 314 , p. $147,150-151$
B 797, p. 30
B 328 , p. 235-236, 242-250,
B 810, p. 40 261-263
B 345 , p. $216-217$
B 379, p. 293-294
B 813, p. 40,47
B 442 , p. $343,360-363$
B 824, p. 46,53
B 836, p. 47,54
B 449 ; p. 117-123, 128
B $844-$ A, p. 49,54
B 520 , p. 342
B 857-A, p. 45, 51
B 592, p. 388, 391-392
B 864-A, p. 50, 57
B 622 , p. 371
B 868-A, p. 51-52, 59
B 662 , p. 452-453, 455
B 880-A, p. 53, 61
B 712, p. 187, 189
B 897-A, p. 65, 71
B 910-A, p. 69, 76
B 714, p. 232-235
B 926-A, p. 63, 71
B 722, p. 63, 206-207
B 739, p. 9
BMB 142, p. 28
BMB 153, p. 56

Ophir Gold Dred. Co. See. (Ophir Cr.).

Oro Dred. Co. See (Elkhorn Cr.), (Goose Cr.).

Osmun. See (Slate Cr.).

(Oversight Cr.) - (3.2-3.3, 15.0-15.05) ; GOLD.

B 379, p. 331

Peck. See (Casadepaga R.).

(Penelope Cr.) - (5.55-5.65, 14.9-15.35) ; GOLD.

B 284, p. 137

B 345, p. 223

B 314, p. 153

B 433, p. 187-188, 193-194

B 328, p. 259, 265

(Penny Cr.) (trib. Iron Cr.) - (3.1-3.25, 15.2-15.25) ; GOLD.

B 379, p. 331

(Penny Cr.) (trib. Solomon R.) - (4.7-4.85, 11.2-11.6) ; GOLD.

B 328, p. 227

B 592, p. 390

B 345, p. 222

B 868-A, p. 52

B 433, p. 179

(Post Cr.) - (S 1/2 7, 17) ; GOLD.

B 314 , p. 155

(Problem Gulch) or (Cr.) - (4.0-4.1, 11.3-11.35) ; GOLD.

B 328, p. 226

B 662, p. 455

B 433, p. 173, 175

(Puckmummie Cr.) - (6.95-7.0, 16.7-16.75) ; GOLD.

B 433, p. 190 
(Puzzle Gulch) - (4.15-4.2, 11.35-11.5) ; GOLD.

B 433 , p. 173,175

(Quartz Cr.) . (trib. Casadepaga R.) - (5.0-5.2, 15.5-15.85) ; GOLD.

B 328, p. 258-259

B 433, p. 194, 196-197

B.345, p: 224 .

(Quartz Cr.) (trib. Solomon R.) - (5.1, 11.25-11.35) ; GOLD.

B 433, p. 164-166, 180

Quiggley $=(5.4,11.5) ;$ ANTIMONY. Includes references to Gray Eagle, (Last

Chance Cr.) (trib. Big Hurrah Cr.); not mentioned by name on p. 184, B 722.

B 328, p. 223, 232

C 196, p. 1, 4

B 662, p. 439

TDM 5-R, p. 11

B 722, p. 184, 204

(Rabbit Cr.) (East of Solomon) - $(4.95,10.95)$ approx ; GOLD.

B 379, p. 284-285

B 433, p. 212-213

(Rabbit Cr.) (trib. Iron Cr.) - (2.15-2.2, 15.8-15.85);; GOLD. B 314, p. 163

B 379, p. 329

(Rapid Cr.) - (2.1-2.15, 15.75) ; GOLD.

B 314, p. 163

(Ready Bullion Cr.) - (3.1, 5.1-5.2) ; GOLD. B 379, p. 331

(Richter Cr.) - (9.0-9.05, 16.15-16.3) ; GOLD.

B 314, p. 151

B 328, p. 263

(Ridgeway Cr.) - $(4.3-4.45,14.1-14.3)$; GOLD.

B 433, p. 202

(Rock Cr.) (Iron Cr. dist.) - (0.9-1.1,15.55-15.9) ; GOLD. B 379, p. 320

(Rock Cr.) (trib. Fish R.) - (13, 15) ; GOLD.

B 797, p. 26

B 824 , p. 46

B 813, p. 41

B 836, p. 47

(Rock Cr.) (trib. Solomon R.) - (.4.65-4.8, 10.45-10.55) ; GOLD.

B 433 , p. $170-171$

B 864-A, p. 50

B 592, p. 390

B 868-A, p. 52

B 857-A, p. 45

(Rocky Cr.) - (2.1-2.15, 15.75-15.8) ; GOLD.

B 314, p. 163

B 379, p. 329

Rolando. See (Game Cr.).

(Rover Cr.) - (4.8, 13.8-13.95) ; GOLD.

B 433, p. 202

(Ruby Cr.) (5.0-5.5, 14.0-14.3) ; GOLD.

B 284, p. 137

B 314, p. 154

B 442 , p. 360

B 328, p. 260,266

B 345, p. 225

B 433, p. 156, 199-201

B 836, p. 48

B 844-A, p. 48,55

B 857-A, p. 45, 51

B 868-A, p. 52

Ruby Dred. Co. See (Casadepaga R.), (Ruby Cr.).

(Ryan Cr.) - (8.9, 10.1-10.2) ; GOLD.

B 328, p. 283, 288-289, 293

B 442, p. 343

B 449, p. 123, 125

(San Jose Cr.) - (0.3-0.8, 12.9-13.1) ; GOLD.

B 328, p. 221 
(Sapphire Gulch) - $(4.8,11.85)$; GOLD (?).

B 433, p. 179

Scott. See (Iron Cr.).

Scott-Newburg. See (Solomon R.).

Sea Gull. See (Daniels Cr.).

Seward Dred. Co. See (Solomon R.).

(Sherrette Cr.) (lode) - $(2.8,15.85)$; COPPER. See also Wheeler (Sherrette Cr.).

B 722, p. 181, 215

TDM 5-R, p. 21

(Sherrette Cr.) (placer) - (3.0-3.2, 16.5-16.55) ; GOLD.
B 379, p. 331-333
B 917-A, p. 65

B 592, p. 393

(Shoal Cr.) - (3.0-3.15, 15.2-15.4) ; GOLD.

B 379, p. 331

(Shovel Cr.) $-(4.25-4.6,11.2-12.2)$; GOLD.

B 259, p. 22

B 328, p. 226

B 755, p. 14

B 345, p. 221

B 773, p. 27

B 379, p. 286

B 783, p. 18

B 433 , p. 156, 171-179

B 792, p. 25

B 442 , p. 359

B 797, p. 31

B 520 , p. 342

B 810, p. 34,40

B 592, p: $387,390-391$

B 813, p. 41

B 712, p. 187

B. 824, p. 46

B 722, p. 63

B 836, p. 47

B 739, p. 9

B 917-A; p. 70

Shovel Cr. Dred. Co. See (Shovel Cr.).

Shovel Cr. Min. Co. See (Shovel Cr.).

(Sidney Cr.) - (2.25-2.35, 15.8-15.85) ; GOLD.

B 379, p. 329

Sievertsen (\& Johnson). See (Solomion R.).

Silver - $(5.7,12.0)(?)$; GOLD, TUNGSTEN.

B 917-A p. 31

TDM 5-R, p. 43

B 926-A, p. 28

(Silver Bow Cr.). See (Little Anvil Cr.).

Sivertsen \& Johnson Min. \& Dred. Co. See (Solomon R.).

(Slate Cr.) - (1.1-1.25, 15.6-16.05) ; GOLD. Includes references to Osmun.

B 328, p. 267-268

B 379 , p. 320-321

B 592, p. 405-406

B 722, p. 256

(Snowball Cr.) - (10.3-10.4, 17.45-17.6); GOLD. See also (Snowball Cr.) (Bendeleben quad.).
B 284, p. 138-139
B 328, p. 251

Solomon Dred. Co. See (Solomon R.).

(Solomon R.) - (4.6-5.35, 10.3-13.55) ; GOLD, TUNGSTEN.

B 225, p. 53-54

B 263, p. 14, 164, 166, 209

B 284, p. 136-137

B 314, p. 146-147

B 328, p. 224-225, 233

B 345, p. 222

B 379, p. 286-291
B 433, p. 156-170

B 520, p. 342

B 592 , p. 387, 390-391

B 622, p. 37

B 662, p. 452,454

B 712 , p. 187

B 714, p. 233 
(Solomon R.)-Continued
B 722, p. 63
B 739, p. 9
B 755, p. 14
B 773, p. 27
B 783, p. 18
B 792 , p: 25
B 797, p. 26,31
B 810, p. 34,40
B 813, p. 41,48
B 824, p. 46,53
B 844-A, p. 47-48, 55
B $857-A$, p. 45,51
B 864-A, p. 50
B 868-A, p. 52
B 897-A, p. 65-66, 71
B 910-A, p. 69-70, 77
B 917-A, p. 69-70
B 926-A, p. 65, 71
B 933-A, p. 62,68
BMB 153, p. 55-56

B 836, p. 47,54

Solomon R. Dred. Co. See (Solomon R.).

Solomon Valley Dred. Co. See (Solomon R.).

(Spruce Cr.), (east of Solomon) - (6.4-6.55, 10.4-10.5) ; GOLD. Includes reference to (Cache Cr.).

B 328, p. 233

B 433 , p. 203-204

B 824, p. 46

B 836, p. 47,54

B $844-A$, p. 47,55

B 857-A, p. 44, 51

(Spruce Cr.) (trib. Casadepaga R.) - (5.85-5.9, 15.45-15.6) ; GOLD, COPPER.
B $864-A$, p. 50,57

B 868-A, p. 52, 59

B $880-$ A, p. $55-56,62$

B $897-$ A, p. 65,71 .

B 910-A, 69-70, 77
B 328, p. 258-259
B 345, p. 219-221, 238, 243
B 722, p. 181
B 433, p. 188, 193
TDM 5-R, p. 19

Spruce Cr. Dred. Co. See (Spruce Cr.) (east of Solomon).

Star Dred. Co. See (Mystery Cr.) (trib. Shovel Cr.).

Sullivan Min. Co., Crabtree \& others. See (Daniels Cr.).

Sullivan, Ryan \& others. See (Daniels Cr.).

(Sunshine Cr.) - (4.75-4.8, 15.0-15.1) ; GOLD.
B 433, p. 198
B 868-A, p. 52
B 712, p. 189

(Swede Cr.) or (Gulch) - $(9.8,10.0)$; GOLD, MERCURY.
B 328 , p. 288-289
B 836 ; p. 49,81
B 442, p. 343
B 449, p. 123
B $844-$ A, p. 50,79
B 722, p. 196
B $857-A$, p. 46,74
B 813 , p. 41
B $864-A$, p. 51
B $926-A$, p. 66
B 824, p. 47,80

Swede Cr. Min. Co. See (Swede Cr.).

(Sweetcake Cr.) - (9.3-9.45, 16.3-16.8) ; GOLD.
B 284, p. 138
B 314 , p. 150-151
B. 592 , p. 392
B 328, p. 244, 250-251, 262
B 813 , p. 41
B 442 , p. 362
B $880-$ A, p. 53
B $897-$ A, p. 65

B 449, p. 119

(Telegram Cr.). See (Iron Cr.).

(Telegraph Cr.). See (Iron Cr.).

(Thorpe Cr.) or Gulch) - (5.85-5.95, 15.6-15.65) ; GOLD.

B 328 , p. 258

B 433 , p. 192-193

Three Friends Min. Co. See (Solomon R.). 
Topkok Chief Min. Co. See (Daniels Cr.).

Topkok Ditch Co. See (Daniels Cr.).

(Tubutulik R.) - $(21.4,15.3)$; GOLD.
B 442, p. 341-342
B 712, p. 189

B 449 , p. 115-116

(Uncle Sam Mtn.) - (5.5, 10.85-10.9) ; GOLD (?).

B 433, p. 147

B 722, p. 198-199

Uplift Min. Co. See (Camp Cr.), (Niukluk R.).

(Venetia Cr.) - (1.1-1.5, 13.9-14.55) ; GOLD.
B 225, p. 53
B 379, p. $337-338$
B 328, p. 221-222

(Walla Walla Cr.) - $(19.0,11.0)$ approx ; GOLD.

B 449, p. 129

(Warm Cr.) - (8.1-8.8, 16.7-17.1) ; GOLD, SILVER, TUNGSTEN.
B 263, p. 14,53
B 284, p. 138
B 314, p. 151
B 328, p. 254-256
B 345, p. 217
B 379, p. 295
B. 442 , p. 363
B 520, p. 342

Warm Cr. Dred. Co. See (Warm Cr.).
B 592, p. 388, 392
B 622 , p. 371
B 662, p: 452
B 712, p. 189
B 739, p. 9
B 755 , p. 14
BMB 142, $\mathrm{p}_{:}: 28$
BMB 153, p. 56

(West Cr.) : $(3.75-4.3,11.8-12.0)$; GOLD. Includes reference to Alden.
B 328, p. 226
B 345, p. 221-222, 238
B 722, p. 198, 205
B 379, p. 286, 288
B 433, p. 93, 148, 175-177
B 857-A, p. 45
B. 864-A, p. 50
B 868-A, p. 52

B 520, p. 342

Wheeler (Iron Cr.) - (W $1 / 2$ 2, 16) ; COPPER.

B 662, p. 441

TDM 5-R, p. 21

Wheeler (Kruzgamepa -R.) - (2.6-2.7, 17.15-17.2) ; LEAD, SILVER, ZINO.

Note.-Not mentioned by name in $B 345,379,662$.
B 345, p. 246-247
B 722, p. 182-183, 210-211
B 379, p. 343-344
TDM 5-R, p. 28
B 662, p. 446

Wheeler (Sherrette Cr.) - $(2.7,15.95)$; COPPER, GOLD, SILVER. NOTE.-Not mentioned by name in $B \mathbf{3 4 5}, 379$.
B 345, p. 242-243
B 379, p. 343
B 722, p. 212, 214-215
TDM 5-R, p. 21

B 662, p. $441-442$

Wild Goose (Min. \& Trad. Co.). See (Dutch Cr.), (Ophir Cr.).

(Willow Cr.) (trib. Casadepaga R.). See (Lower Willow Cr.).

(Willow Cr.) (trib. Kruzgamepa R.) - (1.4-1.5, 15.8-16.15) ; GOLD.
B 328 , p. 267
B 379 , p. 321
B 622, p. 372
BMB 142, p. 27

Willow Dred. Co. See (Lower Willow Cr.).

(Wilson Cr.) - (3.8-3.9, 14.3-14.55) ; GOLD.

B 379 , p. 336-337

(Winnebago Cr.) - $(5.15,12.3)$; GOLD.

B 433, p. 168-169

(-.--) (copper, gold) - $(5.65,11.6)$; GOLD, COPPER.

B 722, p. 199-200

TDM 5-R, p. 19 


\section{STEPOVAK BAY QUADRANGLE}

(-_---) (sulfur) - (1.0,17.0) approx; SULFUR.

B 692 , p. 297-298

\section{SUMDUM QUADRANGLE}

Alaska Bond \& Dev. Co. See (Windham Bay). Alaska Copper (Min.) Co. See (Pt. Astley).

Alaska-Peerless (Gold Min. Co.) - (6.5-6.55, 10.45-10.55) ; GOLD. Includes references to Peerless, Yellow Jacket (Min. Co.) ; see also California-Alaska Min. Co., Helvetia.
B 287, p. 41
B 773 , p. 126
B 712, p. 30
B 792 , p. 10
B 722 , p. 36
BMB 153, p. 20
B 739, p. 21

Alaska Snettisham Gold Min. Co. See Crystal, Friday. Alaska-Windham Gold Min. Co. See (Windham Bay). Apache. See Windham Chief Gold Min. Co.

Apollo. See (Pt. Astley).

Bald Eagle. See Sumdum.

Basin Queen. See Alaska Peerless.

Butterbaugh. See (Tracy Arm).

California-Alaska Min. Co. - $(6.5,10.5)$; GOLD. See also Alaska Peerless.

B 287 , p. 41

(Chuck R.). See (Shuck R.).

Colp \& Lee - $(11,1)$; GOLD, LEAD, ZINC. COPPER.

B 739, p. 68

Cook - $(3.45,16.75)$; GOLD, LEAD. NotE.-Not mentioned by name in B 287.

B 284, p. 41

B 287, p. 47

Coughlin \& Pekovich. See (Snettisham) (iron).

Crystal - $(1.85,16.85)$; GOLD. Includes references to Daisy Bell, (Snettisham) (gold).
B 225, p. 36
B 480 , p. 97
B 259, p. 53
B 502, p. $39-40$
B 284 , p. $40-41$
B 542, p. 33
B 287, p. $.47-48$
B 712, p. 30
B 314, p. 58
B 722, p. 36
B 345, p. 90
B 739, p. 21
B 379, p. $71-72$
B 755, p. 24
B 442 , p. 139
RI 5195, p. 6

Daisy Bell. See Crystal.

Doctor. See California-Alaska Min. Co.

Durer. See Alaska Peerless.

Evening Star. See California-Alaska Min. Co.

Fairview. See Rowe.

Friday - $(1.8,16.95)$; GOLD, IRON.

B 287, p. 47

RI 5195, p. 6

(Gambier Bay) - (0, 7-8) ; MARBLE.

B 682 , p. 55

Golden R. Min. Co. See (Shuck R.).

Great Mine. See Jensen. 
Heins. See Crystal.

Helvetia (Gold Min. Co.) - $(6.45-6.5,10.55-10.6)$; GOLD. See also Alaska Peerless.

$\begin{array}{ll}\text { B 284, p. } 41 & \text { B 314, p. 58-59 } \\ \text { B 287, p. } 41 & \text { B 773, p. 125-126 }\end{array}$

Holkham Bay - $(6.05,11.15)$ approx ; GOLD, LEAD, COPPER.

B 284, p. 41

B 345, p. 90

B 287, p. 45

B 379, p. 72

B 314, p. 58

Independent (Gold) Min. Co. - $(6.2-6.3,10.35-10.5)$; GOLD, LEAD, ZINC.

Includes reference to Mildred.
B 287, p. 41-42
B 773, p. 127
B 722, p. 36

Jangle. See (Tracy Arm).

Jenny'Reed Gold Min. Co. - $(6.4,10.6)$; GOLD.

B 287, p. 41

Jensen - $(6.55 ; 10.45)$; GOLD.

B 773, p. 126-127

Jingle. See (Tracy Arm).

Lost Rocker. See (Slate Cr.).

Magnetite. See (Snettisham) (iron):

Marty (Mines). See (Windham Bay).

Mildred (Group Min. Co.). See Independent Min. Co.

Navajo. See Windham Chief Gold Min. Co.

Neglected Prize. See (Tracy Arm).

Oceanic Min. Co. See (Pt.:Astley).

Owens. See (Tracy Arm).

Peerless. See Alaska Peerless.

(Pt. Astley) - (3.5-3.55, 12.4-12.45); COPPER, SILVER, ZINC, LEA.D. Includes references to Alaska Copper Co., Sunny Day.

$\begin{array}{ll}\text { B 287, p. 44-45 } & \text { B } 800, \text { p. 318, 323, 327 } \\ \text { B 379, p. 72 } & \text { B 1058-A, p. 25, 27 } \\ \text { B 712, p. 30 } & \text { C 248, p. 6, 10 } \\ \text { B 773, p. 131-133 } & \end{array}$

(Port Houghton) - $(8.8,5.65)$ approx ; COPPER, GARNET.

B 773, p. $128-130 \quad$ : IC 7844, p. 11

Portland - $(6.0-6.3,11.75-12.05)$; GOLD, SILVER, LEAD, ZINC.

B 284, p. 41

B 287, p. 45

(Powers Cr.) - (4.5-4.7, 13.3-13.5) ; GOLD.

B 287, p. 2, 45

IC 7844, p. 7

Red Wing. See Windham Bay Gold Min. Co.

Rowe - $(6.55,10.45)$ approx ; GOLD.

B 773, p. 126

(Shuck R.) - (6.6-6.8, 9.25-9.3) approx; GOLD. NoTE.-Shown on recent maps as Chuck $\mathbf{R}$.

B 225, p. 37

B 792, p. 15

B 287, p. 42-43

(Slate Cr.) - (6.25-6.3, 9.5-9.55) ; GOLD.

B 287, p. 42

(Snettisham) (gold). See Crystal. 
(Snettisham) (iron) - (1.85-2.2, 17.15-17.45) ; IRON, COPPER:
B 773, p. 133-134
IC 7844, p. 11
B 800, p. 352
RI 5195, p. 6, 8-9, 11, 23-36, 39-40
C 252, p. 10

(Spruce Cr.) - (6.4-6.5, 10.5-10.55) ; GOLD.

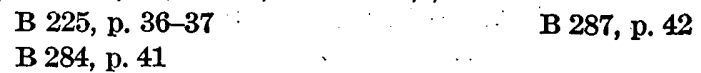

Spruce Cr. Min. Co. See (Spruce Cr.).

Sumdum (Chief) - $(5.0,11.8)$; GOLD, LEAD, ZINC. Includes reference to Bald Eagle.
AR 18, p. 62-63, 75-76
B 287, p. 4,44
B 225, p. 29,36
B 314, p. 58
B 259, p. 53
B 345, p. 90
B 284, p. 40-41
B 800, p. 317

Sunny Day See (Pt. Astley).

Townsend. See (Tracy Arm).

(Tracy Arm) - (3.95, 15.8) ; ZINC, COPPER, SILVER, GOLD, LEAD. Includès references to Neglected Prize.
B 642 , p. 99
B 773, p. 130-131
B 800, p. 362
C 252, p. 6
IC 7379, p. $41-42$
IC 7844, p. 12
B 998-A, p. 1-3, 7-11

(Windham Bay) - $(6.2-6.8,9.25-10.6)$; GOLD. Includes references to AlaskaWindham Gold Min. Co., Marty (Mines) ; see also Alaska Peerless, CaliforniaAlaska Min. Co., Helvetia, Independent Min. Co., Jenny Reed Gold Min. Co., Jensen, Rowe, (Shuck R.), (Slate Cr.), (Spruce Cr.), Windham Bay Gold Min. Co., Windham Chief Gold Min. Co., Yates.
B 225, p. 46
B 857-A, p. 15
B 259 , p. 53
B 864-A; p. 17
B 379, p. 72
B $868-$ A, p. 16
B 642 , p. 76
B 880-A, p. 17
B 797, p. 10-11
B $897-A$, p. 18
B 810, p. 13
B $910-A$, p. 20
B 813, p. 15
B 933-A, p. 32
B 824, p. 16
IC 7844, p. 7

Windham Bay Gold Min. Co. - $(6.45,10.5)$; GOLD. Includes reference to Red Wing.

B 287, p. 40-41 $\quad$ B 314, p. 59

Windham Chief Gold Min. Co. - $(6.75,10.45) ;$ GOLD.

B 287, p. 41

Yates - $(6.55,10.45)$ approx; GOLD.

B 773, p. 126

Yellow Jacket (Min. Co.). See Alaska Peerless.

\section{SURVEY PASS QUADRANGLE}

(Alatna R.) - (13.5-18.7, 0.4-9.2) ; GOLD.
P 10 , p. 50
B $910-A$, p. 56
B 813, p. 45
B $926-A$, p. 52
B 815, p. 334-335

(Allen R.). See (Alatna R.).

Arctic Prospecting \& Dev. Co. See (Alatna R.), (Walker L.). 
Copper King - $(7-8,10)(?) ;$ GOLD, COPPER.
P 20, p. 103-104
B 815 , p. $337-338,342$

Copper Queen. See Copper King.

Gray Eagle. See Copper King. .

Ground Hog. See Copper King.

(Helpmejack Cr.) - (14.5-16.5, 0.3-2.3) ; GOLD.

P 10, p. 50

Iowa. See Copper King.

Lucky Six. See Copper King.

(Lucky Six Cr.). - (6.85-7.8, 9.6-10.9) ; GOLD, SILVER, GRAPHITE.

B 520, p. $335-336$

B 536, p. 140-141

B 815 , p. 331-332

B 773, p. 51

B 824 , p: 50

TDM 5-R, p. 25; 32

Mammoth. See Copper King.

(Mauneluk R.) - (SW1/4 quad.) ; SILVER. TDM 5-R, p. 32

(Mecklenberg Cr.) - (19.8-20.5, 2.0-2.5) ; GOLD.

B 536, p. 143-144

B 815, p. 334-335

(Mettenpherg Cr.). See (Mecklenberg Cr.).

(Pingaluk R.) - (14.7-17.5; 8.5-13.0) ; GOLD (?).

B $844-A$, p. 39

(Reed R.) - (7-8, 0-3) ; GOLD.(?).

B 824, p. 50

B 836, p. 51

Silver King. See Copper King.

(Walker L.) - (9.5-12.0, 1.0-4.0) ; GOLD (?).

B 813, p. $44-45$

B 824, p. 50

B $910-A$, p. 73

\section{TAKU RIVER QUADRANGLE}

Arizona - $(0.3,0.8)$ approx; GOLD, LEAD, ZINC.

B 379, p. 72

Bach. See Enterprise.

Enterprise - $(0.3,0.8)$ approx; GOLD, LEAD, ZINC. Includes references to

Bach, (Limestone Inlet) (metals), Williams \& Leak.

B 345 , p. 90

B 379, p. 72

B 480 , p. $97-98$

B 442, p. 139

B 662, p. 77-78

BMB 153, p. 24

(Limestone Inlet) (marble) - $(0.7,0.8)$; MARBLE.

B 314, p. 77

B 480 , p. 94

B 592, p. 98,107

B 682, p. $40-41,112-113$

(Limestone Inlet) (metals). See Enterprise.

Lost Charlie Ross. See (Whiting R.).

Montana - $(0.3,0.8)$ approx; GOLD (?).

BMB 153, p. 24

Northern Exp. Co. See Enterprise, Montana.

(Whiting R.) - (5.15, 0.9) ; SILVER, GOLD, LEAD, ZINC, COPPER.

Includes reference to Lost Charlie Ross.

B 442 , p. 139

B 642 , p. 98-99

B 773, p. 135-136

Williams \& Leak. See Enterprise.
B 800, p. 362

IC 7844, p. 12 


\section{TALKEETNA QUADRANGIE}

Adams. See (Falls Cr.).

Alaska Continental Gold Mines. See (Lake Cr.).

Alaska Exp. Min. Co. See (Bird Cr.).

Anna Bub. See (Dollar Cr.).

Battle-Axe Assn. See (Thunder Cr.).

(Bear Cr.) - (16.3-17.45, 11.2-12.0) ; GOLD.

B 810, p. 22

(Big Boulder Cr.) - (NE 1/4 11, 5) ;GOLD. See also (Twin Cr.).

B 520, p. $198 \quad$ B 534, p. 70

(Bird Cr.) - (16.7-17.05, 10.4-10.45) ; GOLD, TUNGSTEN, COPPER.

B 520, p. 182, 194-195

B 534, p. 65

B 592, p. 66

B 692, p. 260-261

B 864-A, p. 31

B 755, p. 131

B 868-A, p. 32-33

B 897-A, p. $42-43$

B 810, p. 22

B 910-A, p. 41

B 917-A, p. 39

B 813, p. 25

B 926-A, p. 35

B 933-A, p. 35

B 824 , p. 29

B 1024-A, p. 2, 13, 18-19

B 836, p. 29

B 857-A, p. 27

Boedeker - $(23.1,16.85)$; GOLD.

B 824, p. 23

B 836, p. 23

BMB 153, p. 42

TDM 1, p. 39

B 857-C, p. 138-139

(Boulder Bench). See (Kahiltna R.).

(Boulder Cr.) - (NE1/4 11, 5) ; GOLD. See also (Big Boulder Cr.), (Little Boulder Cr.).

B 917-A, p. 39

Bradley. See (Cache Cr.).

Bubb \& Bahern. See (Dollar Cr.).

(Cache Cr.) - (15.95-17.25, 7.1-10.2) ; GOLD, PLATINUM, TIN, TUNGSTEN.

Includes references to Cache Cr. Min. Co. if definitely to this creek; Murray \& Harper.

P 70, p. 167

B 442, p. 43

B 480 , p. 38

B 520, p. 182-187

B 534, p. 54-57

B 592, p. 65

B 622 , p. 49

B 662, p. $48-49$

B 692, p. 32-33, 243-248

B 712, p. 35

B 714, p. 38,78

B 722, p. 42

B 739, p. 9, 26

B 755, p. 14, 31-32, 129-130

B 773, p. 27, 43

B 783, p. 18

B 792, p. 15,25

Cache Cr. Dred. Co. See (Cache Cr.).
B 797, p. 17

B 810, p. 22

B 813, p. 25

B 824, p. 29

B 836, p. 28

B 844-A, p. 29

B 857-A, p. 27

B 864-A; p. 31, 56

B 868-A; p. 32, 59

B 880-A, p. 29,36

B 897-A, p. 42-43

B 910-A, p. 41-42

B 917-A, p. 39

B 926-A, p. 36

B 1024-A, p. 2, 13, 18

BMB 153, p. 38,42 .

TDM 1, p. 20, 34, 39 
Cache Cr. Min. Co. See (Cache Cr.), (Nugget Cr.).

(Camp Cr.) - (11-12, 6-7) ;.GOLD, PLATINUM. See also (Cottonwood Cr.) (trib. Camp Cr.), (Mills Cr.).
B 534, p. 71
B 692 , p. 32-33

(Canyon Cr.) - $(18.1,10.8)$ approx ; GOLD, TIN, PLATINUM. B 692, p. 261-262

Carlson. See (Falls Cr.).

(Chechako Gulch) - $(\mathbf{1 6 . 1 5}, \mathbf{8 . 7})$; GOLD.

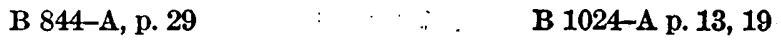

(Chicago Gulch) - (NE1/4 11, 5) ; GOLD. See also (Mills Cr.). B 520, p. 198

B 534, p. 68-69

Conhart Min. Co. See (Dollar Cr.).

(Cottonwood Cr.) (trib. Camp. Cr.) : (11.7-12.6, 6.2-6.9) ; GOLD.
B 797, p. 17
B 910-A, p. 42

(Cottonwood Cr.) (trib. Peters Cr.) - $(17,10)$; GOLD. See also (Poorman Cr.), (Willow Cr.).

B 810, p. 22

(Curry). See (Mile 247, ARR).

Devault, Devault \& Leitz. See (Pass Cr.).

(Dog Cr.). See (Long Cr.).

(Dollar Cr.) - $(15.7,8.9)$ approx ; GOLD, TIN.

$\begin{array}{ll}\text { B. 314, p. 119 } & \text { B 836,p. 28-29 } \\ \text { B 520, p. 174, 182, 191-192 } & \text { B 844-A, p. 29 } \\ \text { B 534, p. 62-63 } & \text { B 857-A, p. 27 } \\ \text { B 542, p. 44 } & \text { B 864-A; p. 31 } \\ \text { B 592, p. 66 } & \text { B 868-A, p. 33 } \\ \text { B 692, p. 252-254 } & \text { B 917-A, p. 39 } \\ \text { B 755, p. 130-131 } & \text { B 1024-A,p. 2 } \\ \text { B 773, p. 54-57, 59 } & \text { BMB 153, p. 42 } \\ \text { B 824, p. 29 } & \end{array}$

(Dutch Cr.) - (14.7-16.0, 10.0-11.3) ; GOLD, TUNGSTEN.
B, 868-A; p. 33
B 880-A, p. 36
B 933-A, p. 35
B 910-A, p. 41
RI 4174, p. 28
TDM 2, p. 20

B 917-A, p. 39

Dutch Cr. Min. Co. See (Dutch Cr.), (First Cr.).

(Eagle Cr.) - (7-9, 4-5) (?) ; GOLD.

P 70, p. 167

B 480 , p. 38

Ebheart \& Anderson. See (Falls Cr.).

Eiderstein. See (Peters Cr.).

Erickson. See (Cache Cr.).

(Falls Cr.) - (15.9-16.6, 8.75-9.4) ; GOLD.
B 314, p. 119
B 824, p. 29
B 345, p. 38
B 520, p. 182,191
B 836, p. 28
B 534, p. 62
B 592 , p. 66
B 692, p. 251-252
B 844-A, p. 29
B 857-A, p. 27.
B 864-A, p. 31
B 755, p. 130
B 868-A, p. 33
B 797, p. 17
B 917-A, p. 39
B 810, p. 22
B 933-A, p. 35
B 813, p. 25
B 1024-A, p. 13
BMB 153, p. 42

$593018-61-20$ 
(Falls Gulch) - (17.3-17.45, 10.3-10.65) ; GOLD. See also (Willow Cr.). B 520, p. $195-196$ B. 534, p. 66

(First Cr.) - (15, 9-10) (?) ; GOLD. B 897-A, p. 43

B 917-A, p. 39

Francis \& Foster. See (Long Cr.).

Funk. See (Falls Cr.).

Gage \& Mack. See (Thunder Cr.).

(Gold Cr.) - $(16.75,9.75)$ GOLD, SILVER.

B 520, p. 182, 187

B 755, p. 131

B 534, p. 58

BMB 153, p. 42

B 592, p. 65

Gold Placers, Ltd. See (Cache Cr.).

(Gopher Cr.) :: $(17.3,10.7)$ approx ; GOLD, LEAD.

B 692, p. 260

B 810, p. 22

Gray. See (Treasure Cr.).

Gredakin. See (Bird Cr.).

Hamberg \& Gliska. See (Pass Cr.).

Hamersmith. See (Bird Cr.).

Hansen. See (Willow Cr.).

Harper Bros. See (Nugget Cr.).

Haugham. See (Thunder Cr.).

(Independence Cr.) - $(9,4)(?)$; GOLD.
P 70, p. 167
B 520, p. 199
B 480, p. 38
B 534, p. 71

J \& K - $(7.05,15.65)$; SILVER, LEAD, ZINC, COPPER.

B 792, p. 108-109

Jenkins. See (Willow Cr.).

Jiles, Knudson \& Cators. See J \& K.

(Johns Cr.) - (NE1/4 11, 5) ; GOLD. See also (Twin Cr.). B 520, p. 198 B 534, p. 70

Jumping Jack. See (Nugget Cr.).

(Kahiltna R.) - (17.3-18.1, 0.3-2.85) ; GOLD, PLATINUM, TUNGSTEN, TIN. Includes references to (Kahiltna R.) under Tyonek quad.
B 520, p. 199
B 813 , p. 60
B 534, p. 71
B 824 , p. 29
B 662, p. 23
B 836, p. 29
B 666, p. 96
B 864-A, p. 31
B 692, p. 32-33, 262-263
B 868-A, p. 33
B 714, p. 38
B 1024-A, p. 21-22
B 810 , p. 53
TDM 1, p. 20

Kast, Nelson \& Larson. See (Poorman Cr.).

(Kichatna R.) - (10.2, 2.6) approx ; GOLD, PLATINUM.
B 520, p. 199
B 692, p. 32-33
B 534, p. 70-71

Krummenacher. See (Chechako Gulch).

(Lake Cr.) - $(11.5-17.0,2.0-6.0)$; GOLD, PLATINUM. See also (Mills Cr.), (Twin Cr.).

B 314, p. 119

B 520, p. 197,199

B 534, p. 67-68, 71

B 692, p. 32-33
B 755, p. 131

B 857-A p. 28

B $880-$ A, p. 36 
(Little Boulder Cr.) - (NE1/4 11, 5) ; GOLD. See also (Twin Cr.).
B 520, p. 198
B 534, p. 70

(Long Cr.) - $(18.1,10.8)$ approx; GOLD, PLATINUM, TIN. See also (Canyon Cr.).

B 520, p. 196

B 534, p. 67

B 692, p. 261-262

B 714, p. 38

(Lucky Gulch) - $(16.65,9.05)$; GOLD.

B 520, p. 190
B 810, p. 22

B 824, p. 29

B 857-A, p. 27

BMB 153, p. 42

B 534, p. 60

McDonald, Pat, Inc. See (Peters Cr.).

McElroy \& Remmer See (Falls Cr.).

(Mile 247, ARR) - $(24.0,10.9)$; MOLYBDENUM, BUILDING STONE. Includes reference to (Curry).

B 926-C, p. 189-190

C 18, p. 3, 5

(Mills Cr.) - (NE 1/4 11,5) ; GOLD. See also (Chicago Gulch), (Wagner Gulch). B 520, p. 182, 197-199

B 534, p. 69-70

B 592 , p. 66

B 755, p. 131

B 797, p. 17

B 810, p. 22

B 836, p. 29

B 844-A, p. 29

B $910-A$, p. 42

B 917-A, p. 39

B 933-A, p. 35

B 1024-A, p. 2

Morgan. See (Cache Cr.), (Nugget Cr.).

Murray. See (Cache Cr.), (Nugget Cr.).

Murray \& Harper. See (Cache Cr.).

(Nakochna R.) - (10.2, 2.6) (?) ; GOLD.

P 70, p. 166

B 520 , p. 199

B 480, p. 38

B 534, p. 70-71

Nagley and assoc. See (Falls Cr.).

(Notobac Cr.) - (11-12, 5-6) ; GOLD.

B 810, p. 22

B $844-A$, p. 29

B 813, p. 25

B 917-A, p. 39

B 836, p. 29

(Nugget Cr.) - (16.55-16.9, 9.25-9.8) ; GOLD, TIN, TUNGSTEN.

B 314, p. 119

B 345, p. 38

B 810, p. 22

B 824, p. 29

B 480 , p. 38

B 844-A, p. 29

B 520, p. 182, 187-189

B 857-A, p. 27

B 534, p. 58-60

B 864-A, p. 31 .

B 542,p. 44

B 592, p. 65-66

B 868-A, p. 33

B 662, p. 49

B 692, p. 248-249

B 910-A p. 41

B 917-A, p. 39

B 755, p. 130

P. 933-A, p. 35

B 797, p. 17

B 1024-A, p. 2, 13, 18

BMB 153, p. 42

Obermiller \& Englehorn. See (Cache Cr.).

(Pass Cr.) - (11.15-12.0, 6.6-7.1) ; GOLD.

B 797, p. 17

B 844-A, p. 29

B 917-A, p. 39

B 897-A, p. 43

B 926-A, p. 36

B 910-A, p. 42

B 933-A, p. 35 
(Peters Cr.) -. (16.9-18.0, 9:1-10.5) ; GOLD, PLATINUM.

$\begin{array}{lll}\text { P 70, p. 167 } & & \text { B 836, p. 29 } \\ \text { B 284, p. 7 } & & \text { B 844-A, p. 29 } \\ \text { B 345, p. 38 } & \text { B 857-A, p. 27 } \\ \text { B 442, p. 43 } & & \text { B 864-A, p. 31 } \\ \text { B 480, p. 38 } & & \text { B 868-A, p. 32 } \\ \text { B 520, p. 182, 193-194 } & & \\ \text { B 534, p. 64-65 } & \text { B 880-A, p. 35-36 } \\ \text { B 592, p. 65-66 } & \text { B 897-A, p. 42 } \\ \text { B 662, p. 49 } & \text { B 910-A; p. 41 } \\ \text { B 692, p. 32-33, 255-257 } & & \text { B 917-A, p. 39 } \\ \text { B 755, p. 129, 131 } & & \text { B 926-A, p. 36 } \\ \text { B 810, p. 22 } & & \text { B 1024-A, p. 2, 13, 19 } \\ \text { B 813, p. 25 } & & \text { BMB 153, p. 42 } \\ \text { B 824, p } & & \text { TDM1, p. 20 }\end{array}$

B 824, p. 29

Peters Cr. Min. Co. See (Peters Cr.).

Peters Cr. Placer Co. See (Peters Cr.).

Peterson. See (Cache Cr.).

(Pineo Bar). See (Cache Cr.).

(Poorman Cr.) - (17.45-17.65, 10.5-10.8) ; GOLD, PLATINUM, TIN.
B 314, p. 119
B 345, p. 38
B 520, p. 182, 196
B 534, p. 66-67
B 592, p. 66
B 692, p. 257-259
B 714, p. 38
B 755, p. 131

B 810, p. 22

B 813, p. 25

B 824, p. 29

B. 836, p. 29

B 917-A, p. 39

B 926-A, p. 35

BMB 153, p. 42

Price. See (Nugget Cr.).

(Rambler Cr.) or (Gulch) - (16.55, 9.0) ; GOLD.

B 520, p. 182, 186-187, 190

B 592, p. 65

B 534, p. 57, 60-61

(Ramsdyke Cr.) - (17.1-17.9, 10.8-11.5) ; GOLD.

B 810 , p. 22

BMB 153, p. 42

B 880-A, p. 36

Raymond. See (Nugget Cr.).

(Red Hill Bar). See (Kahiltna R.).

Rice. See (Willow Cr.).

Richardson. See (Ramsdyke Cr.).

(Rocky Gulch) - (17.3-17.45, 10.3-10.65) ; GOLD. See also (Willow Cr.). B 520, p. 195-196

B 534, p. 66

(Round Bar Bend). See (Kahiltna R.).

(Ruby Cr.) - (17.3, 10.7) approx.; GOLD, TIN, PLATINUM.

B 692, p. 259-260 B 917-A, p. 39

St. Louis. See (Bird Cr.).

(Sholan Bar). See (Kahiltna R.).

(Short Cr.) - (15.95-16.0, 8.45-8.55) ; GOLD.
B 755, p. 130
B 836, p. 28

(Slate Gulch) - (17.3-17.45, 10.3-10.6) ; GOLD. See also (Willow Cr.).

B 520, p. 195-196

B 534, p. 66

Smith \& Hogan. See (Nugget Cr.). 
(Snow Gulch) - (17.3-17.45, 10.3-10.65) ; GOLD. See also (Willow Cr.). B 520, p. 195-196.

Spokane-Peters Cr. Min. Co. See (Peters Cr.).

(Spruce Cr.) - (16.0-16.3, 7.7-8.1) ; GOLD. BMB 153, p. 42

(Susitna R.) - (23.0-24.3, 0.5-11.7) ; GOLD. P 70, p. 164-165.

Taraski. See (Cache Cr.).

Tesmer \& Beidermann. See (Cache Cr.).

(Thunder Cr.) - (16.3-16.35, 9.0-9.35) ; GOLD, TIN, TUNGSTEN.

B 314, p. 119

B 520, p. 182, 190-191

B 836, p. 28

B 534, p. 61

B 844-A, p. 29

B 542, p. 44

B 857-A, p. 27

B 592, p. 66

B 864-A, p. 31

B 692, p. 249-251

B 868-A, p. 33

B 755, p. 130

B 910-A, p. 41

B 773, p. 55, 57-59

B 917-A, p. 39

B 810 , p. 22

B 933-A, p. 35

B 813, p. 25

B 1024-A, p. 2, 13, 19

B 824, p. 29

BMB 153, p. 42

Thunder Cr. Min. Co.' See (Falls Cr.), (Thunder Cr.), (Windy Cr.).

(Tokichitna R.) - (17.1-18.1, 10.8-11.5) approx; GOLD See also (Long Cr.),

(Ramsdyke Cr.).

B 813, p. 25

B 836, p. 29

B 824, p. 29

B 844-A, p. 31

(Treasure Cr.) - (15.9-16.6, 8.75-9.05) approx; GOLD. See also (Falls Cr.). B 314, p. 119

BMB 153,p. 42

(Twin Cr.) - (NE 1/4 11, 5) ; GOLD. See also (Big Boulder Cr.), (Boulder Cr.),

(Johns Cr.), (Little Boulder Cr.).

B 592, p. 66

B 933-A, p. 35

B 755, p. 131

(Twin Mills Cr.) - (NE 1/4 11, 5) ; GOLD. See also (Mills Cr.), (Twin Cr.). B 542, p. 44

Wagner. See (Bird Cr.).

Wagner \& Co. See (Cottonwood Cr.) (trib. Camp Cr.), (Mills Cr.).

(Wagner Gulch) or (Cr.) - (NE 1/4 11, 5) ; GOLD. See also (Mills Cr.). B 442, p. 43

B 534, p. 68

B 520, p. 197

Weatherall \& Andresen. See (Gold Cr.).

Wetherell. See (Cache Cr.).

(Willow Cr.) - (17.3-17.45, 10.3-10.65) ; GOLD, PLATINUM, TIN.

$\begin{array}{ll}\text { B 314, p. 119 } & \text { B 813, p. 25 } \\ \text { B 520, p. 182, 195 } & \text { B 824, p. 29 } \\ \text { B 534, p. 66 } & \text { B 836, p. 29 } \\ \text { B 542, p. 44 } & \text { B 857-A, p. 27 } \\ \text { B 592, p. 68 } & \text { B 864-A, p. 31 } \\ \text { B 692, p. 259 } & \text { B 917-A, p. 39 } \\ \text { B 714, p. 38 } & \text { B 926-A, p. 35 } \\ \text { B 755, p. 131 } & \text { B 933-A, p. 35 } \\ \text { B 773, p. 55, 58-59 } & \text { B 1024-A, p. 13 } \\ \text { B 810, p. 22 } & \text { BMB 153, p. 42 }\end{array}$


(Wilson Cr.). See (Willow Cr.).

(Windy Cr.) - (16.1, 8.2) ; GOLD, TIN, TUNGSTEN, COPPER.
B 692, p. 254
B 1024-A, p. 2
B 755, p. 131
BMB 153, p. 42
B 810, p. 22
TDM 1, p. 39

B 933-A, p. 35

Wolf \& Maloche. See (Spruce Cr.).

Yentna Dred. Co. See (Cache Cr.).

\title{
TALKEETNA MOUNTAINS QUADRANGLE
}

(Ilbert Cr.) - (21.8-22.25, 0.5-0.55) ; GOLD, PLATINUM.

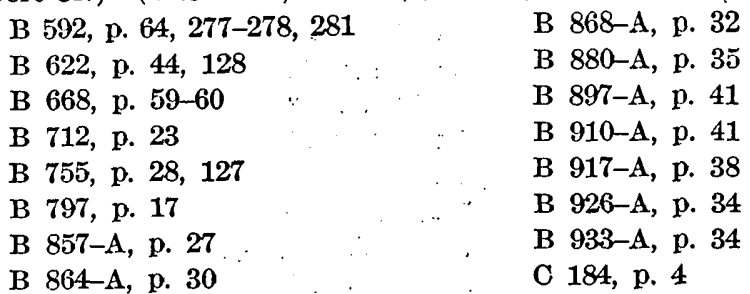

Blue Lode - $(7.65,6.15)$ approx ; COPPER.

B 692 , p. $202-203$

B 755 , p. $123-124$

(Chunilna Cr.) - (0.25-1.4, 7.95-9.35) ; GOLD.
P 70, p. 165
B 327, p. 64

Copper King - $(6.75,6.05)$ approx ; COPPER, GOLD, SILVER.

\author{
B 692 , p. $201 \quad$ B 755, p. 122
}

Copper Queen -. $(6.45,6.35)$ approx ; COPPER, GOLD.
B 692, p. 199-201
B 755 , p. $121-122$

Copper Wonder - (7.1, 5.95) approx; COPPER, IRON.
B 692 , p. 201
B 755 , p. 122-123

(Cottonwood Cr.) See (Sleigh Cr.).

(Crooked Cr.) - $(22.9,1.5)$ approx; GoLD.
B 592, p. 278,281
B 668 , p. $60-61$
B 622 , p. $127-128$
B 755, p. 126

(Daisy Cr.) - (23.05-23.1, 5.15-5.4) ; GOLD. B 622 , p. 129

B 668, p. 64

Eastview - $(7.7,6.05)$ approx ; COPPER. B 692 , p. 203

B 755 , p. 124

Ella B. - (5.1, 15.1) approx; Commodity, if any, not known. B 783 , p. 90

(Fourth of July Cr.) - $(22.3,5.3)$; GOLD.
B 622, p. 129
B 668 , p. 64

(Gold Cr.) (trib. Oshetna R.) - $(18.6,4.5)$; GOLD.
B 622, p. 130
B 668 , p. 64

(Gold Cr.) (trib. Susitna R.) - (2.6-3.6, 13.25-13.6) ; GOLD. B 692, p. 231

(Granite Cr.) - $(\mathbf{1 7 . 7}, \mathbf{5 . 5 5})$; GOLD. B 622 , p. 130

B 668, p. 64

(Indian R.) - $(2.85,14.8)$; HAYDITE.

RI 4932 , p. $68-70,80$ 
(Iron Cr.) - (6.45-8.0, 5.95-6.5) (?) ; COPPER; GOLD. See also Blue Lode, Copper King, Copper Queen, Copper Wonder, Eastview, Phoenix, Talkeetna. B 480 , p. 32-33 B 622, p. 47

B 520, p. 28

Johnson (\& Wertz). See Mint.

(Little Nelchina R.) - (18-24, 2) ; GOLD (?). B 622, p. 129

B 668, p. $63-64$

(Mazuma Cr.) - (17.7-17.8, 1.1-1.7) ; GOLD.

B. 592, p. 279-281

B.668, p. 63:

Mint - $(5.2,15.3)$; SILVER, ANTIMONY, COPPER, LEAD, MOLYBDENUM (?). Includes references to (Portage Cr.).

B 773, p. 13, 43-44

B 783, p. 89-95

B 836, p. 23

B 797, p. 37

B $880-$ A, p. 66

B 810 , p. 50

B $910-A$, p. 81

B 824, p. 23

B $926-$ C, p. 190

(North Cr.) - (22.15-22.3, 0.65-0.75) ; GOLD.

B 592, p. 278

Olsen, Getchel (, Palmer, McCormack). See (Albert Cr.).

Phoenix - (7.5, 6.0) approx; COPPER. B 692 , p. 202

(Portage Cr.). See Mint.

B 755, p. 123

(Roaring Cr.) - (17.2,4.85) ; GOLD.

B 622, p. 130

B 668, p. 64

(Sleigh Cr.) - (22.7-22.95, 0.6-1.05) ; GOLD(?). Includes reference to (Cottonwood Cr.).

B 592, p. 278

B 622, p. 129

(Susitna R.) - $(0.0-2.75,11.0-13.85)$; GOLD.

P 70, p. 164-165

B 692 , p. 231

Talkeetna - $(8.0,6.5)$ ( ?) ; COPPER, IRON, GOLD, SILVER. B 692, p. 203-204

B 755, p. 124-125

Wells, Wells \& Coffee. See Copper Queen.

(Willow Cr.) - $(22.45,1.2)$; GOLD (?). B 622 , p. 129

(Ýacko Cr.) - (21.6-21.8, 4.0-4.8) ; GOLD. B 622, p. 129

B 668 , p. 64

\section{TANACROSS QUADRANGLE}

(Boulder Cr.) - $(1.55,4.4)$; ANTIMONY, GOLD. Includes reference to (Stibnite Cr.), (Tok R.).
B 649, p. 63
B 904, p. $43-45$
B 933-A, p. 89
B 943-B, p. 44-45

B 989-D, p. 190, 201, 207-208

RI 4173, p. 4, 30-35

TDM 1, p. 13 .

Caulk. See (Boulder Cr.).

(Fortymile R., South Fork of Denison Fork) - $(13,8)(?)$; GOLD (?). B 542, p. 215

Gamblin (\& Murhoffer). See (Boulder Cr.).

Rambler. See (Boulder Cr.). 
(Sixtymile R.) - (21-23, 15-17) (?) ; GOLD. May be in Yukon Territory, Canada.

B 520, p. 212

(Stibnite Cr.). See (Boulder Cr.).

(Timber Cr.) - (SW 1/4 quad.) ; ASBESTOS(?).

TDM 1, p. 44

(Tok R.). See (Boulder Cr.).

\section{TANANA: QUADRANGIE}

Abe Lincoln. See (Sullivan Cr.).

Albrecht \& Hanson (Co.). See (Deep Cr.), (Woodchopper Cr.).

(American Cr.) - $(13,1)$; GOLD, CHROMITE. Includes references to American Cr. Dred. Co., American Cr. Operating Co.

B 520, p. 281, 283-284

B 535, p. 29, 33, 35, 37

B 824, p. 36, 52

B 542, p. 220

B 592, p. 68, 362

B 836, p. 36

B 622, p. 239, 245

B 844-A, p. 37

B 642, p. 64

B 662 , p. 57

B 692, p. 37, 335

B 714, p. 82

B 755, p. 149-150

B 783, p. 14

B 797, p. 22

B 810, p. 28,40

B 813, p. 32,47

American Cr. Dred. Co. See (American Cr.).

B 844-D, p. 213-214, 241

B 857-A, p. 33, 51

B 864-A, p. 39

B 868-A, p. 41, 59

B 880-A, p. 42,61

B $897-A$, p. 51,71

B 910-A, p. 52, 71

B 917-A, p. 50

B 926-A, p. 47, 71

B 933-A, p. 43, 67

BMB 153, p. 52

American Cr. Min. Co. See (American Cr.).

American Cr. Operating Co. See (American Cr.).

(American Gulch) - $(0.55,4.95)(?)$; GOLD.

B 520, p. 282

B 692, p. 39

B 535, p. 34

Barrett - $(16.75,0.9)$; GOLD, SILVER, LEAD, COBALT, COPPER, NICKEL(?).

B 844-D, p. 215-216 TDM 1, p. 18-19

C 317, p. 3-4

(Bear Cr.) - (12.75-14.6, 6.6-7.55) ( ?) ; GOLD.

B 844-D, p. $192 \quad$ B 880-A, p. 47

B 868-A, p. 44

Beardsley, Belsea \& Dillon. See (Glen Cr.).

Benson. See (Cache Cr.).

Bensonen. See (Deep Cr.).

(Big Boulder Cr.). See (Boulder Cr.).

(Big Denver Cr.) - $(16,1)(?)$; GOLD (?).

B 844-D, p. 216

(Big Minook Cr.). See (Minook Cr.).

Bock. See (Deep Cr.), (Hokeley Gulch), (Woodchopper Cr.).

Bock \& Co. See (Woodchopper Cr.).

Bock \& Hanson. See (Deep Cr.). 
(Bonanza Cr.) - (12.05-12.3, 5.85-6.3) ; TIN, GOLD.

B 844-D, p. 192

RI 4322, p. 7

(Boothby Cr.) - $(20.8,3.95)$ approx ; GOLD.

B. 314, p. 37

"Boston Boys." See (Eureka Cr.).

(Boulder Cr.) - (14.0-14.7, 2.6-2.75) approx; GOLD. Includes references to (Big Boulder Cr.).

B 642, p. 64

B 880-A, p. 42

B $662 ;$ p. 57

B $897-A$, p. 51

B 692, p. 37-38, 331, 335

B $910-A$, p. 53

B 836, p. 36

B 844-A, p. 37

B 844-D, p. 214-215, 241

B. $917-A$, p. 50

B 857-A, p. 33

Brock and assoc. See (Pioneer Cr.).

Brock \& Johnson. ' See (Eureka Cr.).

(Cache Cr.) - (15.9-16.15; 1.8-2.2) approx; GOLD, SILVER, TIN, RARE

EARTHS.

B 442, p. 243

B 797, p. 22

B 480,p. 166

B 926-A, p. 47

C 317, p. 6

B 520, p. 92, 283-284

B 535, p. 33, 35-36

B 542, p. 221

B 622, p. 242, 244

B 642, p. 64

B 662, p. 57

B 692, p. 334

B 844-D, p. 212, 240

B 910-A, p. 53

B $917-$ A, p. 50

B 926-A, p. 47

B 933-A, p. 43

BMB 142, p. 25

RI 5373, p. 6-8, 48

T'DM 1, p. 34

(California Cr.) -(18.95-19.65, 3.85-4.5) ; GOLD.

B 857-A, p. 33

(Chapman Cr.) - $(20.9-21.05,6.15)$; GOLD.

B 442, p. 241

B 542, p. 222

(Chicago Cr.) - $(19.35,3.35)$ approx ; GOLD.

B 280, p. 45

B 337, p. 92

B 880-A, p. 42

B 692, p. 334

B 897-A, p. 51

B 910-A, p. 53

Cleary Hill Mines, Inc. See (Sullivan Cr.), (Tofty Gulch).

(Colorado Cr.) - $(13,1)$; GOLD.

B 910-A, p. 53

(Columbe Cr.) - $(1,6)(?)$; GOLD. See also (Tozimoran Cr.) ; may be the same stream.

B 345, p. 46

(Cooney Cr.) - (17.0-17.05, 2.2-2.7) ; GOLD

B 542, p. 221

B 917-A, p. 50

B 592, p. 362

Cunningham and assoc. See (Little Minook Cr.).

(Dakota Bar). See (Sullivan Cr.).

(Dalton Cr.) or (Gulch) - $(16.1,2.05)$ approx; GOLD, TIN.

B 642, p. 64

RI 5373, p. 7-8, 48

Dean. See (Miller Gulch). 
(Deep Cr.) - (14.7-15.25, 1.2-1.35) approx; GOLD, TIN, SILVER, RARE EARTHS, CHROMITE', NICKEL. Includes references to Bock \& Hanson; see also (Hokeley Gulch), (Innesvale Gulch).

B 824, p. 37, 68

B 836, p. 36,71

B 844-A, p. 69

B 844-D, p. 238-239

B 868-A, p. 41

B 910-A, p. 52-53

Deep Cr. Min. Co. See (Deep Cr.).

(Deep Miller Cr.) - (14.7-15.3, 1.2-1.6) (?) ; GOLD. See also (Deep Cr.), (Miller Cr.) ; this reference may be to either or both.

BMB 142, p. 25

Donnelly - $(\mathrm{NE} 1 / 4,16,0)$; GOLD (?).

B 844-D, p. 216

(Doric Cr.) - $(20.65,3.85)$ approx ; GOLD. See also (Pioneer Cr.).

B 259, p. 116

B 280 , p. $41-42,48-50$

B 337, p. $86-87, .96,98$

B 824, p. 37

(Dry Cr.) - (S 1/2 13, 3) ; GOLD.

B 692, p. 335

Eagle Min. Co. See (Gien Cr.).

(Eureka Cr.) - (20.4-20.8, 3.8-4.25) ; GOLD. See also Farmer \& Jones, B 213, p. 51, 55

B 259, p. 115, 117

B 263, p. 52

B 280 , p. $38-40,49-50$

B 284, p. 126

B 337, p. $82-85,98$

B 379, p. 56

B 442, p. 242

B 480, p. 166

B 520, p. 283

B 535, p. 35

B 542, p. 221

B 592, p. 362

B 622, p. 242

B 642 , p. 64

B 692, p. 37,334

Farmer \& Jones - (20.4-20.8, 3.6-4.25) approx; GOLD. See also (Eureka Cr.), (McCaskey Bar).

B 813, p. 32

Farrell and assoc. See (Idaho Bar).

(Ferguson Gulch) - $(16.15,2.1)$ approx; GOLD, TIN.

RI 5373, p. 8

Fisher (\& Fisher). See (Grant:Cr.).

(Florida Cr.) - (20.95-21.2, 7.75-7.9) ; GOLD.

B 259, p. 111, 113

B 280, p. 36-37, 48, 50

B 337, p. 79,98
B 836, p. 36

B 844-A, p. 37

B 844-D, p. 197, 201
B 783, p. 14

B 797, p. 22

B 824, p. 37

B 836, p. 36

B 844-A, p. 37

B 844-D, p. 166, 192-195

B 857-A, p. 33

B 868-A, p. 41

B 880-A, p. 42

B 897-A, p. 51

B 910-A, p. 53

B 917-A, p. 50

B 926-A,p. 47

B 933-A, p. 43

BMB 153, p. 52
B 926-A; p. 47 .

, p. 43

p. $6-9,12,17,45-48,55$

TDM 1, p. 34 
- Frank \& Co. See (Doric Cr.), (Eureka. Cr.), (Pioneer Cr.), (Seattle Jr. Cr.), (Skookum Cr.).

Frank \& Graham. See (Pioneer Cr.).

Gill. See (Eureka Cr.), (Last Bench), (Skoolkum Cr.).

(Glen Cr.) or (Gulch) - (20.15-20.2, 3.75-3.85) approx ; GOLD.

B 213, p. 47, 51-52

B 836, p. 36

B 259, p. 115, 117-118

B 844-D, p. 203

B 280 , p. 38-39, 42-43, 48-50

B 284 , p. 126

B 857-A, p. 33

B. 337, p. $82-83,87-89,96,98$

B 868-A, p. 41

B 345, p. 49

B 880-A, p. 42

B 379, p. 56

B 897-A, p. 51

B 592, p. 362

B 910-A, p. 53

B 773, p. 24

B 917-A, p. 50

B 926-A, p. 47

(Gold Basin Cr.) - $(16,2)$; TIN, GOLD.

B 692, p. 38

(Gold Hill) - $(1.45,3.55)$; GOLD, SILVER.
AR 18, p. 293
B 379, p. 236
P. 70, p. 184
B 410, p. 82
B 345, p. 46
B 631, p. 82

(Gold Run) - (20.05-20.1, 3.8-3.9) approx ; GOLD.

$\begin{array}{ll}\text { B 213, p. 51-53 } & \text { B } 622, \text { p. } 242 \\ \text { B 259, p. 115, 118 } & \text { B } 642, \text { p. } 64 \\ \text { B 263, p. 53, 208 } & \text { B 836, p. } 36 \\ \text { B 280, p. 38, 42-44, 48-50 } & \text { B 844-D, p. 200-201 } \\ \text { B 337, p. 82, 87, 89-90, 96, 98 } & \text { B 910-A, p. 53 } \\ \text { B 592, p. 362 } & \text { B 917-A, p. } 50\end{array}$

(Grant Cr) - (0.4-0.5, 3.4-4.4) ; GOLD, TIN.
P 70, p. 184
B 824 , p. 40,68
B 379, p. 235
B 836, p. 42,70
B 410, p. 81,83
B 844-A, p. 41
B 442, p. 44-45
B 864-A, p. 43
B 520, p. 281
B $897-A$, p. 56
B 535, p. 34
B 910-A, p. 57
B 592 , p. 383
B 917-A, p. 56
B 631, p. 82
C 331, p. 33, 35
B 692 , p. 39
TDM 1, p. 34

Hansen. See (Deep Cr.).

Hansen \& Albright. See (Innesvale Gulch).

Hansen \& Linberg. See (Woodchopper Cr.).

(Harter Gulch) - $(15.85,2.0)$ approx ; GOLD, TIN.

RI 5373, p. 8, 48

Hartner. 'See (Sullivan Cr.).

Hillside Assn. See (American Cr.).

(Hokeley Gulch) - $(\mathbf{1 4 . 9 5 , 1 . 5 )}$ approx ; GOLD, TIN.

B 622, p. 241-243

RI 5373, p. 7

(Homestake Bar.) See (Quartz Cr.) (trib. Sullivan Cr.).

(Homestake Cr.) - $(11.95,5.75)$ approx ; GOLD.

P 70, p. 184 
(Hoosier Cr.) - (21.05-21.45, 8.15-8.4) ; GOLD, TUNGSTEN, MERCURY, COPPER.

B 259, p. 111-113

B 263, p. 52

B 280, p. 27, 36, 50

B 284, p. 126

B 314, p. 37

B 337, p. 65, 79, 98

B 379 , p. 55

B 442 , p. 240

B 480, p. 166-167

B 622 , p. 64

B 642 , p. 64

B 797, p. 23
B. 813 , p. 35

B 836, p: 42

B 844-A, p. 41 .

B 844-D, p. 165, 186-187, 234

Ii $857-\mathrm{A}$, p. 39

B $864-$ A, p. 43

B 868-A, p. 44

B 880-A, p. 47

B 910-A, p. 57

B 917-A, p. 56

TDM 1, p. 27, 39

(Hosiana Cr.) - (18-22, 6-8) (?) ; GOLD(?). . May be in Livengood quad. B 480, p. 167

Howell \& Cleveland. See (Boulder Cr.), (Sullivan Cr.).

Hunter. See (Hunter Cr.).

(Hunter Cr.) - (21.2-21.85, 8.95). See (Hunter Cr.) under Livengood quad.

(Hutlina Cr.) See (Hutlinana Cr.).

(Hutlinana Cr.) - (20.9-22.1, 3.1-4.2) approx; GOLD. Includes references to (Hutlina Cr.).
B 259, p. 115
B 280, p. 46
B 520, p. 283
B 337, p. 93
B 535, p. 35-36
B 844-D, p. 205

(Idaho Bar) - (21.8-21.9; 8.65-8.75) ; GOLD. Includes reference to Farrell and assoc.

$\begin{array}{ll}\text { B 259, p. } 113 & \text { B 844-D, p. 183-184, 234-235 } \\ \text { B 592, p. 362 } & \text { B 857-A, p. 39 } \\ \text { B 813, p. 35 } & \text { B 864-A, p. 43 } \\ \text { B 836, p. 42 } & \text { B 868-A, p. 44 } \\ \text { B 844-A, p. 41 } & \text { B 880-A, p. 47 }\end{array}$

(Idaho Gulch) or (Cr.) - $(15.4,1.75)$ approx; GOLD, TIN. See also (Sullivan Cr.).
B 662, p. 57
RI 5373, p. 6-8, 12, 24-26, 47, 55

B 844-D, p. 212

(Innesvale Gulch) - $(\mathbf{1 5 . 0 , 1 . 3 )}$ approx ; GOLD, TIN, SILVER.

B 844-A, p. 37

B 844-D, p. 210-212

Johnson \& Hensley - $(20.15,3.8)$ ( ?) ; GOLD. See also (Glen Cr.).

B 813, p. 32

Johnson \& Johnson. See (Glen Cr.).

Johnson \& Toftaker. See (Glen Cr.), (Shirley Bar.).

(Jordan Bar) - (21.0, 4.15) approx; GOLD. See also (Pioneer Cr.).

B 844-D, p. $198 \quad$ B 868-A, p. 41

Junction. See (Sullivan Cr.).

(Killarney Cr.) - $(\mathbf{1 6 . 7 5}, 2.7)$ approx ; TIN, GOLD (?).

B 592, p. 362

RI 5373, p. 8

B 642, p. 28

(Lancaster Cr.) - $(1.65,3.65)$ approx ; GOLD.

B 692, p. 39

Langford. See (Little Minook Cr.). 
Larsen. See (Deep Cr.).

(Last Bench) - (21.0-21.15, 4.1-4.2) approx; GOLD. See also (Pioneer Cr.). B 844-A, p: 37

B 844-D, p. 197-198

Lieber \& File. See (Sullivan Cr.).

(Little Boulder Cr.) - $(13,2)$; GOLD. B 662, p. 57

B 692, p. 335

(Little Minook Cr.) - (21.25-21.8, 8.45-8.65) ; GOLD, TUNGSTEN, COPPER, LEAD, CHROMITE, MERCURY(?). Includes reference to (Little Mynook Cr.).

AR 18, p. 356-357.

B 797, p. 23

B 213, p. 55

B 810, p. 30

B 259, p. 111-112

B 813, p. 35

B 263, p. 52, 54, 208

B 824, p. 40

B 280, p. 26-27, 33-35, 37, 48, 50

B 836, p. 42

B 337, p. 65, 77, 98 .

B :844-A, p. 41

B 379, p. 55

B 442, p. 239-240

B 844-D, p. 165, 181-183, 232-234.

B 480 , p. 167

B 520, p. 282-283

B 857-A, p. 39

B 535, p. 28-29, 35

B 864-A, p. 43

B 868-A, p. 44

B 542, p. 222

B 880-A, p. 47

B 592, p. 362

B 897-A, p. 56

B 622, p. 64

B 910-A, p. 57

B 642, p. 64

B 917-A, p. 56-57

B 662 , p. 57

B 926-A, p. 53

B 692 , p. 37

B 933-A, p. 49

B 739, p. 32

W 342, p. 229

B 755, p. 37

BMB 153, p. 53

B 783, p. 14

TDM 1, p. 27, 39

(Little Minook Jr. Cr.) - (21.1-21.45, 8.45-8.5) ; GOLD, LEAD.

B 259, p. 111-112

B 542, p. 222

B 263, p. 55-56, 208

B 592, p. 362

B 280, p. $27,35-36,50$

B 824 A, p. 40

B 337, p. 65, 77-78, 98

B 836-A, p. 42

B 379 , p. 55

B 442 , p. $240-241$

B 844-D, p: 165, 175, 184-185, 234

B 480 , p. 167

B 868-A, p. 44

B 880-A, p. 47

(Little Mynook Cr.). See (Little Minook Cr.).

(Lynx Cr.) - (0.5-0.95, 3.9-4.1) ; GOLD.

B 520, p. 281-282

B 535, p. 34

(McCaskey Bar) - $(20.55,3.58)$ approx; GOLD, MERCURY. See also Farmer \& Jones.

B 844-A, p. 37

B 917-A, $p_{i}^{\prime} \mathbf{5 0}$

B 844-D, p. 193, 198-199, 237

B 926-A, p. 47

B 910-A, p. 53

TDM 1, p. 27

(McDonald Bar) - (21.1, 7.75) approx; GOLD.

B 259 , p. 113

McGee. See (Idaho Gulch), (Sullivan Cr.), (Tofty Gulch).

(McKinley Cr.) - (19.8-20.0, 2.2-3.4) approx; GOLD.

B 213, p. 55

McLean. See (Little Minook Jr. Cr.). 
McVicar, Snyder \& Marshall. See (Sullivan Cr.).

Midnight Sun. See (Sullivan Cr.).

(Miller Gulch) or (Cr.) - $(15.3,1.6)$ approx; GOLD, TIN, SILVER, RARE EARTHS. See also (Sullivan Cr.).
B 442, p. 240
B 542, p. 221
B. 622 , p. 241-244
B 642 ; p. 64
B 692, p. 334
B 824, p. 68
B 836, p. 36, 71
B 844-A, p. 69

B. 844-D, p. 212

B 910-A, p. 53

B 917-A, p. 50.

B 933-A, p: 43

C 317, p. 5

RI 5373, p. 6-8, 12-13, 17, 23, 45-46

TDM 1, p. 34

Millianich. See (Miller Gulch).

Minook. See (Little Minook Cr.).

(Minook Cr.) - (20.1-21.05, 7.0-8.95) ; GOLD, SILVER. Includes references to (Big Minook Cr.), (Mynook Cr.).
AR 18, p. 355
P 70, p. 183
B 213, p. 55
B 259, p. 114
B 263, p. 52
B 284, p. 126
B 442 , p. 241
B 480 , p. 167
B 520, p. 276, 278
B 535, p. 28, 30
B 542, p. 222

B 280, p. $26-30,37,48,50$

B 337, p. 65, 69-70, 97-98

B 622, p. 64
B 642, p. 64
B 755, p. 37
B 773, p. 22
B $844-D$, p. $176-177$
B $857-A$, p. 39
B $864-A$, p. 43
B $868-A$, p. 44
B $880-A$, p. 47
B $910-A$, p. 57
B $917-A$, p. 56
B $933-A$, p. 49
BMB 153, p. 53

Minook, Ltd. See (Little Minook C̣r.).

Mohawk Assn. See (Woodchopper Gr.).

Montana Min. Co. See (Omega Cr.).

(Moraine Cr.) - (0-1,3-6) (?);; GOLD. May be in Melozitna quad.

B 824, p. 40

(Morelock Cr.) - (12.05-12.2, 5.75-5.9) ; GOLD, TIN.

$\begin{array}{ll}\text { B } 379, \text { p. } 55 & \text { B 880-A, p. 47 } \\ \text { B } 592, \text { p. } 383 & \text { B 910-A, p. 57-58 } \\ \text { B 631, p. } 82 & \text { B 917-A, p. 56 } \\ \text { B 844-D, p. } 192 & \text { RI } 4322, \text { p. 1-8 } \\ \text { B 864-A, p. } 43 & \text { TDM 2, p. 18-19 } \\ \text { B } 868-A, \text { p. } 45 & \end{array}$

(Mynook Cr.). See (Minook Cr.).

Ness - (19-20, 3-4) ; GOLD.

B 813, p. 32

(New York Gulch) or (Cr.) - $(13,1)$; GOLD. NoTE.-Trib. American Cr.

B 797, p. 22

B 844-D, p. 214, 241

B 910-A, p. 53

(Oakley Cr.) - (S 1/2 SE $1 / 4$ quad.); GOLD. See also (Hokeley Gulch); may. be same.

B 642, p. 64 
(Omega Cr.) - (19.35-19.55, 3.3-3.5) approx ; GOțD, TUNGSTEN, MERCURY. See also (Orange Cr.).

P 70, p. 183

B 213, p. 55

B 259, p. 115, 118

B 263, p. 53

B 280, p. 38, 44-45, 48-50

B 284, p. 126

B 337, p. $82,91,96,98$

B 520, p. 283

B 535, p. 35

B 592, p. 362

B 692, p. 334

B 783, p. 14

\author{
B 797, p. 22 \\ B 836, p. 36 \\ B 844-A, p. 37 \\ B 844-D, p. 203-204, 238 \\ B 857-A, p. 33 \\ B 880-A, p. 42 \\ B $897-A$, p. 51 \\ B 910-A, p. 53 \\ B 917-A, p. 50 \\ B 926-A, p. 47 \\ B 933-A, p. 43 \\ TDM 1, p. 27, 39
}

(Orange Cr.) - (19.35-19.55, 3.3-3.5) approx (?) ; GOLD. See also (Omega Cr.) ; may be the same.

B 868-A, p. 41

(Patterson Cr.) - (14-16, 1-2) ; GOLD, TIN, SILVER. See also (Cache Cr.), (Deep Cr.), (Sullivan Cr.), (Woodchopper Cr.).
P 70, p. 183
B 712, p. 40
B 337, p. 93
B 714, p. 82
B 345, p. 49
RI 5373, p. 7
B 592, p. 68
TDM 1, p. 34

B 642, p. 64

(Pioneer Cr.) - (20.55-21.15, 3.8-4.2) approx; GOLD, TUNGSTEN, MERCURY.

See also (Doric Cr.), (Jordän Bar), (Last Bench), (Seattle Bar), (Seattle Jr. Cr.), (Skookum Cr.), (What Cheer Bar).
P 70, p. 183
B 797, p. 22
B 213, p. 55
B 836, p. 36
B. 259, p. 115-117
B 844-A, p. 37
B 280, p. 38-41
B 284, p. 126
B 857-A, p. 33
B 337, p. 82-83, 85-86
B 880-A, p. 42
B 480 , p. 166
B 897-A, p. 51
B 520, p. 280, 283
B 535, p. 32, 35-36
B $910-$ A, p. 53
B 542, p. 221
B 917-A, p. 50
B 622, p. 242
B 926-A, p. 47
B 692, p. 334
B 933-A, p. 43
TDM 1, p. 27, 39

B 844-D, p. 195-198, 236-237

(Poker Cr.) - (19.8, 3.35) approx; GOLD.

B 844-A, p. 37

B 844-D, p. 205

Pringle and assoc. See (Rhode Island $\mathrm{Cr}$.).

Purkeypile \& Webories. See (Tozimoran Cr.).

(Quartz Cr.) (trib. Sullivan Cr.) - (15.7-15.8, 2.55) approx; GOLD.
B 442, p. 243
B 622 , p. 244-245
B 480 , p. 166
B 836, p. 36
B 520, p. 280, 283-284
B 844-D, p. 212-213

B 535, p. 32, 35-37

(Quartz Cr.) (trib. Yukon R.) - (11.9, 4.9) ; SILVER, LEAD, GOLD.

B 631, p. 82-83

Rampart Gold Min. Co. See (Minook Cr.). 
(Rhode Island Cr.) - (19.95-20.1; 3.6-3.9) approx; GOLD.
P 70, p. 183
B 213, p. 51, 53-54
B 259, p. 115, 118
B 263, p. 53
B 280, p. 42,44
B 337, p. $82,87,90$
B 692, p. 334
B 844-A, p. 37

B 844-D, p. 199-201

B 868-D, p. 41

B $880-A$, p. 42

B $897-$ A, p. 51

B 910-A, p. 53

B 917-A, p. 50

B 926-A, p. 47

B 933-A, p. 43

Rolke. See (Woodchopper Cr.).

(Ruby Cr.) - (20.4-20.85, 7.45-7.7) ; GOLD, SILVER.
B 213, p. 55
B 259, p. 114
B 280, p. $27,37-38,48,50$
B 284, p. 126
B 314, p. 37
B 337, p. $65,80-81,96-98$
B 379, p. 55
B 442, p. 241
B 642, p. 64
B 844-D, p. 188-189
BMB 153, p. 53

(Schieffelin Cr.) See (Shevlin Cr.).

(Seattle Bar) - (21.0-21.15, 4.1-4.2) approx; GOLD: Sèe also (Pioneer Cr.). B 259, p. 116

B 280, p. 42

B 379, p. 56

B 337, p. 87

B 844-D, p. 196

(Seattle Cr.) - (19.9, 3.9) approx; GOLD. See also (Seattle Jr. Cr.).

B 263, p. 53

B 280, p. $42,44,48-50$

B 337, p. $87,90,96,98$

B 824, p. 37

B 836, p. 36

B $844-D$, p. 200

(Seattle Jr. Cr.) - $(21.0,4.1)$ approx; GOLD. See also (Seattle Cr.).

B 542, p. 221

B 824, p. 37

B 592, p. 362

B 844-D, p. 197

B 622 , p. 242

(Shevlin Cr.) - (10.3-11.5, 4.15-5.9); GOLD. Nore.-Schieffelin is correct spelling.

B 379, p. 55

(Shirley Bar) or (Bench) - (20.1, 3.8) approx ; GOLD, MERCURY, LEAD.

B 259 , p. 118

B 280, p. 42,48

B 337, p. $87-88,96$

B $844-A$, p. 37

B 844-D, p. 193, 201-202, 237-238

B 910-A, p. 53

B 917-A, p. 50

TDM 1, p. 27

(Skookum Cr.) or (Gulch) - $(21: 15,4.2)$ approx ; GOLD.

B 314 , p. 37

B 824, p. 37

B 836, p. 36

B 857-A, p. 33

(Slate Cr.) - (20.5-20.75, 6.8-6.95) ; GOLD, SILVER, COPPER (?).

B 213, p. 55

B 259, p. 114

B 642 , p. 64

B 280, p. $27,38,48,50$

B 797, p. 23

B 337, p. 65, 81-82, 96-98

B 813, p. 35

B 824 , p. 40

B 379, p. 55

B 442, p. 241

B 480 , p. 167

B 542, p. 222

B 592, p. 362

B 836, p. 42

B 844-A, p. 41

B 844-D, p. 165, 187-188

B 857-A, p. 39

B 622, p. 64

B 864-A, p. 43

B 868-A, p. 44 
(Slate Cr.)-Continued
B 880-A, p. 47
B $917-\mathrm{A}$, p. 56
B 910-A, p. 57
BMB 153, p. 53

Snyder \& Kempter. See (Sullivan Cr.).

Snyder, Harter \& Kempter. See (Tofty Gulch).

Strandberg Mines, Inc. See (Deep Cr.), (Idaho Gulch), (Sullivan Cr.), (Tofty Gulch), (Woodchopper Cr.).

(Sullivan Cr.) - (15.3-15.6, 1.3-2.0) approx; GOLD, TIN, SILVER, RARE

EARTHS, COPPER. See also (Idaho Gulch), (Miller Gulch), (Tofty Gulch).

B 379, p. 56

B 844-A, p. 37

B 442, p. 39, 242-243

B 844-D, p. 209-210, 239-240

B 480 , p. 166

B 857-A, p. 33

B 520, p. 89, 92, 276-277, 283-285 B 868-A, p. 41

B 535, p. 28-30, 33, 35-37

B 880-A, p. 42

B 542, p. 221

B 897-A, p. 51

B 592, p. 68,362

B 910-A, p. 52-53

B 622, p. 58, 92-93, 240-241

B 917-A, p. 50

B 642 , p. 64

B 926-A, p. 47

B 662, p. 57

B 933-A, p. 43

B 692, p. 37-38, 331, 333-334

BMB 142, p. 25

B 712, p. 40

BMB 153, p. 52

B 755, p. 149

RI 4346, p. 1-8

B 783, p. 14

RI 5373, p. 7-8, 22, 27-31, 55

B 824 , p. $36-37$

TDM 1, p. 34

B 836, p. 36

(Thanksgiving Cr.) - (SE 1/4 18,3) ; GOLD, SILVER.
P 70, p. 183
B 259, p. 115, 118-119
B 535, p. 35
B 542, p. 221
B 263, p. 53, 208
B 592 , p. 362
B 280 , p. $38-39,45-46,48-50$
B 622, p. 242
B 337, p. 82-83, 92-93, 97-98
B 692, p. 334
B 345, p. 49
B 836, p. 36
B 379, p. 56
B 844-A, p. 37
B 442 , p. 242
B 844-D, p. 204-205
B 480, p. 166
B 857-A, p. 33
B 520, p. 283
B $897-A$, p. 51

Tilleson \& L'Heureux. See (Sullivan Cr.).

(Tofty Gulch) - (15.45-15.55, 1.8-1.9) approx ; GOLD, TIN, SILVER. Includes reference to (Tufty Gulch) ; see also (Sullivan Cr.).

B 379, p. 56

B 442, p. 242-243

B 692 , p. 334

B 480 , p. 166

B 844-D, p. 166, 209, 212

B 520, p. 92,280

C 317 , p. 5

B 542, p. 221

RI 5373, p. 7-8, 24-26, 55

B 535, p. 32-33

TDM 1, p. 34

(Tozimoran Cr.) - (1.4-1.9, 6.55-6.7) ; TIN, GOLD, LEAD.

C 331, p. 35 RI 4323, p. 1-11

(Tufty Gulch). See (Tofty Gulch).

U.S. Assoc. See (Miller Gulch).

Vogt. See (Bonanza Cr.), (Morelock Cr.).

Webories \& Purkeypile. See (Tozimoran Cr.).

$593018-61-21$ 
(What Cheer Bar) - $(20.6,3.85)$ approx ; GOLD.

$\begin{array}{ll}\text { B 259, p. 115-116 } & \text { B 520, p. 277, 279-280 } \\ \text { B 280, p. 41 } & \text { B 535, p. 30, 32 } \\ \text { B 284, p. } 126 & \text { B 542, p. 221 } \\ \text { B 337, p. } 86 & \text { B 622, p. 239-240 } \\ \text { B 379, p. 56 } & \text { B 844-D, p. 193, 195-196 }\end{array}$

B 442 , p. 242

Wild Goose. See (Hokeley Gulch).

Wild Goose Assn. See (American Cr.).

(Woodchopper Cr.) - $(14,1)$; GOLD, TIN, SILVER. Includes references to

Bock \& Co. and to Albrecht \& Hanson if not definitely to some other creek.

B 592, p. 362

B 642, p. 64

B 662, p. 57

B 692, p. 37, 331-332, 334

B 755, p. 149-150

B 797, p. 22

B 810, p. 28,53

B 836, p. 36

B $844-\mathrm{D}$, p. 212

B $857-$ A, p. 33

(----) (antimony) - (SE $1 / 220,5)$; ANTIMONY. NorE.-On ridge between Granite Cr. and Upper Minook Cr.

B 844-D, p. 217

(-_-_-) (silver, lead) - $(21,4)(?)$; SILVER, LEAD.

B 844-D, p. 217

\section{TAYLOR MOUNTAINS QUADRANGLE}

(Alder Gulch) - $(1.25,12.9-12.95)$; MERCURY.

RI 4719, p. 1,3

Bristol Bay Min. Co. See (Cinnabar Cr.).

Broken Shovel - $(1.2,14.2)$; MERCURY, ANTIMONY.

P 268, p. 115

RI 4065, p. 44-46

(Canary Gulch) - $(1.25,12.95-13.05)$; MERCURY. P 268, p. 115

RI 4719, p. 1, 3

(Caribou Cr.) - (east central 1/4 quad.) (?) ; GOLD. NotE.-Headwater trib. Nushagak $\mathbf{R}$.

B 903, p. 91

(Cinnabar Cr.) - (1.0-1.2, 13.9-14.2) ; MERCURY. Includes references to (Cinnabar Gulch), (Cinnabar Run).

P 268, p. 66, 108, 115

RI 4065, p. 4, 44-46, 48

TDM 1, p. 23-24

TDM 2, p. 17

RI 4719, p. 1, 3-4, 9

(Cinnabar Gulch). See (Cinnabar Cr.).

(Cinnabar Run). See (Cinnabar Cr.).

(King Salmon R.) - (SW 1/4 quad.) ; GOLD.

B 903, p. 91

Landru. See Broken Shovel, (Cinnabar Cr.).

(Little Taylor Mtns.) - (S. $1 / 2$ 15, 15) ; COPPER.

P 268, p. 122 
Lucky Day - $(1.25,13.1)$; MERCURY, ANTIMONY.

P 268, p. 113-115

RI 4065, p. 4, 44-49

(Mulchatna R.)-(SE1/4 quad.) ; GOLD.

B 442, p. 202

New York-Alaska (Gold Dred.) Corp. See Broken Shovel, (Cinnabar Cr.).

(Pulchatnachakcharak R.) - (SE 1/3 quad.) ; GOLD. NotE.-North-flowing headwater trib. Nushagak $\mathbf{R}$.

B 903, p. 91

Redskin - $(1.2,12.85)$; MERCURY.

P 268, p. 115

RI 4065, p. 4, 44-47

Schaefer. See Broken Shovel, (Canary Gulch), (Cinnabar Cr.), Lucky Day, Redskin.

Schaefer \& Winchell. See (Cinnabar Cr.), Lucky Day, Redskin.

(Sleitat Mtn.) - (16-17, 0-1) ; GOLD.

B 903 , p. 91

(Stevens Cr.) - $(13.8,16.8)$ approx ; TUNGSTEN.

P 268, p. 83, 121

(Taylor Cr.) - (14.7-15.25, 15.6-16.65) ; GOLD, MERCURY, TIN. P 268, p. 71, 116, 119

(Tikchik Mtn.) - $(6,0)$; GOLD.

B 903, p. 91

\section{TELLER QUADRANGLE}

Adams. See (Cape Mtn.).

(Agiapuk R.) - (SE $1 / 1$ quad.) ; GOLD. See also (Allene Cr.), (American R.), (Budd Cr.), (Igloo Cr.), (Windy Cr.) (trib. American R.).
B 813, p. 42
B 836, p. 49
B 824, p. 48
B $933-$ A, p. 63

Alan Dale. See (Potato Mtn.).

Alaska Chief - $(12.8,7.8)$ approx; LEAD. NotE.-Not mentioned bु name in

B 692, 722 .
B 345, p. $268-269$
B 358, p. 58-59
B 722, p. 182
B 733 , p. 81
B 442, p. 38
TDM 5-R, p. 29

B 692 , p. 359

Alaska Graphite (Min.) Co. See (Graphite Cr.), (Ruby Cr.).

Alaska Tin Corp. See (Ear Mtn.), (Eldorado Cr.), (Kreuger Cr.), (Tuttle Cr.).

(Alder Cr.) - (20.65-21.0, 1.55-1.75) ; GOLD.

B 225, p. 54

C 244, p. 1

B 328, p. $273,279-280$

(Allene Cr.) - (21.55-21.65, 5.95-6.6) ; GOLD. See also (Ilene Cr.), (Swanson Cr.).

American Gold Dred. Co. See (Anikovik R.), (Buck Cr.), (Swanson Cr.), (Windy Cr.) (trib. American R.).
B 592, p. 393
B 857-A, p. 47
B 810, p. 36
B 864-A, p. 52

(American R.) - (22-25, 10-14) ; GOLD. See also (Budd Cr.), (Windy Cr.) (trib. American R.).
B 592, p. 393
B 857-A, p. 47
B 810, p. 36
B 864-A, p. 52 


\section{INDEX OF METALLIC AND NONMETALLIC MINERAL DEPOSITS}

American Tin (Dred.) Co. See (Buck Cr.).

American Tin Min. Co. See (Buck Cr.), (Grouse Cr.).

American Tinfields, Inc. - (7.7-8.1, 10.05-10.4); TIN. See also (Cape Cr.). (First Chance Cr.), (Goodwin Gulch).
B 880-A, p. 75
B 917-A, p. 90-91
B 897-A, p. 85
B 926-A, p. 83
B 910-A, p. 91
B $933-$ A, p. 80

Andrews. See (Sunset Cr.)

Anglo-American Gold Dred. Corp. See (Sunset Cr.).

(Anikovik R.) - (9.65-10.1, 8.7-9.3) ; GOLD, TIN.

P 2, p. 48-51

B 213 , p. 93

B 225; p. 158, 165-167

B 229, p. 16, 35-36

B 259, p. 120

B 263, p. 209

April. See (Ear Mtn.).
B 328, p. 281

B 622, p. $23,83-84,89,91-92,367,372-$ 373

B 642, p. 28

B 662, p. 451

B 692, p. 353, 360-361

BMB 142, p. 28

Arctic. See (Cape Mtn.).

Arkansas. See Ward.

Aspen. See (Cape Mtn.).

August. See (Ear Mtn.).

Aurora. See (Cape Mtn.).

(Baituk Cr.) - (8.8-9.4, 9.2-10.1) ; TIN.

P 2, p. 49-51

B 225, p. 166

B 229, p. 36

Bald Eagle. See Lost $R$.

(Banner Cr.) - (10.1-11.0, 9.05-9.4) ; TIN. B 225 , p. 166

B 229, p. 36

Bartels (Tin Min.) Co. See (Cape Mtn.).

Bartels and assoc. See (Cape Mtn.).

Bartholomae Oil Corp. See (Alder Cr.), (Gold Run).

Bear. See (Cape Mtn.).

(Bering Cr.) - $(2,19-20)$ (?) ; GOLD.

B 225, p. 54

B 328, p. 273, 280-281

Bering Straits Tin Min. Co. See (Boulder Cr.), (Goodwin Cr.), (Granite Cr.), (Village Cr.).

Bessie. See Bessie \& Maple.

Fessie \& Maple - $(13.15,8.05)$; SILVER, LFAD, ANTIMONY, TIN, TUNGSTEN, COPPER. Includes reference to Southern Cross.
B 692 , p. 359
IC 7871, p. 9
B 733, p. 78-80
TDM 5-R, p. 30

Birthday. See (Cape Mtn.).

Blomquist \& Goodwin. See Cow Boy, Yankee Girl.

(Bluestone R.) - (20.5-21.8, 2.0-3.7) ; GOLD. See also (Gold Run).
B 225, p. 54
B 783 , p. 17
B 328, p. 269, 270, 273-275
B 792, p. 23
B 379, p. 297-298
B 797 , p. 27
B 712, p. 189
B 810 , p. 36 
(Bluestone R.)-Continued
B 813, p. $42-43$
B 836 , p. 49

B 824 , p. 47

(Bluestone R., Right Fork) - (19.0-20.5, 1.8-2.3). GOLD.

B 328 , p. 280

B 379 , p. 299

(Boulder Cr.) - (7.4, 10.75-10.9) ; TIN, RARE EARTHS. Includes reference to (Lagoon Cr.).
P 2, p. 49
RI 3978 , p. 9
IC 7878, p. $1-2,9,13-14,17-18$,
RI 4345, p. 4-7, 12

$20,43,47-48,50-65,67$

Bronx. See (Cape Mtn.).

(Brooks Mtn.) - (13.4-13.6, 9.05-9.35) ; ANTIMONY, BISMUTH, COPPER, FLUORITE, LEAD, SILVER, TIN, TUNGSTEN, ZINC. Includes reference to Luther, Reed.
B 225 , p. 166
B 229 , p. 17,26
B 259 , p. 120,125
B 260 , p. 181
B 314, p. 28
B 345, p. 270-271
B 358, p. $41-44$
B 442 , p. 39
B 490 , p. $9-10$
B 520, p. 89,92

\author{
B 592, p. 407 \\ B 649, p. 59 \\ B 692 , p. 359 \\ B 722 , p. 182,185 \\ B 733 , p. $86-87$ \\ C 196, p. 1,3 \\ C 214, p. 1-7 \\ IC 7871 , p. 4 \\ TDM 5-R, p. 13, 17, 29
}

(Buck Cr.) - (10.55-11.25, 11.05-11.65) ; TIN, GOLD. NoTE-Includes all forks of creek.
P 2, p. 48-49
B 225, p. $156,162-165$
B 592, p. $388,393,407$
B 229, p. $16,29-35,39$
B 622, p. 23, 83-84, 89-91, 372
B 259 , p. 126
B 642, p. 28
B 260 , p. 181
B 662 , p. 456
B 284 , p. 155
B 692 , p. $41,353-354,360-361$
B 314, p. 28
B 712, p. 51-52, 195
B 328 , p. 281
B 714, p. 71, 236
B 345 , p. 265
B 722, p. 22
B 358, p. 9, 16-17, 32-35, 61-62
B 733, p. 90, 94-96
B 442 , p. 39
B 520, p. 89-91, 340-342
BMB 142, p. 27-28
IC 7871, p. 4
B 542, p. 50
RI 4418, p. 4, 6-8, 10-14, 21

(Budd Cr.) - (25.2, 11.4) ; GOLD, MERCURY, COPPER, TIN(?).

B 592, p. 388,393

B 662 , p. 452

B 836 , p. 49

B 722, p. 63,185

B 824 , p. 48

B $926-$ A, p. 65

C 265, p. 4,6

TDM 5-R, p. 22

Budd Cr. (Gold) Dred. Co. See (American R.), (Budd Cr.).

(Buhner Cr.) - (10.1, 9.25) approx ; TIN, GOLD.
P 2, p. 48-49
B 213, p. 92-93
B 520, p. 89
B 225, p. 157, 165-166
B 622, p. 81
B 229, p. $16,35-36$
B 692, p. 353
IC 7871, p. 4

B 328, p. 269 
(Burke Cr.) - (24.2-24.8, 12.65-12.7) ; GOLD( ?).

B 836, p. 49

B 864-A, p. 52

B $857-A$, p. 47

Butte. See Ward.

Cameron. See (Brooks Mtn.).

(Camp Cr.). See Lost R.

Canoe. See (Cape Mtn.).

(Canyon Cr.) - (21.55-22.3, 0.5-2.3) ; GOLD. B 810, p. 36

(Cape Cr.) - (7.7-7.8, 10.05-10.3) ; TIN. Includes references to (Tin City Cr.) ; see also (First Chance Cr.).

B 692, p. 41

B 722, p. 22

B 733, p. 102

B 844-A, p. 69

C 248, p. 13
IC 7871, p. 4

IC 7878, p. 1-2, 7, 9, 13-15, 17-43, $46-47,67$

RI 3978, p. 9

RI 4345, p. 4-9

(Cape Mtn.) - (7.35-7.7, 10.25-10.55) ; TIN, FLUORITE, TUNGSTEN, ZINC. Includes references to Bartels (Tin Min.) Co., Empire Tin Min. Co., U.S. Alaska Tin (Min.) Co.

B 225, p. 157-158, 160-162, 166-167

B 229, p. 16, 24-25

B 259, p. 124-125

B 260 , p. 181

B 284, p. 150-155

B 314, p. 28-29

B 345, p. 260-261

B 358, p. $35-41$.

B 442 , p. 39

B 520, p. 89,91
B 542, p. 50

B 592, p. 407

B 642, p. 28

B 692, p. 358

B 733, p. 97-102

B 824 , p. 68

BMB 142, p. 27

IC 7871, p. 4

RI 3978, p. 5-15

TDM 5-R, p. 39-40

Carlson \& Goodwin. See (Cape Mtn.).

Carry Gow. See Lost R.

Cassiterite. See Lost R.

(Cassiterite Cr.). See Lost R.

Champion. See (Cape Mtn.).

Christensen. See (Goodwin Cr.).

(Christophosen Cr.) - $(25.15,1.15)$; GRAPHITE. Includes references to (Graphite Bay), Uncle. Sam Alaska Min. Syn., Uncle Sam Graphite Min. Co. (or) Syn.

B 662, p. 40,449

B 692, p. 42, 363-365

B 722, p. 185, 221-223

TDM 5-R, p. 25

Chloride. See (Ear Mtn.).

(Clara Cr.) - (12.8-13.3;10.3-10.8) ; TIN( ?). B 225 p. 166

B 229, p. 36

(Cobblestone R.) - $(26.2,0.85)$ approx ; GOLD.

B 345, p. 234

Collier. See Lost R.

(Collin Cr.) - (23-25, 13-15) ; GOLD.

B 328, p. 272-273

(Columbia Cr.) - (27.75-28.55, 12.7-13.5) ; GOLD.

B 328, p. 326

Compass. See (Cape Mtn.).

Comstock. See (Cape Mtn.). 
Cow Boy - $(13.6,8.3)$ approx ; barren claim.

B 733, p. 80

(Coyote Cr.) - (19.8-20.0, 3.8-4.9) ; GOLD.

B 712, p. 189

B 783, p. 17

B 836, p. 49

B 792, p. 23

B 797, p. 27

B 810, p. 36

B 844-A, p. 50

B 813, p. 42

B 824, p. 48

B 857-A, p. 47

B $864-A$, p. 52

B $926-A$, p. 65

B 933-A, p. 63

Crim, Randt \& O'Brien. See Lost R.

(Crosby Cr.) - $(20.3,16.05)$; TIN.

RI 5493, p. 1-2, 29-30, 32

Daisy. See (Cape Mtn.), (Potato Mtn.). Note.-Two separate claims.

December. See (Ear Mtn.).

(Deer Cr.) - $(19.95,16.05)$; TIN.

RI 5493, p. 1-2, 29-30, 32

(De (a) se Cr.) - (20.2-21.1,.3.4-4.55) ; GOLD.

B 810 , p. 36

B 910-A, p. 71, 77

B 824, p. 48

B 868-A, p. 54, 59

B 880-A, p. 56, 62

B $917-A$, p. 69

B 897-A, p. 64, 71

B 926-A, p. 65,72

C 244, p. 2

(Dewey Cr.) - (20.85-21.2, 5.55-5.95) ; GOLD.

B 328, p. 270-271

Dieter. See (Cape Mtn.).

(Diomede Cr.) - (10.05-10.3, 11.65-11.85) ; TIN.

RI 4418, p. 7, 20

Dodson. See (Budd Cr.).

Dolcoath. See Lost R.

Douglas and assoc. See (Goodwin Cr.), (Goodwin Gulch).

(Ear Mtn.) - (19.7-20.1, 16.65-17.0) ; TIN, COPPER, LEAD, GOLD, SILVER, FLUORITE, ZINC, TUNGSTEN, ANTIMONY, MERCURY. Includes references to Eunson's shaft, Vatney, (Vatney Gulch), Winfleld.

B 225, p. 166

B 229, p. 17, 26-28

B 259, p. 120, 125

B 260, p. 181

B 284, p. 155-157

B 314, p. 28-29

B 345, p. 252, 254-256

B 358, p. 9, 25-32

B 490, p. 10, 21

B 520, p. 89

(Eldorado Cr.) - (20.3-20.8, 16.7-16.75) ; TIN, TUNGSTEN, FLUORITE.

B 345, p. 252

B 358, p. 26

B 692 , p. 359
B 592, p. 58,407

B 692, p. $41,355,357$

B 722, p. 181

B 733, p. 103-111

B 1024-C, p. 59, 66-70, 87

IC 7871, p. 4

RI 5493, p. 2-3, 8, 11, 13, 17, 19,

$21,33-52$

TDM 5-R, p. 22

Elgin. See (Cape Mtn.).

Empire Tin Min. Co. See (Cape Mtn.).

Engineer. See Lost R.

Estabrook. See (Windy Cr.) (trib. American R.). 
Eunson's shaft. See (Ear Mtn.).

Eureka. See (Potato Mtn.).

Excelsior. See (Cape Mtn.).

Excelsor. See Ward.

Fairview. See (Cape Mtn.).

Fidgeland Bros. See (Gold Run).

(First Chance Cr.) - (7.7-7.75, 10.1-10.15) ; TIN.

IC 7878 , p. 1, 15, 17-21, 44

RI 4345, p. 4-7

RI 3978, p. 9

Foggy Day. See (Brooks Mtn.).

Fourth of July. See (Cape Mtn.).

Gem. See Ward.

Gertrude. See Lost R.

(Glacier Cr.). See (Graphite Cr.).

(Gold Run) - (19.5-20.85, 0.95-2.0) ; GOLD, TUNGSTEN. Sce also (Bluestone R.).

B 225, p. 54

B 263, p. 209

B 284, p. 139

B 328, p. 273, 275-279

B 345, p. 229

B 379, p. 298-299

B 662, p. 455

B 712, p. 189

B 783, p. 17

B 797, p. 27

B 810, p. 36

B 813, p. 42-43

B 824, p. 48

B 836, p. 49

B 844-A, p. 50

B 857-A, p. 47

B 864-A, p. 52

B 868-A, p. 54

B 880-A, p. 56-57, 62

B 897-A, p. 64, 71

B 910-A, p. 71, 77

B 917-A, p. 69

B 926-A, p. 65, 71

B 933-A, p. 63, 68

C 244, p. 1

Goodwin \& Carlson. See (Cape Mtn.).

(Goodwin Cr.) - (8.0-8.1, 10.35-10.65) ; TIN.

B 722, p. 22

RI 4345, p. 4, 6-7, 11

IC 7878, p. 1-2, 14-15, 18, 67

(Goodwin Gulch) - $(8.0-8.1,10.4)$; TIN.

B 783, p. 24

B 797, p. 40

C 248, p. 13

B 810, p. 53

B 813 , p. 61

IC 7871, p. 4

B 844-A, p. 69

IC 7878, p. $1,7,14-15,17-18,67$

RI 3978, p. 9

RI 4345, p. 4,6

Granite. See (Ear Mtn.).

(Granite Cr.) - (7.65-7.7, 10.55-10.8) ; TIN(?). RI 3978, p. 9

RI 4345, p. 4, 6, 10

(Graphite Bay). See (Christophosen Cr.).

(Graphite Cr.) - $(25.5,1.35)$; GRAPHITE. Includes references to Alaska Graphite (Min.) Co., (Glacier Cr.).

B 345, p. 250

B 662, p. 40,449

Green. See Lost $\mathbf{R}$.

Greenstone. See Lost R.
B 692, p. 363-364, 366

B 722, p. 185, 221-223 
(Grouse Cr.) - (11.25-11.45, 11.05-11.15) ; TIN, GOLD. Includes references to (Skookum Cr.), (Sterling Cr.).
P 2, p. 48-51
B 225, p. 164
B 229, p. 32
B 284, p. 157
B 345, p. 266
B 358, p. 62

\author{
B 622, p. 89,91 \\ B 662, p. 456 \\ B 692, p. 41, 354, 360 \\ B 714, p. 71,236 \\ B 722, p. 22 \\ RI 4418, p. 4-5, 7-9
}

Halpin (and assoc.). See Lost R.

Hellerich and assoc. See (Brooks Mtn.).

Hinton. See (Cape Mtn.).

I. See Bessie \& Maple.

Ida Bell. See Lost R.

Idaho - $(13.4,8.2)$ approx ; COPPER, FLUORITE. See also Yankee Girl ; not mentioned by name in $B \mathbf{7 5 5}$.
B 345, p. 269
B 733, p. 81
B 358, p. 17, 59
TDM 5-R, p. 22

B 722, p. 181

(Igloo) - $(29,3)$; BISMUTH (?).

TDM 5-R, p. 17

(Igloo Cr.) - (20.45-21.0, 5.7-6.4) ; GOLD. Note.-Now called Moonlight Cr. P 2, p. 44-46

C 244, p. 1

B 328, p. 270-271

(Ilene Cr.) - (21.55-21.65, 5.95-6.6) (?) ; GOLD. See also (Allene Cr.) ; may be same creek.

B 836, p. 49

B 844-A, p. 50

(Imuruk Basin) (garnet) - (23-26, 2-3) ; GARNET.

B 722, p. 33

(Imuruk Basin) (graphite) - (25.15-25.5, 1.15-1.35) ; GRAPHITE. See also (Christophosen Cr.), (Graphite Cr.), (Ruby Cr.).

B 520, p. 344

B 714, p. 54-55

Iron Cap. See (Brooks Mtn.).

(Iron Cr.) - (10.5, 11.35-11.45) ; TIN.

B 692, p. 41, 360

RI 4418 , p. 5, 7-8, 15, 21

B 733, p. 94

J. See Bessie \& Maple.

Jamme Syn. See Lost R.

January. See (Ear. Mtn.).

Jefferson. See (Cape Mtn.).

Jenney Lyn. See Lost R.

(Joe Cr.) - (25.35-26.5, 11.2-11.5) ; GOLD( ?).

B 926-A, p. 65

Johnson. See (Alder Cr.).

Johnson and assoc. Bee Lost $\mathbf{R}$.

Johnstone. See (Budd Cr.).

July. See (War Mtn.).

June. See (Ear Mtn.).

Jupiter. See (Cape Mtn.), Lost R. Nort.-Two separate claims.

(Kanauguk R.) - (10.3-11.8, 8.1-8.8) ; TIN(?).

P 2, p. 49

Kendall. See (Buck Cr.). 
(Kigezruk Cr.) - (9.2-9.7, 9.0-9.2) ; TIN (?).
B 225, p. 166
B $229 ;$ p. 36

Klondike. See Lost R.

(Kreuger Cr.) - (20.6-20.8, 16.55-17.0) ; TIN.

RI 5493, p. 2-3, 8, 11, 21-22, 24, 33

(Lagoon Cr.). See (Boulder Cr.).

(Lawson Cr.) - (23-25, 12-14) (?) ; GOLD. NotE.-Trib. American R. B 328, p. 271-272

Iincoln. See Lost R.

Lomen \& Gabrielson. See (Boulder Cr.), (Cape Cr.), (First Chance Cr.), (Goodwin Cr.), (Goodwin Gulch).

Lomen Comm. Co. See (Boulder Cr.), (Cape Cr.), (First Chance Cr.), (Goodwin Cr.), (Goodwin Gulch).

Lost R. - (13.55-13.6, 8.7-8.95) ; TIN, TUNGSTEN, FLUORITE, MOLYBDENUM, LEAD, ZINC, ANTIMONY, COPPER, BISMUTH. Includes references to (Camp Cr.) Cassiterite, (Cassiterite Cr.), Dolcoath, Greenstone, Ida Bell, National Tin Min. Co.
B 225, p. 157-160, 166-167
B 229, p. 18-23
B 259, p. 121-123
B 260, p. 181
B 262, p. 129-130
B. 284 , p. 146-150
B 314, p. 28-29
B 345, p. 262-263
B 358, p. 44, 49-50, 52-55
B 442 , p. 39
B 520, p. 89, 91-92
B 542, p. 50
B 592, p. 58, 71, 407
B 622, p. $68,84-89$
B 642 , p. 28
B 662, p. $19,62,436-437$.
B 714, p. 37-38, 71, 95, 236
B 722, p. 22, 65, 163, 185
B 733, p. 51-74, 76-78
B 813, p. 61-62
B 824 , p. 68
B 926-C, p. 203
C 248, p. 13
C 252, p. 7
C 319 , p. 1, 3-4
BMB 142, p. 27
BMB 153, p. 4
IC 7379 , p. 66
IC 7871 , p. 1-4, 7-76
RI 3902, p. 2-44
RI 3978, p. 9-10
B 692, p. 41, 354-357, 359
RI 4174, p. 31
B 712, p. 21-22, 52, 194
TDM 5-R, p. 35, 41, 43-44, 47

(Lost R.) - (SW 1/8 13, 8) ; SILVER, LEAD, TUNGSTEN, FLUORITE, TIN.

NotE.-Lode west of Lost R. opposite mouth of Tin Cr.; includes references to. "wolframite-topaz" lode; not the same as Bessie \& Maple.

B 259, p. 123-124

B 345, p. 263-264

B 733, p. 81

B 358, p. 57-58

C 196, p. 3

TDM 5-R, p. 13, 22, 29, 44

Lost R. Tin Min. Co. See Lost R.

Lucky Queen. See (Cape Mtn.).

Luther. See (Brooks Mtn.).

(McKinley Cr.) - (21.5-21.75, 5.0-5.5) ; GOLD.

B 328, p. 270-271

Madison. See (Cape Mtn.).

Maple. See Bessie \& Maple.

March. See (Ear Mtn.).

Margaret. See Lost $\mathbf{R}$.

Mars. See (Cape Mtn.), Lost R. Note.-Two separate claims.

Martha. See (Cape Mtn.). 
Martinson (and assoc.). See (Gold Run).

May. See (Ear Mtn.).

Metalsmith Mines Corp. See (Bluestone R.), (Gold Run).

Midnight. See (Cape Mtn.).

Midnight Sun. See (Brooks Mtn.).

(Mint Cr.) or (R.) - (12.0-12.8, 10.0-12.0) ; TIN(?). See also (Clara Cr.). P 2, p. 49-51

B 284, p. 157

Mispickel. See (Cape Mtn.).

Monroe. See (Cape Mtn.).

(Moonlight Cr.). See (Igloo Cr.).

Munz, Edwards (\& Worm). See (Boulder Cr.).

National Tin Min. Co. See Lost R.

Noble. See (Cape Mtn.).

North Star. See (Cape Mtn.), Lost R. Nore.-Two separate claims.

November. See (Ear Mtn.).

O'Brien. See Lost R.

October. See (Ear Mtn.).

(Offield Cr.) - (21.85-22.05, 4.95-5.3) ; GOLD.
B 328, p. 270-271
B $910-A$, p. 71
B $844-$ A, p. 50
B $917-A$, p. 69
B $857-$ A, p. 47
B 926-A, p. 65
B 864-A, p. 52
C 244, p. 2

Pageite. See (Brooks Mtn.).

Palmer. See (Boulder Cr.), (Cape Cr.).

Pasadena Gold Dred. Co. See (Budd Cr.).

(Pauline Gulch) - (7.3-7.4, 9.9-10.1) ; TIN.

C 248, p. 13

Peck. See (Swanson Cr.).

(Peluk Cr.) - $(10.65,11: 65-11.7)$; TIN.

B 345, p. 257

B 358, p. 33-34

RI 4418 , p. 7,16

Percy. See (Cape Mtn.).

(Percy Gulch) - (8.05, 10.3-10.7) approx; TIN.

B 722, p. 22

Peterson (and assoc.). See (Cape Cr.).

(Pinguk R.) - (16-18, 10-11) ; TUNGSTEN.

TDM 5-R, p. 44

(Pinnacle Cr.) - $(20.0-20.2,16.2-16.6)$; TIN.

B 1024-C, p. 82-83

RI 5493, p. 1-2, 29-30, 32

Planet. See (Cape Mtn.).

Poorman. See Bessie \& Maple.

(Potato Cr.) - (9.7-9.95, 11.5-11.8) ; TIN.

B 712, p. $22,52,195$

B 722 , p. 22

(Potato Mtn.) - (10.4-10.5, 11.65-11.85) ; TIN. Includes references to Alan

Dale, Daisy (Potato Mtn.), Eureka, Red Fox.

B 284, p. 157

B 733, p. 88-94

B 345, p. 257

IC 7871, p. 4

B 692, p. 355,357

TDM 5-R, p. 39-40

(Quartz Cr.) - (19.55-19.8, 16.6-16.7) ; RARE EARTHS.

B 1024-C, p. 82

Randt, Crim \& O'Brien. See Lost R.

RI 5493, p. 30 
Read. See (Brooks Mtn.).

Reaf. See Lost R.

Red Fox. See (Potato Mtn.).

Rice. See (Sunset Cr.), (Swanson Cr.).

Rob Roy. See Lost R.

Rogers. See (Cape Mtn.).

(Ruby Cr.) - $(25.35,1.25)$; GRAPHITE. Includes references to Alaska Graphite (Min.) Co.

B 662, p. 40,449

B 692, p. 363-364, 366

B 722, p. 185, 221-223

Rusty. Bee (Cape Mtn.).

Saturn. See (Cape Mtn.).

September. See (Ear Mtn.).

Seward Tin Min. Co. See (Cape Mtn.).

Shon Rue. See Lost R.

Side. See Ward.

(Skookum Cr.). See (Grouse Cr.).

Southern Cross. See Bessie \& Maple.

Square Zero. See (Brooks Mtn.).

(Step Gulch) - (19.8-19.95, 16.5-16.65) ; TIN.

B 1024-C, p. 83

RI 5493, p. 1-2, 29-30, 32

(Sterling Cr.) See (Grouse Cr.).

Stines \& Kirklin. See (Cape Cr.).

Sullivan \& Dobson. See (American R.).

Sun. See (Cape Mtn.).

Sun Drum. See (Cape Mtn.).

Sunny Day. See (Brooks Mtn.).

Sunrise. See (Cape Mtn.).

(Sunset Cr.) - (20.0-20.45, 6.15-6.4) ; GOLD, TUNGSTEN.

$\begin{array}{ll}\text { B 263, p. } 209 & \text { B } 714, \text { p. 232-233 } \\ \text { B 328, p. 270-271 } & \text { B 880-A, p. 57 } \\ \text { B 345, p. 229 } & \text { B 910-A, p. 71 } \\ \text { B 379, p. 297, 299-300 } & \text { B 917-A, p. 69 } \\ \text { B 592, p. 388, 393 } & \text { B 926-A, p. 65 } \\ \text { B 622, p. 372 } & \text { B 933-A, p. 62-63 } \\ \text { B 662, p. 457 } & \text { C 244, p. 2 } \\ \text { B 692, p. } 41 & \text { BMB 142, p. } 28\end{array}$

Surprise. See (Ear Mtn.).

Surveyor. See Lost R.

(Sutter Cr.) - (10.7-10.85, 11.25-11.3) ; TIN, GOLD.
B 225, p. 163-164
B 345, p. 265
B 358, p. 62
B 622, p. 89,91
B 692 , p. 360
B 733, p. 95-96
BMB 142, p. 27
RI 4418, p. 7, 17-18, 21

B 642, p. 28

(Swanson Cr.) - (21.0-21.65, 6.55-6.7) ; GOLD.

B 662, p. 451
B 692, p. 353
B 739, p. 9

B $880-A$, p. 56,62

B 897-A, p. 64

C 244, p. 2

Thrassa. See Lost R.

Three Prospectors. See Lost R.

Tiger. See Bessie \& Maple. 
(Tin City Cr.). See (Cape Cr.).

(Tin Cr.) - $(13.75,8.25)$ approx ; ANTIMONY, FLUORITE.
B 345, p. 269
B 662, p. 440
B 649 , p. 59
TDM 5-R, p. 13

Tin Quartz. See (Cape Mtn.).

Tourmaline. See (Brooks Mtn.).

Tremont. See (Cape Mtn.).

Triangle. See Lost $\mathrm{R}$.

(Trilby Cr.). See (Windy Cr.).

(Tuttle Cr.) - (18.0-19.7, 16.0-17.1) ; TIN, GOLD, TUNGSTEN, RARE EARTHS.

See also (Ear Mtn.).

B 692 , p. $359-360$

RI 5493, p. 1-3, 11, 21-23, 30, 32-33

B $1024-C$, p. 69,82

Tweet (\& Sons). See (Coyote Cr.), (Dese Cr.), (Graphite Cr.), (Ruby Cr.).

Tweet Bros. See (Igloo Cr.).

Uncle Sam Alaska Min. Syn. See (Christophosen Cr.).

Uncle Sam Graphite Min. Co. (or) Syn. See (Christophosen Cr.).

U.S. Alaska Tin (Min.) Co. See (Cape Mtn.).

U.S. Smelt., Ref. \& Min. Co. See (Brooks Mtn.).

U.S. Tin Corp. See Bessie \& Maple, Lost R.

Vatney. See (Ear Mtn.).

(Vatney Gulch). See (Ear Mtn.).

Venus. See (Cape Mtn.).

Victor. See Ward.

(Village Cr.) - $(6.7-6.95,10.8-10.9 ;$ TIN

B 314, p. 28-29

RI 4345, p. $4,6,13$

RI 3978, p. 9

Vogen. See (Swanson Cr.).

Walker, Lovell \& Co. See (Cape Mtn.).

Ward (Copper Co.) - $(27.15,13.75)$; COPPER. Note.-Includes references in which name is not used, but in which identity of prospect is unmistakable.

B 284, p. 139

B 345, p. 244

B 662, p. 440-441

B 722, p. 65, 181
C 265, p. 4

RI 4110, p. 1-4

TDM 5-R, p. 20-21

Washington. See (Cape Mtn.).

Welch \& Doran. See (Budd Cr.).

(Windy Cr.) (trib. American R.) - (25.2, 11.4) approx; GOLD, TIN (?).

B 345, p. 229

B 622, p. 372

C 265, p. 4

BMB 142, p. 28

B 662, p. 452

(Windy Cr.) (trib. Bluestone R.) - (19.4-19.75, 1.55-2.2) ; GOLD.

B 783, p. 17

B 797, p. 27

B 824, p. 47

B 836, p. 49

B 810, p. 36

B 844-A, p. 50

B 813, p. 42

Winfleld. See (Ear Mtn.).

Worcester - $(27.8,12.0)$ approx; COPPER, LEAD.

B 662, p. 442

TDM 5-R, p. 21, 28

(Yankee Cr.) or (R.) - (12.3-13.55, 10.5-12.5) ; TIN (?).

P 2, p. 49 
Yankee Girl - (13.6, 8.3) approx ; LEAD, FLUORITE, TIN, COPPER, SILVER, GOLD (?). Note.-Not mentioned by name in B $284,345, .358,722$.
B 284, p. 150
B 722, p. 182
B 345 , p. 269
B 733, p. 80
B 358, p. 59-60
TDM 5-R, p. 30

(York Cr.) - (13.5-14.5, 9.7-12.0) approx ; TIN.

B 225, p. 166

B 259, p. 126

B 229, p. 36

York Dred. Co. See (Buck Cr.), (Grouse Cr.).

York Tin Dred. Co. See (Grouse Cr.).

York Tin Min. Co. See (Grouse Cr.).

Zenda Gold Min. Co. See (Boulder Cr.), (Cape Cr.), (First Chance Cr.), (Goodwin Cr.), (Goodwin Gulch).

(

RI 5493, p. 30, 32

\section{TRINITY ISLANDS QUADRANGLE}

Alaska Westward Min. Co. See (Cape Alitak).

(Cape Alitak) - $(16.4,15.1)$; GOLD (?).

B 880-C, p. 172-173

\section{TYONEK QUADRANGLE}

(Beluga R.) - (13.0-17.5, 4.0-7.2) ; GOLD.

P 70, p. 163

B 662, p. 45

B 442, p. 42

Bessie. See (Lewis R.).

(Boulder Bench). See (Kahiltna R.) under Talkeetna quad.

Daisy. See (Lewis R.).

Granite. See (Lewis R.).

(Kahiltna R.) - (18.1-19.0, 16.65-17.85). See (Kahiltna R.) under Talkeetna quad.

(Leslies Bar). See (Kahiltna R.) under Talkeetna quad.

(Lewis R.) - (17.55-17.75, 6.4-7.3) ; GOLD. B 662, p. 45-47

(Skwentna R.) - $(5.8,16.9)$; GOLD, SILVER, COPPER, LEAD. AR 20, p. 111-112, 259

(Straight Cr.) - $(8.55,3.9)$ approx ; PUMICE. B 862, p. $87-88$

\section{UNALAKLEET QUADRANGLE}

(Anvik R.) - (south central $1 / 3$ quad.) (?) ; GOLD, PLATINUM (?). See also (Anvik R.) under Holy. Cross quad. B 683 , p. $62-63$ B 692, p. 22,40

McLeod - $(21.4,5.3)$; MOLYBDENUM. Note.-Not mentioned by name in B 868-D, 926-C.

B 868-D, p. 174

B 926-C, p. 197-198

C 328, p. 2, 9-10

C 248, p. 2-4

TDM 2, p. 18

\section{UNALASKA QUADRANGLE}

Biorka. See (Sedanka I.).

(Captain's Bay) - (15.1, 10.8) approx; GOLD. See also Hague, (Pyramid Peak). B 259, p. 103 
Centralia. See (Makushin Volcano).

Dumond. See (Makushin Volcano).

Hague - $(15.1,10.8)$ approx; GOLD, COPPER. . See also .(Captain's Bay), (Pyramid Peak).

AR 18, p. 62

Johnstone \& Brown. See (Sedanka I.).

(Makushin Volcano) - $(11.0,11.0)$ approx ; SULFUR.

B 480 , p. 42

B 692, p. 284-292

B 642, p. 56

IC 7379, p. 82

B 666, p. 102

(Pyramid Peak) - (15.1, 10:8) approx; GOLD, COPPER. See also (Captain's

Bay), Hague.

AR 18, p. 85

(Sedanka I.) - (18.7, 9.75) ; ZINC, SILVER, COPPER, LEAD, CADMIUM.

C 252, p. 7

RI 3967, p. 2-15

IC 7379, p. $42-43$

Social. See (Makushin Volcano).

Welcome. See (Makushin Volcano)."

\section{UNIMAK QUADRANGLE}

(Akun Head). See (Akun I.).

(Akun I.) - $(3.9,4.8)$; SULFUR.

B 692, p. 292-297

B 712, p. 35

B 714, p. 55, 75, 78

B 722, p. 33, 43

B 739, p. 20

Akun King. See (Akun I.).

B 755, p. 22-23

B 836, p. 82

B $844-\mathrm{A}, \mathrm{p} .82$

B $857-A$, p. 76

B $880-$ A, p. 88

Akun Queen. See (Akun I.).

Alaska (Northwest) Sulphur Co. Sce (Akun I.).

Dorothy. See (Akun I.).

Matrona. See (Akun I.).

Pacific Sulphur Co. See (Akun I.).

Paula. See (Akun I.).

\section{VALDEZ QUADRANGLE}

Addison Powell - $(7.6,0.6)$; COPPER, GOLD.

B 642, p. 140

B $963-B$, p. 52-53

B 662 , p. 183

Alaska Chugach Mines, Inc. See Cliff (Gold Min: Co.).

Alaska Dev. \& Min. Co. See Midas.

Alaska Finley Co. See Ramsay-Rutherford.

Alaska Gold Hill - (3.8-4.4, 2.3-2.5) approx; GOLD. Includes references to Black Diamond.

B 622, p. 152,183

B 692, p. 151

B 662, p. 191

Alaska Mayfield (Mines, Inc.). See Mayfield.

Alaska - (SE 1/1 5, 3) ; GOLD, LEAD, ZINC, COPPER.

B 622, p. 169

Albert Johnson. See (Elliott Cr.). 
Alder - $(2.8,2.35) ;$ GOLD (?).

B 520, p. 124

Alice (Mines, Ltd.) - $(3.4,2.4)$; GOLD, SILVER, ZINC, COPPER, LEAD.

B 520, p. 123

B 542, p. 35

B 642, p. 144

B 592, p. 238-239

B 662 , p. 190-191

B 622, p. 152, 175-176

BMB 153, p. 43

All-American. See Midas.

American Eagle. See Eagle.

Amman - (24.35, 12.3) ; COPPER. See also Blue Bird (copper), Bunker Hill (copper), Cave (Copper Cr.), Forget-me-not, Mountain Boy, Mountain Sheep,

Peacock.

B 947-G, p. 125-126

Bald Mtn. See Olson.

Bayview - $(6.15,0.1)$ approx; COPPER.

B 692, p. 165, 171

(Bear Cr.) - (23.0, 15.0) approx; COPPER.

P 15, p. 18

P 41, p. 94 .

Bence-McDonald - $(3.35,3.9)$ ( ?) ; GOLD.

B 592, p. 238

B 642, p. 144

B 622, p. 183

(Benito Cr.) - (23.85, 11.35) ; GOLD, SILVER.
B 622, p. 115
B 714, p. 193-194
B 745, p. 142-143, 146
B 755, p. 69

Bertha. See Knowles.

Bessie Williams - (W 1/2 1,2); GOLD (?). B 622, p. 153, 186

Big Four (McAllister Cr.) - $(2.95,2.35)$; GOLD, LEAD. B 520, p. 124

Big Four (Mineral Cr.) - (5.25, 3.9) ; GOLD, LEAD, ZINC.
B 520, p. 125
B 592, p. 239
B 755, p. 28-29
B 622, p. 153, 164-165
B 810, p. 16
B 642, p. 144
B 880-A, p. 23-24
B 662, p. 190
B 897-A, p. 24
B 712, p. 33
B 910-A, p. 27-28
B 739, p. 24
B 926-A, p. 26-27
BM:B 142, p. 29, 52

Black Diamond. See Alaska Gold Hill.

Blackney - $(22.4,8.3)$; COPPER.

B 520, p. 101-102

B 576, p. 51-52

Blue Bird (copper) - $(24.65,12.1)$; COPPER.

B 745, p. 103

B $947-G$, p. 131

Blue Ribbon - $(6.2,3.45)$; GOID.

B 520, p. 127

B 592, p. 239

Bluebird (gold) - $(3.25,2.2)$; GOLD, LEAD, COPPER.

B 520, p. 120, 123

B 622, p. 182

B 542, p. 35

B 662, p. 191

B 592, p. 239

Bluff - $(3,2)$ (?) ; GOLD (?). Note--In Port Valdez area.

B 542, p. 35 
(Boulder Cr.) - (13.4-13.8, 13.75-13.9) approx; GOLD. See also Eagle, Ross.

B 592, p. 237

B 797, p. 11

B 662 , p. $178-179$

C 184, p. 3

Budd Min. Co. See (Gold Cr.).

Bunker Hill (copper) - $(24.85,12.2)$; COPPER.

B 745, p. 104

B 947-G, p. 131-132

Bunkerhill (gold) - $(3.5,2.85)$; GOLD, LEAD, ZINC. B 622, p. 153,181

B 662 , p. 192

Buster - $(5.5,4.05)$; GOLD (?).

B 520 , p. 125

B 622, p. 165

Cameron-Johnson (Gold Min. Co.) - (1.95-2.0, 3.45-3.7) ; GOLD, LEAD, ZINC.

Includes references to Valdez Gold (Co.).

B 542, p. 36

B 592, p. 63,238

B 622 , p. $45,153,172-174$

B 662 , p. 190,192

B 692 , p. $150-151$

B 722, p. 39

B 739 , p. 24

B $880-A$, p. 24

BMB 142, p. 41, 52

BMB 153, p. 51

Canning \& Centino. See (Benito Cr.).

(Canyon Cr.). See Spirit Mtn.

Cave - $(24.45,12.25)$; COPPER.

B 745, p. 102-103

B $947-G$, p. $129-130$

Chance. See (Elliott Cr.).

(Cheshnina R.). See (Bear Cr.), (Mt. Chitty).

Chesna - $(5.65,4.05)$; GOLD (?).

B 520, p. 126

B 622 , p. 165

Cliff. See (Elliott Cr.).

Cliff (Gold) Mines, Inc. See Cliff (Gold Min. Co.).

Cliff (Gold Min. Co.) - $(3.65,2.3)$; GOLD, ZINO, LEAD.

B 442 , p. 165

B 443, p. $72-74,76-77$

B 480 , p. 29-31, 62

B 520 , p. $27,108,112,116-118$, 121-123

B 542 , p. 35

B 592, p. 63,238

B 783, p. 8

B 797, p. 11

B $864-A$, p. $23-24$

B 880-A, p. 23

B 897-A, p. 24

B $910-A$, p. $26-27$

B 917-A, p. 27

B 622 , p. $45,135,152-153,156$,

B 926-A, p. 26 170-172

B 642 , p. $143-144$

B 662 , p. $190-191$

B $933-A$, p. 26

B 989-E, p. 304-306

B 692 , p. $149-150$

BMB 142, p. 39,52

B 712, p. 33

B 722, p. 14,40

BMB 153, p. 44

IC 7379, p. 22

Cook \& Barrett. See Monte Carlo.

(Copper Cr.) - (24.35-24.85, 12.1-12.35); COPPER. Includes references to Hoffman; see also Amman, Blue Bird (copper), Bunker Hill (copper), Cave, Forget-me-not, Mountain Boy, Mountain Sheep, Mullen, Peacock.
P 15, p. 18, 21-22
P 41, p. 94, 97
B 810 , p. $46-47$
B 345, p. 144-145
B 813 , p. 54
B 824, p. 60
B 745, p. 101
B 836, p. 63
B 797, p. 36
B 897-A, p. 41

Copper Cr. Copper Min. Co. See Mullen. 
Copper King. See (Elliott Cr.).

Copper Queen. See (Elliott Cr.).

(Copper R.) - (E $1 / 2$ quad.) (?) ; CORUNDUM. NoTE.-Location uncertain; may be in Cordova or Gulkana quad.

B 180, p. 88

B 269, p. 150

Crawford \& Amman. See Amman, Blue Bird (copper), Bunker Hill (copper), Cave, (Copper Cr.), Forget-me-not, Mountain Boy, Mountain Sheep, Peacock.

Cube (Min. Co.) - $(3.85,2.5)$; GOLD, ZINC, LEAD, COPPË. Includes references to Three-in-One.
B 592, p. 239
B 662 , p. $190-191$
B 622, p. 176-177
B 692 , p. $150-151$
B 642 , p. 144
BMB 153, p. 44

Cube Mines Co. See Cube.

Curley Kidney - $(3.2,0.05)$; GOLD.

B 692 , p. 172

Curtis. See (Elliott Cr.).

Denby. See Midas.

Devinney \& Dolan - (5, 3-4) ; GOLD.

B 824, p. 22

B 836, p. 22

(Divide Cr.) - $(22.9,6.75)$; COPPER.

B 576, p. 52

Dorothy - $(3.2,2.4)$; GOLD.

B 520, p. 124

Eagle - $(13.4,5.9)$; GOLD, LEAD. Includes references to American Eagle, Meckem.

B 662, p. 178-179

B 866, p. 29-32

B 692, p. 31

Elizabeth. See (Elliott Cr.).

(Elliott Cr.) - (23.55-24.7, 11.7-12.15) ; COPPER, GOLD. Includes references to Albert Johnson, Chance, Cliff, Copper King, Copper Queen, Curtis, Elizabeth, Fog, Goodyear, Guthrie, Henry Prather, Hubbard Elliott (Copper Mines Dev. Co.), Kings, Lawton, Leland, Lizzie G., Louise, Marie Antoinette, Marmot, Mary Ellen, Mineral King (copper), Swazie.

$\begin{array}{ll}\text { P 15, p. 16-19, 23-26 } & \text { B 592, p. 61 } \\ \text { P 41, p. 92-93, 99-102 } & \text { B 622, p. 44, 112-113 } \\ \text { B 213, p. 145 } & \text { B 642, p. 54 } \\ \text { B 314, p. 28 } & \text { B 662, p. 156-157 } \\ \text { B 345, p. 146-152 } & \text { B 745, p. 82-83, 89-90, 115-125, 146 } \\ \text { B 374, p. 65-71 } & \text { B 755, p. 26 } \\ \text { B 379, p. 155 } & \text { B 783, p. 21 } \\ \text { B 442, p. 161 } & \text { BMB 142, p. 37, 52 } \\ \text { B 542, p. 83 } & \text { BMB 153, p. 30 }\end{array}$

Ellis. See Cliff (Gold Min. Co.), Eagle, Midas.

Ellis \& Meckem. See Eagle.

Ellis Imperial. See Imperial.

(Ernestine Cr.) - (15.85-16.3, 6.1-7.85) ; GOLD.

AR 21, p. 436-437

Ethel (Min. Co) . - (5, 3-4) ; GOLD.

B 797, p. 11

B 813, p. 18

B 810, p. 16 
(Fall Cr.) - (15.65-15.75, 6.9-7.4) ; GOLD.
AR 21, p. 436-437
B 662, p. 181-182
P 15, p. 62
B 866, p. 36
P 41, p. 121
C 184, p. 3

B 592, p. 237

(Falls Cr.) - (23.0, 6.55) ; COPPER.
B 520, p. 103
B 576, p. 52

Foaming Falls. See Thompson-Ford.

Fog. See (Elliott Cr.).

Ford \& Thomson Min. Co. See Thompson-Ford.

Forget-me-Not - $(24.6,12.1)$; COPPER.
B 745, p. 103
B 947-G, p. 130-131

45 - (5.5, 3.75) ; GOLD, LEAD, ZINC, COPPER. B 622, p. 166-167

(Fourth of July Cr.) - (13-15, 5-7) (?) ; GOLD. NotE.-Near Tiekel ; placer. B 824, p. 28 B 836, p. 28

Galena Bay Min. Co. See Mullen.

Genzler. See Williams-Gentzler.

(Glorious Fourth Cr.) - (13-15, 5-7) (?) ; GOLD. NOTE.-Near Tiekel; lode. B 592, p. 237

Gold Bluff - $(3.5,2.3)$; GOLD.

B 520, p. 124

13622 , p. 182

B 542, p. 35

(Gold Cr.) - (4.4, 2.2-2.65) ; GOLD.

B 284, p. 86

B 379 , p. 97

B 622, p. 187

B 642 , p. 145

B 443 , p. 71

Is $989-\mathrm{E}$, p. 307

B 520, p. 121, 125

C 184, p. 4

B 592, p. 62

Gold King (Min. Co.) - $(2.25,3.65)$; GOLD, ANTIMONY, LEAD, ZINO, COPPER.

B 592, p. 63, 237-238

B 622, p. $45,152,157,183-185$

B 692, p. 151

B 642, p. 143-145

B 649 , p. 61

B 662 , p. 190, 192

B 712 , p. 33

B 755, p. 28-29

BMB 142, p. 40, 52

BMB 153, p. 46

Gold Standard - (SW 1/4 quad.) ; GOLD ( ?). B 662, p. 191

Gold Sunlight - $(5.65,3.55)$; GOLD (?). See also High Grade. B 520, p. 126

Golden Dollar - $(5.65,3.35)$ approx ; GOLD ( ?). B 520, p. 127

Goodyear. See (Elliott Cr.).

Granby Consol. Min., Smelt. \& Power Co. (Ltd.). See Midas.

Great Northern Dev. Co. See (Iron Mtn.).

Guthrie. See (Elliott Cr.).

Guthrie \& Belloli - $(3.65,2.75)$; GOLD, ZINC, LEAD, COPPER.

B 592, p. 239

B 662, p. 191

B 622, p. 181

Hecla (near Mineral Cr.) - $(5.85,3.3)$; GOLD ( ?).

B 622, p. $169-170$ 
Hecla (near Shoup Glacier) - $(2.7,2.2)$ approx; SILVER, GOLD, LEAD.

B 520, p. 124

Henry Prather. See (Elliott Cr.).

Hercules - $(5.35,4.0)$; GOLD, LEAD, ZINC.
B 520, p. 125
B 622 , p. 165
B 542, p. 35
B 662, p. 190

B 592, p. 239

Hewitt. See Cameron-Johnson.

Hickey. See Mountain View.

High Grade - $(5.65,3.55)$ approx; GOLD, LEAD. See also Gold Sunlight. B 622 , p. 166

Hoffman. See (Copper Cr.).

Hogan. See Mayfield.

Hubbard Elliott (Copper Mines Dev. Co.). See (Elliott Cr.).

Hughes. See Cliff (Gold Min. Co.).

(Hurdle Cr.). See (Hurtle Cr.).

(Hurtle Cr.) - (13.15-13.25, 6.7-6.85) approx; GOLD. Includes reference to (Hurdle Cr.) ; see also Telluride, Wetzler.

B 592, p. 237

C 184, p. 3

B 797, p. 11

Ibex - $(6.65,3.8)$; GOLD.
B 520, p. 117, 128
B 622 , p. 152, 162

Imperial - $(4.05,2.35)$; GOLD. Includes reference to Ellis Imperial.

B 443 , p. $74-75$

B 520, p. 123

B 480, p. 31

B 622 , pp. 152, 183

(Iron Mtn.) - (SE $1 / 424,11)$; COPPER.

$$
\text { B 379, p. } 156
$$

B 442, p. 161

Ivanhoe - $(3.35,3.9)(?)$; GOLD. B 622 , p. 183

I.X.L. - $(3.2,2.4)$; GOLD. B 520, p. 124

(Jack Bay) - (3.35-3.7, 0.5-0.85) ; COPPER, ZINC, LEAD. NotE.-Two prospects.
B 692, p. 171-172
B 963-B p. 53

Janes. See July, Little Giant, Rose.

July - (5.95, 3.95) approx ; GOLD, LEAD, ZINC.

B 622, p. 168-169

Jumbo. See Midas.

King Solomon's Copper Mines. See Midas.

Kings. See (Elliott Cr.).

Knowles (\& Backman) - $(13.05,5.75)$; GOLD, LEAD. Includes reference to (Stuart Cr.).
B 792, p. 11
B 866, p. 32-35

B 810 , p. $16-17$

Lawson. See (Elliott Cr.).

Layton \& Nelson. See Telluride, Wetzler.

Leland. See (Elliott Cr.).

(Little Bremmer R.) - (21.8-22.3, 2.35-3.05) ; GOLD.
P 41, p. 120
B 576 p. $43-44,48-49$
B 520, p. 94, 100-101 
Little Giant - $(5.95,3.95)$ approx ; GOLD, LEAD, ZINC.

B 592, p. 239

B 622, p. 168-169

B 662, p. 190

B 783, p. 8

B 792, p. 10

B 797, p. 11

B 810, p. 16
B 813, p. 18

B 824, p. 22

B 836, p. 22

B 868-A, p. 22

B $880-$ A, p. 22, 24

B 910-A, p. 27-28

C 184, p. 4

Lizzie G. See (Elliott Cr.).

Lost Hope. See Ramsay-Rutherford.

Louise See (Elliott Cr.).

(Lowe R.) - (9.5-11.0, 1.75-2.1) ; GOLD.

B 622 , p. 186

B 989-E, p. 308

B 642, p. 145

Lucky Six - (E 1/2 quad.) (?) ; GOLD (?).

B 810 , p. 17

Lucky Strike. See Knowles

McCallum - (4.6, 3.05) (?) ; GOLD.

B 642, p. 144

B 662, p. 191

McDonald - $(3,2)$ (?) ; GOLD (?).

B 692, p. 151

McIntosh - (5.5, 3.45) ; GOLD.

B 520, p. 127

(Mahlo R.) - (9.7-11.15, 10.45-13.0) ; GOLD.

AR 20, p. 422

B 866, p. 36

Mammoth Min. Co. See Bessie Williams.

(Manker Cr.) - (10.0-11.85, 8.5-13.5) ; GOLD.

AR 20, p. 422

Marie Antoinette. See (Elliott Cr.).

Marmot. See (Elliott Cr.).

Mary Ellen. See (Elliott Cr.).

Mayfield (Gold Min. Co.) - (1.5, 2.7) ; GOLD, LEAD, ZINC, COPPER. Includes references to Alaska Mayfield (Mines, Inc.).
B 520, p. 125
B 542 , p. 35
B $880-A$, p. 24-25
B 897-A, p. 24
B 622, p. 153, 185-186
B $910-A$, p. 27
B 642, p. 145
B 917-A, p. 27
B 692 , p. 151
B 926-A, p. 26

B 824, p. 22

Mazuma. See Cameron-Johnson.

Meckem. See Eagle.

Meckem \& Reis. See (Fall Cr.).

Midas (Copper Co.) - $(6.1,0.4)$; COPPER, GOLD, SILVYER, ZINC. Includes reference to (Solomon Gulch) (copper).

B 284, p. $82-83$

B 520, p. 117

B 542, p. 36

B 592, p. 62,240

B 622, p. 45, 132-133, 151-153, :

156-157, 187-188

B 642 , p. 144-145

$\therefore$ B 662, p. 44, 184-185

$593018-61-23$
B 692, p. 31, 144, 147, 157

B 712, p. 33

B 714, p. 22

B 739, p. 24

B 963-B, p. 51-52

B 989-E, p. 298, 302

BMB 142 , p. $38,40,52$

BMB 153, p. 46-47 
(Mill Cr.) See Knowles.

Millionaire - $(5.45,4.1)$; GOLD, LEAD, COPPER.
B 520, p. 125
B 542, p. 35
B 622 , p. 152, 165-166
B 592, p. 239
B 662, p. 190
B $910-$ A, p. 28
(Mineral Cr.) - (5.0-5.7, 2.4-4.0) ; GOLD.
AR 20, p. 421
B 284, p. 86
B 622, p. 186
B 379, p. 97
B 443, p. 71-72
B 642 , p. 145
B 989-E, p. 283,307
C 184, p. 4

Mineral Cr. Dev. Co. See Hercules, Millionaire.

Mineral Cr. Min. Co. See Buster, Chesna, Hercules, Millionaire, Mineral King (gold), Mountain View, Sunshine.

Mineral King (copper). See (Elliott Cr.).

Mineral King (gold) - $(5.65,3.9)$; GOLD. B 520, p. 126

Minnie - $(3.2,3.65)$; GOLD.

B 592, p. 63, 238

B 622, p. 168

B 622, p. 180

B 662, p. 192

Montana Boy. See Mountain Boy.

Monte Carlo - $(5.5,4.15)$; GOLD, LEAD. B 520, p. 126

B 622, p. 168

Mountain Boy - $(24.75,12.1)$; COPPER, GOLD(?). Includes reference to Montana Boy. B 745, p. 103

B 947-G, p. 131

Mountain King (Min. Co.) - $(5.95,4.0)$; GOLD, LEAD, ZINC, COPPER. B 592, p. 63,239

B 622, p. $45,153,163-164$

B 642,p. 144

BMB 142, p. 42, 52

Mountain Sheep - $(24.5,12.2)$; COPPER. B 745, p. 103

B 947-G, p. 130

Mountain View - $(5.8,3.95)$; GOLD, LEAD. B 520, p. 126

B 622, p. 167-168

(Mt. Chitty) - $(23.0,15.0)$ approx ; COPPER, GOLD, SILVER. P 15, p. 18

P 41, p. 94

Mullen - $(24.4,12.35)$; COPPER.

B 345, p. 144-145

B 745, p. 101-102

B 374, p. 62

B 947-G, p. 126-129

B 622, p. 111-112

Mystic. See Cliff (Gold Min. Co.).

(Nadina R.) - (17.35-17.9, 17.0-17.85) ; PLATINUM(?). May be in Gulkana quad.

P 15, p. 63-64

P 41, p. 121-122

National - $(1.0-3.5,2.0-4.0)$; GOLD.

B 642, p. 143,145

North Star. See Knowles.

Nymond - $(3,2)(?)$; GOLD.

B 642, p. 144

B 662, p. 191

B 692, p. 151

Olsen, Gustafson \& Anderson. See Gold King. .

Olson - (3.1, 3.55) ; GOLD, ZINC, LEAD. Includes references to Bald Mtn.

B 592, p. 238

B 622, p. 153, 179-180

B 662, p. 191

B 642, p. 144

B 692, p. 151 
Olson \& Woods - $(5.65,3.3)$; GOLD.

B 520, p. 126-127

B 622, p. 170

B 592, p. 239

Oregon. See Owl.

Orion - $(4.15,0.1)$; GOLD.

B 692, p. 165, 173

Owl (Min. Co.) - $(3.85,2.65)$; GOLD, LEAD, ZINC, COPPEIR.

B 542, p. 35

B 622 , p. 180-181

Palmer - $(3.05,2.45)$; GOLD.

B 520, p. 124

Patten Min. Co. - $(5.0,1.6)$; GOLD ( ?).

B 692, p. 151

Peabody Alaska Copper Corp. See Addison Powell.

Peacock - $(24.45,12.25)$; COPPER.

B 745, p. 103

B 947-G, p. 130

Peterson, Young \& Halversen. See Spirit Mtn.

Pinochle - $(7.35,3.7)$; GOLD.

B 622, p. 153, 161-162 $\quad$ B 662, p. 190

B 642, p. 143

Portland (near Tiekel) - (W 1/2, 14, 5) ; GOLD.

B 662, p. 177

Portland (near Valdez). See Seacoast.

Poy. See Big Four (Mineral Cr.), Little Giant, Rose, Rose Quartz, Star.

Pulver \& Baker. See Owl.

Quail. See Wetzler.

(Quartz Cr.) - (13.0-14.1, 7.0-9.25) ; GOLD, LEAD, COPPER.
AR 20, p. 422
P 41, p. 121
AR 21, p. 402, 436-437
B 662, p. 179-180
P 15, p. 63
B 866, p. 35-36

Quartz Cr. Gold Min. Co. See (Quartz Cr.).

Queen of Sheba - (SE 1/4 5, 3) ; GOLD.

B 622, p. 169-170

Quitsch - $(5.75,4.75)$ approx ; GOLD, LEAD. See also Little Giant. B 622, p. 167

Rambler (Gold Min. Co.) - $(3.25,3.75)$ approx ; GOLD.

B 592, p. 238

B 662, p. 190,192

B 622 , p. 153, 183

Ramsay-Rutherford (Gold Min. Co.) - (7.55, 3.65) ; GOLD, SILVER, ZINO,

LEAD, COPPER.

B 542, p. 35

B 592 , p. 239

B 622 , p. $45,153,159-161$

B 642, p. 141, 143

B 662, p. 44,190

B 692 , p. $149-151$

B 755, p. 28-29

B 783, p. 8

B 792, p. 10

B 797, p. 11

B 810, p. 16
B 813, p. 18

B 824 , p. 22

B 836 , p. 22

B 868-A, p. 22

B 880-A, p. 22, 24

B $910-A$, p. 28

B 926-A, p. 26

B 989-E, p. 304, 308

BMB 142, p. 42,52

BMB 153, p. 49

Rankin and assoc. See Cameron-Johnson.

Reeve \& Thompson. See Rough \& Tough. 
Reis - (SW 1/4 14, 5) ; GOLD.

B 662, p. 177

Reynolds. See (Fall Cr.).

Rose - (5.95, 3.95) approx; GOLD, LEAD. See also Rose Quartz; probably the same.
B 622, p. 168-169
B 868-A, p. 22
B 662 , p. 190
B $880-A$, p. 22

Rose Johnson - $(7.05,3.25)$; GOLD, LEAD, COPPER.

B 622, p. 162-163

Rose Quartz - (5.95, 3.95) approx ; GOLD, LEAD, COPPER. See also Rose. B 880-A, p. 24

Ross - $(13.8,5.75)$ approx ; GOLD, LEAD.

B 662, p. 178

Rough \& Tough - $(1.75,3.6)$ approx; GOLD, SILVER.

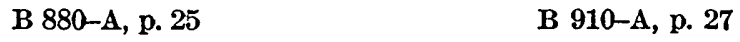

Seacoast (Min. Co.) - $(3.55,2.9)$; GOLD, LEAD, ZINC, COPPER.

B 592, p. 239

B 662, p. 191

B 622, p. 178-179

BMB 153, p. 49

B 642, p. 144

Sealy-Davis (Min. Co.) - (3.6-3.65, 2.4-2.45) ; GOLD, LEAD, ZINC, COPPER. B 520, p. 124

B 542, p. 35

B 592; p. 239

B 642, p. 144

BMB 142, p. 42,52

B 622, p. 152, 174-175

BMB 153, p. 49-50

Shoup Bay Min. Co. - $(3.35,2.35)$; SILVER, GOLD.

B 520, p. 123

Shoup Glacier Co. See Palmer.

Silver Falls. See Thompson-Ford.

Silver Gem - (3.35, 2.45) ; GOLD, ANTIMONY, SILVER, ZINC, LEAD. B 520, p. 123

B 649, p. 61

B 622, p. 152, 157, 179

Slide - $(\mathrm{E} 1 / 25,4)(?)$; GOLD.

B 692 , p. 150-151

Smith. See Mountain King.

(Solomon Basin). See (Solomon Gulch) (gold).

(Solomon Gulch) (copper). See Midas.

(Solomon Gulch) (gold) - $(5.9-6.25,0.1-1.2)$; GOLD. Includes reference to (Solomon Basin).

AR 20, p. 421

B 989-E, p. 308

B 379, p. 97

Spanish - $(3.25,2.4)$; GOLD.

B 520, p. 124

Spirit Mtn. - $(22.9,5.75)$; COPPER, NICKEL, COBALT, ZINC. Includes references to (Canyon Cr.).

B 520, p. 103-104

B 576, p. 52-53

B 662, p. 25, 43

B 666, p. 97.

B 692, p. 23

B 712, p. 91-98

Star - (5.95, 3.95) ( ?) ; GOLD.

B 868-A, p. 22

B 714, p. 40

B 943-C, p. 49-56

C 252, p. 7

IC 7379, p. 70-71

RI 3913, p. 2-8

B 880-A; p. 22 
Stevens - (3.55, 2.9) approx; GOLD(?).

B 622, p. 183

(Stuart Cr.). See Knowles.

(Sulphide Gulch). See Addison Powell.

Sunshine - $(5.5,4.05)$; GOLD, LEAD. B 520, p. 125

B 622, p. 165

(Surprise Cr.) - $(22.95,7.55)$; COPPER. B 520, p. 102-103

B 576, p. 52

Swazie. See (Elliott Cr.).

Telluride - $(13.25,6.7)$ approx ; GOLD. See also (Hurtle Cr.). B 662, p. 180

Thompson-Ford (Min. Co.) - $(3.9,2.75)$; GOLD, LEAD, ZINO, COPPER. Includes reference to Ford-Thomson Min. Co.

B 520, p. 125

B 622, p. 177

B 542, p. 35

B 662 , p. 192

B 592, p. 239

Three-in-One. See Cube.

Tiger - $(5.85,3.35)$; GOLD ( ?).

B 622, p. 170

Townsend \& Holland - $(\mathbf{1 3 . 4 5 , 3 . 9 ) ~ ; ~ G O L D . ~}$

B 866, p. 35-36

Treasury Note. See Cameron-Johnson.

Tuscarora - $(3,2)(?)$; GOLD.

B 662 , p. 192

B 783, p. 8

B 739, p. 24

B 792, p. 10 .

B 773, p. 15, 38

Valdez (Min. Co.) - (6.7, 3.57) ; GOLD.

B 520, p. 117, 127-128

B 592 , p. 239

B 622, p. 153, 162

B 642 , p. 143

B 662, p. 190

B 692 , p. 151

B 722, p. 40

BMB 153, p. 51

Valdez Bonanza - $(6.4,3.4)$; GOLD.

B.520,p. 117, 127

B 592, p. 239

Valdez Gold (Co.). See Cameron-Johnson.

von Gunther - $(5.7,4.3)$; GOLD.

B 622 , p. 167

Washington. See Owl.

Wetzler - $(13.15,6.85)$; GOLD, LEAD, ZINC, COPPER. See also (Burtle Cr.). B 662 , p. $180-181$

B 866, p. 27-29

Whistler - $(3.25,2.2)$ approx ; GOLD.

B 520, p. 123

Williams-Gentzler - $(6.1,3.85)$; GOLD. Includes references to Genzler.

B 480 , p. 31

B 592, p. 239

B 520, p. 127

\section{WISEMAN QUADRANGLE}

Anglich. See (Hammond R.).

(Archibald Gulch) or (Cr.) - (18.65-18.9, 8.9-8.95) ; GOLD; ANTIMONY.

B 442, p. 292, 301-303

B 880-A, p. 45

B 532, p. 69, 92

B 810, p. 27

B 864-A, p. 41

B 868-A, p. 42

B 897-A, p. 54

B 910-A, p. 56

B $917-A$, p. 55

RI 4173, p. 38 
(Birch Cr.) - (10.1-10.75, 7.1-9.0) (?) ; GOLD. Note.-Probably the stream called Flat Cr. on recent maps.
B 442, p. 292, 314
B 714, p. 90
B 532, p. 70, 109
B 815, p. 333
B 662 , p. 59

Boyle. See (Smith Cr.).

Brady. See (Hammond R.).

(Buckeye Gulch) - (19.5, 9.4) ; GOLD. B 442, p. 292, 305

B 532, p. 69, 96-97

(Chapman Cr.) - (17.0-17.25, 1.75-1.8) ; GOLD.

B 532, p. 85

Chappell. See (Nolan Cr.).

(Clara Gulch) or (Cr.) - (19.1-19.9, 5.3-5.5) ; GOLD.
AR 21, p. 486
B 532, p. 69,89
P 20, p. 99, 101-102
B 868-A, p. 43

B 442, p. 292

Collins. See (Hammond R.).

(Confederate Gulch) - (19.5-19.75, 8.3-8.6) ; GOLD.

B 532, p. 95

(Crevice Cr.) - (6.5-8.5, 6.2-7.0) ; GOLD.

B 442, p. 292, 314

B 642, p. 65

B 532, p. 70, 110

B 815, p. 334

B 536, p. 144

Detroit Min. Co. See (Hammond R.).

Dow \& Chappell. See (Archibald Gulch).

(Eagle Bluff) or (Cliff) - $(20.1,1.35)$ approx ; GOLD.

P 20, p. 102

I 532 , p. 70,107

B 442, p. 292, 312

(Emma Cr.) - (18.8-19.1, 6.1-6.15) ; GOLD.

AR 21, p. 486

P 20, p. 99, 102

B 532, p. 69, 90-91

B 345, p. 45

B 542, p. 45

B 442, p. 292, 300

B 622 , p. 59

B 662, p. 59

(Fay Gulch) or (Cr.) - (18.7-18.9, 9.0-9.05) ; GOLD, ANTIMONY.

B 345, p. 45

B 442, p. 292, 301-303

RI 4173, p. 37

TDM 2, p. 16-17

B 532, p. 69, 92-94

Ferguson - $(19.1,8.5)$; ANTIMONY.

RI 4173, p. 37

(Fool Cr.) - (5.2-6.4, 6.35-6.6) ; GOLD. See also (Sixtymile R.).

B 532, p. 110

B 815, p. 334

B 536, p. 144

Gamblin \& Ferguson. See (Fay Cr.).

(Gold Bench) - (17.55, 0.35) ; GOLD, TIN, MERCURY, TUNGSTEN, COPPER, LEAD, BISMUTH (?).
P 20, p. 101-102
C 195, p. 8, 10-11
B 442, p. 292, 311-312
C 248, p. 3, 5, 13, 15
B 532, p. 70, 105-107
C 348, p. 16

(Goldbottom Gulch) - $(19.7,9.0)$; GOLD.

B 442, p. 292, 305

B 532, p. 69, 96-97

(Grubstake Bar) - $(19.5,1.5)$ approx ; GOLD.

B 442, p. 292, 312

B 532, p. 70, 107 
(Hammond R.) or (Cr.) - (19.5-19.95, 8.55-9.8) ; GOLD. See also (Buckeye Gulch), (Goldbottom Gulch), (Swift Gulch), (Vermont Cr.).

P 20, p. 100-102

B 225, p. 58

B 442, p. 288, 292, 304-305

B 520, p. 38

B 532 , p. $69,95-96$

B 542, p 45

B 592, p. 69

B 622 , p. 58-60

B 642 , p. 64

B 662 , p. 59

B 755, p. 46

B 792, p. 19

B 797, p. 21-22
B 810, p. 27-28

B 813, p. 33

B 844 A, p. 39

B 857-A, p. 36

B 864-A, p. 41

B 868-A, p. 42

B 880-A, p. 45

B 897-A, p. 54

B 926-A, p. 52

B 933-A, p. 47

BMB 142, p. 25

BMB 153, p. 54

Harvey. See (Clara Gulch):

Haslen and assoc. See (Nolan Cr.).

(Ironside Bench) or (Bar) - $(17.7,0.5)$ approx ; GOLD.

P 20, p. 102

B 532, p. 70, 107

B 442, p. 292, 312

(Jay Cr.) - (11.05-11.4, 7.4-7.8) ; GOLD.

B 642, p. 65

B 815, p. 333

B 662, p. 59

BMB 142, p. 25 .

B 722, p. 59

(John R.) - (4.8-8.5, 5.0-7.0) approx ; GOLD, COPPER, ANTIMONY. : Sce also (Crevice Cr.), (Fool Cr.), (Midas Cr.).

P 20, p. 104

B 259, p. 30-31

B 836, p. 40

B 815, p. 342

B 844-A, p. 39

TDM 1, p. 14

Jones \& Boyle - $(18.65,8.8)$; ANTIMONY. RI 4173, p. 37

TDM 2, p. 16-17

Jones, White \& O'Leary. See ('Smith Cr.).

Kelley, Foley \& Burke. See (Hammond R.).

(Kelly Gulch) - (18.7-19.05, 5.6-5.7) ; GOLD. B 442, p. 292

B 532, p. 69, 89

Knorr. See (Mascot Cr.).

(Koyukuk R., North Fork) - (16, 8-9) (?) ; GOLD. See also (Mascot Cr.). B 836, p. 39

(Koyukuk R., South Fork) - (17-20, 0-1); GOLD. See also (Eagle. Cliff), (Gold Bench).

B 836, p. 39

B 897-A, p. 54

B 880, p. 46

(Lake Cr.) - (9.4-9.85, 8.45-8.6) ; GOLD, TUNGSTEN, ANTIMONY, BISMUTH, COPPER.

B 442 p. 292, 314

B 532 , p. 70,109

B $917-A$, p. 55

B 815, p. 333

RI 4174, p. 28

B 857-A, p. 36

TDM 1, p. 39

B 868-A, p. 42

TDM 2, p. 17,20

Le Boyteau. See (Nolan Cr.).

(McKinley Cr.) - (4.2-6.2, 7.2-8.8) (?) ; GOLD.

AR 21, p. 485 
(Mailbox Cr.) - (17.0-17.2, 2.15-2.7) ; GOLD.

B 880-A, p. 45

Mangles. See (Lake Cr.).

(Mascot Cr.) - (16.3-16.55, 8.4-9.2) ; GOLD.
P 20, p. 100
B 345, p. 45
B 532, p. 70, 108-109
B 868-A, p. $42-43$

B 442, p. 290-292, 313

(Michigan Cr.) - (10.8-12.5, 5.1-8.2) approx ; LEAD, GOLD, SILVER.
P 20, p. 105
B 815, p. 343

B 739, p. 42

(Midas Cr.) - (4.8-5.2, 5.0-6.2) ; GOLD.

B 442 , p. 314

B 815, p. 334

B 532, p. 110

(Minnie Cr.) - (19.6-20.25, 7.7-7.8) ; GOLD. NoTE.-Production may have been

from part of creek in Chandalar quad.
B 442, p. 292
B 662 , p. 59
B 532, p. 69, 94-95
B 836, p. 39

B 542, p. 45

(Moose Gulch) - (18.8-19.2, 6.65-6.8) ; GOLD.

B 532, p. 91

Neck. See (Porcupine Or.).

(Nolan Cr.) - (18.45-18.75, 8.6-9.1) ; GOLD. See also (Archibald Gulch), (Fay Gulch), (Smith Gulch).
P 20, p. 100
B 345, p. 45
B 379, p. 57
B 442, p. 290, 292, 301-304
B 480 , p. 39
B 520, p. 38
B 532, p. 69, 92-94
B 542, p. 45
B 622, p. 59-60
B 810, p. 27
B 813, p. 33
B 824, p. 38
B 836, p. 39
B 844-A, p. 39
B 857-A, p. 36
B 864-A, p. 41
B 868-A, p. 42
B 642, p. 64
B 880-A, p. 45
B 662, p. 59
B 897-A, p. 54
B 714, p. 90
B 910-A, p. 56
B 722, p. 59
B 917-A, p. 55
B 739, p. 42
B 926-A, p. 52
B 755, p. 46
B 933-A, p. 47
B 797, p. 21
BMB 142, p. 25
BMB 153, p. 54

(Old Man Cr.) - (SE 1/8 quad.) (?) ; GOLD.

B 480, p. 39

(Pasco Cr.) - $(17,8)(?)$; GOLD.

AR 21, p. 485

(Porcupine Cr.) - (17.7-18.5, 4.6-5.2) ; GOLD.
AR 21, p. 485
P 20, p. 102
B 824, p. 38
B 442, p. 292
B 836 p. 39
B 532, p. 69, 86
B 868-A, p. 43
B 662, p. 59
B 880-A, p. 46
B 897-A, p. 54

B 810, p. 28

Rooney. See (Lake Cr.). 
(Rose Cr.) - (18.7-18.9, 3.9-3.95) ; GOLD. Nore-Shown as Rasie Or. on recent maps.

B 532, p. 86

(Rye Cr.) - (10.6-11.3, 7.0-7.55) ; GOLD, TUNGSTEN, LEAD, COPPER.

B 642 , p. 65

C 195, p. 8-11

B 662, p. 59

(Sawyer Gulch) - (18.55-19.2, 6.4-6.7) ; GOLD.

B 532, p. 91

'(Sixtymile R.) - (3.0-5.2, 6.2-8.0) ; GOLD. See also (Fool Cr.). B 755, p. 46

(Smith Cr.) or (Gulch) - (18.6-18.75, 8.75-8.8) ; GOLD. See also Jones \& Boyle.

B 345, p. 45

B 442, p. 292, 301-303

B 532, p. 69, 92-94

B 542, p. 45

B 622, p. 59

B 642, p. 65

B 662, p. 59
B 722, p. 59

B 810, p. 27

B 824, p. 38

B 836, p. 39

B 868-A, p. 42

B 880-A, p. 45

B 897-A, p. 54

Spinks \& Irish. See (Hammond R.).

(Spring Cr.) - (9.6-10.1, 9.1-9.15) ; GOLD.
B 442, p. 292, 314
B 836, p. 39-40
B 532, p. 70, 110
B $910-A$, p. 56
B 815, p. 333
B 917-A, p. 55

Standish. See (Nolan Cr.).

Standish Bros. See (Porcuplne Cr.).

(Summit Cr.) - (9.6-10.05, 9.2-9.4) (?) ; GOLD. B 824, p. 38-39

(Surprise Cr.) - $(9.85-10.25,9.15-9.45) ;$ GOLD.

B 836, p. 39-40

(Swift Gulch) or (Cr.) - (19.4-19.55, 9.0-9.15) ; GOLD.
P 20, p. 100
B 532, p. 69, 96-97
B 345, p. 45
B 542, p. 45
B 442, p. 292, 305
B 810, p. 27

(Tramway Bar) - $(16.9,1.7)$; GOLD.
AR 21, p. 483
P 20, p. 98, 102
B 532 , p. $69,84-85$
B 926-A, p. 52
B 933-A, p. 47
B 442, p. 291-292, 297
(Twelvemile Cr.) - (17.0-17.85, 3.6-4.0) ; GOLD.
AR 21, p. 485
B 532, p. 69, 85
P 20, p. 102
B 868-A, p. 43
B 442, p. 292
B $880-$ A, p. 46

Ulen. See (Nolan Cr.).

(Union Gulch) or (Cr.) - (19.3-19.65, 8.15-8.45) ; GOLD.
P 20, p. 99, 101-102
B 532, p. 69, 95
B 442, p. 292, 304
B 868-A, p. 42

(Vermont Cr.) - (19.15-19.4, 9.8) ; GOLD.

$\begin{array}{ll}\text { P } 20, \text { p. } 100 & \text { B } 810, \text { p. } 27 \\ \text { B } 345, \text { p. } 45 & \text { B } 824, \text { p. } 38 \\ \text { B } 442, \text { p. 292, 305-306 } & \text { B 836, p. 39 } \\ \text { B 532, p. 69, 97-98 } & \text { B 880-A, p. 45 } \\ \text { B 622, p. 59 } & \text { B 897-A, p. 54 }\end{array}$

B 662, p. 59 


\section{INDEX OF METALLIC AND NONMETALLIC MINERAL DEPOSITS}

Wanamaker (, Allen \& Eaton).: See (Smith Cr.).

Wannemaker \& Wortman - $(18.55,8.6)$; ANTIMONY. RI 4173, p. 38

(Washington Cr.) - (17.3-18.1, 9.3-10.0) ; GOLD.
P 20, p. 100
B 532, p. 70, 108
B 442, p. 292, 313

Watts. See (Union Cr.).

Watts, Harvey \& Neck. See (Hammond R.).

(Wild R.) or (Cr.) - (9.4-10.75, 7.1-9.4); GOLD. See also (Birch Cr.), (Lake Cr.), (Spring Cr.), (Summit Cr.).
B 259, p. 30-31
B 622, p. 59
B $864-A$, p. 41
B 824, p. 38
B 880-A, p. 46
B 836, p. 39-40
B 897-A, p 54
B 844-A, p. 39
B 926-A, p. 52
B 933-A, p. 47

B 857-A, p. 36

(Wilson Cr.) - (17.6-17.8, 0.4-0.9) ( ?) ; GOLD

B 532, p. 107

(Wiseman Cr.) - (17.8-19.5, 7.5-9.2) ; GOLD. See also (Nolan Cr.)

AR 21, p. 485

B 259, p. 30-31

B 836, p. 39

B 532, p. 91-92

B 917-A, p. 55

B 824 , p. 38

B 926-A, p. 52

Wooll. See (Nolan Cr.).

\section{YAKUTAT QUADRANGLE}

(Black Sand I.) - (North-central part of quad.) ; GOLD. B 314, p. 86-87

(Khantaak Beach) - (10.6-11.3, 10.35-11.3) ; GOLD.

P 64, p. 167

B 662, p. 23

B 284, p. 64

(Logan Beach) - (12.2-12.6, 13.7-14.9) ; GOLD. P 64, p. 165-167

B 284, p. 64

(Russell Fiord) - (13-15, 10-17) ; COPPER. B 314, p. 87

(Yakutat Bay) - (10-12, 10-14) ; GOLD, PLATINUM(?). P 64, p. 165-168

B 225, p. 46

B 662, p. 23

B 773, p. 23 


\section{LIST OF REPORTS CITED}

The preceding index contains references to the following reports.

\section{U.S. GEOLOGICAI SURVEY}

\section{ANNUAL REPORTS}

18. Part III. Economic geology. 1897. Contains: Reconnaissance of the gold fields of southern Alaska, with some notes on general geology, by G. F. Becker, p. 1-86; Geology of the Yukon gold district, Alaska, by J. E. Spurr, with an introductory chapter on the history and condition of the district to 1897 , by H. B. Goodrich, p. 87-392.

20. Part VII. Explorations in Alaska in 1898. 1899. Contains: A reconnaissance in the Sushitna basin and adjacent territory, Alaska, in 1898 , by G. H. Eldridge, p. 1-29; A reconnaissance in southwestern Alaska in 1898, by J. E. Spurr, p. 31-264; A reconnaissance from Resurrection Bay to the Tánana River, Alaska, in 1898, by W. C. Mendenhall, p. 265-340; A reconnaissance of a part of Prince William Sound and the Copper River district, Alaska, in 1898, by F. C. Schrader, p. 341-423; A reconnaissance in the White and Tanana river basins, Alaska, in 1898, by A. H. Brooks, p. 425-494.

21. Part II. General geology, economic geology, Alaska. 1900. Contains: A reconnaissance from Pyramid Harbor to Eagle City, Alaska, including a description of the copper deposits of the upper White and Tanana Rivers, by A. H. Brooks, p. 331-391; A reconnaissance of the Chitina River and the Skolai Mountains, Alaska, by Oscar Rohn, p. 393-440; Preliminary report on a reconnaissance along the Chandalar and Koyukuk Rivers, Alaska, in 1899, by F. C. Schrader, p. 441-486.

\section{PROFESTIONAL PAPERS}

1. Preliminary report on the Ketchikan mining district, Alaska, with an introductory sketch of the geology of southeastern Alaska, by A. B. Brooks. 1902 . $120 \mathrm{p}$.

2. A reconnaissance of the northwestern portion of Seward Peninsula, Alaska, by A. J. Collier. $1902.70 \mathrm{p}$.

10. Reconnaissance from Fort Hamlin to Kotzebue Sound, Alaska, by way Dall, Kanuti, Allen, and Kowak Rivers, by W. C. Mendenhall. 1902. $68 \mathrm{p}$.

15. The mineral resources of the Mount Wrangell district, Alaska, by W. C. Mendenhall and F. C. Schrader. $1903.71 \mathrm{p}$.

20. A reconnaissance in northern Alaska across the Rocky Mountains along Koyukuk, John, Anaktuvuk, and Colville Rivers and the Arctic Coast to Cape Lisburne, in 1901, by F. C. Schrader, with notes by W. J. Peters. 1904: 139 p.

41. Geology of the central Copper River region, Alaska, by W. C. Mendenhall. $1905.133 \mathrm{p}$.

64. The Yakutat Bay region, Alaska: Physiography and glacial geology, by R. S. Tarr; Areal geology, by R. S. Tarr and B. S. Butler. 1909. $183 \mathrm{p}$.

70. The Mount McKinley region, Alaska, by A. H. Brooks, with descriptions of the igneous rocks and of the Bonnifield and Kantishna districts, by L. M. Prindle. 1911. $234 \mathrm{p}$. 
87. Geology and ore deposits of Copper Mountain and Kasaan Peninsula, Alaska, by C. W. Wright. $1915.110 \mathrm{p}$.

251. Geology and mineral deposits of Jumbo Basin, southeastern Alaska, by G. C. Kennedy. 1953. 46 p.

268. The central Kuskokwim region, Alaska, by W. M. Cady, R. E. Wallace, J. M. Hoare, and E. J. Webber. 1955. 132 p.

293. Quaternary and engineering geology in the central part of the Alaska Range. Contains: (a) Quaternary geology of the Nenana River valley and adjacent parts of the Alaska Range; by Clyde Wahrhaftig; (b) Engineering geology along part of the Alaska Railroad, by Clyde Wahrhaftig and R. F. Black. 1958. $118 \mathrm{p}$.

302-A. Phosphate deposits in northern Alaska, by W. W. Patton, Jr., and J. J. Matzko. 1959. p. 1-17.

\section{BUILETINS}

180. The occurrence and distribution of corundum in the United States, by J. H. Pratt. 1901. 98 p.

213. Contributions to economic geology, 1902; S. F. Emmons and C. W. Hayes, geologists in charge. 1903. $449 \mathrm{p}$. Contains: Placer gold mining in Alaska in 1902; by A. H. Brooks, p. 41-48; The Glenn Creek gold-mining district, Alaska, by A. J. Collier, p. 49-56; The Chistochina gold field, Alaska, by W. C. Mendenhall, p. 71-75; Stream tin in Alaska, by A. H. Brooks, p. 92-93; Copper deposits of the Mount Wrangell region; Alaska, by W. C. Mendenhall and F. C. Schrader. p. 141-148.

225. Contributions to económic geology, 1903; S. F. Emmons and C. W. Hayes, geologists in charge. 1904. 527 p. Contains: The Juneau gold belt, Alaska, by A. C. Spencer, p. 28-42; Placer mining in Alaska in 1903 , by A. H. Brooks, p. 43-59; The Porcupine placer mining district, Alaska, by C. W. Wright, p. 60-63; Gold placers of the Fairbanks district, Alaska, by L. M. Prindle, p. 64-73; The Kotzebue placer gold field of Seward Peninsula, Alaska, by F. H. Moffit, p. 7480; Tin deposits of the York region, Alaska, by A. J. Collier, p. 154167.

229. The tin deposits of the York region, Alaska, by A: J. Collier. 1904. $61 \mathrm{p}$.

236. The Porcupine placer district, Alaska, by C. W. Wright. 1904.35 p.

247. The Fairhaven gold placers, Seward Peninsula, Alaska, by F. H. Moffit. 1905. $85 \mathrm{p}$.

250. The petroleum fields of the Pacific Coast of Alaska, with an account of the Bering River coal deposits, by G. C. Martin. $1905.64 \mathrm{p}$.

251. The gold placers of the Fortymile, Birch Creek, and Fairbanks regions, Alaska, by L. M. Prindle. 1905. 98 p.

259. Report on progress of investigations of mineral resources of Alaska in 1904, by A. H. Brooks and others. 1905. 196 p. Contains: Placer mining in Alaska in 1904, by A. H. Brooks, p. 18-31; Methods and costs of gravel and placer mining in Alaska, by C. W. Purington, p. 32-46; Economic developments in southeastern Alaska, by F. E. and C. W. Wright, p. 47-68; The Treadwell ore deposits, Douglas Island, by A. C. Spencer, p. 69-87; The Cape Yaktag placers, by G. C. Martin, p. 88-89; Gold placers of Turnagain Arm, Cook Inlet, by F. H. Moffit, p. 90-99; Gold deposits of the Shumagin Islands, by G. C. Martin, 
p. 100-101; Auriferous quartz veins on Unalaska Island, by A. J. Collier, p. 102-103; Rampart placer region, by L. M. Prindle and F. L. Hess, p. 104-119; Recent development of Alaskan tin deposits, by A. J. Collier, p. 120-127.

260. Contributions to economic geology, 1904; S. F. Emmons and C. W. Hayes, geologists in charge. $1905.620 \mathrm{p}$. Contains: The occurrence and distribution of tin, by L. C. Graton and F. L. Hess, p. 161-187.

262. Contributions to mineralogy from the United States Geological Survey, by .F. W. Clarke and others. 1905. 147 p. Contains: Mineralogical notes, by W. T. Schaller, p. 121-144.

263. Methods and costs of gravel and placer mining in Alaska, by C. W. Purington. 1905. $273 \mathrm{p}$.

269. Corundum and its occurrence and distribution in the United States, by J. H. Pratt. 1906.175 p.

277. Mineral resources of Kenai Peninsula, Alaska: Gold fields of the Turnagain Arm region, by F. H. Moffit; Coal fields of the Kachemak Bay region, by $R$. W. Stone. 1906.80 p.

280. The Rampart gold placer region, Alaska, by L. M. Prindle and F. L Hess. 1906. 54 p.

284. Report on investigations of mineral resources of Alaska in 1905, by A. H. Brooks and others. 1906.169 p. Contains: The mining industry in 1905, by A. H. Brooks, p. 4-9; Lode mining in southeastern Alaska, by F. E. and C. W. Wright, p. 30-54; Nonmetallic deposits of southeastern Alaska, by C. W. Wright, p. 55-60; Copper and other mineral resources of Prince William Sound, by U. S. Grant, p. 78-87; Yukon placer fields, by L. M. Prindle, p. 109-127; Gold mining on Seward Peninsula, by F. H. Moffit, p. 132-144; The York tin region, by F. L. Hess, p. 145-157.

287. The Juneau gold belt, Alaska, by A. C. Spencer, and a reconnaissance of Admiralty Island, Alaska, by C. W. Wright. 1906. $161 \mathrm{p}$.

295. The Yukon-Tanana region, Alaska: Description of Circle quadrangle, by L. M. Prindle. $1906.27 \mathrm{p}$.

314. Report on progress of investigations of mineral resources of Alaska in 1906, by A. H. Brooks and others. 1907. 234 p. Contains: The mining industry in 1906, by A. H. Brooks, p. 19-39; Lode mining in southeastern Alaska, by C. W. Wright, p. 47-72; Nonmetalliferous mineral resources of southeastern Alaska, by C. W. Wright, p. 73-81; Reconnaissance on the Pacific coast from Yakutat to Alsek River, by Eliot Blackwelder, p. 82-83; Reconnaissance in the Matanuska and Talkeetna Basins, Alaska, with notes on the placers of the adjacent regions, by Sidney Paige and Adolph Knopf, p. 104-125; The Nome region, Alaska, by F. H. Moffit, p. 126-145; Gold fields of the Solomon and Niukluk river basins, by P. S. Smith, p. 146-156; Geology and mineral resources of Iron Creek, by P. S. Smith, p. 157-163; The Kougarok region, Alaska, by A. H. Brooks, p. 164-181; The Circle precinct, Alaska, by A. H. Brooks, p. 187-204; The Bonnifield and Kantishna regions, Alaska, by L. M. Prindle, p. 205-226.

327. Geologic reconnaissance in the Matanuska and Talkeetna basins, Alaska, by Sidney Paige and Adólph Knopf. 1907. $71 \mathrm{p}$.

328. The gold placers of parts of Seward Peninsula, Alaska, including the Nome, Council, Kougarok, Port Clarence, and Goodhope precincts, by..A. J. Collier, F. L. Hess,. P. S. Smith, and A. H. Brooks. 1.908. $343 \mathrm{p}$. 
337. The Fairbanks and Rampart quadrangles; Yukon-Tanana region, Alaska, by $\mathrm{L}$. M. Prindle, with a section on the Rampart placers, by F. L. Hess, and a paper on the water supply of the Fairbanks region, by C. C. Covert. $1908.102 \mathrm{p}$.

345. Mineral resources of Alaska, report on progress of investigations in 1907, by A. H. Brooks and others. 1908. 294 p. Contains: The distribution of mineral resources in Alaska, by A. H. Brooks, p. 18-29; The mining industry in 1907, by A. H. Brooks, p. 30-53; Lode mining in southeastern Alaska, 1907, by C. W. Wright, p. 79-97; Copper deposits on Kasaan Peninsula; Prince of Wales Island, by C. W. Wright and Sidney Paige; p. 98-115; The building stones and materials of southeastern Alaska, by C. W. Wright, p. 116-126; The mineral resources of the Kotsina and. Chitina valleys, Copper River region, by F. H. Moffit and A. G. Maddren, p. 127-175; Notes on copper prospects of Prince William Sound, by F. H. Moffit, p. 176-178; Occurrence of gold in the Yukon-Tanana region, by L. M. Prindle, p. 179-186; The Fortymile gold placer district, by L. M. Prindle, p. 187-197; Investigations of the mineral deposits of Seward Peninsula, by P. S. Smith, p. 206-250; The Seward Peninsula tin deposits, by Adolph Knopf, p. 251-267; The mineral deposits of the Lost River and Brooks Mountain region, Seward Peninsula, by Adolph Knopf, p. 268-271.

347. The Ketchikan and Wrangell mining districts, Alaska, by F. E. and C. W. Wright. 1908. $210 \mathrm{p}$.

358. Geology of the Seward Peninsula tin deposits, Alaska, by Adolph Knopf. 1908. $71 \mathrm{p}$.

374. Mineral resources of the Kotsina-Chitina region, Alaska, by F. H. Moffit and A. G. Maddren. 1909. 103 p.

375. The Fortymile quadrangle, Yukon-Tanana region, Alaska, by. L. M. Prindle. $1909.52 \mathrm{p}$.

379. Mineral resources of Alaska, report on progress of investigations in 1908, by A. H. Brooks and others. 1909. 418 p. Contains: The mining industry in 1908, by A. H. Brooks, p. 21-62; Mining in southeastern Alaska, by C. W. Wright, p. 67-86; Copper mining and prospecting on Prince William Sound, by U. S. Grant and D. F. Higgins, Jr., p. 87-96; Gold on Prince William Sound, by U. S. Grant, p. 97; Notes on geology and mineral prospects in the vicinity of Seward, Kenai Peninsula, by U. S. Grant and D. F. Higgins, Jr., p. 98-107; Mineral resources of southwestern Alaska, by W: W. Atwood, p. 108-152; Mining in the Kotsina-Chitina, Chistochina, and Valdez Creek regions, by F. H. Moffit, p. 153-160; Mineral resources of the Nabesna-White River district, by F. H. Moffit and Adolph Knopf, p. 161-180; The Fairbanks gold-placer region, by L. M. Prindle and F. J. Katz, p. 181-200; Gold placers of the Ruby Creek district, by. A. G. Maddren, p. 229-233; Placers of the Gold Hill district, by A: G. Maddren, p. 234-237; Gold placers of the Innoko district, by A. G. Maddren, p. 238-266; Recent developments in southern Seward Peninsula, by P. S. Smith, p. 267-301; The Iron Creek region, by P. S. Smith, p. 302-354; Mining in the Fairhaven precinct, by F. F. Henshaw, p. 355-369.

394. Papers on the conservation of mineral resources (reprinted from report - of the National Conservation:Commission, February 1909). 1909. 214 p. Contains: Mineral resources of Alaska, by A. H. Brooks, p. 172-207. 
410. The Innoko gold-placer district, Alaska, with accounts of the central

- Kuskokwim Valley and the Ruby Creek and Gold Hill placers, by A. G. Maddren. $1910.87 \mathrm{p}$.

417. Mineral resources of the Nabesna-White River district, Alaska, by F. H. Moffit and Adolph Knopf, with a section on the Quaternary; by S. R. Capps: 1910.64 p.

433. Geology and mineral resources of the Solomon and Casadepaga quadrangles, Seward Peninsula, Alaska, by P. S. Smith. 1910. 234 p.

442. Mineral resources of Alaska, report on progress of investigations in 1909, by A. H. Brooks and others. 1910. $432 \mathrm{p}$. Contains: The mining industry in 1909 , by A. H. Brooks, p. 20-46; Mining in southeastern Alaska, by Adolph Knopf, p. 133-143; Occurrence of iron ore near Haines, by Adolph Knopf, p. 144-146; Mining in the Chitina district, by F. H. Moffit, p. 158-163; Mining and prospecting on Prince William Sound in 1909, by U. S. Grant, p. 164-165; Preliminary report on the mineral resources of the southern part of Kenai Peninsula, by U. S. Grant and D. F. Higgins, p. 166-178; Outline of the geology and mineral resources of the Iliamna and Clark lakes region, by G. C. Martin and F. J. Katz, p. 179-200; Gold placers of the Mulchatna, by F. J. Katz, p. 201-202; Sketch of the geology of the northeastern part of the Fairbanks quadrangle, by L. M. Prindle, p. 203-209; Auriferous quartz veins in the Fairbanks district, by L. M. Prindle, p. 210-229; Placer mining in the Yukon-Tanana region, by C. E. Ellsworth, p. 230-245; Occurrence of wolframite and cassiterite in the gold placers of Deadwood Creek, Birch Creek district, by B. L. Johnson, p. 246-250; The Koyukuk-Chandalar gold region, by A. G. Maddren, p. 284-315; Mineral resources of the Nulato-Council region, by P. S. Smith and H. M. Eakin, p. 316-352; Mining in Seward Peninsula, by F. F. Henshaw; p. 353-371.

443. Reconnaissance of the geology and mineral resources of Prince William Sound, Alaska, by U. S. Grant and D. F. Higgins. 1910.89 p.

446. Geology of the Berners Bay region, Alaska, by Adolph Knopf. 1911. $58 \mathrm{p}$.

448. Geology and mineral resources of the Nizina district, Alaska, by F. H. Moffit and S. R. Capps. 1911. $111 \mathrm{p}$.

449. A geologic reconnaissance in southeastern Seward Peninsula and the Norton Bay-Nulato region, Alaska, by P. S. Smith and H. M. Eakin. 1911. $146 \mathrm{p}$.

467. Geology and mineral resources of parts of the Alaska Peninsula, by W. W. Atwood. 1911. $137 \mathrm{p}$.

480. Mineral resources of Alaska, report on progress of investigations in 1910, by A. H. Brooks and others. 1911. 333.p. Contains: The mining industry in 1910, by A. H. Brooks; p. 21-42; Geologic features of Alaskan metalliferous lodes by A. H. Brooks, p. 43-93; Mining in southeastern Alaska, by Adolph Knopf, p. 94-102; The Eagle River region, by Adolph Knopf, p. 103-111; The upper Susitna and Chistochina districts, by F. H. Moffit, p. 112-127; A reconnaissance of Willow Creek gold region, by F. J. Katz, p. 139-152; Placer mining in the Yukon-Tanana region, by C. E. Ellsworth and G. L. Parker, p. 153-172; Mineral resources of the Bonnifield region, by S. R. Capps, p. 218-235; Gold placer mining developments in the Innoko-Iditarod region, by A. G. Maddren, p: 236-270; The Shungnak region,!Kobuk 
Valley, by P. S. Smith and H. M. Eakin, p. 271-305; The Squirrel River placers, by P. S. Smith, p. 306-319.

485. A geologic reconnaissance of the Iliamna region, Alaska, by G. C. Martin and F. J. Katz. 1912.138 p.

490. Mineralogical notes, series 1, by W. T. Schaller. 1911. $109 \mathrm{p}$.

498. Headwater regions of Gulkana and Susitna Rivers, Alaska, with accounts of the Valdez Creek and Chistochina placer districts, by F. H. Moffit. 1912. $82 \mathrm{p}$.

501. The Bonnifield region, Alaska, by S. R. Capps. 1912 . $64 \mathrm{p}$.

502. The Eagle River region, southeastern Alaska, by Adolph Knopf. 1912. $61 \mathrm{p}$.

504. The Sitka mining district, Alaska, by Adolph Knopf. $1912.32 \mathrm{p}$.

520. Mineral resources of Alaska, report on progress of investigations in 1911, by A. H. Brooks and others. $1912.360 \mathrm{p}$. Contains: The mining industry in 1911, by A. H. Brooks, p. 17-44; Tin resources of Alaska, by F. L. Hess, p. 89-92; The Taral and Bremner River districts, by F. H. Moffit, p. 93-104; The Chitina copper district, by F. H. Moffit, p. 105-107; Gold deposits near Valdez, by A. H. Brooks, p. 108-130; Gold deposits of the Seward-Sunrise region, Kenai Peninsula, by B. L. Johnson, p. 131-173; Gold placers of the Yentna district, by S. R. Capps, p. 174-200; Gold placers between Woodchopper and Fourth of July Creeks, upper Yukon River, by L. M. Prindle and J. B. Mertie, Jr., p. 201-210; Placer mining in the Fortymile, Eagle, and Seventymile River districts, by E. A. Porter, p. 211-218; Placer mining in the Fairbanks and Circle districts; by .C. E. Ellsworth, p. 240-245; The Rampart and Hot Springs regions, by H. M. Eakin, p. 271-286; The Ruby placer district, by A. G. Maddren, p. 287-296; The AlatnaNoatak region, by P. S. Smith, p. 315-358; Notes on mining in Seward Peninsula, by P. S. Smith, p. 339-344.

525. A geologic reconnaissance of the Fairbanks quadrangle, Alaska, by L. M. Prindle, with a detailed description of the Fairbanks district, by L. M. Prindle and F. J. Katz, and an account of lode mining near Fairbanks, by P. S. Smith. 1913. 220 p.

532. The Koyukuk-Chandalar region, Alaska, by A. G. Maddren. 1913. $119 \mathrm{p}$.

533. Geology of the Nome and Grand Central quadrangles, Alaska; by F. H. Moffit. $1913.140 \mathrm{p}$.

534. The Yentna district, Alaska, by S. R. Capps. $1913.75 \mathrm{p}$.

535. A geologic reconnaissance of a part of the Rampart quadrangle, Alaska, by H. M. Eakin. 1913. 38 p.

536. The Noatak-Kobuk region, Alaska, by P. S. Smith. 1913.160 p.

538. A geologic reconnaissance of the Circle quadrangle, Alaska, by L. M. Prindle. 1913. $82 \mathrm{p}$.

542. Mineral resources of Alaska, report on progress of investigations in 1912, by A. H. Brooks and others. 1913. 308 p. Contains: The mining industry in 1912, by A. H. Brooks, p. 18-51; Marble resources of the Ketchikan and Wrangell districts, by E. F. Burchard, p. 52-77; The McKinley Lake district, by Theodore Chapin, p. 78-80; Mining in Chitina Valley, by F. H. Moffit, p. 81-85; Mineral deposits of the Ellamar district, by S. R. Capps and B. L. Johnson, p. 86-124; Mineral deposits of Kodiak and the neighboring islands, by G. C. Martin, p. 125-136; Lode mining near Fairbanks, by. P. S. Smith, p. 137-202; Placer mining in the Yukon-Tanana region, by 
C. E. Ellsworth and R. W. Davenport, p. 203-222; Gold placers of the Ruby district; by H. M. Eakin, p. 279-292; Gold placers of the InnokoIditarod region, by H. M. Eakin, p. 293-303.

576. Geology of the Hanagita-Bremner region, Alaska, by F. H. Moffit. 1914. $56 \mathrm{p}$.

578. The Iditarod-Ruby region, Alaska, by H. M. Eakin. $1914.45 \mathrm{p}$.

587. Geology and mineral resources of Kenai Peninsula, Alaska, by G. C. Martin, B. L. Johnson, and U. S. Grant. 1915. 243 p.

592. Mineral resources of Alaska, report on progress of investigations in 1913, by A. H. Brooks and others. 1914. 413 p. Contains: The Alaskan mining industry in 1913, by A. H. Brooks, p. 45-74; Lode mining in the Ketchikan region, by P. S. Smith, p. 75-94; Marble resources of the Juneau, Skagway, and Sitka districts, by E. F. Burchard, p. 95107; A barite deposit near Wrangell, by E. F. Burchard, p. 109-117; Mineral deposits of the Yakataga district, by A. G. Maddren, p. 119153; The Port Wells gold-lode district, by B. L. Johnson, p. 195-236; Mining on Prince William Sound, by B. L. Johnson, p. 237-243; Gold lodes and placers of the Willow Creek district, by S. R. Capps, p. 245272; Mineral resources of the upper Matanuska and Nelchina valleys, by G. C. Martin and J. B. Mertie, Jr., p. 273-299; Preliminary report. on the Broad Pass region, by F. H. Moffit, p. 301-305; Mining in the Valdez Creek placer distrist, by F. H. Moffit, p. 307-308; The Chisana. placer district, by A. H. Brooks, p. 309-320; Lode mining near. Fairbanks, by Theodore Chapin, p. 321-355; Placer mining in the YukonTanana region, by Theodore Chapin, p. 357-362; Placer mining in the Ruby district, by H. M. Eakin, p. 363-369; Mineral resources of the Yukon-Koyukuk region, by H. M. Eakin, p. 371-384; Placer mining: on Seward Peninsula, by Theodore Chapin, p. 385-395; Lode developments on Seward Peninsula, by Theodore Chapin, p. 397-407.

605. The Elamar district, Alaska, by S. R. Capps and B. L. Johnson. 1915. $125 \mathrm{p}$.

607. The Willow Creek district, Alaska, by S. R. Capps. 1915.86 p.

608. The Broad Pass region, Alaska, by F. H. Moffit, with sections on Quaternary deposits, igneous rocks, and glaciation, by J. E. Pogue. 1915. $80 \mathrm{p}$.

622. Mineral resources of Alaska, report on progress of investigations in 1914, by A. H. Brooks and others. 1915. 380 p. Contains: The Alaskan mining industry in 1914 , by $\mathrm{A}$. H. Brooks, p. 15-68; Tin mining in Alaska, by H. M. Eakin, p. 81-94; Mining in the Juneau region, by H. M. Eakin, p. 95-102; Mineral deposits of the KotsinaKuskulana district, with notes on mining in Chitina Valley, by F. H. Moffit, p. 103-117; Auriferous gravels of the Nelchina-Susitna region, by Theodore Chapin, p. 118-130; Mining on Prince William Sound, by B. L. Johnson, p. 131-139; The gold and copper deposits of the Port Valdez district, by B. L. Johnson, p. 140-188; Mineral resources. of the Chisana-White River district, by S. R. Capps, p. 189-228; Mining in the Fairbanks district, by H. M. Eakin, p. 229-238; Mining in the Hot Springs district, by H. M. Eakin, p. 239-245; Mineral resources of the Lake Clark-Iditarod region, by P. S. Smith, p. 247271; Quicksilver deposits of the Kuskokwim region, by $\mathrm{P}$ S. Smith and A. G. Maddren, p. 272-291; Gold placers of the lower Kuskokwim, with a note on copper in the Russian Mountains, by A. G. Maddren, 
p. 292-360; Iron-ore deposits near Nome, by H. M: Eakin, p. 361365; Placer mining in Seward Peninsula, by H. M. Eakin, p. 366-373.

630. The Chisana-White-River district, Alaska, by-S. R. Capps. 1916.130 p. 631. The Yukon-Koyukuk region, Alaska, by H. M. Eakin. 1916: 88 p. 642. Mineral resources of Alaska, report on progress of investigations in 1915, by A. H. Brooks and others. 1916. $279 \cdot \dot{p}$. Contains: The Alaskan mining industry in 1915 , by A. H. Brooks, p. 16-71; Mining developments in southeastern Alaska, by Theodore Chapin, p. 73104; Mineral resources of the upper Chitina Valley, by F. H. - Moffit, p. 129-136; Mining on Prince William Sound, by B. L. Johnson, p. 137-145; The Turnagain-Knik region, by S. R. Capps, p. 147-194; Gold mining in the Willow Creek district, by S. R. Capps, p. 195200; Preliminary report on the Tolovana district, by A. H. Brooks, p. 201-209; Exploration in the Cosna-Nowitna region, by H. M. Eakin, p. 211-222; Mineral resources of the Ruby-Kuskokwim region, by J. B. Mertie, Jr., and G. L. Harrington, p. 223-266.

649. Antimony deposits of Alaska, by A. H. Brooks. 1916. $67 \mathrm{p}$.

655. The Lake Clark-central Kuskokwim region, Alaska, by P. S. Smith. 1917. $162 \mathrm{p}$.

662. Mineral resources of Alaska, report on progress of investigations in 1916, by A. H. Brooks and others. 1918.469 p. Contains: The Alaskan mining industry in 1916, by A. H. Brooks, p. 11-62; Mining developments in the Ketchikan and Wrangell mining districts, by Theodore Chapin, p. 63-75; Lode mining in the Juneau gold belt, by H. M. Eakin, p. 77-92; Gold-placer mining in the Porcupine district, by H. M. Eakin, p. 93-100; Mining in the lower Copper River basin, by F. H. Moffit, p. 155-182; Mining on Prince William Sound, by B. L. Johnson, p. 183-192; Copper deposits of the Latouche and Knight Island districts, Prince William Sound, by B. L. Johnson, p. 193-220; The gold placers of the Tolovana district, by J. B. Mertie; Jr., p. 221-277; Mineral resources of the Kantishna region; by S. R. Capps, p. 279-331; Gold placers of the Anvik-Andreafski region, by G. L. Harrington, p. 333-349; Lode deposits near the Nenana coal field, by R. M. Overbeck, p. 351-362; Gold placers near the Nenana coal field, by A. G. Maddren, p. 363-402; Lode mining in the Fairbanks district, by $\mathrm{J}$ : B. Mertie, Jr., p. 403-424; Lode mining and prospecting on Seward Peninsula, by J. B. Mertie, Jr., p. 425-449; Placer mining on Seward Peninsula, by J. B. Mertie, Jr., p. 451-458.

666. Our mineral supplies; H. D. McCaskey and E. F. Burchard, geologists in charge. 1919. 278 p. Contains: Alaska's mineral supplies, by A. H. Brooks, p. 89-102.

667. The Cosna-Nowitna region, Alaska, by H. M. Eakin. 1918.54 p.

668. The Nelchina-Susitna region, Alaska, by Theodore Chapin. $1918.67 \mathrm{p}$.

675. The upper Chitina Valley, Alaska, by F. H. Moffit, with a description of the igneous rocks, by R. M. Overbeck. $1918.82 \mathrm{p}$.

682. Marble resources of southeastern Alaska, by E. F. Burchard, with a section on the geography and geology, by Theodore Chapin. 1920. $118 \mathrm{p}$.

683. The Anvik-Andreafski region, Alaska (including the Marshall district), by G. L. Harrington. $1918.70 \mathrm{p}$.

687. The Kantishna region, Alaska, by S. R. Capps. 1919.118 p. 
692. Mineral resources of Alaska, report on progress of investigations in 19:17, by G. C. Martin and others. 1919. 408 p. ' Contains: The Alaskan mining industry in 1917, by G. C. Martin, p. 11-42; Mining developments in the Ketchikan district, by Theodore Chapin, p. 8589; Geology and mineral resources of the west coast of Chichagof Island, by R. M. Overbeck, p. 91-136; Platinum-bearing auriferous gravels of the Chistochina River; by Theodore Chapin, p. 137-141; Mining on Prince William Sound, by B. L. Johnson, p. 143-151; Mineral resources of Jack Bay district and vicinity, Prince William Sound, by B. L. Johnson, p. 153-173; Mining in central and northern Kenai Peninsula, by B. L. Johnson, p. 175-176; Gold lode mining in the Willow Creek district, by S. R. Capps, p. 177-186; Mineral resources of the western Talkeetna Mountains, by S. R. Capps, p. 187-205; Mineral resources of the upper Chulitna region, by S. R. Capps, p. 207-232; Platinum-bearing gold placers of the Kahiltna Valley, by J. B.. Mertie, Jr.; p. 233-264; Chromite deposits in Alaska, by J. B. Mertie, Jr., p. 265-267; Sulphur on Unalaska and Akun Islands and near Stepovak Bay, by A. G. Maddren, p. 283-298; The beach placers of the west coast of Kodiak Island, by A. G. Maddren, p. 299-319; Mining in the Fairbanks district, by Theodore Chapin, p. 321-327; A molybdenite lode on Healy River, by Theodore Chapin, p. 329; Mining in the Hot Springs district, by Theodore Chapin, p. 331-335; Tin deposits. of the Ruby district, by Theodor. Chapin, $\mathrm{p}$. 337; The gold and platinum placers of the Tolstoi district, by G. L. Harrington, p. 339-351; Tin mining in Seward Peninsula, by G. L. Harrington, p. 353-361; Graphite mining in Seward Peninsula, by G. L. Harrington, p. 363-367; The gold and platinum placers of the Kiwalik-Koyuk region, by G. L. Harrington, p. 369-400.

697. Gypsum deposits of the United States, by R. W. Stone and others. 1920. $326 \mathrm{p}$.

699. The Porcupine gold placer district, Alaska, by H. M. Eakin. 1919.29 p. 712. Mineral resources of Alaska, report on progress of investigations in 1918, by G. C. Martin and others. 1920. 204 p. Contains: The Alaskan mining industry in 1918, by G. C. Martin, p. 11-52; Nickel deposits in the lower Copper River valley, by R. M. Overbeck, p. 91-98; Preliminary report on the chromite of Kenai. Peninsula, by A. C. Gill, p. 99-129; Lode developments in the Willow Creek district, by Theodore Chapin, p. 169-176; Placer mining in the Tolovana district, by R. M. Overbeck, p. 177-184; Mining in northwestern Alaska, by S. H. Cathcart, p. 185-198.

714. Mineral resources of Alaska, report on progress of investigations in 1919, by A. H.: Brooks and others. 1921. 244 p. Contains: The future of Alaska mining, by A. H. Brooks, p. 5-57; The Alaskan mining industry in 1919, by A. H. Brooks and G. C. Martin, p. 59-95; Lode mining $\mathrm{un}$ the Juneau and Ketchikan districts, by J. B. Mertie, Jr., p. 105-128; Notes on the Salmon-Unuk River region, compiled by J. B. Mertie, Jr., p. 129-142; Mining in Chitina Valley, by F. H.. Moffit, p. 189-196; Lode developments in the Willow Creek district, by Theodore Chapin, p. 201-206; Mineral resources of the Goodnews Bay region, by G. L. Harrington, p. 207-228; Mining on Seward Peninsula; by. G. L. Harrington, p. 229-244. 
722. Mineral resources of Alaska, report on progress of investigations in 1920, by A. H. Brooks and others. 1922. 266 p. Contains: The Alaskan mining industry in 1920, by A. H. Brooks, p. 7-67; Ore deposits of the Salmon River district, Portland Canal region, by L. G. Westgate, p. 117-140; Geology of the vicinity of Tuxedni Bay, Cook Inlet, by F. H.: Moffit, p. 141-147; Gold lodes in the upper Kuskokwim region, by G. C. Martin, p. 149-161; Metalliferous lodes in southern Seward Peninsula, Alaska, by S. H. Cathcart, p. 163-261.

733. Geology of the York tin deposits, Alaska, by Edward Steidtmann and S. H. Cathcart. 1922 . $130 \mathrm{p}$.

739. Mineral resources of Alaska, report on progress of investigations in 1921, by A. H. Brooks and others. 1923, 169 p. Contains: The Alaskan mining industry in 1921, by A. H. Brooks, p. 1-44; Minerak deposits of the Wrangell district, southeastern Alaska, by A. F. Budr dington, p. 51-75; : The occurrence of metalliferous deposits in the Yukon and Kuskokwim regions, by J. B. Mertie, Jr., p. 149-165.

742. Chromite of Kenai Peninsula, Alaska, by A. C. Gill. 1922. 52 p.

745. The Kotsina-Kuskulana district, Alaska, by F. H. Moffit and J. B, Mertie, Jr. 1923.149 p.

754. The Ruby-Kuskokwim region, Alaska, by J. B. Mertie, Jr., and G. L, Harrington. $1924.129 \mathrm{p}$.

755. Mineral resources of Alaska, report on progress of investigations in 1922 , by A. H. Brooks and others. 1924. 222 p. Contains: The Alaskan. mining industry in 1922 , by A. H. Brooks and S. R. Capps, p. 3-49; The metalliferous deposits of Chitina Valley, Alaska, by F. H. Moffit, p. 57-72; Geology and mineral resources of the region traversed by the Alaska Railroad, by S. R. Capps, p. 73-150.

761. Molybdenum deposits, a short review, by F. L. Hess. 1924. $35 \mathrm{p}$.

773. Mineral resources of Alaska, report on progress of investigations in 1923, by A. H. Brooks and others. 1925. 267 p. Contains: Alaska's min'eral resources and production, 1923, by A. H. Brooks, p. 3-52; An. early Tertiary placer deposit in the Yentna district, by S. R. Capps, p. 53-61; Mineral investigations in southeastern Alaska, by A. F. Buddington, p. 71-139; The occurrence of copper on Prince William Sound, Alaska, by F. H. Moffit, p. 141-158; Mineral resources of the Kamishak Bay region, by K. F. Mather, p. 159-181; The Cold BayKatmai district, by W. R. Smith; p. 183-207; Geology and gold placers: of the Chandalar district, Alaska, by J. B. Mertie, Jr., p. 215-263.

783. Mineral resources of Alaska, report on progress of investigations in 1924, by $\mathrm{P}$. S. Smith and others. $1926.168 \mathrm{p}$. Contains: Mineral industry of Alaska in 1924, by P. S. Smith, p. 1-30; Mineral investigations in southeastern Alaska, by A. F. Buddington, p. 41-62; A ruby silver prospect in Alaska, by S. R. Capps and M. N. Short, p. 89-95; The Nixon Fork country and Silver-lead prospects near Ruby, by J. S. Brown, p. 97-150.

789. The Iniskin-Chinitna Peninsula and the Snug Harbor district, Alaska, b.y F. H. Moffit. 1927. 71 p.

791. Geology of the Upper Matanuska Valley, Alaska, by S. R. Capps, with a section on the igneous rocks, by J. B. Mertie, Jr. 1927. $92 \mathrm{p}$.

792. Mineral resources of Alaska, report on progress of investigations in 1925, by F. H. Moffit and others. 1927. 122. p. Contains: Mineral. industry of Alaska in 1925, by F. H. Moffit, p. 1-39; Geology. of the. 
Knik-Matanuska district, Alaska, by K. K. Landes, p. 51-72; The Toklat-Tonzona River region, by S. R. Capps, p. 73-110.

797. Mineral resources of Alaska, report on progress of investigations in 1926, by P.S. Smith and others. '1929. 227 p. Contains: Mineral industry of Alaska in 1926, by P. S. Smith, p. 1-50; Preliminary report on the Sheenjek River district, by J. B. Mertie, Jir., p. 99-123; Geology and mineral resources of the Aniakchak district, by R. S. Knappen, p. 161-227.

800. Geology and mineral deposits of southeastern Alaska, by A. F. Buddington and Theodore Chapin. 1929. $398 \mathrm{p}$.

807. Geology of Hyder and vicinity, southeastern Alaska, with a reconnaissance of Chickamin River, by A. F. Buddington. 1929.' 124 p.

810. Mineral resources of Alaska, report on progress of investigations in 1927, by P. S. Smith and others. 1930.:174 p. Contains: Mineral industry of Alaska in 1927, by P. S. Smith, p. 1-64.

813. Mineral resources of Alaska, report on progress of investigations in 1928, by P. S. Smith and others. 1930. 166 p.' Contains: Mineral industry of Alaska in 1928, by P.S. Smith, p. 1-72; Mining in the Fortymile district, by J. B. Mertie, Jr., p. 125-142.

815. Geology and mineral resources of northwestern Alaska, by P. S. Smith and J. B. Mertie, Jr. 1930: 351 p.

816. Geology of the Eagle-Circle district, Alaska, by J.: B. Mertie, Jr: 1930. $168 \mathrm{p}$.

824. Mineral resources of Alaska, report on progress of investigations in 1929, by P. S. Smith and others. 1932. 181 p. Contains: Mineral industry of Alaska in 1929, by P. S. Smith; p. 1-81; The Slana district, upper Copper River region, by F. H. Moffit, p. 111-124; .The Lake Clark-Mulchatna region, by S. R. Capps, p. 125-154; Mining in the Circle district, by J. B. Mertie, Jr., p. 155-172; The occurrence of gypsum at Iyoukeen Cove, Chichagof Island, by B. D. Stewart, p. 173-181.

827. A geologic reconnaissance of the Dennison Fork district, Alaska, by J. B. Mertie, Jr. $1931.44 \mathrm{p}$.

836. Mineral resources of Alaska, report on progress of investigations in 1930, by $P$. S. Smith and others. 1933. 454 p. Contains: Mineral industry of Alaska in 1930 , by P. S. Smith, p. 1-83; Notes on the geography and geology of Lituya Bay, by J. B. Mertie, Jr., p. 117-135; The eastern portion of Mount McKinley National Park, by S. R. Capps, p. 219-300; The Kantishna district, by F. H. Moffit, p. 301338; Mining development in the Tatlanika and Totatlanika Basins, by F. H. Moffit, p. 339-345.

844-A. Mineral industry of Alaska in 1931 and Administrative report, by P. S. Smith. 1933. p. 1-117.

844-C. The Suslota Pass district, upper Copper River region, Alaska, by F. H. Moffit. 1938. p. 137-162.

844-D. Mineral deposits of the Rampart and Hot Springs districts, Alaska, by J. B. Mertie, Jr.; Placer concentrates of the Rampart and Hot Springs districts, by A. E. Waters, Jr. 1934. p. 163-246.

849-B. Lode deposits of the Fairbanks district, Alaska, by J. M. Hill. 1933. p. 29-163.

849-C. The Willow Creek gold-lode district, Alaska, by J. C. Ray. 1933. p. 165-229. 
849-D. The Mount Eielson district, Alaska; by. J. C. Reed. $1933 . \quad$ p. 231-287. 849-E. Mineral deposits near the West Fork of the Chulitna River, Alaska, by C. P. Ross. 1933 . p. 289-333.

849-F. Lode deposits of Eureka and vicinity, Kantishna district, Alaska, by F. G. Wells. 1933 . p. 335-37.9.

849-G. The Girdwood district, Alaska, by C. F. Park, Jr. 1933 . p. 381-424. 849-H. The Valdez Creek mining district, Alaska, by C. P. Ross. 1933. p. 425-468.

849-I. The Moose Pass-Hope district, Kenai Peninsula, Alaska, by Ralph Tuck. 1933. p. $469-530$.

857-A. Mineral industry of Alaska in 1932, by P. S. Smith. 1934. p. 1-91.

857-C. The Curry district, Alaska, by Ralph Tuck. 1934 . p. 99-140.

857-D. Notes on the geology of the Alaska Peninsula and Aleutian Islands, by S. R. Capps. 1934... p. 141-153.

862. The southern Alaska Range, by S. R. Capps. $1935.101 \mathrm{p}$.

864-A. Mineral industry of Alaska in 1933, by P. S. Smith. 1934. p. 1-94.

864-B. The Willow Creek-Kashwitna district, Alaska, by S. R. Capps and Ralph Tuck. 1935. p. 95-113.

864-C. Mineral deposits of the Ruby-Kuskokwim region, by J. B. Mertie, Jr. 1936. p. 115-255.

866. Geology of the Tonsina district, Alaska, by F. H. Moffit. 1935.38 p. 868-A. Mineral industry of Alaska in 1934, by P. S. Smith. 1936. p. 1-91. 868-B. Kodiak and vicinity by S. R. Capps. 1937. p. 93-134.

868-C. Upper Copper and Tanana Rivers, by F. H. Moffit. 1936. p. 135-143.

868-D. The Kaiyuh Hills, by J. B. Mertie, Jr., 1937. p. 145-178.

872. The Yukon-Tanana region, Alaska, by J. B. Mertie, Jr., 1937. 276 p.

880-A. Mineral industry of Alaska in 1935, by P. S. Smith. 1937. p. 1-95.

880-B. Recent mineral developments in the Copper River region, by F. H. Moffit. 1937. p. 97-109.

880-C. Kodiak and adjacent islands, by S.' R. Capps. 1937. p. 111-184.

897-A. Mineral industry of Alaska in 1936, by P. S. Smith. 1938. p. 1-107.

897-B. The Valdez Creek mining district: in 1936, by Ralph Tuck. 1938. p. 109-131.

897-C. Gold placers of the Fortymile, Eagle, and Circle districts, Alaska, by J. B. Mertie, Jr. 1938. p. 133-261.

897-D. Nickel content of an Alaskan basic rock, by J. C. Reed. 1939. p. 263-268.

903. The Nushagak district, Alaska, by J. B. Mertie, Jr. 1938.96 p.

904. Geology of the Slana-Tok district, Alaska, by F. H. Moffit. 1938. 54 p.

910-A. Mineral industry of Alaska in 1937, by P. S. Smith. 1939. p. 1-113.

910-B. Platinum deposits of the Goodnews Bay district, Alaska, by J. B. Mertie, Jr. 1939. p. 115-145.

917-A. Mineral industry of Alaska in 1938, by P. S. Smith. 1939. p. 1-113.

917-B. Geology of the upper. Tetling River district, Alaska, by F. H. Moffit. 1941. p. 115-157.

917-D. Tertiary deposits of the Eagle-Circle district, Alaska, by J. B. Mertie, Jr. 1942. p. 213-264.

918. The Goodnews platinum deposits, Alaska, by J. B. Mertie, Jr. 1940. $97 \mathrm{p}$.

926-A. Mineral industry of Alaska in 1939, by P. S. Smith. 1941. p. 1-106.

926-B. Geology of the Gerstle River district, Alaska, with a report on the Black Rapids Glacier, by F. H. Moffit. 1942. p. 107-160. 
926-C. Occurrences of molybdenum minerals in Alaska, by P. S. Smith. 1942. p. 161-210.

926-D. Geology of the Portage Pass area, Alaska, by F. F. Barnes. 1943. p. 211-235.

929. Geology and ore deposits of the Chichagof mining district, Alaska, by J. C. Reed and R. R. Coats. 1942. 148.p.

931-F. Nickel deposits of Bohemia Basin and vicinity, Yakobi Island, Alaska, by J. C. Reed and J. V. N. Dorr 2d. 1942. p. 105-138.

931-G. Chromite deposits of Kenai Peninsula, Alaska, by P. W. Guild. 1942. p. 139-175.

933-A. Mineral industry of Alaska in 1940, by P. S. Smith. 1942. p. 1-102.

933-B. Geology of the Nutzotin Mountains, Alaska, by F. H. Moffit, with a section on the igneous rocks, by R. G. Wayland; Gold deposits near Nabesna, by R. G. Wayland. 1943. p. 103-199.

933-C. Relations of structure to mineral deposition at the Independence mine, Alaska, by W. C. Stoll. 1944. p. 201-217.

936-G. Chromite deposits of Red Bluff Bay and vicinity, Baranof Island, Alaska, by P. W. Guild and J. R. Balsley, Jr. 1942. p. 171-187.:

936-I. Nickel-copper deposits on the west coast of Chichagof Island, Alaska, by W. T. Pecora. 1942. p. 221-243.

936-M. Nickel-copper deposits at Snipe Bay, Baranof Island, Alaska, by J. C. Reed and G. O. Gates. 1942 . p. 321-330.

936-N. Antimony deposits of the Stampede Creek area, Kantishna district, Alaska, by D. E. White. 1942. p. $331-348$.

936-0. Nickel-copper deposits at Funter. Bay; Admiralty Island, Alaska, by J. C. Reed. 1942 . p. 349-361.

943-A. Mineral industry of Alaska in 1941 and 1942, by P. S. Smith. 1944. p. 1-23.

943-B. Mining in the northern Copper River region, Alaska, by F. H. Moffit. 1944. p. 25-47.

943-C. Nickel-copper prospect near Spirit Mountain, Copper River region, Alaska, by Jack Kingston and D. J. Miller. 1945. p. 49-57.

947-B. Molybdenite investigations in southeastern Alaska, by W. S. Twenhofel, G. D. Robinson, and H. R. Gault. 1946. p. 7-38.

947-C. Nickel investigations in southeastern Alaska, by G. C. Kennedy and M. S. Walton, Jr. 1946. p. 39-64.

947-D. Geology and associated mineral deposits of some ultrabasic rock bodies in southeastern Alaska, by G. C. Kennedy and M. S. Walton, Jr. 1946. p. 65-84.

947-E. Copper Bullion claims, Rua Cove, Knight Island, Alaska, by Karl Stefansson and R. M. Moxham. 1946. p. 85-92.

947-F. Copper deposits of the Nizina district, Alaska, by D. J. Miller, with an introduction by F. H. Moffit. 1946. p. 93-120.

947-G. Copper deposits of the Kotsina-Kuskulana district, Alaska, by R. E. Van Alstine and R. F. Black, with an introduction by F. H. Moffit. 1946. p. 121-141.

963-A. Some mineral investigations in southeastern Alaska, by W. S. Twenhofel, J. C. Reed, and G. O. Gates. 1949 . p. 1-45.

963-B. Copper deposits of the Prince William Sound district, Alaska, by F. H. Moffit and R. E. Fellows. 1950. p. 47-80. 
963-C. Garnet deposits near Wrangell, southeastern Alaska, by C. T. Bressler. 1950. p. 81-93.

963-E. Coal investigations in south-central Alaska, 1944-1946, by F. F. Barnes and others, with a section on clay deposits on Healy Creek, by E. H. Cobb. 1951. p. 137-213.

989-B. Gypsum deposits near Iyoukeen Cove, Chichagof Island, southeastern Alaska, by G. M. Flint, Jr., and E. H. Cobb. 1953. p. 27-37.

989-C. Gypsiferous deposits on Sheep Mountain, Alaska, by R. A. Eckhart. 1953. p. 39-61.

989-D. Geology of the eastern part of the Alaska Range and adjacent area, by F. H. Moffit. 1954. p. 65-218.

989-E. Geology of the Prince William Sound region, Alaska, by F. H. Moffit. 1954. p. 225-310.

998-A. Zinc-copper deposit at Tracy Arm, Petersburg district, Alaska, by $\boldsymbol{H}$. $R$. Gault and R. E. Fellows. 1953. p. 1-13.

998-B. Some zinc-lead deposits of the Wrangell district, Alaska, by H. R. Gault, D. L. Rossman, G. M. Flint, Jr., and R. G. Ray. 1953. p. 15-58.

998-C. Some lead-zinc and zinc-copper deposits of the Ketchikan and Wales districts, Alaska, by G. D. Robinson and W. S. Twenhofel. 1953. p. 59-84.

1004. Geology and ore deposits of the Willow Creek mining district, Alaska, by R. G. Ray. $1954.86 \mathrm{p}$.

1024-A: Radioactivity investigations in the Cache Creek area, Yentna district, Alaska, 1945, by G. D. Robinson, Helmuth Wedow, Jr., and J. B. Lyons. 1955 . p. 1-23.

1024-B. Investigations for radioactive deposits in southeastern Alaska, by W. S. West and P. D. Benson. ' 1955. p. 25-57.

1024-C: Radioactivity investigations at Ear Mountain, Seward Peninsula, Alaska, 1945, by P. L. Killeen and R. J. Ordway. 1955. p. 59-94.

1024-D. Magnetite deposits at Tuxedni Bay, Alaska, by Arthur Grantz. 1956. p. 95-106.

1024-E. Pyrite deposits at Horseshoe Bay, Latouche Island, Alaska, by F. A. Stejer. 1956. p. 107-122.

1024-F. Tungsten deposits of the Hyder district, Alaska, by F. M. Byers, Jr., and C. L. Sainsbury. 1956. p. 123-140.

1024-G. Some pegmatite deposits in southeastern Alaska, by C. L. Sainsbury. 1957. p. 141-161.

1024-H. A geochemical exploration for antimony in southeastern Alaska, by C. L. Sainsbury. 1957. p. 163-178.

1024-I. Tungsten deposits in the Fairbanks district, Alaska, by F. M. Byers, Jr. 1957. p. 179-216.

1028-A. Geology of Pavlof Volcano and vicinity, Alaska, by G. C. Kennedy and H. H. Waldron. 1956. p. 107-122.

1028-H. Geology of Little Sitkin Island, Alaska, by G. L. Snyder. 1959. p. 169-210.

1039-A. Marl deposits in the Knik Arm area, Alaska, by R. M. Moxham and R. A. Eckhart. 1956. p. 1-23.

1039-B. Occurrence of diatomaceous earth near Kenai, Alaska, by George Plafker. 1956. p. 25-31.

1039-C. Haydite raw material in the Kings River, Sutton and Lawing areas, Alaska, by R. A. Eckhart and George Plafker. 1959. p. 33-65.

1058-A. Reconnaissance for radioactive deposits in southeastern Alaska, 1952, by J. R. Houston, R. G. Bates, R. S. Velikanje, and Helmuth Wedow, Jr. 1958 . p. 1-31. 
1058-B. Geology and ore deposits, Reid Inlet area, Glacier Bay, Alaska, by D. L. Rossman. 1958. p. 33-59.

1058-F. Geology and coal resources of the Homer district, Kenai coal field, Alaska, by F. F. Barnes and E. H. Cobb. 1959. p. 217-260.

\section{WATTER-SUPPLY PAPER}

342. Surface water supply of the Yukon-Tanana region, Alaska, by C. E. Ellsworth and R. W. Davenport. 1915. 343 p.

\section{CIRCULARS}

18. Nonmetalliferous deposits in the Alaska Railroad belt, by G. A. Waring. 1947. $10 \mathrm{p}$.

184. Reconnaissance for radioactive deposits in south-central Alaska, 1947-49, by R. M. Moxham and A. E. Nelson. 1952. 14 p.

195. Radioactivity of selected rocks and placer concentrates from northeastern Alaska, by M. G. White. $1952.12 \mathrm{p}$.

196. Preliminary summary of reconnaissance for uranium in Alaska, 1951, by M. G. White, W. S. West, G. E. Tolbert, A. E. Nelson, and J. R. Houston. $1952.17 \mathrm{p}$.

207. Reconnaissance for radioactive deposits in the southern Cook Inlet region, Alaska, 1949, by R. M. Moxham and A. E. Nelson. 1952. $22 \mathrm{p}$.

214. The occurrence of zeunerite at Brooks Mountain, Seward Peninsula, Alaska, by W. S. West and M. G. White. 1952.7 p.

244. Reconnaissance for radioactive deposits in the vicinity of Teller and Cape Nome, Seward Peninsula, Alaska, 1946-47, by M. G. White, W. S. West, and J. J. Matzko. 1953. 8 p.

248. Preliminary summary of reconnaissance for uranium and thorium in Alaska, 1952, by Helmuth Wedow, Jr., and others. $1953.15 \mathrm{p}$.

250. Reconnaissance for radioactive deposits in the northeastern part of the Seward Peninsula, Alaska, 1945-47 and 1951, by H. R. Gault, P. L. Killeen, W. S. West, and others. $1953.31 \mathrm{p}$.

252. Potential Alaskan mineral resources for proposed electrochemical and electrometallurgical industries in the upper Lynn Canal area, Alaska, by W. S. Twenhofel. 1953. $14 \mathrm{p}$.

255. Reconnaissance for radioactive deposits in the lower. Yukon-Kuskokwim Highlands region, Alaska, 1947, by M. G. White and P. L. Killeen. 1953. $17 \mathrm{p}$.

265. Radioactivity investigations in the Serpentine-Kougarok area, Seward Peninsula, Alaska, 1946, by R. M. Moxham and W. S. West. 1953. $11 \mathrm{p}$.

279. Reconnaissance for radioactive deposits in the Ruby-Poorman and Nixon Fork districts, west-central Alaska, by M. G. White and J. M. Stevens. 1953. $19 \mathrm{p}$.

300. Reconnaissance for radioactive deposits in the Darby Mountains, Seward Peninsula, Alaska, 1948, by. W. S. West. 1953. 7 p.

316. Reconnaissance for radioactive deposits in the Eagle-Nation area, eastcentral Alaska, 1948, by Helmuth Wedow, Jr. 1954. 9 p.

317. Reconnaissance for radioactive deposits in the Manly Hot SpringsRampart district, east-central Alaska, 1948, by R. M. Moxham. 1954. 6 p. 
319. Reconnaissance for uranium in the Lost River area, Alaska, by M. G. White and W. S. West. 1954. 4 p.

328. Reconnaissance for radioactive deposits in the lower Yukon-Kuskokwim region, Alaska, 1952, by .W. S. West. 1954 . 10 p.

331. Reconnaissance for radioactive deposits in eastern interior Alaska, 1946, by Helmuth Wedow, Jr., P. L. Killeen, and others. 1954. $36 \mathrm{p}$.

332. The Kathleen-Margaret (K-M) copper prospect on the upper Maclaren River, Alaska, by.R. M. Chapman and R. H. Saunders. 1954.5 p.

335. Reconnaissance for radioactive deposits in east-central Alaska, 1949, by Helmuth Wedow, Jr., M. G. White, and others. 1954. 22 p.

348. Reconnaissance for radioactive deposits in eastern Alaska, 1952, by A. E. Nelson, W. S. West, and J. J. Matzko. 1954. 21 p.

\section{GEOLOGIC QUADRANGLE MAPS}

GQ 100: Geology of the Juneau (B-3) quadrangle, Alaska, by Fred Barker. 1957. Scale, $1: 63,360$.

GQ 110: Geology of the Fairbanks (D-2) quadrangle, Alaska, by T. L. Péwé. 1958. Scale, 1:63,360.

\section{MISCELLANEOUS GEOLOGIC INVESTIGATIONS MAPS}

I-230: Photogeologic map of the Salt Chuck area, Prince of Wales Island, Alaska, showing linear features as seen on aerial photographs, by C. L. Pillmore and Kathleen McQueen. 1956. Scale, 1:12,000.

I-231. Map of the Hollis area, Prince of Wales Island, Alaska, showing linear features as seen on aerial photographs, part 1, by C. L. Pillmore and Kathleen McQueen. 1956. Scale, 1:12,000.

I-232. Map of the Hollis area; Prince of Wales Island, Alaska, showing linear features as seen on aerial photographs, part 2, by C. L. Pillmore and Kathleen McQueen. 1956. Scale, 1:12,000.

I-273. Map of a part of the Prince William Sound area, Alaska, showing linear geologic features as shown on aerial photographs, by W. H. Condon and J. T. Cass. 1958. Scale, 1:125,000.

I-286. Reconnaissance geologic map of the Norton Bay quadrangle, Alaska, by J. T. Cass. 1959. Scale, 1:250,000.

I-287. Reconnaissance geologic map of the Candle quadrangle, Alaska, by J. T. Cass. 1959. Scale, 1:250,000.

I-289. Reconnaissance geologic map of the Ruby quadrangle, Alaska, by J. T. Cass. 1959. Scale, 1:250,000.

1-291.' Reconnaissance geologic map of the Nulato quadrangle, Alaska, by J. T. Cass. 1959. Scale, $1: 250,000$.

I-303. Progress map of the geology of the Juneau quadrangle, Alaska, by E. H. Lathram, R. A. Loney, W. H. Condon, and H. C. Berg. 1959. Scale, I: 250,000 .

\section{U.S. BUREAU OF MINES}

\section{BULLETINS}

142. The mining industry in the Territory of Alaska during the calendar year 1915 , by S. S. Smith. 1917. 66 p.

153. The mining industry in the Territory of Alaska during the calendar year 1916, by S. S. Smith. 1917. 89 p.

405. Copper mining in North America, by E. D. Gardner, C. H. Johnson, and B. S. Butler. 1938. 300 p. 


\section{INFORMATION CIRCULARS}

6186. Mining methods and costs, Alaska-Juneau Gold Mining Co., Juneau, Alaska, by P. R. Bradley. 1929.18 p.

6236. Milling practice at the Alaska Juneau concentrator, by. P. R. Bradley. 1930. $16 \mathrm{p}$.

7313. Paligorskite, a possible asbestos substitute, by R. B. Fisher, R. L. Thorne, and Corbin Van Cott. 1945. 5 p.

7379. Alaska's minerals as a basis for industry, by H. F. Bain. 1946. 89 p.

7784. Molybdenum. A materials survey, by Wilmer McInnis, with a chapter on geology and resources by S. C. Creasey. 1957. $77 \mathrm{p}$.

7844. Southeastern Alaska's mineral industry, by Alvin Kaufman. 1958. $37 \mathrm{p}$.

7871. Lode-tin mining at Lost River, Seward Peninsula, Alaska, by S. H. Lorain, R. R. Wells, Miro Mihelich, J. J. Mulligan, R. L. Thorne, and J. A. Herdlick. $1959.76 \mathrm{p}$.

7878. Tin-placer sampling methods and results, Cape Mountain district, Seward Peninsula, Alaska, by J. J. Mulligan and R. L. Thorne. . 1959. $69 \mathrm{p}$.

\section{REPORTS OF INVESTIGATIONS}

2158. Recovery of gold from a magnetic black sand, by J. A. Davis and John Gross. $1920.5 \mathrm{p}$.

2192. Comparison of methods of gold recovery from black sand, by John Gross. 1920. $4 \mathrm{p}$.

2731. Analysis of copper-palladium-gold-silver concentrates, by C. W. Davis. 1926. $5 \mathrm{p}$.

3885. Exploration of Red Mountain chromite deposits, Kenai Peninsula, Alaska, by. F. A. Rutledge. 1946.26 p.

3902. Investigation of the Lost River tin deposit, Seward Peninsula, Alaska, by H. E. Heide. 1946.57 p.

3913. Exploration of Spirit Mountain. nickel prospect, Canyon Creek, lower Copper River Region, Alaska, by H. C. Pierce. 1946.8 p...

3940. Exploration of argentiferous lead-copper deposits of the Slana District, Alaska, by. R. L. Thorne. $1946.9 \mathrm{p}$.

3942. Exploration of the copper-sulfur deposit; Khayyam and Stumble-On properties, Prince of Wales Island, Alaska, by E. L. Fosse. 1946. $8 \mathrm{p}$.

3944. Exploration of Mountain V.iew tungsten deposit, Hyder, Alaska, by 7. A. W. Erickson. 1946. 10 p.

3950. Exploration of a nickel-copper-cobalt deposit at Funter. Bay, Admiralty Island, Alaska, by S. P. Holt and J. M. Moss. 1946 . 15. p.

3952. Exploration of the Jumbo Basin iron deposit, Prince of Wales Island, southeastern Alaska, by W. S. Wright and E. L. Fosse. 1946. 9 p.

3956. Exploration of Poor Man iron deposit; Kasaan Peninsula, Prince of W.ales Island, southeastern Alaska, by S. P. Holt and R. S. Sanford. 1946. $8 \mathrm{p}$.

3967. Exploration of Sedanka zinc deposit, Sedanka Island, Alaska, by B. S. Webber, J. M. Moss, and F. A. Rutledge. 1946.15 p.

3978. Exploration of Cape Mountain lode-tin deposits, Seward Peninsula, Alaska, by H. E. Heide, W. S. Wright, and R. S. Sanford. · 1946.16 p.

4065. Mercury deposits of southwestern Alaska, by B. S. Webber, S. C. Bjorklund, F. A. Rutledge, B. I. Thomas, and W. S. Wright. 1947. $57 \mathrm{p}$. 
4110. Ward copper deposit, Seward Peninsula, Alaska, by W. S. Wright. 1947. $4 \mathrm{p}$.

4121. Investigation of the Mount Eielson zinc-lead deposits, Mount McKinley National Park, Alaska, by N. M. Muir, B. I. Thomas, and R. S. Sanford. 1947. $13 \mathrm{p}$.

4129. Mount Andrew iron deposit, Kasaan Peninsula, Prince of Wales Island, southeastern Alaska, by W. S. Wright and A. W. Tolonen. 1947. $27 \mathrm{p}$.

4168. Mirror Harbor nickel deposits, Chichagof Island, Alaska, by W. M. Traver, Jr. $1948.13 \mathrm{p}$.

4173. Antimony deposits in Alaska, by Norman Ebbley, Jr., and W. S. Wright, 1948. $41 \mathrm{p}$.

4174. Tungsten deposits in Alaska, by R. L. Thorne, N. M. Muir, A. W. Erickson, B. I. Thomas, H. E. Heide, and W. S. Wright. 1948.22 p.

4182. Yakobi Island nickel deposit, Sitka Mining District, Alaska, by J. H. East, Jr., W. S. Traver, Jr., R. S. Sanford, and W. S. Wright. 1948. $28 \mathrm{p}$.

4322. Investigation of the Morelock Creek tin placer deposits, Fort Gibbon district, Alaska, by B. I. Thomas and W. S. Wright. 1948. 8 p.

4323. Investigation of the Tozimoran Creek tin placer deposits, Fort Gibbon District, Alaska, by B. I. Thomas and W. S. Wright. $1948.11 \mathrm{p}$.

4345. Churn drilling at Cape Mountain tin placer deposits, Seward Peninsula, Alaska, by H. E. Heide and R. S. Sanford. 1948.14 p.

4346. Sampling methods and results at the Sullivan Creek tin placer deposits, Manley Hot Springs, Tofty, Alaska, by R. L. Thorne and. W. S. Wright. 1948. 8 p.

4349. Diamond drilling at Rush and Brown copper mine, Kasaan Bay, Prince of Wales Island, southeastern Alaska, by S. P. Holt, J. G. Shepard, R. L. Thorne, A. W. Tolonen, and E. L. Fosse. 1948. 7 p.

4356. Investigation of Knik Valley chromite deposits, Palmer, Alaska, by Stuart Bjorklund and W. S. Wright. $1948.5 \mathrm{p}$.

4358. Investigation of the Salt Chuck copper mine, Kasaan Peninsula, Prince of Wales Island, southeastern Alaska, by S. P. Holt, J. G. Shepard, R. L. Thorne, A. W. Tolonen, and E. L. Fosse. 1948.16 p.

4361. Investigation of the Rainy Creek mercury prospect. Bethel District, Kuskokwim Region, southwestern Alaska, by F. A. Rutledge. 1948. $7 \mathrm{p}$.

4373. Investigation of Tolstoi Mountain iron deposits, Kasaan Peninsula, Prince of Wales Island, southeastern Alaska, by A. W. Erickson. 1948. $5 \mathrm{p}$.

4414. Investigations of the Kobuk River asbestos deposits, Kobuk District, northwestern Alaska, by H. E. Heide, W. S. Wright, and F. A. Rutledge. 1949. $25 \mathrm{p}$.

4418. Investigation of Potato Mountain tin placer deposits, Seward Peninsula, northwestern Alaska, by H. E. Heide and F. A. Rutledge. 1949. 21 p.

4419. Investigation of Claim Point chromite deposits, Kenai Peninsula, Alaska, by R. S. Sanford and J. W. Cole. 1949. $11 \mathrm{p}$.

4421. Investigation of Muir Inlet or Nunatak molybdenum deposits, Glacier Bay, southeastern Alaska, by R. S. Sanford, G. A. Apell, and F. A. Rutledge. $1949.6 \mathrm{p}$.

4669. Investigation of Taylor Creek lead-zinc deposit, Kupreanof Island, Petersburg, Alaska, by W. H. Kerns. 1950. 13 p. 
4719. Investigation of mercury deposits, Cinnabar Creek area, Georgetown and Akiak Districts, Kuskokwim Region, southwestern Alaska, by F. A. Rutledge. $1950.9 \mathrm{p}$.

4828. Investigation of Kasna Creek copper prospect, Lake Kontrashibuna, Lake Clark Region, Alaska, by R. S. Warfield and F. A. Rutledge. 1951. $10 \mathrm{p}$.

4852. Diamond drilling the Gypsum-Camel prospect, Iyoukeen Cove, Chichagof Island, southeastern Alaska, by G. D. Jermain and F. A. Rutledge. $1952.6 \mathrm{p}$.

4890. Investigation of the Millett copper deposit, Iliamna Lake, southwestern Alaska, by F. A. Rutledge and J. J. Mulligan. 1952. 22 p.

4932. Preliminary report: Nonmetallic deposits accessible to The Alaska Railroad as possible sources of raw materials for the construction industry, by F. A. Rutledge, R. L. Thorne, W. H. Kerns, and J. J. Mulligan. 1953. $129 \mathrm{p}$.

4984. Concentration of Klukwan, Alaska, magnetite ore, by R. R. Wells and R. L. Thorne. $1953.15 \mathrm{p}$.

4986. Investigation of the Copper Bullion claims, Rua Cove, Knight Island, Alaska, by F. A. Rutledge. 1953. $6 \mathrm{p}$.

5195. Studies of the Snettisham magnetite deposit, southeastern Alaska, by R. L. Thorne and R. R. Wells. 1956. $41 \mathrm{p}$.

5243. Selective extraction of mercury and antimony from cinnabar-stibnite ore, by E. G. Erspamer and R. R. Wells. 1956. 15 p.

5245. Laboratory concentration of various Alaska copper ores, by $R$. $R$. Wells. 1956. $9 \mathrm{p}$.

5312. Beneficiation of iron-copper ores from Kasaan Peninsula, Prince of Wales Island, Alaska, by R. R. Wells, E. G. Erspamer, and F. T. Sterling. 1957. $15 \mathrm{p}$.

5320. Copper mines and prospects adjacent to Landlocked Bay, Prince William Sound, Alaska, by Miro Mihelich and R. R. Wells. 1957. 21 p.

5373. Tin-bearing placer deposits near Tofty, Hot Springs district, centrol Alaska, by B. I. Thomas. 1957. $56 \mathrm{p}$.

5377. Laboratory concentration of chromite ores, Red Mountain District, Kenai Peninsula, Alaska, by R. R. Wells, F. T. Sterling, E. G. Erspamer, and W. A. Stickney. 1957. $22 \mathrm{p}$.

5433. Recovering mercury from cinnabar-stibnite ore by flotation and fluidizedbed roasting, by R. R. Wells, M. M. Johnson, and F. T. Sterling. 1958. $19 \mathrm{p}$.

549.3. Tin placer and lode investigations Ear Mountain area, Seward Peninsula, Alaska, by J. J. Mulligan. 1959. 53 p.

\section{TERRITORY OF ALASKA DEPARTMENT OF MINES PAMPHIETS}

1. Strategic mineral occurrences in interior Alaska, by H. R. Joesting. 1942. $46 \mathrm{p}$.

2. Supplement to Pamphlet No. 1-Strategic mineral occurrences in interior Alaska, by H. R. Joesting. 1943. $28 \mathrm{p}$.

3-R. Asbestos and jade occurrences in the Kobuk River region, Alaska, by Eskil Anderson. $1945.26 \mathrm{p}$.

5-R. Mineral occurrences other than gold deposits in northwestern Alaska, by Eskil Anderson. 1944. $48 \mathrm{p}$.

6. Some high calcium limestone deposits in southeastern Alaska, by J. C. Roehm. 1946. 85 p. 